Prepared for the U.S. Department of Energy under Contract DE-AC05-76RL01830

\title{
Biological Sampling and Analysis in Sinclair and Dyes Inlets, Washington: Chemical Analyses for 2007 Puget Sound Biota Study
}

JM Brandenberger CR Suslick

RK Johnson

October 2008 


\title{
DISCLAIMER
}

This report was prepared as an account of work sponsored by an agency of the United States Government. Neither the United States Government nor any agency thereof, nor Battelle Memorial Institute, nor any of their employees, makes any warranty, express or implied, or assumes any legal liability or responsibility for the accuracy, completeness, or usefulness of any information, apparatus, product, or process disclosed, or represents that its use would not infringe privately owned rights. Reference herein to any specific commercial product, process, or service by trade name, trademark, manufacturer, or otherwise does not necessarily constitute or imply its endorsement, recommendation, or favoring by the United States Government or any agency thereof, or Battelle Memorial Institute. The views and opinions of authors expressed herein do not necessarily state or reflect those of the United States Government or any agency thereof.

\author{
PACIFIC NORTHWEST NATIONAL LABORATORY \\ operated by \\ BATTELLE \\ for the \\ UNITED STATES DEPARTMENT OF ENERGY \\ under Contract DE-AC05-76RL01830
}

Printed in the United States of America
Available to DOE and DOE contractors from the Office of Scientific and Technical Information,
P.O. Box 62, Oak Ridge, TN 37831-0062;
ph: (865) 576-8401
fax: $(865) 576-5728$
email: reports@adonis.osti.gov

\footnotetext{
Available to the public from the National Technical Information Service, U.S. Department of Commerce, 5285 Port Royal Rd., Springfield, VA 22161 ph: (800) 553-6847 fax: $(703) 605-6900$ email: orders@ntis.fedworld.gov online ordering: http://www.ntis.gov/ordering.htm
}

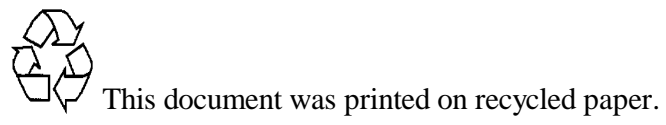


Biological Sampling and Analysis in Sinclair and Dyes Inlets, Washington: Chemical Analyses for 2007 Puget Sound Biota Study

J. M. Brandenberger

C.R. Suslick

Battelle Marine Sciences Laboratory

Sequim, Washington

R. K. Johnston

Space and Naval Warfare Systems Center

Bremerton, Washington

September 2008

Prepared for

Puget Sound Naval Shipyard and

Intermediate Maintenance Facility

Project ENVVEST

Bremerton, Washington

Under Contract No. DE-AC06-76RLO 1830

Pacific Northwest National Laboratory

Richland, Washington 99352 


\begin{abstract}
Evaluating spatial and temporal trends in contaminant residues in Puget Sound fish and macroinvertebrates are the objectives of the Puget Sound Ambient Monitoring Program (PSAMP). In a cooperative effort between the ENVironmental inVESTment group (ENVVEST) and Washington State Department of Fish and Wildlife, additional biota samples were collected during the 2007 PSAMP biota survey and analyzed for chemical residues and stable isotopes of carbon $\left(\delta^{13} \mathrm{C}\right)$ and nitrogen $\left(\delta^{15} \mathrm{~N}\right)$. Approximately three specimens of each species collected from Sinclair Inlet, Georgia Basin, and reference locations in Puget Sound were selected for whole body chemical analysis. The muscle tissue of specimens selected for chemical analyses were also analyzed for $\delta^{13} \mathrm{C}$ and $\delta^{15} \mathrm{~N}$ to provide information on relative trophic level and food sources. This data report summarizes the chemical residues for the 2007 PSAMP fish and macro-invertebrate samples. In addition, six Spiny Dogfish (Squalus acanthias) samples were necropsied to evaluate chemical residue of various parts of the fish (digestive tract, liver, embryo, muscle tissue), as well as, a weight proportional whole body composite (WBWC). Whole organisms were homogenized and analyzed for silver, arsenic, cadmium, chromium, copper, nickel, lead, zinc, mercury, 19 polychlorinated biphenyl (PCB) congeners, PCB homologues, percent moisture, percent lipids, $\delta^{13} \mathrm{C}$, and $\delta^{15} \mathrm{~N}$.
\end{abstract}




\section{$\underline{\text { Table of Contents }}$}

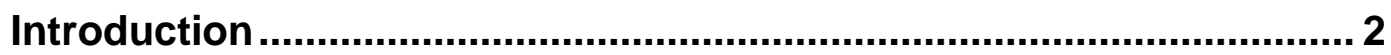

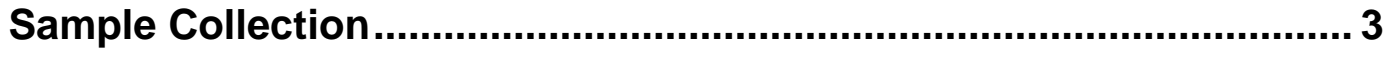

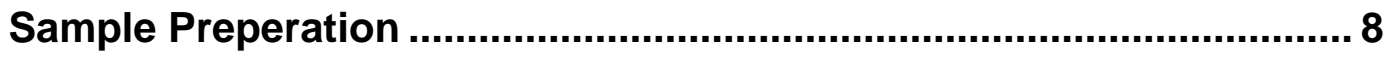

Field Data Summary ........................................................................... 11

Metals Results, Wet Weight Basis .................................................. 12

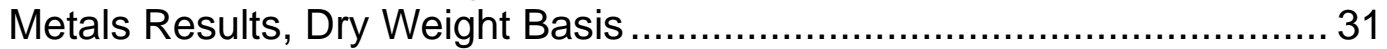

PCB Results, Wet Weight Basis ........................................................ 51

Stable Isotopes, Dry Weight Basis ................................................. 75

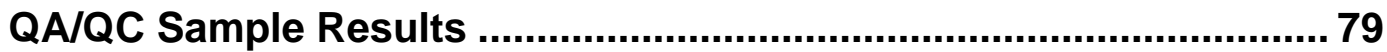

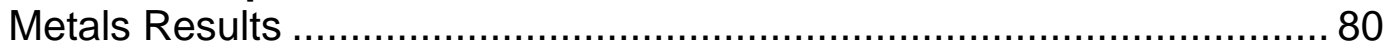

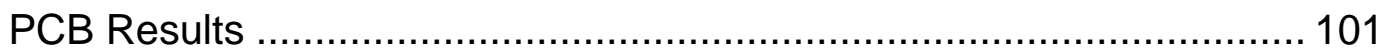

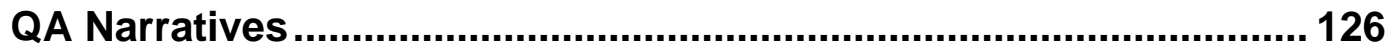

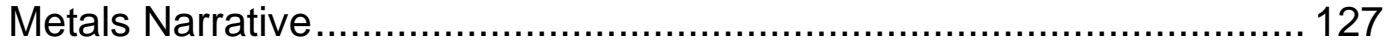

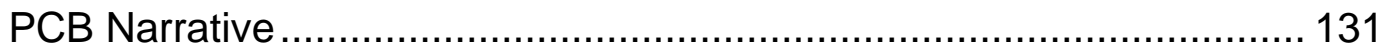

Stable Isotopes Narrative .......................................................... 150

Sample Custody Records .......................................................... 155

Field Collection Worksheets ........................................................ 156

Sample Selection for Analysis ....................................................... 162

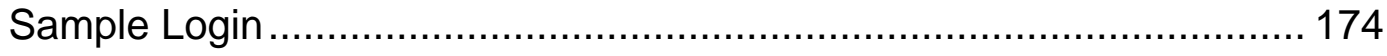

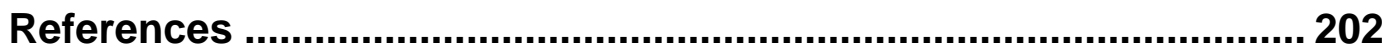




\section{INTRODUCTION}

Sinclair Inlet and Dyes Inlet were listed on the State of Washington's 1998 Section 303(d) list of impaired waters because of fecal coliform contamination in marine waters and tributary streams, heavy metals and toxic organics in the bottom sediments, and polycyclic aromatic hydrocarbons (PAHs), polychlorinated biphenyl (PCBs), aldrin, dieldrin, mercury $(\mathrm{Hg})$, and arsenic (As) in the tissues of marine organisms. A cooperative watershed agreement for the inlets was established among the Puget Sound Naval Shipyard (PSNS) and Intermediate Maintenance Facility (IMF); the Environmental Protection Agency (EPA); the Washington State Department of Ecology (Ecology); and other technical stakeholders. The ENVironmental inVESTment group (ENVVEST) was formed to assist regulatory agencies in developing total maximum daily loads (TMDLs) and to assess ecological risk within the watershed. Ultimately, the effectiveness of pollution abatement, cleanup, and restoration programs will be reflected by the health and status of marine organisms living within the inlets. Furthermore, information about bioaccumulation and ecological stress of organisms resident in the inlets will support decisions about setting priorities for implementing TMDLs and determining what contaminants should be on the 303(d) List.

Assessment of the deposition and transport of contaminants within Sinclair and Dyes Inlets, and the impact of these contaminants to the local marine biota require the cooperation of the various stakeholders that are conducting environmental monitoring programs in the region. The Puget Sound Ambient Monitoring Program (PSAMP) is a multi-agency effort to monitor the health of Puget Sound. The spatial and temporal trends in contaminant exposure in Puget Sound fish and macroinvertebrates, and the effects of contaminant exposure on the health of these resources, are assessed by trawl sampling at various stations throughout Puget Sound (WDFW 2003). In order to increase the data yield from these efforts, ENVVEST collaborated with Washington State Department of Fish and Wildlife (WDFW) to collect additional biological samples for contaminant bioaccumulation analysis and stable isotopes of carbon $\left(\delta^{13} \mathrm{C}\right)$ and nitrogen $\left(\delta^{15} \mathrm{~N}\right)$. This data report summarizes the analytical chemistry conducted on the fish and 
macroinvertebrate samples. Whole organisms were homogenized and analyzed for silver $(\mathrm{Ag})$, As, cadmium $(\mathrm{Cd})$, chromium $(\mathrm{Cr})$, copper $(\mathrm{Cu})$, nickel $(\mathrm{Ni})$, lead $(\mathrm{Pb})$, zinc $(\mathrm{Zn})$, $\mathrm{Hg}, 19$ PCB congeners, PCB homologues, percent moisture, percent lipids, $\delta^{13} \mathrm{C}$, and $\delta^{15} \mathrm{~N}$.

For additional project information see the following documents:

- Biological Sampling and Analysis in Sinclair and Dyes Inlets, WA 2005 Quality Assurance Project Plan (Johnston et al. 2005).

- Monitoring DNA Damage in Caged Mussels Deployed in Sinclair and Dyes

Inlets, Puget Sound, WA, USA (Steinert et al. 2006).

- Using Caged Mussels to Characterize Exposure and Effects Over Small Spatial Scales in Sinclair/Dyes Inlet, WA (Salazar et al. 2006).

- 2003 Sinclair and Dyes Inlet TMDL Study: Biological Sampling and Analysis for Metals and PCBs (Brandenberger et al. 2003)

- Biological Sampling and Analysis in Sinclair and Dyes Inlets, Washington:

Chemical Analyses for 2005 Puget Sound Biota Study (Brandenberger et al. 2005)

- Contaminate Residues in Demersal Fish, Invetebrates, and Deployed Mussels in Selected Areas of The Puget Sound, WA (Johnston et al. 2007).

\section{Sample Collection}

The demersal fish and macroinvertebrate samples were collected from the 2007 PSAMP otter trawls. Approximately three specimens of each species collected from Sinclair Inlet, Georgia Basin, and reference locations in Puget Sound were selected for whole body chemical analyses. Individual specimens were analyzed with the exception of a few samples (noted as COMP) where the mass was limited and composites of more than specimen were required. The muscle tissue of specimens selected for chemical analyses were also analyzed for $\delta^{13} \mathrm{C}$ and $\delta^{15} \mathrm{~N}$ to provide information on relative trophic level and food sources. Table 1 summarizes the organisms collected, PSAMP station name, weight, length, and laboratory codes. Fish species collected by PSAMP were English sole 
(Parophrys vetulus), Pacific staghorn sculpin (Leptocottus armatus), spotted ratfish (Hydrolagus collieii), sand sole (Psettichthys melanostictus), rock sole (Lepidopsetta bilineata), and Shiner Surfperch (Cymatogaster aggregate). Invertebrate species collected were sea cucumber (Parasticopus californicus) and graceful crab (Cancer gracilis). In addition, University of Washington provided six specimens of Spiny Dogfish (Squalus acanthias). Necropsies were performed on the dogfish and samples were collected from various parts of the fish (digestive tract, liver, embryo, muscle tissue), as well as, a weight proportional whole body composite (WBWC).

Table 1. The organisms sampled in 2007 biota study with weights, lengths, and PSAMP station information.

\begin{tabular}{|c|c|c|c|c|c|}
\hline Organism & Station & $\begin{array}{c}\text { Weight } \\
\text { (Kg) }\end{array}$ & $\begin{array}{c}\text { Length } \\
\text { or } \\
\text { carapice } \\
\text { width } \\
\text { (cm) } \\
\end{array}$ & $\begin{array}{c}\text { PSAMP } \\
\text { Code }\end{array}$ & $\begin{array}{l}\text { MSL } \\
\text { Code }\end{array}$ \\
\hline English sole adult 1 & Vendovi & 0.083 & 19.1 & V-ES-1 & $2838-1$ \\
\hline English sole adult 2 & Vendovi & 0.092 & 20.3 & V-ES-2 & $2838-2$ \\
\hline English sole adult3 & Vendovi & 0.115 & 23.5 & V-ES-3 & $2838-3$ \\
\hline English sole adult4 & Vendovi & 0.196 & 27.3 & V-ES-4 & $2838-4$ \\
\hline English sole adult5 & Vendovi & 0.224 & 27.9 & V-ES-5 & $2838-5$ \\
\hline English sole adult6 & Vendovi & 0.250 & 27.9 & V-ES-6 & $2838-6$ \\
\hline English sole adult7 & Vendovi & 0.096 & 21.0 & V-ES-7 & $2838-7$ \\
\hline English sole adult8 & Vendovi & 0.197 & 27.3 & V-ES-8 & $2838-8$ \\
\hline English sole adult9 & Vendovi & 0.420 & 33.0 & V-ES-9 & $2838-9$ \\
\hline Rock sole1 & Vendovi & 0.119 & 20.3 & V-RS-1 & $2838-10$ \\
\hline Rock sole 2 & Vendovi & 0.142 & 21.0 & V-RS-2 & 2838-11 \\
\hline Rock sole3 & Vendovi & 0.510 & 31.1 & V-RS-3 & $2838-12$ \\
\hline Staghorn sculpin1 & Vendovi & 0.094 & 17.8 & V-SSc-1 & $2838-13$ \\
\hline Staghorn sculpin2 & Vendovi & 0.298 & 24.8 & V-SSc-2 & $2838-14$ \\
\hline Staghorn sculpin3 & Vendovi & 0.294 & 25.4 & V-SSc-3 & $2838-15$ \\
\hline Shiner perch1 & Vendovi & 0.016 & 7.6 & V-SP-1 & $2838-16$ \\
\hline Shiner perch2 & Vendovi & 0.015 & 8.9 & V-SP-2 & $2838-17$ \\
\hline Shiner perch3 & Vendovi & 0.016 & 8.9 & V-SP-3 & $2838-18$ \\
\hline Sea cucumber (3)COMP & Vendovi & $\begin{array}{l}0.55 \\
1.20\end{array}$ & NA & $\begin{array}{l}\text { V-SC- } \\
\text { COMP }\end{array}$ & 2838-19 \\
\hline English sole adult1 & Strait of Georgia & 0.270 & 30.5 & SG-ES-1 & $2838-20$ \\
\hline English sole adult2 & Strait of Georgia & 0.138 & 23.5 & SG-ES-2 & 2838-21 \\
\hline
\end{tabular}




\begin{tabular}{|c|c|c|c|c|c|}
\hline Organism & Station & $\begin{array}{l}\text { Weight } \\
\text { (Kg) }\end{array}$ & $\begin{array}{c}\text { Length } \\
\text { or } \\
\text { carapice } \\
\text { width } \\
\text { (cm) } \\
\end{array}$ & $\begin{array}{c}\text { PSAMP } \\
\text { Code }\end{array}$ & $\begin{array}{l}\text { MSL } \\
\text { Code }\end{array}$ \\
\hline English sole adult3 & Strait of Georgia & 0.184 & 26.7 & SG-ES-3 & $2838-22$ \\
\hline English sole adult4 & Strait of Georgia & 0.189 & 26.0 & SG-ES-4 & $2838-23$ \\
\hline English sole adult5 & Strait of Georgia & 0.192 & 26.7 & SG-ES-5 & $2838-24$ \\
\hline English sole adult6 & Strait of Georgia & 0.510 & 37.5 & SG-ES-6 & $2838-25$ \\
\hline English sole adult7 & Strait of Georgia & 0.290 & 29.8 & SG-ES-7 & $2838-26$ \\
\hline English sole adult 8 & Strait of Georgia & 0.254 & 26.0 & SG-ES-8 & $2838-27$ \\
\hline English sole adult 9 & Strait of Georgia & 0.360 & 33.0 & SG-ES-9 & $2838-28$ \\
\hline Spotted Ratfish1 & Strait of Georgia & 0.115 & 27.9 & SG-RF-1 & $2838-29$ \\
\hline Spotted Ratfish2 & Strait of Georgia & 0.320 & 35.6 & SG-RF-2 & $2838-30$ \\
\hline Spotted Ratfish3 & Strait of Georgia & 0.770 & 48.3 & SG-RF-3 & 2838-31 \\
\hline Sea cucumber (6)COMP & Strait of Georgia & 0.290 & NA & $\begin{array}{l}\text { SG-SC- } \\
\text { COMP }\end{array}$ & $2838-32$ \\
\hline English sole adult1 & Hood Canal & 0.131 & 22.9 & HC-ES-1 & $2838-33$ \\
\hline English sole adult2 & Hood Canal & 0.133 & 22.9 & HC-ES-2 & 2838-34 \\
\hline English sole adult3 & Hood Canal & 0.220 & 26.7 & HC-ES-3 & $2838-35$ \\
\hline Rock sole1 & Hood Canal & 0.460 & 31.8 & HC-RS-1 & $2838-36$ \\
\hline Spotted Ratfish1 & Hood Canal & 0.480 & 39.4 & HC-RF-1 & $2838-37$ \\
\hline Spotted Ratfish2 & Hood Canal & 0.340 & 38.1 & $\mathrm{HC}-\mathrm{RF}-2$ & 2838-38 \\
\hline Spotted Ratfish3 & Hood Canal & 0.450 & 42.5 & HC-RF-3 & 2838-39 \\
\hline Shiner perch1 & Hood Canal & 0.016 & 9.5 & HC-SP1 & $2838-40$ \\
\hline Shiner perch2 & Hood Canal & 0.020 & 10.2 & HC-SP2 & $2838-41$ \\
\hline Shiner perch3 & Hood Canal & 0.010 & 8.3 & HC-SP3 & $2838-42$ \\
\hline Graceful Crab1 & Hood Canal & 0.237 & 10.2 & HC-GC-1 & $2838-43$ \\
\hline Graceful Crab2 & Hood Canal & 0.064 & 6.4 & HC-GC-2 & $2838-44$ \\
\hline English sole adult1 & Elliott Bay & 0.030 & 14.0 & EB-ES-1 & $2838-45$ \\
\hline English sole adult 2 & Elliott Bay & 0.058 & 17.8 & EB-ES-2 & $2838-46$ \\
\hline English sole adult3 & Elliott Bay & 0.157 & 26.0 & EB-ES-3 & $2838-47$ \\
\hline Spotted Ratfish1 & Elliott Bay & 0.350 & 40.6 & EB-RF-1 & $2838-48$ \\
\hline Spotted Ratfish2 & Elliott Bay & 0.370 & 41.9 & EB-RF-2 & $2838-49$ \\
\hline Spotted Ratfish3 & Elliott Bay & 0.810 & 53.3 & EB-RF-3 & $2838-50$ \\
\hline English sole adult1 & Eagle Harbor & 0.055 & 19.1 & EH-ES-1 & $2838-51$ \\
\hline English sole adult2 & Eagle Harbor & 0.420 & 35.6 & EH-ES-2 & $2838-52$ \\
\hline English sole adult 3 & Eagle Harbor & 0.089 & 22.2 & EH-ES-3 & $2838-53$ \\
\hline
\end{tabular}




\begin{tabular}{|c|c|c|c|c|c|}
\hline Organism & Station & $\begin{array}{c}\text { Weight } \\
\text { (Kg) }\end{array}$ & $\begin{array}{c}\text { Length } \\
\text { or } \\
\text { carapice } \\
\text { width } \\
\text { (cm) } \\
\end{array}$ & $\begin{array}{c}\text { PSAMP } \\
\text { Code }\end{array}$ & $\begin{array}{l}\text { MSL } \\
\text { Code }\end{array}$ \\
\hline English sole adult4 & Eagle Harbor & 0.199 & 27.3 & EH-ES-4 & $2838-54$ \\
\hline Spotted Ratfish1 & Eagle Harbor & 0.230 & 33.0 & EH-RF-1 & $2838-55$ \\
\hline Spotted Ratfish2 & Eagle Harbor & 0.380 & 38.1 & EH-RF-2 & $2838-56$ \\
\hline Spotted Ratfish3 & Eagle Harbor & 0.760 & 50.8 & EH-RF-3 & $2838-57$ \\
\hline English sole adult1 & Sinclair Inlet & 0.330 & 29.2 & SI-ES-1 & $2838-58$ \\
\hline English sole adult2 & Sinclair Inlet & 0.330 & 29.2 & SI-ES-2 & $2838-59$ \\
\hline English sole adult 3 & Sinclair Inlet & 0.530 & 35.6 & SI-ES-3 & $2838-60$ \\
\hline English sole adult4 & Sinclair Inlet & 0.192 & 25.4 & SI-ES-4 & $2838-61$ \\
\hline English sole adult5 & Sinclair Inlet & 0.069 & 18.4 & SI-ES-5 & $2838-62$ \\
\hline English sole adult6 & Sinclair Inlet & 0.178 & 25.4 & SI-ES-6 & $2838-63$ \\
\hline English sole adult7 & Sinclair Inlet & 0.179 & 25.4 & SI-ES-7 & $2838-64$ \\
\hline English sole adult8 & Sinclair Inlet & 0.261 & 29.2 & SI-ES-8 & $2838-65$ \\
\hline English sole adult 9 & Sinclair Inlet & 0.460 & 33.0 & SI-ES-9 & $2838-66$ \\
\hline Rock sole1 & Sinclair Inlet & 0.024 & 10.2 & SI-RS-1 & $2838-67$ \\
\hline Rock sole2 & Sinclair Inlet & 0.021 & 10.2 & SI-RS-2 & $2838-68$ \\
\hline Rock sole3 & Sinclair Inlet & 0.320 & 27.3 & SI-RS-3 & $2838-69$ \\
\hline Rock sole4 & Sinclair Inlet & 0.220 & 26.7 & SI-RS-4 & $2838-70$ \\
\hline Sand sole1 & Sinclair Inlet & 0.270 & 25.4 & SI-SS-1 & $2838-71$ \\
\hline Sand sole 2 & Sinclair Inlet & 0.290 & 27.3 & SI-SS-2 & $2838-72$ \\
\hline Sand sole 3 & Sinclair Inlet & 0.320 & 29.8 & SI-SS-3 & $2838-73$ \\
\hline Sand sole4 & Sinclair Inlet & 0.180 & 25.4 & SI-SS-4 & $2838-74$ \\
\hline Sand sole 5 & Sinclair Inlet & 0.190 & 24.8 & SI-SS-5 & $2838-75$ \\
\hline Sand sole6 & Sinclair Inlet & 0.190 & 24.1 & SI-SS-6 & $2838-76$ \\
\hline Staghorn sculpin1 & Sinclair Inlet & 0.045 & 14.6 & SI-SSc-1 & $2838-77$ \\
\hline Staghorn sculpin2 & Sinclair Inlet & 0.051 & 15.2 & SI-SSc-2 & $2838-78$ \\
\hline Staghorn sculpin3 & Sinclair Inlet & 0.047 & 14.0 & SI-SSc-3 & $2838-79$ \\
\hline Staghorn sculpin5 & Sinclair Inlet & 0.059 & 14.6 & SI-SSc-5 & $2838-81$ \\
\hline Staghorn sculpin 4 & Sinclair Inlet & 0.112 & 17.8 & SI-SSc-4 & $2838-80$ \\
\hline Staghorn sculpin6 & Sinclair Inlet & 0.037 & 14.6 & SI-SSc-6 & $2838-82$ \\
\hline Spotted Ratfish1 & Sinclair Inlet & 0.550 & 41.3 & SI-RF-1 & $2838-83$ \\
\hline Spotted Ratfish2 & Sinclair Inlet & 0.380 & 36.8 & SI-RF-2 & $2838-84$ \\
\hline Shiner perch1 & Sinclair Inlet & NA & NA & SI-SP-1 & $2838-85$ \\
\hline Shiner perch4 & Sinclair Inlet & 0.013 & 8.3 & SI-SP-4 & $2838-88$ \\
\hline
\end{tabular}




\begin{tabular}{|c|c|c|c|c|c|}
\hline Organism & Station & $\begin{array}{c}\text { Weight } \\
\text { (Kg) }\end{array}$ & $\begin{array}{l}\text { Length } \\
\text { or } \\
\text { carapice } \\
\text { width } \\
\text { (cm) } \\
\end{array}$ & $\begin{array}{c}\text { PSAMP } \\
\text { Code } \\
\end{array}$ & $\begin{array}{l}\text { MSL } \\
\text { Code }\end{array}$ \\
\hline Shiner perch5 & Sinclair Inlet & 0.012 & 7.0 & SI-SP-5 & $2838-89$ \\
\hline Shiner perch6 & Sinclair Inlet & 0.017 & 7.6 & SI-SP-6 & $2838-90$ \\
\hline Shiner perch2 & Sinclair Inlet & 0.006 & 5.7 & SI-SP-2 & $2838-86$ \\
\hline Shiner perch7 & Sinclair Inlet & 0.015 & 8.3 & SI-SP-7 & $2838-91$ \\
\hline Shiner perch3 & Sinclair Inlet & 0.007 & 6.4 & SI-SP-3 & $2838-87$ \\
\hline Graceful Crab2 & Sinclair Inlet & 0.104 & 7.6 & SI-GC-2 & $2838-93$ \\
\hline Graceful Crab6 & Sinclair Inlet & 0.103 & 7.6 & SI-GC-6 & $2838-97$ \\
\hline Graceful Crab3 & Sinclair Inlet & 0.062 & 5.7 & SI-GC-3 & 2838-94 \\
\hline Graceful Crab4 & Sinclair Inlet & 0.071 & 8.3 & SI-GC-4 & $2838-95$ \\
\hline Graceful Crab7 & Sinclair Inlet & 0.076 & 7.0 & SI-GC-7 & $2838-98$ \\
\hline Graceful Crab1 & Sinclair Inlet & 0.045 & 6.4 & SI-GC-1 & $2838-92$ \\
\hline Graceful Crab5 & Sinclair Inlet & 0.100 & 7.0 & SI-GC-5 & $2838-96$ \\
\hline Graceful Crab8 & Sinclair Inlet & 0.084 & 8.3 & SI-GC-8 & 2838-99 \\
\hline Sea cucumber (6)COMP & Sinclair Inlet & 4.10 & NA & $\begin{array}{l}\text { SI-SC- } \\
\text { COMP } \\
\end{array}$ & $2838-100$ \\
\hline English sole adult1 & Port Gardner & 0.158 & 24.8 & PG-ES-1 & $2838-101$ \\
\hline English sole adult 2 & Port Gardner & 0.101 & 21.6 & PG-ES-2 & $2838-102$ \\
\hline English sole adult3 & Port Gardner & 0.128 & 24.8 & PG-ES-3 & $2838-103$ \\
\hline English sole adult1 & Nisqually & 0.166 & 25.4 & NIS-ES-1 & 2838-104 \\
\hline English sole adult 2 & Nisqually & 0.214 & 26.7 & NIS-ES-2 & $2838-105$ \\
\hline English sole adult3 & Nisqually & 0.290 & 30.5 & NIS-ES-3 & $2838-106$ \\
\hline Spotted Ratfish1 & Nisqually & 0.620 & 48.3 & NIS-RF-1 & $2838-107$ \\
\hline Spotted Ratfish2 & Nisqually & 0.430 & 40.6 & NIS-RF-2 & $2838-108$ \\
\hline Spotted Ratfish3 & Nisqually & 0.450 & 38.1 & NIS-RF-3 & 2838-109 \\
\hline English sole adult1 & $\begin{array}{l}\text { Commencement } \\
\text { Bay }\end{array}$ & 0.145 & 22.9 & CB-ES-1 & $2838-110$ \\
\hline English sole adult2 & $\begin{array}{l}\text { Commencement } \\
\text { Bay }\end{array}$ & 0.157 & 24.8 & CB-ES-2 & 2838-111 \\
\hline English sole adult 3 & $\begin{array}{l}\text { Commencement } \\
\text { Bay }\end{array}$ & 0.162 & 24.8 & CB-ES-3 & $2838-112$ \\
\hline Spotted Ratfish1 & $\begin{array}{l}\text { Commencement } \\
\text { Bay }\end{array}$ & 0.320 & 40.6 & CB-RF-1 & $2838-113$ \\
\hline Spotted Ratfish2 & $\begin{array}{l}\text { Commencement } \\
\text { Bay }\end{array}$ & 0.350 & 38.7 & CB-RF-2 & $2838-114$ \\
\hline Spotted Ratfish3 & $\begin{array}{l}\text { Commencement } \\
\text { Bay }\end{array}$ & 0.390 & 38.7 & CB-RF-3 & $2838-115$ \\
\hline English sole adult1 & Duwamish & 0.075 & 17.8 & DU-ES-1 & $2838-116$ \\
\hline English sole adult2 & Duwamish & 0.096 & 21.6 & DU-ES-2 & $2838-117$ \\
\hline
\end{tabular}




\begin{tabular}{|c|c|c|c|c|c|}
\hline Organism & Station & $\begin{array}{l}\text { Weight } \\
\text { (Kg) }\end{array}$ & $\begin{array}{l}\text { Length } \\
\text { or } \\
\text { carapice } \\
\text { width } \\
\text { (cm) } \\
\end{array}$ & $\begin{array}{l}\text { PSAMP } \\
\text { Code } \\
\end{array}$ & $\begin{array}{l}\text { MSL } \\
\text { Code }\end{array}$ \\
\hline English sole adult3 & Duwamish & 0.156 & 26.0 & DU-ES-3 & $2838-118$ \\
\hline English sole adult4 & Duwamish & 0.264 & 30.5 & DU-ES-4 & 2838-119 \\
\hline \multicolumn{6}{|l|}{ Dogfish Study: } \\
\hline embryo mass (g) 1 & ADUW-DOG01 & 0.410 & & DF-EM-1 & $2838-126$ \\
\hline embryo mass $(\mathrm{g}) 2$ & ADUW-DOG02 & 0.440 & & DF-EM-2 & $2838-127$ \\
\hline embryo mass (g)4 & ADUW-DOG04 & 0.188 & & DF-EM-4 & $2838-129$ \\
\hline embryo mass (g)6 & ADUW-DOG06 & 0.322 & & DF-EM-6 & 2838-131 \\
\hline liver $(\mathrm{g}) 1$ & ADUW-DOG01 & 0.498 & & DF-LV-1 & $2838-132$ \\
\hline liver $(\mathrm{g}) 2$ & ADUW-DOG02 & 0.260 & & DF-LV-2 & $2838-133$ \\
\hline liver $(\mathrm{g}) 4$ & ADUW-DOG04 & 0.790 & & DF-LV-4 & $2838-135$ \\
\hline liver $(\mathrm{g}) 6$ & ADUW-DOG06 & 0.434 & & DF-LV-6 & $2838-137$ \\
\hline digestive tract - All 1 & ADUW-DOG01 & 0.319 & & DF-DIG-1 & $2838-138$ \\
\hline digestive tract - All 2 & ADUW-DOG02 & 0.194 & & DF-DIG-2 & $2838-139$ \\
\hline digestive tract - All 4 & ADUW-DOG04 & 0.320 & & DF-DIG-4 & 2838-141 \\
\hline digestive tract - All 6 & ADUW-DOG06 & 0.327 & & DF-DIG-6 & $2838-143$ \\
\hline Section Weighted-Comp1 & ADUW-DOG01 & & & DF-SWC-1 & $2838-174$ \\
\hline Section Weighted-Comp2 & ADUW-DOG02 & & & DF-SWC-2 & $2838-175$ \\
\hline Section Weighted-Comp3 & ADUW-DOG06 & & & DF-SWC-6 & $2838-176$ \\
\hline Section Weighted-Comp4 & ADUW-DOG04 & & & DF-SWC-4 & 2838-183 \\
\hline $\begin{array}{l}\text { Whole Body Weighted- } \\
\text { Comp1 }\end{array}$ & ADUW-DOG01 & 5.40 & & $\begin{array}{c}\text { DF-WBWC- } \\
1\end{array}$ & $2838-177$ \\
\hline $\begin{array}{l}\text { Whole Body Weighted- } \\
\text { Comp2 }\end{array}$ & ADUW-DOG02 & 4.29 & & $\begin{array}{c}\text { DF-WBWC- } \\
2\end{array}$ & $2838-178$ \\
\hline $\begin{array}{l}\text { Whole Body Weighted- } \\
\text { Comp3 }\end{array}$ & ADUW-DOG03 & 3.14 & & $\begin{array}{c}\text { DF-WBWC- } \\
3\end{array}$ & $2838-179$ \\
\hline $\begin{array}{l}\text { Whole Body Weighted- } \\
\text { Comp4 }\end{array}$ & ADUW-DOG04 & 5.42 & & $\begin{array}{c}\text { DF-WBWC- } \\
4\end{array}$ & $2838-180$ \\
\hline $\begin{array}{l}\text { Whole Body Weighted- } \\
\text { Comp5 }\end{array}$ & ADUW-DOG05 & 3.02 & & $\begin{array}{c}\text { DF-WBWC- } \\
5\end{array}$ & $2838-181$ \\
\hline $\begin{array}{l}\text { Whole Body Weighted- } \\
\text { Comp6 }\end{array}$ & ADUW-DOG06 & 4.09 & & $\begin{array}{c}\text { DF-WBWC- } \\
6 \\
\end{array}$ & $2838-182$ \\
\hline Sequim Bay & eelgrass shoots & & & & $2838-184$ \\
\hline
\end{tabular}

\section{Sample Preparation}

At least three specimens for each species and sampling location were selected for chemical analyses (if available). Specimens were selected with similar weights and 
lengths to approximate relative age. In general, individual fish specimen were homogenized to create a whole body composite (WBC) for each specimen. Due to sample size limitations, a few specimens were combined into a single sample. These samples are noted on the table as COMP sample type or composite sample type. The fish were rinsed with deionized water (DI) to remove external debris and patted dry with paper towel. They were sectioned using ceramic tools and a Teflon cutting board into portions that fit into a titanium-bladed tissue homogenizer to prevent metals contamination. The samples were homogenized to a uniform color. All homogenization equipment was decontaminated between each sample using a laboratory detergent, hot water rinse, methanol rinse (if necessary due to oily samples), and 3x deionized water rinse. Gloves and work surface papers were changed between samples. Homogenized material was subsampled into three separate containers:

1. $\sim 30 \mathrm{~g}$ into a precleaned $4 \mathrm{oz}$. glass jar for PCBs

2. $\sim 20 \mathrm{~g}$ in a tarred, precleaned $4 \mathrm{oz}$. polyprolyene jar for metals

3. $\sim 5 \mathrm{~g}$ in a tarred $2 \mathrm{oz}$. polyprolyene jar for isotopes

The sea cucumbers were stored in a single zip-top bag and frozen. Therefore, individual specimens were not achievable and the entire bag was used to generate a composite sample for each location. The number of individual specimens in each composite sample is noted in Table 1. Whole individual crabs were thawed, rinsed with DI water, and homogenized to a uniform color. The crabs collected from Sinclair Inlet were too small for individual analyses; therefore, two crabs were composited for this location (see Table 1).

Samples were collected from the various body parts of each of the six dogfish specimens. Due to the sizes of the dogfish, a whole body weighted composite was prepared instead of homogenizing the whole organism. The weight of each body part sampled is listed in Table 2 along with the percentage of the total body weight. 
Table 2. Dogfish samples collected from Admiralty Inlet on September 19, 2007. The percentage of each body part that was homogenized to generate the whole body weighted composite for each specimen.

\begin{tabular}{|c|c|c|c|c|c|c|c|c|c|c|c|c|}
\hline \multirow[b]{2}{*}{ Body Parts } & \multicolumn{12}{|c|}{ WEIGHTS OF INDIVIDUAL BODY PARTS (g) AND PERCENTAGE OF WHOLE BODY COMPOSITE } \\
\hline & $\begin{array}{l}\text { ADUW- } \\
\text { DOG01 }\end{array}$ & $\begin{array}{l}\% \text { of } \\
\text { Total }\end{array}$ & $\begin{array}{l}\text { ADUW- } \\
\text { DOG02 }\end{array}$ & $\begin{array}{l}\% \text { of } \\
\text { Total }\end{array}$ & $\begin{array}{l}\text { ADUW- } \\
\text { DOG03 }\end{array}$ & $\begin{array}{c}\% \text { of } \\
\text { Total }\end{array}$ & $\begin{array}{l}\text { ADUW- } \\
\text { DOG04 }\end{array}$ & $\begin{array}{l}\% \text { of } \\
\text { Total }\end{array}$ & $\begin{array}{l}\text { ADUW- } \\
\text { DOG05 }\end{array}$ & $\begin{array}{l}\% \text { of } \\
\text { Total }\end{array}$ & $\begin{array}{l}\text { ADUW- } \\
\text { DOG06 }\end{array}$ & $\begin{array}{l}\% \text { of } \\
\text { Total }\end{array}$ \\
\hline Embryo & 410 & $7.6 \%$ & 440 & $10 \%$ & 97 & $3.1 \%$ & 188 & $3.5 \%$ & 151.6 & $5.0 \%$ & 321.7 & $7.9 \%$ \\
\hline Liver & 498 & $9.2 \%$ & 260 & $6.1 \%$ & 318 & $10 \%$ & 790 & $15 \%$ & 382.9 & $13 \%$ & 433.5 & $11 \%$ \\
\hline Digestive tract & 319 & $5.9 \%$ & 194 & $4.5 \%$ & 288.8 & $9.2 \%$ & 320 & $5.9 \%$ & 190.6 & $6.3 \%$ & 326.7 & $8.0 \%$ \\
\hline Organ? & 47.2 & $0.9 \%$ & 48.8 & $1.1 \%$ & 37.7 & $1.2 \%$ & 65.2 & $1.2 \%$ & 49.1 & $1.6 \%$ & 46.7 & $1.1 \%$ \\
\hline Kidney & 14.4 & $0.3 \%$ & 10.5 & $0.2 \%$ & 8.2 & $0.3 \%$ & 18.5 & $0.3 \%$ & 12.7 & $0.4 \%$ & & $0.0 \%$ \\
\hline $\begin{array}{l}\text { Head Section } \\
\text { Pectral }\end{array}$ & 613.3 & $11 \%$ & 730 & $17 \%$ & 490 & $16 \%$ & 790 & $15 \%$ & 510 & $17 \%$ & 510 & $12 \%$ \\
\hline Section & 1027 & $19 \%$ & 720 & $17 \%$ & 510 & $16 \%$ & 610 & $11 \%$ & 420 & $14 \%$ & 620 & $15 \%$ \\
\hline Mid-section & 1590 & $29 \%$ & 1240 & $29 \%$ & 940 & $30 \%$ & 1800 & $33 \%$ & 900 & $30 \%$ & 1370 & $33 \%$ \\
\hline Tail Section & 670 & $12 \%$ & 450 & $10 \%$ & 320 & $10 \%$ & 600 & $11 \%$ & 320 & $11 \%$ & 450 & $11 \%$ \\
\hline
\end{tabular}


Field Data Summary 


\section{Field Data Summary: Metals in Biota Tissue}

Wet Weight Basis 


\begin{tabular}{|c|c|c|c|c|c|c|c|c|c|}
\hline Client Code & Client Code & & & \multicolumn{6}{|c|}{ Units $=\mu \mathrm{g} / \mathrm{g}$ WET weight } \\
\hline & PSAMP Code & Organism & $\begin{array}{l}\text { Sample } \\
\text { Type }\end{array}$ & MSL Code & $\begin{array}{l}\text { Length } \\
\text { (cm) }\end{array}$ & $\begin{array}{l}\text { Weight } \\
\text { (g) }\end{array}$ & $\begin{array}{l}\text { Collection } \\
\text { Date }\end{array}$ & $\begin{array}{c}\text { Percent } \\
\text { Dry } \\
\text { Weight }\end{array}$ & Ag \\
\hline Station & & & & & & & & Instrument: & ICP-MS \\
\hline \multicolumn{7}{|c|}{$\begin{array}{l}\text { Laboratory Achieved Method Detection Limits (converted to wet weight) } \\
\text { Reporting Limit, Wet Weight }\end{array}$} & Average & 27.01 & 0.0006 \\
\hline Vendovi & V-ES-5 & English Sole & WBC & $2838-5$ & 27.9 & 224 & 05/01/07 & 18.59 & 0.00569 \\
\hline Vendovi & V-ES-6 & English Sole & WBC & $2838-6$ & 27.9 & 250 & 05/01/07 & 17.79 & 0.00302 \\
\hline Vendovi & V-ES-8 & English Sole & WBC & $2838-8$ & 27.3 & 197 & 05/01/07 & 22.64 & 0.00611 \\
\hline Vendovi & V-RS-1 & Rock sole & WBC & $2838-10$ & 20.3 & 119 & $05 / 01 / 07$ & 21.79 & 0.00445 \\
\hline Vendovi & V-RS-2 & Rock sole & WBC & $2838-11$ & 21.0 & 142 & $05 / 01 / 07$ & 21.23 & 0.00306 \\
\hline Vendovi & V-RS-3 & Rock sole & WBC & $2838-12$ & 31.1 & 510 & 05/01/07 & 20.30 & 0.00487 \\
\hline Vendovi & V-SSc-1 & Staghorn sculpin & WBC & $2838-13$ & 17.8 & 94 & $05 / 01 / 07$ & 19.54 & 0.0244 \\
\hline Vendovi & V-SSc-2 & Staghorn sculpin & WBC & $2838-14$ & 24.8 & 298 & 05/01/07 & 20.54 & 0.00778 \\
\hline Vendovi & V-SSc-3 & Staghorn sculpin & WBC & $2838-15$ & 25.4 & 294 & 05/01/07 & 19.90 & 0.00503 \\
\hline Vendovi & V-SP-1 & Shinner Surfperch & WBC & $2838-16$ & 7.62 & 16 & 05/01/07 & 23.28 & 0.00484 \\
\hline Vendovi & V-SP-2 & Shinner Surfperch & WBC & $2838-17$ & 8.89 & 15 & 05/01/07 & 22.19 & $0.00175 \mathrm{~J}$ \\
\hline Vendovi & V-SP-3 & Shinner Surfperch & WBC & $2838-18$ & 8.89 & 16 & 05/01/07 & 24.13 & 0.00524 \\
\hline Vendovi & V-SC-COMP & Sea cucumber (3) & COMP & 2838-19 & & 5501 & 05/01/07 & 13.20 & 0.00961 \\
\hline Strait of Georgia & SG-ES-3 & English Sole & WBC & $2838-22$ & 26.7 & 184 & $05 / 02 / 07$ & 20.54 & $0.00137 \mathrm{~J}$ \\
\hline Strait of Georgia & SG-ES-4 & English Sole & WBC & $2838-23$ & 26.0 & 189 & $05 / 02 / 07$ & 20.95 & 0.00237 \\
\hline Strait of Georgia & SG-ES-5 & English Sole & WBC & $2838-24$ & 26.7 & 192 & $05 / 02 / 07$ & 21.39 & 0.00447 \\
\hline Strait of Georgia & SG-RF-1 & Ratfish & WBC & $2838-29$ & 27.9 & 115 & $05 / 02 / 07$ & 19.86 & 0.0427 \\
\hline Strait of Georgia & SG-RF-2 & Ratfish & WBC & $2838-30$ & 35.6 & 320 & $05 / 02 / 07$ & 27.99 & 0.100 \\
\hline Strait of Georgia & SG-RF-3 & Ratfish & WBC & $2838-31$ & 48.3 & 770 & $05 / 02 / 07$ & 33.64 & 0.128 \\
\hline Strait of Georgia & SG-SC-COMP & Sea cucumber (6) & COMP & $2838-32$ & & 290 & $05 / 02 / 07$ & 7.70 & 0.00590 \\
\hline Hood Canal & HC-ES-1 & English Sole & WBC & $2838-33$ & 22.9 & 131 & 05/03/07 & 17.91 & $0.00190 \mathrm{~J}$ \\
\hline Hood Canal & HC-ES-2 & English Sole & WBC & $2838-34$ & 22.9 & 133 & 05/03/07 & 20.28 & $0.0006 \mathrm{U}$ \\
\hline Hood Canal & HC-ES-3 & English Sole & WBC & $2838-35$ & 26.7 & 220 & 05/03/07 & 21.19 & 0.00415 \\
\hline Hood Canal & HC-RS-1 & Rock sole & WBC & $2838-36$ & 31.8 & 460 & $05 / 03 / 07$ & 22.07 & 0.00267 \\
\hline Hood Canal & HC-RF-1 & Ratfish & WBC & $2838-37$ & 39.4 & 480 & 05/03/07 & 25.63 & 0.326 \\
\hline Hood Canal & HC-RF-2 & Ratfish & WBC & $2838-38$ & 38.1 & 340 & $05 / 03 / 07$ & 27.04 & 0.270 \\
\hline Hood Canal & HC-RF-3 & Ratfish & WBC & 2838-39 & 42.5 & 450 & 05/03/07 & 27.61 & 1.62 \\
\hline Hood Canal & HC-SP1 & Shinner Surfperch & WBC & $2838-40$ & 9.53 & 16 & 05/03/07 & 27.84 & 0.0162 \\
\hline Hood Canal & HC-SP2 & Shinner Surfperch & WBC & $2838-41$ & 10.2 & 20 & $05 / 03 / 07$ & 34.05 & 0.0197 \\
\hline Hood Canal & HC-GC-1 & Graceful Crab & WBC & $2838-43$ & 10.2 & 237 & 05/03/07 & 19.14 & 0.212 \\
\hline Elliott Bay & EB-ES-1/2 & English Sole (2) & COMP & $2838-45-46$ & 15.8 & 44 & $05 / 16 / 07$ & 21.44 & 0.0170 \\
\hline Elliott Bay & EB-ES-3 & English Sole & WBC & $2838-47$ & 26.0 & 157 & $05 / 16 / 07$ & 19.72 & 0.0107 \\
\hline Elliott Bay & EB-RF-1 & Ratfish & WBC & $2838-48$ & 40.6 & 350 & $05 / 16 / 07$ & 26.43 & 2.08 \\
\hline Elliott Bay & EB-RF-2 & Ratfish & WBC & $2838-49$ & 41.9 & 370 & $05 / 16 / 07$ & 27.23 & 0.178 \\
\hline Elliott Bay & EB-RF-3 & Ratfish & WBC & $2838-50$ & 53.3 & 810 & $05 / 16 / 07$ & 27.11 & 0.835 \\
\hline Eagle Harbor & EH-ES-2 & English Sole & WBC & $2838-52$ & 35.6 & 420 & 05/19/07 & 17.35 & 0.00606 \\
\hline Eagle Harbor & EH-ES-3 & English Sole & WBC & $2838-53$ & 22.2 & 89 & $05 / 19 / 07$ & 23.38 & 0.00491 \\
\hline Eagle Harbor & EH-ES-4 & English Sole & WBC & $2838-54$ & 27.3 & 199 & 05/19/07 & 21.69 & 0.00315 \\
\hline Eagle Harbor & EH-RF-1 & Ratfish & WBC & $2838-55$ & 33.0 & 230 & 05/19/07 & 26.50 & 0.248 \\
\hline Eagle Harbor & EH-RF-2 & Ratfish & WBC & $2838-56$ & 38.1 & 380 & $05 / 19 / 07$ & 23.39 & 0.386 \\
\hline Eagle Harbor & EH-RF-3 & Ratfish & WBC & $2838-57$ & 50.8 & 760 & $05 / 19 / 07$ & 31.68 & 0.243 \\
\hline Sinclair Inlet & SI-ES-1 & English Sole & WBC & $2838-58$ & 29.2 & 330 & $05 / 17 / 07$ & 22.19 & 0.00581 \\
\hline Sinclair Inlet & SI-ES-2 & English Sole & WBC & $2838-59$ & 29.2 & 330 & 05/17/07 & 19.35 & 0.00526 \\
\hline Sinclair Inlet & SI-ES-4 & English Sole & WBC & $2838-61$ & 25.4 & 192 & 05/17/07 & 40.66 & 0.0147 \\
\hline Sinclair Inlet & SI-ES-6 & English Sole & WBC & $2838-63$ & 25.4 & 178 & 05/17/07 & 20.31 & 0.00230 \\
\hline Sinclair Inlet & SI-ES-7 & English Sole & WBC & $2838-64$ & 25.4 & 179 & 05/17/07 & 20.51 & 0.00224 \\
\hline Sinclair Inlet & SI-ES-8 & English Sole & WBC & $2838-65$ & 29.2 & 261 & $05 / 17 / 07$ & 20.71 & 0.00565 \\
\hline Sinclair Inlet & SI-RS-1/2 & Rock sole (2) & COMP & 2838-67-68 & 10.2 & 23 & $05 / 17 / 07$ & 24.28 & 0.00750 \\
\hline Sinclair Inlet & SI-RS-3 & Rock sole & WBC & $2838-69$ & 27.3 & 320 & $05 / 17 / 07$ & 21.12 & 0.00589 \\
\hline Sinclair Inlet & SI-RS-4 & Rock sole & WBC & $2838-70$ & 26.7 & 220 & $05 / 17 / 07$ & 21.17 & $0.0006 \mathrm{U}$ \\
\hline Sinclair Inlet & SI-SS-1 & Sand sole & WBC & $2838-71$ & 25.4 & 270 & 05/17/07 & 21.66 & 0.00942 \\
\hline Sinclair Inlet & SI-SS-2 & Sand sole & WBC & $2838-72$ & 27.3 & 290 & $05 / 17 / 07$ & 22.13 & $0.0006 \mathrm{U}$ \\
\hline Sinclair Inlet & SI-SS-3 & Sand sole & WBC & $2838-73$ & 29.8 & 320 & 05/17/07 & 21.05 & 0.00722 \\
\hline
\end{tabular}




\begin{tabular}{|c|c|c|c|c|c|c|c|c|c|}
\hline Client Code & Client Code & & & \multicolumn{6}{|c|}{ Units $=\mu \mathrm{g} / \mathrm{g}$ WET weight } \\
\hline Station & PSAMP Code & Organism & $\begin{array}{l}\text { Sample } \\
\text { Type }\end{array}$ & MSL Code & $\begin{array}{l}\text { Length } \\
\text { (cm) }\end{array}$ & $\begin{array}{l}\text { Weight } \\
\text { (g) }\end{array}$ & $\begin{array}{l}\text { Collection } \\
\text { Date }\end{array}$ & $\begin{array}{c}\text { Percent } \\
\text { Dry } \\
\text { Weight }\end{array}$ & Ag \\
\hline & & & & & & & & Instrument: & ICP-MS \\
\hline $\begin{array}{l}\text { Laboratory Achieve } \\
\text { Reporting Limit, W }\end{array}$ & $\begin{array}{l}\text { Method Detect } \\
\text { t Weight }\end{array}$ & tion Limits (conver & ed to wet & weight) & & & Average & 27.01 & $\begin{array}{r}0.0006 \\
0.002\end{array}$ \\
\hline Sinclair Inlet & SI-SSc-1/2 & Staghorn sculpin (2) & COMP & $2838-77-78$ & 14.9 & 48 & 05/17/07 & 20.66 & 0.0283 \\
\hline Sinclair Inlet & SI-SSc-3/5 & Staghorn sculpin (2) & COMP & $2838-79-81$ & 14.3 & 53 & 05/17/07 & 20.03 & 0.0130 \\
\hline Sinclair Inlet & SI-SSc-4/6 & Staghorn sculpin (2) & COMP & $2838-80-82$ & 16.2 & 75 & $05 / 17 / 07$ & 19.51 & 0.0277 \\
\hline Sinclair Inlet & SI-RF-1 & Ratfish & WBC & $2838-83$ & 41.3 & 550 & $05 / 17 / 07$ & 32.67 & 0.735 \\
\hline Sinclair Inlet & SI-RF-2 & Ratfish & WBC & $2838-84$ & 36.8 & 380 & $05 / 17 / 07$ & 26.61 & 0.258 \\
\hline Sinclair Inlet & SI-SP-4/5 & Shinner Surfperch $(2$ & ) COMP & $2838-88-89$ & 7.62 & 13 & 05/17/07 & 24.47 & 0.00881 \\
\hline Sinclair Inlet & SI-SP-6/2 & Shinner Surfperch (2 & COMP & $2838-90-86$ & 6.67 & 11 & 05/17/07 & 24.68 & 0.0108 \\
\hline Sinclair Inlet & SI-SP-7/3 & Shinner Surfperch (2 & ) COMP & $2838-91-87$ & 7.3 & 11 & $05 / 17 / 07$ & 25.12 & 0.0191 \\
\hline Sinclair Inlet & SI-GC-2/6 & Graceful Crab (2) & COMP & 2838-93-97 & 7.62 & 103 & $05 / 17 / 07$ & 28.75 & 0.296 \\
\hline Sinclair Inlet & SI-GC-3/4 & Graceful Crab (2) & COMP & 2838-94-95 & 6.99 & 67 & 05/17/07 & 33.60 & 0.221 \\
\hline Sinclair Inlet & SI-GC-7/1 & Graceful Crab (2) & COMP & $2838-98-92$ & 6.67 & 60 & $05 / 17 / 07$ & 54.85 & 0.435 \\
\hline Sinclair Inlet & SI-GC-5/8 & Graceful Crab (2) & COMP & 2838-96-99 & 7.62 & 92 & $05 / 17 / 07$ & 30.89 & 0.383 \\
\hline Sinclair Inlet & SI-SC-COMP & Sea cucumber (6) & COMP & $2838-100$ & & 4100 & $05 / 17 / 07$ & 9.52 & 0.0131 \\
\hline Port Gardner & PG-ES-1 & English Sole & WBC & $2838-101$ & 24.8 & 158 & $05 / 29 / 07$ & 19.23 & $0.00165 \mathrm{~J}$ \\
\hline Port Gardner & PG-ES-2 & English Sole & WBC & $2838-102$ & 21.6 & 101 & $05 / 29 / 07$ & 20.82 & $0.000849 \mathrm{~J}$ \\
\hline Port Gardner & PG-ES-3 & English Sole & WBC & $2838-103$ & 24.8 & 128 & $05 / 29 / 07$ & 19.95 & $0.00123 \mathrm{~J}$ \\
\hline Nisqually & NIS-ES-1 & English Sole & WBC & 2838-104 & 25.4 & 166 & $05 / 30 / 07$ & 20.67 & 0.00589 \\
\hline Nisqually & NIS-ES-2 & English Sole & WBC & $2838-105$ & 26.7 & 214 & $05 / 30 / 07$ & 21.52 & 0.0125 \\
\hline Nisqually & NIS-ES-3 & English Sole & WBC & 2838-106 & 30.5 & 290 & $05 / 30 / 07$ & 21.61 & 0.00501 \\
\hline Nisqually & NIS-RF-1 & Ratfish & WBC & 2838-107 & 48.3 & 620 & $05 / 30 / 07$ & 31.21 & 1.53 \\
\hline Nisqually & NIS-RF-2 & Ratfish & WBC & 2838-108 & 40.6 & 430 & $05 / 30 / 07$ & 32.53 & 0.238 \\
\hline Nisqually & NIS-RF-3 & Ratfish & WBC & 2838-109 & 38.1 & 450 & $05 / 30 / 07$ & 29.35 & 0.628 \\
\hline Commencement Bay & CB-ES-1 & English Sole & WBC & $2838-110$ & 22.9 & 145 & $05 / 31 / 07$ & 21.50 & 0.00598 \\
\hline Commencement Bay & CB-ES-2 & English Sole & WBC & $2838-111$ & 24.8 & 157 & $05 / 31 / 07$ & 23.68 & 0.0135 \\
\hline Commencement Bay & CB-ES-3 & English Sole & WBC & $2838-112$ & 24.8 & 162 & $05 / 31 / 07$ & 23.16 & 0.0148 \\
\hline Commencement Bay & CB-RF-1 & Ratfish & WBC & $2838-113$ & 40.6 & 320 & $05 / 31 / 07$ & 30.17 & 0.272 \\
\hline Commencement Bay & CB-RF-2 & Ratfish & WBC & $2838-114$ & 38.7 & 350 & $05 / 31 / 07$ & 28.54 & 0.973 \\
\hline Commencement Bay & CB-RF-3 & Ratfish & WBC & $2838-115$ & 38.7 & 390 & $05 / 31 / 07$ & 30.58 & 0.266 \\
\hline Duwamish & DU-ES-1 & English Sole & WBC & $2838-116$ & 17.8 & 75 & 05/18/07 & 22.95 & 0.00523 \\
\hline Duwamish & DU-ES-2 & English Sole & WBC & $2838-117$ & 21.6 & 96 & $05 / 18 / 07$ & 20.32 & 0.00711 \\
\hline Duwamish & DU-ES-3 & English Sole & WBC & $2838-118$ & 26.0 & 156 & $05 / 18 / 07$ & 23.00 & 0.00213 \\
\hline Admiralty Inlet & DF-EM-1 & Dogfish 1 & EM & $2838-126$ & & 410 & 09/19/07 & 52.97 & 0.0107 \\
\hline Admiralty Inlet & DF-EM-2 & Dogfish 2 & EM & $2838-127$ & & 440 & 09/19/07 & 52.87 & 0.0129 \\
\hline Admiralty Inlet & DF-LV-1 & Dogfish 1 & $\mathrm{LV}$ & $2838-132$ & & 498 & $09 / 19 / 07$ & 55.21 & 0.282 \\
\hline Admiralty Inlet & DF-LV-2 & Dogfish 2 & LV & $2838-133$ & & 260 & 09/19/07 & 52.95 & 0.0604 \\
\hline Admiralty Inlet & DF-DIG-1 & Dogfish 1 & DIG & $2838-138$ & & 319 & 09/19/07 & 22.67 & 0.0465 \\
\hline Admiralty Inlet & DF-DIG-2 & Dogfish 2 & DIG & 2838-139 & & 194 & 09/19/07 & 21.16 & 0.00214 \\
\hline Admiralty Inlet & DF-SWC-1 & Dogfish 1 & SWC & $2838-174$ & & & 09/19/07 & 37.19 & $0.0006 \mathrm{U}$ \\
\hline Admiralty Inlet & DF-SWC-2 & Dogfish 2 & SWC & $2838-175$ & & & 09/19/07 & 28.81 & $0.0006 \mathrm{U}$ \\
\hline Admiralty Inlet & DF-WBWC-1 & Dogfish 1 & WBWC & $2838-177$ & 110.5 & 5400 & 09/19/07 & 39.76 & 0.0145 \\
\hline Admiralty Inlet & DF-WBWC-3 & Dogfish 3 & WBWC & 2838-179 & 93.03 & 3140 & 09/19/07 & 36.70 & $0.00150 \mathrm{~J}$ \\
\hline Admiralty Inlet & DF-WBWC-2 & Dogfish 2 & WBWC & $2838-178$ & 104.8 & 4290 & 09/19/07 & 35.83 & 0.00566 \\
\hline Admiralty Inlet & DF-EM-4 & Dogfish 4 & EM & $2838-129$ & & 188 & 09/19/07 & 52.59 & 0.00360 \\
\hline Admiralty Inlet & DF-EM-6 & Dogfish 6 & EM & $2838-131$ & & 322 & 09/19/07 & 56.33 & 0.00242 \\
\hline Admiralty Inlet & DF-LV-4 & Dogfish 4 & LV & $2838-135$ & & 790 & 09/19/07 & 77.18 & 0.0486 \\
\hline Admiralty Inlet & DF-LV-6 & Dogfish 6 & LV & $2838-137$ & & 434 & 09/19/07 & 31.93 & 0.0354 \\
\hline Admiralty Inlet & DF-DIG-4 & Dogfish 4 & DIG & $2838-141$ & & 320 & $09 / 19 / 07$ & 15.59 & 0.00451 \\
\hline Admiralty Inlet & DF-DIG-6 & Dogfish 6 & DIG & $2838-143$ & & 327 & 09/19/07 & 17.06 & 0.00677 \\
\hline Admiralty Inlet & DF-SWC-6 & Dogfish 6 & SWC & $2838-176$ & & & 09/19/07 & 35.23 & $0.0006 \mathrm{U}$ \\
\hline Admiralty Inlet & DF-WBWC-4 & Dogfish 4 & WBWC & $2838-180$ & 111.1 & 5420 & 09/19/07 & 40.36 & 0.00266 \\
\hline Admiralty Inlet & DF-WBWC-5 & Dogfish 5 & WBWC & $2838-181$ & 93.98 & 3020 & 09/19/07 & 41.03 & 0.0185 \\
\hline Admiralty Inlet & DF-WBWC-6 & Dogfish 6 & WBWC & $2838-182$ & 98.11 & 4090 & 09/19/07 & 37.03 & 0.00644 \\
\hline Admiralty Inlet & DF-SWC-4 & Dogfish 4 & SWC & $2838-183$ & & & 09/19/07 & 29.84 & $0.0006 \mathrm{U}$ \\
\hline
\end{tabular}




\begin{tabular}{|c|c|c|c|c|c|c|c|c|c|}
\hline Client Code & Client Code & & & \multicolumn{6}{|c|}{ Units $=\mu \mathrm{g} / \mathrm{g}$ WET weight } \\
\hline Station & PSAMP Code & Organism & \begin{tabular}{|l} 
Sample \\
Type
\end{tabular} & MSL Code & $\begin{array}{l}\text { Length } \\
\text { (cm) }\end{array}$ & $\begin{array}{l}\text { Weight } \\
\text { (g) }\end{array}$ & $\begin{array}{l}\text { Collection } \\
\text { Date }\end{array}$ & $\begin{array}{c}\text { Percent } \\
\text { Dry } \\
\text { Weight }\end{array}$ & As \\
\hline & & & & & & & & Instrument: & ICP-MS \\
\hline $\begin{array}{l}\text { Laboratory Ach } \\
\text { Reporting Limit }\end{array}$ & $\begin{array}{l}\text { Method Detec } \\
\text { t Weight }\end{array}$ & tion Limits (conves & d to we & weight) & & & Average & 27.01 & $\begin{array}{r}0.03 \\
0.1\end{array}$ \\
\hline Vendovi & V-ES-5 & English Sole & WBC & $2838-5$ & 27.9 & 224 & 05/01/07 & 18.59 & 5.93 \\
\hline Vendovi & V-ES-6 & English Sole & WBC & $2838-6$ & 27.9 & 250 & 05/01/07 & 17.79 & 8.06 \\
\hline Vendovi & V-ES-8 & English Sole & WBC & $2838-8$ & 27.3 & 197 & 05/01/07 & 22.64 & 6.32 \\
\hline Vendovi & V-RS-1 & Rock sole & WBC & $2838-10$ & 20.3 & 119 & 05/01/07 & 21.79 & 2.94 \\
\hline Vendovi & V-RS-2 & Rock sole & WBC & 2838-11 & 21.0 & 142 & 05/01/07 & 21.23 & 2.61 \\
\hline Vendovi & V-RS-3 & Rock sole & WBC & $2838-12$ & 31.1 & 510 & 05/01/07 & 20.30 & 3.69 \\
\hline Vendovi & V-SSc-1 & Staghorn sculpin & WBC & $2838-13$ & 17.8 & 94 & 05/01/07 & 19.54 & 2.66 \\
\hline Vendovi & V-SSc-2 & Staghorn sculpin & WBC & $2838-14$ & 24.8 & 298 & 05/01/07 & 20.54 & 2.94 \\
\hline Vendovi & V-SSc-3 & Staghorn sculpin & WBC & $2838-15$ & 25.4 & 294 & 05/01/07 & 19.90 & 3.04 \\
\hline Vendovi & V-SP-1 & Shinner Surfperch & WBC & $2838-16$ & 7.62 & 16 & 05/01/07 & 23.28 & 0.957 \\
\hline Vendovi & V-SP-2 & Shinner Surfperch & WBC & $2838-17$ & 8.89 & 15 & 05/01/07 & 22.19 & 0.708 \\
\hline Vendovi & V-SP-3 & Shinner Surfperch & WBC & $2838-18$ & 8.89 & 16 & 05/01/07 & 24.13 & 1.25 \\
\hline Vendovi & V-SC-COMP & Sea cucumber (3) & COMP & $2838-19$ & & 5501 & 05/01/07 & 13.20 & 1.43 \\
\hline Strait of Georgia & SG-ES-3 & English Sole & WBC & $2838-22$ & 26.7 & 184 & $05 / 02 / 07$ & 20.54 & 2.24 \\
\hline Strait of Georgia & SG-ES-4 & English Sole & WBC & $2838-23$ & 26.0 & 189 & $05 / 02 / 07$ & 20.95 & 3.79 \\
\hline Strait of Georgia & SG-ES-5 & English Sole & WBC & $2838-24$ & 26.7 & 192 & $05 / 02 / 07$ & 21.39 & 3.79 \\
\hline Strait of Georgia & SG-RF-1 & Ratfish & WBC & $2838-29$ & 27.9 & 115 & $05 / 02 / 07$ & 19.86 & 5.86 \\
\hline Strait of Georgia & SG-RF-2 & Ratfish & WBC & $2838-30$ & 35.6 & 320 & $05 / 02 / 07$ & 27.99 & 4.28 \\
\hline Strait of Georgia & SG-RF-3 & Ratfish & WBC & 2838-31 & 48.3 & 770 & $05 / 02 / 07$ & 33.64 & 10.7 \\
\hline Strait of Georgia & SG-SC-COMP & Sea cucumber (6) & COMP & $2838-32$ & & 290 & $05 / 02 / 07$ & 7.70 & 1.29 \\
\hline Hood Canal & HC-ES-1 & English Sole & WBC & $2838-33$ & 22.9 & 131 & 05/03/07 & 17.91 & 5.03 \\
\hline Hood Canal & HC-ES-2 & English Sole & WBC & $2838-34$ & 22.9 & 133 & 05/03/07 & 20.28 & 10.4 \\
\hline Hood Canal & HC-ES-3 & English Sole & WBC & $2838-35$ & 26.7 & 220 & 05/03/07 & 21.19 & 4.60 \\
\hline Hood Canal & HC-RS-1 & Rock sole & WBC & $2838-36$ & 31.8 & 460 & 05/03/07 & 22.07 & 2.47 \\
\hline Hood Canal & HC-RF-1 & Ratfish & WBC & $2838-37$ & 39.4 & 480 & 05/03/07 & 25.63 & 7.23 \\
\hline Hood Canal & HC-RF-2 & Ratfish & WBC & $2838-38$ & 38.1 & 340 & $05 / 03 / 07$ & 27.04 & 6.90 \\
\hline Hood Canal & HC-RF-3 & Ratfish & WBC & 2838-39 & 42.5 & 450 & 05/03/07 & 27.61 & 9.80 \\
\hline Hood Canal & HC-SP1 & Shinner Surfperch & WBC & $2838-40$ & 9.53 & 16 & 05/03/07 & 27.84 & 1.14 \\
\hline Hood Canal & HC-SP2 & Shinner Surfperch & WBC & $2838-41$ & 10.2 & 20 & 05/03/07 & 34.05 & 1.28 \\
\hline Hood Canal & HC-GC-1 & Graceful Crab & WBC & $2838-43$ & 10.2 & 237 & 05/03/07 & 19.14 & 3.37 \\
\hline Elliott Bay & EB-ES-1/2 & English Sole (2) & COMP & $2838-45-46$ & 15.8 & 44 & $05 / 16 / 07$ & 21.44 & 3.24 \\
\hline Elliott Bay & EB-ES-3 & English Sole & WBC & $2838-47$ & 26.0 & 157 & 05/16/07 & 19.72 & 5.34 \\
\hline Elliott Bay & EB-RF-1 & Ratfish & WBC & $2838-48$ & 40.6 & 350 & 05/16/07 & 26.43 & 13.0 \\
\hline Elliott Bay & EB-RF-2 & Ratfish & WBC & $2838-49$ & 41.9 & 370 & $05 / 16 / 07$ & 27.23 & 8.22 \\
\hline Elliott Bay & EB-RF-3 & Ratfish & WBC & $2838-50$ & 53.3 & 810 & 05/16/07 & 27.11 & 6.70 \\
\hline Eagle Harbor & EH-ES-2 & English Sole & WBC & $2838-52$ & 35.6 & 420 & $05 / 19 / 07$ & 17.35 & 2.76 \\
\hline Eagle Harbor & EH-ES-3 & English Sole & WBC & $2838-53$ & 22.2 & 89 & 05/19/07 & 23.38 & 4.86 \\
\hline Eagle Harbor & EH-ES-4 & English Sole & WBC & $2838-54$ & 27.3 & 199 & 05/19/07 & 21.69 & 3.38 \\
\hline Eagle Harbor & EH-RF-1 & Ratfish & WBC & $2838-55$ & 33.0 & 230 & 05/19/07 & 26.50 & 10.0 \\
\hline Eagle Harbor & EH-RF-2 & Ratfish & WBC & $2838-56$ & 38.1 & 380 & 05/19/07 & 23.39 & 6.29 \\
\hline Eagle Harbor & EH-RF-3 & Ratfish & WBC & $2838-57$ & 50.8 & 760 & $05 / 19 / 07$ & 31.68 & 7.35 \\
\hline Sinclair Inlet & SI-ES-1 & English Sole & WBC & $2838-58$ & 29.2 & 330 & 05/17/07 & 22.19 & 2.64 \\
\hline Sinclair Inlet & SI-ES-2 & English Sole & WBC & $2838-59$ & 29.2 & 330 & 05/17/07 & 19.35 & 3.41 \\
\hline Sinclair Inlet & SI-ES-4 & English Sole & WBC & $2838-61$ & 25.4 & 192 & 05/17/07 & 40.66 & 8.86 \\
\hline Sinclair Inlet & SI-ES-6 & English Sole & WBC & $2838-63$ & 25.4 & 178 & 05/17/07 & 20.31 & 2.30 \\
\hline Sinclair Inlet & SI-ES-7 & English Sole & WBC & $2838-64$ & 25.4 & 179 & 05/17/07 & 20.51 & 2.54 \\
\hline Sinclair Inlet & SI-ES-8 & English Sole & WBC & $2838-65$ & 29.2 & 261 & 05/17/07 & 20.71 & 3.52 \\
\hline Sinclair Inlet & SI-RS-1/2 & Rock sole (2) & COMP & 2838-67-68 & 10.2 & 23 & 05/17/07 & 24.28 & 3.11 \\
\hline Sinclair Inlet & SI-RS-3 & Rock sole & WBC & $2838-69$ & 27.3 & 320 & 05/17/07 & 21.12 & 5.07 \\
\hline Sinclair Inlet & SI-RS-4 & Rock sole & WBC & $2838-70$ & 26.7 & 220 & 05/17/07 & 21.17 & 6.96 \\
\hline Sinclair Inlet & SI-SS-1 & Sand sole & WBC & $2838-71$ & 25.4 & 270 & $05 / 17 / 07$ & 21.66 & 1.74 \\
\hline Sinclair Inlet & SI-SS-2 & Sand sole & WBC & $2838-72$ & 27.3 & 290 & $05 / 17 / 07$ & 22.13 & 1.59 \\
\hline Sinclair Inlet & SI-SS-3 & Sand sole & WBC & $2838-73$ & 29.8 & 320 & 05/17/07 & 21.05 & 1.61 \\
\hline
\end{tabular}




\begin{tabular}{|c|c|c|c|c|c|c|c|c|c|}
\hline Client Code & Client Code & & & \multicolumn{6}{|c|}{ Units $=\mu \mathrm{g} / \mathrm{g}$ WET weight } \\
\hline Station & PSAMP Code & Organism & \begin{tabular}{|l} 
Sample \\
Type
\end{tabular} & MSL Code & $\begin{array}{l}\text { Length } \\
\text { (cm) }\end{array}$ & $\begin{array}{l}\text { Weight } \\
\text { (g) }\end{array}$ & $\begin{array}{c}\text { Collection } \\
\text { Date }\end{array}$ & $\begin{array}{c}\text { Percent } \\
\text { Dry } \\
\text { Weight }\end{array}$ & As \\
\hline & & & & & & & & Instrument: & $I C P-M S$ \\
\hline $\begin{array}{l}\text { Laboratory Achievec } \\
\text { Reporting Limit, We }\end{array}$ & $\begin{array}{l}\text { Method Detec } \\
\text { t Weight }\end{array}$ & tion Limits (conver & d to wet & weight) & & & Average & 27.01 & $\begin{array}{r}0.03 \\
0.1\end{array}$ \\
\hline Sinclair Inlet & SI-SSc-1/2 & Staghorn sculpin (2) & COMP & $2838-77-78$ & 14.9 & 48 & $05 / 17 / 07$ & 20.66 & 1.73 \\
\hline Sinclair Inlet & SI-SSc-3/5 & Staghorn sculpin (2) & COMP & $2838-79-81$ & 14.3 & 53 & $05 / 17 / 07$ & 20.03 & 1.18 \\
\hline Sinclair Inlet & SI-SSc-4/6 & Staghorn sculpin (2) & COMP & $2838-80-82$ & 16.2 & 75 & 05/17/07 & 19.51 & 1.88 \\
\hline Sinclair Inlet & SI-RF-1 & Ratfish & WBC & $2838-83$ & 41.3 & 550 & 05/17/07 & 32.67 & 9.54 \\
\hline Sinclair Inlet & SI-RF-2 & Ratfish & WBC & $2838-84$ & 36.8 & 380 & 05/17/07 & 26.61 & 9.29 \\
\hline Sinclair Inlet & SI-SP-4/5 & Shinner Surfperch (2) & COMP & $2838-88-89$ & 7.62 & 13 & 05/17/07 & 24.47 & 0.670 \\
\hline Sinclair Inlet & SI-SP-6/2 & Shinner Surfperch (2) & COMP & $2838-90-86$ & 6.67 & 11 & 05/17/07 & 24.68 & 0.876 \\
\hline Sinclair Inlet & SI-SP-7/3 & Shinner Surfperch (2) & COMP & $2838-91-87$ & 7.3 & 11 & $05 / 17 / 07$ & 25.12 & 1.07 \\
\hline Sinclair Inlet & SI-GC-2/6 & Graceful Crab (2) & COMP & 2838-93-97 & 7.62 & 103 & $05 / 17 / 07$ & 28.75 & 5.26 \\
\hline Sinclair Inlet & SI-GC-3/4 & Graceful Crab (2) & COMP & 2838-94-95 & 6.99 & 67 & 05/17/07 & 33.60 & 4.17 \\
\hline Sinclair Inlet & SI-GC-7/1 & Graceful Crab (2) & COMP & 2838-98-92 & 6.67 & 60 & $05 / 17 / 07$ & 54.85 & 8.12 \\
\hline Sinclair Inlet & SI-GC-5/8 & Graceful Crab (2) & COMP & 2838-96-99 & 7.62 & 92 & $05 / 17 / 07$ & 30.89 & 4.48 \\
\hline Sinclair Inlet & SI-SC-COMP & Sea cucumber (6) & COMP & $2838-100$ & & 4100 & 05/17/07 & 9.52 & 1.03 \\
\hline Port Gardner & PG-ES-1 & English Sole & WBC & $2838-101$ & 24.8 & 158 & $05 / 29 / 07$ & 19.23 & 2.98 \\
\hline Port Gardner & PG-ES-2 & English Sole & WBC & $2838-102$ & 21.6 & 101 & $05 / 29 / 07$ & 20.82 & 2.58 \\
\hline Port Gardner & PG-ES-3 & English Sole & WBC & $2838-103$ & 24.8 & 128 & 05/29/07 & 19.95 & 2.91 \\
\hline Nisqually & NIS-ES-1 & English Sole & WBC & 2838-104 & 25.4 & 166 & $05 / 30 / 07$ & 20.67 & 4.86 \\
\hline Nisqually & NIS-ES-2 & English Sole & WBC & $2838-105$ & 26.7 & 214 & $05 / 30 / 07$ & 21.52 & 5.40 \\
\hline Nisqually & NIS-ES-3 & English Sole & WBC & $2838-106$ & 30.5 & 290 & $05 / 30 / 07$ & 21.61 & 4.41 \\
\hline Nisqually & NIS-RF-1 & Ratfish & WBC & $2838-107$ & 48.3 & 620 & $05 / 30 / 07$ & 31.21 & 10.9 \\
\hline Nisqually & NIS-RF-2 & Ratfish & WBC & 2838-108 & 40.6 & 430 & $05 / 30 / 07$ & 32.53 & 8.75 \\
\hline Nisqually & NIS-RF-3 & Ratfish & WBC & 2838-109 & 38.1 & 450 & $05 / 30 / 07$ & 29.35 & 7.98 \\
\hline Commencement Bay & CB-ES-1 & English Sole & WBC & $2838-110$ & 22.9 & 145 & 05/31/07 & 21.50 & 4.45 \\
\hline Commencement Bay & CB-ES-2 & English Sole & WBC & 2838-111 & 24.8 & 157 & $05 / 31 / 07$ & 23.68 & 1.61 \\
\hline Commencement Bay & CB-ES-3 & English Sole & WBC & $2838-112$ & 24.8 & 162 & $05 / 31 / 07$ & 23.16 & 4.56 \\
\hline Commencement Bay & CB-RF-1 & Ratfish & WBC & $2838-113$ & 40.6 & 320 & $05 / 31 / 07$ & 30.17 & 5.55 \\
\hline Commencement Bay & CB-RF-2 & Ratfish & WBC & $2838-114$ & 38.7 & 350 & $05 / 31 / 07$ & 28.54 & 5.91 \\
\hline Commencement Bay & CB-RF-3 & Ratfish & WBC & $2838-115$ & 38.7 & 390 & $05 / 31 / 07$ & 30.58 & 4.92 \\
\hline Duwamish & DU-ES-1 & English Sole & WBC & $2838-116$ & 17.8 & 75 & 05/18/07 & 22.95 & 3.79 \\
\hline Duwamish & DU-ES-2 & English Sole & WBC & $2838-117$ & 21.6 & 96 & 05/18/07 & 20.32 & 2.28 \\
\hline Duwamish & DU-ES-3 & English Sole & WBC & $2838-118$ & 26.0 & 156 & 05/18/07 & 23.00 & 5.47 \\
\hline Admiralty Inlet & DF-EM-1 & Dogfish 1 & EM & $2838-126$ & & 410 & 09/19/07 & 52.97 & 5.77 \\
\hline Admiralty Inlet & DF-EM-2 & Dogfish 2 & EM & $2838-127$ & & 440 & 09/19/07 & 52.87 & 4.11 \\
\hline Admiralty Inlet & DF-LV-1 & Dogfish 1 & LV & $2838-132$ & & 498 & 09/19/07 & 55.21 & 9.72 \\
\hline Admiralty Inlet & DF-LV-2 & Dogfish 2 & LV & $2838-133$ & & 260 & 09/19/07 & 52.95 & 4.14 \\
\hline Admiralty Inlet & DF-DIG-1 & Dogfish 1 & DIG & $2838-138$ & & 319 & 09/19/07 & 22.67 & 5.80 \\
\hline Admiralty Inlet & DF-DIG-2 & Dogfish 2 & DIG & 2838-139 & & 194 & 09/19/07 & 21.16 & 1.41 \\
\hline Admiralty Inlet & DF-SWC-1 & Dogfish 1 & SWC & $2838-174$ & & & $09 / 19 / 07$ & 37.19 & 3.69 \\
\hline Admiralty Inlet & DF-SWC-2 & Dogfish 2 & SWC & $2838-175$ & & & 09/19/07 & 28.81 & 1.59 \\
\hline Admiralty Inlet & DF-WBWC-1 & Dogfish 1 & WBWC & $2838-177$ & 110.5 & 5400 & 09/19/07 & 39.76 & 4.41 \\
\hline Admiralty Inlet & DF-WBWC-3 & Dogfish 3 & WBWC & $2838-179$ & 93.03 & 3140 & $09 / 19 / 07$ & 36.70 & 2.73 \\
\hline Admiralty Inlet & DF-WBWC-2 & Dogfish 2 & WBWC & $2838-178$ & 104.8 & 4290 & 09/19/07 & 35.83 & 2.82 \\
\hline Admiralty Inlet & DF-EM-4 & Dogfish 4 & EM & 2838-129 & & 188 & $09 / 19 / 07$ & 52.59 & 5.73 \\
\hline Admiralty Inlet & DF-EM-6 & Dogfish 6 & EM & 2838-131 & & 322 & 09/19/07 & 56.33 & 5.42 \\
\hline Admiralty Inlet & DF-LV-4 & Dogfish 4 & LV & $2838-135$ & & 790 & 09/19/07 & 77.18 & 8.49 \\
\hline Admiralty Inlet & DF-LV-6 & Dogfish 6 & LV & $2838-137$ & & 434 & 09/19/07 & 31.93 & 4.69 \\
\hline Admiralty Inlet & DF-DIG-4 & Dogfish 4 & DIG & $2838-141$ & & 320 & 09/19/07 & 15.59 & 3.32 \\
\hline Admiralty Inlet & DF-DIG-6 & Dogfish 6 & DIG & $2838-143$ & & 327 & 09/19/07 & 17.06 & 1.89 \\
\hline Admiralty Inlet & DF-SWC-6 & Dogfish 6 & SWC & $2838-176$ & & & 09/19/07 & 35.23 & 3.73 \\
\hline Admiralty Inlet & DF-WBWC-4 & Dogfish 4 & WBWC & $2838-180$ & 111.1 & 5420 & 09/19/07 & 40.36 & 4.88 \\
\hline Admiralty Inlet & DF-WBWC-5 & Dogfish 5 & WBWC & $2838-181$ & 93.98 & 3020 & 09/19/07 & 41.03 & 5.17 \\
\hline Admiralty Inlet & DF-WBWC-6 & Dogfish 6 & WBWC & $2838-182$ & 98.11 & 4090 & 09/19/07 & 37.03 & 4.18 \\
\hline Admiralty Inlet & DF-SWC-4 & Dogfish 4 & SWC & $2838-183$ & & & 09/19/07 & 29.84 & 3.49 \\
\hline
\end{tabular}




\begin{tabular}{|c|c|c|c|c|c|c|c|c|c|}
\hline Client Code & Client Code & & & \multicolumn{6}{|c|}{ Units $=\mu \mathrm{g} / \mathrm{g}$ WET weight } \\
\hline Station & PSAMP Code & Organism & \begin{tabular}{|l} 
Sample \\
Type
\end{tabular} & MSL Code & $\begin{array}{l}\text { Length } \\
\text { (cm) }\end{array}$ & $\begin{array}{l}\text { Weight } \\
\text { (g) }\end{array}$ & $\begin{array}{l}\text { Collection } \\
\text { Date }\end{array}$ & $\begin{array}{c}\text { Percent } \\
\text { Dry } \\
\text { Weight }\end{array}$ & Cd \\
\hline & & & & & & & & Instrument: & $I C P-M S$ \\
\hline $\begin{array}{l}\text { Laboratory Ach } \\
\text { Reporting Limit }\end{array}$ & $\begin{array}{l}\text { Method Detec } \\
\text { t Weight }\end{array}$ & tion Limits (conves & dtow & weight) & & & Average & 27.01 & $\begin{array}{r}0.0006 \\
0.002 \\
\end{array}$ \\
\hline Vendovi & V-ES-5 & English Sole & WBC & $2838-5$ & 27.9 & 224 & 05/01/07 & 18.59 & 0.0164 \\
\hline Vendovi & V-ES-6 & English Sole & WBC & $2838-6$ & 27.9 & 250 & 05/01/07 & 17.79 & 0.0117 \\
\hline Vendovi & V-ES-8 & English Sole & WBC & $2838-8$ & 27.3 & 197 & 05/01/07 & 22.64 & 0.0159 \\
\hline Vendovi & V-RS-1 & Rock sole & WBC & $2838-10$ & 20.3 & 119 & 05/01/07 & 21.79 & 0.00708 \\
\hline Vendovi & V-RS-2 & Rock sole & WBC & 2838-11 & 21.0 & 142 & 05/01/07 & 21.23 & 0.0134 \\
\hline Vendovi & V-RS-3 & Rock sole & WBC & $2838-12$ & 31.1 & 510 & 05/01/07 & 20.30 & 0.0280 \\
\hline Vendovi & V-SSc-1 & Staghorn sculpin & WBC & $2838-13$ & 17.8 & 94 & 05/01/07 & 19.54 & 0.0326 \\
\hline Vendovi & V-SSc-2 & Staghorn sculpin & WBC & $2838-14$ & 24.8 & 298 & 05/01/07 & 20.54 & 0.0401 \\
\hline Vendovi & V-SSc-3 & Staghorn sculpin & WBC & $2838-15$ & 25.4 & 294 & 05/01/07 & 19.90 & 0.0190 \\
\hline Vendovi & V-SP-1 & Shinner Surfperch & WBC & $2838-16$ & 7.62 & 16 & 05/01/07 & 23.28 & 0.0300 \\
\hline Vendovi & V-SP-2 & Shinner Surfperch & WBC & $2838-17$ & 8.89 & 15 & 05/01/07 & 22.19 & 0.0322 \\
\hline Vendovi & V-SP-3 & Shinner Surfperch & WBC & $2838-18$ & 8.89 & 16 & 05/01/07 & 24.13 & 0.0490 \\
\hline Vendovi & V-SC-COMP & Sea cucumber (3) & COMP & $2838-19$ & & 5501 & 05/01/07 & 13.20 & 0.0594 \\
\hline Strait of Georgia & SG-ES-3 & English Sole & WBC & $2838-22$ & 26.7 & 184 & $05 / 02 / 07$ & 20.54 & 0.00690 \\
\hline Strait of Georgia & SG-ES-4 & English Sole & WBC & $2838-23$ & 26.0 & 189 & $05 / 02 / 07$ & 20.95 & 0.0127 \\
\hline Strait of Georgia & SG-ES-5 & English Sole & WBC & $2838-24$ & 26.7 & 192 & $05 / 02 / 07$ & 21.39 & 0.0162 \\
\hline Strait of Georgia & SG-RF-1 & Ratfish & WBC & $2838-29$ & 27.9 & 115 & $05 / 02 / 07$ & 19.86 & 0.00546 \\
\hline Strait of Georgia & SG-RF-2 & Ratfish & WBC & $2838-30$ & 35.6 & 320 & $05 / 02 / 07$ & 27.99 & 0.00982 \\
\hline Strait of Georgia & SG-RF-3 & Ratfish & WBC & 2838-31 & 48.3 & 770 & $05 / 02 / 07$ & 33.64 & 0.0228 \\
\hline Strait of Georgia & SG-SC-COMP & Sea cucumber (6) & COMP & $2838-32$ & & 290 & $05 / 02 / 07$ & 7.70 & 0.120 \\
\hline Hood Canal & HC-ES-1 & English Sole & WBC & $2838-33$ & 22.9 & 131 & 05/03/07 & 17.91 & 0.00947 \\
\hline Hood Canal & HC-ES-2 & English Sole & WBC & $2838-34$ & 22.9 & 133 & 05/03/07 & 20.28 & 0.0105 \\
\hline Hood Canal & HC-ES-3 & English Sole & WBC & $2838-35$ & 26.7 & 220 & 05/03/07 & 21.19 & 0.00949 \\
\hline Hood Canal & HC-RS-1 & Rock sole & WBC & $2838-36$ & 31.8 & 460 & 05/03/07 & 22.07 & 0.0243 \\
\hline Hood Canal & HC-RF-1 & Ratfish & WBC & $2838-37$ & 39.4 & 480 & 05/03/07 & 25.63 & 0.0779 \\
\hline Hood Canal & HC-RF-2 & Ratfish & WBC & $2838-38$ & 38.1 & 340 & $05 / 03 / 07$ & 27.04 & 0.0525 \\
\hline Hood Canal & HC-RF-3 & Ratfish & WBC & 2838-39 & 42.5 & 450 & 05/03/07 & 27.61 & 0.0555 \\
\hline Hood Canal & HC-SP1 & Shinner Surfperch & WBC & $2838-40$ & 9.53 & 16 & 05/03/07 & 27.84 & 0.0476 \\
\hline Hood Canal & HC-SP2 & Shinner Surfperch & WBC & $2838-41$ & 10.2 & 20 & 05/03/07 & 34.05 & 0.0385 \\
\hline Hood Canal & HC-GC-1 & Graceful Crab & WBC & $2838-43$ & 10.2 & 237 & 05/03/07 & 19.14 & 0.463 \\
\hline Elliott Bay & EB-ES-1/2 & English Sole (2) & COMP & $2838-45-46$ & 15.8 & 44 & $05 / 16 / 07$ & 21.44 & 0.0236 \\
\hline Elliott Bay & EB-ES-3 & English Sole & WBC & $2838-47$ & 26.0 & 157 & 05/16/07 & 19.72 & 0.0199 \\
\hline Elliott Bay & EB-RF-1 & Ratfish & WBC & $2838-48$ & 40.6 & 350 & $05 / 16 / 07$ & 26.43 & 0.0682 \\
\hline Elliott Bay & EB-RF-2 & Ratfish & WBC & $2838-49$ & 41.9 & 370 & 05/16/07 & 27.23 & 0.0438 \\
\hline Elliott Bay & EB-RF-3 & Ratfish & WBC & $2838-50$ & 53.3 & 810 & 05/16/07 & 27.11 & 0.0211 \\
\hline Eagle Harbor & EH-ES-2 & English Sole & WBC & $2838-52$ & 35.6 & 420 & 05/19/07 & 17.35 & 0.0361 \\
\hline Eagle Harbor & EH-ES-3 & English Sole & WBC & $2838-53$ & 22.2 & 89 & 05/19/07 & 23.38 & 0.0933 \\
\hline Eagle Harbor & EH-ES-4 & English Sole & WBC & $2838-54$ & 27.3 & 199 & $05 / 19 / 07$ & 21.69 & 0.00701 \\
\hline Eagle Harbor & EH-RF-1 & Ratfish & WBC & $2838-55$ & 33.0 & 230 & 05/19/07 & 26.50 & 0.0228 \\
\hline Eagle Harbor & EH-RF-2 & Ratfish & WBC & $2838-56$ & 38.1 & 380 & 05/19/07 & 23.39 & 0.0547 \\
\hline Eagle Harbor & EH-RF-3 & Ratfish & WBC & $2838-57$ & 50.8 & 760 & 05/19/07 & 31.68 & 0.0295 \\
\hline Sinclair Inlet & SI-ES-1 & English Sole & WBC & $2838-58$ & 29.2 & 330 & 05/17/07 & 22.19 & 0.0104 \\
\hline Sinclair Inlet & SI-ES-2 & English Sole & WBC & $2838-59$ & 29.2 & 330 & 05/17/07 & 19.35 & 0.0163 \\
\hline Sinclair Inlet & SI-ES-4 & English Sole & WBC & $2838-61$ & 25.4 & 192 & $05 / 17 / 07$ & 40.66 & 0.0312 \\
\hline Sinclair Inlet & SI-ES-6 & English Sole & WBC & $2838-63$ & 25.4 & 178 & 05/17/07 & 20.31 & 0.00526 \\
\hline Sinclair Inlet & SI-ES-7 & English Sole & WBC & $2838-64$ & 25.4 & 179 & 05/17/07 & 20.51 & 0.0172 \\
\hline Sinclair Inlet & SI-ES-8 & English Sole & WBC & $2838-65$ & 29.2 & 261 & 05/17/07 & 20.71 & 0.0112 \\
\hline Sinclair Inlet & SI-RS-1/2 & Rock sole (2) & COMP & 2838-67-68 & 10.2 & 23 & $05 / 17 / 07$ & 24.28 & 0.00799 \\
\hline Sinclair Inlet & SI-RS-3 & Rock sole & WBC & $2838-69$ & 27.3 & 320 & $05 / 17 / 07$ & 21.12 & 0.0124 \\
\hline Sinclair Inlet & SI-RS-4 & Rock sole & WBC & $2838-70$ & 26.7 & 220 & 05/17/07 & 21.17 & 0.00726 \\
\hline Sinclair Inlet & SI-SS-1 & Sand sole & WBC & $2838-71$ & 25.4 & 270 & $05 / 17 / 07$ & 21.66 & 0.0290 \\
\hline Sinclair Inlet & SI-SS-2 & Sand sole & WBC & $2838-72$ & 27.3 & 290 & 05/17/07 & 22.13 & 0.00476 \\
\hline Sinclair Inlet & SI-SS-3 & Sand sole & WBC & $2838-73$ & 29.8 & 320 & 05/17/07 & 21.05 & 0.00747 \\
\hline
\end{tabular}




\begin{tabular}{|c|c|c|c|c|c|c|c|c|c|}
\hline Client Code & Client Code & & & \multicolumn{6}{|c|}{ Units $=\mu \mathrm{g} / \mathrm{g}$ WET weight } \\
\hline Station & PSAMP Code & Organism & \begin{tabular}{|l} 
Sample \\
Type
\end{tabular} & MSL Code & $\begin{array}{l}\text { Length } \\
\text { (cm) }\end{array}$ & $\begin{array}{l}\text { Weight } \\
\text { (g) }\end{array}$ & $\begin{array}{c}\text { Collection } \\
\text { Date }\end{array}$ & $\begin{array}{c}\text { Percent } \\
\text { Dry } \\
\text { Weight }\end{array}$ & Cd \\
\hline & & & & & & & & Instrument: & ICP-MS \\
\hline $\begin{array}{l}\text { Laboratory Achievec } \\
\text { Reporting Limit, We }\end{array}$ & $\begin{array}{l}\text { Method Detec } \\
\text { t Weight }\end{array}$ & tion Limits (conver & d to wet & weight) & & & Average & 27.01 & $\begin{array}{r}0.0006 \\
0.002 \\
\end{array}$ \\
\hline Sinclair Inlet & SI-SSc-1/2 & Staghorn sculpin (2) & COMP & $2838-77-78$ & 14.9 & 48 & $05 / 17 / 07$ & 20.66 & 0.0141 \\
\hline Sinclair Inlet & SI-SSc-3/5 & Staghorn sculpin (2) & COMP & $2838-79-81$ & 14.3 & 53 & $05 / 17 / 07$ & 20.03 & 0.00995 \\
\hline Sinclair Inlet & SI-SSc-4/6 & Staghorn sculpin (2) & COMP & $2838-80-82$ & 16.2 & 75 & $05 / 17 / 07$ & 19.51 & 0.0124 \\
\hline Sinclair Inlet & SI-RF-1 & Ratfish & WBC & $2838-83$ & 41.3 & 550 & 05/17/07 & 32.67 & 0.0376 \\
\hline Sinclair Inlet & SI-RF-2 & Ratfish & WBC & $2838-84$ & 36.8 & 380 & 05/17/07 & 26.61 & 0.0325 \\
\hline Sinclair Inlet & SI-SP-4/5 & Shinner Surfperch (2) & COMP & $2838-88-89$ & 7.62 & 13 & 05/17/07 & 24.47 & 0.0489 \\
\hline Sinclair Inlet & SI-SP-6/2 & Shinner Surfperch (2) & COMP & $2838-90-86$ & 6.67 & 11 & 05/17/07 & 24.68 & 0.0358 \\
\hline Sinclair Inlet & SI-SP-7/3 & Shinner Surfperch (2) & COMP & 2838-91-87 & 7.3 & 11 & 05/17/07 & 25.12 & 0.0332 \\
\hline Sinclair Inlet & SI-GC-2/6 & Graceful Crab (2) & COMP & 2838-93-97 & 7.62 & 103 & $05 / 17 / 07$ & 28.75 & 0.160 \\
\hline Sinclair Inlet & SI-GC-3/4 & Graceful Crab (2) & COMP & 2838-94-95 & 6.99 & 67 & 05/17/07 & 33.60 & 0.130 \\
\hline Sinclair Inlet & SI-GC-7/1 & Graceful Crab (2) & COMP & 2838-98-92 & 6.67 & 60 & $05 / 17 / 07$ & 54.85 & 0.177 \\
\hline Sinclair Inlet & SI-GC-5/8 & Graceful Crab (2) & COMP & 2838-96-99 & 7.62 & 92 & $05 / 17 / 07$ & 30.89 & 0.113 \\
\hline Sinclair Inlet & SI-SC-COMP & Sea cucumber (6) & COMP & $2838-100$ & & 4100 & 05/17/07 & 9.52 & 0.100 \\
\hline Port Gardner & PG-ES-1 & English Sole & WBC & $2838-101$ & 24.8 & 158 & $05 / 29 / 07$ & 19.23 & 0.0127 \\
\hline Port Gardner & PG-ES-2 & English Sole & WBC & $2838-102$ & 21.6 & 101 & $05 / 29 / 07$ & 20.82 & 0.0115 \\
\hline Port Gardner & PG-ES-3 & English Sole & WBC & $2838-103$ & 24.8 & 128 & 05/29/07 & 19.95 & 0.0110 \\
\hline Nisqually & NIS-ES-1 & English Sole & WBC & 2838-104 & 25.4 & 166 & $05 / 30 / 07$ & 20.67 & 0.0294 \\
\hline Nisqually & NIS-ES-2 & English Sole & WBC & $2838-105$ & 26.7 & 214 & 05/30/07 & 21.52 & 0.0334 \\
\hline Nisqually & NIS-ES-3 & English Sole & WBC & $2838-106$ & 30.5 & 290 & $05 / 30 / 07$ & 21.61 & 0.0341 \\
\hline Nisqually & NIS-RF-1 & Ratfish & WBC & $2838-107$ & 48.3 & 620 & $05 / 30 / 07$ & 31.21 & 0.0758 \\
\hline Nisqually & NIS-RF-2 & Ratfish & WBC & 2838-108 & 40.6 & 430 & $05 / 30 / 07$ & 32.53 & 0.0249 \\
\hline Nisqually & NIS-RF-3 & Ratfish & WBC & 2838-109 & 38.1 & 450 & $05 / 30 / 07$ & 29.35 & 0.156 \\
\hline Commencement Bay & CB-ES-1 & English Sole & WBC & $2838-110$ & 22.9 & 145 & 05/31/07 & 21.50 & 0.0144 \\
\hline Commencement Bay & CB-ES-2 & English Sole & WBC & 2838-111 & 24.8 & 157 & $05 / 31 / 07$ & 23.68 & 0.0419 \\
\hline Commencement Bay & CB-ES-3 & English Sole & WBC & $2838-112$ & 24.8 & 162 & $05 / 31 / 07$ & 23.16 & 0.0217 \\
\hline Commencement Bay & CB-RF-1 & Ratfish & WBC & $2838-113$ & 40.6 & 320 & $05 / 31 / 07$ & 30.17 & 0.0168 \\
\hline Commencement Bay & CB-RF-2 & Ratfish & WBC & $2838-114$ & 38.7 & 350 & $05 / 31 / 07$ & 28.54 & 0.0291 \\
\hline Commencement Bay & CB-RF-3 & Ratfish & WBC & $2838-115$ & 38.7 & 390 & $05 / 31 / 07$ & 30.58 & 0.0228 \\
\hline Duwamish & DU-ES-1 & English Sole & WBC & $2838-116$ & 17.8 & 75 & 05/18/07 & 22.95 & 0.0169 \\
\hline Duwamish & DU-ES-2 & English Sole & WBC & $2838-117$ & 21.6 & 96 & 05/18/07 & 20.32 & 0.0184 \\
\hline Duwamish & DU-ES-3 & English Sole & WBC & $2838-118$ & 26.0 & 156 & 05/18/07 & 23.00 & 0.0207 \\
\hline Admiralty Inlet & DF-EM-1 & Dogfish 1 & EM & $2838-126$ & & 410 & 09/19/07 & 52.97 & 0.0202 \\
\hline Admiralty Inlet & DF-EM-2 & Dogfish 2 & EM & $2838-127$ & & 440 & 09/19/07 & 52.87 & 0.0124 \\
\hline Admiralty Inlet & DF-LV-1 & Dogfish 1 & LV & 2838-132 & & 498 & 09/19/07 & 55.21 & 3.46 \\
\hline Admiralty Inlet & DF-LV-2 & Dogfish 2 & LV & $2838-133$ & & 260 & 09/19/07 & 52.95 & 2.20 \\
\hline Admiralty Inlet & DF-DIG-1 & Dogfish 1 & DIG & $2838-138$ & & 319 & 09/19/07 & 22.67 & 4.26 \\
\hline Admiralty Inlet & DF-DIG-2 & Dogfish 2 & DIG & 2838-139 & & 194 & 09/19/07 & 21.16 & 0.459 \\
\hline Admiralty Inlet & DF-SWC-1 & Dogfish 1 & SWC & $2838-174$ & & & $09 / 19 / 07$ & 37.19 & 0.00792 \\
\hline Admiralty Inlet & DF-SWC-2 & Dogfish 2 & SWC & $2838-175$ & & & 09/19/07 & 28.81 & 0.00959 \\
\hline Admiralty Inlet & DF-WBWC-1 & Dogfish 1 & WBWC & $2838-177$ & 110.5 & 5400 & 09/19/07 & 39.76 & 0.290 \\
\hline Admiralty Inlet & DF-WBWC-3 & Dogfish 3 & WBWC & $2838-179$ & 93.03 & 3140 & $09 / 19 / 07$ & 36.70 & 0.153 \\
\hline Admiralty Inlet & DF-WBWC-2 & Dogfish 2 & WBWC & $2838-178$ & 104.8 & 4290 & 09/19/07 & 35.83 & 0.142 \\
\hline Admiralty Inlet & DF-EM-4 & Dogfish 4 & EM & 2838-129 & & 188 & $09 / 19 / 07$ & 52.59 & 0.0368 \\
\hline Admiralty Inlet & DF-EM-6 & Dogfish 6 & EM & $2838-131$ & & 322 & $09 / 19 / 07$ & 56.33 & 0.0117 \\
\hline Admiralty Inlet & DF-LV-4 & Dogfish 4 & LV & $2838-135$ & & 790 & 09/19/07 & 77.18 & 2.84 \\
\hline Admiralty Inlet & DF-LV-6 & Dogfish 6 & LV & $2838-137$ & & 434 & 09/19/07 & 31.93 & 2.40 \\
\hline Admiralty Inlet & DF-DIG-4 & Dogfish 4 & DIG & $2838-141$ & & 320 & 09/19/07 & 15.59 & 3.37 \\
\hline Admiralty Inlet & DF-DIG-6 & Dogfish 6 & DIG & $2838-143$ & & 327 & 09/19/07 & 17.06 & 2.03 \\
\hline Admiralty Inlet & DF-SWC-6 & Dogfish 6 & SWC & $2838-176$ & & & 09/19/07 & 35.23 & 0.0170 \\
\hline Admiralty Inlet & DF-WBWC-4 & Dogfish 4 & WBWC & $2838-180$ & 111.1 & 5420 & 09/19/07 & 40.36 & 0.900 \\
\hline Admiralty Inlet & DF-WBWC-5 & Dogfish 5 & WBWC & $2838-181$ & 93.98 & 3020 & 09/19/07 & 41.03 & 0.0726 \\
\hline Admiralty Inlet & DF-WBWC-6 & Dogfish 6 & WBWC & $2838-182$ & 98.11 & 4090 & 09/19/07 & 37.03 & 0.974 \\
\hline Admiralty Inlet & DF-SWC-4 & Dogfish 4 & SWC & $2838-183$ & & & 09/19/07 & 29.84 & 0.0164 \\
\hline
\end{tabular}




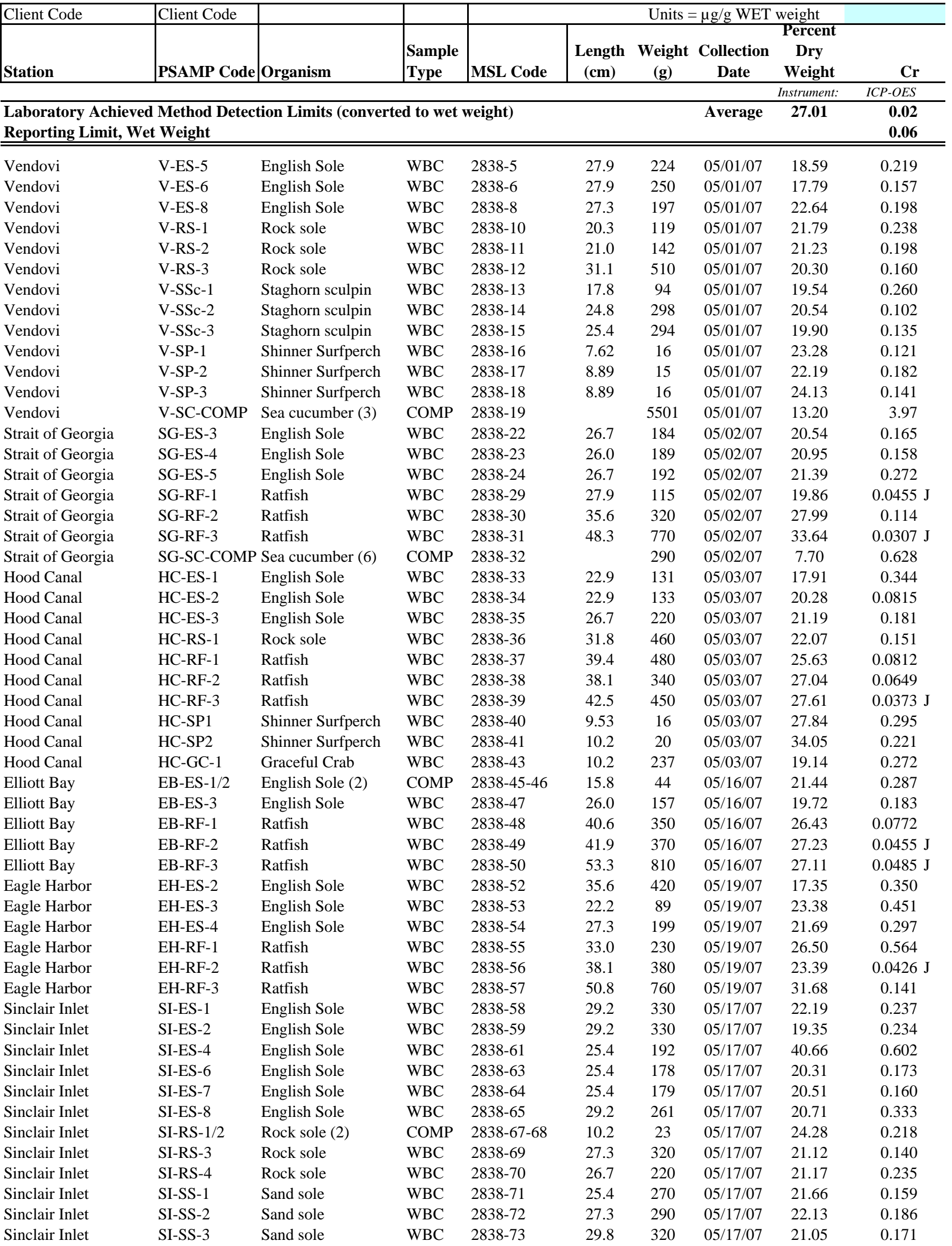




\begin{tabular}{|c|c|c|c|c|c|c|c|c|c|}
\hline Client Code & Client Code & & & \multicolumn{6}{|c|}{ Units $=\mu \mathrm{g} / \mathrm{g}$ WET weight } \\
\hline \multirow[t]{2}{*}{ Station } & PSAMP Code & Organism & $\begin{array}{l}\text { Sample } \\
\text { Type }\end{array}$ & MSL Code & $\begin{array}{l}\text { Length } \\
\text { (cm) }\end{array}$ & $\begin{array}{l}\text { Weight } \\
\text { (g) }\end{array}$ & $\begin{array}{l}\text { Collection } \\
\text { Date }\end{array}$ & $\begin{array}{c}\text { Percent } \\
\text { Dry } \\
\text { Weight }\end{array}$ & $\mathrm{Cr}$ \\
\hline & & & & & & & \multicolumn{2}{|r|}{ Instrument: } & ICP-OES \\
\hline \multicolumn{7}{|c|}{$\begin{array}{l}\text { Laboratory Achieved Method Detection Limits (converted to wet weight) } \\
\text { Reporting Limit, Wet Weight }\end{array}$} & Average & 27.01 & 0.02 \\
\hline Sinclair Inlet & SI-SSc-1/2 & Staghorn sculpin (2) & COMP & $2838-77-78$ & 14.9 & 48 & 05/17/07 & 20.66 & 0.300 \\
\hline Sinclair Inlet & SI-SSc-3/5 & Staghorn sculpin (2) & COMP & $2838-79-81$ & 14.3 & 53 & 05/17/07 & 20.03 & 0.200 \\
\hline Sinclair Inlet & SI-SSc-4/6 & Staghorn sculpin (2) & COMP & $2838-80-82$ & 16.2 & 75 & $05 / 17 / 07$ & 19.51 & 0.203 \\
\hline Sinclair Inlet & SI-RF-1 & Ratfish & WBC & $2838-83$ & 41.3 & 550 & $05 / 17 / 07$ & 32.67 & 0.104 \\
\hline Sinclair Inlet & SI-RF-2 & Ratfish & WBC & $2838-84$ & 36.8 & 380 & $05 / 17 / 07$ & 26.61 & $0.0431 \mathrm{~J}$ \\
\hline Sinclair Inlet & SI-SP-4/5 & Shinner Surfperch (2) & ) COMP & $2838-88-89$ & 7.62 & 13 & 05/17/07 & 24.47 & 0.257 \\
\hline Sinclair Inlet & SI-SP-6/2 & Shinner Surfperch (2) & COMP & $2838-90-86$ & 6.67 & 11 & 05/17/07 & 24.68 & 0.149 \\
\hline Sinclair Inlet & SI-SP-7/3 & Shinner Surfperch (2) & COMP & $2838-91-87$ & 7.3 & 11 & $05 / 17 / 07$ & 25.12 & 0.274 \\
\hline Sinclair Inlet & SI-GC-2/6 & Graceful Crab (2) & COMP & 2838-93-97 & 7.62 & 103 & $05 / 17 / 07$ & 28.75 & 0.702 \\
\hline Sinclair Inlet & SI-GC-3/4 & Graceful Crab (2) & COMP & 2838-94-95 & 6.99 & 67 & 05/17/07 & 33.60 & 0.336 \\
\hline Sinclair Inlet & SI-GC-7/1 & Graceful Crab (2) & COMP & $2838-98-92$ & 6.67 & 60 & $05 / 17 / 07$ & 54.85 & 0.581 \\
\hline Sinclair Inlet & SI-GC-5/8 & Graceful Crab (2) & COMP & 2838-96-99 & 7.62 & 92 & $05 / 17 / 07$ & 30.89 & 0.794 \\
\hline Sinclair Inlet & SI-SC-COMP & Sea cucumber (6) & COMP & $2838-100$ & & 4100 & $05 / 17 / 07$ & 9.52 & 0.971 \\
\hline Port Gardner & PG-ES-1 & English Sole & WBC & $2838-101$ & 24.8 & 158 & $05 / 29 / 07$ & 19.23 & 0.410 \\
\hline Port Gardner & PG-ES-2 & English Sole & WBC & $2838-102$ & 21.6 & 101 & $05 / 29 / 07$ & 20.82 & 0.579 \\
\hline Port Gardner & PG-ES-3 & English Sole & WBC & 2838-103 & 24.8 & 128 & $05 / 29 / 07$ & 19.95 & 0.367 \\
\hline Nisqually & NIS-ES-1 & English Sole & WBC & 2838-104 & 25.4 & 166 & $05 / 30 / 07$ & 20.67 & 0.339 \\
\hline Nisqually & NIS-ES-2 & English Sole & WBC & 2838-105 & 26.7 & 214 & 05/30/07 & 21.52 & 0.433 \\
\hline Nisqually & NIS-ES-3 & English Sole & WBC & 2838-106 & 30.5 & 290 & $05 / 30 / 07$ & 21.61 & 0.141 \\
\hline Nisqually & NIS-RF-1 & Ratfish & WBC & 2838-107 & 48.3 & 620 & $05 / 30 / 07$ & 31.21 & $0.0241 \mathrm{~J}$ \\
\hline Nisqually & NIS-RF-2 & Ratfish & WBC & 2838-108 & 40.6 & 430 & $05 / 30 / 07$ & 32.53 & 0.0654 \\
\hline Nisqually & NIS-RF-3 & Ratfish & WBC & 2838-109 & 38.1 & 450 & $05 / 30 / 07$ & 29.35 & $0.0326 \mathrm{~J}$ \\
\hline Commencement Bay & CB-ES-1 & English Sole & WBC & $2838-110$ & 22.9 & 145 & $05 / 31 / 07$ & 21.50 & 0.169 \\
\hline Commencement Bay & CB-ES-2 & English Sole & WBC & $2838-111$ & 24.8 & 157 & $05 / 31 / 07$ & 23.68 & 0.310 \\
\hline Commencement Bay & CB-ES-3 & English Sole & WBC & $2838-112$ & 24.8 & 162 & $05 / 31 / 07$ & 23.16 & 0.340 \\
\hline Commencement Bay & CB-RF-1 & Ratfish & WBC & $2838-113$ & 40.6 & 320 & $05 / 31 / 07$ & 30.17 & 0.0941 \\
\hline Commencement Bay & CB-RF-2 & Ratfish & WBC & $2838-114$ & 38.7 & 350 & $05 / 31 / 07$ & 28.54 & 0.0990 \\
\hline Commencement Bay & CB-RF-3 & Ratfish & WBC & $2838-115$ & 38.7 & 390 & $05 / 31 / 07$ & 30.58 & 0.0798 \\
\hline Duwamish & DU-ES-1 & English Sole & WBC & $2838-116$ & 17.8 & 75 & $05 / 18 / 07$ & 22.95 & 0.282 \\
\hline Duwamish & DU-ES-2 & English Sole & WBC & $2838-117$ & 21.6 & 96 & $05 / 18 / 07$ & 20.32 & 0.455 \\
\hline Duwamish & DU-ES-3 & English Sole & WBC & $2838-118$ & 26.0 & 156 & 05/18/07 & 23.00 & 0.237 \\
\hline Admiralty Inlet & DF-EM-1 & Dogfish 1 & EM & $2838-126$ & & 410 & 09/19/07 & 52.97 & 0.105 \\
\hline Admiralty Inlet & DF-EM-2 & Dogfish 2 & EM & $2838-127$ & & 440 & 09/19/07 & 52.87 & 0.113 \\
\hline Admiralty Inlet & DF-LV-1 & Dogfish 1 & LV & 2838-132 & & 498 & 09/19/07 & 55.21 & $0.0348 \mathrm{~J}$ \\
\hline Admiralty Inlet & DF-LV-2 & Dogfish 2 & LV & $2838-133$ & & 260 & 09/19/07 & 52.95 & 0.0757 \\
\hline Admiralty Inlet & DF-DIG-1 & Dogfish 1 & DIG & $2838-138$ & & 319 & 09/19/07 & 22.67 & 0.140 \\
\hline Admiralty Inlet & DF-DIG-2 & Dogfish 2 & DIG & 2838-139 & & 194 & 09/19/07 & 21.16 & $0.0480 \mathrm{~J}$ \\
\hline Admiralty Inlet & DF-SWC-1 & Dogfish 1 & SWC & $2838-174$ & & & 09/19/07 & 37.19 & 0.0625 \\
\hline Admiralty Inlet & DF-SWC-2 & Dogfish 2 & SWC & $2838-175$ & & & 09/19/07 & 28.81 & $0.0383 \mathrm{~J}$ \\
\hline Admiralty Inlet & DF-WBWC-1 & Dogfish 1 & WBWC & $2838-177$ & 110.5 & 5400 & 09/19/07 & 39.76 & 0.0676 \\
\hline Admiralty Inlet & DF-WBWC-3 & Dogfish 3 & WBWC & $2838-179$ & 93.03 & 3140 & $09 / 19 / 07$ & 36.70 & $0.0499 \mathrm{~J}$ \\
\hline Admiralty Inlet & DF-WBWC-2 & Dogfish 2 & WBWC & $2838-178$ & 104.8 & 4290 & 09/19/07 & 35.83 & 0.0656 \\
\hline Admiralty Inlet & DF-EM-4 & Dogfish 4 & $\mathrm{EM}$ & 2838-129 & & 188 & 09/19/07 & 52.59 & 0.0915 \\
\hline Admiralty Inlet & DF-EM-6 & Dogfish 6 & EM & $2838-131$ & & 322 & 09/19/07 & 56.33 & 0.118 \\
\hline Admiralty Inlet & DF-LV-4 & Dogfish 4 & LV & $2838-135$ & & 790 & 09/19/07 & 77.18 & $0.0410 \mathrm{~J}$ \\
\hline Admiralty Inlet & DF-LV-6 & Dogfish 6 & $\mathrm{LV}$ & $2838-137$ & & 434 & 09/19/07 & 31.93 & $0.0227 \mathrm{~J}$ \\
\hline Admiralty Inlet & DF-DIG-4 & Dogfish 4 & DIG & $2838-141$ & & 320 & $09 / 19 / 07$ & 15.59 & $0.0499 \mathrm{~J}$ \\
\hline Admiralty Inlet & DF-DIG-6 & Dogfish 6 & DIG & $2838-143$ & & 327 & 09/19/07 & 17.06 & 0.665 \\
\hline Admiralty Inlet & DF-SWC-6 & Dogfish 6 & SWC & $2838-176$ & & & 09/19/07 & 35.23 & $0.0571 \mathrm{~J}$ \\
\hline Admiralty Inlet & DF-WBWC-4 & Dogfish 4 & WBWC & $2838-180$ & 111.1 & 5420 & 09/19/07 & 40.36 & 0.102 \\
\hline Admiralty Inlet & DF-WBWC-5 & Dogfish 5 & WBWC & $2838-181$ & 93.98 & 3020 & 09/19/07 & 41.03 & $0.0529 \mathrm{~J}$ \\
\hline Admiralty Inlet & DF-WBWC-6 & Dogfish 6 & WBWC & $2838-182$ & 98.11 & 4090 & 09/19/07 & 37.03 & 0.214 \\
\hline Admiralty Inlet & DF-SWC-4 & Dogfish 4 & SWC & $2838-183$ & & & $09 / 19 / 07$ & 29.84 & $0.0477 \mathrm{~J}$ \\
\hline
\end{tabular}




\begin{tabular}{|c|c|c|c|c|c|c|c|c|c|}
\hline Client Code & Client Code & & & \multicolumn{6}{|c|}{ Units $=\mu \mathrm{g} / \mathrm{g}$ WET weight } \\
\hline & PSAMP Code & Organism & $\begin{array}{l}\text { Sample } \\
\text { Type }\end{array}$ & MSL Code & $\begin{array}{l}\text { Length } \\
\text { (cm) }\end{array}$ & $\begin{array}{l}\text { Weight } \\
\text { (g) }\end{array}$ & $\begin{array}{c}\text { Collection } \\
\text { Date }\end{array}$ & $\begin{array}{c}\text { Percent } \\
\text { Dry } \\
\text { Weight } \\
\end{array}$ & $\mathbf{C u}$ \\
\hline Station & & & & & & & & Instrument: & ICP-MS \\
\hline \multicolumn{7}{|c|}{$\begin{array}{l}\text { Laboratory Achieved Method Detection Limits (converted to wet weight) } \\
\text { Reporting Limit, Wet Weight }\end{array}$} & Average & 27.01 & $\begin{array}{r}0.03 \\
0.1\end{array}$ \\
\hline Vendovi & V-ES-5 & English Sole & WBC & $2838-5$ & 27.9 & 224 & 05/01/07 & 18.59 & 0.638 \\
\hline Vendovi & V-ES-6 & English Sole & WBC & $2838-6$ & 27.9 & 250 & 05/01/07 & 17.79 & 0.397 \\
\hline Vendovi & V-ES-8 & English Sole & WBC & $2838-8$ & 27.3 & 197 & 05/01/07 & 22.64 & 0.629 \\
\hline Vendovi & V-RS-1 & Rock sole & WBC & $2838-10$ & 20.3 & 119 & 05/01/07 & 21.79 & 0.525 \\
\hline Vendovi & V-RS-2 & Rock sole & WBC & 2838-11 & 21.0 & 142 & 05/01/07 & 21.23 & 0.465 \\
\hline Vendovi & V-RS-3 & Rock sole & WBC & $2838-12$ & 31.1 & 510 & 05/01/07 & 20.30 & 0.382 \\
\hline Vendovi & V-SSc-1 & Staghorn sculpin & WBC & $2838-13$ & 17.8 & 94 & 05/01/07 & 19.54 & 1.99 \\
\hline Vendovi & V-SSc-2 & Staghorn sculpin & WBC & $2838-14$ & 24.8 & 298 & 05/01/07 & 20.54 & 0.776 \\
\hline Vendovi & V-SSc-3 & Staghorn sculpin & WBC & $2838-15$ & 25.4 & 294 & 05/01/07 & 19.90 & 0.746 \\
\hline Vendovi & V-SP-1 & Shinner Surfperch & WBC & $2838-16$ & 7.62 & 16 & 05/01/07 & 23.28 & 0.622 \\
\hline Vendovi & V-SP-2 & Shinner Surfperch & WBC & $2838-17$ & 8.89 & 15 & 05/01/07 & 22.19 & 0.810 \\
\hline Vendovi & V-SP-3 & Shinner Surfperch & WBC & $2838-18$ & 8.89 & 16 & 05/01/07 & 24.13 & 0.630 \\
\hline Vendovi & V-SC-COMP & Sea cucumber (3) & COMP & $2838-19$ & & 5501 & 05/01/07 & 13.20 & 6.24 \\
\hline Strait of Georgia & SG-ES-3 & English Sole & WBC & $2838-22$ & 26.7 & 184 & $05 / 02 / 07$ & 20.54 & 0.503 \\
\hline Strait of Georgia & SG-ES-4 & English Sole & WBC & $2838-23$ & 26.0 & 189 & $05 / 02 / 07$ & 20.95 & 0.614 \\
\hline Strait of Georgia & SG-ES-5 & English Sole & WBC & $2838-24$ & 26.7 & 192 & 05/02/07 & 21.39 & 0.811 \\
\hline Strait of Georgia & SG-RF-1 & Ratfish & WBC & $2838-29$ & 27.9 & 115 & $05 / 02 / 07$ & 19.86 & 0.504 \\
\hline Strait of Georgia & SG-RF-2 & Ratfish & WBC & $2838-30$ & 35.6 & 320 & $05 / 02 / 07$ & 27.99 & 0.680 \\
\hline Strait of Georgia & SG-RF-3 & Ratfish & WBC & $2838-31$ & 48.3 & 770 & 05/02/07 & 33.64 & 0.801 \\
\hline Strait of Georgia & SG-SC-COMP & Sea cucumber (6) & COMP & $2838-32$ & & 290 & $05 / 02 / 07$ & 7.70 & 1.00 \\
\hline Hood Canal & HC-ES-1 & English Sole & WBC & $2838-33$ & 22.9 & 131 & 05/03/07 & 17.91 & 0.51 \\
\hline Hood Canal & HC-ES-2 & English Sole & WBC & $2838-34$ & 22.9 & 133 & 05/03/07 & 20.28 & 0.41 \\
\hline Hood Canal & HC-ES-3 & English Sole & WBC & 2838-35 & 26.7 & 220 & 05/03/07 & 21.19 & 0.608 \\
\hline Hood Canal & HC-RS-1 & Rock sole & WBC & $2838-36$ & 31.8 & 460 & 05/03/07 & 22.07 & 0.461 \\
\hline Hood Canal & HC-RF-1 & Ratfish & WBC & $2838-37$ & 39.4 & 480 & 05/03/07 & 25.63 & 0.925 \\
\hline Hood Canal & HC-RF-2 & Ratfish & WBC & $2838-38$ & 38.1 & 340 & 05/03/07 & 27.04 & 0.717 \\
\hline Hood Canal & HC-RF-3 & Ratfish & WBC & $2838-39$ & 42.5 & 450 & 05/03/07 & 27.61 & 1.27 \\
\hline Hood Canal & HC-SP1 & Shinner Surfperch & WBC & $2838-40$ & 9.53 & 16 & 05/03/07 & 27.84 & 0.735 \\
\hline Hood Canal & HC-SP2 & Shinner Surfperch & WBC & $2838-41$ & 10.2 & 20 & 05/03/07 & 34.05 & 0.916 \\
\hline Hood Canal & HC-GC-1 & Graceful Crab & WBC & $2838-43$ & 10.2 & 237 & 05/03/07 & 19.14 & 8.98 \\
\hline Elliott Bay & EB-ES-1/2 & English Sole (2) & COMP & $2838-45-46$ & 15.8 & 44 & 05/16/07 & 21.44 & 0.866 \\
\hline Elliott Bay & EB-ES-3 & English Sole & WBC & $2838-47$ & 26.0 & 157 & $05 / 16 / 07$ & 19.72 & 0.710 \\
\hline Elliott Bay & EB-RF-1 & Ratfish & WBC & $2838-48$ & 40.6 & 350 & $05 / 16 / 07$ & 26.43 & 1.14 \\
\hline Elliott Bay & EB-RF-2 & Ratfish & WBC & $2838-49$ & 41.9 & 370 & $05 / 16 / 07$ & 27.23 & 0.869 \\
\hline Elliott Bay & EB-RF-3 & Ratfish & WBC & $2838-50$ & 53.3 & 810 & $05 / 16 / 07$ & 27.11 & 1.26 \\
\hline Eagle Harbor & EH-ES-2 & English Sole & WBC & $2838-52$ & 35.6 & 420 & 05/19/07 & 17.35 & 0.847 \\
\hline Eagle Harbor & EH-ES-3 & English Sole & WBC & $2838-53$ & 22.2 & 89 & 05/19/07 & 23.38 & 1.12 \\
\hline Eagle Harbor & EH-ES-4 & English Sole & WBC & $2838-54$ & 27.3 & 199 & 05/19/07 & 21.69 & 0.798 \\
\hline Eagle Harbor & EH-RF-1 & Ratfish & WBC & $2838-55$ & 33.0 & 230 & 05/19/07 & 26.50 & 1.17 \\
\hline Eagle Harbor & EH-RF-2 & Ratfish & WBC & $2838-56$ & 38.1 & 380 & 05/19/07 & 23.39 & 1.03 \\
\hline Eagle Harbor & EH-RF-3 & Ratfish & WBC & $2838-57$ & 50.8 & 760 & 05/19/07 & 31.68 & 1.25 \\
\hline Sinclair Inlet & SI-ES-1 & English Sole & WBC & $2838-58$ & 29.2 & 330 & 05/17/07 & 22.19 & 0.890 \\
\hline Sinclair Inlet & SI-ES-2 & English Sole & WBC & $2838-59$ & 29.2 & 330 & 05/17/07 & 19.35 & 0.846 \\
\hline Sinclair Inlet & SI-ES-4 & English Sole & WBC & $2838-61$ & 25.4 & 192 & 05/17/07 & 40.66 & 1.86 \\
\hline Sinclair Inlet & SI-ES-6 & English Sole & WBC & $2838-63$ & 25.4 & 178 & 05/17/07 & 20.31 & 0.559 \\
\hline Sinclair Inlet & SI-ES-7 & English Sole & WBC & $2838-64$ & 25.4 & 179 & 05/17/07 & 20.51 & 0.541 \\
\hline Sinclair Inlet & SI-ES-8 & English Sole & WBC & $2838-65$ & 29.2 & 261 & 05/17/07 & 20.71 & 0.874 \\
\hline Sinclair Inlet & SI-RS-1/2 & Rock sole (2) & COMP & $2838-67-68$ & 10.2 & 23 & 05/17/07 & 24.28 & 1.05 \\
\hline Sinclair Inlet & SI-RS-3 & Rock sole & WBC & $2838-69$ & 27.3 & 320 & 05/17/07 & 21.12 & 0.957 \\
\hline Sinclair Inlet & SI-RS-4 & Rock sole & WBC & $2838-70$ & 26.7 & 220 & 05/17/07 & 21.17 & 0.377 \\
\hline Sinclair Inlet & SI-SS-1 & Sand sole & WBC & 2838-71 & 25.4 & 270 & 05/17/07 & 21.66 & 0.977 \\
\hline Sinclair Inlet & SI-SS-2 & Sand sole & WBC & $2838-72$ & 27.3 & 290 & 05/17/07 & 22.13 & 0.480 \\
\hline Sinclair Inlet & SI-SS-3 & Sand sole & WBC & $2838-73$ & 29.8 & 320 & 05/17/07 & 21.05 & 0.861 \\
\hline
\end{tabular}




\begin{tabular}{|c|c|c|c|c|c|c|c|c|c|}
\hline Client Code & Client Code & & & \multicolumn{6}{|c|}{ Units $=\mu \mathrm{g} / \mathrm{g}$ WET weight } \\
\hline Station & PSAMP Code & Organism & \begin{tabular}{|l} 
Sample \\
Type
\end{tabular} & MSL Code & $\begin{array}{l}\text { Length } \\
\text { (cm) }\end{array}$ & $\begin{array}{l}\text { Weight } \\
\text { (g) }\end{array}$ & $\begin{array}{c}\text { Collection } \\
\text { Date }\end{array}$ & $\begin{array}{c}\text { Percent } \\
\text { Dry } \\
\text { Weight }\end{array}$ & $\mathbf{C u}$ \\
\hline & & & & & & & & Instrument: & $I C P-M S$ \\
\hline $\begin{array}{l}\text { Laboratory Achieve } \\
\text { Reporting Limit, W }\end{array}$ & $\begin{array}{l}\text { Method Detec } \\
\text { t Weight }\end{array}$ & tion Limits (conver & d to wet & weight) & & & Average & 27.01 & $\begin{array}{r}0.03 \\
0.1 \\
\end{array}$ \\
\hline Sinclair Inlet & SI-SSc-1/2 & Staghorn sculpin (2) & COMP & $2838-77-78$ & 14.9 & 48 & $05 / 17 / 07$ & 20.66 & 2.04 \\
\hline Sinclair Inlet & SI-SSc-3/5 & Staghorn sculpin (2) & COMP & $2838-79-81$ & 14.3 & 53 & $05 / 17 / 07$ & 20.03 & 1.27 \\
\hline Sinclair Inlet & SI-SSc-4/6 & Staghorn sculpin (2) & COMP & $2838-80-82$ & 16.2 & 75 & 05/17/07 & 19.51 & 1.64 \\
\hline Sinclair Inlet & SI-RF-1 & Ratfish & WBC & $2838-83$ & 41.3 & 550 & 05/17/07 & 32.67 & 1.52 \\
\hline Sinclair Inlet & SI-RF-2 & Ratfish & WBC & $2838-84$ & 36.8 & 380 & 05/17/07 & 26.61 & 0.913 \\
\hline Sinclair Inlet & SI-SP-4/5 & Shinner Surfperch (2) & COMP & $2838-88-89$ & 7.62 & 13 & 05/17/07 & 24.47 & 0.940 \\
\hline Sinclair Inlet & SI-SP-6/2 & Shinner Surfperch (2) & COMP & $2838-90-86$ & 6.67 & 11 & 05/17/07 & 24.68 & 0.827 \\
\hline Sinclair Inlet & SI-SP-7/3 & Shinner Surfperch (2) & COMP & $2838-91-87$ & 7.3 & 11 & $05 / 17 / 07$ & 25.12 & 1.40 \\
\hline Sinclair Inlet & SI-GC-2/6 & Graceful Crab (2) & COMP & 2838-93-97 & 7.62 & 103 & $05 / 17 / 07$ & 28.75 & 19.0 \\
\hline Sinclair Inlet & SI-GC-3/4 & Graceful Crab (2) & COMP & 2838-94-95 & 6.99 & 67 & 05/17/07 & 33.60 & 16.8 \\
\hline Sinclair Inlet & SI-GC-7/1 & Graceful Crab (2) & COMP & 2838-98-92 & 6.67 & 60 & $05 / 17 / 07$ & 54.85 & 29.9 \\
\hline Sinclair Inlet & SI-GC-5/8 & Graceful Crab (2) & COMP & 2838-96-99 & 7.62 & 92 & $05 / 17 / 07$ & 30.89 & 17.8 \\
\hline Sinclair Inlet & SI-SC-COMP & Sea cucumber (6) & COMP & $2838-100$ & & 4100 & 05/17/07 & 9.52 & 1.54 \\
\hline Port Gardner & PG-ES-1 & English Sole & WBC & $2838-101$ & 24.8 & 158 & $05 / 29 / 07$ & 19.23 & 0.735 \\
\hline Port Gardner & PG-ES-2 & English Sole & WBC & $2838-102$ & 21.6 & 101 & $05 / 29 / 07$ & 20.82 & 0.743 \\
\hline Port Gardner & PG-ES-3 & English Sole & WBC & $2838-103$ & 24.8 & 128 & $05 / 29 / 07$ & 19.95 & 0.720 \\
\hline Nisqually & NIS-ES-1 & English Sole & WBC & 2838-104 & 25.4 & 166 & $05 / 30 / 07$ & 20.67 & 1.30 \\
\hline Nisqually & NIS-ES-2 & English Sole & WBC & $2838-105$ & 26.7 & 214 & 05/30/07 & 21.52 & 1.68 \\
\hline Nisqually & NIS-ES-3 & English Sole & WBC & $2838-106$ & 30.5 & 290 & $05 / 30 / 07$ & 21.61 & 0.795 \\
\hline Nisqually & NIS-RF-1 & Ratfish & WBC & $2838-107$ & 48.3 & 620 & $05 / 30 / 07$ & 31.21 & 1.49 \\
\hline Nisqually & NIS-RF-2 & Ratfish & WBC & 2838-108 & 40.6 & 430 & $05 / 30 / 07$ & 32.53 & 1.17 \\
\hline Nisqually & NIS-RF-3 & Ratfish & WBC & 2838-109 & 38.1 & 450 & $05 / 30 / 07$ & 29.35 & 1.81 \\
\hline Commencement Bay & CB-ES-1 & English Sole & WBC & $2838-110$ & 22.9 & 145 & 05/31/07 & 21.50 & 0.866 \\
\hline Commencement Bay & CB-ES-2 & English Sole & WBC & 2838-111 & 24.8 & 157 & $05 / 31 / 07$ & 23.68 & 1.30 \\
\hline Commencement Bay & CB-ES-3 & English Sole & WBC & $2838-112$ & 24.8 & 162 & $05 / 31 / 07$ & 23.16 & 1.51 \\
\hline Commencement Bay & CB-RF-1 & Ratfish & WBC & $2838-113$ & 40.6 & 320 & $05 / 31 / 07$ & 30.17 & 0.881 \\
\hline Commencement Bay & CB-RF-2 & Ratfish & WBC & $2838-114$ & 38.7 & 350 & $05 / 31 / 07$ & 28.54 & 1.24 \\
\hline Commencement Bay & CB-RF-3 & Ratfish & WBC & $2838-115$ & 38.7 & 390 & $05 / 31 / 07$ & 30.58 & 1.09 \\
\hline Duwamish & DU-ES-1 & English Sole & WBC & $2838-116$ & 17.8 & 75 & $05 / 18 / 07$ & 22.95 & 1.10 \\
\hline Duwamish & DU-ES-2 & English Sole & WBC & $2838-117$ & 21.6 & 96 & 05/18/07 & 20.32 & 1.78 \\
\hline Duwamish & DU-ES-3 & English Sole & WBC & $2838-118$ & 26.0 & 156 & 05/18/07 & 23.00 & 0.922 \\
\hline Admiralty Inlet & DF-EM-1 & Dogfish 1 & EM & $2838-126$ & & 410 & 09/19/07 & 52.97 & 1.04 \\
\hline Admiralty Inlet & DF-EM-2 & Dogfish 2 & EM & $2838-127$ & & 440 & 09/19/07 & 52.87 & 1.19 \\
\hline Admiralty Inlet & DF-LV-1 & Dogfish 1 & LV & $2838-132$ & & 498 & $09 / 19 / 07$ & 55.21 & 6.13 \\
\hline Admiralty Inlet & DF-LV-2 & Dogfish 2 & LV & $2838-133$ & & 260 & 09/19/07 & 52.95 & 2.40 \\
\hline Admiralty Inlet & DF-DIG-1 & Dogfish 1 & DIG & $2838-138$ & & 319 & 09/19/07 & 22.67 & 1.01 \\
\hline Admiralty Inlet & DF-DIG-2 & Dogfish 2 & DIG & 2838-139 & & 194 & 09/19/07 & 21.16 & 0.753 \\
\hline Admiralty Inlet & DF-SWC-1 & Dogfish 1 & SWC & $2838-174$ & & & 09/19/07 & 37.19 & 0.269 \\
\hline Admiralty Inlet & DF-SWC-2 & Dogfish 2 & SWC & $2838-175$ & & & 09/19/07 & 28.81 & 0.314 \\
\hline Admiralty Inlet & DF-WBWC-1 & Dogfish 1 & WBWC & $2838-177$ & 110.5 & 5400 & 09/19/07 & 39.76 & 0.612 \\
\hline Admiralty Inlet & DF-WBWC-3 & Dogfish 3 & WBWC & 2838-179 & 93.03 & 3140 & 09/19/07 & 36.70 & 0.440 \\
\hline Admiralty Inlet & DF-WBWC-2 & Dogfish 2 & WBWC & $2838-178$ & 104.8 & 4290 & 09/19/07 & 35.83 & 0.555 \\
\hline Admiralty Inlet & DF-EM-4 & Dogfish 4 & EM & 2838-129 & & 188 & $09 / 19 / 07$ & 52.59 & 0.752 \\
\hline Admiralty Inlet & DF-EM-6 & Dogfish 6 & EM & $2838-131$ & & 322 & $09 / 19 / 07$ & 56.33 & 0.822 \\
\hline Admiralty Inlet & DF-LV-4 & Dogfish 4 & LV & $2838-135$ & & 790 & 09/19/07 & 77.18 & 3.37 \\
\hline Admiralty Inlet & DF-LV-6 & Dogfish 6 & LV & $2838-137$ & & 434 & 09/19/07 & 31.93 & 1.86 \\
\hline Admiralty Inlet & DF-DIG-4 & Dogfish 4 & DIG & $2838-141$ & & 320 & 09/19/07 & 15.59 & 0.702 \\
\hline Admiralty Inlet & DF-DIG-6 & Dogfish 6 & DIG & $2838-143$ & & 327 & 09/19/07 & 17.06 & 1.28 \\
\hline Admiralty Inlet & DF-SWC-6 & Dogfish 6 & SWC & $2838-176$ & & & 09/19/07 & 35.23 & 0.285 \\
\hline Admiralty Inlet & DF-WBWC-4 & Dogfish 4 & WBWC & $2838-180$ & 111.1 & 5420 & 09/19/07 & 40.36 & 0.545 \\
\hline Admiralty Inlet & DF-WBWC-5 & Dogfish 5 & WBWC & $2838-181$ & 93.98 & 3020 & 09/19/07 & 41.03 & 0.882 \\
\hline Admiralty Inlet & DF-WBWC-6 & Dogfish 6 & WBWC & $2838-182$ & 98.11 & 4090 & 09/19/07 & 37.03 & 1.01 \\
\hline Admiralty Inlet & DF-SWC-4 & Dogfish 4 & SWC & $2838-183$ & & & 09/19/07 & 29.84 & 0.230 \\
\hline
\end{tabular}




\begin{tabular}{|c|c|c|c|c|c|c|c|c|c|}
\hline Client Code & Client Code & & & \multicolumn{6}{|c|}{ Units $=\mu \mathrm{g} / \mathrm{g}$ WET weight } \\
\hline & PSAMP Code & Organism & $\begin{array}{l}\text { Sample } \\
\text { Type }\end{array}$ & MSL Code & $\begin{array}{l}\text { Length } \\
\text { (cm) }\end{array}$ & $\begin{array}{l}\text { Weight } \\
\text { (g) }\end{array}$ & $\begin{array}{l}\text { Collection } \\
\text { Date }\end{array}$ & $\begin{array}{c}\text { Percent } \\
\text { Dry } \\
\text { Weight }\end{array}$ & Hg \\
\hline Station & & & & & & & \multicolumn{2}{|c|}{ Instrument: } & CVAA \\
\hline \multicolumn{7}{|c|}{$\begin{array}{l}\text { Laboratory Achieved Method Detection Limits (converted to wet weight) } \\
\text { Reporting Limit, Wet Weight }\end{array}$} & Average & 27.01 & 0.002 \\
\hline Vendovi & V-ES-5 & English Sole & WBC & $2838-5$ & 27.9 & 224 & 05/01/07 & 18.59 & 0.0424 \\
\hline Vendovi & V-ES-6 & English Sole & WBC & $2838-6$ & 27.9 & 250 & 05/01/07 & 17.79 & 0.0352 \\
\hline Vendovi & V-ES-8 & English Sole & WBC & $2838-8$ & 27.3 & 197 & 05/01/07 & 22.64 & 0.0324 \\
\hline Vendovi & V-RS-1 & Rock sole & WBC & $2838-10$ & 20.3 & 119 & $05 / 01 / 07$ & 21.79 & 0.0325 \\
\hline Vendovi & V-RS-2 & Rock sole & WBC & $2838-11$ & 21.0 & 142 & 05/01/07 & 21.23 & 0.0297 \\
\hline Vendovi & V-RS-3 & Rock sole & WBC & $2838-12$ & 31.1 & 510 & 05/01/07 & 20.30 & 0.0548 \\
\hline Vendovi & V-SSc-1 & Staghorn sculpin & WBC & $2838-13$ & 17.8 & 94 & 05/01/07 & 19.54 & 0.0586 \\
\hline Vendovi & V-SSc-2 & Staghorn sculpin & WBC & 2838-14 & 24.8 & 298 & 05/01/07 & 20.54 & 0.0641 \\
\hline Vendovi & V-SSc-3 & Staghorn sculpin & WBC & $2838-15$ & 25.4 & 294 & 05/01/07 & 19.90 & 0.0702 \\
\hline Vendovi & V-SP-1 & Shinner Surfperch & WBC & $2838-16$ & 7.62 & 16 & 05/01/07 & 23.28 & 0.0442 \\
\hline Vendovi & V-SP-2 & Shinner Surfperch & WBC & $2838-17$ & 8.89 & 15 & 05/01/07 & 22.19 & 0.0592 \\
\hline Vendovi & V-SP-3 & Shinner Surfperch & WBC & $2838-18$ & 8.89 & 16 & 05/01/07 & 24.13 & 0.0432 \\
\hline Vendovi & V-SC-COMP & Sea cucumber (3) & COMP & 2838-19 & & 5501 & 05/01/07 & 13.20 & 0.00677 \\
\hline Strait of Georgia & SG-ES-3 & English Sole & WBC & $2838-22$ & 26.7 & 184 & $05 / 02 / 07$ & 20.54 & 0.0271 \\
\hline Strait of Georgia & SG-ES-4 & English Sole & WBC & $2838-23$ & 26.0 & 189 & $05 / 02 / 07$ & 20.95 & 0.0373 \\
\hline Strait of Georgia & SG-ES-5 & English Sole & WBC & $2838-24$ & 26.7 & 192 & $05 / 02 / 07$ & 21.39 & 0.0379 \\
\hline Strait of Georgia & SG-RF-1 & Ratfish & WBC & $2838-29$ & 27.9 & 115 & $05 / 02 / 07$ & 19.86 & 0.0304 \\
\hline Strait of Georgia & SG-RF-2 & Ratfish & WBC & $2838-30$ & 35.6 & 320 & $05 / 02 / 07$ & 27.99 & 0.0635 \\
\hline Strait of Georgia & SG-RF-3 & Ratfish & WBC & 2838-31 & 48.3 & 770 & $05 / 02 / 07$ & 33.64 & 0.139 \\
\hline Strait of Georgia & SG-SC-COMP & Sea cucumber (6) & COMP & $2838-32$ & & 290 & $05 / 02 / 07$ & 7.70 & $0.00256 \mathrm{~J}$ \\
\hline Hood Canal & HC-ES-1 & English Sole & WBC & $2838-33$ & 22.9 & 131 & 05/03/07 & 17.91 & 0.0380 \\
\hline Hood Canal & HC-ES-2 & English Sole & WBC & $2838-34$ & 22.9 & 133 & $05 / 03 / 07$ & 20.28 & 0.0312 \\
\hline Hood Canal & HC-ES-3 & English Sole & WBC & $2838-35$ & 26.7 & 220 & $05 / 03 / 07$ & 21.19 & 0.0506 \\
\hline Hood Canal & HC-RS-1 & Rock sole & WBC & $2838-36$ & 31.8 & 460 & 05/03/07 & 22.07 & 0.0477 \\
\hline Hood Canal & HC-RF-1 & Ratfish & WBC & $2838-37$ & 39.4 & 480 & $05 / 03 / 07$ & 25.63 & 0.0764 \\
\hline Hood Canal & HC-RF-2 & Ratfish & WBC & $2838-38$ & 38.1 & 340 & $05 / 03 / 07$ & 27.04 & 0.141 \\
\hline Hood Canal & HC-RF-3 & Ratfish & WBC & 2838-39 & 42.5 & 450 & 05/03/07 & 27.61 & 0.164 \\
\hline Hood Canal & HC-SP1 & Shinner Surfperch & WBC & $2838-40$ & 9.53 & 16 & 05/03/07 & 27.84 & 0.0432 \\
\hline Hood Canal & HC-SP2 & Shinner Surfperch & WBC & $2838-41$ & 10.2 & 20 & 05/03/07 & 34.05 & 0.0381 \\
\hline Hood Canal & HC-GC-1 & Graceful Crab & WBC & $2838-43$ & 10.2 & 237 & 05/03/07 & 19.14 & 0.0173 \\
\hline Elliott Bay & EB-ES-1/2 & English Sole (2) & COMP & $2838-45-46$ & 15.8 & 44 & 05/16/07 & 21.44 & 0.0232 \\
\hline Elliott Bay & EB-ES-3 & English Sole & WBC & $2838-47$ & 26.0 & 157 & $05 / 16 / 07$ & 19.72 & 0.0874 \\
\hline Elliott Bay & EB-RF-1 & Ratfish & WBC & $2838-48$ & 40.6 & 350 & $05 / 16 / 07$ & 26.43 & 0.194 \\
\hline Elliott Bay & EB-RF-2 & Ratfish & WBC & $2838-49$ & 41.9 & 370 & $05 / 16 / 07$ & 27.23 & 0.0937 \\
\hline Elliott Bay & EB-RF-3 & Ratfish & WBC & $2838-50$ & 53.3 & 810 & $05 / 16 / 07$ & 27.11 & 0.210 \\
\hline Eagle Harbor & EH-ES-2 & English Sole & WBC & $2838-52$ & 35.6 & 420 & $05 / 19 / 07$ & 17.35 & 0.0704 \\
\hline Eagle Harbor & EH-ES-3 & English Sole & WBC & $2838-53$ & 22.2 & 89 & $05 / 19 / 07$ & 23.38 & 0.0825 \\
\hline Eagle Harbor & EH-ES-4 & English Sole & WBC & $2838-54$ & 27.3 & 199 & $05 / 19 / 07$ & 21.69 & 0.0414 \\
\hline Eagle Harbor & EH-RF-1 & Ratfish & WBC & $2838-55$ & 33.0 & 230 & $05 / 19 / 07$ & 26.50 & 0.0676 \\
\hline Eagle Harbor & EH-RF-2 & Ratfish & WBC & $2838-56$ & 38.1 & 380 & 05/19/07 & 23.39 & 0.156 \\
\hline Eagle Harbor & EH-RF-3 & Ratfish & WBC & $2838-57$ & 50.8 & 760 & $05 / 19 / 07$ & 31.68 & 0.233 \\
\hline Sinclair Inlet & SI-ES-1 & English Sole & WBC & $2838-58$ & 29.2 & 330 & 05/17/07 & 22.19 & 0.0295 \\
\hline Sinclair Inlet & SI-ES-2 & English Sole & WBC & $2838-59$ & 29.2 & 330 & $05 / 17 / 07$ & 19.35 & 0.0244 \\
\hline Sinclair Inlet & SI-ES-4 & English Sole & WBC & 2838-61 & 25.4 & 192 & $05 / 17 / 07$ & 40.66 & 0.0842 \\
\hline Sinclair Inlet & SI-ES-6 & English Sole & WBC & $2838-63$ & 25.4 & 178 & 05/17/07 & 20.31 & 0.0230 \\
\hline Sinclair Inlet & SI-ES-7 & English Sole & WBC & $2838-64$ & 25.4 & 179 & 05/17/07 & 20.51 & 0.0603 \\
\hline Sinclair Inlet & SI-ES-8 & English Sole & WBC & $2838-65$ & 29.2 & 261 & $05 / 17 / 07$ & 20.71 & 0.0373 \\
\hline Sinclair Inlet & SI-RS-1/2 & Rock sole (2) & COMP & $2838-67-68$ & 10.2 & 23 & $05 / 17 / 07$ & 24.28 & 0.0218 \\
\hline Sinclair Inlet & SI-RS-3 & Rock sole & WBC & $2838-69$ & 27.3 & 320 & $05 / 17 / 07$ & 21.12 & 0.0395 \\
\hline Sinclair Inlet & SI-RS-4 & Rock sole & WBC & $2838-70$ & 26.7 & 220 & $05 / 17 / 07$ & 21.17 & 0.0868 \\
\hline Sinclair Inlet & SI-SS-1 & Sand sole & WBC & $2838-71$ & 25.4 & 270 & $05 / 17 / 07$ & 21.66 & 0.111 \\
\hline Sinclair Inlet & SI-SS-2 & Sand sole & WBC & $2838-72$ & 27.3 & 290 & $05 / 17 / 07$ & 22.13 & 0.0879 \\
\hline Sinclair Inlet & SI-SS-3 & Sand sole & WBC & $2838-73$ & 29.8 & 320 & 05/17/07 & 21.05 & 0.101 \\
\hline
\end{tabular}




\begin{tabular}{|c|c|c|c|c|c|c|c|c|c|}
\hline Client Code & Client Code & & & \multicolumn{6}{|c|}{ Units $=\mu \mathrm{g} / \mathrm{g}$ WET weight } \\
\hline \multirow[t]{2}{*}{ Station } & PSAMP Code & Organism & $\begin{array}{l}\text { Sample } \\
\text { Type }\end{array}$ & MSL Code & $\begin{array}{l}\text { Length } \\
\text { (cm) }\end{array}$ & $\begin{array}{l}\text { Weight } \\
\text { (g) }\end{array}$ & $\begin{array}{l}\text { Collection } \\
\text { Date }\end{array}$ & $\begin{array}{c}\text { Percent } \\
\text { Dry } \\
\text { Weight }\end{array}$ & $\mathrm{Hg}$ \\
\hline & & & & & & & \multicolumn{2}{|r|}{ Instrument: } & CVAA \\
\hline \multicolumn{7}{|c|}{$\begin{array}{l}\text { Laboratory Achieved Method Detection Limits (converted to wet weight) } \\
\text { Reporting Limit, Wet Weight }\end{array}$} & Average & 27.01 & $\begin{array}{l}0.002 \\
0.006\end{array}$ \\
\hline Sinclair Inlet & SI-SSc-1/2 & Staghorn sculpin (2) & COMP & $2838-77-78$ & 14.9 & 48 & 05/17/07 & 20.66 & 0.0517 \\
\hline Sinclair Inlet & SI-SSc-3/5 & Staghorn sculpin (2) & COMP & $2838-79-81$ & 14.3 & 53 & 05/17/07 & 20.03 & 0.0499 \\
\hline Sinclair Inlet & SI-SSc-4/6 & Staghorn sculpin (2) & COMP & $2838-80-82$ & 16.2 & 75 & $05 / 17 / 07$ & 19.51 & 0.0864 \\
\hline Sinclair Inlet & SI-RF-1 & Ratfish & WBC & $2838-83$ & 41.3 & 550 & $05 / 17 / 07$ & 32.67 & 0.257 \\
\hline Sinclair Inlet & SI-RF-2 & Ratfish & WBC & $2838-84$ & 36.8 & 380 & $05 / 17 / 07$ & 26.61 & 0.138 \\
\hline Sinclair Inlet & SI-SP-4/5 & Shinner Surfperch (2) & ) COMP & $2838-88-89$ & 7.62 & 13 & 05/17/07 & 24.47 & 0.0587 \\
\hline Sinclair Inlet & SI-SP-6/2 & Shinner Surfperch (2) & COMP & $2838-90-86$ & 6.67 & 11 & 05/17/07 & 24.68 & 0.0536 \\
\hline Sinclair Inlet & SI-SP-7/3 & Shinner Surfperch (2) & COMP & $2838-91-87$ & 7.3 & 11 & $05 / 17 / 07$ & 25.12 & 0.0872 \\
\hline Sinclair Inlet & SI-GC-2/6 & Graceful Crab (2) & COMP & 2838-93-97 & 7.62 & 103 & $05 / 17 / 07$ & 28.75 & 0.0624 \\
\hline Sinclair Inlet & SI-GC-3/4 & Graceful Crab (2) & COMP & 2838-94-95 & 6.99 & 67 & 05/17/07 & 33.60 & 0.0363 \\
\hline Sinclair Inlet & SI-GC-7/1 & Graceful Crab (2) & COMP & $2838-98-92$ & 6.67 & 60 & $05 / 17 / 07$ & 54.85 & 0.103 \\
\hline Sinclair Inlet & SI-GC-5/8 & Graceful Crab (2) & COMP & 2838-96-99 & 7.62 & 92 & $05 / 17 / 07$ & 30.89 & 0.0516 \\
\hline Sinclair Inlet & SI-SC-COMP & Sea cucumber (6) & COMP & $2838-100$ & & 4100 & $05 / 17 / 07$ & 9.52 & 0.0137 \\
\hline Port Gardner & PG-ES-1 & English Sole & WBC & $2838-101$ & 24.8 & 158 & $05 / 29 / 07$ & 19.23 & 0.0306 \\
\hline Port Gardner & PG-ES-2 & English Sole & WBC & $2838-102$ & 21.6 & 101 & $05 / 29 / 07$ & 20.82 & 0.0210 \\
\hline Port Gardner & PG-ES-3 & English Sole & WBC & 2838-103 & 24.8 & 128 & $05 / 29 / 07$ & 19.95 & 0.0269 \\
\hline Nisqually & NIS-ES-1 & English Sole & WBC & 2838-104 & 25.4 & 166 & $05 / 30 / 07$ & 20.67 & 0.0641 \\
\hline Nisqually & NIS-ES-2 & English Sole & WBC & 2838-105 & 26.7 & 214 & 05/30/07 & 21.52 & 0.0603 \\
\hline Nisqually & NIS-ES-3 & English Sole & WBC & 2838-106 & 30.5 & 290 & $05 / 30 / 07$ & 21.61 & 0.0590 \\
\hline Nisqually & NIS-RF-1 & Ratfish & WBC & 2838-107 & 48.3 & 620 & $05 / 30 / 07$ & 31.21 & 0.231 \\
\hline Nisqually & NIS-RF-2 & Ratfish & WBC & 2838-108 & 40.6 & 430 & $05 / 30 / 07$ & 32.53 & 0.158 \\
\hline Nisqually & NIS-RF-3 & Ratfish & WBC & 2838-109 & 38.1 & 450 & $05 / 30 / 07$ & 29.35 & 0.132 \\
\hline Commencement Bay & CB-ES-1 & English Sole & WBC & $2838-110$ & 22.9 & 145 & $05 / 31 / 07$ & 21.50 & 0.0344 \\
\hline Commencement Bay & CB-ES-2 & English Sole & WBC & $2838-111$ & 24.8 & 157 & $05 / 31 / 07$ & 23.68 & 0.0774 \\
\hline Commencement Bay & CB-ES-3 & English Sole & WBC & $2838-112$ & 24.8 & 162 & $05 / 31 / 07$ & 23.16 & 0.0530 \\
\hline Commencement Bay & CB-RF-1 & Ratfish & WBC & $2838-113$ & 40.6 & 320 & $05 / 31 / 07$ & 30.17 & 0.0712 \\
\hline Commencement Bay & CB-RF-2 & Ratfish & WBC & $2838-114$ & 38.7 & 350 & $05 / 31 / 07$ & 28.54 & 0.115 \\
\hline Commencement Bay & CB-RF-3 & Ratfish & WBC & $2838-115$ & 38.7 & 390 & $05 / 31 / 07$ & 30.58 & 0.118 \\
\hline Duwamish & DU-ES-1 & English Sole & WBC & $2838-116$ & 17.8 & 75 & $05 / 18 / 07$ & 22.95 & 0.0390 \\
\hline Duwamish & DU-ES-2 & English Sole & WBC & $2838-117$ & 21.6 & 96 & $05 / 18 / 07$ & 20.32 & 0.0360 \\
\hline Duwamish & DU-ES-3 & English Sole & WBC & $2838-118$ & 26.0 & 156 & 05/18/07 & 23.00 & 0.0458 \\
\hline Admiralty Inlet & DF-EM-1 & Dogfish 1 & EM & $2838-126$ & & 410 & 09/19/07 & 52.97 & 0.0195 \\
\hline Admiralty Inlet & DF-EM-2 & Dogfish 2 & EM & $2838-127$ & & 440 & 09/19/07 & 52.87 & 0.0152 \\
\hline Admiralty Inlet & DF-LV-1 & Dogfish 1 & LV & 2838-132 & & 498 & 09/19/07 & 55.21 & 0.212 \\
\hline Admiralty Inlet & DF-LV-2 & Dogfish 2 & LV & $2838-133$ & & 260 & 09/19/07 & 52.95 & 0.237 \\
\hline Admiralty Inlet & DF-DIG-1 & Dogfish 1 & DIG & $2838-138$ & & 319 & 09/19/07 & 22.67 & 0.162 \\
\hline Admiralty Inlet & DF-DIG-2 & Dogfish 2 & DIG & 2838-139 & & 194 & 09/19/07 & 21.16 & 0.220 \\
\hline Admiralty Inlet & DF-SWC-1 & Dogfish 1 & SWC & $2838-174$ & & & 09/19/07 & 37.19 & 0.829 \\
\hline Admiralty Inlet & DF-SWC-2 & Dogfish 2 & SWC & $2838-175$ & & & 09/19/07 & 28.81 & 0.651 \\
\hline Admiralty Inlet & DF-WBWC-1 & Dogfish 1 & WBWC & $2838-177$ & 110.5 & 5400 & 09/19/07 & 39.76 & 0.577 \\
\hline Admiralty Inlet & DF-WBWC-3 & Dogfish 3 & WBWC & $2838-179$ & 93.03 & 3140 & 09/19/07 & 36.70 & 0.738 \\
\hline Admiralty Inlet & DF-WBWC-2 & Dogfish 2 & WBWC & $2838-178$ & 104.8 & 4290 & 09/19/07 & 35.83 & 0.566 \\
\hline Admiralty Inlet & DF-EM-4 & Dogfish 4 & EM & $2838-129$ & & 188 & 09/19/07 & 52.59 & 0.00657 \\
\hline Admiralty Inlet & DF-EM-6 & Dogfish 6 & EM & $2838-131$ & & 322 & 09/19/07 & 56.33 & 0.0133 \\
\hline Admiralty Inlet & DF-LV-4 & Dogfish 4 & LV & $2838-135$ & & 790 & 09/19/07 & 77.18 & 0.0458 \\
\hline Admiralty Inlet & DF-LV-6 & Dogfish 6 & $\mathrm{LV}$ & $2838-137$ & & 434 & 09/19/07 & 31.93 & 0.0773 \\
\hline Admiralty Inlet & DF-DIG-4 & Dogfish 4 & DIG & $2838-141$ & & 320 & 09/19/07 & 15.59 & 0.0589 \\
\hline Admiralty Inlet & DF-DIG-6 & Dogfish 6 & DIG & $2838-143$ & & 327 & 09/19/07 & 17.06 & 0.0740 \\
\hline Admiralty Inlet & DF-SWC-6 & Dogfish 6 & SWC & $2838-176$ & & & 09/19/07 & 35.23 & 0.860 \\
\hline Admiralty Inlet & DF-WBWC-4 & Dogfish 4 & WBWC & $2838-180$ & 111.1 & 5420 & 09/19/07 & 40.36 & 0.311 \\
\hline Admiralty Inlet & DF-WBWC-5 & Dogfish 5 & WBWC & $2838-181$ & 93.98 & 3020 & 09/19/07 & 41.03 & 0.620 \\
\hline Admiralty Inlet & DF-WBWC-6 & Dogfish 6 & WBWC & $2838-182$ & 98.11 & 4090 & 09/19/07 & 37.03 & 0.500 \\
\hline Admiralty Inlet & DF-SWC-4 & Dogfish 4 & SWC & $2838-183$ & & & $09 / 19 / 07$ & 29.84 & 0.352 \\
\hline
\end{tabular}




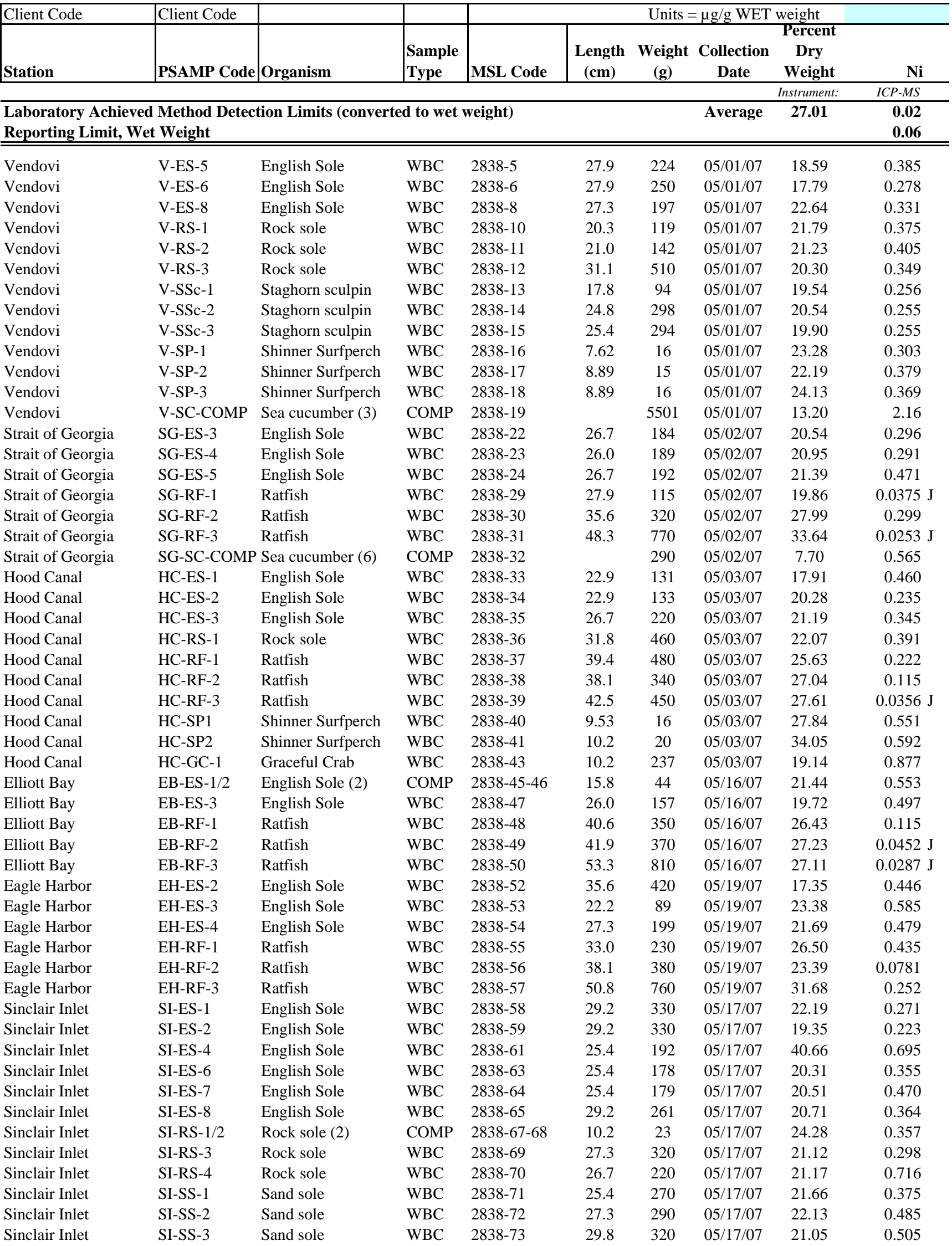




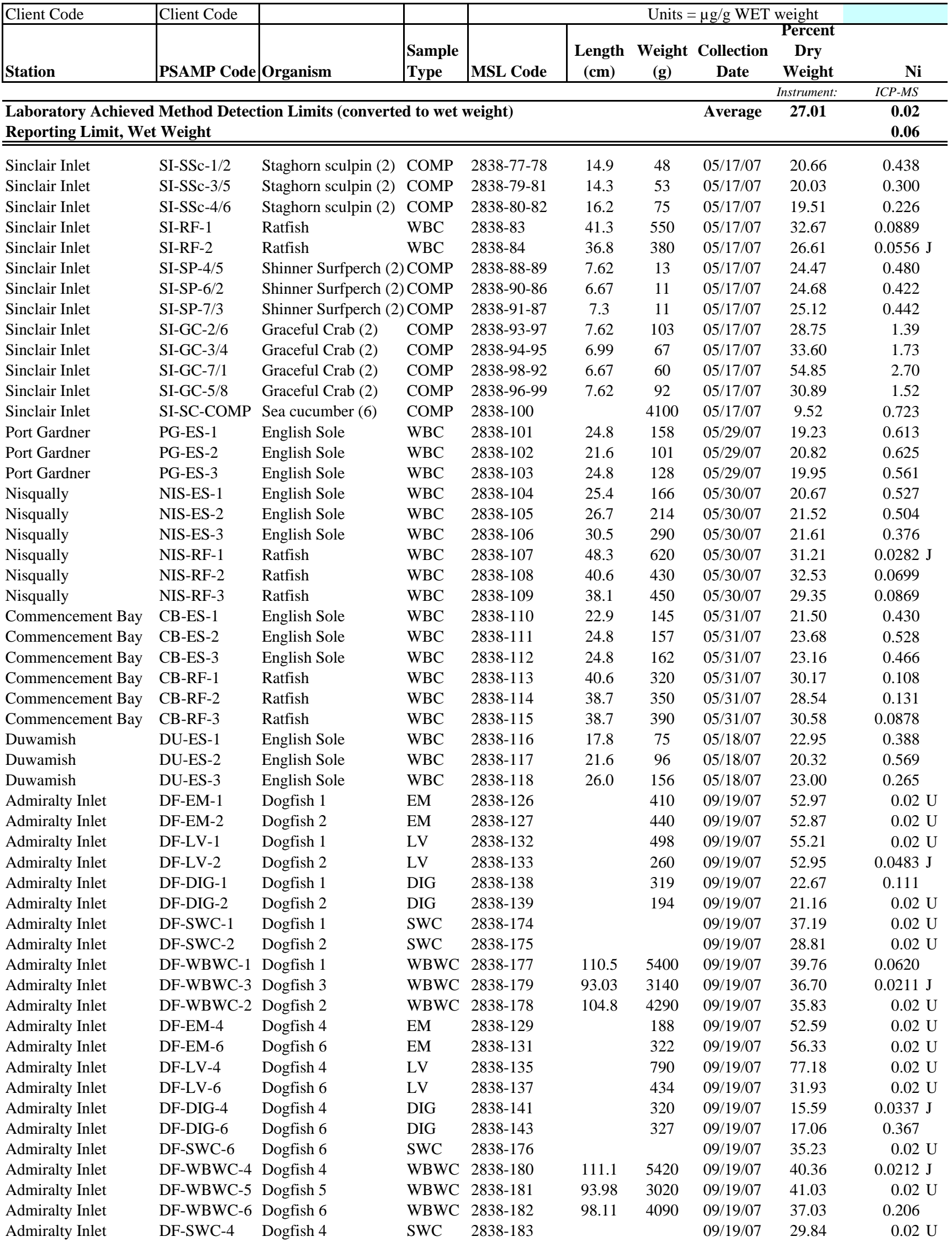




\begin{tabular}{|c|c|c|c|c|c|c|c|c|c|}
\hline Client Code & Client Code & & & \multicolumn{6}{|c|}{ Units $=\mu \mathrm{g} / \mathrm{g}$ WET weight } \\
\hline Station & PSAMP Code & Organism & $\begin{array}{l}\text { Sample } \\
\text { Type }\end{array}$ & MSL Code & $\begin{array}{l}\text { Length } \\
\text { (cm) }\end{array}$ & $\begin{array}{c}\text { Weight } \\
\text { (g) }\end{array}$ & $\begin{array}{c}\text { Collection } \\
\text { Date }\end{array}$ & $\begin{array}{c}\text { Percent } \\
\text { Dry } \\
\text { Weight }\end{array}$ & $\mathbf{P b}$ \\
\hline & & & & & & & & Instrument: & ICP-MS \\
\hline $\begin{array}{l}\text { Laboratory Ach } \\
\text { Reporting Limit }\end{array}$ & $\begin{array}{l}\text { Method Detec } \\
\text { t Weight }\end{array}$ & tion Limits (conver & d to we & weight) & & & Average & 27.01 & $\begin{array}{r}0.0009 \\
0.003 \\
\end{array}$ \\
\hline Vendovi & V-ES-5 & English Sole & WBC & $2838-5$ & 27.9 & 224 & 05/01/07 & 18.59 & 0.127 \\
\hline Vendovi & V-ES-6 & English Sole & WBC & $2838-6$ & 27.9 & 250 & 05/01/07 & 17.79 & 0.0763 \\
\hline Vendovi & V-ES-8 & English Sole & WBC & $2838-8$ & 27.3 & 197 & $05 / 01 / 07$ & 22.64 & 0.118 \\
\hline Vendovi & V-RS-1 & Rock sole & WBC & $2838-10$ & 20.3 & 119 & $05 / 01 / 07$ & 21.79 & 0.0510 \\
\hline Vendovi & V-RS-2 & Rock sole & WBC & $2838-11$ & 21.0 & 142 & 05/01/07 & 21.23 & 0.0431 \\
\hline Vendovi & V-RS-3 & Rock sole & WBC & $2838-12$ & 31.1 & 510 & 05/01/07 & 20.30 & 0.0367 \\
\hline Vendovi & V-SSc-1 & Staghorn sculpin & WBC & $2838-13$ & 17.8 & 94 & $05 / 01 / 07$ & 19.54 & 0.0260 \\
\hline Vendovi & V-SSc-2 & Staghorn sculpin & WBC & $2838-14$ & 24.8 & 298 & $05 / 01 / 07$ & 20.54 & 0.00723 \\
\hline Vendovi & V-SSc-3 & Staghorn sculpin & WBC & $2838-15$ & 25.4 & 294 & $05 / 01 / 07$ & 19.90 & 0.0120 \\
\hline Vendovi & V-SP-1 & Shinner Surfperch & WBC & $2838-16$ & 7.62 & 16 & 05/01/07 & 23.28 & 0.00836 \\
\hline Vendovi & V-SP-2 & Shinner Surfperch & WBC & $2838-17$ & 8.89 & 15 & 05/01/07 & 22.19 & 0.0201 \\
\hline Vendovi & V-SP-3 & Shinner Surfperch & WBC & $2838-18$ & 8.89 & 16 & 05/01/07 & 24.13 & 0.0113 \\
\hline Vendovi & V-SC-COMP & Sea cucumber (3) & COMP & 2838-19 & & 5501 & $05 / 01 / 07$ & 13.20 & 0.570 \\
\hline Strait of Georgia & SG-ES-3 & English Sole & WBC & $2838-22$ & 26.7 & 184 & $05 / 02 / 07$ & 20.54 & 0.0705 \\
\hline Strait of Georgia & SG-ES-4 & English Sole & WBC & $2838-23$ & 26.0 & 189 & $05 / 02 / 07$ & 20.95 & 0.0872 \\
\hline Strait of Georgia & SG-ES-5 & English Sole & WBC & $2838-24$ & 26.7 & 192 & $05 / 02 / 07$ & 21.39 & 0.108 \\
\hline Strait of Georgia & SG-RF-1 & Ratfish & WBC & $2838-29$ & 27.9 & 115 & $05 / 02 / 07$ & 19.86 & 0.00775 \\
\hline Strait of Georgia & SG-RF-2 & Ratfish & WBC & $2838-30$ & 35.6 & 320 & $05 / 02 / 07$ & 27.99 & 0.0243 \\
\hline Strait of Georgia & SG-RF-3 & Ratfish & WBC & $2838-31$ & 48.3 & 770 & $05 / 02 / 07$ & 33.64 & 0.0102 \\
\hline Strait of Georgia & SG-SC-COMP & Sea cucumber (6) & COMP & $2838-32$ & & 290 & $05 / 02 / 07$ & 7.70 & 0.0541 \\
\hline Hood Canal & HC-ES-1 & English Sole & WBC & $2838-33$ & 22.9 & 131 & $05 / 03 / 07$ & 17.91 & 0.0494 \\
\hline Hood Canal & HC-ES-2 & English Sole & WBC & $2838-34$ & 22.9 & 133 & $05 / 03 / 07$ & 20.28 & 0.0131 \\
\hline Hood Canal & HC-ES-3 & English Sole & WBC & $2838-35$ & 26.7 & 220 & $05 / 03 / 07$ & 21.19 & 0.0631 \\
\hline Hood Canal & HC-RS-1 & Rock sole & WBC & $2838-36$ & 31.8 & 460 & 05/03/07 & 22.07 & 0.0349 \\
\hline Hood Canal & HC-RF-1 & Ratfish & WBC & $2838-37$ & 39.4 & 480 & 05/03/07 & 25.63 & 0.0238 \\
\hline Hood Canal & HC-RF-2 & Ratfish & WBC & $2838-38$ & 38.1 & 340 & $05 / 03 / 07$ & 27.04 & 0.00957 \\
\hline Hood Canal & HC-RF-3 & Ratfish & WBC & $2838-39$ & 42.5 & 450 & 05/03/07 & 27.61 & 0.0115 \\
\hline Hood Canal & HC-SP1 & Shinner Surfperch & WBC & $2838-40$ & 9.53 & 16 & 05/03/07 & 27.84 & 0.0888 \\
\hline Hood Canal & HC-SP2 & Shinner Surfperch & WBC & $2838-41$ & 10.2 & 20 & 05/03/07 & 34.05 & 0.0256 \\
\hline Hood Canal & HC-GC-1 & Graceful Crab & WBC & $2838-43$ & 10.2 & 237 & 05/03/07 & 19.14 & 0.0570 \\
\hline Elliott Bay & EB-ES-1/2 & English Sole (2) & COMP & $2838-45-46$ & 15.8 & 44 & $05 / 16 / 07$ & 21.44 & 0.272 \\
\hline Elliott Bay & EB-ES-3 & English Sole & WBC & $2838-47$ & 26.0 & 157 & $05 / 16 / 07$ & 19.72 & 0.306 \\
\hline Elliott Bay & EB-RF-1 & Ratfish & WBC & $2838-48$ & 40.6 & 350 & $05 / 16 / 07$ & 26.43 & 0.0616 \\
\hline Elliott Bay & EB-RF-2 & Ratfish & WBC & $2838-49$ & 41.9 & 370 & $05 / 16 / 07$ & 27.23 & 0.0260 \\
\hline Elliott Bay & EB-RF-3 & Ratfish & WBC & $2838-50$ & 53.3 & 810 & $05 / 16 / 07$ & 27.11 & 0.0382 \\
\hline Eagle Harbor & EH-ES-2 & English Sole & WBC & $2838-52$ & 35.6 & 420 & 05/19/07 & 17.35 & 0.239 \\
\hline Eagle Harbor & EH-ES-3 & English Sole & WBC & $2838-53$ & 22.2 & 89 & 05/19/07 & 23.38 & 0.596 \\
\hline Eagle Harbor & EH-ES-4 & English Sole & WBC & $2838-54$ & 27.3 & 199 & $05 / 19 / 07$ & 21.69 & 0.203 \\
\hline Eagle Harbor & EH-RF-1 & Ratfish & WBC & $2838-55$ & 33.0 & 230 & 05/19/07 & 26.50 & 0.243 \\
\hline Eagle Harbor & EH-RF-2 & Ratfish & WBC & $2838-56$ & 38.1 & 380 & $05 / 19 / 07$ & 23.39 & 0.0255 \\
\hline Eagle Harbor & EH-RF-3 & Ratfish & WBC & $2838-57$ & 50.8 & 760 & $05 / 19 / 07$ & 31.68 & 0.127 \\
\hline Sinclair Inlet & SI-ES-1 & English Sole & WBC & $2838-58$ & 29.2 & 330 & $05 / 17 / 07$ & 22.19 & 0.402 \\
\hline Sinclair Inlet & SI-ES-2 & English Sole & WBC & $2838-59$ & 29.2 & 330 & $05 / 17 / 07$ & 19.35 & 1.14 \\
\hline Sinclair Inlet & SI-ES-4 & English Sole & WBC & $2838-61$ & 25.4 & 192 & $05 / 17 / 07$ & 40.66 & 0.793 \\
\hline Sinclair Inlet & SI-ES-6 & English Sole & WBC & $2838-63$ & 25.4 & 178 & $05 / 17 / 07$ & 20.31 & 0.238 \\
\hline Sinclair Inlet & SI-ES-7 & English Sole & WBC & $2838-64$ & 25.4 & 179 & 05/17/07 & 20.51 & 0.388 \\
\hline Sinclair Inlet & SI-ES-8 & English Sole & WBC & $2838-65$ & 29.2 & 261 & $05 / 17 / 07$ & 20.71 & 0.383 \\
\hline Sinclair Inlet & SI-RS-1/2 & Rock sole (2) & COMP & $2838-67-68$ & 10.2 & 23 & $05 / 17 / 07$ & 24.28 & 0.391 \\
\hline Sinclair Inlet & SI-RS-3 & Rock sole & WBC & $2838-69$ & 27.3 & 320 & $05 / 17 / 07$ & 21.12 & 0.0826 \\
\hline Sinclair Inlet & SI-RS-4 & Rock sole & WBC & $2838-70$ & 26.7 & 220 & $05 / 17 / 07$ & 21.17 & 0.514 \\
\hline Sinclair Inlet & SI-SS-1 & Sand sole & WBC & $2838-71$ & 25.4 & 270 & $05 / 17 / 07$ & 21.66 & 0.105 \\
\hline Sinclair Inlet & SI-SS-2 & Sand sole & WBC & $2838-72$ & 27.3 & 290 & $05 / 17 / 07$ & 22.13 & 0.0398 \\
\hline Sinclair Inlet & SI-SS-3 & Sand sole & WBC & $2838-73$ & 29.8 & 320 & $05 / 17 / 07$ & 21.05 & 0.0680 \\
\hline
\end{tabular}




\begin{tabular}{|c|c|c|c|c|c|c|c|c|c|}
\hline Client Code & Client Code & & & \multicolumn{6}{|c|}{ Units $=\mu \mathrm{g} / \mathrm{g}$ WET weight } \\
\hline Station & PSAMP Code & Organism & $\begin{array}{l}\text { Sample } \\
\text { Type }\end{array}$ & MSL Code & $\begin{array}{l}\text { Length } \\
\text { (cm) }\end{array}$ & $\begin{array}{l}\text { Weight } \\
\text { (g) }\end{array}$ & $\begin{array}{l}\text { Collection } \\
\text { Date }\end{array}$ & $\begin{array}{c}\text { Percent } \\
\text { Dry } \\
\text { Weight }\end{array}$ & $\mathbf{P b}$ \\
\hline & & & & & & & & Instrument: & ICP-MS \\
\hline $\begin{array}{l}\text { Laboratory Achievec } \\
\text { Reporting Limit, We }\end{array}$ & $\begin{array}{l}\text { Method Detec } \\
\text { t Weight }\end{array}$ & tion Limits (conver & ed to wet & weight) & & & Average & 27.01 & $\begin{array}{r}0.0009 \\
0.003\end{array}$ \\
\hline Sinclair Inlet & SI-SSc-1/2 & Staghorn sculpin (2) & COMP & $2838-77-78$ & 14.9 & 48 & 05/17/07 & 20.66 & 0.242 \\
\hline Sinclair Inlet & SI-SSc-3/5 & Staghorn sculpin (2) & COMP & $2838-79-81$ & 14.3 & 53 & 05/17/07 & 20.03 & 0.126 \\
\hline Sinclair Inlet & SI-SSc-4/6 & Staghorn sculpin (2) & COMP & $2838-80-82$ & 16.2 & 75 & $05 / 17 / 07$ & 19.51 & 0.144 \\
\hline Sinclair Inlet & SI-RF-1 & Ratfish & WBC & $2838-83$ & 41.3 & 550 & $05 / 17 / 07$ & 32.67 & 0.0725 \\
\hline Sinclair Inlet & SI-RF-2 & Ratfish & WBC & $2838-84$ & 36.8 & 380 & $05 / 17 / 07$ & 26.61 & 0.0359 \\
\hline Sinclair Inlet & SI-SP-4/5 & Shinner Surfperch $(2$ & ) COMP & $2838-88-89$ & 7.62 & 13 & 05/17/07 & 24.47 & 0.123 \\
\hline Sinclair Inlet & SI-SP-6/2 & Shinner Surfperch $(2$ & COMP & $2838-90-86$ & 6.67 & 11 & $05 / 17 / 07$ & 24.68 & 0.0513 \\
\hline Sinclair Inlet & SI-SP-7/3 & Shinner Surfperch (2 & COMP & $2838-91-87$ & 7.3 & 11 & 05/17/07 & 25.12 & 0.168 \\
\hline Sinclair Inlet & SI-GC-2/6 & Graceful Crab (2) & COMP & 2838-93-97 & 7.62 & 103 & $05 / 17 / 07$ & 28.75 & 1.67 \\
\hline Sinclair Inlet & SI-GC-3/4 & Graceful Crab (2) & COMP & 2838-94-95 & 6.99 & 67 & 05/17/07 & 33.60 & 0.763 \\
\hline Sinclair Inlet & SI-GC-7/1 & Graceful Crab (2) & COMP & $2838-98-92$ & 6.67 & 60 & $05 / 17 / 07$ & 54.85 & 2.68 \\
\hline Sinclair Inlet & SI-GC-5/8 & Graceful Crab (2) & COMP & 2838-96-99 & 7.62 & 92 & $05 / 17 / 07$ & 30.89 & 0.778 \\
\hline Sinclair Inlet & SI-SC-COMP & Sea cucumber (6) & COMP & $2838-100$ & & 4100 & $05 / 17 / 07$ & 9.52 & 0.920 \\
\hline Port Gardner & PG-ES-1 & English Sole & WBC & $2838-101$ & 24.8 & 158 & $05 / 29 / 07$ & 19.23 & 0.107 \\
\hline Port Gardner & PG-ES-2 & English Sole & WBC & $2838-102$ & 21.6 & 101 & $05 / 29 / 07$ & 20.82 & 0.0954 \\
\hline Port Gardner & PG-ES-3 & English Sole & WBC & $2838-103$ & 24.8 & 128 & $05 / 29 / 07$ & 19.95 & 0.101 \\
\hline Nisqually & NIS-ES-1 & English Sole & WBC & 2838-104 & 25.4 & 166 & $05 / 30 / 07$ & 20.67 & 0.482 \\
\hline Nisqually & NIS-ES-2 & English Sole & WBC & $2838-105$ & 26.7 & 214 & $05 / 30 / 07$ & 21.52 & 0.286 \\
\hline Nisqually & NIS-ES-3 & English Sole & WBC & 2838-106 & 30.5 & 290 & $05 / 30 / 07$ & 21.61 & 0.225 \\
\hline Nisqually & NIS-RF-1 & Ratfish & WBC & 2838-107 & 48.3 & 620 & $05 / 30 / 07$ & 31.21 & 0.0337 \\
\hline Nisqually & NIS-RF-2 & Ratfish & WBC & 2838-108 & 40.6 & 430 & $05 / 30 / 07$ & 32.53 & 0.0400 \\
\hline Nisqually & NIS-RF-3 & Ratfish & WBC & 2838-109 & 38.1 & 450 & $05 / 30 / 07$ & 29.35 & 0.0179 \\
\hline Commencement Bay & CB-ES-1 & English Sole & WBC & $2838-110$ & 22.9 & 145 & $05 / 31 / 07$ & 21.50 & 0.301 \\
\hline Commencement Bay & CB-ES-2 & English Sole & WBC & $2838-111$ & 24.8 & 157 & $05 / 31 / 07$ & 23.68 & 0.400 \\
\hline Commencement Bay & CB-ES-3 & English Sole & WBC & $2838-112$ & 24.8 & 162 & $05 / 31 / 07$ & 23.16 & 0.533 \\
\hline Commencement Bay & CB-RF-1 & Ratfish & WBC & $2838-113$ & 40.6 & 320 & $05 / 31 / 07$ & 30.17 & 0.152 \\
\hline Commencement Bay & CB-RF-2 & Ratfish & WBC & $2838-114$ & 38.7 & 350 & $05 / 31 / 07$ & 28.54 & 0.161 \\
\hline Commencement Bay & CB-RF-3 & Ratfish & WBC & $2838-115$ & 38.7 & 390 & $05 / 31 / 07$ & 30.58 & 0.0563 \\
\hline Duwamish & DU-ES-1 & English Sole & WBC & $2838-116$ & 17.8 & 75 & 05/18/07 & 22.95 & 0.308 \\
\hline Duwamish & DU-ES-2 & English Sole & WBC & $2838-117$ & 21.6 & 96 & $05 / 18 / 07$ & 20.32 & 0.264 \\
\hline Duwamish & DU-ES-3 & English Sole & WBC & $2838-118$ & 26.0 & 156 & $05 / 18 / 07$ & 23.00 & 0.146 \\
\hline Admiralty Inlet & DF-EM-1 & Dogfish 1 & EM & $2838-126$ & & 410 & 09/19/07 & 52.97 & 0.00561 \\
\hline Admiralty Inlet & DF-EM-2 & Dogfish 2 & EM & $2838-127$ & & 440 & 09/19/07 & 52.87 & $0.0009 \mathrm{U}$ \\
\hline Admiralty Inlet & DF-LV-1 & Dogfish 1 & LV & $2838-132$ & & 498 & 09/19/07 & 55.21 & 0.00436 \\
\hline Admiralty Inlet & DF-LV-2 & Dogfish 2 & LV & $2838-133$ & & 260 & 09/19/07 & 52.95 & 0.00381 \\
\hline Admiralty Inlet & DF-DIG-1 & Dogfish 1 & DIG & $2838-138$ & & 319 & 09/19/07 & 22.67 & 0.0117 \\
\hline Admiralty Inlet & DF-DIG-2 & Dogfish 2 & DIG & 2838-139 & & 194 & 09/19/07 & 21.16 & $0.00226 \mathrm{~J}$ \\
\hline Admiralty Inlet & DF-SWC-1 & Dogfish 1 & SWC & $2838-174$ & & & 09/19/07 & 37.19 & 0.00315 \\
\hline Admiralty Inlet & DF-SWC-2 & Dogfish 2 & SWC & $2838-175$ & & & 09/19/07 & 28.81 & $0.00198 \mathrm{~J}$ \\
\hline Admiralty Inlet & DF-WBWC-1 & Dogfish 1 & WBWC & $2838-177$ & 110.5 & 5400 & 09/19/07 & 39.76 & 0.00331 \\
\hline Admiralty Inlet & DF-WBWC-3 & Dogfish 3 & WBWC & 2838-179 & 93.03 & 3140 & 09/19/07 & 36.70 & 0.00320 \\
\hline Admiralty Inlet & DF-WBWC-2 & Dogfish 2 & WBWC & $2838-178$ & 104.8 & 4290 & 09/19/07 & 35.83 & 0.00351 \\
\hline Admiralty Inlet & DF-EM-4 & Dogfish 4 & EM & $2838-129$ & & 188 & 09/19/07 & 52.59 & $0.0009 \mathrm{U}$ \\
\hline Admiralty Inlet & DF-EM-6 & Dogfish 6 & EM & $2838-131$ & & 322 & 09/19/07 & 56.33 & $0.0009 \mathrm{U}$ \\
\hline Admiralty Inlet & DF-LV-4 & Dogfish 4 & LV & $2838-135$ & & 790 & 09/19/07 & 77.18 & $0.00290 \mathrm{~J}$ \\
\hline Admiralty Inlet & DF-LV-6 & Dogfish 6 & LV & $2838-137$ & & 434 & 09/19/07 & 31.93 & $0.00101 \mathrm{~J}$ \\
\hline Admiralty Inlet & DF-DIG-4 & Dogfish 4 & DIG & $2838-141$ & & 320 & $09 / 19 / 07$ & 15.59 & 0.00479 \\
\hline Admiralty Inlet & DF-DIG-6 & Dogfish 6 & DIG & $2838-143$ & & 327 & 09/19/07 & 17.06 & 0.0432 \\
\hline Admiralty Inlet & DF-SWC-6 & Dogfish 6 & SWC & $2838-176$ & & & 09/19/07 & 35.23 & $0.00237 \mathrm{~J}$ \\
\hline Admiralty Inlet & DF-WBWC-4 & Dogfish 4 & WBWC & $2838-180$ & 111.1 & 5420 & 09/19/07 & 40.36 & 0.00412 \\
\hline Admiralty Inlet & DF-WBWC-5 & Dogfish 5 & WBWC & $2838-181$ & 93.98 & 3020 & 09/19/07 & 41.03 & $0.00223 \mathrm{~J}$ \\
\hline Admiralty Inlet & DF-WBWC-6 & Dogfish 6 & WBWC & $2838-182$ & 98.11 & 4090 & 09/19/07 & 37.03 & 0.0201 \\
\hline Admiralty Inlet & DF-SWC-4 & Dogfish 4 & SWC & $2838-183$ & & & 09/19/07 & 29.84 & $0.00126 \mathrm{~J}$ \\
\hline
\end{tabular}




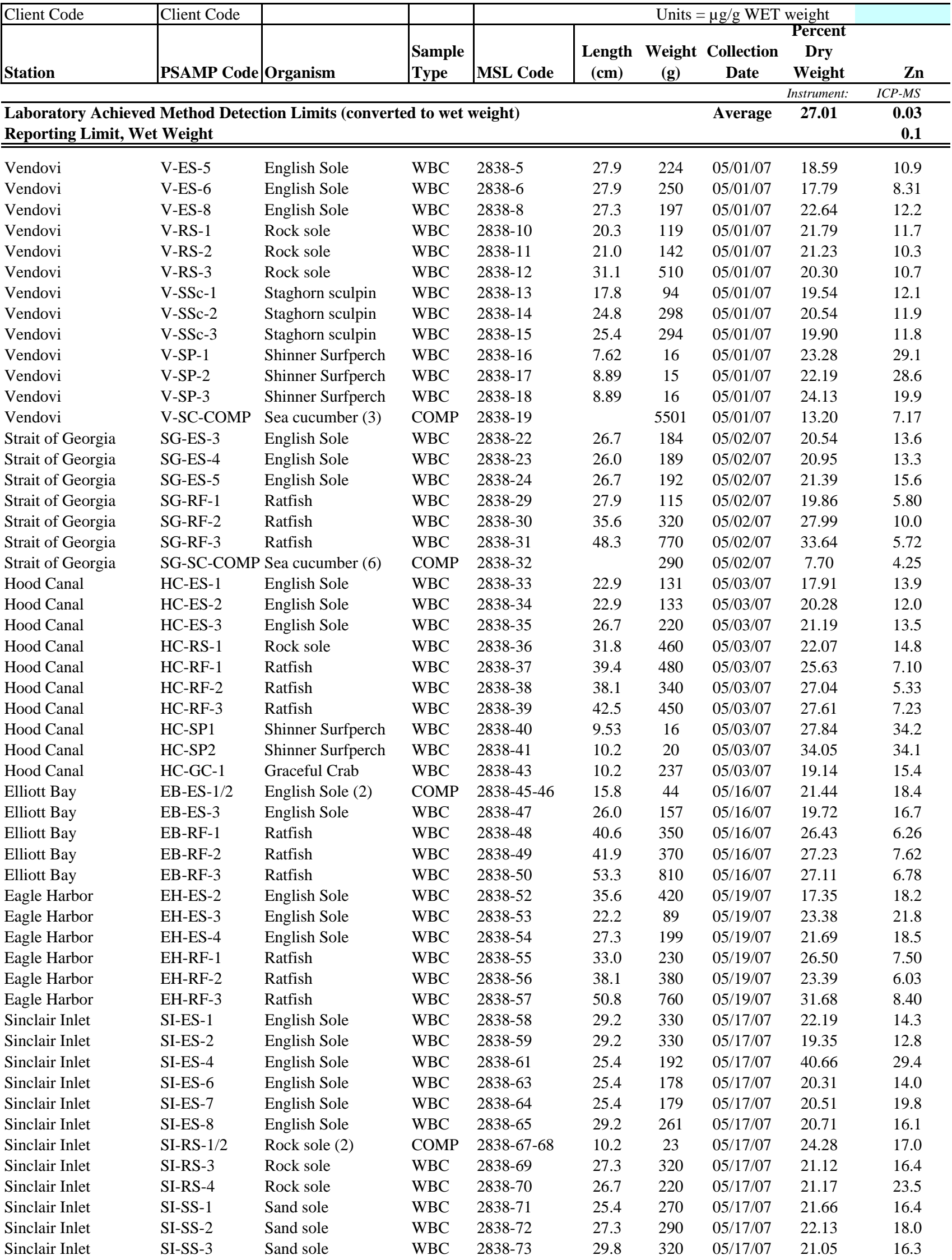




\begin{tabular}{|c|c|c|c|c|c|c|c|c|c|}
\hline Client Code & Client Code & & & \multicolumn{6}{|c|}{ Units $=\mu \mathrm{g} / \mathrm{g}$ WET weight } \\
\hline \multirow[t]{2}{*}{ Station } & PSAMP Code & Organism & $\begin{array}{l}\text { Sample } \\
\text { Type }\end{array}$ & MSL Code & $\begin{array}{l}\text { Length } \\
\text { (cm) }\end{array}$ & $\begin{array}{l}\text { Weight } \\
\text { (g) }\end{array}$ & $\begin{array}{l}\text { Collection } \\
\text { Date }\end{array}$ & $\begin{array}{c}\text { Percent } \\
\text { Dry } \\
\text { Weight }\end{array}$ & Zn \\
\hline & & & & & & & \multicolumn{2}{|r|}{ Instrument: } & ICP-MS \\
\hline \multicolumn{7}{|c|}{$\begin{array}{l}\text { Laboratory Achieved Method Detection Limits (converted to wet weight) } \\
\text { Reporting Limit, Wet Weight }\end{array}$} & Average & 27.01 & $\begin{array}{r}0.03 \\
0.1\end{array}$ \\
\hline Sinclair Inlet & SI-SSc-1/2 & Staghorn sculpin (2) & COMP & $2838-77-78$ & 14.9 & 48 & 05/17/07 & 20.66 & 15.2 \\
\hline Sinclair Inlet & SI-SSc-3/5 & Staghorn sculpin (2) & COMP & $2838-79-81$ & 14.3 & 53 & $05 / 17 / 07$ & 20.03 & 12.7 \\
\hline Sinclair Inlet & SI-SSc-4/6 & Staghorn sculpin (2) & COMP & $2838-80-82$ & 16.2 & 75 & $05 / 17 / 07$ & 19.51 & 12.8 \\
\hline Sinclair Inlet & SI-RF-1 & Ratfish & WBC & $2838-83$ & 41.3 & 550 & $05 / 17 / 07$ & 32.67 & 7.55 \\
\hline Sinclair Inlet & SI-RF-2 & Ratfish & WBC & $2838-84$ & 36.8 & 380 & $05 / 17 / 07$ & 26.61 & 6.04 \\
\hline Sinclair Inlet & SI-SP-4/5 & Shinner Surfperch $(2$ & ) COMP & $2838-88-89$ & 7.62 & 13 & 05/17/07 & 24.47 & 30.6 \\
\hline Sinclair Inlet & SI-SP-6/2 & Shinner Surfperch (2 & COMP & $2838-90-86$ & 6.67 & 11 & 05/17/07 & 24.68 & 29.4 \\
\hline Sinclair Inlet & SI-SP-7/3 & Shinner Surfperch (2 & ) COMP & $2838-91-87$ & 7.3 & 11 & $05 / 17 / 07$ & 25.12 & 25.0 \\
\hline Sinclair Inlet & SI-GC-2/6 & Graceful Crab (2) & COMP & 2838-93-97 & 7.62 & 103 & $05 / 17 / 07$ & 28.75 & 43.7 \\
\hline Sinclair Inlet & SI-GC-3/4 & Graceful Crab (2) & COMP & 2838-94-95 & 6.99 & 67 & 05/17/07 & 33.60 & 32.8 \\
\hline Sinclair Inlet & SI-GC-7/1 & Graceful Crab (2) & COMP & $2838-98-92$ & 6.67 & 60 & $05 / 17 / 07$ & 54.85 & 77.9 \\
\hline Sinclair Inlet & SI-GC-5/8 & Graceful Crab (2) & COMP & 2838-96-99 & 7.62 & 92 & $05 / 17 / 07$ & 30.89 & 32.4 \\
\hline Sinclair Inlet & SI-SC-COMP & Sea cucumber (6) & COMP & $2838-100$ & & 4100 & $05 / 17 / 07$ & 9.52 & 6.92 \\
\hline Port Gardner & PG-ES-1 & English Sole & WBC & $2838-101$ & 24.8 & 158 & $05 / 29 / 07$ & 19.23 & 15.6 \\
\hline Port Gardner & PG-ES-2 & English Sole & WBC & $2838-102$ & 21.6 & 101 & $05 / 29 / 07$ & 20.82 & 15.0 \\
\hline Port Gardner & PG-ES-3 & English Sole & WBC & 2838-103 & 24.8 & 128 & $05 / 29 / 07$ & 19.95 & 15.6 \\
\hline Nisqually & NIS-ES-1 & English Sole & WBC & 2838-104 & 25.4 & 166 & $05 / 30 / 07$ & 20.67 & 16.6 \\
\hline Nisqually & NIS-ES-2 & English Sole & WBC & $2838-105$ & 26.7 & 214 & $05 / 30 / 07$ & 21.52 & 18.0 \\
\hline Nisqually & NIS-ES-3 & English Sole & WBC & 2838-106 & 30.5 & 290 & $05 / 30 / 07$ & 21.61 & 14.5 \\
\hline Nisqually & NIS-RF-1 & Ratfish & WBC & 2838-107 & 48.3 & 620 & $05 / 30 / 07$ & 31.21 & 7.02 \\
\hline Nisqually & NIS-RF-2 & Ratfish & WBC & 2838-108 & 40.6 & 430 & $05 / 30 / 07$ & 32.53 & 7.90 \\
\hline Nisqually & NIS-RF-3 & Ratfish & WBC & 2838-109 & 38.1 & 450 & $05 / 30 / 07$ & 29.35 & 8.22 \\
\hline Commencement Bay & CB-ES-1 & English Sole & WBC & $2838-110$ & 22.9 & 145 & $05 / 31 / 07$ & 21.50 & 17.0 \\
\hline Commencement Bay & CB-ES-2 & English Sole & WBC & $2838-111$ & 24.8 & 157 & $05 / 31 / 07$ & 23.68 & 18.8 \\
\hline Commencement Bay & CB-ES-3 & English Sole & WBC & $2838-112$ & 24.8 & 162 & $05 / 31 / 07$ & 23.16 & 17.2 \\
\hline Commencement Bay & CB-RF-1 & Ratfish & WBC & $2838-113$ & 40.6 & 320 & $05 / 31 / 07$ & 30.17 & 6.00 \\
\hline Commencement Bay & CB-RF-2 & Ratfish & WBC & $2838-114$ & 38.7 & 350 & $05 / 31 / 07$ & 28.54 & 5.88 \\
\hline Commencement Bay & CB-RF-3 & Ratfish & WBC & $2838-115$ & 38.7 & 390 & $05 / 31 / 07$ & 30.58 & 6.36 \\
\hline Duwamish & DU-ES-1 & English Sole & WBC & $2838-116$ & 17.8 & 75 & 05/18/07 & 22.95 & 17.3 \\
\hline Duwamish & DU-ES-2 & English Sole & WBC & $2838-117$ & 21.6 & 96 & $05 / 18 / 07$ & 20.32 & 16.3 \\
\hline Duwamish & DU-ES-3 & English Sole & WBC & $2838-118$ & 26.0 & 156 & 05/18/07 & 23.00 & 17.4 \\
\hline Admiralty Inlet & DF-EM-1 & Dogfish 1 & EM & $2838-126$ & & 410 & 09/19/07 & 52.97 & 13.0 \\
\hline Admiralty Inlet & DF-EM-2 & Dogfish 2 & EM & $2838-127$ & & 440 & 09/19/07 & 52.87 & 12.2 \\
\hline Admiralty Inlet & DF-LV-1 & Dogfish 1 & LV & 2838-132 & & 498 & 09/19/07 & 55.21 & 21.4 \\
\hline Admiralty Inlet & DF-LV-2 & Dogfish 2 & LV & $2838-133$ & & 260 & 09/19/07 & 52.95 & 13.3 \\
\hline Admiralty Inlet & DF-DIG-1 & Dogfish 1 & DIG & $2838-138$ & & 319 & 09/19/07 & 22.67 & 44.0 \\
\hline Admiralty Inlet & DF-DIG-2 & Dogfish 2 & DIG & 2838-139 & & 194 & 09/19/07 & 21.16 & 17.7 \\
\hline Admiralty Inlet & DF-SWC-1 & Dogfish 1 & SWC & $2838-174$ & & & $09 / 19 / 07$ & 37.19 & 4.35 \\
\hline Admiralty Inlet & DF-SWC-2 & Dogfish 2 & SWC & $2838-175$ & & & 09/19/07 & 28.81 & 4.06 \\
\hline Admiralty Inlet & DF-WBWC-1 & Dogfish 1 & WBWC & $2838-177$ & 110.5 & 5400 & 09/19/07 & 39.76 & 8.51 \\
\hline Admiralty Inlet & DF-WBWC-3 & Dogfish 3 & WBWC & $2838-179$ & 93.03 & 3140 & $09 / 19 / 07$ & 36.70 & 5.06 \\
\hline Admiralty Inlet & DF-WBWC-2 & Dogfish 2 & WBWC & $2838-178$ & 104.8 & 4290 & 09/19/07 & 35.83 & 6.34 \\
\hline Admiralty Inlet & DF-EM-4 & Dogfish 4 & EM & $2838-129$ & & 188 & 09/19/07 & 52.59 & 15.7 \\
\hline Admiralty Inlet & DF-EM-6 & Dogfish 6 & EM & $2838-131$ & & 322 & 09/19/07 & 56.33 & 14.5 \\
\hline Admiralty Inlet & DF-LV-4 & Dogfish 4 & LV & $2838-135$ & & 790 & 09/19/07 & 77.18 & 15.3 \\
\hline Admiralty Inlet & DF-LV-6 & Dogfish 6 & $\mathrm{LV}$ & $2838-137$ & & 434 & 09/19/07 & 31.93 & 8.08 \\
\hline Admiralty Inlet & DF-DIG-4 & Dogfish 4 & DIG & $2838-141$ & & 320 & 09/19/07 & 15.59 & 50.0 \\
\hline Admiralty Inlet & DF-DIG-6 & Dogfish 6 & DIG & $2838-143$ & & 327 & 09/19/07 & 17.06 & 37.0 \\
\hline Admiralty Inlet & DF-SWC-6 & Dogfish 6 & SWC & $2838-176$ & & & 09/19/07 & 35.23 & 3.84 \\
\hline Admiralty Inlet & DF-WBWC-4 & Dogfish 4 & WBWC & $2838-180$ & 111.1 & 5420 & 09/19/07 & 40.36 & 15.8 \\
\hline Admiralty Inlet & DF-WBWC-5 & Dogfish 5 & WBWC & $2838-181$ & 93.98 & 3020 & 09/19/07 & 41.03 & 6.73 \\
\hline Admiralty Inlet & DF-WBWC-6 & Dogfish 6 & WBWC & $2838-182$ & 98.11 & 4090 & 09/19/07 & 37.03 & 18.5 \\
\hline Admiralty Inlet & DF-SWC-4 & Dogfish 4 & SWC & $2838-183$ & & & 09/19/07 & 29.84 & 3.25 \\
\hline
\end{tabular}




\section{Field Data Summary: Metals in Biota Tissue}

Dry Weight Basis 


\begin{tabular}{|c|c|c|c|c|c|c|c|c|c|}
\hline Client Code & Client Code & & & & Units $=$ & $\mu \mathrm{g} / \mathrm{g}$ dry & weight & & \\
\hline Station & PSAMP Code & Organism & \begin{tabular}{|l} 
Sample \\
Type
\end{tabular} & MSL Code & $\begin{array}{c}\text { Length } \\
\text { (cm) }\end{array}$ & $\begin{array}{c}\text { Weight } \\
\text { (g) }\end{array}$ & $\begin{array}{c}\text { Collection } \\
\text { Date }\end{array}$ & $\begin{array}{c}\text { Percent } \\
\text { Moisture }\end{array}$ & Ag \\
\hline & & & & & & & & Instrument: & ICP-MS \\
\hline Laboratory Achi & Method Detect & tion Limits & & & & & & & 0.002 \\
\hline Reporting Limit & $\left.L^{*} 3.18\right)$ & & & & & & & & 0.01 \\
\hline Vendovi & V-ES-5 & English Sole & WBC & $2838-5$ & 27.9 & 224 & 05/01/07 & 81.41 & 0.0306 \\
\hline Vendovi & V-ES-6 & English Sole & WBC & $2838-6$ & 27.9 & 250 & 05/01/07 & 82.21 & 0.0170 \\
\hline Vendovi & V-ES-8 & English Sole & WBC & $2838-8$ & 27.3 & 197 & 05/01/07 & 77.36 & 0.0270 \\
\hline Vendovi & V-RS-1 & Rock sole & WBC & $2838-10$ & 20.3 & 119 & 05/01/07 & 78.21 & 0.0204 \\
\hline Vendovi & V-RS-2 & Rock sole & WBC & 2838-11 & 21.0 & 142 & 05/01/07 & 78.77 & 0.0144 \\
\hline Vendovi & V-RS-3 & Rock sole & WBC & $2838-12$ & 31.1 & 510 & 05/01/07 & 79.70 & 0.0240 \\
\hline Vendovi & V-SSc-1 & Staghorn sculpin & WBC & $2838-13$ & 17.8 & 94 & 05/01/07 & 80.46 & 0.125 \\
\hline Vendovi & V-SSc-2 & Staghorn sculpin & WBC & $2838-14$ & 24.8 & 298 & 05/01/07 & 79.46 & 0.0379 \\
\hline Vendovi & V-SSc-3 & Staghorn sculpin & WBC & $2838-15$ & 25.4 & 294 & 05/01/07 & 80.10 & 0.0253 \\
\hline Vendovi & V-SP-1 & Shiner perch & WBC & $2838-16$ & 7.62 & 16 & $05 / 01 / 07$ & 76.72 & 0.0208 \\
\hline Vendovi & V-SP-2 & Shiner perch & WBC & $2838-17$ & 8.89 & 15 & 05/01/07 & 77.81 & $0.00787 \mathrm{~J}$ \\
\hline Vendovi & V-SP-3 & Shiner perch & WBC & $2838-18$ & 8.89 & 16 & 05/01/07 & 75.87 & 0.0217 \\
\hline Vendovi & V-SC-COMP & Sea cucumber (3) & COMP & 2838-19 & & 5501 & 05/01/07 & 86.80 & 0.0728 \\
\hline Strait of Georgia & SG-ES-3 & English Sole & WBC & $2838-22$ & 26.7 & 184 & $05 / 02 / 07$ & 79.46 & $0.00669 \mathrm{~J}$ \\
\hline Strait of Georgia & SG-ES-4 & English Sole & WBC & $2838-23$ & 26.0 & 189 & $05 / 02 / 07$ & 79.05 & 0.0113 \\
\hline Strait of Georgia & SG-ES-5 & English Sole & WBC & $2838-24$ & 26.7 & 192 & $05 / 02 / 07$ & 78.61 & 0.0209 \\
\hline Strait of Georgia & SG-RF-1 & Spotted Ratfish & WBC & $2838-29$ & 27.9 & 115 & $05 / 02 / 07$ & 80.14 & 0.215 \\
\hline Strait of Georgia & SG-RF-2 & Spotted Ratfish & WBC & $2838-30$ & 35.6 & 320 & $05 / 02 / 07$ & 72.01 & 0.359 \\
\hline Strait of Georgia & SG-RF-3 & Spotted Ratfish & WBC & $2838-31$ & 48.3 & 770 & $05 / 02 / 07$ & 66.36 & 0.380 \\
\hline Strait of Georgia & SG-SC-COMP & Sea cucumber (6) & COMP & $2838-32$ & & 290 & $05 / 02 / 07$ & 92.30 & 0.0766 \\
\hline Hood Canal & HC-ES-1 & English Sole & WBC & $2838-33$ & 22.9 & 131 & $05 / 03 / 07$ & 82.09 & 0.0106 \\
\hline Hood Canal & HC-ES-2 & English Sole & WBC & 2838-34 & 22.9 & 133 & $05 / 03 / 07$ & 79.72 & $0.00277 \mathrm{~J}$ \\
\hline Hood Canal & HC-ES-3 & English Sole & WBC & $2838-35$ & 26.7 & 220 & 05/03/07 & 78.81 & 0.0196 \\
\hline Hood Canal & HC-RS-1 & Rock sole & WBC & $2838-36$ & 31.8 & 460 & $05 / 03 / 07$ & 77.93 & 0.0121 \\
\hline Hood Canal & HC-RF-1 & Spotted Ratfish & WBC & $2838-37$ & 39.4 & 480 & 05/03/07 & 74.37 & 1.27 \\
\hline Hood Canal & HC-RF-2 & Spotted Ratfish & WBC & $2838-38$ & 38.1 & 340 & $05 / 03 / 07$ & 72.96 & 0.997 \\
\hline Hood Canal & HC-RF-3 & Spotted Ratfish & WBC & 2838-39 & 42.5 & 450 & 05/03/07 & 72.39 & 5.86 \\
\hline Hood Canal & HC-SP1 & Shiner perch & WBC & $2838-40$ & 9.53 & 16 & $05 / 03 / 07$ & 72.16 & 0.0582 \\
\hline Hood Canal & $\mathrm{HC}-\mathrm{SP} 2$ & Shiner perch & WBC & $2838-41$ & 10.2 & 20 & $05 / 03 / 07$ & 65.95 & 0.0578 \\
\hline Hood Canal & HC-GC-1 & Graceful Crab & WBC & $2838-43$ & 10.2 & 237 & 05/03/07 & 80.86 & 1.11 \\
\hline Elliott Bay & EB-ES-1/2 & English Sole (2) & COMP & $2838-45-46$ & 15.8 & 44 & $05 / 16 / 07$ & 78.56 & 0.0791 \\
\hline Elliott Bay & EB-ES-3 & English Sole & WBC & $2838-47$ & 26.0 & 157 & $05 / 16 / 07$ & 80.28 & 0.0545 \\
\hline Elliott Bay & EB-RF-1 & Spotted Ratfish & WBC & $2838-48$ & 40.6 & 350 & 05/16/07 & 73.57 & 7.86 \\
\hline Elliott Bay & EB-RF-2 & Spotted Ratfish & WBC & $2838-49$ & 41.9 & 370 & $05 / 16 / 07$ & 72.77 & 0.655 \\
\hline Elliott Bay & EB-RF-3 & Spotted Ratfish & WBC & $2838-50$ & 53.3 & 810 & 05/16/07 & 72.89 & 3.08 \\
\hline Eagle Harbor & EH-ES-2 & English Sole & WBC & $2838-52$ & 35.6 & 420 & 05/19/07 & 82.65 & 0.0349 \\
\hline Eagle Harbor & EH-ES-3 & English Sole & WBC & $2838-53$ & 22.2 & 89 & 05/19/07 & 76.62 & 0.0210 \\
\hline Eagle Harbor & EH-ES-4 & English Sole & WBC & $2838-54$ & 27.3 & 199 & 05/19/07 & 78.31 & 0.0145 \\
\hline Eagle Harbor & EH-RF-1 & Spotted Ratfish & WBC & $2838-55$ & 33.0 & 230 & 05/19/07 & 73.50 & 0.934 \\
\hline Eagle Harbor & EH-RF-2 & Spotted Ratfish & WBC & $2838-56$ & 38.1 & 380 & 05/19/07 & 76.61 & 1.65 \\
\hline Eagle Harbor & EH-RF-3 & Spotted Ratfish & WBC & $2838-57$ & 50.8 & 760 & 05/19/07 & 68.32 & 0.768 \\
\hline Sinclair Inlet & SI-ES-1 & English Sole & WBC & $2838-58$ & 29.2 & 330 & 05/17/07 & 77.81 & 0.0262 \\
\hline Sinclair Inlet & SI-ES-2 & English Sole & WBC & $2838-59$ & 29.2 & 330 & 05/17/07 & 80.65 & 0.0272 \\
\hline Sinclair Inlet & SI-ES-4 & English Sole & WBC & $2838-61$ & 25.4 & 192 & 05/17/07 & 59.34 & 0.0362 \\
\hline Sinclair Inlet & SI-ES-6 & English Sole & WBC & $2838-63$ & 25.4 & 178 & 05/17/07 & 79.69 & 0.0113 \\
\hline Sinclair Inlet & SI-ES-7 & English Sole & WBC & $2838-64$ & 25.4 & 179 & 05/17/07 & 79.49 & 0.0109 \\
\hline Sinclair Inlet & SI-ES-8 & English Sole & WBC & $2838-65$ & 29.2 & 261 & 05/17/07 & 79.29 & 0.0273 \\
\hline Sinclair Inlet & SI-RS-1/2 & Rock sole (2) & COMP & $2838-67-68$ & 10.2 & 23 & 05/17/07 & 75.72 & 0.0309 \\
\hline Sinclair Inlet & SI-RS-3 & Rock sole & WBC & $2838-69$ & 27.3 & 320 & 05/17/07 & 78.88 & 0.0279 \\
\hline Sinclair Inlet & SI-RS-4 & Rock sole & WBC & $2838-70$ & 26.7 & 220 & 05/17/07 & 78.83 & $0.002 \mathrm{U}$ \\
\hline Sinclair Inlet & SI-SS-1 & Sand sole & WBC & 2838-71 & 25.4 & 270 & 05/17/07 & 78.34 & 0.0435 \\
\hline Sinclair Inlet & SI-SS-2 & Sand sole & WBC & $2838-72$ & 27.3 & 290 & 05/17/07 & 77.87 & $0.00225 \mathrm{~J}$ \\
\hline Sinclair Inlet & SI-SS-3 & Sand sole & WBC & $2838-73$ & 29.8 & 320 & 05/17/07 & 78.95 & 0.0343 \\
\hline
\end{tabular}




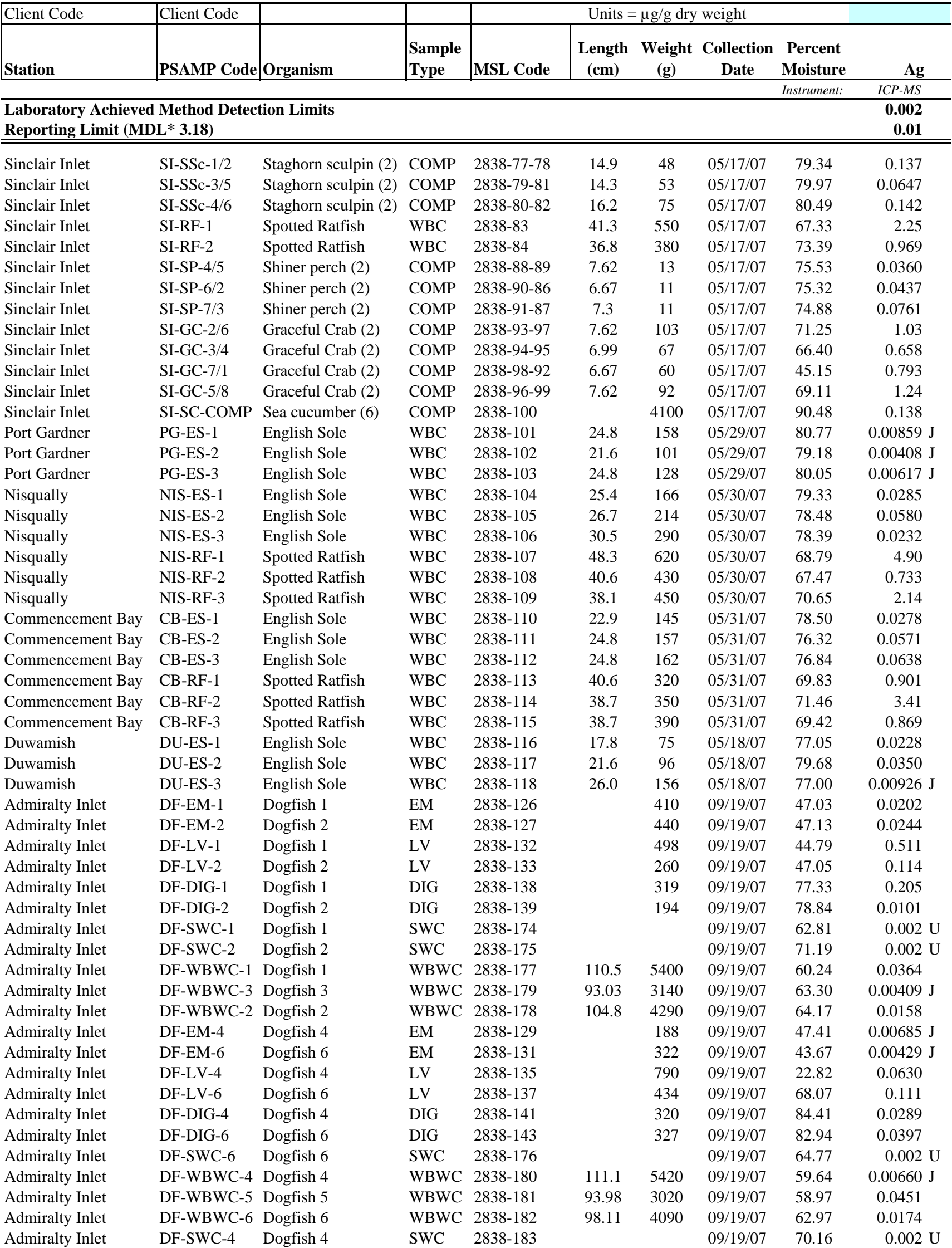




\begin{tabular}{|c|c|c|c|c|c|c|c|c|c|}
\hline Client Code & Client Code & & & & Units $=$ & $=\mu \mathrm{g} / \mathrm{g}$ dry & weight & & \\
\hline Station & PSAMP Code & Organism & $\begin{array}{l}\text { Sample } \\
\text { Type }\end{array}$ & MSL Code & $\begin{array}{c}\text { Length } \\
\text { (cm) }\end{array}$ & $\begin{array}{l}\text { Weight } \\
\text { (g) }\end{array}$ & $\begin{array}{c}\text { Collection } \\
\text { Date }\end{array}$ & $\begin{array}{c}\text { Percent } \\
\text { Moisture }\end{array}$ & As \\
\hline & & & & & & & & Instrument: & $I C P-M S$ \\
\hline Laboratory Ach & Method Detec & tion Limits & & & & & & & 0.1 \\
\hline Reporting Limit & L*3.18) & & & & & & & & 0.3 \\
\hline Vendovi & V-ES-5 & English Sole & WBC & $2838-5$ & 27.9 & 224 & 05/01/07 & 81.41 & 31.9 \\
\hline Vendovi & V-ES-6 & English Sole & WBC & $2838-6$ & 27.9 & 250 & 05/01/07 & 82.21 & 45.3 \\
\hline Vendovi & V-ES-8 & English Sole & WBC & $2838-8$ & 27.3 & 197 & 05/01/07 & 77.36 & 27.9 \\
\hline Vendovi & V-RS-1 & Rock sole & WBC & $2838-10$ & 20.3 & 119 & $05 / 01 / 07$ & 78.21 & 13.5 \\
\hline Vendovi & V-RS-2 & Rock sole & WBC & $2838-11$ & 21.0 & 142 & 05/01/07 & 78.77 & 12.3 \\
\hline Vendovi & V-RS-3 & Rock sole & WBC & 2838-12 & 31.1 & 510 & 05/01/07 & 79.70 & 18.2 \\
\hline Vendovi & V-SSc-1 & Staghorn sculpin & WBC & $2838-13$ & 17.8 & 94 & 05/01/07 & 80.46 & 13.6 \\
\hline Vendovi & V-SSc-2 & Staghorn sculpin & WBC & $2838-14$ & 24.8 & 298 & 05/01/07 & 79.46 & 14.3 \\
\hline Vendovi & V-SSc-3 & Staghorn sculpin & WBC & $2838-15$ & 25.4 & 294 & 05/01/07 & 80.10 & 15.3 \\
\hline Vendovi & V-SP-1 & Shiner perch & WBC & $2838-16$ & 7.62 & 16 & 05/01/07 & 76.72 & 4.11 \\
\hline Vendovi & V-SP-2 & Shiner perch & WBC & $2838-17$ & 8.89 & 15 & 05/01/07 & 77.81 & 3.19 \\
\hline Vendovi & V-SP-3 & Shiner perch & WBC & $2838-18$ & 8.89 & 16 & 05/01/07 & 75.87 & 5.17 \\
\hline Vendovi & V-SC-COMP & Sea cucumber (3) & COMP & 2838-19 & & 5501 & 05/01/07 & 86.80 & 10.8 \\
\hline Strait of Georgia & SG-ES-3 & English Sole & WBC & $2838-22$ & 26.7 & 184 & $05 / 02 / 07$ & 79.46 & 10.9 \\
\hline Strait of Georgia & SG-ES-4 & English Sole & WBC & $2838-23$ & 26.0 & 189 & $05 / 02 / 07$ & 79.05 & 18.1 \\
\hline Strait of Georgia & SG-ES-5 & English Sole & WBC & $2838-24$ & 26.7 & 192 & $05 / 02 / 07$ & 78.61 & 17.7 \\
\hline Strait of Georgia & SG-RF-1 & Spotted Ratfish & WBC & $2838-29$ & 27.9 & 115 & $05 / 02 / 07$ & 80.14 & 29.5 \\
\hline Strait of Georgia & SG-RF-2 & Spotted Ratfish & WBC & $2838-30$ & 35.6 & 320 & $05 / 02 / 07$ & 72.01 & 15.3 \\
\hline Strait of Georgia & SG-RF-3 & Spotted Ratfish & WBC & $2838-31$ & 48.3 & 770 & $05 / 02 / 07$ & 66.36 & 31.9 \\
\hline Strait of Georgia & SG-SC-COMP & Sea cucumber (6) & COMP & $2838-32$ & & 290 & $05 / 02 / 07$ & 92.30 & 16.8 \\
\hline Hood Canal & HC-ES-1 & English Sole & WBC & $2838-33$ & 22.9 & 131 & 05/03/07 & 82.09 & 28.1 \\
\hline Hood Canal & HC-ES-2 & English Sole & WBC & $2838-34$ & 22.9 & 133 & 05/03/07 & 79.72 & 51.4 \\
\hline Hood Canal & HC-ES-3 & English Sole & WBC & $2838-35$ & 26.7 & 220 & $05 / 03 / 07$ & 78.81 & 21.7 \\
\hline Hood Canal & HC-RS-1 & Rock sole & WBC & $2838-36$ & 31.8 & 460 & $05 / 03 / 07$ & 77.93 & 11.2 \\
\hline Hood Canal & HC-RF-1 & Spotted Ratfish & WBC & $2838-37$ & 39.4 & 480 & 05/03/07 & 74.37 & 28.2 \\
\hline Hood Canal & HC-RF-2 & Spotted Ratfish & WBC & $2838-38$ & 38.1 & 340 & 05/03/07 & 72.96 & 25.5 \\
\hline Hood Canal & HC-RF-3 & Spotted Ratfish & WBC & 2838-39 & 42.5 & 450 & 05/03/07 & 72.39 & 35.5 \\
\hline Hood Canal & HC-SP1 & Shiner perch & WBC & $2838-40$ & 9.53 & 16 & 05/03/07 & 72.16 & 4.08 \\
\hline Hood Canal & HC-SP2 & Shiner perch & WBC & $2838-41$ & 10.2 & 20 & 05/03/07 & 65.95 & 3.75 \\
\hline Hood Canal & HC-GC-1 & Graceful Crab & WBC & $2838-43$ & 10.2 & 237 & 05/03/07 & 80.86 & 17.6 \\
\hline Elliott Bay & EB-ES-1/2 & English Sole (2) & COMP & $2838-45-46$ & 15.8 & 44 & $05 / 16 / 07$ & 78.56 & 15.1 \\
\hline Elliott Bay & EB-ES-3 & English Sole & WBC & $2838-47$ & 26.0 & 157 & $05 / 16 / 07$ & 80.28 & 27.1 \\
\hline Elliott Bay & EB-RF-1 & Spotted Ratfish & WBC & $2838-48$ & 40.6 & 350 & 05/16/07 & 73.57 & 49.2 \\
\hline Elliott Bay & EB-RF-2 & Spotted Ratfish & WBC & $2838-49$ & 41.9 & 370 & $05 / 16 / 07$ & 72.77 & 30.2 \\
\hline Elliott Bay & EB-RF-3 & Spotted Ratfish & WBC & $2838-50$ & 53.3 & 810 & $05 / 16 / 07$ & 72.89 & 24.7 \\
\hline Eagle Harbor & EH-ES-2 & English Sole & WBC & $2838-52$ & 35.6 & 420 & 05/19/07 & 82.65 & 15.9 \\
\hline Eagle Harbor & EH-ES-3 & English Sole & WBC & $2838-53$ & 22.2 & 89 & 05/19/07 & 76.62 & 20.8 \\
\hline Eagle Harbor & EH-ES-4 & English Sole & WBC & $2838-54$ & 27.3 & 199 & 05/19/07 & 78.31 & 15.6 \\
\hline Eagle Harbor & EH-RF-1 & Spotted Ratfish & WBC & $2838-55$ & 33.0 & 230 & 05/19/07 & 73.50 & 37.9 \\
\hline Eagle Harbor & EH-RF-2 & Spotted Ratfish & WBC & $2838-56$ & 38.1 & 380 & 05/19/07 & 76.61 & 26.9 \\
\hline Eagle Harbor & EH-RF-3 & Spotted Ratfish & WBC & $2838-57$ & 50.8 & 760 & 05/19/07 & 68.32 & 23.2 \\
\hline Sinclair Inlet & SI-ES-1 & English Sole & WBC & $2838-58$ & 29.2 & 330 & $05 / 17 / 07$ & 77.81 & 11.9 \\
\hline Sinclair Inlet & SI-ES-2 & English Sole & WBC & $2838-59$ & 29.2 & 330 & 05/17/07 & 80.65 & 17.6 \\
\hline Sinclair Inlet & SI-ES-4 & English Sole & WBC & $2838-61$ & 25.4 & 192 & 05/17/07 & 59.34 & 21.8 \\
\hline Sinclair Inlet & SI-ES-6 & English Sole & WBC & $2838-63$ & 25.4 & 178 & 05/17/07 & 79.69 & 11.3 \\
\hline Sinclair Inlet & SI-ES-7 & English Sole & WBC & $2838-64$ & 25.4 & 179 & $05 / 17 / 07$ & 79.49 & 12.4 \\
\hline Sinclair Inlet & SI-ES-8 & English Sole & WBC & $2838-65$ & 29.2 & 261 & 05/17/07 & 79.29 & 17.0 \\
\hline Sinclair Inlet & SI-RS-1/2 & Rock sole (2) & COMP & $2838-67-68$ & 10.2 & 23 & 05/17/07 & 75.72 & 12.8 \\
\hline Sinclair Inlet & SI-RS-3 & Rock sole & WBC & $2838-69$ & 27.3 & 320 & $05 / 17 / 07$ & 78.88 & 24.0 \\
\hline Sinclair Inlet & SI-RS-4 & Rock sole & WBC & $2838-70$ & 26.7 & 220 & 05/17/07 & 78.83 & 32.9 \\
\hline Sinclair Inlet & SI-SS-1 & Sand sole & WBC & $2838-71$ & 25.4 & 270 & 05/17/07 & 78.34 & 8.02 \\
\hline Sinclair Inlet & SI-SS-2 & Sand sole & WBC & $2838-72$ & 27.3 & 290 & 05/17/07 & 77.87 & 7.17 \\
\hline Sinclair Inlet & SI-SS-3 & Sand sole & WBC & $2838-73$ & 29.8 & 320 & $05 / 17 / 07$ & 78.95 & 7.64 \\
\hline
\end{tabular}




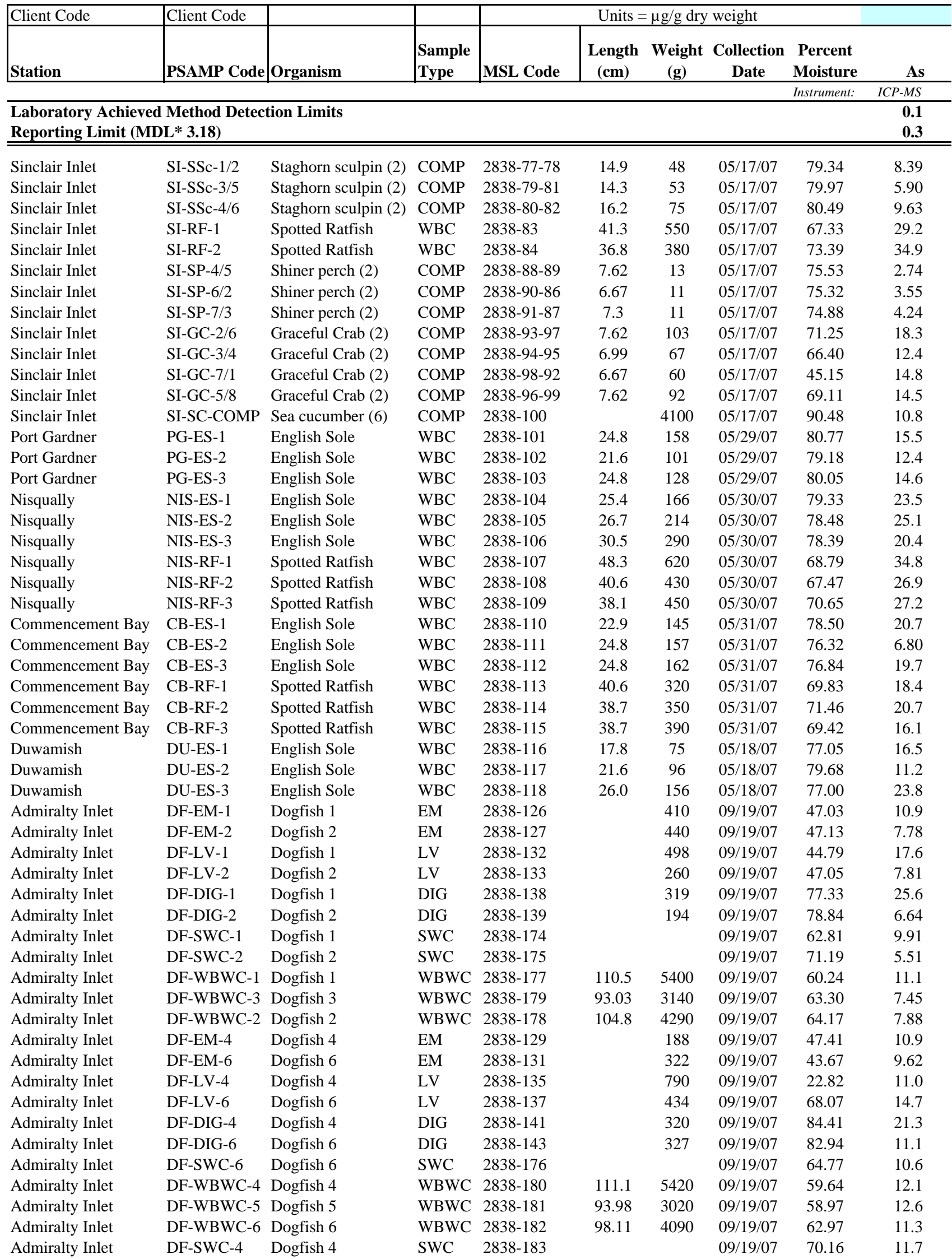




\begin{tabular}{|c|c|c|c|c|c|c|c|c|c|}
\hline Client Code & Client Code & & & & Units $=$ & $\mu \mathrm{g} / \mathrm{g}$ dry & weight & & \\
\hline Station & PSAMP Code & Organism & \begin{tabular}{|l} 
Sample \\
Type
\end{tabular} & MSL Code & $\begin{array}{c}\text { Length } \\
\text { (cm) }\end{array}$ & $\begin{array}{c}\text { Weight } \\
\text { (g) }\end{array}$ & $\begin{array}{c}\text { Collection } \\
\text { Date }\end{array}$ & $\begin{array}{c}\text { Percent } \\
\text { Moisture } \\
\end{array}$ & Cd \\
\hline & & & & & & & & Instrument: & ICP-MS \\
\hline Laboratory Achi & Method Detect & tion Limits & & & & & & & 0.002 \\
\hline Reporting Limit & L*3.18) & & & & & & & & $\mathbf{0 . 0 1}$ \\
\hline Vendovi & V-ES-5 & English Sole & WBC & $2838-5$ & 27.9 & 224 & 05/01/07 & 81.41 & 0.0884 \\
\hline Vendovi & V-ES-6 & English Sole & WBC & $2838-6$ & 27.9 & 250 & 05/01/07 & 82.21 & 0.0657 \\
\hline Vendovi & V-ES-8 & English Sole & WBC & $2838-8$ & 27.3 & 197 & 05/01/07 & 77.36 & 0.0701 \\
\hline Vendovi & V-RS-1 & Rock sole & WBC & $2838-10$ & 20.3 & 119 & 05/01/07 & 78.21 & 0.0325 \\
\hline Vendovi & V-RS-2 & Rock sole & WBC & $2838-11$ & 21.0 & 142 & 05/01/07 & 78.77 & 0.0633 \\
\hline Vendovi & V-RS-3 & Rock sole & WBC & $2838-12$ & 31.1 & 510 & 05/01/07 & 79.70 & 0.138 \\
\hline Vendovi & V-SSc-1 & Staghorn sculpin & WBC & $2838-13$ & 17.8 & 94 & 05/01/07 & 80.46 & 0.167 \\
\hline Vendovi & V-SSc-2 & Staghorn sculpin & WBC & $2838-14$ & 24.8 & 298 & 05/01/07 & 79.46 & 0.195 \\
\hline Vendovi & V-SSc-3 & Staghorn sculpin & WBC & $2838-15$ & 25.4 & 294 & 05/01/07 & 80.10 & 0.0956 \\
\hline Vendovi & V-SP-1 & Shiner perch & WBC & $2838-16$ & 7.62 & 16 & 05/01/07 & 76.72 & 0.129 \\
\hline Vendovi & V-SP-2 & Shiner perch & WBC & $2838-17$ & 8.89 & 15 & 05/01/07 & 77.81 & 0.145 \\
\hline Vendovi & V-SP-3 & Shiner perch & WBC & $2838-18$ & 8.89 & 16 & 05/01/07 & 75.87 & 0.203 \\
\hline Vendovi & V-SC-COMP & Sea cucumber (3) & COMP & $2838-19$ & & 5501 & 05/01/07 & 86.80 & 0.450 \\
\hline Strait of Georgia & SG-ES-3 & English Sole & WBC & $2838-22$ & 26.7 & 184 & 05/02/07 & 79.46 & 0.0336 \\
\hline Strait of Georgia & SG-ES-4 & English Sole & WBC & $2838-23$ & 26.0 & 189 & 05/02/07 & 79.05 & 0.0608 \\
\hline Strait of Georgia & SG-ES-5 & English Sole & WBC & $2838-24$ & 26.7 & 192 & 05/02/07 & 78.61 & 0.0758 \\
\hline Strait of Georgia & SG-RF-1 & Spotted Ratfish & WBC & $2838-29$ & 27.9 & 115 & 05/02/07 & 80.14 & 0.0275 \\
\hline Strait of Georgia & SG-RF-2 & Spotted Ratfish & WBC & $2838-30$ & 35.6 & 320 & 05/02/07 & 72.01 & 0.0351 \\
\hline Strait of Georgia & SG-RF-3 & Spotted Ratfish & WBC & $2838-31$ & 48.3 & 770 & 05/02/07 & 66.36 & 0.0679 \\
\hline Strait of Georgia & SG-SC-COMP & Sea cucumber (6) & COMP & $2838-32$ & & 290 & 05/02/07 & 92.30 & 1.56 \\
\hline Hood Canal & HC-ES-1 & English Sole & WBC & $2838-33$ & 22.9 & 131 & 05/03/07 & 82.09 & 0.0529 \\
\hline Hood Canal & HC-ES-2 & English Sole & WBC & $2838-34$ & 22.9 & 133 & 05/03/07 & 79.72 & 0.0516 \\
\hline Hood Canal & HC-ES-3 & English Sole & WBC & $2838-35$ & 26.7 & 220 & 05/03/07 & 78.81 & 0.0448 \\
\hline Hood Canal & HC-RS-1 & Rock sole & WBC & $2838-36$ & 31.8 & 460 & 05/03/07 & 77.93 & 0.110 \\
\hline Hood Canal & HC-RF-1 & Spotted Ratfish & WBC & $2838-37$ & 39.4 & 480 & 05/03/07 & 74.37 & 0.304 \\
\hline Hood Canal & HC-RF-2 & Spotted Ratfish & WBC & $2838-38$ & 38.1 & 340 & 05/03/07 & 72.96 & 0.194 \\
\hline Hood Canal & HC-RF-3 & Spotted Ratfish & WBC & $2838-39$ & 42.5 & 450 & 05/03/07 & 72.39 & 0.201 \\
\hline Hood Canal & HC-SP1 & Shiner perch & WBC & $2838-40$ & 9.53 & 16 & 05/03/07 & 72.16 & 0.171 \\
\hline Hood Canal & HC-SP2 & Shiner perch & WBC & $2838-41$ & 10.2 & 20 & 05/03/07 & 65.95 & 0.113 \\
\hline Hood Canal & HC-GC-1 & Graceful Crab & WBC & $2838-43$ & 10.2 & 237 & 05/03/07 & 80.86 & 2.42 \\
\hline Elliott Bay & EB-ES-1/2 & English Sole (2) & COMP & 2838-45-46 & 15.8 & 44 & 05/16/07 & 78.56 & 0.110 \\
\hline Elliott Bay & EB-ES-3 & English Sole & WBC & $2838-47$ & 26.0 & 157 & 05/16/07 & 80.28 & 0.101 \\
\hline Elliott Bay & EB-RF-1 & Spotted Ratfish & WBC & $2838-48$ & 40.6 & 350 & 05/16/07 & 73.57 & 0.258 \\
\hline Elliott Bay & EB-RF-2 & Spotted Ratfish & WBC & $2838-49$ & 41.9 & 370 & 05/16/07 & 72.77 & 0.161 \\
\hline Elliott Bay & EB-RF-3 & Spotted Ratfish & WBC & $2838-50$ & 53.3 & 810 & 05/16/07 & 72.89 & 0.0780 \\
\hline Eagle Harbor & EH-ES-2 & English Sole & WBC & $2838-52$ & 35.6 & 420 & 05/19/07 & 82.65 & 0.208 \\
\hline Eagle Harbor & EH-ES-3 & English Sole & WBC & $2838-53$ & 22.2 & 89 & 05/19/07 & 76.62 & 0.399 \\
\hline Eagle Harbor & EH-ES-4 & English Sole & WBC & $2838-54$ & 27.3 & 199 & 05/19/07 & 78.31 & 0.0323 \\
\hline Eagle Harbor & EH-RF-1 & Spotted Ratfish & WBC & $2838-55$ & 33.0 & 230 & 05/19/07 & 73.50 & 0.0862 \\
\hline Eagle Harbor & EH-RF-2 & Spotted Ratfish & WBC & $2838-56$ & 38.1 & 380 & 05/19/07 & 76.61 & 0.234 \\
\hline Eagle Harbor & EH-RF-3 & Spotted Ratfish & WBC & $2838-57$ & 50.8 & 760 & 05/19/07 & 68.32 & 0.0930 \\
\hline Sinclair Inlet & SI-ES-1 & English Sole & WBC & $2838-58$ & 29.2 & 330 & 05/17/07 & 77.81 & 0.0467 \\
\hline Sinclair Inlet & SI-ES-2 & English Sole & WBC & $2838-59$ & 29.2 & 330 & 05/17/07 & 80.65 & 0.0843 \\
\hline Sinclair Inlet & SI-ES-4 & English Sole & WBC & $2838-61$ & 25.4 & 192 & 05/17/07 & 59.34 & 0.0767 \\
\hline Sinclair Inlet & SI-ES-6 & English Sole & WBC & $2838-63$ & 25.4 & 178 & 05/17/07 & 79.69 & 0.0259 \\
\hline Sinclair Inlet & SI-ES-7 & English Sole & WBC & $2838-64$ & 25.4 & 179 & 05/17/07 & 79.49 & 0.0841 \\
\hline Sinclair Inlet & SI-ES-8 & English Sole & WBC & $2838-65$ & 29.2 & 261 & 05/17/07 & 79.29 & 0.0541 \\
\hline Sinclair Inlet & SI-RS-1/2 & Rock sole (2) & COMP & $2838-67-68$ & 10.2 & 23 & 05/17/07 & 75.72 & 0.0329 \\
\hline Sinclair Inlet & SI-RS-3 & Rock sole & WBC & $2838-69$ & 27.3 & 320 & 05/17/07 & 78.88 & 0.0588 \\
\hline Sinclair Inlet & SI-RS-4 & Rock sole & WBC & $2838-70$ & 26.7 & 220 & 05/17/07 & 78.83 & 0.0343 \\
\hline Sinclair Inlet & SI-SS-1 & Sand sole & WBC & $2838-71$ & 25.4 & 270 & 05/17/07 & 78.34 & 0.134 \\
\hline Sinclair Inlet & SI-SS-2 & Sand sole & WBC & $2838-72$ & 27.3 & 290 & 05/17/07 & 77.87 & 0.0215 \\
\hline Sinclair Inlet & SI-SS-3 & Sand sole & WBC & $2838-73$ & 29.8 & 320 & 05/17/07 & 78.95 & 0.0355 \\
\hline
\end{tabular}




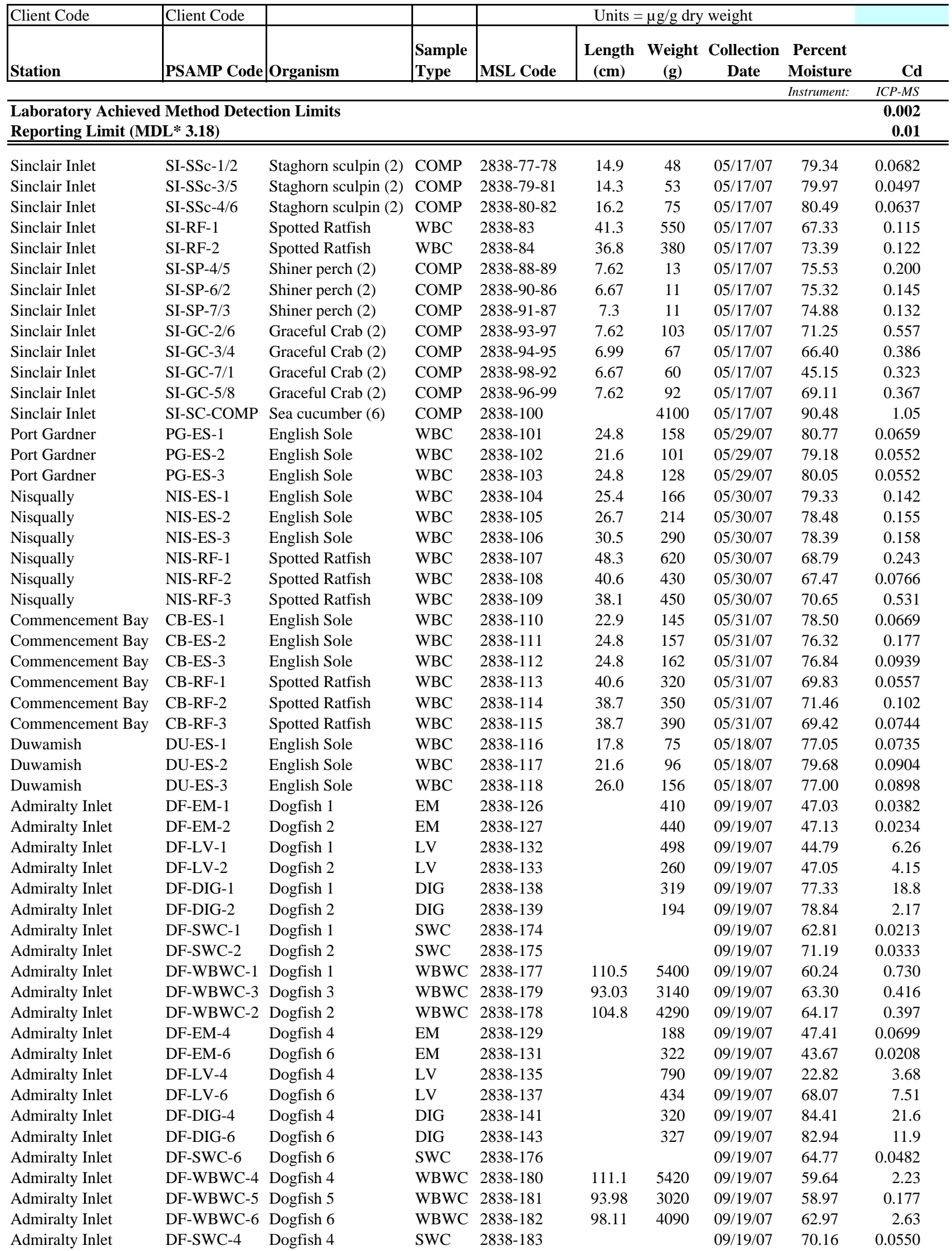




\begin{tabular}{|c|c|c|c|c|c|c|c|c|c|}
\hline Client Code & Client Code & & & & Units $=$ & $\mu \mathrm{g} / \mathrm{g}$ dry & weight & & \\
\hline Station & PSAMP Code & Organism & $\begin{array}{l}\text { Sample } \\
\text { Type }\end{array}$ & MSL Code & $\begin{array}{c}\text { Length } \\
\text { (cm) }\end{array}$ & $\begin{array}{c}\text { Weight } \\
\text { (g) }\end{array}$ & $\begin{array}{c}\text { Collection } \\
\text { Date }\end{array}$ & $\begin{array}{c}\text { Percent } \\
\text { Moisture } \\
\end{array}$ & $\mathrm{Cr}$ \\
\hline & & & & & & & & Instrument: & ICP-OES \\
\hline Laboratory Ach & Method Detect & tion Limits & & & & & & & 0.04 \\
\hline Reporting Limit & L* 3.18) & & & & & & & & 0.1 \\
\hline Vendovi & V-ES-5 & English Sole & WBC & $2838-5$ & 27.9 & 224 & 05/01/07 & 81.41 & 1.18 \\
\hline Vendovi & V-ES-6 & English Sole & WBC & $2838-6$ & 27.9 & 250 & 05/01/07 & 82.21 & 0.880 \\
\hline Vendovi & V-ES-8 & English Sole & WBC & $2838-8$ & 27.3 & 197 & 05/01/07 & 77.36 & 0.874 \\
\hline Vendovi & V-RS-1 & Rock sole & WBC & $2838-10$ & 20.3 & 119 & 05/01/07 & 78.21 & 1.09 \\
\hline Vendovi & V-RS-2 & Rock sole & WBC & 2838-11 & 21.0 & 142 & 05/01/07 & 78.77 & 0.931 \\
\hline Vendovi & V-RS-3 & Rock sole & WBC & $2838-12$ & 31.1 & 510 & 05/01/07 & 79.70 & 0.788 \\
\hline Vendovi & V-SSc-1 & Staghorn sculpin & WBC & $2838-13$ & 17.8 & 94 & 05/01/07 & 80.46 & 1.33 \\
\hline Vendovi & V-SSc-2 & Staghorn sculpin & WBC & $2838-14$ & 24.8 & 298 & 05/01/07 & 79.46 & 0.498 \\
\hline Vendovi & V-SSc-3 & Staghorn sculpin & WBC & $2838-15$ & 25.4 & 294 & 05/01/07 & 80.10 & 0.680 \\
\hline Vendovi & V-SP-1 & Shiner perch & WBC & $2838-16$ & 7.62 & 16 & 05/01/07 & 76.72 & 0.520 \\
\hline Vendovi & V-SP-2 & Shiner perch & WBC & $2838-17$ & 8.89 & 15 & 05/01/07 & 77.81 & 0.821 \\
\hline Vendovi & V-SP-3 & Shiner perch & WBC & $2838-18$ & 8.89 & 16 & 05/01/07 & 75.87 & 0.583 \\
\hline Vendovi & V-SC-COMP & Sea cucumber (3) & COMP & 2838-19 & & 5501 & 05/01/07 & 86.80 & 30.1 \\
\hline Strait of Georgia & SG-ES-3 & English Sole & WBC & $2838-22$ & 26.7 & 184 & $05 / 02 / 07$ & 79.46 & 0.803 \\
\hline Strait of Georgia & SG-ES-4 & English Sole & WBC & $2838-23$ & 26.0 & 189 & 05/02/07 & 79.05 & 0.754 \\
\hline Strait of Georgia & SG-ES-5 & English Sole & WBC & $2838-24$ & 26.7 & 192 & $05 / 02 / 07$ & 78.61 & 1.27 \\
\hline Strait of Georgia & SG-RF-1 & Spotted Ratfish & WBC & $2838-29$ & 27.9 & 115 & $05 / 02 / 07$ & 80.14 & 0.229 \\
\hline Strait of Georgia & SG-RF-2 & Spotted Ratfish & WBC & $2838-30$ & 35.6 & 320 & $05 / 02 / 07$ & 72.01 & 0.407 \\
\hline Strait of Georgia & SG-RF-3 & Spotted Ratfish & WBC & $2838-31$ & 48.3 & 770 & $05 / 02 / 07$ & 66.36 & $0.0914 \mathrm{~J}$ \\
\hline Strait of Georgia & SG-SC-COMP & Sea cucumber (6) & COMP & $2838-32$ & & 290 & $05 / 02 / 07$ & 92.30 & 8.15 \\
\hline Hood Canal & HC-ES-1 & English Sole & WBC & $2838-33$ & 22.9 & 131 & 05/03/07 & 82.09 & 1.92 \\
\hline Hood Canal & HC-ES-2 & English Sole & WBC & $2838-34$ & 22.9 & 133 & 05/03/07 & 79.72 & 0.402 \\
\hline Hood Canal & HC-ES-3 & English Sole & WBC & $2838-35$ & 26.7 & 220 & $05 / 03 / 07$ & 78.81 & 0.852 \\
\hline Hood Canal & HC-RS-1 & Rock sole & WBC & $2838-36$ & 31.8 & 460 & 05/03/07 & 77.93 & 0.684 \\
\hline Hood Canal & HC-RF-1 & Spotted Ratfish & WBC & $2838-37$ & 39.4 & 480 & 05/03/07 & 74.37 & 0.317 \\
\hline Hood Canal & HC-RF-2 & Spotted Ratfish & WBC & $2838-38$ & 38.1 & 340 & 05/03/07 & 72.96 & 0.240 \\
\hline Hood Canal & HC-RF-3 & Spotted Ratfish & WBC & 2838-39 & 42.5 & 450 & 05/03/07 & 72.39 & 0.135 \\
\hline Hood Canal & HC-SP1 & Shiner perch & WBC & $2838-40$ & 9.53 & 16 & 05/03/07 & 72.16 & 1.06 \\
\hline Hood Canal & $\mathrm{HC}-\mathrm{SP} 2$ & Shiner perch & WBC & $2838-41$ & 10.2 & 20 & 05/03/07 & 65.95 & 0.650 \\
\hline Hood Canal & HC-GC-1 & Graceful Crab & WBC & $2838-43$ & 10.2 & 237 & 05/03/07 & 80.86 & 1.42 \\
\hline Elliott Bay & EB-ES-1/2 & English Sole (2) & COMP & $2838-45-46$ & 15.8 & 44 & $05 / 16 / 07$ & 78.56 & 1.34 \\
\hline Elliott Bay & EB-ES-3 & English Sole & WBC & $2838-47$ & 26.0 & 157 & 05/16/07 & 80.28 & 0.930 \\
\hline Elliott Bay & EB-RF-1 & Spotted Ratfish & WBC & $2838-48$ & 40.6 & 350 & 05/16/07 & 73.57 & 0.292 \\
\hline Elliott Bay & EB-RF-2 & Spotted Ratfish & WBC & $2838-49$ & 41.9 & 370 & $05 / 16 / 07$ & 72.77 & 0.167 \\
\hline Elliott Bay & EB-RF-3 & Spotted Ratfish & WBC & $2838-50$ & 53.3 & 810 & 05/16/07 & 72.89 & 0.179 \\
\hline Eagle Harbor & EH-ES-2 & English Sole & WBC & $2838-52$ & 35.6 & 420 & 05/19/07 & 82.65 & 2.02 \\
\hline Eagle Harbor & EH-ES-3 & English Sole & WBC & $2838-53$ & 22.2 & 89 & 05/19/07 & 76.62 & 1.93 \\
\hline Eagle Harbor & EH-ES-4 & English Sole & WBC & $2838-54$ & 27.3 & 199 & 05/19/07 & 78.31 & 1.37 \\
\hline Eagle Harbor & EH-RF-1 & Spotted Ratfish & WBC & $2838-55$ & 33.0 & 230 & 05/19/07 & 73.50 & 2.13 \\
\hline Eagle Harbor & EH-RF-2 & Spotted Ratfish & WBC & $2838-56$ & 38.1 & 380 & 05/19/07 & 76.61 & 0.182 \\
\hline Eagle Harbor & EH-RF-3 & Spotted Ratfish & WBC & $2838-57$ & 50.8 & 760 & $05 / 19 / 07$ & 68.32 & 0.444 \\
\hline Sinclair Inlet & SI-ES-1 & English Sole & WBC & $2838-58$ & 29.2 & 330 & 05/17/07 & 77.81 & 1.07 \\
\hline Sinclair Inlet & SI-ES-2 & English Sole & WBC & $2838-59$ & 29.2 & 330 & 05/17/07 & 80.65 & 1.21 \\
\hline Sinclair Inlet & SI-ES-4 & English Sole & WBC & $2838-61$ & 25.4 & 192 & 05/17/07 & 59.34 & 1.48 \\
\hline Sinclair Inlet & SI-ES-6 & English Sole & WBC & $2838-63$ & 25.4 & 178 & 05/17/07 & 79.69 & 0.854 \\
\hline Sinclair Inlet & SI-ES-7 & English Sole & WBC & $2838-64$ & 25.4 & 179 & 05/17/07 & 79.49 & 0.779 \\
\hline Sinclair Inlet & SI-ES-8 & English Sole & WBC & $2838-65$ & 29.2 & 261 & 05/17/07 & 79.29 & 1.61 \\
\hline Sinclair Inlet & SI-RS-1/2 & Rock sole (2) & COMP & $2838-67-68$ & 10.2 & 23 & 05/17/07 & 75.72 & 0.896 \\
\hline Sinclair Inlet & SI-RS-3 & Rock sole & WBC & $2838-69$ & 27.3 & 320 & 05/17/07 & 78.88 & 0.664 \\
\hline Sinclair Inlet & SI-RS-4 & Rock sole & WBC & $2838-70$ & 26.7 & 220 & 05/17/07 & 78.83 & 1.11 \\
\hline Sinclair Inlet & SI-SS-1 & Sand sole & WBC & $2838-71$ & 25.4 & 270 & 05/17/07 & 78.34 & 0.733 \\
\hline Sinclair Inlet & SI-SS-2 & Sand sole & WBC & $2838-72$ & 27.3 & 290 & 05/17/07 & 77.87 & 0.839 \\
\hline Sinclair Inlet & SI-SS-3 & Sand sole & WBC & $2838-73$ & 29.8 & 320 & 05/17/07 & 78.95 & 0.810 \\
\hline
\end{tabular}




\begin{tabular}{|c|c|c|c|c|c|c|c|c|c|}
\hline Client Code & Client Code & & & & Units $=$ & $\mu \mathrm{g} / \mathrm{g}$ dry & weight & & \\
\hline Station & PSAMP Code & Organism & \begin{tabular}{|l} 
Sample \\
Type
\end{tabular} & MSL Code & $\begin{array}{l}\text { Length } \\
\text { (cm) }\end{array}$ & $\begin{array}{c}\text { Weight } \\
\text { (g) }\end{array}$ & $\begin{array}{c}\text { Collection } \\
\text { Date }\end{array}$ & $\begin{array}{c}\text { Percent } \\
\text { Moisture } \\
\end{array}$ & $\mathrm{Cr}$ \\
\hline & & & & & & & & Instrument: & ICP-OES \\
\hline Laboratory Achievec & Method Detect & tion Limits & & & & & & & 0.04 \\
\hline Reporting Limit (MI & $\left.\mathrm{L}^{*} 3.18\right)$ & & & & & & & & 0.1 \\
\hline Sinclair Inlet & SI-SSc-1/2 & Staghorn sculpin (2) & COMP & 2838-77-78 & 14.9 & 48 & 05/17/07 & 79.34 & 1.45 \\
\hline Sinclair Inlet & SI-SSc-3/5 & Staghorn sculpin (2) & COMP & 2838-79-81 & 14.3 & 53 & $05 / 17 / 07$ & 79.97 & 1.00 \\
\hline Sinclair Inlet & SI-SSc-4/6 & Staghorn sculpin (2) & COMP & $2838-80-82$ & 16.2 & 75 & 05/17/07 & 80.49 & 1.04 \\
\hline Sinclair Inlet & SI-RF-1 & Spotted Ratfish & WBC & $2838-83$ & 41.3 & 550 & $05 / 17 / 07$ & 67.33 & 0.317 \\
\hline Sinclair Inlet & SI-RF-2 & Spotted Ratfish & WBC & $2838-84$ & 36.8 & 380 & 05/17/07 & 73.39 & 0.162 \\
\hline Sinclair Inlet & SI-SP-4/5 & Shiner perch (2) & COMP & $2838-88-89$ & 7.62 & 13 & 05/17/07 & 75.53 & 1.05 \\
\hline Sinclair Inlet & SI-SP-6/2 & Shiner perch (2) & COMP & $2838-90-86$ & 6.67 & 11 & 05/17/07 & 75.32 & 0.602 \\
\hline Sinclair Inlet & SI-SP-7/3 & Shiner perch (2) & COMP & $2838-91-87$ & 7.3 & 11 & 05/17/07 & 74.88 & 1.09 \\
\hline Sinclair Inlet & SI-GC-2/6 & Graceful Crab (2) & COMP & $2838-93-97$ & 7.62 & 103 & $05 / 17 / 07$ & 71.25 & 2.44 \\
\hline Sinclair Inlet & SI-GC-3/4 & Graceful Crab (2) & COMP & 2838-94-95 & 6.99 & 67 & 05/17/07 & 66.40 & 1.00 \\
\hline Sinclair Inlet & SI-GC-7/1 & Graceful Crab (2) & COMP & $2838-98-92$ & 6.67 & 60 & 05/17/07 & 45.15 & 1.06 \\
\hline Sinclair Inlet & SI-GC-5/8 & Graceful Crab (2) & COMP & 2838-96-99 & 7.62 & 92 & 05/17/07 & 69.11 & 2.57 \\
\hline Sinclair Inlet & SI-SC-COMP & Sea cucumber (6) & COMP & $2838-100$ & & 4100 & 05/17/07 & 90.48 & 10.2 \\
\hline Port Gardner & PG-ES-1 & English Sole & WBC & 2838-101 & 24.8 & 158 & 05/29/07 & 80.77 & 2.13 \\
\hline Port Gardner & PG-ES-2 & English Sole & WBC & $2838-102$ & 21.6 & 101 & $05 / 29 / 07$ & 79.18 & 2.78 \\
\hline Port Gardner & PG-ES-3 & English Sole & WBC & $2838-103$ & 24.8 & 128 & 05/29/07 & 80.05 & 1.84 \\
\hline Nisqually & NIS-ES-1 & English Sole & WBC & $2838-104$ & 25.4 & 166 & 05/30/07 & 79.33 & 1.64 \\
\hline Nisqually & NIS-ES-2 & English Sole & WBC & $2838-105$ & 26.7 & 214 & 05/30/07 & 78.48 & 2.01 \\
\hline Nisqually & NIS-ES-3 & English Sole & WBC & 2838-106 & 30.5 & 290 & 05/30/07 & 78.39 & 0.652 \\
\hline Nisqually & NIS-RF-1 & Spotted Ratfish & WBC & $2838-107$ & 48.3 & 620 & 05/30/07 & 68.79 & $0.0771 \mathrm{~J}$ \\
\hline Nisqually & NIS-RF-2 & Spotted Ratfish & WBC & $2838-108$ & 40.6 & 430 & 05/30/07 & 67.47 & 0.201 \\
\hline Nisqually & NIS-RF-3 & Spotted Ratfish & WBC & 2838-109 & 38.1 & 450 & 05/30/07 & 70.65 & 0.111 \\
\hline Commencement Bay & CB-ES-1 & English Sole & WBC & $2838-110$ & 22.9 & 145 & 05/31/07 & 78.50 & 0.786 \\
\hline Commencement Bay & CB-ES-2 & English Sole & WBC & 2838-111 & 24.8 & 157 & 05/31/07 & 76.32 & 1.31 \\
\hline Commencement Bay & CB-ES-3 & English Sole & WBC & $2838-112$ & 24.8 & 162 & 05/31/07 & 76.84 & 1.47 \\
\hline Commencement Bay & CB-RF-1 & Spotted Ratfish & WBC & $2838-113$ & 40.6 & 320 & $05 / 31 / 07$ & 69.83 & 0.312 \\
\hline Commencement Bay & CB-RF-2 & Spotted Ratfish & WBC & $2838-114$ & 38.7 & 350 & 05/31/07 & 71.46 & 0.347 \\
\hline Commencement Bay & CB-RF-3 & Spotted Ratfish & WBC & $2838-115$ & 38.7 & 390 & 05/31/07 & 69.42 & 0.261 \\
\hline Duwamish & DU-ES-1 & English Sole & WBC & $2838-116$ & 17.8 & 75 & 05/18/07 & 77.05 & 1.23 \\
\hline Duwamish & DU-ES-2 & English Sole & WBC & $2838-117$ & 21.6 & 96 & 05/18/07 & 79.68 & 2.24 \\
\hline Duwamish & DU-ES-3 & English Sole & WBC & $2838-118$ & 26.0 & 156 & 05/18/07 & 77.00 & 1.03 \\
\hline Admiralty Inlet & DF-EM-1 & Dogfish 1 & EM & $2838-126$ & & 410 & 09/19/07 & 47.03 & 0.198 \\
\hline Admiralty Inlet & DF-EM-2 & Dogfish 2 & EM & $2838-127$ & & 440 & 09/19/07 & 47.13 & 0.213 \\
\hline Admiralty Inlet & DF-LV-1 & Dogfish 1 & LV & $2838-132$ & & 498 & 09/19/07 & 44.79 & $0.0631 \mathrm{~J}$ \\
\hline Admiralty Inlet & DF-LV-2 & Dogfish 2 & LV & $2838-133$ & & 260 & 09/19/07 & 47.05 & 0.143 \\
\hline Admiralty Inlet & DF-DIG-1 & Dogfish 1 & DIG & $2838-138$ & & 319 & 09/19/07 & 77.33 & 0.616 \\
\hline Admiralty Inlet & DF-DIG-2 & Dogfish 2 & DIG & $2838-139$ & & 194 & 09/19/07 & 78.84 & 0.227 \\
\hline Admiralty Inlet & DF-SWC-1 & Dogfish 1 & SWC & $2838-174$ & & & 09/19/07 & 62.81 & 0.168 \\
\hline Admiralty Inlet & DF-SWC-2 & Dogfish 2 & SWC & $2838-175$ & & & 09/19/07 & 71.19 & 0.133 \\
\hline Admiralty Inlet & DF-WBWC-1 & Dogfish 1 & WBWC & $2838-177$ & 110.5 & 5400 & 09/19/07 & 60.24 & 0.170 \\
\hline Admiralty Inlet & DF-WBWC-3 & Dogfish 3 & WBWC & $2838-179$ & 93.03 & 3140 & 09/19/07 & 63.30 & 0.136 \\
\hline Admiralty Inlet & DF-WBWC-2 & Dogfish 2 & WBWC & 2838-178 & 104.8 & 4290 & 09/19/07 & 64.17 & 0.183 \\
\hline Admiralty Inlet & DF-EM-4 & Dogfish 4 & EM & $2838-129$ & & 188 & 09/19/07 & 47.41 & 0.174 \\
\hline Admiralty Inlet & DF-EM-6 & Dogfish 6 & EM & $2838-131$ & & 322 & 09/19/07 & 43.67 & 0.209 \\
\hline Admiralty Inlet & DF-LV-4 & Dogfish 4 & LV & $2838-135$ & & 790 & 09/19/07 & 22.82 & $0.0531 \mathrm{~J}$ \\
\hline Admiralty Inlet & DF-LV-6 & Dogfish 6 & LV & $2838-137$ & & 434 & 09/19/07 & 68.07 & $0.0710 \mathrm{~J}$ \\
\hline Admiralty Inlet & DF-DIG-4 & Dogfish 4 & DIG & $2838-141$ & & 320 & 09/19/07 & 84.41 & 0.320 \\
\hline Admiralty Inlet & DF-DIG-6 & Dogfish 6 & DIG & $2838-143$ & & 327 & 09/19/07 & 82.94 & 3.90 \\
\hline Admiralty Inlet & DF-SWC-6 & Dogfish 6 & SWC & $2838-176$ & & & 09/19/07 & 64.77 & 0.162 \\
\hline Admiralty Inlet & DF-WBWC-4 & Dogfish 4 & WBWC & $2838-180$ & 111.1 & 5420 & 09/19/07 & 59.64 & 0.253 \\
\hline Admiralty Inlet & DF-WBWC-5 & Dogfish 5 & WBWC & $2838-181$ & 93.98 & 3020 & 09/19/07 & 58.97 & 0.129 \\
\hline Admiralty Inlet & DF-WBWC-6 & Dogfish 6 & WBWC & $2838-182$ & 98.11 & 4090 & 09/19/07 & 62.97 & 0.578 \\
\hline Admiralty Inlet & DF-SWC-4 & Dogfish 4 & SWC & $2838-183$ & & & 09/19/07 & 70.16 & 0.160 \\
\hline
\end{tabular}




\begin{tabular}{|c|c|c|c|c|c|c|c|c|c|}
\hline Client Code & Client Code & & & & Units $=$ & $\mu \mathrm{g} / \mathrm{g}$ dry & weight & & \\
\hline Station & PSAMP Code & Organism & \begin{tabular}{|l} 
Sample \\
Type
\end{tabular} & MSL Code & $\begin{array}{l}\text { Length } \\
\text { (cm) }\end{array}$ & $\begin{array}{c}\text { Weight } \\
\text { (g) }\end{array}$ & $\begin{array}{c}\text { Collection } \\
\text { Date }\end{array}$ & $\begin{array}{l}\text { Percent } \\
\text { Moisture }\end{array}$ & $\mathbf{C u}$ \\
\hline & & & & & & & & Instrument: & $I C P-M S$ \\
\hline Laboratory Achi & Method Detect & tion Limits & & & & & & & 0.09 \\
\hline Reporting Limit & $\left.\mathrm{L}^{*} 3.18\right)$ & & & & & & & & 0.3 \\
\hline Vendovi & V-ES-5 & English Sole & WBC & $2838-5$ & 27.9 & 224 & 05/01/07 & 81.41 & 3.43 \\
\hline Vendovi & V-ES-6 & English Sole & WBC & $2838-6$ & 27.9 & 250 & 05/01/07 & 82.21 & 2.23 \\
\hline Vendovi & V-ES-8 & English Sole & WBC & $2838-8$ & 27.3 & 197 & 05/01/07 & 77.36 & 2.78 \\
\hline Vendovi & V-RS-1 & Rock sole & WBC & $2838-10$ & 20.3 & 119 & 05/01/07 & 78.21 & 2.41 \\
\hline Vendovi & V-RS-2 & Rock sole & WBC & $2838-11$ & 21.0 & 142 & 05/01/07 & 78.77 & 2.19 \\
\hline Vendovi & V-RS-3 & Rock sole & WBC & $2838-12$ & 31.1 & 510 & 05/01/07 & 79.70 & 1.88 \\
\hline Vendovi & V-SSc-1 & Staghorn sculpin & WBC & $2838-13$ & 17.8 & 94 & 05/01/07 & 80.46 & 10.2 \\
\hline Vendovi & V-SSc-2 & Staghorn sculpin & WBC & $2838-14$ & 24.8 & 298 & 05/01/07 & 79.46 & 3.78 \\
\hline Vendovi & V-SSc-3 & Staghorn sculpin & WBC & $2838-15$ & 25.4 & 294 & 05/01/07 & 80.10 & 3.75 \\
\hline Vendovi & V-SP-1 & Shiner perch & WBC & $2838-16$ & 7.62 & 16 & 05/01/07 & 76.72 & 2.67 \\
\hline Vendovi & V-SP-2 & Shiner perch & WBC & $2838-17$ & 8.89 & 15 & 05/01/07 & 77.81 & 3.65 \\
\hline Vendovi & V-SP-3 & Shiner perch & WBC & $2838-18$ & 8.89 & 16 & 05/01/07 & 75.87 & 2.61 \\
\hline Vendovi & V-SC-COMP & Sea cucumber (3) & COMP & 2838-19 & & 5501 & 05/01/07 & 86.80 & 47.3 \\
\hline Strait of Georgia & SG-ES-3 & English Sole & WBC & $2838-22$ & 26.7 & 184 & 05/02/07 & 79.46 & 2.45 \\
\hline Strait of Georgia & SG-ES-4 & English Sole & WBC & $2838-23$ & 26.0 & 189 & 05/02/07 & 79.05 & 2.93 \\
\hline Strait of Georgia & SG-ES-5 & English Sole & WBC & $2838-24$ & 26.7 & 192 & 05/02/07 & 78.61 & 3.79 \\
\hline Strait of Georgia & SG-RF-1 & Spotted Ratfish & WBC & $2838-29$ & 27.9 & 115 & 05/02/07 & 80.14 & 2.54 \\
\hline Strait of Georgia & SG-RF-2 & Spotted Ratfish & WBC & $2838-30$ & 35.6 & 320 & 05/02/07 & 72.01 & 2.43 \\
\hline Strait of Georgia & SG-RF-3 & Spotted Ratfish & WBC & $2838-31$ & 48.3 & 770 & 05/02/07 & 66.36 & 2.38 \\
\hline Strait of Georgia & SG-SC-COMP & Sea cucumber (6) & COMP & $2838-32$ & & 290 & 05/02/07 & 92.30 & 13.0 \\
\hline Hood Canal & HC-ES-1 & English Sole & WBC & $2838-33$ & 22.9 & 131 & 05/03/07 & 82.09 & 2.83 \\
\hline Hood Canal & HC-ES-2 & English Sole & WBC & $2838-34$ & 22.9 & 133 & 05/03/07 & 79.72 & 2.04 \\
\hline Hood Canal & HC-ES-3 & English Sole & WBC & $2838-35$ & 26.7 & 220 & 05/03/07 & 78.81 & 2.87 \\
\hline Hood Canal & HC-RS-1 & Rock sole & WBC & $2838-36$ & 31.8 & 460 & 05/03/07 & 77.93 & 2.09 \\
\hline Hood Canal & HC-RF-1 & Spotted Ratfish & WBC & $2838-37$ & 39.4 & 480 & 05/03/07 & 74.37 & 3.61 \\
\hline Hood Canal & HC-RF-2 & Spotted Ratfish & WBC & $2838-38$ & 38.1 & 340 & 05/03/07 & 72.96 & 2.65 \\
\hline Hood Canal & HC-RF-3 & Spotted Ratfish & WBC & 2838-39 & 42.5 & 450 & 05/03/07 & 72.39 & 4.60 \\
\hline Hood Canal & HC-SP1 & Shiner perch & WBC & $2838-40$ & 9.53 & 16 & 05/03/07 & 72.16 & 2.64 \\
\hline Hood Canal & HC-SP2 & Shiner perch & WBC & $2838-41$ & 10.2 & 20 & 05/03/07 & 65.95 & 2.69 \\
\hline Hood Canal & HC-GC-1 & Graceful Crab & WBC & $2838-43$ & 10.2 & 237 & 05/03/07 & 80.86 & 46.9 \\
\hline Elliott Bay & EB-ES-1/2 & English Sole (2) & COMP & $2838-45-46$ & 15.8 & 44 & 05/16/07 & 78.56 & 4.04 \\
\hline Elliott Bay & EB-ES-3 & English Sole & WBC & $2838-47$ & 26.0 & 157 & 05/16/07 & 80.28 & 3.60 \\
\hline Elliott Bay & EB-RF-1 & Spotted Ratfish & WBC & $2838-48$ & 40.6 & 350 & 05/16/07 & 73.57 & 4.30 \\
\hline Elliott Bay & EB-RF-2 & Spotted Ratfish & WBC & $2838-49$ & 41.9 & 370 & 05/16/07 & 72.77 & 3.19 \\
\hline Elliott Bay & EB-RF-3 & Spotted Ratfish & WBC & $2838-50$ & 53.3 & 810 & 05/16/07 & 72.89 & 4.66 \\
\hline Eagle Harbor & EH-ES-2 & English Sole & WBC & $2838-52$ & 35.6 & 420 & 05/19/07 & 82.65 & 4.88 \\
\hline Eagle Harbor & EH-ES-3 & English Sole & WBC & $2838-53$ & 22.2 & 89 & 05/19/07 & 76.62 & 4.79 \\
\hline Eagle Harbor & EH-ES-4 & English Sole & WBC & $2838-54$ & 27.3 & 199 & 05/19/07 & 78.31 & 3.68 \\
\hline Eagle Harbor & EH-RF-1 & Spotted Ratfish & WBC & $2838-55$ & 33.0 & 230 & 05/19/07 & 73.50 & 4.43 \\
\hline Eagle Harbor & EH-RF-2 & Spotted Ratfish & WBC & $2838-56$ & 38.1 & 380 & 05/19/07 & 76.61 & 4.39 \\
\hline Eagle Harbor & EH-RF-3 & Spotted Ratfish & WBC & $2838-57$ & 50.8 & 760 & 05/19/07 & 68.32 & 3.93 \\
\hline Sinclair Inlet & SI-ES-1 & English Sole & WBC & $2838-58$ & 29.2 & 330 & 05/17/07 & 77.81 & 4.01 \\
\hline Sinclair Inlet & SI-ES-2 & English Sole & WBC & $2838-59$ & 29.2 & 330 & 05/17/07 & 80.65 & 4.37 \\
\hline Sinclair Inlet & SI-ES-4 & English Sole & WBC & $2838-61$ & 25.4 & 192 & 05/17/07 & 59.34 & 4.57 \\
\hline Sinclair Inlet & SI-ES-6 & English Sole & WBC & $2838-63$ & 25.4 & 178 & 05/17/07 & 79.69 & 2.75 \\
\hline Sinclair Inlet & SI-ES-7 & English Sole & WBC & $2838-64$ & 25.4 & 179 & 05/17/07 & 79.49 & 2.64 \\
\hline Sinclair Inlet & SI-ES-8 & English Sole & WBC & $2838-65$ & 29.2 & 261 & 05/17/07 & 79.29 & 4.22 \\
\hline Sinclair Inlet & SI-RS-1/2 & Rock sole (2) & COMP & $2838-67-68$ & 10.2 & 23 & 05/17/07 & 75.72 & 4.34 \\
\hline Sinclair Inlet & SI-RS-3 & Rock sole & WBC & $2838-69$ & 27.3 & 320 & 05/17/07 & 78.88 & 4.53 \\
\hline Sinclair Inlet & SI-RS-4 & Rock sole & WBC & $2838-70$ & 26.7 & 220 & 05/17/07 & 78.83 & 1.78 \\
\hline Sinclair Inlet & SI-SS-1 & Sand sole & WBC & $2838-71$ & 25.4 & 270 & 05/17/07 & 78.34 & 4.51 \\
\hline Sinclair Inlet & SI-SS-2 & Sand sole & WBC & $2838-72$ & 27.3 & 290 & 05/17/07 & 77.87 & 2.17 \\
\hline Sinclair Inlet & SI-SS-3 & Sand sole & WBC & $2838-73$ & 29.8 & 320 & 05/17/07 & 78.95 & 4.09 \\
\hline
\end{tabular}




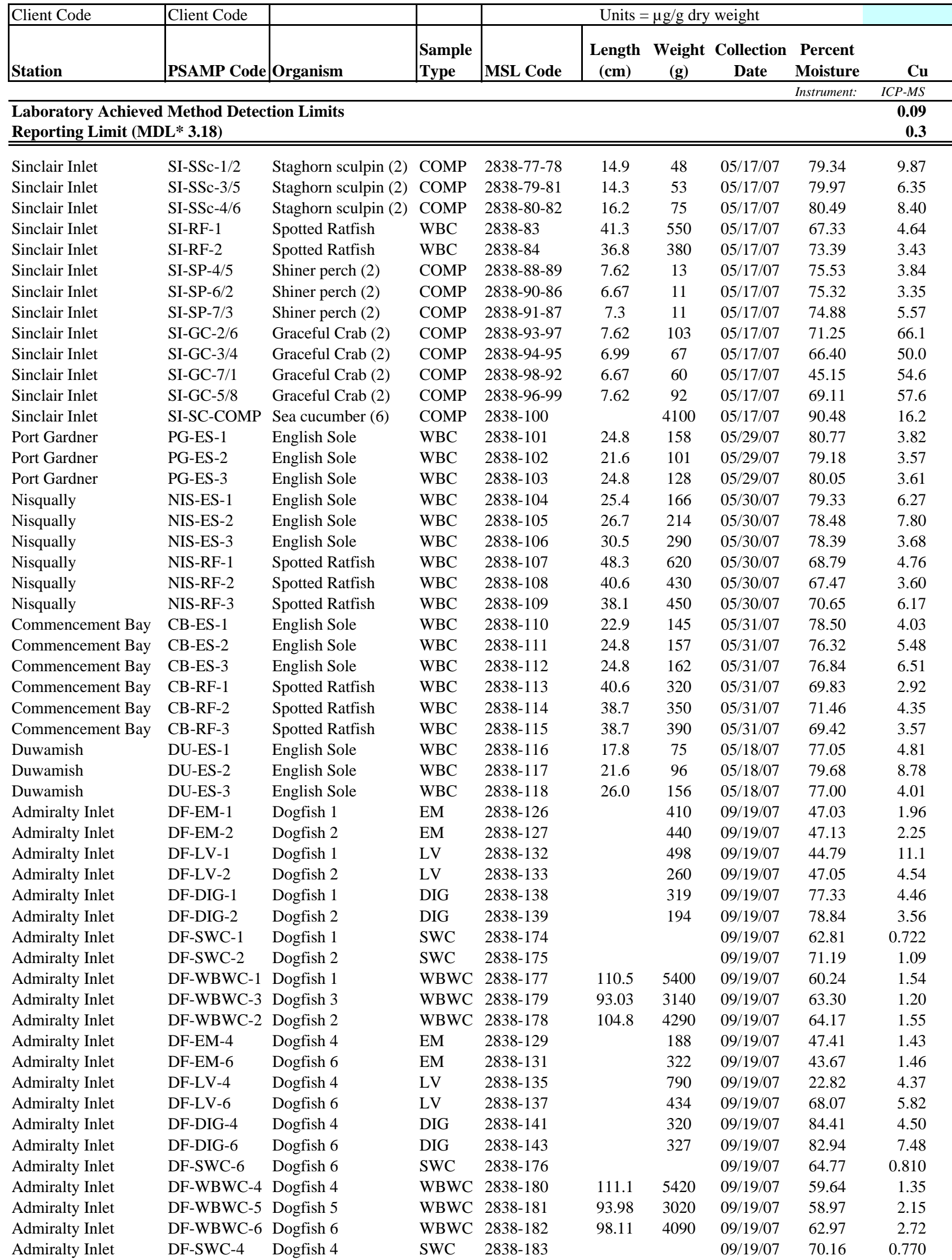




\begin{tabular}{|c|c|c|c|c|c|c|c|c|c|}
\hline Client Code & Client Code & & & & Units $=$ & $\mu \mathrm{g} / \mathrm{g}$ dry & weight & & \\
\hline Station & PSAMP Code & Organism & \begin{tabular}{|l} 
Sample \\
Type
\end{tabular} & MSL Code & $\begin{array}{c}\text { Length } \\
\text { (cm) }\end{array}$ & $\begin{array}{c}\text { Weight } \\
\text { (g) }\end{array}$ & $\begin{array}{c}\text { Collection } \\
\text { Date }\end{array}$ & $\begin{array}{c}\text { Percent } \\
\text { Moisture }\end{array}$ & Hg \\
\hline & & & & & & & & Instrument: & CVAA \\
\hline Laboratory Achi & Method Detect & tion Limits & & & & & & & 0.005 \\
\hline Reporting Limit & $\left.\mathrm{L}^{*} 3.18\right)$ & & & & & & & & 0.02 \\
\hline Vendovi & V-ES-5 & English Sole & WBC & $2838-5$ & 27.9 & 224 & 05/01/07 & 81.41 & 0.228 \\
\hline Vendovi & V-ES-6 & English Sole & WBC & $2838-6$ & 27.9 & 250 & 05/01/07 & 82.21 & 0.198 \\
\hline Vendovi & V-ES-8 & English Sole & WBC & $2838-8$ & 27.3 & 197 & 05/01/07 & 77.36 & 0.143 \\
\hline Vendovi & V-RS-1 & Rock sole & WBC & $2838-10$ & 20.3 & 119 & 05/01/07 & 78.21 & 0.149 \\
\hline Vendovi & V-RS-2 & Rock sole & WBC & $2838-11$ & 21.0 & 142 & 05/01/07 & 78.77 & 0.140 \\
\hline Vendovi & V-RS-3 & Rock sole & WBC & $2838-12$ & 31.1 & 510 & 05/01/07 & 79.70 & 0.270 \\
\hline Vendovi & V-SSc-1 & Staghorn sculpin & WBC & $2838-13$ & 17.8 & 94 & 05/01/07 & 80.46 & 0.300 \\
\hline Vendovi & V-SSc-2 & Staghorn sculpin & WBC & $2838-14$ & 24.8 & 298 & 05/01/07 & 79.46 & 0.312 \\
\hline Vendovi & V-SSc-3 & Staghorn sculpin & WBC & $2838-15$ & 25.4 & 294 & 05/01/07 & 80.10 & 0.353 \\
\hline Vendovi & V-SP-1 & Shiner perch & WBC & $2838-16$ & 7.62 & 16 & $05 / 01 / 07$ & 76.72 & 0.190 \\
\hline Vendovi & V-SP-2 & Shiner perch & WBC & $2838-17$ & 8.89 & 15 & 05/01/07 & 77.81 & 0.267 \\
\hline Vendovi & V-SP-3 & Shiner perch & WBC & $2838-18$ & 8.89 & 16 & 05/01/07 & 75.87 & 0.179 \\
\hline Vendovi & V-SC-COMP & Sea cucumber (3) & COMP & 2838-19 & & 5501 & 05/01/07 & 86.80 & 0.0513 \\
\hline Strait of Georgia & SG-ES-3 & English Sole & WBC & $2838-22$ & 26.7 & 184 & $05 / 02 / 07$ & 79.46 & 0.132 \\
\hline Strait of Georgia & SG-ES-4 & English Sole & WBC & $2838-23$ & 26.0 & 189 & $05 / 02 / 07$ & 79.05 & 0.178 \\
\hline Strait of Georgia & SG-ES-5 & English Sole & WBC & $2838-24$ & 26.7 & 192 & $05 / 02 / 07$ & 78.61 & 0.177 \\
\hline Strait of Georgia & SG-RF-1 & Spotted Ratfish & WBC & $2838-29$ & 27.9 & 115 & $05 / 02 / 07$ & 80.14 & 0.153 \\
\hline Strait of Georgia & SG-RF-2 & Spotted Ratfish & WBC & $2838-30$ & 35.6 & 320 & $05 / 02 / 07$ & 72.01 & 0.227 \\
\hline Strait of Georgia & SG-RF-3 & Spotted Ratfish & WBC & $2838-31$ & 48.3 & 770 & $05 / 02 / 07$ & 66.36 & 0.413 \\
\hline Strait of Georgia & SG-SC-COMP & Sea cucumber (6) & COMP & $2838-32$ & & 290 & $05 / 02 / 07$ & 92.30 & 0.0333 \\
\hline Hood Canal & HC-ES-1 & English Sole & WBC & $2838-33$ & 22.9 & 131 & 05/03/07 & 82.09 & 0.212 \\
\hline Hood Canal & HC-ES-2 & English Sole & WBC & 2838-34 & 22.9 & 133 & $05 / 03 / 07$ & 79.72 & 0.154 \\
\hline Hood Canal & HC-ES-3 & English Sole & WBC & $2838-35$ & 26.7 & 220 & 05/03/07 & 78.81 & 0.239 \\
\hline Hood Canal & HC-RS-1 & Rock sole & WBC & $2838-36$ & 31.8 & 460 & 05/03/07 & 77.93 & 0.216 \\
\hline Hood Canal & HC-RF-1 & Spotted Ratfish & WBC & $2838-37$ & 39.4 & 480 & 05/03/07 & 74.37 & 0.298 \\
\hline Hood Canal & HC-RF-2 & Spotted Ratfish & WBC & $2838-38$ & 38.1 & 340 & 05/03/07 & 72.96 & 0.522 \\
\hline Hood Canal & HC-RF-3 & Spotted Ratfish & WBC & 2838-39 & 42.5 & 450 & 05/03/07 & 72.39 & 0.593 \\
\hline Hood Canal & HC-SP1 & Shiner perch & WBC & $2838-40$ & 9.53 & 16 & 05/03/07 & 72.16 & 0.155 \\
\hline Hood Canal & HC-SP2 & Shiner perch & WBC & $2838-41$ & 10.2 & 20 & 05/03/07 & 65.95 & 0.112 \\
\hline Hood Canal & HC-GC-1 & Graceful Crab & WBC & $2838-43$ & 10.2 & 237 & 05/03/07 & 80.86 & 0.0902 \\
\hline Elliott Bay & EB-ES-1/2 & English Sole (2) & COMP & $2838-45-46$ & 15.8 & 44 & $05 / 16 / 07$ & 78.56 & 0.108 \\
\hline Elliott Bay & EB-ES-3 & English Sole & WBC & $2838-47$ & 26.0 & 157 & $05 / 16 / 07$ & 80.28 & 0.443 \\
\hline Elliott Bay & EB-RF-1 & Spotted Ratfish & WBC & $2838-48$ & 40.6 & 350 & 05/16/07 & 73.57 & 0.734 \\
\hline Elliott Bay & EB-RF-2 & Spotted Ratfish & WBC & $2838-49$ & 41.9 & 370 & $05 / 16 / 07$ & 72.77 & 0.344 \\
\hline Elliott Bay & EB-RF-3 & Spotted Ratfish & WBC & $2838-50$ & 53.3 & 810 & 05/16/07 & 72.89 & 0.776 \\
\hline Eagle Harbor & EH-ES-2 & English Sole & WBC & $2838-52$ & 35.6 & 420 & 05/19/07 & 82.65 & 0.406 \\
\hline Eagle Harbor & EH-ES-3 & English Sole & WBC & $2838-53$ & 22.2 & 89 & $05 / 19 / 07$ & 76.62 & 0.353 \\
\hline Eagle Harbor & EH-ES-4 & English Sole & WBC & $2838-54$ & 27.3 & 199 & 05/19/07 & 78.31 & 0.191 \\
\hline Eagle Harbor & EH-RF-1 & Spotted Ratfish & WBC & $2838-55$ & 33.0 & 230 & 05/19/07 & 73.50 & 0.255 \\
\hline Eagle Harbor & EH-RF-2 & Spotted Ratfish & WBC & $2838-56$ & 38.1 & 380 & 05/19/07 & 76.61 & 0.668 \\
\hline Eagle Harbor & EH-RF-3 & Spotted Ratfish & WBC & $2838-57$ & 50.8 & 760 & $05 / 19 / 07$ & 68.32 & 0.737 \\
\hline Sinclair Inlet & SI-ES-1 & English Sole & WBC & $2838-58$ & 29.2 & 330 & $05 / 17 / 07$ & 77.81 & 0.133 \\
\hline Sinclair Inlet & SI-ES-2 & English Sole & WBC & $2838-59$ & 29.2 & 330 & 05/17/07 & 80.65 & 0.126 \\
\hline Sinclair Inlet & SI-ES-4 & English Sole & WBC & $2838-61$ & 25.4 & 192 & 05/17/07 & 59.34 & 0.207 \\
\hline Sinclair Inlet & SI-ES-6 & English Sole & WBC & $2838-63$ & 25.4 & 178 & 05/17/07 & 79.69 & 0.113 \\
\hline Sinclair Inlet & SI-ES-7 & English Sole & WBC & $2838-64$ & 25.4 & 179 & 05/17/07 & 79.49 & 0.294 \\
\hline Sinclair Inlet & SI-ES-8 & English Sole & WBC & $2838-65$ & 29.2 & 261 & 05/17/07 & 79.29 & 0.180 \\
\hline Sinclair Inlet & SI-RS-1/2 & Rock sole (2) & COMP & $2838-67-68$ & 10.2 & 23 & 05/17/07 & 75.72 & 0.0898 \\
\hline Sinclair Inlet & SI-RS-3 & Rock sole & WBC & $2838-69$ & 27.3 & 320 & 05/17/07 & 78.88 & 0.187 \\
\hline Sinclair Inlet & SI-RS-4 & Rock sole & WBC & $2838-70$ & 26.7 & 220 & 05/17/07 & 78.83 & 0.410 \\
\hline Sinclair Inlet & SI-SS-1 & Sand sole & WBC & 2838-71 & 25.4 & 270 & 05/17/07 & 78.34 & 0.512 \\
\hline Sinclair Inlet & SI-SS-2 & Sand sole & WBC & $2838-72$ & 27.3 & 290 & 05/17/07 & 77.87 & 0.397 \\
\hline Sinclair Inlet & SI-SS-3 & Sand sole & WBC & $2838-73$ & 29.8 & 320 & 05/17/07 & 78.95 & 0.480 \\
\hline
\end{tabular}




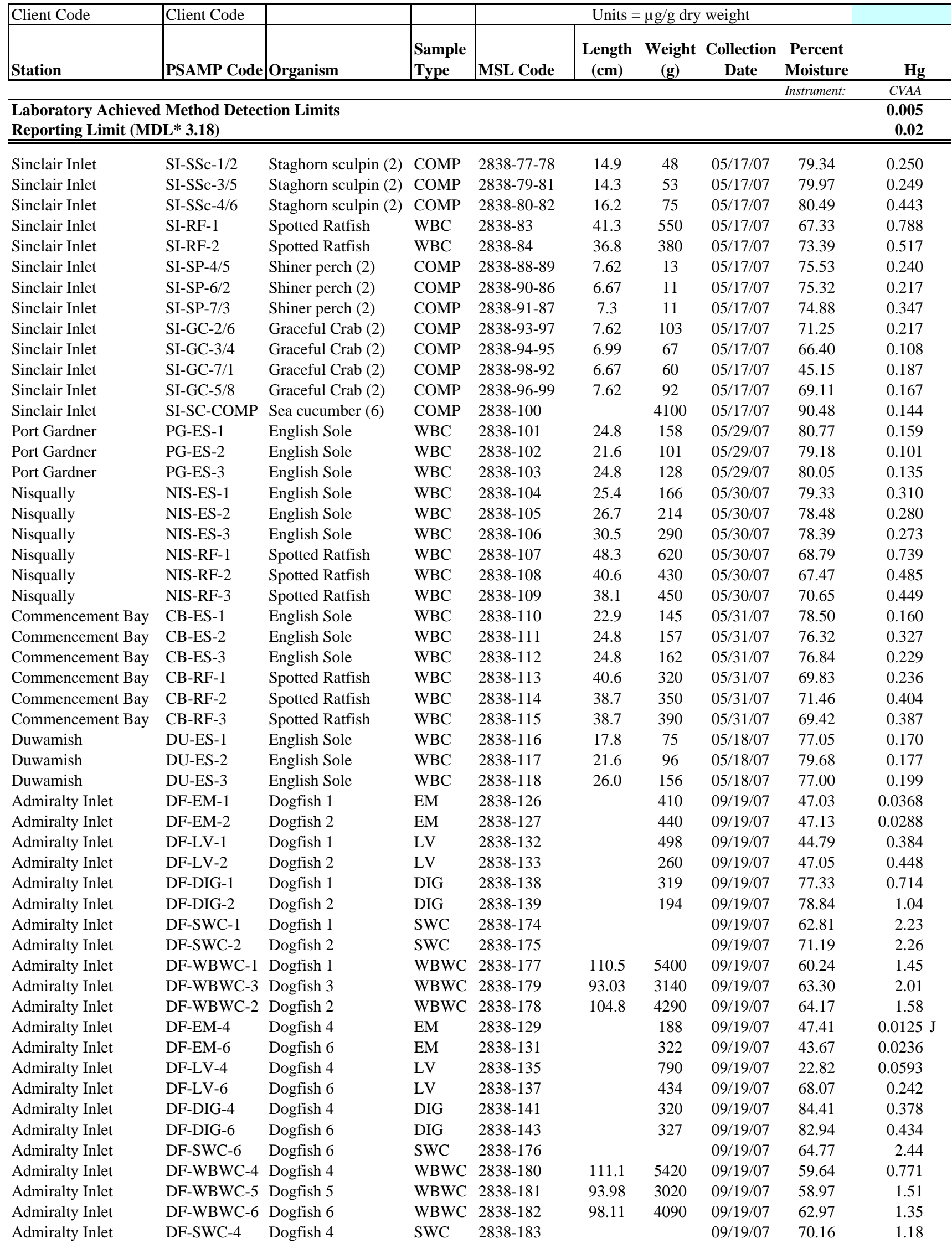




\begin{tabular}{|c|c|c|c|c|c|c|c|c|c|}
\hline Client Code & Client Code & & & & Units = & $=\mu \mathrm{g} / \mathrm{g}$ dry & weight & & \\
\hline Station & PSAMP Code & Organism & \begin{tabular}{|l} 
Sample \\
Type
\end{tabular} & MSL Code & $\begin{array}{c}\text { Length } \\
\text { (cm) }\end{array}$ & $\begin{array}{c}\text { Weight } \\
\text { (g) }\end{array}$ & $\begin{array}{c}\text { Collection } \\
\text { Date }\end{array}$ & $\begin{array}{c}\text { Percent } \\
\text { Moisture }\end{array}$ & $\mathrm{Ni}$ \\
\hline & & & & & & & & Instrument: & ICP-MS \\
\hline Laboratory Ach & 1 Method Detec & tion Limits & & & & & & & 0.04 \\
\hline Reporting Limit & $L * 3.18)$ & & & & & & & & 0.1 \\
\hline Vendovi & V-ES-5 & English Sole & WBC & $2838-5$ & 27.9 & 224 & 05/01/07 & 81.41 & 2.07 \\
\hline Vendovi & V-ES-6 & English Sole & WBC & $2838-6$ & 27.9 & 250 & $05 / 01 / 07$ & 82.21 & 1.56 \\
\hline Vendovi & V-ES-8 & English Sole & WBC & $2838-8$ & 27.3 & 197 & $05 / 01 / 07$ & 77.36 & 1.46 \\
\hline Vendovi & V-RS-1 & Rock sole & WBC & $2838-10$ & 20.3 & 119 & $05 / 01 / 07$ & 78.21 & 1.72 \\
\hline Vendovi & V-RS-2 & Rock sole & WBC & 2838-11 & 21.0 & 142 & 05/01/07 & 78.77 & 1.91 \\
\hline Vendovi & V-RS-3 & Rock sole & WBC & $2838-12$ & 31.1 & 510 & 05/01/07 & 79.70 & 1.72 \\
\hline Vendovi & V-SSc-1 & Staghorn sculpin & WBC & $2838-13$ & 17.8 & 94 & 05/01/07 & 80.46 & 1.31 \\
\hline Vendovi & V-SSc-2 & Staghorn sculpin & WBC & $2838-14$ & 24.8 & 298 & 05/01/07 & 79.46 & 1.24 \\
\hline Vendovi & V-SSc-3 & Staghorn sculpin & WBC & $2838-15$ & 25.4 & 294 & 05/01/07 & 80.10 & 1.28 \\
\hline Vendovi & V-SP-1 & Shiner perch & WBC & $2838-16$ & 7.62 & 16 & 05/01/07 & 76.72 & 1.30 \\
\hline Vendovi & V-SP-2 & Shiner perch & WBC & $2838-17$ & 8.89 & 15 & 05/01/07 & 77.81 & 1.71 \\
\hline Vendovi & V-SP-3 & Shiner perch & WBC & $2838-18$ & 8.89 & 16 & 05/01/07 & 75.87 & 1.53 \\
\hline Vendovi & V-SC-COMP & Sea cucumber (3) & COMP & 2838-19 & & 5501 & 05/01/07 & 86.80 & 16.4 \\
\hline Strait of Georgia & SG-ES-3 & English Sole & WBC & $2838-22$ & 26.7 & 184 & $05 / 02 / 07$ & 79.46 & 1.44 \\
\hline Strait of Georgia & SG-ES-4 & English Sole & WBC & $2838-23$ & 26.0 & 189 & $05 / 02 / 07$ & 79.05 & 1.39 \\
\hline Strait of Georgia & SG-ES-5 & English Sole & WBC & $2838-24$ & 26.7 & 192 & $05 / 02 / 07$ & 78.61 & 2.20 \\
\hline Strait of Georgia & SG-RF-1 & Spotted Ratfish & WBC & $2838-29$ & 27.9 & 115 & $05 / 02 / 07$ & 80.14 & 0.189 \\
\hline Strait of Georgia & SG-RF-2 & Spotted Ratfish & WBC & $2838-30$ & 35.6 & 320 & $05 / 02 / 07$ & 72.01 & 1.07 \\
\hline Strait of Georgia & SG-RF-3 & Spotted Ratfish & WBC & $2838-31$ & 48.3 & 770 & $05 / 02 / 07$ & 66.36 & $0.0753 \mathrm{~J}$ \\
\hline Strait of Georgia & SG-SC-COMP & Sea cucumber (6) & COMP & $2838-32$ & & 290 & $05 / 02 / 07$ & 92.30 & 7.34 \\
\hline Hood Canal & HC-ES-1 & English Sole & WBC & $2838-33$ & 22.9 & 131 & 05/03/07 & 82.09 & 2.57 \\
\hline Hood Canal & HC-ES-2 & English Sole & WBC & $2838-34$ & 22.9 & 133 & $05 / 03 / 07$ & 79.72 & 1.16 \\
\hline Hood Canal & HC-ES-3 & English Sole & WBC & $2838-35$ & 26.7 & 220 & $05 / 03 / 07$ & 78.81 & 1.63 \\
\hline Hood Canal & HC-RS-1 & Rock sole & WBC & $2838-36$ & 31.8 & 460 & 05/03/07 & 77.93 & 1.77 \\
\hline Hood Canal & HC-RF-1 & Spotted Ratfish & WBC & $2838-37$ & 39.4 & 480 & 05/03/07 & 74.37 & 0.866 \\
\hline Hood Canal & HC-RF-2 & Spotted Ratfish & WBC & $2838-38$ & 38.1 & 340 & 05/03/07 & 72.96 & 0.425 \\
\hline Hood Canal & HC-RF-3 & Spotted Ratfish & WBC & 2838-39 & 42.5 & 450 & 05/03/07 & 72.39 & 0.129 \\
\hline Hood Canal & HC-SP1 & Shiner perch & WBC & $2838-40$ & 9.53 & 16 & 05/03/07 & 72.16 & 1.98 \\
\hline Hood Canal & HC-SP2 & Shiner perch & WBC & $2838-41$ & 10.2 & 20 & 05/03/07 & 65.95 & 1.74 \\
\hline Hood Canal & HC-GC-1 & Graceful Crab & WBC & $2838-43$ & 10.2 & 237 & 05/03/07 & 80.86 & 4.58 \\
\hline Elliott Bay & EB-ES-1/2 & English Sole (2) & COMP & $2838-45-46$ & 15.8 & 44 & $05 / 16 / 07$ & 78.56 & 2.58 \\
\hline Elliott Bay & EB-ES-3 & English Sole & WBC & $2838-47$ & 26.0 & 157 & $05 / 16 / 07$ & 80.28 & 2.52 \\
\hline Elliott Bay & EB-RF-1 & Spotted Ratfish & WBC & $2838-48$ & 40.6 & 350 & $05 / 16 / 07$ & 73.57 & 0.435 \\
\hline Elliott Bay & EB-RF-2 & Spotted Ratfish & WBC & $2838-49$ & 41.9 & 370 & $05 / 16 / 07$ & 72.77 & 0.166 \\
\hline Elliott Bay & EB-RF-3 & Spotted Ratfish & WBC & $2838-50$ & 53.3 & 810 & 05/16/07 & 72.89 & 0.106 \\
\hline Eagle Harbor & EH-ES-2 & English Sole & WBC & $2838-52$ & 35.6 & 420 & 05/19/07 & 82.65 & 2.57 \\
\hline Eagle Harbor & EH-ES-3 & English Sole & WBC & $2838-53$ & 22.2 & 89 & $05 / 19 / 07$ & 76.62 & 2.50 \\
\hline Eagle Harbor & EH-ES-4 & English Sole & WBC & $2838-54$ & 27.3 & 199 & 05/19/07 & 78.31 & 2.21 \\
\hline Eagle Harbor & EH-RF-1 & Spotted Ratfish & WBC & $2838-55$ & 33.0 & 230 & 05/19/07 & 73.50 & 1.64 \\
\hline Eagle Harbor & EH-RF-2 & Spotted Ratfish & WBC & $2838-56$ & 38.1 & 380 & 05/19/07 & 76.61 & 0.334 \\
\hline Eagle Harbor & EH-RF-3 & Spotted Ratfish & WBC & $2838-57$ & 50.8 & 760 & 05/19/07 & 68.32 & 0.797 \\
\hline Sinclair Inlet & SI-ES-1 & English Sole & WBC & $2838-58$ & 29.2 & 330 & 05/17/07 & 77.81 & 1.22 \\
\hline Sinclair Inlet & SI-ES-2 & English Sole & WBC & $2838-59$ & 29.2 & 330 & 05/17/07 & 80.65 & 1.15 \\
\hline Sinclair Inlet & SI-ES-4 & English Sole & WBC & $2838-61$ & 25.4 & 192 & 05/17/07 & 59.34 & 1.71 \\
\hline Sinclair Inlet & SI-ES-6 & English Sole & WBC & $2838-63$ & 25.4 & 178 & $05 / 17 / 07$ & 79.69 & 1.75 \\
\hline Sinclair Inlet & SI-ES-7 & English Sole & WBC & $2838-64$ & 25.4 & 179 & 05/17/07 & 79.49 & 2.29 \\
\hline Sinclair Inlet & SI-ES-8 & English Sole & WBC & $2838-65$ & 29.2 & 261 & 05/17/07 & 79.29 & 1.76 \\
\hline Sinclair Inlet & SI-RS-1/2 & Rock sole (2) & COMP & 2838-67-68 & 10.2 & 23 & 05/17/07 & 75.72 & 1.47 \\
\hline Sinclair Inlet & SI-RS-3 & Rock sole & WBC & $2838-69$ & 27.3 & 320 & $05 / 17 / 07$ & 78.88 & 1.41 \\
\hline Sinclair Inlet & SI-RS-4 & Rock sole & WBC & $2838-70$ & 26.7 & 220 & 05/17/07 & 78.83 & 3.38 \\
\hline Sinclair Inlet & SI-SS-1 & Sand sole & WBC & $2838-71$ & 25.4 & 270 & $05 / 17 / 07$ & 78.34 & 1.73 \\
\hline Sinclair Inlet & SI-SS-2 & Sand sole & WBC & $2838-72$ & 27.3 & 290 & 05/17/07 & 77.87 & 2.19 \\
\hline Sinclair Inlet & SI-SS-3 & Sand sole & WBC & $2838-73$ & 29.8 & 320 & 05/17/07 & 78.95 & 2.40 \\
\hline
\end{tabular}




\begin{tabular}{|c|c|c|c|c|c|c|c|c|c|}
\hline Client Code & Client Code & & & & Units $=$ & $=\mu \mathrm{g} / \mathrm{g}$ dry & weight & & \\
\hline Station & PSAMP Code & Organism & \begin{tabular}{|l} 
Sample \\
Type
\end{tabular} & MSL Code & $\begin{array}{l}\text { Length } \\
\text { (cm) }\end{array}$ & $\begin{array}{c}\text { Weight } \\
\text { (g) }\end{array}$ & $\begin{array}{c}\text { Collection } \\
\text { Date }\end{array}$ & $\begin{array}{c}\text { Percent } \\
\text { Moisture }\end{array}$ & $\mathrm{Ni}$ \\
\hline & & & & & & & & Instrument: & ICP-MS \\
\hline Laboratory Achieved & 1 Method Detec & tion Limits & & & & & & & 0.04 \\
\hline Reporting Limit (MI & $L * 3.18)$ & & & & & & & & 0.1 \\
\hline Sinclair Inlet & SI-SSc- $1 / 2$ & Staghorn sculpin (2) & COMP & $2838-77-78$ & 14.9 & 48 & 05/17/07 & 79.34 & 2.12 \\
\hline Sinclair Inlet & SI-SSc-3/5 & Staghorn sculpin (2) & COMP & $2838-79-81$ & 14.3 & 53 & $05 / 17 / 07$ & 79.97 & 1.50 \\
\hline Sinclair Inlet & SI-SSc-4/6 & Staghorn sculpin (2) & COMP & $2838-80-82$ & 16.2 & 75 & $05 / 17 / 07$ & 80.49 & 1.16 \\
\hline Sinclair Inlet & SI-RF-1 & Spotted Ratfish & WBC & $2838-83$ & 41.3 & 550 & $05 / 17 / 07$ & 67.33 & 0.272 \\
\hline Sinclair Inlet & SI-RF-2 & Spotted Ratfish & WBC & $2838-84$ & 36.8 & 380 & $05 / 17 / 07$ & 73.39 & 0.209 \\
\hline Sinclair Inlet & SI-SP-4/5 & Shiner perch (2) & COMP & $2838-88-89$ & 7.62 & 13 & $05 / 17 / 07$ & 75.53 & 1.96 \\
\hline Sinclair Inlet & SI-SP-6/2 & Shiner perch (2) & COMP & $2838-90-86$ & 6.67 & 11 & $05 / 17 / 07$ & 75.32 & 1.71 \\
\hline Sinclair Inlet & SI-SP-7/3 & Shiner perch (2) & COMP & $2838-91-87$ & 7.3 & 11 & $05 / 17 / 07$ & 74.88 & 1.76 \\
\hline Sinclair Inlet & SI-GC-2/6 & Graceful Crab (2) & COMP & 2838-93-97 & 7.62 & 103 & $05 / 17 / 07$ & 71.25 & 4.82 \\
\hline Sinclair Inlet & SI-GC-3/4 & Graceful Crab (2) & COMP & 2838-94-95 & 6.99 & 67 & $05 / 17 / 07$ & 66.40 & 5.14 \\
\hline Sinclair Inlet & SI-GC-7/1 & Graceful Crab (2) & COMP & 2838-98-92 & 6.67 & 60 & $05 / 17 / 07$ & 45.15 & 4.92 \\
\hline Sinclair Inlet & SI-GC-5/8 & Graceful Crab (2) & COMP & 2838-96-99 & 7.62 & 92 & 05/17/07 & 69.11 & 4.92 \\
\hline Sinclair Inlet & SI-SC-COMP & Sea cucumber (6) & COMP & $2838-100$ & & 4100 & $05 / 17 / 07$ & 90.48 & 7.59 \\
\hline Port Gardner & PG-ES-1 & English Sole & WBC & $2838-101$ & 24.8 & 158 & $05 / 29 / 07$ & 80.77 & 3.19 \\
\hline Port Gardner & PG-ES-2 & English Sole & WBC & $2838-102$ & 21.6 & 101 & $05 / 29 / 07$ & 79.18 & 3.00 \\
\hline Port Gardner & PG-ES-3 & English Sole & WBC & $2838-103$ & 24.8 & 128 & $05 / 29 / 07$ & 80.05 & 2.81 \\
\hline Nisqually & NIS-ES-1 & English Sole & WBC & 2838-104 & 25.4 & 166 & $05 / 30 / 07$ & 79.33 & 2.55 \\
\hline Nisqually & NIS-ES-2 & English Sole & WBC & $2838-105$ & 26.7 & 214 & $05 / 30 / 07$ & 78.48 & 2.34 \\
\hline Nisqually & NIS-ES-3 & English Sole & WBC & $2838-106$ & 30.5 & 290 & $05 / 30 / 07$ & 78.39 & 1.74 \\
\hline Nisqually & NIS-RF-1 & Spotted Ratfish & WBC & $2838-107$ & 48.3 & 620 & $05 / 30 / 07$ & 68.79 & $0.0905 \mathrm{~J}$ \\
\hline Nisqually & NIS-RF-2 & Spotted Ratfish & WBC & $2838-108$ & 40.6 & 430 & $05 / 30 / 07$ & 67.47 & 0.215 \\
\hline Nisqually & NIS-RF-3 & Spotted Ratfish & WBC & 2838-109 & 38.1 & 450 & $05 / 30 / 07$ & 70.65 & 0.296 \\
\hline Commencement Bay & CB-ES-1 & English Sole & WBC & $2838-110$ & 22.9 & 145 & $05 / 31 / 07$ & 78.50 & 2.00 \\
\hline Commencement Bay & CB-ES-2 & English Sole & WBC & 2838-111 & 24.8 & 157 & $05 / 31 / 07$ & 76.32 & 2.23 \\
\hline Commencement Bay & CB-ES-3 & English Sole & WBC & $2838-112$ & 24.8 & 162 & $05 / 31 / 07$ & 76.84 & 2.01 \\
\hline Commencement Bay & CB-RF-1 & Spotted Ratfish & WBC & $2838-113$ & 40.6 & 320 & $05 / 31 / 07$ & 69.83 & 0.359 \\
\hline Commencement Bay & CB-RF-2 & Spotted Ratfish & WBC & $2838-114$ & 38.7 & 350 & $05 / 31 / 07$ & 71.46 & 0.459 \\
\hline Commencement Bay & CB-RF-3 & Spotted Ratfish & WBC & $2838-115$ & 38.7 & 390 & $05 / 31 / 07$ & 69.42 & 0.287 \\
\hline Duwamish & DU-ES-1 & English Sole & WBC & $2838-116$ & 17.8 & 75 & 05/18/07 & 77.05 & 1.69 \\
\hline Duwamish & DU-ES-2 & English Sole & WBC & $2838-117$ & 21.6 & 96 & 05/18/07 & 79.68 & 2.80 \\
\hline Duwamish & DU-ES-3 & English Sole & WBC & $2838-118$ & 26.0 & 156 & 05/18/07 & 77.00 & 1.15 \\
\hline Admiralty Inlet & DF-EM-1 & Dogfish 1 & EM & $2838-126$ & & 410 & 09/19/07 & 47.03 & $0.04 \mathrm{U}$ \\
\hline Admiralty Inlet & DF-EM-2 & Dogfish 2 & EM & $2838-127$ & & 440 & 09/19/07 & 47.13 & $0.04 \mathrm{U}$ \\
\hline Admiralty Inlet & DF-LV-1 & Dogfish 1 & LV & 2838-132 & & 498 & 09/19/07 & 44.79 & $0.04 \mathrm{U}$ \\
\hline Admiralty Inlet & DF-LV-2 & Dogfish 2 & LV & $2838-133$ & & 260 & 09/19/07 & 47.05 & $0.0912 \mathrm{~J}$ \\
\hline Admiralty Inlet & DF-DIG-1 & Dogfish 1 & DIG & $2838-138$ & & 319 & 09/19/07 & 77.33 & 0.491 \\
\hline Admiralty Inlet & DF-DIG-2 & Dogfish 2 & DIG & 2838-139 & & 194 & 09/19/07 & 78.84 & $0.0826 \mathrm{~J}$ \\
\hline Admiralty Inlet & DF-SWC-1 & Dogfish 1 & SWC & $2838-174$ & & & 09/19/07 & 62.81 & $0.0447 \mathrm{~J}$ \\
\hline Admiralty Inlet & DF-SWC-2 & Dogfish 2 & SWC & $2838-175$ & & & 09/19/07 & 71.19 & $0.0619 \mathrm{~J}$ \\
\hline Admiralty Inlet & DF-WBWC-1 & Dogfish 1 & WBWC & $2838-177$ & 110.5 & 5400 & 09/19/07 & 60.24 & 0.156 \\
\hline Admiralty Inlet & DF-WBWC-3 & Dogfish 3 & WBWC & 2838-179 & 93.03 & 3140 & 09/19/07 & 63.30 & $0.0576 \mathrm{~J}$ \\
\hline Admiralty Inlet & DF-WBWC-2 & Dogfish 2 & WBWC & $2838-178$ & 104.8 & 4290 & 09/19/07 & 64.17 & $0.0496 \mathrm{~J}$ \\
\hline Admiralty Inlet & DF-EM-4 & Dogfish 4 & $\mathrm{EM}$ & $2838-129$ & & 188 & 09/19/07 & 47.41 & $0.04 \mathrm{U}$ \\
\hline Admiralty Inlet & DF-EM-6 & Dogfish 6 & EM & 2838-131 & & 322 & 09/19/07 & 43.67 & $0.04 \mathrm{U}$ \\
\hline Admiralty Inlet & DF-LV-4 & Dogfish 4 & LV & $2838-135$ & & 790 & 09/19/07 & 22.82 & $0.04 \mathrm{U}$ \\
\hline Admiralty Inlet & DF-LV-6 & Dogfish 6 & LV & 2838-137 & & 434 & 09/19/07 & 68.07 & $0.04 \mathrm{U}$ \\
\hline Admiralty Inlet & DF-DIG-4 & Dogfish 4 & DIG & $2838-141$ & & 320 & $09 / 19 / 07$ & 84.41 & 0.216 \\
\hline Admiralty Inlet & DF-DIG-6 & Dogfish 6 & DIG & $2838-143$ & & 327 & 09/19/07 & 82.94 & 2.15 \\
\hline Admiralty Inlet & DF-SWC-6 & Dogfish 6 & SWC & $2838-176$ & & & 09/19/07 & 64.77 & $0.0465 \mathrm{~J}$ \\
\hline Admiralty Inlet & DF-WBWC-4 & Dogfish 4 & WBWC & $2838-180$ & 111.1 & 5420 & $09 / 19 / 07$ & 59.64 & $0.0525 \mathrm{~J}$ \\
\hline Admiralty Inlet & DF-WBWC-5 & Dogfish 5 & WBWC & $2838-181$ & 93.98 & 3020 & 09/19/07 & 58.97 & $0.0286 \mathrm{U}$ \\
\hline Admiralty Inlet & DF-WBWC-6 & Dogfish 6 & WBWC & $2838-182$ & 98.11 & 4090 & 09/19/07 & 62.97 & 0.555 \\
\hline Admiralty Inlet & DF-SWC-4 & Dogfish 4 & SWC & $2838-183$ & & & 09/19/07 & 70.16 & $0.04 \mathrm{U}$ \\
\hline
\end{tabular}




\begin{tabular}{|c|c|c|c|c|c|c|c|c|c|}
\hline Client Code & Client Code & & & & Units $=$ & $=\mu \mathrm{g} / \mathrm{g}$ dry & weight & & \\
\hline Station & PSAMP Code & Organism & \begin{tabular}{|l} 
Sample \\
Type
\end{tabular} & MSL Code & $\begin{array}{c}\text { Length } \\
\text { (cm) }\end{array}$ & $\begin{array}{c}\text { Weight } \\
\text { (g) }\end{array}$ & $\begin{array}{c}\text { Collection } \\
\text { Date }\end{array}$ & $\begin{array}{c}\text { Percent } \\
\text { Moisture } \\
\end{array}$ & $\mathbf{P b}$ \\
\hline & & & & & & & & Instrument: & ICP-MS \\
\hline Laboratory Achi & Method Detec & tion Limits & & & & & & & 0.003 \\
\hline Reporting Limit & L*3.18) & & & & & & & & 0.01 \\
\hline Vendovi & V-ES-5 & English Sole & WBC & $2838-5$ & 27.9 & 224 & 05/01/07 & 81.41 & 0.683 \\
\hline Vendovi & V-ES-6 & English Sole & WBC & $2838-6$ & 27.9 & 250 & 05/01/07 & 82.21 & 0.429 \\
\hline Vendovi & V-ES-8 & English Sole & WBC & $2838-8$ & 27.3 & 197 & 05/01/07 & 77.36 & 0.522 \\
\hline Vendovi & V-RS-1 & Rock sole & WBC & $2838-10$ & 20.3 & 119 & 05/01/07 & 78.21 & 0.234 \\
\hline Vendovi & V-RS-2 & Rock sole & WBC & $2838-11$ & 21.0 & 142 & 05/01/07 & 78.77 & 0.203 \\
\hline Vendovi & V-RS-3 & Rock sole & WBC & $2838-12$ & 31.1 & 510 & 05/01/07 & 79.70 & 0.181 \\
\hline Vendovi & V-SSc-1 & Staghorn sculpin & WBC & $2838-13$ & 17.8 & 94 & 05/01/07 & 80.46 & 0.133 \\
\hline Vendovi & V-SSc-2 & Staghorn sculpin & WBC & $2838-14$ & 24.8 & 298 & 05/01/07 & 79.46 & 0.0352 \\
\hline Vendovi & V-SSc-3 & Staghorn sculpin & WBC & $2838-15$ & 25.4 & 294 & 05/01/07 & 80.10 & 0.0601 \\
\hline Vendovi & V-SP-1 & Shiner perch & WBC & $2838-16$ & 7.62 & 16 & 05/01/07 & 76.72 & 0.0359 \\
\hline Vendovi & V-SP-2 & Shiner perch & WBC & $2838-17$ & 8.89 & 15 & 05/01/07 & 77.81 & 0.0908 \\
\hline Vendovi & V-SP-3 & Shiner perch & WBC & $2838-18$ & 8.89 & 16 & 05/01/07 & 75.87 & 0.0470 \\
\hline Vendovi & V-SC-COMP & Sea cucumber (3) & COMP & 2838-19 & & 5501 & 05/01/07 & 86.80 & 4.32 \\
\hline Strait of Georgia & SG-ES-3 & English Sole & WBC & $2838-22$ & 26.7 & 184 & 05/02/07 & 79.46 & 0.343 \\
\hline Strait of Georgia & SG-ES-4 & English Sole & WBC & $2838-23$ & 26.0 & 189 & 05/02/07 & 79.05 & 0.416 \\
\hline Strait of Georgia & SG-ES-5 & English Sole & WBC & $2838-24$ & 26.7 & 192 & $05 / 02 / 07$ & 78.61 & 0.503 \\
\hline Strait of Georgia & SG-RF-1 & Spotted Ratfish & WBC & $2838-29$ & 27.9 & 115 & 05/02/07 & 80.14 & 0.0390 \\
\hline Strait of Georgia & SG-RF-2 & Spotted Ratfish & WBC & $2838-30$ & 35.6 & 320 & $05 / 02 / 07$ & 72.01 & 0.0868 \\
\hline Strait of Georgia & SG-RF-3 & Spotted Ratfish & WBC & $2838-31$ & 48.3 & 770 & 05/02/07 & 66.36 & 0.0304 \\
\hline Strait of Georgia & SG-SC-COMP & Sea cucumber (6) & COMP & $2838-32$ & & 290 & 05/02/07 & 92.30 & 0.702 \\
\hline Hood Canal & HC-ES-1 & English Sole & WBC & $2838-33$ & 22.9 & 131 & 05/03/07 & 82.09 & 0.276 \\
\hline Hood Canal & HC-ES-2 & English Sole & WBC & $2838-34$ & 22.9 & 133 & 05/03/07 & 79.72 & 0.0647 \\
\hline Hood Canal & HC-ES-3 & English Sole & WBC & $2838-35$ & 26.7 & 220 & 05/03/07 & 78.81 & 0.298 \\
\hline Hood Canal & HC-RS-1 & Rock sole & WBC & $2838-36$ & 31.8 & 460 & 05/03/07 & 77.93 & 0.158 \\
\hline Hood Canal & HC-RF-1 & Spotted Ratfish & WBC & $2838-37$ & 39.4 & 480 & 05/03/07 & 74.37 & 0.0927 \\
\hline Hood Canal & HC-RF-2 & Spotted Ratfish & WBC & $2838-38$ & 38.1 & 340 & 05/03/07 & 72.96 & 0.0354 \\
\hline Hood Canal & HC-RF-3 & Spotted Ratfish & WBC & $2838-39$ & 42.5 & 450 & 05/03/07 & 72.39 & 0.0417 \\
\hline Hood Canal & HC-SP1 & Shiner perch & WBC & $2838-40$ & 9.53 & 16 & 05/03/07 & 72.16 & 0.319 \\
\hline Hood Canal & HC-SP2 & Shiner perch & WBC & $2838-41$ & 10.2 & 20 & 05/03/07 & 65.95 & 0.0753 \\
\hline Hood Canal & HC-GC-1 & Graceful Crab & WBC & $2838-43$ & 10.2 & 237 & 05/03/07 & 80.86 & 0.298 \\
\hline Elliott Bay & EB-ES-1/2 & English Sole (2) & COMP & $2838-45-46$ & 15.8 & 44 & $05 / 16 / 07$ & 78.56 & 1.27 \\
\hline Elliott Bay & EB-ES-3 & English Sole & WBC & $2838-47$ & 26.0 & 157 & 05/16/07 & 80.28 & 1.55 \\
\hline Elliott Bay & EB-RF-1 & Spotted Ratfish & WBC & $2838-48$ & 40.6 & 350 & 05/16/07 & 73.57 & 0.233 \\
\hline Elliott Bay & EB-RF-2 & Spotted Ratfish & WBC & $2838-49$ & 41.9 & 370 & $05 / 16 / 07$ & 72.77 & 0.0955 \\
\hline Elliott Bay & EB-RF-3 & Spotted Ratfish & WBC & $2838-50$ & 53.3 & 810 & 05/16/07 & 72.89 & 0.141 \\
\hline Eagle Harbor & EH-ES-2 & English Sole & WBC & $2838-52$ & 35.6 & 420 & 05/19/07 & 82.65 & 1.38 \\
\hline Eagle Harbor & EH-ES-3 & English Sole & WBC & $2838-53$ & 22.2 & 89 & $05 / 19 / 07$ & 76.62 & 2.55 \\
\hline Eagle Harbor & EH-ES-4 & English Sole & WBC & $2838-54$ & 27.3 & 199 & 05/19/07 & 78.31 & 0.936 \\
\hline Eagle Harbor & EH-RF-1 & Spotted Ratfish & WBC & $2838-55$ & 33.0 & 230 & $05 / 19 / 07$ & 73.50 & 0.916 \\
\hline Eagle Harbor & EH-RF-2 & Spotted Ratfish & WBC & $2838-56$ & 38.1 & 380 & 05/19/07 & 76.61 & 0.109 \\
\hline Eagle Harbor & EH-RF-3 & Spotted Ratfish & WBC & $2838-57$ & 50.8 & 760 & 05/19/07 & 68.32 & 0.400 \\
\hline Sinclair Inlet & SI-ES-1 & English Sole & WBC & $2838-58$ & 29.2 & 330 & 05/17/07 & 77.81 & 1.81 \\
\hline Sinclair Inlet & SI-ES-2 & English Sole & WBC & $2838-59$ & 29.2 & 330 & 05/17/07 & 80.65 & 5.87 \\
\hline Sinclair Inlet & SI-ES-4 & English Sole & WBC & $2838-61$ & 25.4 & 192 & 05/17/07 & 59.34 & 1.95 \\
\hline Sinclair Inlet & SI-ES-6 & English Sole & WBC & $2838-63$ & 25.4 & 178 & 05/17/07 & 79.69 & 1.17 \\
\hline Sinclair Inlet & SI-ES-7 & English Sole & WBC & $2838-64$ & 25.4 & 179 & 05/17/07 & 79.49 & 1.89 \\
\hline Sinclair Inlet & SI-ES-8 & English Sole & WBC & $2838-65$ & 29.2 & 261 & 05/17/07 & 79.29 & 1.85 \\
\hline Sinclair Inlet & SI-RS-1/2 & Rock sole (2) & COMP & $2838-67-68$ & 10.2 & 23 & 05/17/07 & 75.72 & 1.61 \\
\hline Sinclair Inlet & SI-RS-3 & Rock sole & WBC & $2838-69$ & 27.3 & 320 & 05/17/07 & 78.88 & 0.391 \\
\hline Sinclair Inlet & SI-RS-4 & Rock sole & WBC & $2838-70$ & 26.7 & 220 & 05/17/07 & 78.83 & 2.43 \\
\hline Sinclair Inlet & SI-SS-1 & Sand sole & WBC & $2838-71$ & 25.4 & 270 & 05/17/07 & 78.34 & 0.485 \\
\hline Sinclair Inlet & SI-SS-2 & Sand sole & WBC & $2838-72$ & 27.3 & 290 & 05/17/07 & 77.87 & 0.180 \\
\hline Sinclair Inlet & SI-SS-3 & Sand sole & WBC & $2838-73$ & 29.8 & 320 & 05/17/07 & 78.95 & 0.323 \\
\hline
\end{tabular}




\begin{tabular}{|c|c|c|c|c|c|c|c|c|c|}
\hline Client Code & Client Code & & & & Units $=$ & $=\mu \mathrm{g} / \mathrm{g}$ dry & weight & & \\
\hline Station & PSAMP Code & Organism & \begin{tabular}{|l|} 
Sample \\
Type
\end{tabular} & MSL Code & $\begin{array}{c}\text { Length } \\
\text { (cm) }\end{array}$ & $\begin{array}{c}\text { Weight } \\
\text { (g) }\end{array}$ & $\begin{array}{c}\text { Collection } \\
\text { Date }\end{array}$ & $\begin{array}{c}\text { Percent } \\
\text { Moisture } \\
\end{array}$ & $\mathbf{P b}$ \\
\hline & & & & & & & & Instrument: & ICP-MS \\
\hline Laboratory Achieved & Method Detect & tion Limits & & & & & & & 0.003 \\
\hline Reporting Limit (MI & $\left.L^{*} 3.18\right)$ & & & & & & & & 0.01 \\
\hline Sinclair Inlet & SI-SSc-1/2 & Staghorn sculpin (2) & COMP & $2838-77-78$ & 14.9 & 48 & 05/17/07 & 79.34 & 1.17 \\
\hline Sinclair Inlet & SI-SSc-3/5 & Staghorn sculpin (2) & COMP & $2838-79-81$ & 14.3 & 53 & $05 / 17 / 07$ & 79.97 & 0.628 \\
\hline Sinclair Inlet & SI-SSc-4/6 & Staghorn sculpin (2) & COMP & $2838-80-82$ & 16.2 & 75 & 05/17/07 & 80.49 & 0.736 \\
\hline Sinclair Inlet & SI-RF-1 & Spotted Ratfish & WBC & $2838-83$ & 41.3 & 550 & 05/17/07 & 67.33 & 0.222 \\
\hline Sinclair Inlet & SI-RF-2 & Spotted Ratfish & WBC & $2838-84$ & 36.8 & 380 & 05/17/07 & 73.39 & 0.135 \\
\hline Sinclair Inlet & SI-SP-4/5 & Shiner perch (2) & COMP & $2838-88-89$ & 7.62 & 13 & 05/17/07 & 75.53 & 0.504 \\
\hline Sinclair Inlet & SI-SP-6/2 & Shiner perch (2) & COMP & $2838-90-86$ & 6.67 & 11 & 05/17/07 & 75.32 & 0.208 \\
\hline Sinclair Inlet & SI-SP-7/3 & Shiner perch (2) & COMP & $2838-91-87$ & 7.3 & 11 & 05/17/07 & 74.88 & 0.669 \\
\hline Sinclair Inlet & SI-GC-2/6 & Graceful Crab (2) & COMP & 2838-93-97 & 7.62 & 103 & 05/17/07 & 71.25 & 5.82 \\
\hline Sinclair Inlet & SI-GC-3/4 & Graceful Crab (2) & COMP & 2838-94-95 & 6.99 & 67 & 05/17/07 & 66.40 & 2.27 \\
\hline Sinclair Inlet & SI-GC-7/1 & Graceful Crab (2) & COMP & 2838-98-92 & 6.67 & 60 & 05/17/07 & 45.15 & 4.88 \\
\hline Sinclair Inlet & SI-GC-5/8 & Graceful Crab (2) & COMP & 2838-96-99 & 7.62 & 92 & 05/17/07 & 69.11 & 2.52 \\
\hline Sinclair Inlet & SI-SC-COMP & Sea cucumber (6) & COMP & $2838-100$ & & 4100 & 05/17/07 & 90.48 & 9.66 \\
\hline Port Gardner & PG-ES-1 & English Sole & WBC & $2838-101$ & 24.8 & 158 & $05 / 29 / 07$ & 80.77 & 0.556 \\
\hline Port Gardner & PG-ES-2 & English Sole & WBC & $2838-102$ & 21.6 & 101 & $05 / 29 / 07$ & 79.18 & 0.458 \\
\hline Port Gardner & PG-ES-3 & English Sole & WBC & $2838-103$ & 24.8 & 128 & $05 / 29 / 07$ & 80.05 & 0.507 \\
\hline Nisqually & NIS-ES-1 & English Sole & WBC & $2838-104$ & 25.4 & 166 & 05/30/07 & 79.33 & 2.33 \\
\hline Nisqually & NIS-ES-2 & English Sole & WBC & $2838-105$ & 26.7 & 214 & 05/30/07 & 78.48 & 1.33 \\
\hline Nisqually & NIS-ES-3 & English Sole & WBC & $2838-106$ & 30.5 & 290 & 05/30/07 & 78.39 & 1.04 \\
\hline Nisqually & NIS-RF-1 & Spotted Ratfish & WBC & $2838-107$ & 48.3 & 620 & 05/30/07 & 68.79 & 0.108 \\
\hline Nisqually & NIS-RF-2 & Spotted Ratfish & WBC & $2838-108$ & 40.6 & 430 & 05/30/07 & 67.47 & 0.123 \\
\hline Nisqually & NIS-RF-3 & Spotted Ratfish & WBC & 2838-109 & 38.1 & 450 & 05/30/07 & 70.65 & 0.0609 \\
\hline Commencement Bay & CB-ES-1 & English Sole & WBC & $2838-110$ & 22.9 & 145 & 05/31/07 & 78.50 & 1.40 \\
\hline Commencement Bay & CB-ES-2 & English Sole & WBC & $2838-111$ & 24.8 & 157 & $05 / 31 / 07$ & 76.32 & 1.69 \\
\hline Commencement Bay & CB-ES-3 & English Sole & WBC & $2838-112$ & 24.8 & 162 & 05/31/07 & 76.84 & 2.30 \\
\hline Commencement Bay & CB-RF-1 & Spotted Ratfish & WBC & $2838-113$ & 40.6 & 320 & $05 / 31 / 07$ & 69.83 & 0.504 \\
\hline Commencement Bay & CB-RF-2 & Spotted Ratfish & WBC & $2838-114$ & 38.7 & 350 & 05/31/07 & 71.46 & 0.565 \\
\hline Commencement Bay & CB-RF-3 & Spotted Ratfish & WBC & $2838-115$ & 38.7 & 390 & $05 / 31 / 07$ & 69.42 & 0.184 \\
\hline Duwamish & DU-ES-1 & English Sole & WBC & $2838-116$ & 17.8 & 75 & 05/18/07 & 77.05 & 1.34 \\
\hline Duwamish & DU-ES-2 & English Sole & WBC & $2838-117$ & 21.6 & 96 & 05/18/07 & 79.68 & 1.30 \\
\hline Duwamish & DU-ES-3 & English Sole & WBC & $2838-118$ & 26.0 & 156 & 05/18/07 & 77.00 & 0.634 \\
\hline Admiralty Inlet & DF-EM-1 & Dogfish 1 & EM & $2838-126$ & & 410 & 09/19/07 & 47.03 & 0.0106 \\
\hline Admiralty Inlet & DF-EM-2 & Dogfish 2 & EM & $2838-127$ & & 440 & 09/19/07 & 47.13 & $0.003 \mathrm{U}$ \\
\hline Admiralty Inlet & DF-LV-1 & Dogfish 1 & LV & $2838-132$ & & 498 & 09/19/07 & 44.79 & $0.00789 \mathrm{~J}$ \\
\hline Admiralty Inlet & DF-LV-2 & Dogfish 2 & LV & $2838-133$ & & 260 & 09/19/07 & 47.05 & $0.00719 \mathrm{~J}$ \\
\hline Admiralty Inlet & DF-DIG-1 & Dogfish 1 & DIG & $2838-138$ & & 319 & 09/19/07 & 77.33 & 0.0514 \\
\hline Admiralty Inlet & DF-DIG-2 & Dogfish 2 & DIG & $2838-139$ & & 194 & 09/19/07 & 78.84 & 0.0107 \\
\hline Admiralty Inlet & DF-SWC-1 & Dogfish 1 & SWC & $2838-174$ & & & 09/19/07 & 62.81 & $0.00848 \mathrm{~J}$ \\
\hline Admiralty Inlet & DF-SWC-2 & Dogfish 2 & SWC & $2838-175$ & & & 09/19/07 & 71.19 & $0.00687 \mathrm{~J}$ \\
\hline Admiralty Inlet & DF-WBWC-1 & Dogfish 1 & WBWC & $2838-177$ & 110.5 & 5400 & 09/19/07 & 60.24 & $0.00832 \mathrm{~J}$ \\
\hline Admiralty Inlet & DF-WBWC-3 & Dogfish 3 & WBWC & 2838-179 & 93.03 & 3140 & 09/19/07 & 63.30 & $0.00873 \mathrm{~J}$ \\
\hline Admiralty Inlet & DF-WBWC-2 & Dogfish 2 & WBWC & $2838-178$ & 104.8 & 4290 & 09/19/07 & 64.17 & $0.00979 \mathrm{~J}$ \\
\hline Admiralty Inlet & DF-EM-4 & Dogfish 4 & EM & $2838-129$ & & 188 & 09/19/07 & 47.41 & $0.003 \mathrm{U}$ \\
\hline Admiralty Inlet & DF-EM-6 & Dogfish 6 & EM & 2838-131 & & 322 & 09/19/07 & 43.67 & $0.003 \mathrm{U}$ \\
\hline Admiralty Inlet & DF-LV-4 & Dogfish 4 & LV & $2838-135$ & & 790 & 09/19/07 & 22.82 & $0.00376 \mathrm{~J}$ \\
\hline Admiralty Inlet & DF-LV-6 & Dogfish 6 & LV & $2838-137$ & & 434 & 09/19/07 & 68.07 & $0.00316 \mathrm{~J}$ \\
\hline Admiralty Inlet & DF-DIG-4 & Dogfish 4 & DIG & $2838-141$ & & 320 & 09/19/07 & 84.41 & 0.0307 \\
\hline Admiralty Inlet & DF-DIG-6 & Dogfish 6 & DIG & $2838-143$ & & 327 & 09/19/07 & 82.94 & 0.253 \\
\hline Admiralty Inlet & DF-SWC-6 & Dogfish 6 & SWC & 2838-176 & & & 09/19/07 & 64.77 & $0.00672 \mathrm{~J}$ \\
\hline Admiralty Inlet & DF-WBWC-4 & Dogfish 4 & WBWC & $2838-180$ & 111.1 & 5420 & 09/19/07 & 59.64 & 0.0102 \\
\hline Admiralty Inlet & DF-WBWC-5 & Dogfish 5 & WBWC & $2838-181$ & 93.98 & 3020 & 09/19/07 & 58.97 & $0.00543 \mathrm{~J}$ \\
\hline Admiralty Inlet & DF-WBWC-6 & Dogfish 6 & WBWC & $2838-182$ & 98.11 & 4090 & 09/19/07 & 62.97 & 0.0543 \\
\hline Admiralty Inlet & DF-SWC-4 & Dogfish 4 & SWC & $2838-183$ & & & 09/19/07 & 70.16 & $0.00423 \mathrm{~J}$ \\
\hline
\end{tabular}




\begin{tabular}{|c|c|c|c|c|c|c|c|c|c|}
\hline Client Code & Client Code & & & & Units $=$ & $\mu \mathrm{g} / \mathrm{g}$ dry & weight & & \\
\hline Station & PSAMP Code & Organism & \begin{tabular}{|l} 
Sample \\
Type
\end{tabular} & MSL Code & $\begin{array}{c}\text { Length } \\
\text { (cm) }\end{array}$ & $\begin{array}{c}\text { Weight } \\
\text { (g) }\end{array}$ & $\begin{array}{c}\text { Collection } \\
\text { Date }\end{array}$ & $\begin{array}{c}\text { Percent } \\
\text { Moisture }\end{array}$ & Zn \\
\hline & & & & & & & & Instrument: & ICP-MS \\
\hline Laboratory Achi & Method Detect & tion Limits & & & & & & & 0.1 \\
\hline Reporting Limit & $\left.L^{*} 3.18\right)$ & & & & & & & & 0.3 \\
\hline Vendovi & V-ES-5 & English Sole & WBC & $2838-5$ & 27.9 & 224 & 05/01/07 & 81.41 & 58.8 \\
\hline Vendovi & V-ES-6 & English Sole & WBC & $2838-6$ & 27.9 & 250 & $05 / 01 / 07$ & 82.21 & 46.7 \\
\hline Vendovi & V-ES-8 & English Sole & WBC & $2838-8$ & 27.3 & 197 & 05/01/07 & 77.36 & 53.7 \\
\hline Vendovi & V-RS-1 & Rock sole & WBC & $2838-10$ & 20.3 & 119 & 05/01/07 & 78.21 & 53.9 \\
\hline Vendovi & V-RS-2 & Rock sole & WBC & $2838-11$ & 21.0 & 142 & 05/01/07 & 78.77 & 48.4 \\
\hline Vendovi & V-RS-3 & Rock sole & WBC & $2838-12$ & 31.1 & 510 & 05/01/07 & 79.70 & 52.6 \\
\hline Vendovi & V-SSc-1 & Staghorn sculpin & WBC & $2838-13$ & 17.8 & 94 & 05/01/07 & 80.46 & 61.8 \\
\hline Vendovi & V-SSc-2 & Staghorn sculpin & WBC & $2838-14$ & 24.8 & 298 & 05/01/07 & 79.46 & 58.0 \\
\hline Vendovi & V-SSc-3 & Staghorn sculpin & WBC & $2838-15$ & 25.4 & 294 & 05/01/07 & 80.10 & 59.1 \\
\hline Vendovi & V-SP-1 & Shiner perch & WBC & $2838-16$ & 7.62 & 16 & $05 / 01 / 07$ & 76.72 & 125 \\
\hline Vendovi & V-SP-2 & Shiner perch & WBC & $2838-17$ & 8.89 & 15 & 05/01/07 & 77.81 & 129 \\
\hline Vendovi & V-SP-3 & Shiner perch & WBC & $2838-18$ & 8.89 & 16 & 05/01/07 & 75.87 & 82.6 \\
\hline Vendovi & V-SC-COMP & Sea cucumber (3) & COMP & 2838-19 & & 5501 & 05/01/07 & 86.80 & 54.3 \\
\hline Strait of Georgia & SG-ES-3 & English Sole & WBC & $2838-22$ & 26.7 & 184 & $05 / 02 / 07$ & 79.46 & 66.1 \\
\hline Strait of Georgia & SG-ES-4 & English Sole & WBC & $2838-23$ & 26.0 & 189 & $05 / 02 / 07$ & 79.05 & 63.5 \\
\hline Strait of Georgia & SG-ES-5 & English Sole & WBC & $2838-24$ & 26.7 & 192 & $05 / 02 / 07$ & 78.61 & 72.8 \\
\hline Strait of Georgia & SG-RF-1 & Spotted Ratfish & WBC & $2838-29$ & 27.9 & 115 & $05 / 02 / 07$ & 80.14 & 29.2 \\
\hline Strait of Georgia & SG-RF-2 & Spotted Ratfish & WBC & $2838-30$ & 35.6 & 320 & $05 / 02 / 07$ & 72.01 & 35.8 \\
\hline Strait of Georgia & SG-RF-3 & Spotted Ratfish & WBC & $2838-31$ & 48.3 & 770 & $05 / 02 / 07$ & 66.36 & 17.0 \\
\hline Strait of Georgia & SG-SC-COMP & Sea cucumber (6) & COMP & $2838-32$ & & 290 & $05 / 02 / 07$ & 92.30 & 55.2 \\
\hline Hood Canal & HC-ES-1 & English Sole & WBC & $2838-33$ & 22.9 & 131 & 05/03/07 & 82.09 & 77.6 \\
\hline Hood Canal & HC-ES-2 & English Sole & WBC & 2838-34 & 22.9 & 133 & $05 / 03 / 07$ & 79.72 & 59.0 \\
\hline Hood Canal & HC-ES-3 & English Sole & WBC & $2838-35$ & 26.7 & 220 & 05/03/07 & 78.81 & 63.8 \\
\hline Hood Canal & HC-RS-1 & Rock sole & WBC & $2838-36$ & 31.8 & 460 & 05/03/07 & 77.93 & 67.0 \\
\hline Hood Canal & HC-RF-1 & Spotted Ratfish & WBC & $2838-37$ & 39.4 & 480 & 05/03/07 & 74.37 & 27.7 \\
\hline Hood Canal & HC-RF-2 & Spotted Ratfish & WBC & $2838-38$ & 38.1 & 340 & 05/03/07 & 72.96 & 19.7 \\
\hline Hood Canal & HC-RF-3 & Spotted Ratfish & WBC & 2838-39 & 42.5 & 450 & 05/03/07 & 72.39 & 26.2 \\
\hline Hood Canal & HC-SP1 & Shiner perch & WBC & $2838-40$ & 9.53 & 16 & 05/03/07 & 72.16 & 123 \\
\hline Hood Canal & HC-SP2 & Shiner perch & WBC & $2838-41$ & 10.2 & 20 & 05/03/07 & 65.95 & 100 \\
\hline Hood Canal & HC-GC-1 & Graceful Crab & WBC & $2838-43$ & 10.2 & 237 & 05/03/07 & 80.86 & 80.6 \\
\hline Elliott Bay & EB-ES-1/2 & English Sole (2) & COMP & $2838-45-46$ & 15.8 & 44 & $05 / 16 / 07$ & 78.56 & 85.8 \\
\hline Elliott Bay & EB-ES-3 & English Sole & WBC & $2838-47$ & 26.0 & 157 & $05 / 16 / 07$ & 80.28 & 84.7 \\
\hline Elliott Bay & EB-RF-1 & Spotted Ratfish & WBC & $2838-48$ & 40.6 & 350 & 05/16/07 & 73.57 & 23.7 \\
\hline Elliott Bay & EB-RF-2 & Spotted Ratfish & WBC & $2838-49$ & 41.9 & 370 & $05 / 16 / 07$ & 72.77 & 28.0 \\
\hline Elliott Bay & EB-RF-3 & Spotted Ratfish & WBC & $2838-50$ & 53.3 & 810 & 05/16/07 & 72.89 & 25.0 \\
\hline Eagle Harbor & EH-ES-2 & English Sole & WBC & $2838-52$ & 35.6 & 420 & 05/19/07 & 82.65 & 105 \\
\hline Eagle Harbor & EH-ES-3 & English Sole & WBC & $2838-53$ & 22.2 & 89 & 05/19/07 & 76.62 & 93.3 \\
\hline Eagle Harbor & EH-ES-4 & English Sole & WBC & $2838-54$ & 27.3 & 199 & 05/19/07 & 78.31 & 85.1 \\
\hline Eagle Harbor & EH-RF-1 & Spotted Ratfish & WBC & $2838-55$ & 33.0 & 230 & 05/19/07 & 73.50 & 28.3 \\
\hline Eagle Harbor & EH-RF-2 & Spotted Ratfish & WBC & $2838-56$ & 38.1 & 380 & 05/19/07 & 76.61 & 25.8 \\
\hline Eagle Harbor & EH-RF-3 & Spotted Ratfish & WBC & $2838-57$ & 50.8 & 760 & $05 / 19 / 07$ & 68.32 & 26.5 \\
\hline Sinclair Inlet & SI-ES-1 & English Sole & WBC & $2838-58$ & 29.2 & 330 & $05 / 17 / 07$ & 77.81 & 64.6 \\
\hline Sinclair Inlet & SI-ES-2 & English Sole & WBC & $2838-59$ & 29.2 & 330 & 05/17/07 & 80.65 & 66.2 \\
\hline Sinclair Inlet & SI-ES-4 & English Sole & WBC & $2838-61$ & 25.4 & 192 & 05/17/07 & 59.34 & 72.2 \\
\hline Sinclair Inlet & SI-ES-6 & English Sole & WBC & $2838-63$ & 25.4 & 178 & 05/17/07 & 79.69 & 69.1 \\
\hline Sinclair Inlet & SI-ES-7 & English Sole & WBC & $2838-64$ & 25.4 & 179 & 05/17/07 & 79.49 & 96.5 \\
\hline Sinclair Inlet & SI-ES-8 & English Sole & WBC & $2838-65$ & 29.2 & 261 & 05/17/07 & 79.29 & 77.7 \\
\hline Sinclair Inlet & SI-RS-1/2 & Rock sole (2) & COMP & $2838-67-68$ & 10.2 & 23 & 05/17/07 & 75.72 & 70.0 \\
\hline Sinclair Inlet & SI-RS-3 & Rock sole & WBC & $2838-69$ & 27.3 & 320 & 05/17/07 & 78.88 & 77.8 \\
\hline Sinclair Inlet & SI-RS-4 & Rock sole & WBC & $2838-70$ & 26.7 & 220 & 05/17/07 & 78.83 & 111 \\
\hline Sinclair Inlet & SI-SS-1 & Sand sole & WBC & $2838-71$ & 25.4 & 270 & 05/17/07 & 78.34 & 75.6 \\
\hline Sinclair Inlet & SI-SS-2 & Sand sole & WBC & $2838-72$ & 27.3 & 290 & 05/17/07 & 77.87 & 81.2 \\
\hline Sinclair Inlet & SI-SS-3 & Sand sole & WBC & $2838-73$ & 29.8 & 320 & 05/17/07 & 78.95 & 77.6 \\
\hline
\end{tabular}




\begin{tabular}{|c|c|c|c|c|c|c|c|c|c|}
\hline Client Code & Client Code & & & & Units $=$ & $\mu \mathrm{g} / \mathrm{g}$ dry & weight & & \\
\hline Station & PSAMP Code & Organism & $\begin{array}{l}\text { Sample } \\
\text { Type }\end{array}$ & MSL Code & $\begin{array}{c}\text { Length } \\
\text { (cm) }\end{array}$ & $\begin{array}{c}\text { Weight } \\
\text { (g) }\end{array}$ & $\begin{array}{c}\text { Collection } \\
\text { Date }\end{array}$ & $\begin{array}{c}\text { Percent } \\
\text { Moisture } \\
\end{array}$ & Zn \\
\hline & & & & & & & & Instrument: & ICP-MS \\
\hline Laboratory Achieve & Method Detec & tion Limits & & & & & & & 0.1 \\
\hline Reporting Limit (M) & $\left.L^{*} 3.18\right)$ & & & & & & & & 0.3 \\
\hline Sinclair Inlet & SI-SSc-1/2 & Staghorn sculpin (2) & COMP & $2838-77-78$ & 14.9 & 48 & 05/17/07 & 79.34 & 73.4 \\
\hline Sinclair Inlet & SI-SSc-3/5 & Staghorn sculpin (2) & COMP & $2838-79-81$ & 14.3 & 53 & $05 / 17 / 07$ & 79.97 & 63.3 \\
\hline Sinclair Inlet & SI-SSc-4/6 & Staghorn sculpin (2) & COMP & $2838-80-82$ & 16.2 & 75 & 05/17/07 & 80.49 & 65.5 \\
\hline Sinclair Inlet & SI-RF-1 & Spotted Ratfish & WBC & $2838-83$ & 41.3 & 550 & 05/17/07 & 67.33 & 23.1 \\
\hline Sinclair Inlet & SI-RF-2 & Spotted Ratfish & WBC & $2838-84$ & 36.8 & 380 & $05 / 17 / 07$ & 73.39 & 22.7 \\
\hline Sinclair Inlet & SI-SP-4/5 & Shiner perch (2) & COMP & $2838-88-89$ & 7.62 & 13 & $05 / 17 / 07$ & 75.53 & 125 \\
\hline Sinclair Inlet & SI-SP-6/2 & Shiner perch (2) & COMP & $2838-90-86$ & 6.67 & 11 & $05 / 17 / 07$ & 75.32 & 119 \\
\hline Sinclair Inlet & SI-SP-7/3 & Shiner perch (2) & COMP & 2838-91-87 & 7.3 & 11 & 05/17/07 & 74.88 & 99.5 \\
\hline Sinclair Inlet & SI-GC-2/6 & Graceful Crab (2) & COMP & 2838-93-97 & 7.62 & 103 & 05/17/07 & 71.25 & 152 \\
\hline Sinclair Inlet & SI-GC-3/4 & Graceful Crab (2) & COMP & 2838-94-95 & 6.99 & 67 & $05 / 17 / 07$ & 66.40 & 97.6 \\
\hline Sinclair Inlet & SI-GC-7/1 & Graceful Crab (2) & COMP & 2838-98-92 & 6.67 & 60 & $05 / 17 / 07$ & 45.15 & 142 \\
\hline Sinclair Inlet & SI-GC-5/8 & Graceful Crab (2) & COMP & 2838-96-99 & 7.62 & 92 & 05/17/07 & 69.11 & 105 \\
\hline Sinclair Inlet & SI-SC-COMP & Sea cucumber (6) & COMP & $2838-100$ & & 4100 & 05/17/07 & 90.48 & 72.7 \\
\hline Port Gardner & PG-ES-1 & English Sole & WBC & 2838-101 & 24.8 & 158 & $05 / 29 / 07$ & 80.77 & 81.2 \\
\hline Port Gardner & PG-ES-2 & English Sole & WBC & 2838-102 & 21.6 & 101 & $05 / 29 / 07$ & 79.18 & 72.0 \\
\hline Port Gardner & PG-ES-3 & English Sole & WBC & $2838-103$ & 24.8 & 128 & $05 / 29 / 07$ & 80.05 & 78.0 \\
\hline Nisqually & NIS-ES-1 & English Sole & WBC & $2838-104$ & 25.4 & 166 & $05 / 30 / 07$ & 79.33 & 80.4 \\
\hline Nisqually & NIS-ES-2 & English Sole & WBC & $2838-105$ & 26.7 & 214 & 05/30/07 & 78.48 & 83.7 \\
\hline Nisqually & NIS-ES-3 & English Sole & WBC & $2838-106$ & 30.5 & 290 & $05 / 30 / 07$ & 78.39 & 66.9 \\
\hline Nisqually & NIS-RF-1 & Spotted Ratfish & WBC & $2838-107$ & 48.3 & 620 & $05 / 30 / 07$ & 68.79 & 22.5 \\
\hline Nisqually & NIS-RF-2 & Spotted Ratfish & WBC & 2838-108 & 40.6 & 430 & $05 / 30 / 07$ & 67.47 & 24.3 \\
\hline Nisqually & NIS-RF-3 & Spotted Ratfish & WBC & 2838-109 & 38.1 & 450 & $05 / 30 / 07$ & 70.65 & 28.0 \\
\hline Commencement Bay & CB-ES-1 & English Sole & WBC & $2838-110$ & 22.9 & 145 & $05 / 31 / 07$ & 78.50 & 78.9 \\
\hline Commencement Bay & CB-ES-2 & English Sole & WBC & $2838-111$ & 24.8 & 157 & $05 / 31 / 07$ & 76.32 & 79.5 \\
\hline Commencement Bay & CB-ES-3 & English Sole & WBC & $2838-112$ & 24.8 & 162 & $05 / 31 / 07$ & 76.84 & 74.3 \\
\hline Commencement Bay & CB-RF-1 & Spotted Ratfish & WBC & $2838-113$ & 40.6 & 320 & $05 / 31 / 07$ & 69.83 & 19.9 \\
\hline Commencement Bay & CB-RF-2 & Spotted Ratfish & WBC & $2838-114$ & 38.7 & 350 & $05 / 31 / 07$ & 71.46 & 20.6 \\
\hline Commencement Bay & CB-RF-3 & Spotted Ratfish & WBC & $2838-115$ & 38.7 & 390 & $05 / 31 / 07$ & 69.42 & 20.8 \\
\hline Duwamish & DU-ES-1 & English Sole & WBC & $2838-116$ & 17.8 & 75 & 05/18/07 & 77.05 & 75.5 \\
\hline Duwamish & DU-ES-2 & English Sole & WBC & $2838-117$ & 21.6 & 96 & 05/18/07 & 79.68 & 80.2 \\
\hline Duwamish & DU-ES-3 & English Sole & WBC & $2838-118$ & 26.0 & 156 & 05/18/07 & 77.00 & 75.6 \\
\hline Admiralty Inlet & DF-EM-1 & Dogfish 1 & EM & $2838-126$ & & 410 & 09/19/07 & 47.03 & 24.5 \\
\hline Admiralty Inlet & DF-EM-2 & Dogfish 2 & EM & $2838-127$ & & 440 & 09/19/07 & 47.13 & 23.1 \\
\hline Admiralty Inlet & DF-LV-1 & Dogfish 1 & LV & 2838-132 & & 498 & 09/19/07 & 44.79 & 38.7 \\
\hline Admiralty Inlet & DF-LV-2 & Dogfish 2 & LV & $2838-133$ & & 260 & 09/19/07 & 47.05 & 25.2 \\
\hline Admiralty Inlet & DF-DIG-1 & Dogfish 1 & DIG & 2838-138 & & 319 & 09/19/07 & 77.33 & 194 \\
\hline Admiralty Inlet & DF-DIG-2 & Dogfish 2 & DIG & 2838-139 & & 194 & 09/19/07 & 78.84 & 83.6 \\
\hline Admiralty Inlet & DF-SWC-1 & Dogfish 1 & SWC & $2838-174$ & & & 09/19/07 & 62.81 & 11.7 \\
\hline Admiralty Inlet & DF-SWC-2 & Dogfish 2 & SWC & $2838-175$ & & & 09/19/07 & 71.19 & 14.1 \\
\hline Admiralty Inlet & DF-WBWC-1 & Dogfish 1 & WBWC & $2838-177$ & 110.5 & 5400 & 09/19/07 & 60.24 & 21.4 \\
\hline Admiralty Inlet & DF-WBWC-3 & Dogfish 3 & WBWC & $2838-179$ & 93.03 & 3140 & 09/19/07 & 63.30 & 13.8 \\
\hline Admiralty Inlet & DF-WBWC-2 & Dogfish 2 & WBWC & $2838-178$ & 104.8 & 4290 & 09/19/07 & 64.17 & 17.7 \\
\hline Admiralty Inlet & DF-EM-4 & Dogfish 4 & EM & 2838-129 & & 188 & 09/19/07 & 47.41 & 29.8 \\
\hline Admiralty Inlet & DF-EM-6 & Dogfish 6 & EM & 2838-131 & & 322 & 09/19/07 & 43.67 & 25.8 \\
\hline Admiralty Inlet & DF-LV-4 & Dogfish 4 & LV & $2838-135$ & & 790 & 09/19/07 & 22.82 & 19.8 \\
\hline Admiralty Inlet & DF-LV-6 & Dogfish 6 & LV & 2838-137 & & 434 & 09/19/07 & 68.07 & 25.3 \\
\hline Admiralty Inlet & DF-DIG-4 & Dogfish 4 & DIG & $2838-141$ & & 320 & 09/19/07 & 84.41 & 321 \\
\hline Admiralty Inlet & DF-DIG-6 & Dogfish 6 & DIG & $2838-143$ & & 327 & 09/19/07 & 82.94 & 217 \\
\hline Admiralty Inlet & DF-SWC-6 & Dogfish 6 & SWC & $2838-176$ & & & 09/19/07 & 64.77 & 10.9 \\
\hline Admiralty Inlet & DF-WBWC-4 & Dogfish 4 & WBWC & $2838-180$ & 111.1 & 5420 & 09/19/07 & 59.64 & 39.2 \\
\hline Admiralty Inlet & DF-WBWC-5 & Dogfish 5 & WBWC & $2838-181$ & 93.98 & 3020 & 09/19/07 & 58.97 & 16.4 \\
\hline Admiralty Inlet & DF-WBWC-6 & Dogfish 6 & WBWC & $2838-182$ & 98.11 & 4090 & 09/19/07 & 62.97 & 49.9 \\
\hline Admiralty Inlet & DF-SWC-4 & Dogfish 4 & SWC & $2838-183$ & & & 09/19/07 & 70.16 & 10.9 \\
\hline
\end{tabular}


BATTELLE MARINE SCIENCE LABORATORIES

1529 West Sequim Bay Road

Sequim, Washington 98382-9099

360/681-4564

\section{ENVVEST, SINCLAIR AND DYES INLET BIOTA STUDY \\ 2007 PSAMP Trawl Biota \\ Metals in Whole Organisms and Dogfish Parts}

\section{Data Qualifiers}

c Exceeds DQO but meets contingency criteria of either:

1 SRM certified $<10 x$ MDL

2 Insufficient spiking level relative to native sample concentrations

3 Sample concentration $<10 x$ MDL

$\mathrm{U}$ Analyte not detected at or above the MDL, MDL reported

J Analyte detected above the MDL, but less than the RL

-- Not analyzed

NA Not applicable/available

N Spiked sample recovery outside QC criterion of 70-130\%

$\&$ Accuracy result outside QC criterion of $\leq 20 \%$ PD

* Precision result outside QC criterion of $<30 \%$

NS Sample not spiked for this analyte

B Analyte detected in the method blank $>$ RL and sample concentration $<10$ times detected blank value

b Data are blank corrected using the batch specific procedural blank

\section{Legend}

WBC Whole Body Composite of one organism

COMP Composite of several organisms

EM Embryos

LV Liver

DIG Digestive tract + gut contents

SWC Section weighted composite

WBWC Whole body weighted composite

Weight and length of multiple organism complosites are given as the average of individuals incorporated into the com 


\section{Field Data Summary: PCBs in Biota Tissue}

Wet Weight Basis 
BATTELLE MARINE SCIENCE LABORATORIES

1529 West Sequim Bay Road

Sequim, Washington 98382-9099

360/681-4564

\section{SINCLAIR AND DYES INLET \\ 2007 PSAMP Trawl Biota \\ PCBs in Whole Organisms}

\begin{tabular}{|c|c|c|c|c|c|c|}
\hline Sample Name: & V-ES-5 & V-ES-6 & V-ES-8 & V-RS-1 & V-RS-2 & V-RS-3 \\
\hline Station: & Vendovi & Vendovi & Vendovi & Vendovi & Vendovi & Vendovi \\
\hline Organism ID: & ES & ES & ES & $\mathrm{RS}$ & $\mathrm{RS}$ & RS \\
\hline Batch ID: & $08-0004$ & $08-0004$ & $08-0004$ & $08-0004$ & $08-0004$ & 08-0004 \\
\hline Sample Weight (g): & 30.01 & 30.34 & 31.04 & 30.18 & 30 & 30.36 \\
\hline \%Moisture & 81.41 & 82.21 & 77.36 & 78.21 & 78.77 & 79.70 \\
\hline \%Lipids & 0.65 & 0.49 & 1.47 & 0.63 & 0.48 & 0.75 \\
\hline Collection Date: & $05 / 01 / 2007$ & $05 / 01 / 2007$ & $05 / 01 / 2007$ & $05 / 01 / 2007$ & $05 / 01 / 2007$ & $05 / 01 / 2007$ \\
\hline Extraction Date: & 01/10/2008 & 01/10/2008 & 01/10/2008 & 01/10/2008 & 01/10/2008 & 01/10/2008 \\
\hline Analysis Date: & 01/28/2008 & 01/29/2008 & 01/30/2008 & 01/29/2008 & 01/29/2008 & 01/30/2008 \\
\hline Units (wet wt): & ng/g_WET & ng/g_WET & ng/g_WET & ng/g_WET & ng/g_WET & ng/g_WET \\
\hline $\mathrm{Cl} 2(8)$ & $0.02 \mathrm{~J}$ & $0.05 \mathrm{U}$ & $0.05 \mathrm{U}$ & $0.05 \mathrm{U}$ & $0.05 \mathrm{U}$ & $0.05 \mathrm{U}$ \\
\hline $\mathrm{Cl} 3(18)$ & $0.02 \mathrm{~J}$ & $0.06 \mathrm{U}$ & 0.04 & $0.06 \mathrm{U}$ & $0.06 \mathrm{U}$ & $0.06 \mathrm{U}$ \\
\hline $\mathrm{Cl} 3(28)$ & 0.07 & 0.03 & 0.05 & $0.06 \mathrm{U}$ & $0.06 \mathrm{U}$ & $0.06 \mathrm{U}$ \\
\hline $\mathrm{Cl} 4(44)$ & 0.03 & $0.02 \mathrm{~J}$ & 0.05 & $0.12 \mathrm{U}$ & $0.12 \mathrm{U}$ & $0.12 \mathrm{U}$ \\
\hline $\mathrm{Cl} 4(52)$ & 0.09 & 0.05 & 0.13 & $0.12 \mathrm{U}$ & 0.06 & $0.12 \mathrm{U}$ \\
\hline $\mathrm{Cl} 4(66)$ & 0.06 & $0.02 \mathrm{~J}$ & 0.06 & $0.07 \mathrm{U}$ & 0.05 & 0.09 \\
\hline $\mathrm{Cl} 4(77)$ & $0.05 \mathrm{U}$ & $0.05 \mathrm{U}$ & $0.05 \mathrm{U}$ & $0.05 \mathrm{U}$ & $0.05 \mathrm{U}$ & $0.05 \mathrm{U}$ \\
\hline $\mathrm{Cl} 5(101)$ & 0.22 & 0.10 & 0.23 & 0.11 & 0.11 & 0.1 \\
\hline $\mathrm{Cl} 5(105)$ & 0.07 & $0.05 \mathrm{U}$ & 0.07 & 0.08 & $0.05 \mathrm{U}$ & 0.06 \\
\hline $\mathrm{Cl} 5(118)$ & 0.22 & 0.08 & 0.18 & 0.20 & 0.18 & 0.24 \\
\hline $\mathrm{Cl} 5(126)$ & $0.07 \mathrm{U}$ & $0.07 \mathrm{U}$ & $0.07 \mathrm{U}$ & $0.07 \mathrm{U}$ & $0.07 \mathrm{U}$ & $0.07 \mathrm{U}$ \\
\hline $\mathrm{Cl6}(128)$ & 0.05 & $0.02 \mathrm{~J}$ & 0.07 & 0.07 & 0.06 & 0.07 \\
\hline $\mathrm{Cl6}(138)$ & 0.35 & 0.13 & 0.32 & 0.29 & 0.28 & 0.38 \\
\hline $\mathrm{Cl6}(153)$ & 0.66 & 0.24 & 0.58 & 0.47 & 0.49 & 0.71 \\
\hline $\mathrm{Cl} 7(170)$ & 0.09 & 0.03 & 0.07 & 0.07 & 0.06 & 0.10 \\
\hline $\mathrm{Cl}(187)$ & 0.30 & 0.12 & 0.23 & 0.11 & 0.15 & 0.13 \\
\hline $\mathrm{Cl}(188)$ & $0.02 \mathrm{U}$ & $0.02 \mathrm{U}$ & $0.02 \mathrm{U}$ & $0.02 \mathrm{U}$ & $0.02 \mathrm{U}$ & $0.02 \mathrm{U}$ \\
\hline $\mathrm{Cl}(195)$ & $0.05 \mathrm{U}$ & $0.05 \mathrm{U}$ & $0.05 \mathrm{U}$ & $0.05 \mathrm{U}$ & $0.05 \mathrm{U}$ & $0.05 \mathrm{U}$ \\
\hline $\mathrm{Cl}(200)$ & $0.04 \mathrm{U}$ & $0.04 \mathrm{U}$ & $0.04 \mathrm{U}$ & $0.04 \mathrm{U}$ & $0.04 \mathrm{U}$ & $0.04 \mathrm{U}$ \\
\hline $\mathrm{Cl9}(206)$ & 0.06 & $0.04 \mathrm{U}$ & 0.03 & $0.04 \mathrm{U}$ & $0.04 \mathrm{U}$ & $0.04 \mathrm{U}$ \\
\hline Cl10(209) & 0.03 & $0.07 \mathrm{U}$ & $0.02 \mathrm{~J}$ & $0.07 \mathrm{U}$ & $0.07 \mathrm{U}$ & $0.07 \mathrm{U}$ \\
\hline Total PCBs & 5.14 & 2.68 & 4.82 & 4.44 & 4.24 & 5.26 \\
\hline LOC 1 & $0.08 \mathrm{U}$ & $0.02 \mathrm{~J}$ & $0.08 \mathrm{U}$ & $0.08 \mathrm{U}$ & $0.08 \mathrm{U}$ & $0.08 \mathrm{U}$ \\
\hline LOC 2 & $0.05 \mathrm{~J}$ & $0.52 \mathrm{U}$ & $0.52 \mathrm{U}$ & $0.52 \mathrm{U}$ & $0.52 \mathrm{U}$ & $0.52 \mathrm{U}$ \\
\hline LOC 3 & $0.24 \mathrm{~J}$ & $0.07 \mathrm{~J}$ & $0.22 \mathrm{~J}$ & $0.75 \mathrm{U}$ & $0.75 \mathrm{U}$ & $0.75 \mathrm{U}$ \\
\hline LOC 4 & $0.45 \mathrm{~J}$ & $0.2 \mathrm{~J}$ & $0.58 \mathrm{~J}$ & $1.63 \mathrm{U}$ & $0.15 \mathrm{~J}$ & $0.19 \mathrm{~J}$ \\
\hline LOC 5 & 1.15 & $0.42 \mathrm{~J}$ & 1.17 & $0.63 \mathrm{~J}$ & $0.58 \mathrm{~J}$ & 0.76 \\
\hline LOC 6 & 1.82 & $0.64 \mathrm{~J}$ & 1.67 & 1.03 & 1.15 & 1.37 \\
\hline LOC 7 & 1.07 & $0.43 \mathrm{~J}$ & 0.86 & $0.42 \mathrm{~J}$ & $0.54 \mathrm{~J}$ & 0.68 \\
\hline LOC 8 & 0.34 & $0.51 \mathrm{U}$ & $0.12 \mathrm{~J}$ & $0.51 \mathrm{U}$ & $0.51 \mathrm{U}$ & $0.51 \mathrm{U}$ \\
\hline LOC 9 & $0.08 \mathrm{~J}$ & $0.16 \mathrm{U}$ & $0.03 \mathrm{~J}$ & $0.16 \mathrm{U}$ & $0.16 \mathrm{U}$ & $0.16 \mathrm{U}$ \\
\hline Total PCBs & 5.28 & 2.97 & 5.25 & 5.73 & 4.44 & 5.02 \\
\hline $\mathrm{Cl} 3(34)$ & 80 & 41 & 64 & 93 & 76 & 94 \\
\hline $\mathrm{Cl}(152)$ & 82 & 42 & 67 & 83 & 74 & 82 \\
\hline
\end{tabular}


1529 West Sequim Bay Road

Sequim, Washington 98382-9099

360/681-4564

\section{SINCLAIR AND DYES INLET 2007 PSAMP Trawl Biota PCBs in Whole Organisms}

\begin{tabular}{|c|c|c|c|c|}
\hline Sample Name: & V-SSc-1 & V-SSc-2 & V-SSc-3 & SG-ES-3 \\
\hline Station: & Vendovi & Vendovi & Vendovi & Strait of Georgia \\
\hline Organism ID: & STS & STS & STS & ES \\
\hline Batch ID: & $08-0004$ & $08-0004$ & $08-0004$ & $08-0004$ \\
\hline Sample Weight (g): & 30.34 & 30.14 & 31.02 & 30.92 \\
\hline \%Moisture & 80.46 & 79.46 & 80.10 & 79.46 \\
\hline \%Lipids & 0.64 & 0.84 & 0.95 & 1.81 \\
\hline Collection Date: & $05 / 01 / 2007$ & $05 / 01 / 2007$ & $05 / 01 / 2007$ & $05 / 02 / 2007$ \\
\hline Extraction Date: & 01/10/2008 & 01/10/2008 & 01/10/2008 & 01/10/2008 \\
\hline Analysis Date: & 01/29/2008 & 01/29/2008 & 01/29/2008 & 01/29/2008 \\
\hline Units (wet wt): & ng/g_WET & ng/g_WET & ng/g_WET & ng/g_WET \\
\hline $\mathrm{Cl} 2(8)$ & 0.04 & $0.05 \mathrm{U}$ & $0.05 \mathrm{U}$ & $0.02 \mathrm{~J}$ \\
\hline $\mathrm{Cl} 3(18)$ & 0.04 & $0.02 \mathrm{~J}$ & $0.06 \mathrm{U}$ & 0.05 \\
\hline $\mathrm{Cl} 3(28)$ & 0.05 & 0.08 & 0.04 & 0.11 \\
\hline Cl4(44) & 0.04 & 0.04 & 0.03 & 0.10 \\
\hline $\mathrm{Cl} 4(52)$ & 0.07 & 0.14 & 0.06 & 0.24 \\
\hline $\mathrm{Cl} 4(66)$ & 0.05 & 0.14 & 0.06 & 0.15 \\
\hline Cl4(77) & $0.05 \mathrm{U}$ & $0.05 \mathrm{U}$ & $0.05 \mathrm{U}$ & $0.05 \mathrm{U}$ \\
\hline Cl5(101) & 0.17 & 0.34 & 0.19 & 0.49 \\
\hline $\mathrm{Cl5}(105)$ & 0.09 & 0.28 & 0.11 & 0.15 \\
\hline $\mathrm{Cl5}(118)$ & 0.20 & 0.54 & 0.26 & 0.44 \\
\hline $\mathrm{Cl5}(126)$ & $0.07 \mathrm{U}$ & $0.07 \mathrm{U}$ & $0.07 \mathrm{U}$ & $0.07 \mathrm{U}$ \\
\hline $\mathrm{Cl6}(128)$ & 0.07 & 0.19 & 0.10 & 0.13 \\
\hline $\mathrm{Cl6}(138)$ & 0.34 & 0.81 & 0.42 & 0.60 \\
\hline $\mathrm{Cl} 6(153)$ & 0.63 & 1.24 & 0.79 & 1.10 \\
\hline $\mathrm{Cl} 1(170)$ & 0.07 & 0.17 & 0.07 & 0.13 \\
\hline Cl7(187) & 0.19 & 0.37 & 0.22 & 0.40 \\
\hline Cl7(188) & $0.02 \mathrm{U}$ & $0.02 \mathrm{U}$ & $0.02 \mathrm{U}$ & $0.02 \mathrm{U}$ \\
\hline Cl8(195) & $0.05 \mathrm{U}$ & $0.05 \mathrm{U}$ & $0.05 \mathrm{U}$ & $0.05 \mathrm{U}$ \\
\hline $\mathrm{Cl} 18(200)$ & $0.04 \mathrm{U}$ & $0.04 \mathrm{U}$ & $0.04 \mathrm{U}$ & $0.04 \mathrm{U}$ \\
\hline $\mathrm{Cl9}(206)$ & $0.04 \mathrm{U}$ & $0.04 \mathrm{U}$ & $0.04 \mathrm{U}$ & 0.07 \\
\hline Cl10(209) & $0.07 \mathrm{U}$ & $0.07 \mathrm{U}$ & $0.07 \mathrm{U}$ & $0.07 \mathrm{U}$ \\
\hline Total PCBs & 4.78 & 9.50 & 5.60 & 8.96 \\
\hline LOC 1 & $0.08 \mathrm{U}$ & $0.08 \mathrm{U}$ & $0.08 \mathrm{U}$ & $0.08 \mathrm{U}$ \\
\hline LOC 2 & $0.12 \mathrm{~J}$ & $0.52 \mathrm{U}$ & $0.05 \mathrm{~J}$ & $0.02 \mathrm{~J}$ \\
\hline LOC 3 & $0.22 \mathrm{~J}$ & $0.19 \mathrm{~J}$ & $0.07 \mathrm{~J}$ & $0.38 \mathrm{~J}$ \\
\hline LOC 4 & $0.35 \mathrm{~J}$ & $0.75 \mathrm{~J}$ & $0.31 \mathrm{~J}$ & 1.34 \\
\hline LOC 5 & 1.15 & 2.35 & 1.17 & 2.63 \\
\hline LOC 6 & 1.57 & 3.51 & 1.87 & 3.35 \\
\hline LOC 7 & 0.69 & 1.41 & 0.83 & 1.52 \\
\hline LOC 8 & $0.51 \mathrm{U}$ & $0.15 \mathrm{~J}$ & $0.08 \mathrm{~J}$ & 0.32 \\
\hline LOC 9 & $0.16 \mathrm{U}$ & $0.16 \mathrm{U}$ & $0.16 \mathrm{U}$ & $0.07 \mathrm{~J}$ \\
\hline Total PCBs & 4.85 & 9.12 & 4.62 & 9.71 \\
\hline $\mathrm{Cl} 3(34)$ & 60 & 69 & 60 & 56 \\
\hline Cl6(152) & 64 & 69 & 59 & 57 \\
\hline
\end{tabular}


1529 West Sequim Bay Road

Sequim, Washington 98382-9099

360/681-4564

\section{SINCLAIR AND DYES INLET \\ 2007 PSAMP Trawl Biota \\ PCBs in Whole Organisms}

\begin{tabular}{|c|c|c|c|c|c|}
\hline Sample Name: & SG-ES-4 & SG-ES-5 & HC-GC-1 & SI-ES-1 & SI-ES-2 \\
\hline Station: & Strait of Georgia & Strait of Georgia & Hood Canal & Sinclair Inlet & Sinclair Inlet \\
\hline Organism ID: & ES & ES & $\mathrm{GC}$ & ES & ES \\
\hline Batch ID: & $08-0004$ & $08-0004$ & $08-0004$ & $08-0004$ & $08-0004$ \\
\hline Sample Weight (g): & 30.34 & 30.2 & 30.46 & 30.78 & 30.74 \\
\hline \%Moisture & 79.05 & 78.61 & 80.86 & 77.81 & 80.65 \\
\hline \%Lipids & 1.18 & 1.98 & 0.41 & 2.23 & 2.20 \\
\hline Collection Date: & $05 / 02 / 2007$ & $05 / 02 / 2007$ & 05/03/2007 & $05 / 17 / 2007$ & 05/17/2007 \\
\hline Extraction Date: & 01/10/2008 & 01/10/2008 & 01/10/2008 & 01/10/2008 & 01/10/2008 \\
\hline Analysis Date: & 01/30/2008 & 01/29/2008 & 01/29/2008 & 01/30/2008 & 01/30/2008 \\
\hline Units (wet wt): & ng/g_WET & ng/g_WET & ng/g_WET & ng/g_WET & ng/g_WET \\
\hline $\mathrm{Cl} 2(8)$ & $0.05 \mathrm{U}$ & $0.02 \mathrm{~J}$ & $0.05 \mathrm{U}$ & 0.05 & 0.04 \\
\hline $\mathrm{Cl} 3(18)$ & $0.06 \mathrm{U}$ & $0.06 \mathrm{U}$ & $0.06 \mathrm{U}$ & 0.18 & 0.17 \\
\hline $\mathrm{Cl} 3(28)$ & $0.06 \mathrm{U}$ & $0.06 \mathrm{U}$ & $0.06 \mathrm{U}$ & 0.41 & 0.49 \\
\hline Cl4(44) & $0.12 \mathrm{U}$ & $0.12 \mathrm{U}$ & $0.12 \mathrm{U}$ & 0.59 & 0.63 \\
\hline $\mathrm{Cl} 4(52)$ & 0.14 & 0.19 & $0.12 \mathrm{U}$ & 2.00 & 2.68 \\
\hline $\mathrm{Cl} 4(66)$ & 0.11 & 0.13 & $0.07 \mathrm{U}$ & 1.17 & 1.53 \\
\hline Cl4(77) & $0.05 \mathrm{U}$ & $0.05 \mathrm{U}$ & $0.05 \mathrm{U}$ & 0.06 & $0.02 \mathrm{~J}$ \\
\hline $\mathrm{Cl} 5(101)$ & 0.33 & 0.38 & 0.21 & $4.51 \mathrm{D}$ & $5.88 \mathrm{D}$ \\
\hline Cl5(105) & 0.11 & 0.13 & 0.08 & 2.19 & 2.80 \\
\hline Cl5(118) & 0.31 & 0.37 & 0.19 & $6.40 \mathrm{D}$ & $7.89 \mathrm{D}$ \\
\hline Cl5(126) & $0.07 \mathrm{U}$ & $0.07 \mathrm{U}$ & $0.07 \mathrm{U}$ & $0.07 \mathrm{U}$ & $0.07 \mathrm{U}$ \\
\hline Cl6(128) & 0.09 & 0.11 & 0.08 & 2.12 & 2.52 \\
\hline Cl6(138) & 0.46 & 0.49 & 0.34 & $5.82 \mathrm{D}$ & $7.72 \mathrm{D}$ \\
\hline $\mathrm{Cl6}(153)$ & 0.84 & 0.86 & 0.51 & $10.03 \mathrm{D}$ & $13.98 \mathrm{D}$ \\
\hline $\mathrm{Cl7}(170)$ & 0.11 & 0.13 & 0.06 & 2.33 & 3.41 \\
\hline Cl7(187) & 0.32 & 0.33 & 0.16 & 6.65 & $4.82 \mathrm{D}$ \\
\hline Cl7(188) & $0.02 \mathrm{U}$ & $0.02 \mathrm{U}$ & $0.02 \mathrm{U}$ & 0.03 & 0.04 \\
\hline Cl8(195) & $0.05 \mathrm{U}$ & $0.05 \mathrm{U}$ & $0.05 \mathrm{U}$ & 0.46 & 0.61 \\
\hline $\mathrm{Cl} 8(200)$ & $0.04 \mathrm{U}$ & $0.04 \mathrm{U}$ & $0.04 \mathrm{U}$ & 0.18 & 0.20 \\
\hline C19(206) & 0.07 & $0.04 \mathrm{U}$ & $0.04 \mathrm{U}$ & 1.82 & 3.66 \\
\hline Cl10(209) & 0.03 & $0.07 \mathrm{U}$ & $0.07 \mathrm{U}$ & 0.53 & 1.00 \\
\hline Total PCBs & 6.88 & 7.44 & 4.90 & 95.2 & 120.32 \\
\hline LOC 1 & $0.08 \mathrm{U}$ & $0.08 \mathrm{U}$ & $0.08 \mathrm{U}$ & $0.08 \mathrm{U}$ & $0.08 \mathrm{U}$ \\
\hline LOC 2 & $0.52 \mathrm{U}$ & $0.02 \mathrm{~J}$ & $0.52 \mathrm{U}$ & $0.05 \mathrm{~J}$ & $0.06 \mathrm{~J}$ \\
\hline LOC 3 & $0.75 \mathrm{U}$ & $0.75 \mathrm{U}$ & $0.75 \mathrm{U}$ & 0.98 & 1.21 \\
\hline LOC 4 & $0.57 \mathrm{~J}$ & $0.74 \mathrm{~J}$ & $1.63 \mathrm{U}$ & 9.74 & 12.37 \\
\hline LOC 5 & 1.75 & 2.20 & 0.76 & 37.17 & 41.07 \\
\hline LOC 6 & 2.48 & 2.73 & 1.55 & 43.68 & 54.44 \\
\hline LOC 7 & 1.18 & 1.27 & $0.58 \mathrm{~J}$ & 28.47 & 32.37 \\
\hline LOC 8 & $0.15 \mathrm{~J}$ & $0.12 \mathrm{~J}$ & $0.51 \mathrm{U}$ & 9.26 & 14.62 \\
\hline LOC 9 & $0.07 \mathrm{~J}$ & $0.16 \mathrm{U}$ & $0.16 \mathrm{U}$ & 2.41 & 4.58 \\
\hline Total PCBs & 7.55 & 8.07 & 6.54 & 131.84 & 160.8 \\
\hline $\mathrm{Cl} 3(34)$ & 75 & 59 & 92 & 76 & 70 \\
\hline Cl6(152) & 62 & 55 & 83 & 76 & 74 \\
\hline
\end{tabular}


BATTELLE MARINE SCIENCE LABORATORIES

1529 West Sequim Bay Road

Sequim, Washington 98382-9099

360/681-4564

\section{SINCLAIR AND DYES INLET 2007 PSAMP Trawl Biota PCBs in Whole Organisms}

\begin{tabular}{|c|c|c|c|c|c|}
\hline Sample Name: & SI-ES-4 & SI-ES-6 & SI-ES-7 & SI-ES-8 & SI-RS-3 \\
\hline Station: & Sinclair Inlet & Sinclair Inlet & Sinclair Inlet & Sinclair Inlet & Sinclair Inlet \\
\hline Organism ID: & ES & ES & ES & ES & RS \\
\hline Batch ID: & 08-0004 & 08-0004 & $08-0004$ & $08-0004$ & $08-0004$ \\
\hline Sample Weight (g): & 30.2 & 30.29 & 31.18 & 30.34 & 30.16 \\
\hline \%Moisture & 59.34 & 79.69 & 79.49 & 79.29 & 78.88 \\
\hline \%Lipids & 1.01 & 1.18 & 1.05 & 1.54 & 0.71 \\
\hline Collection Date: & $05 / 17 / 2007$ & $05 / 17 / 2007$ & $05 / 17 / 2007$ & $05 / 17 / 2007$ & $05 / 17 / 2007$ \\
\hline Extraction Date: & 01/10/2008 & 01/10/2008 & 01/10/2008 & 01/10/2008 & 01/10/2008 \\
\hline Analysis Date: & 01/29/2008 & 01/30/2008 & 01/30/2008 & 01/30/2008 & 01/29/2008 \\
\hline Units (wet wt): & ng/g_WET & ng/g_WET & ng/g_WET & ng/g_WET & ng/g_WET \\
\hline $\mathrm{Cl} 2(8)$ & 0.04 & $0.02 \mathrm{~J}$ & $0.02 \mathrm{~J}$ & 0.04 & $0.05 \mathrm{U}$ \\
\hline $\mathrm{Cl} 3(18)$ & 0.09 & 0.07 & $0.06 \mathrm{U}$ & 0.19 & $0.06 \mathrm{U}$ \\
\hline $\mathrm{Cl} 3(28)$ & 0.23 & 0.17 & $0.06 \mathrm{U}$ & 0.50 & $0.06 \mathrm{U}$ \\
\hline $\mathrm{Cl} 4(44)$ & 0.30 & 0.22 & 0.21 & 0.80 & $0.12 \mathrm{U}$ \\
\hline $\mathrm{Cl} 4(52)$ & 1.24 & 0.83 & 0.82 & 2.68 & 0.32 \\
\hline $\mathrm{Cl} 4(66)$ & 0.73 & 0.52 & 0.50 & 1.48 & 0.23 \\
\hline $\mathrm{Cl} 4(77)$ & $0.05 \mathrm{U}$ & $0.02 \mathrm{~J}$ & $0.05 \mathrm{U}$ & $0.05 \mathrm{U}$ & $0.05 \mathrm{U}$ \\
\hline $\mathrm{Cl} 5(101)$ & $2.58 \mathrm{D}$ & 4.92 & 5.41 & $4.85 \mathrm{D}$ & 0.97 \\
\hline $\mathrm{Cl}$ (105) & 1.35 & 1.22 & 1.36 & 2.69 & 0.32 \\
\hline $\mathrm{Cl} 5(118)$ & 4.56 & 4.31 & 4.67 & $6.21 \mathrm{D}$ & 1.26 \\
\hline Cl5(126) & $0.07 \mathrm{U}$ & $0.07 \mathrm{U}$ & $0.07 \mathrm{U}$ & $0.07 \mathrm{U}$ & $0.07 \mathrm{U}$ \\
\hline $\mathrm{Cl6}(128)$ & 1.29 & 1.32 & 2.19 & 2.54 & 0.30 \\
\hline $\mathrm{Cl} 6(138)$ & $3.20 \mathrm{D}$ & $3.48 \mathrm{D}$ & $5.36 \mathrm{D}$ & $6.06 \mathrm{D}$ & 1.63 \\
\hline Cl6(153) & $6.48 \mathrm{D}$ & $6.94 \mathrm{D}$ & $9.6 \mathrm{D}$ & $11.71 \mathrm{D}$ & 2.98 \\
\hline $\mathrm{Cl}(170)$ & 1.26 & 1.40 & 2.71 & 2.79 & 0.26 \\
\hline $\mathrm{Cl} 7(187)$ & 4.08 & 4.30 & $4.76 \mathrm{D}$ & $4.28 \mathrm{D}$ & 1.03 \\
\hline $\mathrm{Cl} 7(188)$ & $0.02 \mathrm{~J}$ & $0.02 \mathrm{~J}$ & 0.08 & 0.05 & $0.02 \mathrm{U}$ \\
\hline $\mathrm{Cl}(195)$ & 0.24 & 0.28 & 0.75 & 0.56 & $0.05 \mathrm{U}$ \\
\hline $\mathrm{Cl} 8(200)$ & 0.11 & 0.12 & 0.29 & 0.25 & $0.04 \mathrm{U}$ \\
\hline $\mathrm{Cl9}(206)$ & 0.8 & 1.06 & 2.28 & 2.01 & 0.16 \\
\hline Cl10(209) & 0.34 & 0.37 & 0.96 & 0.73 & 0.09 \\
\hline Total PCBs & 58.12 & 63.32 & 84.42 & 101.08 & 20.14 \\
\hline LOC 1 & $0.08 \mathrm{U}$ & $0.08 \mathrm{U}$ & $0.08 \mathrm{U}$ & $0.08 \mathrm{U}$ & $0.08 \mathrm{U}$ \\
\hline LOC 2 & $0.05 \mathrm{~J}$ & $0.02 \mathrm{~J}$ & $0.08 \mathrm{~J}$ & $0.04 \mathrm{~J}$ & $0.52 \mathrm{U}$ \\
\hline LOC 3 & 0.75 & $0.37 \mathrm{~J}$ & $0.75 \mathrm{U}$ & 1.22 & $0.75 \mathrm{U}$ \\
\hline LOC 4 & 5.99 & 4.09 & 4.29 & 12.4 & 1.21 \\
\hline LOC 5 & 23.01 & 24.19 & 27.6 & 35.62 & 4.96 \\
\hline LOC 6 & 27.25 & 28.31 & 40.29 & 50.48 & 7.99 \\
\hline LOC 7 & 16.69 & 17.77 & 29.37 & 27.41 & 3.70 \\
\hline LOC 8 & 4.76 & 5.62 & 14.49 & 11.19 & 0.98 \\
\hline LOC 9 & 1.17 & 1.46 & 3.45 & 2.86 & 0.22 \\
\hline Total PCBs & 79.75 & 81.91 & 120.4 & 141.3 & 20.41 \\
\hline $\mathrm{Cl} 3(34)$ & 63 & 74 & 94 & 73 & 92 \\
\hline $\mathrm{Cl}(152)$ & 67 & 73 & 89 & 72 & 78 \\
\hline
\end{tabular}




\begin{tabular}{|c|c|c|c|c|c|}
\hline Sample Name: & V-SP-1 & V-SP-2 & V-SP-3 & HC-SP1 & HC-SP2 \\
\hline Station: & Vendovi & Vendovi & Vendovi & Hood Canal & Hood Canal \\
\hline Organism ID: & SP & SP & SP & SP & SP \\
\hline Batch ID: & $08-0013$ & 08-0013 & $08-0013$ & $08-0013$ & $08-0013$ \\
\hline Sample Weight (g): & 7.47 & 7.45 & 7.56 & 8.07 & 10.17 \\
\hline \%Moisture & 76.72 & 77.81 & 75.87 & 72.16 & 65.95 \\
\hline \%Lipids & 4.15 & 2.79 & 3.98 & 5.35 & 6.71 \\
\hline Collection Date: & $05 / 01 / 2007$ & $05 / 01 / 2007$ & $05 / 01 / 2007$ & $05 / 03 / 2007$ & $05 / 03 / 2007$ \\
\hline Extraction Date: & $01 / 22 / 2008$ & $01 / 22 / 2008$ & $01 / 22 / 2008$ & $01 / 22 / 2008$ & $01 / 22 / 2008$ \\
\hline Analysis Date: & $02 / 05 / 2008$ & $02 / 05 / 2008$ & 02/05/2008 & 02/05/2008 & $02 / 05 / 2008$ \\
\hline Units (wet wt): & ng/g_WET & ng/g_WET & ng/g_WET & ng/g_WET & ng/g_WET \\
\hline $\mathrm{Cl} 2(8)$ & $0.12 \mathrm{~J}$ & $0.05 \mathrm{U}$ & $0.16 \mathrm{~J}$ & $0.21 \mathrm{~J}$ & $0.06 \mathrm{~J}$ \\
\hline $\mathrm{Cl} 3(18)$ & $0.12 \mathrm{~J}$ & $0.06 \mathrm{U}$ & $0.15 \mathrm{~J}$ & 0.26 & $0.06 \mathrm{U}$ \\
\hline $\mathrm{Cl} 3(28)$ & $0.21 \mathrm{~J}$ & $0.13 \mathrm{~J}$ & 0.34 & 0.29 & $0.06 \mathrm{U}$ \\
\hline $\mathrm{Cl} 4(44)$ & $0.17 \mathrm{~J}$ & $0.11 \mathrm{~J}$ & 0.27 & $0.18 \mathrm{~J}$ & $0.10 \mathrm{~J}$ \\
\hline $\mathrm{Cl} 4(52)$ & 0.52 & 0.32 & 0.73 & 0.57 & 0.35 \\
\hline $\mathrm{Cl} 4(66)$ & $0.19 \mathrm{~J}$ & $0.16 \mathrm{~J}$ & 0.48 & $0.14 \mathrm{~J}$ & $0.10 \mathrm{~J}$ \\
\hline $\mathrm{Cl} 4(77)$ & $0.05 \mathrm{U}$ & $0.05 \mathrm{U}$ & $0.08 \mathrm{~J}$ & $0.05 \mathrm{~J}$ & $0.05 \mathrm{U}$ \\
\hline $\mathrm{Cl5}(101)$ & 1.06 & 0.84 & 1.23 & 2.61 & 1.62 \\
\hline $\mathrm{Cl5}(105)$ & 0.38 & 0.31 & 0.39 & 0.61 & 0.38 \\
\hline $\mathrm{Cl5}(118)$ & 1.58 & 1.42 & 1.84 & 3.74 & 2.62 \\
\hline $\mathrm{Cl5}(126)$ & $0.07 \mathrm{U}$ & $0.07 \mathrm{U}$ & $0.07 \mathrm{U}$ & $0.07 \mathrm{U}$ & $0.07 \mathrm{U}$ \\
\hline Cl6(128) & $0.18 \mathrm{~J}$ & $0.18 \mathrm{~J}$ & $0.23 \mathrm{~J}$ & 0.36 & 0.28 \\
\hline Cl6(138) & 0.92 & 0.93 & 1.02 & 2.61 & 2.27 \\
\hline $\mathrm{Cl6}(153)$ & 1.63 & 1.67 & 1.68 & 4.79 & 4.57 \\
\hline $\mathrm{Cl}(170)$ & $0.17 \mathrm{~J}$ & $0.15 \mathrm{~J}$ & $0.18 \mathrm{~J}$ & 0.58 & 0.47 \\
\hline $\mathrm{Cl}(187)$ & 0.48 & 0.53 & 0.52 & 1.62 & 1.51 \\
\hline $\mathrm{Cl} 7(188)$ & $0.02 \mathrm{U}$ & $0.02 \mathrm{~J}$ & $0.02 \mathrm{U}$ & $0.02 \mathrm{U}$ & $0.02 \mathrm{U}$ \\
\hline $\mathrm{Cl}(195)$ & $0.05 \mathrm{U}$ & $0.05 \mathrm{U}$ & $0.05 \mathrm{U}$ & $0.05 \mathrm{~J}$ & $0.08 \mathrm{~J}$ \\
\hline $\mathrm{Cl}(200)$ & $0.04 \mathrm{U}$ & $0.04 \mathrm{U}$ & $0.04 \mathrm{U}$ & $0.04 \mathrm{U}$ & $0.04 \mathrm{U}$ \\
\hline $\mathrm{Cl9}(206)$ & $0.04 \mathrm{U}$ & $0.04 \mathrm{U}$ & $0.04 \mathrm{U}$ & $0.19 \mathrm{~J}$ & $0.17 \mathrm{~J}$ \\
\hline Cl10(209) & $0.07 \mathrm{U}$ & $0.07 \mathrm{U}$ & $0.07 \mathrm{U}$ & $0.09 \mathrm{~J}$ & $0.06 \mathrm{~J}$ \\
\hline Total PCBs & 16.14 & 14.40 & 19.18 & 38.16 & 29.88 \\
\hline LOC 1 & $0.08 \mathrm{U}$ & $0.08 \mathrm{U}$ & $0.08 \mathrm{U}$ & $0.04 \mathrm{~J}$ & $0.08 \mathrm{U}$ \\
\hline LOC 2 & $0.12 \mathrm{~J}$ & $0.17 \mathrm{~J}$ & $0.16 \mathrm{~J}$ & $0.37 \mathrm{~J}$ & $0.10 \mathrm{~J}$ \\
\hline LOC 3 & $0.48 \mathrm{~J}$ & $0.20 \mathrm{~J}$ & $0.72 \mathrm{~J}$ & $0.79 \mathrm{~J}$ & $0.75 \mathrm{U}$ \\
\hline LOC 4 & $1.88 \mathrm{~J}$ & $1.37 \mathrm{~J}$ & $3.38 \mathrm{~J}$ & $2.20 \mathrm{~J}$ & $1.31 \mathrm{~J}$ \\
\hline LOC 5 & 6.52 & $4.95 \mathrm{~J}$ & 7.34 & 13.07 & 8.03 \\
\hline LOC 6 & $4.68 \mathrm{~J}$ & $4.71 \mathrm{~J}$ & $5.09 \mathrm{~J}$ & 14.00 & 11.94 \\
\hline LOC 7 & $1.72 \mathrm{~J}$ & $1.68 \mathrm{~J}$ & $1.87 \mathrm{~J}$ & 6.05 & 5.40 \\
\hline LOC 8 & $0.29 \mathrm{~J}$ & $0.1 \mathrm{~J}$ & $0.37 \mathrm{~J}$ & $1.08 \mathrm{~J}$ & $1.33 \mathrm{~J}$ \\
\hline LOC 9 & $0.16 \mathrm{U}$ & $0.16 \mathrm{U}$ & $0.16 \mathrm{U}$ & $0.27 \mathrm{~J}$ & $0.23 \mathrm{~J}$ \\
\hline Total PCBs & 15.93 & 13.42 & 19.17 & 37.87 & 29.17 \\
\hline $\mathrm{Cl} 3(34)$ & 77 & 61 & 66 & 47 & 58 \\
\hline $\mathrm{Cl6}(152)$ & 80 & 72 & 69 & 45 & 49 \\
\hline
\end{tabular}


1529 West Sequim Bay Road

Sequim, Washington 98382-9099

360/681-4564

\section{SINCLAIR AND DYES INLET \\ 2007 PSAMP Trawl Biota \\ PCBs in Whole Organisms}

\begin{tabular}{|c|c|c|c|c|c|}
\hline Sample Name: & SI-RS-1_2 & SI-RS-4 & SI-SS-1 & SI-SS-2 & SI-SS-3 \\
\hline Station: & Sinclair Inlet & Sinclair Inlet & Sinclair Inlet & Sinclair Inlet & Sinclair Inlet \\
\hline Organism ID: & RS & RS & SS & SS & SS \\
\hline Batch ID: & 08-0013 & $08-0013$ & $08-0013$ & $08-0013$ & $08-0013$ \\
\hline Sample Weight (g): & 17.6 & 30 & 30.63 & 30.55 & 30.01 \\
\hline \%Moisture & 75.72 & 78.83 & 78.34 & 77.87 & 78.95 \\
\hline \%Lipids & 3.10 & 0.47 & 0.85 & 0.72 & 0.54 \\
\hline Collection Date: & $05 / 17 / 2007$ & $05 / 17 / 2007$ & $05 / 17 / 2007$ & $05 / 17 / 2007$ & $05 / 17 / 2007$ \\
\hline Extraction Date: & $01 / 22 / 2008$ & $01 / 22 / 2008$ & $01 / 22 / 2008$ & $01 / 22 / 2008$ & $01 / 22 / 2008$ \\
\hline Analysis Date: & $02 / 12 / 2008$ & $02 / 05 / 2008$ & $02 / 13 / 2008$ & $02 / 14 / 2008$ & $02 / 14 / 2008$ \\
\hline Units (wet wt): & ng/g_WET & ng/g_WET & ng/g_WET & ng/g_WET & ng/g_WET \\
\hline $\mathrm{Cl} 2(8)$ & $0.05 \mathrm{U}$ & $0.05 \mathrm{U}$ & $0.05 \mathrm{U}$ & $0.05 \mathrm{U}$ & $0.05 \mathrm{U}$ \\
\hline $\mathrm{Cl} 3(18)$ & $0.06 \mathrm{U}$ & $0.06 \mathrm{U}$ & $0.06 \mathrm{U}$ & $0.06 \mathrm{U}$ & $0.06 \mathrm{U}$ \\
\hline $\mathrm{Cl} 3(28)$ & $0.06 \mathrm{U}$ & $0.06 \mathrm{~J}$ & 0.29 & 0.23 & $0.06 \mathrm{U}$ \\
\hline $\mathrm{Cl} 4(44)$ & 0.23 & $0.12 \mathrm{U}$ & 0.12 & 0.10 & $0.12 \mathrm{U}$ \\
\hline $\mathrm{Cl} 4(52)$ & 1.09 & 0.29 & 2.21 & 2.11 & 1.00 \\
\hline $\mathrm{Cl} 4(66)$ & 0.62 & 0.49 & 0.94 & 0.54 & $0.07 \mathrm{U}$ \\
\hline $\mathrm{Cl} 4(77)$ & $0.05 \mathrm{U}$ & $0.03 \mathrm{~J}$ & 0.04 & $0.05 \mathrm{U}$ & $0.05 \mathrm{U}$ \\
\hline $\mathrm{Cl5}(101)$ & 3.91 & 1.53 & 8.99 & 7.10 & 3.36 \\
\hline $\mathrm{Cl5}(105)$ & 0.50 & 1.38 & 1.44 & 1.12 & 0.40 \\
\hline $\mathrm{Cl} 5(118)$ & 3.18 & $3.78 \mathrm{D}$ & 8.98 & 7.00 & 3.14 \\
\hline $\mathrm{Cl} 5(126)$ & $0.07 \mathrm{U}$ & $0.07 \mathrm{U}$ & $0.07 \mathrm{U}$ & $0.07 \mathrm{U}$ & $0.07 \mathrm{U}$ \\
\hline $\mathrm{Cl6}(128)$ & 0.59 & 2.21 & 1.20 & 0.93 & $0.05 \mathrm{U}$ \\
\hline Cl6(138) & 3.41 & $7.58 \mathrm{D}$ & 9.59 & 6.17 & 2.43 \\
\hline Cl6(153) & 8.31 & $14.25 \mathrm{D}$ & $8.89 \mathrm{D}$ & 14.31 & 5.96 \\
\hline $\mathrm{Cl}(170)$ & 0.71 & 2.71 & 1.90 & 1.14 & 0.66 \\
\hline $\mathrm{Cl} 7(187)$ & 3.21 & 1.79 & 6.65 & 4.65 & 2.08 \\
\hline $\mathrm{Cl} 7(188)$ & $0.02 \mathrm{U}$ & 0.10 & $0.02 \mathrm{U}$ & 0.05 & $0.02 \mathrm{U}$ \\
\hline $\mathrm{Cl} 8(195)$ & $0.05 \mathrm{U}$ & 0.68 & 0.25 & 0.18 & $0.05 \mathrm{U}$ \\
\hline $\mathrm{Cl}(200)$ & $0.07 \mathrm{~J}$ & $0.02 \mathrm{~J}$ & $0.04 \mathrm{U}$ & $0.04 \mathrm{U}$ & $0.04 \mathrm{U}$ \\
\hline $\mathrm{Cl}(206)$ & 0.36 & 2.44 & 1.07 & 0.51 & $0.04 \mathrm{U}$ \\
\hline Cl10(209) & 0.19 & 1.12 & 0.42 & 0.22 & 0.16 \\
\hline Total PCBs & 53.48 & 81.52 & 106.44 & 93.26 & 39.74 \\
\hline LOC 1 & $0.08 \mathrm{U}$ & $0.08 \mathrm{U}$ & $0.08 \mathrm{U}$ & $0.08 \mathrm{U}$ & $0.08 \mathrm{U}$ \\
\hline LOC 2 & $0.52 \mathrm{U}$ & $0.52 \mathrm{U}$ & $0.52 \mathrm{U}$ & $0.52 \mathrm{U}$ & $0.52 \mathrm{U}$ \\
\hline LOC 3 & $0.75 \mathrm{U}$ & $0.06 \mathrm{~J}$ & 0.50 & 0.55 & $0.75 \mathrm{U}$ \\
\hline LOC 4 & 4.37 & $1.91 \mathrm{~J}$ & 7.96 & 7.01 & 2.47 \\
\hline LOC 5 & 19.7 & 15.78 & 43.49 & 32.36 & 13.83 \\
\hline LOC 6 & 25.66 & 33.36 & 45.28 & 39.06 & 16.25 \\
\hline LOC 7 & 11.17 & 17.8 & 24.66 & 15.94 & 8.19 \\
\hline LOC 8 & 3.28 & 9.36 & 7.76 & 3.96 & 0.79 \\
\hline LOC 9 & 0.62 & 3.15 & 1.65 & 0.78 & $0.16 \mathrm{U}$ \\
\hline Total PCBs & 66.15 & 82.02 & 131.9 & 100.26 & 43.04 \\
\hline $\mathrm{Cl} 3(34)$ & 100 & 65 & 106 & 91 & 94 \\
\hline Cl6(152) & 65 & 63 & 60 & 64 & 60 \\
\hline
\end{tabular}




\section{SINCLAIR AND DYES INLET 2007 PSAMP Trawl Biota PCBs in Whole Organisms}

\begin{tabular}{|c|c|c|c|c|c|}
\hline Sample Name: & SI-SSc-1_2 & SI-SSc-3_5 & SI-SSc-4_6 & SI-SP-4_5 & SI-SP-6_2 \\
\hline Station: & Sinclair Inlet & Sinclair Inlet & Sinclair Inlet & Sinclair Inlet & Sinclair Inlet \\
\hline Organism ID: & STS & STS & STS & $\mathrm{SP}$ & $\mathrm{SP}$ \\
\hline Batch ID: & $08-0013$ & $08-0013$ & $08-0013$ & $08-0013$ & $08-0013$ \\
\hline Sample Weight (g): & 30.46 & 30.6 & 33.78 & 10.65 & 10.01 \\
\hline \%Moisture & 79.34 & 79.97 & 80.49 & 75.53 & 75.32 \\
\hline \%Lipids & 1.14 & 1.01 & 1.17 & 4.01 & 2.83 \\
\hline Collection Date: & 05/17/2007 & 05/17/2007 & 05/17/2007 & 05/17/2007 & 05/17/2007 \\
\hline Extraction Date: & 01/22/2008 & 01/22/2008 & 01/22/2008 & 01/22/2008 & 01/22/2008 \\
\hline Analysis Date: & 02/14/2008 & 02/15/2008 & 02/14/2008 & 03/08/2008 & 02/12/2008 \\
\hline Units (wet wt): & ng/g_WET & ng/g_WET & ng/g_WET & ng/g_WET & ng/g_WET \\
\hline $\mathrm{Cl} 2(8)$ & $0.05 \mathrm{U}$ & $0.05 \mathrm{U}$ & $0.05 \mathrm{U}$ & $0.05 \mathrm{UT}$ & $0.05 \mathrm{U}$ \\
\hline $\mathrm{Cl} 3(18)$ & $0.06 \mathrm{U}$ & $0.06 \mathrm{U}$ & 0.06 & $0.05 \mathrm{~T}$ & $0.06 \mathrm{U}$ \\
\hline $\mathrm{Cl} 3(28)$ & $0.06 \mathrm{U}$ & 0.07 & 0.12 & $0.17 \mathrm{~T}$ & 0.29 \\
\hline Cl4(44) & $0.12 \mathrm{U}$ & 0.15 & 0.16 & $0.19 \mathrm{~T}$ & $0.12 \mathrm{U}$ \\
\hline Cl4(52) & 0.42 & 0.65 & 0.59 & $1.19 \mathrm{~T}$ & 1.29 \\
\hline Cl4(66) & 0.20 & 0.21 & 0.22 & $0.18 \mathrm{~T}$ & $0.18 \mathrm{~J}$ \\
\hline Cl4(77) & $0.05 \mathrm{U}$ & $0.05 \mathrm{U}$ & $0.01 \mathrm{~J}$ & $0.07 \mathrm{~T}$ & $0.03 \mathrm{~J}$ \\
\hline $\mathrm{Cl} 5(101)$ & 1.66 & 2.38 & 1.69 & $5.80 \mathrm{~T}$ & 4.96 \\
\hline $\mathrm{Cl} 5(105)$ & 0.38 & 0.54 & 0.41 & $1.16 \mathrm{~T}$ & 0.75 \\
\hline Cl5(118) & 2.78 & 3.49 & 2.43 & $5.32 \mathrm{~T}$ & 4.89 \\
\hline $\mathrm{Cl} 5(126)$ & $0.07 \mathrm{U}$ & $0.07 \mathrm{U}$ & $0.07 \mathrm{U}$ & $0.07 \mathrm{UT}$ & $0.07 \mathrm{U}$ \\
\hline Cl6(128) & 0.39 & $0.05 \mathrm{U}$ & 0.31 & $0.59 \mathrm{~T}$ & 0.51 \\
\hline Cl6(138) & 2.42 & 3.11 & 2.02 & $6.04 \mathrm{~T}$ & 4.05 \\
\hline $\mathrm{Cl6}(153)$ & 6.09 & 6.68 & 4.12 & $15.52 \mathrm{~T}$ & 11.19 \\
\hline Cl7(170) & 0.67 & 0.58 & 0.49 & $0.96 \mathrm{~T}$ & 0.73 \\
\hline $\mathrm{Cl} 7(187)$ & 2.76 & 2.63 & 1.70 & $5.79 \mathrm{~T}$ & 3.55 \\
\hline $\mathrm{Cl} 7(188)$ & $0.02 \mathrm{U}$ & 0.04 & $0.02 \mathrm{U}$ & $0.02 \mathrm{UT}$ & $0.02 \mathrm{U}$ \\
\hline Cl8(195) & 0.09 & 0.14 & 0.08 & $0.05 \mathrm{UT}$ & $0.05 \mathrm{U}$ \\
\hline $\mathrm{Cl} 8(200)$ & $0.04 \mathrm{U}$ & $0.04 \mathrm{U}$ & $0.04 \mathrm{U}$ & $0.04 \mathrm{UT}$ & $0.04 \mathrm{U}$ \\
\hline $\mathrm{Cl} 9(206)$ & 0.50 & 0.34 & 0.28 & $0.72 \mathrm{~T}$ & 0.37 \\
\hline Cl10(209) & 0.17 & 0.13 & 0.09 & $0.34 \mathrm{~T}$ & 0.23 \\
\hline Total PCBs & 38.00 & 42.92 & 29.92 & 88.64 & 66.86 \\
\hline LOC 1 & $0.08 \mathrm{U}$ & $0.08 \mathrm{U}$ & $0.08 \mathrm{U}$ & $0.08 \mathrm{UT}$ & $0.08 \mathrm{U}$ \\
\hline LOC 2 & $0.52 \mathrm{U}$ & $0.52 \mathrm{U}$ & $0.52 \mathrm{U}$ & $0.15 \mathrm{~T}$ & $0.52 \mathrm{U}$ \\
\hline LOC 3 & $0.75 \mathrm{U}$ & 0.07 & $0.24 \mathrm{~J}$ & $0.56 \mathrm{~T}$ & $0.29 \mathrm{~J}$ \\
\hline LOC 4 & 1.36 & 2.12 & 1.94 & $3.35 \mathrm{~T}$ & $3.87 \mathrm{~J}$ \\
\hline LOC 5 & 10.47 & 14.07 & 9.60 & $24.89 \mathrm{~T}$ & 21.67 \\
\hline LOC 6 & 17.44 & 18.91 & 12.83 & $36.28 \mathrm{~T}$ & 27.61 \\
\hline LOC 7 & 9.62 & 9.05 & 6.49 & $16.75 \mathrm{~T}$ & 11.43 \\
\hline LOC 8 & 3.08 & 2.54 & 2.05 & $5.43 \mathrm{~T}$ & 2.69 \\
\hline LOC 9 & 0.72 & 0.54 & 0.41 & $1.17 \mathrm{~T}$ & 0.62 \\
\hline Total PCBs & 44.04 & 47.9 & 34.16 & 88.66 & 68.78 \\
\hline $\mathrm{Cl} 3(34)$ & 82 & 65 & 66 & 70 & 106 \\
\hline Cl6(152) & 59 & 49 & 52 & 74 & 68 \\
\hline
\end{tabular}


1529 West Sequim Bay Road

Sequim, Washington 98382-9099

360/681-4564

\section{SINCLAIR AND DYES INLET 2007 PSAMP Trawl Biota PCBs in Whole Organisms}

\begin{tabular}{|c|c|c|c|c|c|}
\hline Sample Name: & SI-SP-7_3 & SI-GC-2_6 & SI-GC-3_4 & SI-GC-7_1 & SI-GC-5_8 \\
\hline Station: & Sinclair Inlet & Sinclair Inlet & Sinclair Inlet & Sinclair Inlet & Sinclair Inlet \\
\hline Organism ID: & SP & GC & GC & $\mathrm{GC}$ & $\mathrm{GC}$ \\
\hline Batch ID: & $08-0013$ & 08-0013 & $08-0013$ & $08-0013$ & $08-0013$ \\
\hline Sample Weight (g): & 9.07 & 31.73 & 30.43 & 30.81 & 30.07 \\
\hline$\%$ Moisture & 74.88 & 71.25 & 66.40 & 45.15 & 69.11 \\
\hline \%Lipids & 3.16 & 0.92 & 0.94 & 0.92 & 1.00 \\
\hline Collection Date: & $05 / 17 / 2007$ & $05 / 17 / 2007$ & $05 / 17 / 2007$ & $05 / 17 / 2007$ & $05 / 17 / 2007$ \\
\hline Extraction Date: & 01/22/2008 & 01/22/2008 & 01/22/2008 & 01/22/2008 & 01/22/2008 \\
\hline Analysis Date: & 02/12/2008 & 03/08/2008 & 02/12/2008 & 03/08/2008 & 02/12/2008 \\
\hline Units (wet wt): & ng/g_WET & ng/g_WET & ng/g_WET & ng/g_WET & ng/g_WET \\
\hline $\mathrm{Cl} 2(8)$ & $0.05 \mathrm{U}$ & $0.05 \mathrm{UT}$ & $0.05 \mathrm{U}$ & $0.05 \mathrm{UT}$ & $0.05 \mathrm{U}$ \\
\hline $\mathrm{Cl} 3(18)$ & $0.19 \mathrm{~J}$ & $0.06 \mathrm{UT}$ & $0.06 \mathrm{U}$ & $0.06 \mathrm{UT}$ & $0.06 \mathrm{U}$ \\
\hline $\mathrm{Cl} 3(28)$ & 0.34 & $0.22 \mathrm{~T}$ & 0.13 & $0.13 \mathrm{~T}$ & 0.12 \\
\hline Cl4(44) & 0.60 & $0.30 \mathrm{~T}$ & $0.12 \mathrm{U}$ & $0.12 \mathrm{UT}$ & 0.13 \\
\hline $\mathrm{Cl} 4(52)$ & 2.09 & $1.07 \mathrm{~T}$ & 0.67 & $0.64 \mathrm{~T}$ & 0.43 \\
\hline $\mathrm{Cl} 4(66)$ & 0.37 & $0.49 \mathrm{~T}$ & 0.57 & $0.40 \mathrm{~T}$ & 0.26 \\
\hline $\mathrm{Cl} 4(77)$ & $0.05 \mathrm{U}$ & $0.06 \mathrm{~T}$ & $0.05 \mathrm{U}$ & $0.05 \mathrm{UT}$ & $0.05 \mathrm{U}$ \\
\hline $\mathrm{Cl} 5(101)$ & 7.09 & $3.90 \mathrm{~T}$ & 2.93 & $2.74 \mathrm{~T}$ & 1.44 \\
\hline $\mathrm{Cl} 5(105)$ & 1.00 & $0.80 \mathrm{~T}$ & 0.58 & $0.56 \mathrm{~T}$ & 0.26 \\
\hline $\mathrm{Cl5}(118)$ & 5.76 & $3.69 \mathrm{~T}$ & 2.91 & $2.67 \mathrm{~T}$ & 1.40 \\
\hline $\mathrm{Cl} 5(126)$ & $0.07 \mathrm{U}$ & $0.07 \mathrm{UT}$ & $0.07 \mathrm{U}$ & $0.07 \mathrm{UT}$ & $0.07 \mathrm{U}$ \\
\hline $\mathrm{Cl6}(128)$ & 0.60 & $0.49 \mathrm{~T}$ & 0.43 & $0.46 \mathrm{~T}$ & 0.24 \\
\hline Cl6(138) & 4.91 & $3.38 \mathrm{~T}$ & 2.22 & $3.00 \mathrm{~T}$ & 1.25 \\
\hline Cl6(153) & 12.38 & $6.21 \mathrm{~T}$ & 5.10 & $6.12 \mathrm{~T}$ & 2.46 \\
\hline $\mathrm{Cl} 7(170)$ & 0.96 & $0.57 \mathrm{~T}$ & 0.51 & $0.58 \mathrm{~T}$ & 0.25 \\
\hline $\mathrm{Cl7}(187)$ & 3.82 & $2.31 \mathrm{~T}$ & 2.24 & $2.35 \mathrm{~T}$ & 1.22 \\
\hline $\mathrm{Cl7}(188)$ & $0.02 \mathrm{U}$ & $0.02 \mathrm{UT}$ & $0.02 \mathrm{U}$ & $0.02 \mathrm{UT}$ & $0.03 \mathrm{~J}$ \\
\hline $\mathrm{Cl}(195)$ & $0.05 \mathrm{U}$ & $0.05 \mathrm{UT}$ & 0.08 & $0.05 \mathrm{UT}$ & $0.05 \mathrm{U}$ \\
\hline $\mathrm{Cl}(200)$ & $0.04 \mathrm{U}$ & $0.11 \mathrm{~T}$ & $0.06 \mathrm{~J}$ & $0.04 \mathrm{UT}$ & $0.03 \mathrm{~J}$ \\
\hline $\mathrm{Cl} 9(206)$ & 0.62 & $0.45 \mathrm{~T}$ & 0.36 & $0.42 \mathrm{~T}$ & 0.22 \\
\hline Cl10(209) & 0.25 & $0.23 \mathrm{~T}$ & 0.16 & $0.19 \mathrm{~T}$ & 0.15 \\
\hline Total PCBs & 82.52 & 49.06 & 38.64 & 41.44 & 20.34 \\
\hline LOC 1 & $0.08 \mathrm{U}$ & $0.08 \mathrm{UT}$ & $0.08 \mathrm{U}$ & $0.08 \mathrm{UT}$ & $0.08 \mathrm{U}$ \\
\hline LOC 2 & $0.52 \mathrm{U}$ & $0.52 \mathrm{UT}$ & $0.52 \mathrm{U}$ & $0.52 \mathrm{UT}$ & $0.52 \mathrm{U}$ \\
\hline LOC 3 & $0.82 \mathrm{~J}$ & $0.38 \mathrm{~T}$ & $0.22 \mathrm{~J}$ & $0.22 \mathrm{~T}$ & $0.18 \mathrm{~J}$ \\
\hline LOC 4 & 7.02 & $3.87 \mathrm{~T}$ & 2.43 & $1.88 \mathrm{~T}$ & $1.63 \mathrm{~J}$ \\
\hline LOC 5 & 31.08 & $17.13 \mathrm{~T}$ & 12.16 & $12.08 \mathrm{~T}$ & 7.32 \\
\hline LOC 6 & 34.03 & $19.68 \mathrm{~T}$ & 17.04 & $17.71 \mathrm{~T}$ & 9.25 \\
\hline LOC 7 & 13.53 & $9.25 \mathrm{~T}$ & 7.76 & $8.96 \mathrm{~T}$ & 4.28 \\
\hline LOC 8 & 3.12 & $2.62 \mathrm{~T}$ & 2.11 & $2.68 \mathrm{~T}$ & 1.39 \\
\hline LOC 9 & 0.80 & $0.65 \mathrm{~T}$ & 0.50 & $0.56 \mathrm{~T}$ & 0.36 \\
\hline Total PCBs & 91 & 54.18 & 42.82 & 44.69 & 25.01 \\
\hline $\mathrm{Cl} 3(34)$ & 70 & 89 & 81 & 58 & 78 \\
\hline $\mathrm{Cl6}(152)$ & 44 & 84 & 52 & 45 & 48 \\
\hline
\end{tabular}




\section{SINCLAIR AND DYES INLET 2007 PSAMP Trawl Biota PCBs in Whole Organisms}

\begin{tabular}{|c|c|c|c|c|c|}
\hline Sample Name: & V-SC-COMP & SG-RF-1 & SG-RF-2 & SG-RF-3 & SG-SC-COMP \\
\hline Station: & Vendovi & Strait of Georgia & Strait of Georgia & 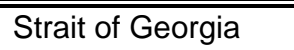 & $\overline{\text { Strait of Georgia }}$ \\
\hline Organism ID: & SC & RF & RF & $\mathrm{RF}$ & $\mathrm{SC}$ \\
\hline Batch ID: & 08-0015 & 08-0015 & 08-0015 & 08-0015 & 08-0015 \\
\hline Sample Weight (g): & 27.63 & 29.92 & 30.42 & 29.92 & 31.26 \\
\hline \%Moisture & 86.80 & 80.14 & 72.01 & 66.36 & 92.30 \\
\hline \%Lipids & 0.10 & 4.06 & 11.50 & 15.36 & 0.34 \\
\hline Collection Date: & $05 / 01 / 2007$ & $05 / 02 / 2007$ & $05 / 02 / 2007$ & $05 / 02 / 2007$ & $05 / 02 / 2007$ \\
\hline Extraction Date: & $01 / 28 / 2008$ & 01/28/2008 & $01 / 28 / 2008$ & $01 / 28 / 2008$ & $01 / 28 / 2008$ \\
\hline Analysis Date: & $02 / 16 / 2008$ & $02 / 16 / 2008$ & $02 / 16 / 2008$ & $02 / 16 / 2008$ & $02 / 16 / 2008$ \\
\hline Units (wet wt): & ng/g_WET & ng/g_WET & ng/g_WET & ng/g_WET & ng/g_WET \\
\hline $\mathrm{Cl} 2(8)$ & $0.05 \mathrm{U}$ & $0.05 \mathrm{U}$ & $0.01 \mathrm{~J}$ & $0.04 \mathrm{~J}$ & $0.05 \mathrm{U}$ \\
\hline $\mathrm{Cl} 3(18)$ & $0.01 \mathrm{~J}$ & $0.01 \mathrm{~J}$ & $0.01 \mathrm{~J}$ & $0.06 \mathrm{U}$ & $0.06 \mathrm{U}$ \\
\hline $\mathrm{Cl} 3(28)$ & $0.02 \mathrm{~J}$ & $0.04 \mathrm{~J}$ & 0.12 & 0.28 & $0.06 \mathrm{U}$ \\
\hline $\mathrm{Cl} 4(44)$ & $0.04 \mathrm{~J}$ & $0.12 \mathrm{U}$ & $0.12 \mathrm{U}$ & $0.12 \mathrm{U}$ & $0.12 \mathrm{U}$ \\
\hline $\mathrm{Cl} 4(52)$ & $0.03 \mathrm{~J}$ & $0.01 \mathrm{~J}$ & $0.01 \mathrm{~J}$ & 0.11 & $0.12 \mathrm{U}$ \\
\hline $\mathrm{Cl} 4(66)$ & $0.07 \mathrm{U}$ & 0.11 & 0.30 & 0.29 & $0.07 \mathrm{U}$ \\
\hline Cl4(77) & $0.05 \mathrm{U}$ & $0.05 \mathrm{U}$ & $0.05 \mathrm{U}$ & $0.05 \mathrm{U}$ & $0.05 \mathrm{U}$ \\
\hline Cl5(101) & $0.02 \mathrm{~J}$ & $0.02 \mathrm{~J}$ & $0.02 \mathrm{~J}$ & 0.09 & 0.06 \\
\hline $\mathrm{Cl} 5(105)$ & $0.05 \mathrm{U}$ & 0.15 & 0.29 & 0.46 & $0.05 \mathrm{U}$ \\
\hline $\mathrm{Cl5}(118)$ & $0.04 \mathrm{~J}$ & 0.66 & 1.45 & 1.17 & 0.09 \\
\hline $\mathrm{Cl5}(126)$ & $0.07 \mathrm{U}$ & $0.07 \mathrm{U}$ & $0.07 \mathrm{U}$ & $0.07 \mathrm{U}$ & $0.07 \mathrm{U}$ \\
\hline $\mathrm{Cl6}(128)$ & $0.05 \mathrm{U}$ & 0.18 & 0.29 & 0.46 & $0.05 \mathrm{U}$ \\
\hline Cl6(138) & $0.03 \mathrm{~J}$ & 0.78 & 1.29 & 1.97 & 0.15 \\
\hline $\mathrm{Cl6}(153)$ & $0.06 \mathrm{~J}$ & 1.31 & 1.97 & 3.43 & 0.27 \\
\hline $\mathrm{Cl}(170)$ & $0.04 \mathrm{U}$ & 0.20 & 0.33 & 0.43 & $0.04 \mathrm{U}$ \\
\hline Cl7(187) & $0.03 \mathrm{~J}$ & $0.02 \mathrm{~J}$ & $0.05 \mathrm{~J}$ & 0.14 & 0.13 \\
\hline $\mathrm{Cl}(188)$ & $0.02 \mathrm{U}$ & $0.02 \mathrm{U}$ & $0.02 \mathrm{U}$ & $0.02 \mathrm{U}$ & $0.02 \mathrm{U}$ \\
\hline $\mathrm{Cl}(195)$ & $0.05 \mathrm{U}$ & $0.04 \mathrm{~J}$ & 0.08 & $0.05 \mathrm{U}$ & $0.05 \mathrm{U}$ \\
\hline $\mathrm{Cl} 8(200)$ & $0.04 \mathrm{U}$ & $0.04 \mathrm{U}$ & $0.04 \mathrm{U}$ & $0.04 \mathrm{U}$ & $0.04 \mathrm{U}$ \\
\hline $\mathrm{Cl9}(206)$ & $0.04 \mathrm{U}$ & 0.08 & 0.17 & 0.18 & $0.04 \mathrm{U}$ \\
\hline Cl10(209) & $0.07 \mathrm{U}$ & $0.03 \mathrm{~J}$ & 0.07 & $0.06 \mathrm{~J}$ & $0.07 \mathrm{U}$ \\
\hline Total PCBs & 1.76 & 7.98 & 13.52 & 19.04 & 3.32 \\
\hline LOC 1 & $0.08 \mathrm{U}$ & $0.08 \mathrm{U}$ & $0.08 \mathrm{U}$ & $0.08 \mathrm{U}$ & $0.08 \mathrm{U}$ \\
\hline LOC 2 & $0.52 \mathrm{U}$ & $0.52 \mathrm{U}$ & $0.01 \mathrm{~J}$ & $0.04 \mathrm{~J}$ & $0.52 \mathrm{U}$ \\
\hline LOC 3 & $0.07 \mathrm{~J}$ & $0.06 \mathrm{~J}$ & $0.17 \mathrm{~J}$ & $0.50 \mathrm{~J}$ & $0.75 \mathrm{U}$ \\
\hline LOC 4 & $0.12 \mathrm{~J}$ & $0.31 \mathrm{~J}$ & $0.81 \mathrm{~J}$ & $1.61 \mathrm{~J}$ & $1.63 \mathrm{U}$ \\
\hline LOC 5 & $0.11 \mathrm{~J}$ & $1.34 \mathrm{~J}$ & 2.83 & 3.06 & $0.30 \mathrm{~J}$ \\
\hline LOC 6 & $0.15 \mathrm{~J}$ & 2.70 & 4.23 & 6.71 & $0.62 \mathrm{~J}$ \\
\hline LOC 7 & $0.07 \mathrm{~J}$ & $1.14 \mathrm{~J}$ & 1.78 & 2.29 & $0.34 \mathrm{~J}$ \\
\hline LOC 8 & $0.51 \mathrm{U}$ & $0.31 \mathrm{~J}$ & $0.55 \mathrm{~J}$ & $0.51 \mathrm{U}$ & $0.51 \mathrm{U}$ \\
\hline LOC 9 & $0.16 \mathrm{U}$ & $0.10 \mathrm{~J}$ & $0.20 \mathrm{~J}$ & $0.18 \mathrm{~J}$ & $0.16 \mathrm{U}$ \\
\hline Total PCBs & 1.79 & 6.56 & 10.66 & 14.98 & 4.91 \\
\hline $\mathrm{Cl} 3(34)$ & 64 & 60 & 54 & 80 & 78 \\
\hline Cl6(152) & 70 & 55 & 51 & 88 & 96 \\
\hline
\end{tabular}


1529 West Sequim Bay Road

Sequim, Washington 98382-9099

360/681-4564

\section{SINCLAIR AND DYES INLET 2007 PSAMP Trawl Biota PCBs in Whole Organisms}

\begin{tabular}{|c|c|c|c|c|}
\hline Sample Name: & HC-ES-1 & HC-ES-2 & HC-ES-3 & HC-RS-1 \\
\hline Station: & Hood Canal & Hood Canal & Hood Canal & Hood Canal \\
\hline Organism ID: & ES & ES & ES & RS \\
\hline Batch ID: & 08-0015 & 08-0015 & 08-0015 & 08-0015 \\
\hline Sample Weight (g): & 30.79 & 30.26 & 30.27 & 30.05 \\
\hline \%Moisture & 82.09 & 79.72 & 78.81 & 77.93 \\
\hline \%Lipids & 0.52 & 1.69 & 1.63 & 0.79 \\
\hline Collection Date: & $05 / 03 / 2007$ & $05 / 03 / 2007$ & $05 / 03 / 2007$ & $05 / 03 / 2007$ \\
\hline Extraction Date: & $01 / 28 / 2008$ & $01 / 28 / 2008$ & $01 / 28 / 2008$ & $01 / 28 / 2008$ \\
\hline Analysis Date: & $02 / 16 / 2008$ & $02 / 16 / 2008$ & $02 / 17 / 2008$ & $02 / 17 / 2008$ \\
\hline Units (wet wt): & ng/g_WET & ng/g_WET & ng/g_WET & ng/g_WET \\
\hline $\mathrm{Cl} 2(8)$ & $0.05 \mathrm{U}$ & $0.05 \mathrm{U}$ & $0.01 \mathrm{~J}$ & $0.05 \mathrm{U}$ \\
\hline $\mathrm{Cl} 3(18)$ & $0.06 \mathrm{U}$ & $0.06 \mathrm{U}$ & $0.03 \mathrm{~J}$ & $0.06 \mathrm{U}$ \\
\hline $\mathrm{Cl} 3(28)$ & $0.02 \mathrm{~J}$ & $0.06 \mathrm{~J}$ & 0.07 & $0.03 \mathrm{~J}$ \\
\hline $\mathrm{Cl} 4(44)$ & $0.12 \mathrm{U}$ & $0.06 \mathrm{~J}$ & 0.09 & $0.12 \mathrm{U}$ \\
\hline $\mathrm{Cl} 4(52)$ & $0.03 \mathrm{~J}$ & 0.16 & 0.25 & 0.09 \\
\hline $\mathrm{Cl} 4(66)$ & $0.02 \mathrm{~J}$ & 0.09 & 0.12 & $0.04 \mathrm{~J}$ \\
\hline $\mathrm{Cl} 4(77)$ & $0.05 \mathrm{U}$ & $0.05 \mathrm{U}$ & $0.05 \mathrm{U}$ & $0.05 \mathrm{U}$ \\
\hline $\mathrm{Cl5}(101)$ & 0.12 & 0.44 & 0.72 & 0.18 \\
\hline $\mathrm{Cl} 15(105)$ & $0.05 \mathrm{U}$ & 0.16 & 0.21 & 0.08 \\
\hline $\mathrm{Cl5}(118)$ & 0.13 & 0.53 & 0.77 & 0.32 \\
\hline $\mathrm{Cl} 5(126)$ & $0.07 \mathrm{U}$ & $0.07 \mathrm{U}$ & $0.07 \mathrm{U}$ & $0.07 \mathrm{U}$ \\
\hline $\mathrm{Cl6}(128)$ & $0.04 \mathrm{~J}$ & 0.15 & 0.16 & 0.12 \\
\hline Cl6(138) & 0.27 & 0.88 & 1.13 & 0.67 \\
\hline Cl6(153) & 0.56 & 1.72 & 2.33 & 1.16 \\
\hline $\mathrm{Cl} 7(170)$ & 0.09 & 0.20 & 0.26 & 0.12 \\
\hline $\mathrm{Cl} 7(187)$ & 0.27 & 0.72 & 0.89 & 0.38 \\
\hline Cl7(188) & $0.02 \mathrm{U}$ & $0.02 \mathrm{U}$ & $0.02 \mathrm{U}$ & $0.02 \mathrm{U}$ \\
\hline $\mathrm{Cl}(195)$ & $0.05 \mathrm{U}$ & $0.05 \mathrm{U}$ & $0.05 \mathrm{U}$ & $0.05 \mathrm{U}$ \\
\hline $\mathrm{Cl} 8(200)$ & $0.04 \mathrm{U}$ & $0.04 \mathrm{U}$ & $0.04 \mathrm{U}$ & $0.04 \mathrm{U}$ \\
\hline $\mathrm{Cl9}(206)$ & $0.04 \mathrm{~J}$ & 0.09 & 0.13 & 0.09 \\
\hline Cl10(209) & $0.04 \mathrm{~J}$ & $0.03 \mathrm{~J}$ & $0.05 \mathrm{~J}$ & $0.03 \mathrm{~J}$ \\
\hline Total PCBs & 4.28 & 11.26 & 14.90 & 7.54 \\
\hline LOC 1 & $0.08 \mathrm{U}$ & $0.08 \mathrm{U}$ & $0.08 \mathrm{U}$ & $0.08 \mathrm{U}$ \\
\hline LOC 2 & $0.52 \mathrm{U}$ & $0.52 \mathrm{U}$ & $0.01 \mathrm{~J}$ & $0.52 \mathrm{U}$ \\
\hline LOC 3 & $0.02 \mathrm{~J}$ & $0.11 \mathrm{~J}$ & $0.19 \mathrm{~J}$ & $0.06 \mathrm{~J}$ \\
\hline LOC 4 & $0.09 \mathrm{~J}$ & $0.71 \mathrm{~J}$ & $1.07 \mathrm{~J}$ & $0.30 \mathrm{~J}$ \\
\hline LOC 5 & $0.48 \mathrm{~J}$ & 2.56 & 3.98 & $1.07 \mathrm{~J}$ \\
\hline LOC 6 & $1.26 \mathrm{~J}$ & 4.61 & 6.57 & 2.63 \\
\hline LOC 7 & $0.91 \mathrm{~J}$ & 2.52 & 3.22 & 1.51 \\
\hline LOC 8 & $0.51 \mathrm{U}$ & $0.67 \mathrm{~J}$ & 0.96 & $0.47 \mathrm{~J}$ \\
\hline LOC 9 & $0.04 \mathrm{~J}$ & $0.13 \mathrm{~J}$ & 0.22 & $0.13 \mathrm{~J}$ \\
\hline Total PCBs & 3.91 & 11.91 & 16.3 & 6.77 \\
\hline $\mathrm{Cl} 3(34)$ & 78 & 67 & 71 & 78 \\
\hline Cl6(152) & 89 & 73 & 73 & 84 \\
\hline
\end{tabular}


1529 West Sequim Bay Road

Sequim, Washington 98382-9099

360/681-4564
SINCLAIR AND DYES INLET

2007 PSAMP Trawl Biota

PCBs in Whole Organisms

\begin{tabular}{|c|c|c|c|c|c|c|}
\hline Sample Name: & HC-RF-1 & HC-RF-2 & HC-RF-3 & EB-ES-1_2 & EB-ES-3 & EB-RF-1 \\
\hline Station: & Hood Canal & Hood Canal & Hood Canal & Elliot Bay & Elliot Bay & Elliot Bay \\
\hline Organism ID: & $\mathrm{RF}$ & $\mathrm{RF}$ & $\mathrm{RF}$ & ES & ES & RF \\
\hline Batch ID: & 08-0015 & $08-0015$ & 08-0015 & $08-0015$ & $08-0015$ & $08-0015$ \\
\hline Sample Weight (g): & 30.98 & 30.58 & 30.38 & 21 & 30.33 & 30.99 \\
\hline \%Moisture & 74.37 & 72.96 & 72.39 & 78.56 & 80.28 & 73.57 \\
\hline \%Lipids & 7.66 & 12.00 & 12.70 & 0.69 & 0.81 & 10.60 \\
\hline Collection Date: & $05 / 03 / 2007$ & $05 / 03 / 2007$ & $05 / 03 / 2007$ & $05 / 16 / 2007$ & $05 / 16 / 2007$ & $05 / 16 / 2007$ \\
\hline Extraction Date: & $01 / 28 / 2008$ & 01/28/2008 & 01/28/2008 & 01/28/2008 & 01/28/2008 & 01/28/2008 \\
\hline Analysis Date: & 02/17/2008 & 02/17/2008 & 02/17/2008 & 02/17/2008 & $02 / 17 / 2008$ & $02 / 17 / 2008$ \\
\hline Units (wet wt): & ng/g_WET & ng/g_WET & ng/g_WET & ng/g_WET & ng/g_WET & ng/g_WET \\
\hline $\mathrm{Cl} 2(8)$ & $0.01 \mathrm{~J}$ & $0.02 \mathrm{~J}$ & $0.03 \mathrm{~J}$ & $0.05 \mathrm{U}$ & $0.05 \mathrm{U}$ & $0.04 \mathrm{~J}$ \\
\hline $\mathrm{Cl} 3(18)$ & $0.06 \mathrm{U}$ & $0.06 \mathrm{U}$ & $0.06 \mathrm{U}$ & $0.06 \mathrm{U}$ & 0.09 & $0.06 \mathrm{U}$ \\
\hline $\mathrm{Cl} 3(28)$ & 0.19 & 0.18 & 0.27 & $0.07 \mathrm{~J}$ & 0.20 & 3.74 \\
\hline $\mathrm{Cl} 4(44)$ & $0.12 \mathrm{U}$ & $0.12 \mathrm{U}$ & $0.12 \mathrm{U}$ & $0.08 \mathrm{~J}$ & 0.37 & $0.12 \mathrm{U}$ \\
\hline $\mathrm{Cl} 4(52)$ & $0.04 \mathrm{~J}$ & $0.12 \mathrm{U}$ & $0.12 \mathrm{U}$ & 0.20 & 1.26 & 0.27 \\
\hline $\mathrm{Cl} 4(66)$ & 0.48 & 0.38 & 1.23 & 0.15 & 0.79 & $21.14 \mathrm{D}$ \\
\hline $\mathrm{Cl}(77)$ & $0.05 \mathrm{U}$ & $0.05 \mathrm{U}$ & $0.05 \mathrm{U}$ & $0.05 \mathrm{U}$ & $0.03 \mathrm{~J}$ & $0.05 \mathrm{U}$ \\
\hline $\mathrm{Cl5}(101)$ & $0.03 \mathrm{~J}$ & $0.05 \mathrm{U}$ & $0.05 \mathrm{~J}$ & 0.69 & 7.56 & 1.11 \\
\hline $\mathrm{Cl} 5(105)$ & 1.06 & 0.76 & 2.82 & 0.26 & 2.03 & $62.2 \mathrm{D}$ \\
\hline $\mathrm{Cl5}(118)$ & 2.82 & 1.87 & 7.36 & 0.92 & 6.70 & 186.45 D \\
\hline $\mathrm{Cl} 5(126)$ & $0.07 \mathrm{U}$ & $0.07 \mathrm{U}$ & $0.07 \mathrm{U}$ & $0.07 \mathrm{U}$ & $0.07 \mathrm{U}$ & $0.07 \mathrm{U}$ \\
\hline $\mathrm{Cl}(128)$ & 1.45 & 1.04 & 3.89 & 0.28 & 2.76 & $85.04 \mathrm{D}$ \\
\hline Cl6(138) & 6.68 & 3.65 & 13.34 & 1.55 & 12.04 & $412.93 \mathrm{D}$ \\
\hline $\mathrm{Cl6}(153)$ & 10.3 & 5.65 & $27.65 \mathrm{D}$ & 2.97 & $29.96 \mathrm{D}$ & $604.75 \mathrm{D}$ \\
\hline $\mathrm{Cl}(170)$ & 1.59 & 0.81 & 4.68 & 0.38 & 4.91 & $128.18 \mathrm{D}$ \\
\hline $\mathrm{Cl}(187)$ & 0.06 & 0.07 & 0.14 & 1.02 & 10.53 & 3.24 \\
\hline $\mathrm{Cl}(188)$ & $0.02 \mathrm{U}$ & $0.02 \mathrm{U}$ & $0.02 \mathrm{U}$ & $0.02 \mathrm{U}$ & $0.04 \mathrm{~J}$ & 0.10 \\
\hline $\mathrm{Cl} 8(195)$ & 0.23 & 0.15 & 1.01 & $0.05 \mathrm{U}$ & 0.92 & $23.1 \mathrm{D}$ \\
\hline $\mathrm{Cl}(200)$ & $0.04 \mathrm{U}$ & $0.04 \mathrm{U}$ & $0.04 \mathrm{U}$ & $0.04 \mathrm{U}$ & 0.36 & $0.04 \mathrm{U}$ \\
\hline $\mathrm{Cl} 9(206)$ & 0.63 & 0.39 & 1.96 & 0.18 & 2.34 & $29.33 \mathrm{D}$ \\
\hline Cl10(209) & 0.29 & 0.19 & 0.80 & $0.05 \mathrm{~J}$ & 1.02 & 8.59 \\
\hline Total PCBs & 52.44 & 31.38 & 131.42 & 18.28 & 168.06 & 3141.10 \\
\hline LOC 1 & $0.08 \mathrm{U}$ & $0.08 \mathrm{U}$ & $0.08 \mathrm{U}$ & $0.08 \mathrm{U}$ & $0.08 \mathrm{U}$ & $0.08 \mathrm{U}$ \\
\hline LOC 2 & $0.01 \mathrm{~J}$ & $0.02 \mathrm{~J}$ & $0.03 \mathrm{~J}$ & $0.52 \mathrm{U}$ & $0.52 \mathrm{U}$ & $0.04 \mathrm{~J}$ \\
\hline LOC 3 & $0.22 \mathrm{~J}$ & $0.21 \mathrm{~J}$ & $0.31 \mathrm{~J}$ & $0.11 \mathrm{~J}$ & $0.45 \mathrm{~J}$ & 3.96 \\
\hline LOC 4 & $1.49 \mathrm{~J}$ & $1.10 \mathrm{~J}$ & 3.77 & $1.09 \mathrm{~J}$ & 6.96 & 62.8 \\
\hline LOC 5 & 6.43 & 5.06 & 17.33 & 4.95 & 41.16 & 475.97 \\
\hline LOC 6 & 20.59 & 11.86 & 50.62 & 8.87 & 90.81 & 1261.94 \\
\hline LOC 7 & 8.43 & 4.35 & 23.73 & 4.48 & 52.38 & 707.03 \\
\hline LOC 8 & 2.31 & 1.23 & 8.03 & $0.97 \mathrm{~J}$ & 14.61 & 197.02 \\
\hline LOC 9 & 0.72 & 0.44 & 2.20 & $0.18 \mathrm{~J}$ & 3.35 & 32.82 \\
\hline Total PCBs & 40.28 & 24.35 & 106.1 & 21.25 & 210.32 & 2741.66 \\
\hline $\mathrm{Cl} 3(34)$ & 70 & 78 & 78 & 76 & 80 & 75 \\
\hline Cl6(152) & 75 & 77 & 79 & 78 & 83 & 76 \\
\hline
\end{tabular}




\begin{tabular}{|c|c|c|c|c|c|}
\hline Sample Name: & EB-RF-2 & EB-RF-3 & EH-ES-2 & EH-ES-3 & EH-ES-4 \\
\hline Station: & Elliot Bay & Elliot Bay & Eagle Harbor & Eagle Harbor & Eagle Harbor \\
\hline Organism ID: & $\mathrm{RF}$ & RF & ES & ES & ES \\
\hline Batch ID: & 08-0015 & 08-0015 & 08-0015 & 08-0015 & 08-0015 \\
\hline Sample Weight (g): & 30.03 & 30.65 & 31.54 & 20.71 & 30.45 \\
\hline \%Moisture & 72.77 & 72.89 & 82.65 & 76.62 & 78.31 \\
\hline \%Lipids & 12.10 & 9.17 & 0.31 & 0.79 & 1.31 \\
\hline Collection Date: & $05 / 16 / 2007$ & $05 / 16 / 2007$ & $05 / 19 / 2007$ & $05 / 19 / 2007$ & $05 / 19 / 2007$ \\
\hline Extraction Date: & $01 / 28 / 2008$ & 01/28/2008 & $01 / 28 / 2008$ & 01/28/2008 & 01/28/2008 \\
\hline Analysis Date: & $02 / 17 / 2008$ & $02 / 28 / 2008$ & $02 / 17 / 2008$ & $02 / 17 / 2008$ & $02 / 17 / 2008$ \\
\hline Units (wet wt): & ng/g_WET & ng/g_WET & ng/g_WET & ng/g_WET & ng/g_WET \\
\hline $\mathrm{Cl} 2(8)$ & $0.04 \mathrm{~J}$ & 0.11 & $0.05 \mathrm{U}$ & $0.05 \mathrm{U}$ & $0.05 \mathrm{U}$ \\
\hline $\mathrm{Cl} 3(18)$ & $0.06 \mathrm{U}$ & $0.06 \mathrm{U}$ & $0.06 \mathrm{U}$ & $0.06 \mathrm{U}$ & $0.06 \mathrm{U}$ \\
\hline $\mathrm{Cl} 3(28)$ & 1.65 & 4.29 & 0.09 & 0.21 & 0.12 \\
\hline Cl4(44) & $0.12 \mathrm{U}$ & $0.12 \mathrm{U}$ & 0.09 & 0.51 & 0.18 \\
\hline $\mathrm{Cl} 4(52)$ & 0.23 & 0.70 & 0.52 & 2.27 & 0.97 \\
\hline $\mathrm{Cl} 4(66)$ & 4.94 & $6.48 \mathrm{D}$ & 0.31 & 1.32 & 0.51 \\
\hline $\mathrm{Cl} 4(77)$ & $0.05 \mathrm{U}$ & $0.05 \mathrm{U}$ & $0.05 \mathrm{U}$ & $0.02 \mathrm{~J}$ & $0.01 \mathrm{~J}$ \\
\hline $\mathrm{Cl} 5(101)$ & 0.76 & 2.64 & 2.43 & 7.85 & 4.14 \\
\hline $\mathrm{Cl5}(105)$ & 8.58 & $11.88 \mathrm{D}$ & 0.79 & 2.54 & 1.58 \\
\hline $\mathrm{Cl} 5(118)$ & $21.6 \mathrm{D}$ & $36.87 \mathrm{D}$ & 2.94 & 9.46 & 5.12 \\
\hline $\mathrm{Cl} 5(126)$ & $0.07 \mathrm{U}$ & $0.07 \mathrm{U}$ & $0.07 \mathrm{U}$ & $0.07 \mathrm{U}$ & $0.07 \mathrm{U}$ \\
\hline $\mathrm{Cl} 6(128)$ & 7.74 & $13.34 \mathrm{D}$ & 1.01 & 2.62 & 1.46 \\
\hline $\mathrm{Cl6}(138)$ & $43.35 \mathrm{D}$ & $78.74 \mathrm{D}$ & 4.85 & 9.71 & 5.48 \\
\hline $\mathrm{Cl6}(153)$ & $67.83 \mathrm{D}$ & $146.65 \mathrm{ED}$ & 9.52 & 19.48 & 9.71 \\
\hline $\mathrm{Cl}(170)$ & 8.94 & $27.46 \mathrm{D}$ & 1.57 & 2.92 & 1.13 \\
\hline $\mathrm{Cl}(187)$ & 1.89 & 3.86 & 4.92 & 8.30 & 3.01 \\
\hline $\mathrm{Cl} 7(188)$ & $0.02 \mathrm{~J}$ & 0.12 & $0.04 \mathrm{~J}$ & $0.05 \mathrm{~J}$ & $0.03 \mathrm{~J}$ \\
\hline $\mathrm{Cl}(195)$ & 1.14 & 8.06 & 0.41 & 0.72 & 0.18 \\
\hline $\mathrm{Cl}(200)$ & $0.04 \mathrm{U}$ & $0.04 \mathrm{U}$ & 0.14 & 0.29 & 0.08 \\
\hline $\mathrm{Cl}(206)$ & 2.09 & 9.29 & 1.77 & 2.91 & 1.34 \\
\hline Cl10(209) & 0.95 & 3.50 & 0.78 & 1.29 & 0.96 \\
\hline Total PCBs & 344.18 & 708.66 & 64.82 & 145.30 & 72.38 \\
\hline LOC 1 & $0.08 \mathrm{U}$ & $0.08 \mathrm{U}$ & $0.08 \mathrm{U}$ & $0.08 \mathrm{U}$ & $0.08 \mathrm{U}$ \\
\hline LOC 2 & $0.04 \mathrm{~J}$ & 0.11 & $0.52 \mathrm{U}$ & $0.52 \mathrm{U}$ & $0.52 \mathrm{U}$ \\
\hline LOC 3 & 2.15 & 6.57 & $0.14 \mathrm{~J}$ & $0.45 \mathrm{~J}$ & $0.17 \mathrm{~J}$ \\
\hline LOC 4 & 14.74 & 39.13 & 2.66 & 10.33 & 3.94 \\
\hline LOC 5 & 65.12 & 116.03 & 14.75 & 49.41 & 24.09 \\
\hline LOC 6 & 144.2 & 307.12 & 28.02 & 61.42 & 30.17 \\
\hline LOC 7 & 63.91 & 173.16 & 19.34 & 36.17 & 12.88 \\
\hline LOC 8 & 12.0 & 50.77 & 7.62 & 12.7 & 4.24 \\
\hline LOC 9 & 2.41 & 11.03 & 2.53 & 4.12 & 2.01 \\
\hline Total PCBs & 304.65 & 704 & 75.66 & 175.2 & 78.1 \\
\hline $\mathrm{Cl} 3(34)$ & 79 & 79 & 78 & 54 & 75 \\
\hline $\mathrm{Cl6}(152)$ & 78 & 66 & 76 & 75 & 75 \\
\hline
\end{tabular}




\begin{tabular}{|c|c|c|c|c|c|}
\hline Sample Name: & EH-RF-1 & EH-RF-2 & EH-RF-3 & SI-RF-1 & SI-RF-2 \\
\hline Station: & Eagle Harbor & Eagle Harbor & Eagle Harbor & Sinclair Inlet & Sinclair Inlet \\
\hline Organism ID: & $\mathrm{RF}$ & $\mathrm{RF}$ & $\mathrm{RF}$ & $\mathrm{RF}$ & $\mathrm{RF}$ \\
\hline Batch ID: & $08-0016$ & 08-0016 & 08-0016 & 08-0016 & 08-0016 \\
\hline Sample Weight (g): & 30.03 & 30.44 & 30.67 & 30.79 & 31.19 \\
\hline \%Moisture & 73.50 & 76.61 & 68.32 & 67.33 & 73.39 \\
\hline \%Lipids & 12.30 & 10.00 & 13.50 & 13.40 & 10.20 \\
\hline Collection Date: & $05 / 19 / 2007$ & $05 / 19 / 2007$ & $05 / 19 / 2007$ & $05 / 17 / 2007$ & $05 / 17 / 2007$ \\
\hline Extraction Date: & $01 / 28 / 2008$ & 01/28/2008 & $01 / 28 / 2008$ & $01 / 28 / 2008$ & 01/28/2008 \\
\hline Analysis Date: & 03/14/2008 & $02 / 27 / 2008$ & 03/15/2008 & 03/14/2008 & 03/14/2008 \\
\hline Units (wet wt): & ng/g_WET & ng/g_WET & ng/g_WET & ng/g_WET & ng/g_WET \\
\hline $\mathrm{Cl} 2(8)$ & $0.05 \mathrm{UT}$ & $0.02 \mathrm{~J}$ & 0.05 UTME & $0.06 \mathrm{JT}$ & $0.05 \mathrm{UT}$ \\
\hline $\mathrm{Cl} 3(18)$ & $0.06 \mathrm{UT}$ & $0.06 \mathrm{U}$ & 0.06 UTME & $0.06 \mathrm{UT}$ & $0.06 \mathrm{UT}$ \\
\hline $\mathrm{Cl} 3(28)$ & $0.44 \mathrm{~T}$ & 0.62 & $1.72 \mathrm{TME}$ & $1.60 \mathrm{~T}$ & $0.61 \mathrm{~T}$ \\
\hline $\mathrm{Cl} 4(44)$ & $0.12 \mathrm{UT}$ & $0.12 \mathrm{U}$ & 0.12 UTME & $0.12 \mathrm{UT}$ & $0.12 \mathrm{UT}$ \\
\hline $\mathrm{Cl} 4(52)$ & $0.12 \mathrm{UT}$ & 0.20 & 0.12 UTME & $0.29 \mathrm{~T}$ & $0.12 \mathrm{UT}$ \\
\hline $\mathrm{Cl} 4(66)$ & $1.01 \mathrm{~T}$ & 2.63 & 4.13 TME & $5.71 \mathrm{~T}$ & $2.31 \mathrm{~T}$ \\
\hline $\mathrm{Cl} 4(77)$ & $0.05 \mathrm{UT}$ & $0.05 \mathrm{U}$ & 0.05 UTME & $0.05 \mathrm{UT}$ & $0.05 \mathrm{UT}$ \\
\hline $\mathrm{Cl} 5(101)$ & $0.29 \mathrm{~T}$ & 0.57 & 0.6 TME & $1.30 \mathrm{~T}$ & $0.28 \mathrm{~T}$ \\
\hline $\mathrm{Cl5}(105)$ & $1.06 \mathrm{~T}$ & 3.11 & 11.72 TME & $11.58 \mathrm{~T}$ & $3.39 \mathrm{~T}$ \\
\hline $\mathrm{Cl} 5(118)$ & $4.94 \mathrm{~T}$ & 13.46 & $11.32 \mathrm{TME}$ & $21.39 \mathrm{DT}$ & $12.87 \mathrm{~T}$ \\
\hline $\mathrm{Cl} 5(126)$ & $0.07 \mathrm{UT}$ & $0.07 \mathrm{U}$ & 0.07 UTME & $0.07 \mathrm{UT}$ & $0.07 \mathrm{UT}$ \\
\hline $\mathrm{Cl6}(128)$ & $0.10 \mathrm{~T}$ & 3.03 & 7.1 TME & $13.07 \mathrm{~T}$ & $3.72 \mathrm{~T}$ \\
\hline Cl6(138) & $11.32 \mathrm{~T}$ & $7.96 \mathrm{D}$ & 19.44 TME & $38.6 \mathrm{DT}$ & $10.37 \mathrm{DT}$ \\
\hline Cl6(153) & $22.61 \mathrm{~T}$ & $17.57 \mathrm{D}$ & 56.99 TME & $82.8 \mathrm{DT}$ & 19.71 DT \\
\hline $\mathrm{Cl}(170)$ & $1.94 \mathrm{~T}$ & 3.22 & $2.96 \mathrm{TME}$ & $14.32 \mathrm{~T}$ & $4.26 \mathrm{~T}$ \\
\hline $\mathrm{Cl} 7(187)$ & $0.30 \mathrm{~T}$ & 0.65 & $0.98 \mathrm{TME}$ & $1.77 \mathrm{~T}$ & $0.32 \mathrm{~T}$ \\
\hline $\mathrm{Cl} 7(188)$ & $0.04 \mathrm{JT}$ & 0.07 & 0.02 UTME & $0.12 \mathrm{~T}$ & $0.09 \mathrm{~T}$ \\
\hline $\mathrm{Cl}(195)$ & $0.41 \mathrm{~T}$ & 0.63 & 0.05 UTME & $3.15 \mathrm{~T}$ & $1.02 \mathrm{~T}$ \\
\hline $\mathrm{Cl}(200)$ & $0.04 \mathrm{UT}$ & $0.04 \mathrm{U}$ & 0.04 UTME & 0.04 UT & $0.04 \mathrm{UT}$ \\
\hline $\mathrm{Cl} 9(206)$ & $0.92 \mathrm{~T}$ & 1.69 & $1.58 \mathrm{TME}$ & $7.34 \mathrm{~T}$ & $3.98 \mathrm{~T}$ \\
\hline Cl10(209) & $0.48 \mathrm{~T}$ & 1.05 & $0.98 \mathrm{TME}$ & $3.51 \mathrm{~T}$ & $2.97 \mathrm{~T}$ \\
\hline Total PCBs & 92.74 & 113.64 & 240.20 & 413.90 & 132.82 \\
\hline LOC 1 & $0.08 \mathrm{UT}$ & $0.08 \mathrm{U}$ & 0.08 UTME & $0.08 \mathrm{UT}$ & $0.08 \mathrm{UT}$ \\
\hline LOC 2 & $0.52 \mathrm{UT}$ & $0.02 \mathrm{~J}$ & 0.52 UTME & $0.06 \mathrm{JT}$ & $0.52 \mathrm{UT}$ \\
\hline LOC 3 & $0.44 \mathrm{JT}$ & $0.69 \mathrm{~J}$ & 1.72 TME & $2.23 \mathrm{~T}$ & $0.61 \mathrm{JT}$ \\
\hline LOC 4 & $4.56 \mathrm{~T}$ & 8.05 & 12.29 TME & $18.11 \mathrm{~T}$ & $7.97 \mathrm{~T}$ \\
\hline LOC 5 & $16.22 \mathrm{~T}$ & 39.13 & 47.19 TME & $74.92 \mathrm{~T}$ & $38.35 \mathrm{~T}$ \\
\hline LOC 6 & $37.89 \mathrm{~T}$ & 38.55 & 101.42 TME & $165.32 \mathrm{~T}$ & $44.16 \mathrm{~T}$ \\
\hline LOC 7 & $14.79 \mathrm{~T}$ & 22.24 & $22.56 \mathrm{TME}$ & $70.13 \mathrm{~T}$ & $29.71 \mathrm{~T}$ \\
\hline LOC 8 & $4.01 \mathrm{~T}$ & 7.15 & 0.51 UTME & $30.43 \mathrm{~T}$ & $10.6 \mathrm{~T}$ \\
\hline LOC 9 & $1.05 \mathrm{~T}$ & 2.13 & $1.58 \mathrm{TME}$ & $8.75 \mathrm{~T}$ & $4.66 \mathrm{~T}$ \\
\hline Total PCBs & 79.56 & 118.04 & 187.87 & 370.03 & 136.66 \\
\hline $\mathrm{Cl} 3(34)$ & 88 & 86 & $117 \mathrm{ME}$ & 66 & 82 \\
\hline Cl6(152) & 66 & 64 & 147 \#ME & 67 & 65 \\
\hline
\end{tabular}


1529 West Sequim Bay Road

Sequim, Washington 98382-9099

360/681-4564

\section{SINCLAIR AND DYES INLET 2007 PSAMP Trawl Biota PCBs in Whole Organisms}

\begin{tabular}{|c|c|c|c|c|}
\hline Sample Name: & SI-SC-COMP & PG-ES-1 & PG-ES-2 & PG-ES-3 \\
\hline Station: & "Sinclair Inlet & Port Gardner & Port Gardner & Port Gardner \\
\hline Organism ID: & $\mathrm{SC}$ & ES & ES & ES \\
\hline Batch ID: & $08-0016$ & 08-0016 & 08-0016 & 08-0016 \\
\hline Sample Weight (g): & 11.12 & 30.75 & 30.34 & 31.51 \\
\hline \%Moisture & 90.48 & 80.77 & 79.18 & 80.05 \\
\hline \%Lipids & 0.21 & 0.81 & 0.48 & 0.73 \\
\hline Collection Date: & $05 / 17 / 2007$ & $05 / 29 / 2007$ & $05 / 29 / 2007$ & $05 / 29 / 2007$ \\
\hline Extraction Date: & 01/28/2008 & 01/28/2008 & 01/28/2008 & 01/28/2008 \\
\hline Analysis Date: & 02/28/2008 & $02 / 28 / 2008$ & $02 / 28 / 2008$ & 02/28/2008 \\
\hline Units (wet wt): & ng/g_WET & ng/g_WET & ng/g_WET & ng/g_WET \\
\hline $\mathrm{Cl} 2(8)$ & $0.05 \mathrm{U}$ & $0.05 \mathrm{U}$ & $0.05 \mathrm{U}$ & $0.05 \mathrm{U}$ \\
\hline $\mathrm{Cl} 3(18)$ & $0.06 \mathrm{U}$ & $0.06 \mathrm{U}$ & $0.06 \mathrm{U}$ & $0.06 \mathrm{U}$ \\
\hline $\mathrm{Cl} 3(28)$ & $0.06 \mathrm{U}$ & $0.04 \mathrm{~J}$ & $0.06 \mathrm{U}$ & $0.05 \mathrm{~J}$ \\
\hline $\mathrm{Cl} 4(44)$ & $0.12 \mathrm{U}$ & 0.13 & $0.12 \mathrm{U}$ & 0.08 \\
\hline $\mathrm{Cl} 4(52)$ & $0.12 \mathrm{U}$ & 0.51 & 0.14 & 0.22 \\
\hline $\mathrm{Cl} 4(66)$ & $0.07 \mathrm{U}$ & 0.22 & 0.10 & 0.25 \\
\hline $\mathrm{Cl} 4(77)$ & $0.05 \mathrm{U}$ & $0.02 \mathrm{~J}$ & $0.05 \mathrm{U}$ & $0.04 \mathrm{~J}$ \\
\hline $\mathrm{Cl} 5(101)$ & 0.33 & 1.93 & 0.92 & 1.04 \\
\hline $\mathrm{Cl5}(105)$ & $0.13 \mathrm{~J}$ & 0.44 & 0.20 & 0.28 \\
\hline $\mathrm{Cl5}(118)$ & 0.33 & 1.89 & 0.76 & 1.01 \\
\hline $\mathrm{Cl} 5(126)$ & $0.07 \mathrm{U}$ & $0.07 \mathrm{U}$ & $0.07 \mathrm{U}$ & $0.07 \mathrm{U}$ \\
\hline Cl6(128) & 0.20 & 0.30 & 0.22 & 0.26 \\
\hline Cl6(138) & 0.60 & 2.01 & 1.65 & 1.49 \\
\hline Cl6(153) & 1.11 & 4.84 & 3.83 & 3.47 \\
\hline $\mathrm{Cl} 7(170)$ & $0.11 \mathrm{~J}$ & 0.55 & 0.55 & 0.55 \\
\hline Cl7(187) & 0.53 & 1.61 & 1.52 & 1.26 \\
\hline $\mathrm{Cl}(188)$ & $0.02 \mathrm{U}$ & $0.02 \mathrm{U}$ & $0.01 \mathrm{~J}$ & $0.02 \mathrm{U}$ \\
\hline Cl8(195) & $0.05 \mathrm{U}$ & 0.11 & 0.10 & 0.09 \\
\hline $\mathrm{Cl}(200)$ & $0.04 \mathrm{U}$ & $0.04 \mathrm{U}$ & $0.03 \mathrm{~J}$ & $0.04 \mathrm{U}$ \\
\hline $\mathrm{Cl9}(206)$ & $0.10 \mathrm{~J}$ & 0.13 & 0.08 & 0.12 \\
\hline Cl10(209) & $0.05 \mathrm{~J}$ & $0.04 \mathrm{~J}$ & $0.03 \mathrm{~J}$ & $0.03 \mathrm{~J}$ \\
\hline Total PCBs & 8.40 & 30.02 & 21.10 & 20.96 \\
\hline LOC 1 & $0.08 \mathrm{U}$ & $0.08 \mathrm{U}$ & $0.08 \mathrm{U}$ & $0.08 \mathrm{U}$ \\
\hline LOC 2 & $0.52 \mathrm{U}$ & $0.52 \mathrm{U}$ & $0.52 \mathrm{U}$ & $0.52 \mathrm{U}$ \\
\hline LOC 3 & $0.75 \mathrm{U}$ & $0.07 \mathrm{~J}$ & $0.02 \mathrm{~J}$ & $0.11 \mathrm{~J}$ \\
\hline LOC 4 & $0.05 \mathrm{~J}$ & 2.16 & $0.63 \mathrm{~J}$ & $1.41 \mathrm{~J}$ \\
\hline LOC 5 & $1.93 \mathrm{~J}$ & 10.48 & 4.29 & 5.67 \\
\hline LOC 6 & $3.23 \mathrm{~J}$ & 13.5 & 10.59 & 9.73 \\
\hline LOC 7 & $1.72 \mathrm{~J}$ & 7.42 & 7.21 & 6.41 \\
\hline LOC 8 & $0.43 \mathrm{~J}$ & 1.55 & 1.49 & 1.48 \\
\hline LOC 9 & $0.10 \mathrm{~J}$ & $0.19 \mathrm{~J}$ & $0.13 \mathrm{~J}$ & $0.15 \mathrm{~J}$ \\
\hline Total PCBs & 8.81 & 35.97 & 24.96 & 25.56 \\
\hline $\mathrm{Cl} 3(34)$ & 69 & 72 & 67 & 70 \\
\hline $\mathrm{Cl6}(152)$ & 73 & 77 & 72 & 73 \\
\hline
\end{tabular}


BATTELLE MARINE SCIENCE LABORATORIES

1529 West Sequim Bay Road

Sequim, Washington 98382-9099

360/681-4564

SINCLAIR AND DYES INLET

2007 PSAMP Trawl Biota

PCBs in Whole Organisms

\begin{tabular}{|c|c|c|c|c|c|c|}
\hline Sample Name: & NIS-ES-1 & NIS-ES-2 & NIS-ES-3 & NIS-RF-1 & NIS-RF-2 & NIS-RF-3 \\
\hline Station: & Nisqually & Nisqually & Nisqually & Nisqually & Nisqually & Nisqually \\
\hline Organism ID: & ES & ES & ES & RF & RF & RF \\
\hline Batch ID: & 08-0016 & 08-0016 & $08-0016$ & 08-0016 & $08-0016$ & 08-0016 \\
\hline Sample Weight (g): & 30.59 & 30.71 & 30.24 & 31.44 & 30.88 & 30.69 \\
\hline \%Moisture & 79.33 & 78.48 & 78.39 & 68.79 & 67.47 & 70.65 \\
\hline \%Lipids & 1.09 & 1.71 & 1.57 & 13.40 & 13.45 & 16.62 \\
\hline Collection Date: & $05 / 30 / 2007$ & $05 / 30 / 2007$ & $05 / 30 / 2007$ & $05 / 30 / 2007$ & $05 / 30 / 2007$ & $05 / 30 / 2007$ \\
\hline Extraction Date: & $01 / 28 / 2008$ & $01 / 28 / 2008$ & $01 / 28 / 2008$ & $01 / 28 / 2008$ & $01 / 28 / 2008$ & $01 / 28 / 2008$ \\
\hline Analysis Date: & $02 / 28 / 2008$ & $02 / 28 / 2008$ & $02 / 28 / 2008$ & $03 / 14 / 2008$ & $03 / 14 / 2008$ & $03 / 14 / 2008$ \\
\hline Units (wet wt): & ng/g_WET & ng/g_WET & ng/g_WET & ng/g_WET & ng/g_WET & ng/g_WET \\
\hline $\mathrm{Cl} 2(8)$ & $0.05 \mathrm{U}$ & $0.05 \mathrm{U}$ & $0.05 \mathrm{U}$ & $0.05 \mathrm{UT}$ & $0.05 \mathrm{UT}$ & $0.05 \mathrm{UT}$ \\
\hline $\mathrm{Cl} 3(18)$ & $0.06 \mathrm{U}$ & $0.06 \mathrm{U}$ & $0.06 \mathrm{U}$ & $0.06 \mathrm{UT}$ & $0.06 \mathrm{UT}$ & $0.06 \mathrm{UT}$ \\
\hline $\mathrm{Cl} 3(28)$ & $0.06 \mathrm{U}$ & 0.08 & $0.05 \mathrm{~J}$ & $0.80 \mathrm{~T}$ & $0.50 \mathrm{~T}$ & $0.88 \mathrm{~T}$ \\
\hline $\mathrm{Cl} 4(44)$ & $0.12 \mathrm{U}$ & 0.18 & 0.07 & $0.12 \mathrm{UT}$ & $0.12 \mathrm{UT}$ & $0.12 \mathrm{UT}$ \\
\hline $\mathrm{Cl} 4(52)$ & 0.10 & 0.26 & 0.21 & $0.12 \mathrm{UT}$ & $0.12 \mathrm{UT}$ & $0.12 \mathrm{UT}$ \\
\hline $\mathrm{Cl} 4(66)$ & 0.08 & 0.18 & 0.14 & $5.67 \mathrm{~T}$ & $1.26 \mathrm{~T}$ & $1.92 \mathrm{~T}$ \\
\hline $\mathrm{Cl}(77)$ & $0.05 \mathrm{U}$ & $0.05 \mathrm{U}$ & $0.05 \mathrm{U}$ & $0.05 \mathrm{UT}$ & $0.05 \mathrm{UT}$ & $0.05 \mathrm{UT}$ \\
\hline $\mathrm{Cl5}(101)$ & 0.51 & 1.12 & 1.00 & $0.05 \mathrm{UT}$ & $0.05 \mathrm{UT}$ & $0.28 \mathrm{~T}$ \\
\hline $\mathrm{Cl} 5(105)$ & 0.12 & 0.24 & 0.25 & $9.20 \mathrm{~T}$ & $1.70 \mathrm{~T}$ & $2.40 \mathrm{~T}$ \\
\hline $\mathrm{Cl} 5(118)$ & 0.50 & 0.98 & 0.94 & 16.77 DT & $7.20 \mathrm{~T}$ & $9.37 \mathrm{~T}$ \\
\hline $\mathrm{Cl} 5(126)$ & $0.07 \mathrm{U}$ & $0.07 \mathrm{U}$ & $0.07 \mathrm{U}$ & $0.07 \mathrm{UT}$ & $0.07 \mathrm{UT}$ & $0.07 \mathrm{UT}$ \\
\hline $\mathrm{Cl6}(128)$ & 0.15 & 0.27 & 0.31 & $10.85 \mathrm{~T}$ & $1.75 \mathrm{~T}$ & $2.85 \mathrm{~T}$ \\
\hline Cl6(138) & 1.03 & 1.97 & 1.88 & $29.91 \mathrm{DT}$ & $15.7 \mathrm{~T}$ & $27.56 \mathrm{~T}$ \\
\hline Cl6(153) & 2.43 & 4.54 & 4.51 & $70.43 \mathrm{DT}$ & $24.97 \mathrm{~T}$ & 18.87 DT \\
\hline $\mathrm{Cl} 7(170)$ & 0.26 & 0.45 & 0.41 & $14.05 \mathrm{~T}$ & $2.62 \mathrm{~T}$ & $3.49 \mathrm{~T}$ \\
\hline $\mathrm{Cl}(187)$ & 1.39 & 2.16 & 2.10 & $0.23 \mathrm{~T}$ & $0.37 \mathrm{~T}$ & $0.46 \mathrm{~T}$ \\
\hline $\mathrm{Cl}(188)$ & $0.01 \mathrm{~J}$ & $0.01 \mathrm{~J}$ & $0.02 \mathrm{U}$ & $0.09 \mathrm{~T}$ & $0.06 \mathrm{~T}$ & $0.07 \mathrm{~T}$ \\
\hline $\mathrm{Cl} 8(195)$ & 0.09 & 0.13 & 0.12 & $3.65 \mathrm{~T}$ & $0.75 \mathrm{~T}$ & $0.93 \mathrm{~T}$ \\
\hline $\mathrm{Cl}(200)$ & $0.04 \mathrm{U}$ & $0.04 \mathrm{~J}$ & $0.04 \mathrm{~J}$ & $0.04 \mathrm{UT}$ & 0.04 UT & $0.04 \mathrm{UT}$ \\
\hline $\mathrm{Cl}(206)$ & 0.27 & 0.36 & 0.37 & $10.6 \mathrm{~T}$ & $2.69 \mathrm{~T}$ & $3.11 \mathrm{~T}$ \\
\hline Cl10(209) & 0.14 & 0.15 & 0.16 & $5.96 \mathrm{~T}$ & $1.51 \mathrm{~T}$ & $1.71 \mathrm{~T}$ \\
\hline Total PCBs & 15.06 & 26.70 & 25.62 & 357.54 & 123.28 & 148.82 \\
\hline LOC 1 & $0.08 \mathrm{U}$ & $0.08 \mathrm{U}$ & $0.08 \mathrm{U}$ & $0.08 \mathrm{UT}$ & $0.08 \mathrm{UT}$ & $0.08 \mathrm{UT}$ \\
\hline LOC 2 & $0.52 \mathrm{U}$ & $0.52 \mathrm{U}$ & $0.52 \mathrm{U}$ & $0.52 \mathrm{UT}$ & $0.52 \mathrm{UT}$ & $0.52 \mathrm{UT}$ \\
\hline LOC 3 & $0.75 \mathrm{U}$ & $0.08 \mathrm{~J}$ & $0.08 \mathrm{~J}$ & $0.80 \mathrm{JT}$ & $0.50 \mathrm{JT}$ & $0.88 \mathrm{JT}$ \\
\hline LOC 4 & $0.36 \mathrm{~J}$ & $1.16 \mathrm{~J}$ & $1.00 \mathrm{~J}$ & $17.69 \mathrm{~T}$ & $4.18 \mathrm{~T}$ & $6.39 \mathrm{~T}$ \\
\hline LOC 5 & 2.44 & 5.89 & 5.17 & $52.55 \mathrm{~T}$ & $20.12 \mathrm{~T}$ & $29.63 \mathrm{~T}$ \\
\hline LOC 6 & 6.14 & 12.2 & 11.82 & $134.88 \mathrm{~T}$ & $47.85 \mathrm{~T}$ & $57.90 \mathrm{~T}$ \\
\hline LOC 7 & 4.41 & 7.13 & 7.12 & $71.6 \mathrm{~T}$ & $18.2 \mathrm{~T}$ & $25.83 \mathrm{~T}$ \\
\hline LOC 8 & 1.80 & 2.63 & 2.50 & $33.55 \mathrm{~T}$ & $7.82 \mathrm{~T}$ & $10.32 \mathrm{~T}$ \\
\hline LOC 9 & 0.42 & 0.53 & 0.54 & $12.01 \mathrm{~T}$ & $3.18 \mathrm{~T}$ & $3.65 \mathrm{~T}$ \\
\hline Total PCBs & 16.92 & 30.22 & 28.83 & 323.68 & 102.45 & 135.2 \\
\hline $\mathrm{Cl} 3(34)$ & 66 & 70 & 67 & 77 & 65 & 84 \\
\hline Cl6(152) & 70 & 73 & 73 & 60 & 55 & 70 \\
\hline
\end{tabular}


BATTELLE MARINE SCIENCE LABORATORIES

1529 West Sequim Bay Road

Sequim, Washington 98382-9099

360/681-4564

\section{SINCLAIR AND DYES INLET \\ 2007 PSAMP Trawl Biota \\ PCBs in Whole Organisms}

\begin{tabular}{|c|c|c|c|}
\hline Sample Name: & CB-ES-1 & CB-ES-2 & CB-ES-3 \\
\hline Station: & Commencement Bay & Commencement Bay & Commencement Bay \\
\hline Organism ID: & ES & ES & ES \\
\hline Batch ID: & $08-0016$ & 08-0016 & 08-0016 \\
\hline Sample Weight (g): & 30.87 & 30.54 & 30.7 \\
\hline \%Moisture & 78.50 & 76.32 & 76.84 \\
\hline \%Lipids & 0.74 & 2.11 & 2.41 \\
\hline Collection Date: & $05 / 31 / 2007$ & $05 / 31 / 2007$ & $05 / 31 / 2007$ \\
\hline Extraction Date: & 01/28/2008 & $01 / 28 / 2008$ & 01/28/2008 \\
\hline Analysis Date: & 02/28/2008 & $02 / 28 / 2008$ & 02/28/2008 \\
\hline Units (wet wt): & ng/g_WET & ng/g_WET & ng/g_WET \\
\hline $\mathrm{Cl} 2(8)$ & $0.05 \mathrm{U}$ & $0.05 \mathrm{U}$ & $0.05 \mathrm{U}$ \\
\hline $\mathrm{Cl} 3(18)$ & $0.06 \mathrm{U}$ & $0.06 \mathrm{U}$ & $0.06 \mathrm{U}$ \\
\hline $\mathrm{Cl} 3(28)$ & 0.13 & 0.16 & 0.49 \\
\hline $\mathrm{Cl} 4(44)$ & $0.12 \mathrm{U}$ & 0.48 & 1.11 \\
\hline $\mathrm{Cl} 4(52)$ & 0.60 & 0.66 & 1.59 \\
\hline $\mathrm{Cl} 4(66)$ & 0.50 & 0.59 & 1.13 \\
\hline $\mathrm{Cl}(77)$ & $0.03 \mathrm{~J}$ & $0.05 \mathrm{U}$ & $0.05 \mathrm{U}$ \\
\hline $\mathrm{Cl5}(101)$ & 4.46 & 2.64 & 8.20 \\
\hline $\mathrm{Cl} 5(105)$ & 1.34 & 0.89 & 2.42 \\
\hline $\mathrm{Cl5}(118)$ & 3.86 & 2.40 & 6.88 \\
\hline $\mathrm{Cl} 5(126)$ & $0.07 \mathrm{U}$ & $0.07 \mathrm{U}$ & $0.07 \mathrm{U}$ \\
\hline $\mathrm{Cl6}(128)$ & 1.19 & 0.68 & 2.43 \\
\hline $\mathrm{Cl6}(138)$ & $6.24 \mathrm{D}$ & 3.56 & $11.71 \mathrm{D}$ \\
\hline $\mathrm{Cl} 6(153)$ & $13.06 \mathrm{D}$ & 7.20 & $27.78 \mathrm{D}$ \\
\hline $\mathrm{Cl}(170)$ & 2.93 & 0.98 & 3.03 \\
\hline $\mathrm{Cl}(187)$ & 6.83 & 3.10 & $12.94 \mathrm{D}$ \\
\hline $\mathrm{Cl}(188)$ & $0.02 \mathrm{~J}$ & $0.01 \mathrm{~J}$ & 0.13 \\
\hline $\mathrm{Cl}(195)$ & 0.56 & 0.20 & 0.78 \\
\hline $\mathrm{Cl}(200)$ & 0.18 & 0.08 & 0.49 \\
\hline $\mathrm{Cl9}(206)$ & 2.38 & 0.53 & 3.20 \\
\hline Cl10(209) & 0.47 & 0.18 & 1.19 \\
\hline Total PCBs & 90.16 & 49.14 & 171.46 \\
\hline LOC 1 & $0.08 \mathrm{U}$ & $0.08 \mathrm{U}$ & $0.08 \mathrm{U}$ \\
\hline LOC 2 & $0.52 \mathrm{U}$ & $0.52 \mathrm{U}$ & $0.52 \mathrm{U}$ \\
\hline LOC 3 & $0.13 \mathrm{~J}$ & $0.16 \mathrm{~J}$ & $0.49 \mathrm{~J}$ \\
\hline LOC 4 & 2.90 & 3.97 & 8.86 \\
\hline LOC 5 & 23.64 & 16.87 & 42.91 \\
\hline LOC 6 & 40.99 & 22.67 & 80.59 \\
\hline LOC 7 & 34.98 & 13.52 & 50.76 \\
\hline LOC 8 & 12.85 & 4.17 & 19.28 \\
\hline LOC 9 & 2.94 & 0.75 & 4.29 \\
\hline Total PCBs & 119.03 & 62.71 & 207.78 \\
\hline $\mathrm{Cl} 3(34)$ & 69 & 68 & 69 \\
\hline Cl6(152) & 76 & 69 & 75 \\
\hline
\end{tabular}


1529 West Sequim Bay Road

Sequim, Washington 98382-9099

360/681-4564

\section{SINCLAIR AND DYES INLET 2007 PSAMP Trawl Biota \\ PCBs in Whole Organisms}

\begin{tabular}{|c|c|c|c|}
\hline Sample Name: & CB-RF-1 & CB-RF-2 & CB-RF-3 \\
\hline Station: & Commencement Bay & Commencement Bay & Commencement Bay \\
\hline Organism ID: & RF & RF & RF \\
\hline Batch ID: & 08-0016 & 08-0016 & 08-0017 \\
\hline Sample Weight (g): & 30.09 & 31.65 & 29.73 \\
\hline \%Moisture & 69.83 & 71.46 & 69.42 \\
\hline \%Lipids & 16.10 & 12.40 & 15.52 \\
\hline Collection Date: & $05 / 31 / 2007$ & $05 / 31 / 2007$ & $05 / 31 / 2007$ \\
\hline Extraction Date: & $01 / 28 / 2008$ & $01 / 28 / 2008$ & 02/07/2008 \\
\hline Analysis Date: & $02 / 28 / 2008$ & 03/14/2008 & $02 / 29 / 2008$ \\
\hline Units (wet wt): & ng/g_WET & ng/g_WET & ng/g_WET \\
\hline $\mathrm{Cl} 2(8)$ & $0.05 \mathrm{U}$ & $0.05 \mathrm{UT}$ & $0.03 \mathrm{~J}$ \\
\hline $\mathrm{Cl} 3(18)$ & $0.06 \mathrm{U}$ & $0.06 \mathrm{UT}$ & $0.06 \mathrm{U}$ \\
\hline $\mathrm{Cl} 3(28)$ & 0.56 & $0.76 \mathrm{~T}$ & 0.77 \\
\hline $\mathrm{Cl} 4(44)$ & $0.12 \mathrm{U}$ & $0.12 \mathrm{UT}$ & $0.12 \mathrm{U}$ \\
\hline $\mathrm{Cl} 4(52)$ & $0.12 \mathrm{U}$ & $0.17 \mathrm{~T}$ & 0.10 \\
\hline $\mathrm{Cl} 4(66)$ & 1.50 & $3.18 \mathrm{~T}$ & 3.02 \\
\hline $\mathrm{Cl}(77)$ & $0.05 \mathrm{U}$ & $0.05 \mathrm{UT}$ & $0.05 \mathrm{U}$ \\
\hline $\mathrm{Cl5}(101)$ & 0.47 & $0.54 \mathrm{~T}$ & 0.20 \\
\hline $\mathrm{Cl5}(105)$ & 2.56 & $7.16 \mathrm{~T}$ & $4.19 \mathrm{D}$ \\
\hline $\mathrm{Cl5}(118)$ & 5.77 & $18.5 \mathrm{DT}$ & $10.04 \mathrm{D}$ \\
\hline $\mathrm{Cl} 5(126)$ & $0.07 \mathrm{U}$ & $0.07 \mathrm{UT}$ & $0.07 \mathrm{U}$ \\
\hline Cl6(128) & 2.27 & $8.81 \mathrm{~T}$ & $4.46 \mathrm{D}$ \\
\hline Cl6(138) & $10.2 \mathrm{D}$ & $43.00 \mathrm{DT}$ & $20.19 \mathrm{D}$ \\
\hline $\mathrm{Cl6}(153)$ & $19.09 \mathrm{D}$ & $83.12 \mathrm{DT}$ & $32.57 \mathrm{D}$ \\
\hline $\mathrm{Cl}(170)$ & 1.88 & $8.35 \mathrm{~T}$ & $4.81 \mathrm{D}$ \\
\hline $\mathrm{Cl}(187)$ & 0.37 & $0.48 \mathrm{~T}$ & 0.22 \\
\hline $\mathrm{Cl}(188)$ & $0.03 \mathrm{~J}$ & $0.05 \mathrm{JT}$ & $0.02 \mathrm{~J}$ \\
\hline Cl8(195) & 0.44 & $2.01 \mathrm{~T}$ & 1.38 \\
\hline $\mathrm{Cl}(200)$ & $0.04 \mathrm{U}$ & 0.04 UT & $0.04 \mathrm{U}$ \\
\hline $\mathrm{Cl9}(206)$ & 1.30 & $6.29 \mathrm{~T}$ & $3.77 \mathrm{D}$ \\
\hline Cl10(209) & 0.82 & $4.19 \mathrm{~T}$ & 2.43 \\
\hline Total PCBs & 95.54 & 374 & 177.08 \\
\hline LOC 1 & $0.08 \mathrm{U}$ & $0.08 \mathrm{UT}$ & $0.08 \mathrm{U}$ \\
\hline LOC 2 & $0.52 \mathrm{U}$ & $0.52 \mathrm{UT}$ & $0.03 \mathrm{~J}$ \\
\hline LOC 3 & $0.56 \mathrm{~J}$ & $0.76 \mathrm{JT}$ & $0.77 \mathrm{~J}$ \\
\hline LOC 4 & 4.69 & $9.96 \mathrm{~T}$ & 8.23 \\
\hline LOC 5 & 20.54 & $53.14 \mathrm{~T}$ & 30.2 \\
\hline LOC 6 & 38.43 & $151.45 \mathrm{~T}$ & 67.15 \\
\hline LOC 7 & 12.62 & $56.35 \mathrm{~T}$ & 27.21 \\
\hline LOC 8 & 4.35 & $17.31 \mathrm{~T}$ & 14.2 \\
\hline LOC 9 & 1.57 & $7.19 \mathrm{~T}$ & 4.22 \\
\hline Total PCBs & 83.36 & 296.76 & 152.09 \\
\hline $\mathrm{Cl} 3(34)$ & 52 & 73 & 79 \\
\hline Cl6(152) & 50 & 84 & 68 \\
\hline
\end{tabular}


BATTELLE MARINE SCIENCE LABORATORIES

1529 West Sequim Bay Road

Sequim, Washington 98382-9099

360/681-4564

\section{SINCLAIR AND DYES INLET 2007 PSAMP Trawl Biota PCBs in Whole Organisms}

\begin{tabular}{|c|c|c|c|}
\hline Sample Name: & DU-ES-1 & DU-ES-2 & DU-ES-3 \\
\hline Station: & Duwamish & Duwamish & Duwamish \\
\hline Organism ID: & ES & ES & ES \\
\hline Batch ID: & 08-0017 & $08-0017$ & 08-0017 \\
\hline Sample Weight (g): & 20.82 & 30.12 & 30.56 \\
\hline \%Moisture & 77.05 & 79.68 & 77.00 \\
\hline \%Lipids & 2.27 & 0.77 & 3.75 \\
\hline Collection Date: & $05 / 18 / 2007$ & $05 / 18 / 2007$ & $05 / 18 / 2007$ \\
\hline Extraction Date: & $02 / 07 / 2008$ & 02/07/2008 & 02/07/2008 \\
\hline Analysis Date: & $02 / 29 / 2008$ & 02/29/2008 & $02 / 29 / 2008$ \\
\hline Units (wet wt): & ng/g_WET & ng/g_WET & ng/g_WET \\
\hline $\mathrm{Cl} 2(8)$ & $0.09 \mathrm{~J}$ & $0.03 \mathrm{~J}$ & 0.31 \\
\hline $\mathrm{Cl} 3(18)$ & 0.76 & 0.12 & 2.80 \\
\hline $\mathrm{Cl} 3(28)$ & 2.42 & 0.34 & $7.90 \mathrm{D}$ \\
\hline $\mathrm{Cl} 4(44)$ & 3.01 & 0.42 & $9.52 \mathrm{D}$ \\
\hline $\mathrm{Cl} 4(52)$ & 11.84 & 1.17 & $29.25 \mathrm{D}$ \\
\hline $\mathrm{Cl} 4(66)$ & 9.97 & 0.79 & $31.64 \mathrm{D}$ \\
\hline $\mathrm{Cl} 4(77)$ & 0.20 & $0.04 \mathrm{~J}$ & 0.48 \\
\hline $\mathrm{Cl5}(101)$ & $27.83 \mathrm{D}$ & 3.30 & $62.38 \mathrm{D}$ \\
\hline $\mathrm{Cl} 5(105)$ & $9.33 \mathrm{D}$ & 0.92 & $30.85 \mathrm{D}$ \\
\hline $\mathrm{Cl5}(118)$ & $19.73 \mathrm{D}$ & 2.92 & $53.93 \mathrm{D}$ \\
\hline $\mathrm{Cl} 5(126)$ & $0.07 \mathrm{U}$ & $0.07 \mathrm{U}$ & $0.07 \mathrm{U}$ \\
\hline Cl6(128) & 7.69 & 0.83 & $16.43 \mathrm{D}$ \\
\hline $\mathrm{Cl6}(138)$ & $32.45 \mathrm{D}$ & 4.13 & $68.59 \mathrm{D}$ \\
\hline Cl6(153) & $80.63 \mathrm{D}$ & $7.08 \mathrm{D}$ & $146.87 \mathrm{D}$ \\
\hline $\mathrm{Cl} 7(170)$ & $9.64 \mathrm{D}$ & 1.16 & 18.64 D \\
\hline $\mathrm{Cl}(187)$ & $21.51 \mathrm{D}$ & 3.09 & $36.27 \mathrm{D}$ \\
\hline $\mathrm{Cl} 7(188)$ & $0.05 \mathrm{~J}$ & $0.01 \mathrm{~J}$ & 0.08 \\
\hline Cl8(195) & 2.08 & 0.20 & 3.46 \\
\hline $\mathrm{Cl}(200)$ & 0.61 & 0.08 & 1.14 \\
\hline $\mathrm{Cl9}(206)$ & 1.63 & 0.47 & 2.81 \\
\hline Cl10(209) & 0.28 & 0.17 & 0.49 \\
\hline Total PCBs & 483.64 & 54.68 & 1047.82 \\
\hline LOC 1 & $0.08 \mathrm{U}$ & $0.08 \mathrm{U}$ & $0.08 \mathrm{U}$ \\
\hline LOC 2 & $0.09 \mathrm{~J}$ & $0.03 \mathrm{~J}$ & $0.45 \mathrm{~J}$ \\
\hline LOC 3 & 7.81 & 1.14 & 25.93 \\
\hline LOC 4 & 67.03 & 6.37 & 190.66 \\
\hline LOC 5 & 159.96 & 20.01 & 432.25 \\
\hline LOC 6 & 246.18 & 24.41 & 483.21 \\
\hline LOC 7 & 117.24 & 14.09 & 202.88 \\
\hline LOC 8 & 28.66 & 3.87 & 34.28 \\
\hline LOC 9 & 2.26 & 0.67 & 3.84 \\
\hline Total PCBs & 629.31 & 70.67 & 1373.58 \\
\hline $\mathrm{Cl} 3(34)$ & 88 & 71 & 73 \\
\hline $\mathrm{Cl}(152)$ & 81 & 67 & 67 \\
\hline
\end{tabular}


BATTELLE MARINE SCIENCE LABORATORIES

1529 West Sequim Bay Road

Sequim, Washington 98382-9099

360/681-4564

\section{SINCLAIR AND DYES INLET \\ 2007 PSAMP Trawl Biota \\ PCBs in Whole Organisms}

\begin{tabular}{|c|c|c|c|c|}
\hline Sample Name: & DF-EM-1 & DF-EM-2 & DF-EM-4 & DF-EM-6 \\
\hline Station: & Embryo & Embryo & Embryo & Embryo \\
\hline Organism ID: & DF1 & DF & DF & DF \\
\hline Batch ID: & 08-0017 & 08-0017 & 08-0017 & 08-0017 \\
\hline Sample Weight (g): & 10.52 & 10.59 & 10.53 & 10.44 \\
\hline \%Moisture & 47.03 & 47.13 & 47.41 & 43.67 \\
\hline \%Lipids & 22.40 & 23.05 & 23.00 & 26.30 \\
\hline Collection Date: & $09 / 18 / 2007$ & $09 / 18 / 2007$ & $09 / 18 / 2007$ & $09 / 18 / 2007$ \\
\hline Extraction Date: & 02/07/2008 & 02/07/2008 & 02/07/2008 & 02/07/2008 \\
\hline Analysis Date: & 03/01/2008 & 03/01/2008 & 03/01/2008 & 03/01/2008 \\
\hline Units (wet wt): & ng/g_WET & ng/g_WET & ng/g_WET & ng/g_WET \\
\hline $\mathrm{Cl} 2(8)$ & $0.05 \mathrm{U}$ & $0.05 \mathrm{U}$ & $0.05 \mathrm{U}$ & $0.05 \mathrm{U}$ \\
\hline Cl3(18) & $0.06 \mathrm{U}$ & $0.06 \mathrm{U}$ & $0.06 \mathrm{U}$ & $0.06 \mathrm{U}$ \\
\hline Cl3(28) & 0.51 & 0.41 & 0.28 & 0.52 \\
\hline $\mathrm{Cl} 4(44)$ & 0.23 & $0.12 \mathrm{U}$ & $0.12 \mathrm{U}$ & 0.37 \\
\hline $\mathrm{Cl} 4(52)$ & 1.93 & 1.42 & 1.08 & 2.38 \\
\hline $\mathrm{Cl} 4(66)$ & 0.97 & 0.72 & 0.25 & 1.39 \\
\hline $\mathrm{Cl} 4(77)$ & $0.05 \mathrm{U}$ & $0.05 \mathrm{U}$ & $0.05 \mathrm{U}$ & $0.05 \mathrm{U}$ \\
\hline Cl5(101) & 3.75 & 2.73 & 1.19 & 7.37 \\
\hline $\mathrm{Cl}(105)$ & 1.08 & 0.66 & $0.18 \mathrm{~J}$ & 1.68 \\
\hline $\mathrm{Cl5}(118)$ & 5.43 & 4.19 & 0.91 & 7.96 \\
\hline $\mathrm{Cl5}(126)$ & $0.07 \mathrm{U}$ & $0.07 \mathrm{U}$ & $0.07 \mathrm{U}$ & $0.07 \mathrm{U}$ \\
\hline $\mathrm{Cl}(128)$ & 0.78 & 0.43 & $0.06 \mathrm{~J}$ & 1.05 \\
\hline Cl6(138) & 6.33 & 4.18 & 0.79 & 8.07 \\
\hline Cl6(153) & 13.69 & 9.18 & 1.86 & 16.99 \\
\hline Cl7(170) & 1.34 & 0.72 & $0.14 \mathrm{~J}$ & 1.41 \\
\hline $\mathrm{Cl}(187)$ & 2.27 & 2.62 & 0.36 & 4.97 \\
\hline $\mathrm{Cl}(188)$ & $0.02 \mathrm{U}$ & $0.02 \mathrm{U}$ & $0.02 \mathrm{U}$ & $0.02 \mathrm{U}$ \\
\hline $\mathrm{Cl} 18(195)$ & $0.05 \mathrm{U}$ & $0.05 \mathrm{U}$ & $0.05 \mathrm{U}$ & $0.05 \mathrm{U}$ \\
\hline $\mathrm{Cl}(200)$ & $0.04 \mathrm{U}$ & $0.04 \mathrm{U}$ & $0.04 \mathrm{U}$ & $0.04 \mathrm{U}$ \\
\hline $\mathrm{Cl9}(206)$ & 0.28 & $0.17 \mathrm{~J}$ & $0.04 \mathrm{U}$ & 0.29 \\
\hline Cl10(209) & $0.16 \mathrm{~J}$ & $0.08 \mathrm{~J}$ & $0.07 \mathrm{U}$ & $0.10 \mathrm{~J}$ \\
\hline Total PCBs & 78.18 & 55.94 & 15.34 & 109.78 \\
\hline LOC 1 & $0.08 \mathrm{U}$ & $0.08 \mathrm{U}$ & $0.08 \mathrm{U}$ & $0.08 \mathrm{U}$ \\
\hline LOC 2 & $0.52 \mathrm{U}$ & $0.52 \mathrm{U}$ & $0.52 \mathrm{U}$ & $0.52 \mathrm{U}$ \\
\hline LOC 3 & $0.51 \mathrm{~J}$ & $0.41 \mathrm{~J}$ & $0.28 \mathrm{~J}$ & $0.52 \mathrm{~J}$ \\
\hline LOC 4 & 5.82 & $3.85 \mathrm{~J}$ & $2.21 \mathrm{~J}$ & 7.96 \\
\hline LOC 5 & 22.01 & 14.57 & 4.88 & 35.94 \\
\hline LOC 6 & 29.94 & 20.68 & $3.88 \mathrm{~J}$ & 44.31 \\
\hline LOC 7 & 12.9 & 9.81 & $1.27 \mathrm{~J}$ & 18.71 \\
\hline LOC 8 & 3.05 & 2.39 & $0.51 \mathrm{U}$ & 3.92 \\
\hline LOC 9 & $0.36 \mathrm{~J}$ & $0.31 \mathrm{~J}$ & $0.16 \mathrm{U}$ & $0.45 \mathrm{~J}$ \\
\hline Total PCBs & 75.19 & 52.62 & 13.79 & 112.41 \\
\hline $\mathrm{Cl} 3(34)$ & 93 & 74 & 85 & 90 \\
\hline Cl6(152) & 70 & 52 & 68 & 65 \\
\hline
\end{tabular}


BATTELLE MARINE SCIENCE LABORATORIES

1529 West Sequim Bay Road

Sequim, Washington 98382-9099

360/681-4564

\section{SINCLAIR AND DYES INLET \\ 2007 PSAMP Trawl Biota \\ PCBs in Whole Organisms}

\begin{tabular}{|c|c|c|c|c|}
\hline Sample Name: & DF-LV-1 & DF-LV-2 & $\begin{array}{l}\text { DF-LV-4 } \\
\end{array}$ & $\begin{array}{l}\text { DF-LV-6 } \\
\end{array}$ \\
\hline Station: & Liver & Liver & Liver & Liver \\
\hline Organism ID: & DF & DF & DF & DF \\
\hline Batch ID: & 08-0017 & $08-0017$ & 08-0017 & $08-0017$ \\
\hline Sample Weight (g): & 5.03 & 5.49 & 5.7 & 5.42 \\
\hline \%Moisture & 44.79 & 47.05 & 22.82 & 68.07 \\
\hline \%Lipids & 44.76 & 62.26 & 63.43 & 70.50 \\
\hline Collection Date: & $09 / 18 / 2007$ & $09 / 18 / 2007$ & $09 / 18 / 2007$ & $09 / 18 / 2007$ \\
\hline Extraction Date: & $02 / 07 / 2008$ & 02/07/2008 & $02 / 07 / 2008$ & $02 / 07 / 2008$ \\
\hline Analysis Date: & $02 / 29 / 2008$ & 02/29/2008 & $02 / 29 / 2008$ & 03/01/2008 \\
\hline Units (wet wt): & ng/g_WET & ng/g_WET & ng/g_WET & ng/g_WET \\
\hline $\mathrm{Cl} 2(8)$ & $0.05 \mathrm{U}$ & $0.05 \mathrm{U}$ & $0.05 \mathrm{U}$ & $0.05 \mathrm{U}$ \\
\hline $\mathrm{Cl} 3(18)$ & $0.06 \mathrm{U}$ & $0.06 \mathrm{U}$ & $0.06 \mathrm{U}$ & $0.06 \mathrm{U}$ \\
\hline $\mathrm{Cl} 3(28)$ & 1.25 & 1.77 & 1.10 & 2.19 \\
\hline $\mathrm{Cl} 4(44)$ & 0.51 & 0.66 & 0.41 & 1.41 \\
\hline $\mathrm{Cl} 4(52)$ & 2.69 & 4.53 & 3.16 & 7.13 \\
\hline $\mathrm{Cl} 4(66)$ & 3.45 & 4.58 & 1.36 & 7.45 \\
\hline $\mathrm{Cl}(777)$ & $0.21 \mathrm{~J}$ & 0.37 & $0.05 \mathrm{~J}$ & 0.45 \\
\hline $\mathrm{Cl5}(101)$ & 7.07 & 12.39 & 4.32 & 26.8 \\
\hline $\mathrm{Cl} 5(105)$ & 6.88 & 10.16 & 1.64 & 15.82 \\
\hline $\mathrm{Cl5}(118)$ & 18.42 & $19.59 \mathrm{D}$ & 4.59 & $21.52 \mathrm{D}$ \\
\hline $\mathrm{Cl} 5(126)$ & $0.26 \mathrm{~J}$ & $0.32 \mathrm{~J}$ & $0.07 \mathrm{U}$ & 0.37 \\
\hline $\mathrm{Cl}(128)$ & 5.67 & 6.30 & $0.23 \mathrm{~J}$ & 10.09 \\
\hline Cl6(138) & $23.01 \mathrm{D}$ & $30.44 \mathrm{D}$ & 4.67 & $38.9 \mathrm{D}$ \\
\hline $\mathrm{Cl6}(153)$ & $43.05 \mathrm{D}$ & $65.42 \mathrm{D}$ & 10.61 & $87.52 \mathrm{D}$ \\
\hline $\mathrm{Cl}(170)$ & 6.64 & 10.57 & 0.86 & 11.31 \\
\hline $\mathrm{Cl7}(187)$ & 6.86 & 21.22 & 2.22 & $26.3 \mathrm{D}$ \\
\hline $\mathrm{Cl}(188)$ & $0.05 \mathrm{~J}$ & $0.07 \mathrm{~J}$ & $0.02 \mathrm{U}$ & $0.08 \mathrm{~J}$ \\
\hline Cl8(195) & 1.02 & 1.29 & $0.05 \mathrm{U}$ & 1.14 \\
\hline $\mathrm{Cl}(200)$ & $0.04 \mathrm{U}$ & $0.04 \mathrm{U}$ & $0.04 \mathrm{U}$ & $0.25 \mathrm{~J}$ \\
\hline $\mathrm{Cl9}(206)$ & 1.11 & 2.17 & $0.11 \mathrm{~J}$ & 1.56 \\
\hline Cl10(209) & $0.30 \mathrm{~J}$ & 0.63 & $0.05 \mathrm{~J}$ & $0.34 \mathrm{~J}$ \\
\hline Total PCBs & 257.20 & 385.26 & 71.34 & 521.48 \\
\hline LOC 1 & $0.08 \mathrm{U}$ & $0.08 \mathrm{U}$ & $0.08 \mathrm{U}$ & $0.08 \mathrm{U}$ \\
\hline LOC 2 & $0.52 \mathrm{U}$ & $0.06 \mathrm{~J}$ & $0.52 \mathrm{U}$ & $0.52 \mathrm{U}$ \\
\hline LOC 3 & $1.25 \mathrm{~J}$ & $1.77 \mathrm{~J}$ & $1.27 \mathrm{~J}$ & $2.19 \mathrm{~J}$ \\
\hline LOC 4 & 13.88 & 19.62 & $9.18 \mathrm{~J}$ & 32.7 \\
\hline LOC 5 & 65.18 & 83.98 & 22.49 & 144.64 \\
\hline LOC 6 & 100.77 & 156.14 & 25.33 & 235.61 \\
\hline LOC 7 & 49.6 & 93.17 & 8.48 & 102.91 \\
\hline LOC 8 & 11.42 & 22.79 & $0.47 \mathrm{~J}$ & 20.97 \\
\hline LOC 9 & 1.41 & 3.08 & $0.11 \mathrm{~J}$ & 2.20 \\
\hline Total PCBs & 244.11 & 380.69 & 67.93 & 541.82 \\
\hline $\mathrm{Cl} 3(34)$ & 63 & 73 & 75 & 77 \\
\hline Cl6(152) & 62 & 69 & 72 & 72 \\
\hline
\end{tabular}




\section{SINCLAIR AND DYES INLET 2007 PSAMP Trawl Biota PCBs in Whole Organisms}

\begin{tabular}{|c|c|c|c|c|}
\hline Sample Name: & DF-DIG-1 & DF-DIG-2 & DF-DIG-4 & DF-DIG-6 \\
\hline Station: & Digestive & Digestive & Digestive & Digestive \\
\hline Organism ID: & DF & DF & DF & DF \\
\hline Batch ID: & 08-0017 & 08-0017 & 08-0017 & 08-0017 \\
\hline Sample Weight (g): & 18.5 & 19.38 & 10.68 & 19.14 \\
\hline \%Moisture & 77.33 & 78.84 & 84.41 & 82.94 \\
\hline \%Lipids & 12.40 & 3.53 & 1.75 & 2.63 \\
\hline Collection Date: & $09 / 18 / 2007$ & $09 / 18 / 2007$ & $09 / 18 / 2007$ & $09 / 18 / 2007$ \\
\hline Extraction Date: & $02 / 07 / 2008$ & 02/07/2008 & 02/07/2008 & $02 / 07 / 2008$ \\
\hline Analysis Date: & 03/01/2008 & 03/01/2008 & 03/01/2008 & 03/01/2008 \\
\hline Units (wet wt): & ng/g_WET & ng/g_WET & ng/g_WET & ng/g_WET \\
\hline $\mathrm{Cl} 2(8)$ & $0.02 \mathrm{~J}$ & $0.01 \mathrm{~J}$ & $0.03 \mathrm{~J}$ & $0.05 \mathrm{U}$ \\
\hline $\mathrm{Cl} 3(18)$ & $0.02 \mathrm{~J}$ & $0.02 \mathrm{~J}$ & $0.06 \mathrm{U}$ & $0.06 \mathrm{U}$ \\
\hline $\mathrm{Cl} 3(28)$ & $0.09 \mathrm{~J}$ & $0.04 \mathrm{~J}$ & $0.07 \mathrm{~J}$ & $0.04 \mathrm{~J}$ \\
\hline $\mathrm{Cl} 4(44)$ & $0.12 \mathrm{U}$ & $0.12 \mathrm{U}$ & $0.12 \mathrm{U}$ & $0.12 \mathrm{U}$ \\
\hline $\mathrm{Cl} 4(52)$ & $0.05 \mathrm{~J}$ & $0.05 \mathrm{~J}$ & $0.09 \mathrm{~J}$ & 0.12 \\
\hline $\mathrm{Cl} 4(66)$ & 0.18 & $0.06 \mathrm{~J}$ & $0.06 \mathrm{~J}$ & 0.15 \\
\hline $\mathrm{Cl} 4(77)$ & $0.02 \mathrm{~J}$ & $0.05 \mathrm{U}$ & $0.04 \mathrm{~J}$ & $0.05 \mathrm{~J}$ \\
\hline $\mathrm{Cl5}(101)$ & $0.05 \mathrm{~J}$ & $0.06 \mathrm{~J}$ & $0.06 \mathrm{~J}$ & 0.32 \\
\hline $\mathrm{Cl} 5(105)$ & 0.35 & $0.07 \mathrm{~J}$ & $0.06 \mathrm{~J}$ & 0.33 \\
\hline $\mathrm{Cl} 5(118)$ & 0.72 & 0.10 & $0.07 \mathrm{~J}$ & 0.53 \\
\hline $\mathrm{Cl} 5(126)$ & $0.07 \mathrm{U}$ & $0.07 \mathrm{U}$ & $0.07 \mathrm{U}$ & $0.07 \mathrm{U}$ \\
\hline $\mathrm{Cl}(128)$ & 0.30 & $0.05 \mathrm{U}$ & $0.05 \mathrm{U}$ & $0.07 \mathrm{~J}$ \\
\hline Cl6(138) & 1.49 & 0.10 & $0.07 \mathrm{~J}$ & 0.80 \\
\hline Cl6(153) & 2.74 & 0.20 & $0.13 \mathrm{~J}$ & 1.70 \\
\hline $\mathrm{Cl} 7(170)$ & 0.33 & $0.04 \mathrm{U}$ & $0.04 \mathrm{U}$ & 0.18 \\
\hline Cl7(187) & $0.06 \mathrm{~J}$ & $0.05 \mathrm{~J}$ & $0.05 \mathrm{~J}$ & 0.51 \\
\hline $\mathrm{Cl}(188)$ & $0.02 \mathrm{U}$ & $0.02 \mathrm{U}$ & $0.02 \mathrm{U}$ & $0.02 \mathrm{U}$ \\
\hline Cl8(195) & $0.05 \mathrm{U}$ & $0.05 \mathrm{U}$ & $0.05 \mathrm{U}$ & $0.05 \mathrm{U}$ \\
\hline $\mathrm{Cl}(200)$ & $0.04 \mathrm{U}$ & $0.04 \mathrm{U}$ & $0.04 \mathrm{U}$ & $0.04 \mathrm{U}$ \\
\hline $\mathrm{Cl9}(206)$ & 0.13 & $0.04 \mathrm{U}$ & $0.04 \mathrm{U}$ & $0.07 \mathrm{~J}$ \\
\hline Cl10(209) & $0.05 \mathrm{~J}$ & $0.07 \mathrm{U}$ & $0.07 \mathrm{U}$ & $0.06 \mathrm{~J}$ \\
\hline Total PCBs & 13.80 & 2.62 & 2.58 & 10.68 \\
\hline LOC 1 & $0.08 \mathrm{U}$ & $0.08 \mathrm{U}$ & $0.08 \mathrm{U}$ & $0.08 \mathrm{U}$ \\
\hline LOC 2 & $0.02 \mathrm{~J}$ & $0.01 \mathrm{~J}$ & $0.03 \mathrm{~J}$ & $0.52 \mathrm{U}$ \\
\hline LOC 3 & $0.19 \mathrm{~J}$ & $0.12 \mathrm{~J}$ & $0.12 \mathrm{~J}$ & $0.05 \mathrm{~J}$ \\
\hline LOC 4 & $0.58 \mathrm{~J}$ & $0.19 \mathrm{~J}$ & $0.29 \mathrm{~J}$ & $0.6 \mathrm{~J}$ \\
\hline LOC 5 & $2.21 \mathrm{~J}$ & $0.35 \mathrm{~J}$ & $0.26 \mathrm{~J}$ & 2.36 \\
\hline LOC 6 & 5.24 & $0.45 \mathrm{~J}$ & $0.28 \mathrm{~J}$ & 4.08 \\
\hline LOC 7 & $1.82 \mathrm{~J}$ & $0.18 \mathrm{~J}$ & $0.10 \mathrm{~J}$ & $2.00 \mathrm{~J}$ \\
\hline LOC 8 & $0.57 \mathrm{~J}$ & $0.51 \mathrm{U}$ & $0.51 \mathrm{U}$ & $0.47 \mathrm{~J}$ \\
\hline LOC 9 & $0.13 \mathrm{~J}$ & $0.16 \mathrm{U}$ & $0.16 \mathrm{U}$ & $0.09 \mathrm{~J}$ \\
\hline Total PCBs & 10.84 & 2.05 & 1.83 & 10.25 \\
\hline $\mathrm{Cl} 3(34)$ & 50 & 41 & 71 & 66 \\
\hline Cl6(152) & 57 & 45 & 77 & 74 \\
\hline
\end{tabular}


1529 West Sequim Bay Road

Sequim, Washington 98382-9099

360/681-4564

\section{SINCLAIR AND DYES INLET \\ 2007 PSAMP Trawl Biota \\ PCBs in Whole Organisms}

\begin{tabular}{|c|c|c|c|c|c|}
\hline Sample Name: & DF-SWC-1 & DF-SWC-2 & DF-SWC-4 & DF-SWC-6 & DF-WBWC-1 \\
\hline Station: & Sec. Comp. & Sec. Comp. & Sec. Comp. & Sec. Comp. & W.Body Comp. \\
\hline Organism ID: & DF & DF & DF & DF & DF \\
\hline Batch ID: & 08-0017 & 08-0017 & 08-0018 & $08-0017$ & 08-0017 \\
\hline Sample Weight (g): & 15.32 & 15.35 & 15.5 & 15.78 & 15.57 \\
\hline \%Moisture & 62.81 & 71.19 & 70.16 & 64.77 & 60.24 \\
\hline \%Lipids & 15.96 & 13.00 & 14.45 & 16.83 & 19.90 \\
\hline Collection Date: & 09/18/2007 & $09 / 18 / 2007$ & $09 / 18 / 2007$ & $09 / 18 / 2007$ & $09 / 18 / 2007$ \\
\hline Extraction Date: & $02 / 07 / 2008$ & 02/07/2008 & 02/07/2008 & 02/07/2008 & 02/07/2008 \\
\hline Analysis Date: & 03/01/2008 & 03/01/2008 & $02 / 29 / 2008$ & 03/01/2008 & 03/01/2008 \\
\hline Units (wet wt): & ng/g_WET & ng/g_WET & ng/g_WET & ng/g_WET & ng/g_WET \\
\hline $\mathrm{Cl} 2(8)$ & $0.02 \mathrm{~J}$ & $0.01 \mathrm{~J}$ & $0.05 \mathrm{U}$ & $0.05 \mathrm{U}$ & $0.05 \mathrm{U}$ \\
\hline $\mathrm{Cl} 3(18)$ & $0.06 \mathrm{U}$ & $0.06 \mathrm{U}$ & $0.06 \mathrm{U}$ & $0.04 \mathrm{~J}$ & $0.06 \mathrm{U}$ \\
\hline $\mathrm{Cl} 3(28)$ & 0.65 & 0.41 & 0.23 & 0.52 & 0.31 \\
\hline $\mathrm{Cl} 4(44)$ & 0.24 & 0.17 & $0.08 \mathrm{~J}$ & 0.41 & 0.13 \\
\hline $\mathrm{Cl} 4(52)$ & 1.47 & 1.22 & 0.58 & 1.94 & 0.72 \\
\hline $\mathrm{Cl} 4(66)$ & 1.60 & 0.86 & 0.19 & 1.66 & 0.84 \\
\hline $\mathrm{Cl} 4(77)$ & $0.05 \mathrm{U}$ & $0.02 \mathrm{~J}$ & $0.05 \mathrm{U}$ & $0.01 \mathrm{~J}$ & $0.04 \mathrm{~J}$ \\
\hline $\mathrm{Cl5}(101)$ & 3.55 & 2.66 & 0.83 & 6.81 & 1.76 \\
\hline $\mathrm{Cl5}(105)$ & 2.94 & 1.94 & 0.26 & 3.55 & 1.75 \\
\hline $\mathrm{Cl5}(118)$ & 8.13 & 5.39 & 0.72 & $4.22 \mathrm{D}$ & 4.19 \\
\hline $\mathrm{Cl} 5(126)$ & $0.07 \mathrm{U}$ & $0.07 \mathrm{U}$ & $0.07 \mathrm{U}$ & $0.07 \mathrm{U}$ & $0.07 \mathrm{U}$ \\
\hline $\mathrm{Cl6}(128)$ & 2.25 & 1.04 & 0.14 & 2.23 & 1.35 \\
\hline Cl6(138) & $8.18 \mathrm{D}$ & 7.79 & 0.86 & $8.02 \mathrm{D}$ & 7.37 \\
\hline $\mathrm{Cl} 6(153)$ & $15.92 \mathrm{D}$ & $9.71 \mathrm{D}$ & 1.74 & $15.59 \mathrm{D}$ & $9.52 \mathrm{D}$ \\
\hline $\mathrm{Cl} 7(170)$ & 1.77 & 1.25 & $0.09 \mathrm{~J}$ & 2.18 & 1.21 \\
\hline $\mathrm{Cl} 7(187)$ & 2.40 & 3.30 & 0.29 & 5.88 & 1.51 \\
\hline $\mathrm{Cl}(188)$ & $0.01 \mathrm{~J}$ & $0.02 \mathrm{U}$ & $0.02 \mathrm{U}$ & $0.02 \mathrm{~J}$ & $0.02 \mathrm{U}$ \\
\hline $\mathrm{Cl}(195)$ & 0.23 & $0.05 \mathrm{U}$ & $0.05 \mathrm{U}$ & 0.19 & 0.18 \\
\hline $\mathrm{Cl} 8(200)$ & $0.04 \mathrm{U}$ & $0.04 \mathrm{U}$ & $0.04 \mathrm{U}$ & $0.04 \mathrm{U}$ & $0.04 \mathrm{U}$ \\
\hline $\mathrm{Cl9}(206)$ & 0.15 & 0.19 & $0.04 \mathrm{U}$ & 0.27 & 0.15 \\
\hline Cl10(209) & $0.04 \mathrm{~J}$ & $0.05 \mathrm{~J}$ & $0.07 \mathrm{U}$ & $0.10 \mathrm{~J}$ & $0.05 \mathrm{~J}$ \\
\hline Total PCBs & 99.54 & 72.50 & 12.92 & 107.60 & 62.64 \\
\hline LOC 1 & $0.08 \mathrm{U}$ & $0.08 \mathrm{U}$ & $0.08 \mathrm{U}$ & $0.08 \mathrm{U}$ & $0.08 \mathrm{U}$ \\
\hline LOC 2 & $0.02 \mathrm{~J}$ & $0.01 \mathrm{~J}$ & $0.52 \mathrm{U}$ & $0.52 \mathrm{U}$ & $0.52 \mathrm{U}$ \\
\hline LOC 3 & $0.82 \mathrm{~J}$ & $0.45 \mathrm{~J}$ & $0.23 \mathrm{~J}$ & $0.56 \mathrm{~J}$ & $0.31 \mathrm{~J}$ \\
\hline LOC 4 & 7.07 & 4.43 & $1.48 \mathrm{~J}$ & 8.07 & $3.60 \mathrm{~J}$ \\
\hline LOC 5 & 30.57 & 18.71 & 3.80 & 34.56 & 15.57 \\
\hline LOC 6 & 38.82 & 29.2 & 4.06 & 48.68 & 25.68 \\
\hline LOC 7 & 14.36 & 13.18 & $1.10 \mathrm{~J}$ & 23.78 & 9.70 \\
\hline LOC 8 & 2.38 & 2.31 & $0.51 \mathrm{U}$ & 4.09 & 1.86 \\
\hline LOC 9 & $0.20 \mathrm{~J}$ & $0.26 \mathrm{~J}$ & $0.16 \mathrm{U}$ & 0.41 & $0.19 \mathrm{~J}$ \\
\hline Total PCBs & 94.32 & 68.63 & 11.94 & 120.75 & 57.51 \\
\hline $\mathrm{Cl} 3(34)$ & 72 & 63 & 75 & 72 & 37 \# \\
\hline $\mathrm{Cl}(152)$ & 75 & 72 & 80 & 73 & 45 \\
\hline
\end{tabular}




\begin{tabular}{|c|c|c|c|c|c|}
\hline Sample Name: & DF-WBWC-2 & DF-WBWC-3 & DF-WBWC-4 & DF-WBWC-5 & DF-WBWC-6 \\
\hline Station: & W.Body Comp. & W.Body Comp. & W.Body Comp. & W.Body Comp. & W.Body Comp. \\
\hline Organism ID: & DF & DF & DF & DF & DF \\
\hline Batch ID: & 08-0018 & 08-0018 & 08-0018 & $08-0018$ & $08-0018$ \\
\hline Sample Weight (g): & 15.65 & 15.1 & 14.94 & 15.65 & 15.71 \\
\hline \%Moisture & 64.17 & 63.30 & 59.64 & 58.97 & 62.97 \\
\hline \%Lipids & 15.32 & 18.06 & 18.09 & 22.21 & 22.21 \\
\hline Collection Date: & 09/18/2007 & $09 / 18 / 2007$ & 09/18/2007 & 09/18/2007 & $09 / 18 / 2007$ \\
\hline Extraction Date: & $02 / 07 / 2008$ & $02 / 07 / 2008$ & $02 / 07 / 2008$ & 02/07/2008 & $02 / 07 / 2008$ \\
\hline Analysis Date: & $02 / 29 / 2008$ & $02 / 29 / 2008$ & $02 / 29 / 2008$ & 02/29/2008 & $02 / 29 / 2008$ \\
\hline Units (wet wt): & ng/g_WET & ng/g_WET & ng/g_WET & ng/g_WET & ng/g_WET \\
\hline $\mathrm{Cl} 2(8)$ & $0.05 \mathrm{U}$ & $0.05 \mathrm{U}$ & $0.05 \mathrm{U}$ & $0.05 \mathrm{U}$ & $0.05 \mathrm{U}$ \\
\hline $\mathrm{Cl} 3(18)$ & $0.06 \mathrm{U}$ & $0.06 \mathrm{U}$ & $0.06 \mathrm{U}$ & $0.06 \mathrm{U}$ & $0.06 \mathrm{U}$ \\
\hline $\mathrm{Cl} 3(28)$ & 0.49 & 0.41 & 0.36 & 0.60 & 0.48 \\
\hline $\mathrm{Cl} 4(44)$ & 0.18 & 0.18 & 0.14 & 0.18 & 0.33 \\
\hline $\mathrm{Cl} 4(52)$ & 1.20 & 1.46 & 0.96 & 0.78 & 1.71 \\
\hline $\mathrm{Cl} 4(66)$ & 0.90 & 1.02 & 0.43 & 2.06 & 1.51 \\
\hline $\mathrm{Cl} 4(77)$ & $0.05 \mathrm{U}$ & $0.05 \mathrm{U}$ & $0.05 \mathrm{U}$ & $0.05 \mathrm{U}$ & 0.13 \\
\hline $\mathrm{Cl5}(101)$ & 3.17 & 4.79 & 1.53 & 2.90 & 6.94 \\
\hline $\mathrm{Cl} 5(105)$ & 1.68 & 2.21 & 0.51 & 4.31 & 3.17 \\
\hline $\mathrm{Cl} 5(118)$ & 5.66 & 6.68 & 1.38 & 12.98 & 8.84 \\
\hline $\mathrm{Cl} 5(126)$ & $0.07 \mathrm{U}$ & $0.07 \mathrm{U}$ & $0.07 \mathrm{U}$ & $0.07 \mathrm{U}$ & $0.07 \mathrm{U}$ \\
\hline $\mathrm{Cl6}(128)$ & 1.08 & 1.45 & 0.21 & 3.99 & 2.10 \\
\hline $\mathrm{Cl6}(138)$ & 8.60 & 9.35 & 1.61 & $15.08 \mathrm{D}$ & 11.95 \\
\hline $\mathrm{Cl6}(153)$ & $11.58 \mathrm{D}$ & $11.89 \mathrm{D}$ & 3.18 & $29.47 \mathrm{D}$ & $13.90 \mathrm{D}$ \\
\hline $\mathrm{Cl}(170)$ & 1.37 & 1.63 & 0.23 & 4.09 & 1.90 \\
\hline $\mathrm{Cl}(187)$ & 3.54 & 4.50 & 0.63 & 3.64 & 5.79 \\
\hline $\mathrm{Cl}(188)$ & $0.01 \mathrm{~J}$ & $0.02 \mathrm{~J}$ & $0.02 \mathrm{U}$ & $0.02 \mathrm{~J}$ & $0.03 \mathrm{~J}$ \\
\hline $\mathrm{Cl}(195)$ & 0.16 & 0.23 & $0.05 \mathrm{U}$ & 0.50 & 0.27 \\
\hline $\mathrm{Cl}(200)$ & $0.04 \mathrm{U}$ & $0.04 \mathrm{U}$ & $0.04 \mathrm{U}$ & $0.04 \mathrm{U}$ & $0.04 \mathrm{U}$ \\
\hline $\mathrm{Cl9}(206)$ & 0.22 & 0.29 & $0.04 \mathrm{U}$ & 0.44 & 0.33 \\
\hline Cl10(209) & $0.08 \mathrm{~J}$ & 0.13 & $0.07 \mathrm{U}$ & $0.12 \mathrm{~J}$ & $0.11 \mathrm{~J}$ \\
\hline Total PCBs & 80.38 & 93.02 & 23.24 & 162.86 & 119.42 \\
\hline LOC 1 & $0.08 \mathrm{U}$ & $0.08 \mathrm{U}$ & $0.08 \mathrm{U}$ & $0.08 \mathrm{U}$ & $0.08 \mathrm{U}$ \\
\hline LOC 2 & $0.52 \mathrm{U}$ & $0.52 \mathrm{U}$ & $0.52 \mathrm{U}$ & $0.52 \mathrm{U}$ & $0.52 \mathrm{U}$ \\
\hline LOC 3 & $0.56 \mathrm{~J}$ & $0.41 \mathrm{~J}$ & $0.41 \mathrm{~J}$ & $0.60 \mathrm{~J}$ & $0.48 \mathrm{~J}$ \\
\hline LOC 4 & 4.73 & 5.65 & $3.09 \mathrm{~J}$ & 6.87 & 6.35 \\
\hline LOC 5 & 18.98 & 25.72 & 7.06 & 38.55 & 36.00 \\
\hline LOC 6 & 31.86 & 37.12 & 7.72 & 64.09 & 49.53 \\
\hline LOC 7 & 13.84 & 17.48 & $2.19 \mathrm{~J}$ & 29.11 & 22.86 \\
\hline LOC 8 & 2.65 & 3.70 & $0.51 \mathrm{U}$ & 6.22 & 4.08 \\
\hline LOC 9 & $0.31 \mathrm{~J}$ & 0.41 & $0.16 \mathrm{U}$ & 0.59 & 0.49 \\
\hline Total PCBs & 73.53 & 91.09 & 21.74 & 146.63 & 120.39 \\
\hline $\mathrm{Cl} 3(34)$ & 78 & 72 & 75 & 62 & 68 \\
\hline $\mathrm{Cl}(152)$ & 81 & 71 & 73 & 61 & 68 \\
\hline
\end{tabular}




\section{Field Data Summary: Stable Isotopes in Biota Tissue}

\section{Dry Weight Basis}




\begin{tabular}{|c|c|c|c|c|c|c|}
\hline PSAMP Station & PSAMP Code & Organism & MSL Code & $\begin{array}{c}\text { Collection } \\
\text { Date }\end{array}$ & $\delta^{15} \mathbf{N}$ & $\delta^{13} \mathrm{C}$ \\
\hline Vendovi & V-ES-1 & English sole adult & $2838-1$ & 05/01/07 & 12.99 & -14.58 \\
\hline Vendovi & V-ES-2 & English sole adult & $2838-2$ & 05/01/07 & 12.83 & -15.34 \\
\hline Vendovi & V-ES-3 & English sole adult & $2838-3$ & 05/01/07 & 12.32 & -15.00 \\
\hline Vendovi & V-ES-4 & English sole adult & $2838-4$ & 05/01/07 & 13.05 & -15.03 \\
\hline Vendovi & V-ES-5 & English sole adult & $2838-5$ & 05/01/07 & 12.55 & -15.65 \\
\hline Vendovi & V-ES-6 & English sole adult & $2838-6$ & 05/01/07 & 12.90 & -14.74 \\
\hline Vendovi & V-ES-7 & English sole adult & $2838-7$ & 05/01/07 & 12.83 & -15.36 \\
\hline Vendovi & V-ES-8 & English sole adult & $2838-8$ & 05/01/07 & 12.79 & -15.48 \\
\hline Vendovi & V-ES-9 & English sole adult & $2838-9$ & 05/01/07 & 13.24 & -15.65 \\
\hline Vendovi & V-RS-1 & Rock sole & $2838-10$ & 05/01/07 & 13.04 & -15.53 \\
\hline Vendovi & V-RS-2 & Rock sole & $2838-11$ & 05/01/07 & 12.80 & -15.95 \\
\hline Vendovi & V-RS-3 & Rock sole & $2838-12$ & 05/01/07 & 13.40 & -15.02 \\
\hline Vendovi & V-SC-COMP & Sea cucumber (3) & $2838-19$ & 05/01/07 & 10.21 & -17.14 \\
\hline Vendovi & V-SP-1 & Shiner perch & $2838-16$ & 05/01/07 & 13.67 & -12.48 \\
\hline Vendovi & V-SP-2 & Shiner perch & $2838-17$ & 05/01/07 & 14.23 & -11.51 \\
\hline Vendovi & V-SP-3 & Shiner perch & $2838-18$ & 05/01/07 & 13.40 & -12.71 \\
\hline Vendovi & V-SSc-1 & Staghorn sculpin & $2838-13$ & 05/01/07 & 15.31 & -13.03 \\
\hline Vendovi & V-SSc-2 & Staghorn sculpin & $2838-14$ & 05/01/07 & 14.97 & -13.67 \\
\hline Vendovi & V-SSc-3 & Staghorn sculpin & $2838-15$ & 05/01/07 & 15.21 & -13.04 \\
\hline Strait of Georgia & SG-ES-1 & English sole adult & $2838-20$ & $05 / 02 / 07$ & 12.27 & -14.83 \\
\hline Strait of Georgia & SG-ES-2 & English sole adult & $2838-21$ & 05/02/07 & 12.30 & -15.98 \\
\hline Strait of Georgia & SG-ES-3 & English sole adult & $2838-22$ & 05/02/07 & 12.36 & -15.11 \\
\hline Strait of Georgia & SG-ES-4 & English sole adult & $2838-23$ & $05 / 02 / 07$ & 13.24 & -15.45 \\
\hline Strait of Georgia & SG-ES-5 & English sole adult & $2838-24$ & $05 / 02 / 07$ & 12.59 & -16.03 \\
\hline Strait of Georgia & SG-ES-6 & English sole adult & $2838-25$ & $05 / 02 / 07$ & 12.50 & -15.36 \\
\hline Strait of Georgia & SG-ES-7 & English sole adult & $2838-26$ & 05/02/07 & 12.50 & -14.93 \\
\hline Strait of Georgia & SG-ES-8 & English sole adult & $2838-27$ & $05 / 02 / 07$ & 12.75 & -15.93 \\
\hline Strait of Georgia & SG-ES-9 & English sole adult & $2838-28$ & 05/02/07 & 12.57 & -15.66 \\
\hline Strait of Georgia & SG-SC-COMP & Sea cucumber (6) & $2838-32$ & $05 / 02 / 07$ & 12.59 & -17.84 \\
\hline Strait of Georgia & SG-RF-1 & Spotted Ratfish & $2838-29$ & 05/02/07 & 14.11 & -15.57 \\
\hline Strait of Georgia & SG-RF-2 & Spotted Ratfish & $2838-30$ & 05/02/07 & 14.13 & -15.16 \\
\hline Strait of Georgia & SG-RF-3 & Spotted Ratfish & $2838-31$ & $05 / 02 / 07$ & 14.92 & -15.16 \\
\hline Sinclair Inlet & SI-ES-1 & English sole adult & $2838-58$ & 05/17/07 & 13.28 & -15.39 \\
\hline Sinclair Inlet & SI-ES-2 & English sole adult & $2838-59$ & 05/17/07 & 13.24 & -15.31 \\
\hline Sinclair Inlet & SI-ES-3 & English sole adult & $2838-60$ & 05/17/07 & 13.08 & -15.77 \\
\hline Sinclair Inlet & SI-ES-4 & English sole adult & $2838-61$ & 05/17/07 & 13.08 & -15.11 \\
\hline Sinclair Inlet & SI-ES-5 & English sole adult & $2838-62$ & 05/17/07 & 13.60 & -15.29 \\
\hline Sinclair Inlet & SI-ES-6 & English sole adult & $2838-63$ & 05/17/07 & 13.10 & -15.24 \\
\hline Sinclair Inlet & SI-ES-7 & English sole adult & $2838-64$ & 05/17/07 & 14.19 & -14.77 \\
\hline Sinclair Inlet & SI-ES-8 & English sole adult & $2838-65$ & 05/17/07 & 12.98 & -15.21 \\
\hline Sinclair Inlet & SI-ES-9 & English sole adult & $2838-66$ & 05/17/07 & 13.19 & -15.55 \\
\hline Sinclair Inlet & SI-GC-1 & Graceful Crab & $2838-92$ & 05/17/07 & 13.30 & -14.88 \\
\hline Sinclair Inlet & SI-GC-2 & Graceful Crab & $2838-93$ & 05/17/07 & 13.47 & -14.54 \\
\hline
\end{tabular}


 \\ Biota Studies 2007 PSAMP Trawl Biota \\ Stable Isotopes}

\begin{tabular}{|c|c|c|c|c|c|c|}
\hline PSAMP Station & PSAMP Code & Organism & MSL Code & \begin{tabular}{|c|}
$\begin{array}{c}\text { Collection } \\
\text { Date }\end{array}$ \\
\end{tabular} & $\delta^{15} \mathbf{N}$ & $\delta^{13} \mathrm{C}$ \\
\hline Sinclair Inlet & SI-GC-3 & Graceful Crab & 2838-94 & $05 / 17 / 07$ & 13.37 & -15.91 \\
\hline Sinclair Inlet & SI-GC-4 & Graceful Crab & $2838-95$ & 05/17/07 & 12.82 & -15.06 \\
\hline Sinclair Inlet & SI-GC-5 & Graceful Crab & $2838-96$ & $05 / 17 / 07$ & 14.22 & -13.52 \\
\hline Sinclair Inlet & SI-GC-6 & Graceful Crab & $2838-97$ & 05/17/07 & 13.52 & -14.62 \\
\hline Sinclair Inlet & SI-GC-7 & Graceful Crab & $2838-98$ & $05 / 17 / 07$ & 12.83 & -15.00 \\
\hline Sinclair Inlet & SI-GC-8 & Graceful Crab & 2838-99 & $05 / 17 / 07$ & 13.41 & -15.04 \\
\hline Sinclair Inlet & SI-RS-1 & Rock sole & $2838-67-68$ & $05 / 17 / 07$ & 12.02 & -16.07 \\
\hline Sinclair Inlet & SI-RS-3 & Rock sole & $2838-69$ & $05 / 17 / 07$ & 13.51 & -14.48 \\
\hline Sinclair Inlet & SI-RS-4 & Rock sole & $2838-70$ & $05 / 17 / 07$ & 14.68 & -14.24 \\
\hline Sinclair Inlet & SI-SS-1 & Sand sole & $2838-71$ & $05 / 17 / 07$ & 13.80 & -14.27 \\
\hline Sinclair Inlet & SI-SS-2 & Sand sole & $2838-72$ & $05 / 17 / 07$ & 13.03 & -14.13 \\
\hline Sinclair Inlet & SI-SS-3 & Sand sole & $2838-73$ & $05 / 17 / 07$ & 13.60 & -14.60 \\
\hline Sinclair Inlet & SI-SS-4 & Sand sole & $2838-74$ & $05 / 17 / 07$ & 12.89 & -14.52 \\
\hline Sinclair Inlet & SI-SS-5 & Sand sole & $2838-75$ & $05 / 17 / 07$ & 13.81 & -14.54 \\
\hline Sinclair Inlet & SI-SS-6 & Sand sole & $2838-76$ & $05 / 17 / 07$ & 13.38 & -14.16 \\
\hline Sinclair Inlet & SI-SC-COMP & Sea cucumber (6) & $2838-100$ & $05 / 17 / 07$ & 13.05 & -16.23 \\
\hline Sinclair Inlet & SI-SP-3 & Shiner perch & $2838-87$ & $05 / 17 / 07$ & 14.05 & -15.62 \\
\hline Sinclair Inlet & SI-SP-4 & Shiner perch & $2838-88$ & $05 / 17 / 07$ & 13.62 & -14.41 \\
\hline Sinclair Inlet & SI-SP-5 & Shiner perch & $2838-89$ & $05 / 17 / 07$ & 13.88 & -16.88 \\
\hline Sinclair Inlet & SI-SP-6 & Shiner perch & $2838-90$ & $05 / 17 / 07$ & 14.07 & -14.70 \\
\hline Sinclair Inlet & SI-SP-7 & Shiner perch & $2838-91$ & $05 / 17 / 07$ & 14.10 & -14.73 \\
\hline Sinclair Inlet & SI-RF-1 & Spotted Ratfish & $2838-83$ & $05 / 17 / 07$ & 15.86 & -13.51 \\
\hline Sinclair Inlet & SI-RF-2 & Spotted Ratfish & $2838-84$ & $05 / 17 / 07$ & 14.53 & -13.82 \\
\hline Sinclair Inlet & SI-SSc-1 & Staghorn sculpin & $2838-77$ & $05 / 17 / 07$ & 15.06 & -14.21 \\
\hline Sinclair Inlet & SI-SSc-2 & Staghorn sculpin & $2838-78$ & $05 / 17 / 07$ & 14.51 & -14.88 \\
\hline Sinclair Inlet & SI-SSc-3 & Staghorn sculpin & $2838-79$ & $05 / 17 / 07$ & 14.89 & -14.70 \\
\hline Sinclair Inlet & SI-SSc-4 & Staghorn sculpin & $2838-80$ & $05 / 17 / 07$ & 15.55 & -14.52 \\
\hline Sinclair Inlet & SI-SSc-5 & Staghorn sculpin & $2838-81$ & $05 / 17 / 07$ & 14.85 & -13.95 \\
\hline Sinclair Inlet & SI-SSc-6 & Staghorn sculpin & $2838-82$ & $05 / 17 / 07$ & 15.11 & -15.27 \\
\hline Port Gardner & PG-ES-1 & English sole adult & 2838-101 & $05 / 29 / 07$ & 12.45 & -16.57 \\
\hline Port Gardner & PG-ES-2 & English sole adult & $2838-102$ & $05 / 29 / 07$ & 12.95 & -14.83 \\
\hline Port Gardner & PG-ES-3 & English sole adult & $2838-103$ & $05 / 29 / 07$ & 12.81 & -15.70 \\
\hline Nisqually & NIS-ES-1 & English sole adult & 2838-104 & $05 / 30 / 07$ & 14.77 & -14.62 \\
\hline Nisqually & NIS-ES-2 & English sole adult & 2838-105 & $05 / 30 / 07$ & 13.72 & -14.82 \\
\hline Nisqually & NIS-ES-3 & English sole adult & $2838-106$ & 05/30/07 & 14.47 & -14.56 \\
\hline Nisqually & NIS-RF-1 & Spotted Ratfish & $2838-107$ & 05/30/07 & 15.24 & -13.08 \\
\hline Nisqually & NIS-RF-2 & Spotted Ratfish & $2838-108$ & 05/30/07 & 15.57 & -13.27 \\
\hline Nisqually & NIS-RF-3 & Spotted Ratfish & 2838-109 & 05/30/07 & 15.38 & -13.43 \\
\hline Hood Canal & HC-ES-1 & English sole adult & $2838-33$ & $05 / 03 / 07$ & 13.04 & -15.91 \\
\hline Hood Canal & HC-ES-2 & English sole adult & $2838-34$ & 05/03/07 & 13.33 & -15.78 \\
\hline Hood Canal & HC-ES-3 & English sole adult & $2838-35$ & 05/03/07 & 12.94 & -15.93 \\
\hline Hood Canal & HC-GC-1 & Graceful Crab & $2838-43$ & 05/03/07 & 12.59 & -15.10 \\
\hline Hood Canal & HC-GC-2 & Graceful Crab & $2838-44$ & 05/03/07 & 12.28 & -16.78 \\
\hline
\end{tabular}




\begin{tabular}{|c|c|c|c|c|c|c|}
\hline PSAMP Station & PSAMP Code & Organism & MSL Code & $\begin{array}{c}\text { Collection } \\
\text { Date } \\
\end{array}$ & $\delta^{15} \mathbf{N}$ & $\delta^{13} \mathrm{C}$ \\
\hline Hood Canal & HC-RS-1 & Rock sole & $2838-36$ & 05/03/07 & 13.18 & -14.96 \\
\hline Hood Canal & HC-SP1 & Shiner perch & $2838-40$ & 05/03/07 & 14.37 & -15.19 \\
\hline Hood Canal & $\mathrm{HC}-\mathrm{SP} 2$ & Shiner perch & $2838-41$ & 05/03/07 & 14.37 & -16.11 \\
\hline Hood Canal & HC-SP3 & Shiner perch & $2838-42$ & 05/03/07 & 14.26 & -16.15 \\
\hline Hood Canal & HC-RF-1 & Spotted Ratfish & $2838-37$ & 05/03/07 & 12.67 & -14.77 \\
\hline Hood Canal & HC-RF-2 & Spotted Ratfish & $2838-38$ & 05/03/07 & 13.36 & -14.96 \\
\hline Hood Canal & HC-RF-3 & Spotted Ratfish & $2838-39$ & 05/03/07 & 13.69 & -14.98 \\
\hline Elliott Bay & EB-ES-1 & English sole adult & $2838-45$ & 05/16/07 & 13.93 & -15.70 \\
\hline Elliott Bay & EB-ES-2 & English sole adult & $2838-46$ & 05/16/07 & 13.59 & -13.47 \\
\hline Elliott Bay & EB-ES-3 & English sole adult & $2838-47$ & 05/16/07 & 13.67 & -15.31 \\
\hline Elliott Bay & EB-RF-1 & Spotted Ratfish & $2838-48$ & 05/16/07 & 15.09 & -15.20 \\
\hline Elliott Bay & EB-RF-2 & Spotted Ratfish & $2838-49$ & 05/16/07 & 14.11 & -14.28 \\
\hline Elliott Bay & EB-RF-3 & Spotted Ratfish & $2838-50$ & 05/16/07 & 15.70 & -14.98 \\
\hline Eagle Harbor & EH-ES-1 & English sole adult & $2838-51$ & 05/19/07 & 13.51 & -14.19 \\
\hline Eagle Harbor & EH-ES-2 & English sole adult & $2838-52$ & 05/19/07 & 14.58 & -14.50 \\
\hline Eagle Harbor & EH-ES-3 & English sole adult & $2838-53$ & 05/19/07 & 13.75 & -14.78 \\
\hline Eagle Harbor & EH-ES-4 & English sole adult & $2838-54$ & $05 / 19 / 07$ & 13.36 & -14.65 \\
\hline Eagle Harbor & EH-RF-1 & Spotted Ratfish & $2838-55$ & 05/19/07 & 13.51 & -14.60 \\
\hline Eagle Harbor & EH-RF-2 & Spotted Ratfish & $2838-56$ & 05/19/07 & 14.33 & -14.26 \\
\hline Eagle Harbor & EH-RF-3 & Spotted Ratfish & $2838-57$ & 05/19/07 & 14.43 & -14.39 \\
\hline Duwamish & DU-ES-1 & English sole adult & $2838-116$ & 05/18/07 & 12.87 & -17.48 \\
\hline Duwamish & DU-ES-2 & English sole adult & $2838-117$ & 05/18/07 & 12.56 & -15.59 \\
\hline Duwamish & DU-ES-3 & English sole adult & $2838-118$ & 05/18/07 & 11.50 & -18.96 \\
\hline Duwamish & DU-ES-4 & English sole adult & $2838-119$ & 05/18/07 & 11.89 & -18.56 \\
\hline Commencement Bay & CB-ES-1 & English sole adult & $2838-110$ & $05 / 31 / 07$ & 12.59 & -16.12 \\
\hline Commencement Bay & CB-ES-2 & English sole adult & $2838-111$ & $05 / 31 / 07$ & 12.81 & -15.60 \\
\hline Commencement Bay & CB-ES-3 & English sole adult & $2838-112$ & $05 / 31 / 07$ & 11.78 & -16.99 \\
\hline Commencement Bay & CB-RF-1 & Spotted Ratfish & $2838-113$ & 05/31/07 & 13.93 & -14.29 \\
\hline Commencement Bay & CB-RF-2 & Spotted Ratfish & $2838-114$ & $05 / 31 / 07$ & 13.36 & -14.58 \\
\hline Commencement Bay & CB-RF-3 & Spotted Ratfish & $2838-115$ & $05 / 31 / 07$ & 13.56 & -14.70 \\
\hline ADUW-DOG01 & DF-SWC-1 & Section Weighted-Composite & $2838-174$ & 09/17/07 & 14.97 & -21.28 \\
\hline ADUW-DOG01 & DF-WBWC-1 & Whole Body Weighted-Composite & $2838-177$ & 09/17/07 & 15.06 & -21.27 \\
\hline ADUW-DOG02 & DF-SWC-2 & Section Weighted-Composite & $2838-175$ & 09/17/07 & 14.81 & -20.87 \\
\hline ADUW-DOG02 & DF-WBWC-2 & Whole Body Weighted-Composite & $2838-178$ & 09/17/07 & 14.46 & -21.05 \\
\hline ADUW-DOG03 & DF-WBWC-3 & Whole Body Weighted-Composite & $2838-179$ & 09/17/07 & 14.54 & -21.08 \\
\hline ADUW-DOG04 & DF-SWC-4 & Section Weighted-Composite & $2838-183$ & 09/17/07 & 14.76 & -20.91 \\
\hline ADUW-DOG04 & DF-WBWC-4 & Whole Body Weighted-Composite & $2838-180$ & 09/17/07 & 14.47 & -21.71 \\
\hline ADUW-DOG05 & DF-WBWC-5 & Whole Body Weighted-Composite & $2838-181$ & 09/17/07 & 14.12 & -20.19 \\
\hline ADUW-DOG06 & DF-SWC-6 & Section Weighted-Composite & $2838-176$ & $09 / 17 / 07$ & 14.60 & -20.17 \\
\hline ADUW-DOG06 & DF-WBWC-6 & Whole Body Weighted-Composite & $2838-182$ & 09/17/07 & 15.04 & -19.96 \\
\hline Sequim Bay & & eelgrass shoots & $2838-184$ & & 8.35 & -9.61 \\
\hline
\end{tabular}




\section{QA/QC Sample Results}




\section{QC Sample Results: Metals in Biota Tissue}

Dry Weight Basis 


\begin{tabular}{|c|c|c|c|c|c|c|c|}
\hline Client Code & Client Code & & & & Units & $\mathrm{ug} / \mathrm{g}$ dry $\mathrm{w}$ & \\
\hline Station & PSAMP Code & Organism & MSL Code & $\begin{array}{l}\text { ICP-MS QC } \\
\text { Batch ID } \\
\end{array}$ & Ag & As & Cd \\
\hline & & & & Instrument: & ICP-MS & ICP-MS & ICP-MS \\
\hline Laboratory & Method Detec & tion Limits & & & 0.002 & 0.1 & 0.002 \\
\hline Reporting I & L*3.18) & & & & 0.01 & 0.3 & 0.01 \\
\hline Procedural & & & & & & & \\
\hline MB 1 & & & Blank 013108 & 020608-6100A & $0.002 \mathrm{U}$ & $0.1 \mathrm{U}$ & $0.002 \mathrm{U}$ \\
\hline MB 2 & & & Blank 020508 & 021908-6100A & $0.002 \mathrm{U}$ & $0.1 \mathrm{U}$ & $0.002 \mathrm{U}$ \\
\hline MB 3 & & & Blank 020708 & 022008-6100A & $0.002 \mathrm{U}$ & $0.1 \mathrm{U}$ & $0.002 \mathrm{U}$ \\
\hline MB 4 & & & Blank 021408 & 022008-6100A & $0.002 \mathrm{U}$ & $0.1 \mathrm{U}$ & $0.002 \mathrm{U}$ \\
\hline MB 5 & & & Blank 041108 & 041608-6100 & $0.002 \mathrm{U}$ & $0.1 \mathrm{U}$ & $0.002 \mathrm{U}$ \\
\hline MB 6 & & & Blank 041508 & 041708-6100 & $0.002 \mathrm{U}$ & $0.138 \mathrm{~J}$ & $0.002 \mathrm{U}$ \\
\hline Laboratory & Sample Results & & & & & & \\
\hline LCS 1 & & & LCS 013108 & 020608-6100A & 4.79 & 5.15 & 5.02 \\
\hline MB 1 & & & Blank 013108 & 020608-6100A & $0.002 \mathrm{U}$ & $0.1 \mathrm{U}$ & $0.002 \mathrm{U}$ \\
\hline & Spike concentrc & ation & & & 5 & 5 & 5 \\
\hline & PERCENT RE & COVERY & & & $96 \%$ & $103 \%$ & $100 \%$ \\
\hline LCS 2 & & & LCS 020508 & 021908-6100A & 4.89 & 5.58 & 4.95 \\
\hline MB 2 & & & Blank 020508 & 021908-6100A & $0.002 \mathrm{U}$ & $0.1 \mathrm{U}$ & $0.002 \mathrm{U}$ \\
\hline & Spike concentrc & Ition & & & 5 & 5 & 5 \\
\hline & PERCENT RF & COVERY & & & $98 \%$ & $112 \%$ & $99 \%$ \\
\hline LCS 3 & & & LCS 020708 & 022008-6100A & 4.96 & 2.67 & 2.70 \\
\hline MB 3 & & & Blank 020708 & 022008-6100A & $0.002 \mathrm{U}$ & $0.1 \mathrm{U}$ & $0.002 \mathrm{U}$ \\
\hline & Spike concentrc & tion & & & 5 & 2.5 & 2.5 \\
\hline & PERCENT RE & COVERY & & & $99 \%$ & $107 \%$ & $108 \%$ \\
\hline LCS 4 & & & LCS 021408 & 022008-6100A & 4.91 & 5.46 & 5.03 \\
\hline MB 4 & & & Blank 021408 & 022008-6100A & $0.002 \mathrm{U}$ & $0.1 \mathrm{U}$ & $0.002 \mathrm{U}$ \\
\hline & Spike concentrc & ation & & & 5 & 5 & 5 \\
\hline & PERCENT RF & COVERY & & & $98 \%$ & $109 \%$ & $101 \%$ \\
\hline LCS 5 & & & Blank 041108 & 041608-6100 & 4.95 & 5.19 & 5.11 \\
\hline MB 5 & & & Blank 041108 & 041608-6100 & $0.002 \mathrm{U}$ & $0.1 \mathrm{U}$ & $0.002 \mathrm{U}$ \\
\hline & Spike concentrc & ition & & & 5 & 5 & 5 \\
\hline & PERCENT RF & COVERY & & & $99 \%$ & $104 \%$ & $102 \%$ \\
\hline LCS 6 & & & LCS 041508 & 041708-6100 & 4.86 & 5.49 & 5.37 \\
\hline MB 6 & & & Blank 041508 & 041708-6100 & $0.002 \mathrm{U}$ & $0.138 \mathrm{~J}$ & $0.002 \mathrm{U}$ \\
\hline & Spike concentrc & ation & & & 5 & 5 & 5 \\
\hline & PERCENT RE & COVERY & & & $97 \%$ & $110 \%$ & $107 \%$ \\
\hline Matrix Spik & & & & & & & \\
\hline MS 1 & V-ES-5 & English Sole & 2838-5 MS & 020608-6100A & 2.00 & 56.0 & 2.23 \\
\hline MSD 1 & V-ES-5 & English Sole & 2838-5 MSD & 020608-6100A & 1.93 & 57.5 & 2.17 \\
\hline Vendovi & V-ES-5 & English Sole & $2838-5$ & 020608-6100A & 0.0306 & 31.9 & 0.0884 \\
\hline & Spike concentrc & tion, $M S$ & & & 2.10 & 24.8 & 2.10 \\
\hline & Spike concentr & ation, $M S D$ & & & 2.04 & 24.6 & 2.04 \\
\hline & PERCENT RF & COVERY, MS & & & $94 \%$ & $97 \%$ & $102 \%$ \\
\hline & PERCENT RF & COVERY, MSD & & & $93 \%$ & $104 \%$ & $102 \%$ \\
\hline & RPD & & & & $1 \%$ & $7 \%$ & 0\% \\
\hline
\end{tabular}




\begin{tabular}{|c|c|c|c|c|c|c|c|}
\hline Client Code & Client Code & & & & Uni & $\lg / \mathrm{g}$ dry & \\
\hline Station & PSAMP Code & Organism & MSL Code & $\begin{array}{l}\text { ICP-MS QC } \\
\text { Batch ID }\end{array}$ & Ag & As & Cd \\
\hline & & & & Instrument: & ICP-MS & ICP-MS & ICP-MS \\
\hline Laboratory Acl & 1 Method Detec & tion Limits & & & 0.002 & 0.1 & 0.002 \\
\hline Reporting Limi & L*3.18) & & & & 0.01 & 0.3 & 0.01 \\
\hline MS 2 & HC-RS-1 & Rock sole & 2838-36 MS & 021908-6100A & 1.88 & 37.5 & 2.17 \\
\hline MSD 2 & HC-RS-1 & Rock sole & 2838-36 MSD & 021908-6100A & 2.04 & 39.5 & 2.09 \\
\hline Hood Canal & HC-RS-1 & Rock sole & $2838-36$ & 021908-6100A & 0.0121 & 11.2 & 0.110 \\
\hline & Spike concentrc & Ition, MS & & & 1.96 & 25.2 & 1.96 \\
\hline & Spike concentrc & ation, $M S D$ & & & 1.98 & 24.5 & 1.98 \\
\hline & PERCENT RF & COVERY, MS & & & $95 \%$ & $104 \%$ & $105 \%$ \\
\hline & PERCENT RF & COVERY, MSD & & & $102 \%$ & $116 \%$ & $100 \%$ \\
\hline & RPD & & & & $7 \%$ & $11 \%$ & $5 \%$ \\
\hline MS 3 & SI-SS-3 & Sand sole & 2838-73 MS & 022008-6100A & 1.97 & 35.6 & 2.12 \\
\hline MSD 3 & SI-SS-3 & Sand sole & 2838-73 MSD & 022008-6100A & 1.96 & 35.2 & 2.18 \\
\hline Sinclair Inlet & SI-SS-3 & Sand sole & $2838-73$ & 022008-6100A & 0.0343 & 7.64 & 0.0355 \\
\hline & Spike concentrc & ation, MS & & & 1.99 & 24.9 & 1.99 \\
\hline & Spike concentrc & Ition, MSD & & & 1.98 & 25.2 & 1.98 \\
\hline & PERCENT RF & COVERY, MS & & & $97 \%$ & $112 \%$ & $105 \%$ \\
\hline & PERCENT RE & COVERY, MSD & & & $97 \%$ & $109 \%$ & $108 \%$ \\
\hline & RPD & & & & $0 \%$ & $3 \%$ & $3 \%$ \\
\hline MS 4 & NIS-RF-2 & Spotted Ratfish & 2838-108 MS & 022008-6100A & 2.81 & 54.1 & 2.21 \\
\hline MSD 4 & NIS-RF-2 & Spotted Ratfish & 2838-108 MSD & 022008-6100A & 2.73 & 55.1 & 2.07 \\
\hline Nisqually & NIS-RF-2 & Spotted Ratfish & $2838-108$ & 022008-6100A & 0.733 & 26.9 & 0.0766 \\
\hline & Spike concentrc & ition, $M S$ & & & 2.00 & 24.1 & 2.00 \\
\hline & Spike concentrc & Ition, $M S D$ & & & 1.91 & 24.6 & 1.91 \\
\hline & PERCENT RF & COVERY, MS & & & $104 \%$ & $113 \%$ & $107 \%$ \\
\hline & PERCENT RF & COVERY, MSD & & & $105 \%$ & $115 \%$ & $104 \%$ \\
\hline & RPD & & & & $1 \%$ & $2 \%$ & $3 \%$ \\
\hline MS 5 & DF-EM-1 & Dogfish 1 (EM) & 2838-126 MS & $041608-6100$ & 2.05 & 38.1 & 2.17 \\
\hline MSD 5 & DF-EM-1 & Dogfish 1 (EM) & 2838-126 MSD & $041608-6100$ & 1.98 & 38.2 & 2.22 \\
\hline Admiralty Inlet & DF-EM-1 & Dogfish 1 (EM) & $2838-126$ & $041608-6100$ & 0.0202 & 10.9 & 0.0382 \\
\hline & Spike concentrc & ition, $M S$ & & & 2.01 & 25.0 & 2.01 \\
\hline & Spike concentrc & Ition, $M S D$ & & & 1.97 & 24.7 & 1.97 \\
\hline & PERCENT RF & COVERY, MS & & & $101 \%$ & $109 \%$ & $106 \%$ \\
\hline & PERCENT RF & COVERY, MSD & & & $99 \%$ & $111 \%$ & $111 \%$ \\
\hline & RPD & & & & $2 \%$ & $2 \%$ & $5 \%$ \\
\hline MS 6 & DF-LV-6 & Dogfish 6 & 2838-137 MS & 041708-6100 & 2.08 & 41.2 & 33.1 \\
\hline MSD 6 & DF-LV-6 & Dogfish 6 & 2838-137 MSD & $041708-6100$ & 2.14 & 42.6 & 33.8 \\
\hline Admiralty Inlet & DF-LV-6 & Dogfish 6 & $2838-137$ & 041708-6100 & 0.111 & 14.7 & 7.51 \\
\hline & Spike concentrc & ation, $M S$ & & & 1.97 & 25.0 & 25.0 \\
\hline & Spike concentrc & Ition, $M S D$ & & & 2.02 & 24.7 & 24.7 \\
\hline & PERCENT RE & COVERY, MS & & & $100 \%$ & $106 \%$ & $102 \%$ \\
\hline & PERCENT RE & COVERY, MSD & & & $100 \%$ & $113 \%$ & $106 \%$ \\
\hline & RPD & & & & 0\% & $6 \%$ & $4 \%$ \\
\hline
\end{tabular}




\begin{tabular}{|c|c|c|c|c|c|c|c|}
\hline Client Code & Client Code & & & & Uni & $\mathrm{ig} / \mathrm{g}$ dry & \\
\hline Station & PSAMP Code & Organism & MSL Code & \begin{tabular}{|l} 
ICP-MS QC \\
Batch ID
\end{tabular} & Ag & As & Cd \\
\hline & & & & Instrument: & ICP-MS & $I C P-M S$ & $I C P-M S$ \\
\hline Laboratory Ach & Method Detect & tion Limits & & & 0.002 & 0.1 & 0.002 \\
\hline Reporting Limi & L*3.18) & & & & 0.01 & 0.3 & 0.01 \\
\hline Laboratory Du & e Results & & & & & & \\
\hline Vendovi & V-SSc-3 & Staghorn sculpin & 2838-15 R1 & 020608-6100A & 0.0253 & 15.3 & 0.0956 \\
\hline Vendovi & V-SSc-3 & Staghorn sculpin & 2838-15 R2 & 020608-6100A & 0.0265 & 15.5 & 0.100 \\
\hline & MEAN & & & & 0.0259 & 15.4 & 0.0978 \\
\hline & RPD & & & & $5 \%$ & $1 \%$ & $4 \%$ \\
\hline Elliot Bay & EB-RF-1 & Spotted Ratfish & 2838-48 R1 & 021908-6100A & 7.86 & 49.2 & 0.258 \\
\hline Elliot Bay & EB-RF-1 & Spotted Ratfish & $2838-48$ R2 & 021908-6100A & 8.23 & 47.6 & 0.256 \\
\hline Elliot Bay & EB-RF-1 & Spotted Ratfish & 2838-48 DUP & 041608-6100 & 8.03 & 44.6 & 0.279 \\
\hline & MEAN & & & & 8.04 & 47.1 & 0.264 \\
\hline & RSD or RPD (N & & & & $2 \%$ & $5 \%$ & $5 \%$ \\
\hline Sinclair Inlet & SI-ES-8 & English Sole & 2838-65 R1 & 022008-6100A & 0.0273 & 17.0 & 0.0541 \\
\hline Sinclair Inlet & SI-ES-8 & English Sole & $2838-65$ R2 & 022008-6100A & 0.0295 & 16.3 & 0.0499 \\
\hline & MEAN & & & & 0.0284 & 16.7 & 0.0520 \\
\hline & RPD & & & & $\mathbf{8 \%}$ & $4 \%$ & $8 \%$ \\
\hline Sinclair Inlet & SI-GC-2/6 & Graceful Crab (2) & 2838-93-97 R1 & 022008-6100A & 1.03 & 18.3 & 0.557 \\
\hline Sinclair Inlet & SI-GC-2/6 & Graceful Crab (2) & 2838-93-97 R2 & 022008-6100A & 0.915 & 17.2 & 0.488 \\
\hline & MEAN & & & & 0.973 & 17.8 & 0.523 \\
\hline & RPD & & & & $12 \%$ & $6 \%$ & $13 \%$ \\
\hline Admiralty Inlet & DF-WBWC-1 & Dogfish 1 & 2838-177 R1 & 041608-6100 & 0.0364 & 11.1 & 0.730 \\
\hline Admiralty Inlet & DF-WBWC-1 & Dogfish 1 & 2838-177 R2 & 041608-6100 & 0.0403 & 11.6 & 0.813 \\
\hline & MEAN & & & & 0.0384 & 11.4 & 0.772 \\
\hline & RPD & & & & $10 \%$ & $4 \%$ & $11 \%$ \\
\hline Admiralty Inlet & DF-WBWC-6 & Dogfish 6 & 2838-182 R1 & 041708-6100 & 0.0174 & 11.3 & 2.63 \\
\hline Admiralty Inlet & DF-WBWC-6 & Dogfish 6 & 2838-182 R2 & 041708-6100 & 0.0142 & 10.7 & 2.33 \\
\hline & MEAN & & & & 0.0158 & 11.0 & 2.48 \\
\hline & RPD & & & & $20 \%$ & $5 \%$ & $12 \%$ \\
\hline Vendovi & V-SSc-1 & Staghorn sculpin & $2838-13$ & 020608-6100A & 0.125 & 13.6 & 0.167 \\
\hline Vendovi & V-SSc-1 & Staghorn sculpin & 2838-13 DUP & 041608-6100 & 0.121 & 12.9 & 0.153 \\
\hline & MEAN & & & & 0.123 & 13.3 & 0.160 \\
\hline & RPD & & & & $3 \%$ & $5 \%$ & $9 \%$ \\
\hline Hood Canal & HC-RF-1 & Spotted Ratfish & 2838-37 & 021908-6100A & 1.27 & 28.2 & 0.304 \\
\hline Hood Canal & HC-RF-1 & Spotted Ratfish & 2838-37 DUP & 041608-6100 & 1.18 & 27.8 & 0.350 \\
\hline & MEAN & & & & 1.23 & 28.0 & 0.327 \\
\hline & RPD & & & & $7 \%$ & $1 \%$ & $14 \%$ \\
\hline Hood Canal & HC-RF-3 & Spotted Ratfish & 2838-39 & 021908-6100A & 5.86 & 35.5 & 0.201 \\
\hline Hood Canal & HC-RF-3 & Spotted Ratfish & 2838-39 DUP & 041608-6100 & 4.42 & 30.6 & 0.181 \\
\hline & MEAN & & & & 5.14 & 33.1 & 0.191 \\
\hline & RPD & & & & $28 \%$ & $15 \%$ & $10 \%$ \\
\hline
\end{tabular}




\begin{tabular}{|c|c|c|c|c|c|c|c|}
\hline Client Code & Client Code & & & & Units = & $\mathrm{g} / \mathrm{g}$ dry & \\
\hline Station & PSAMP Code & Organism & MSL Code & $\begin{array}{l}\text { ICP-MS QC } \\
\text { Batch ID }\end{array}$ & Ag & As & Cd \\
\hline & & & & Instrument: & ICP-MS & ICP-MS & ICP-MS \\
\hline Laboratory Achieve & Method Detect & tion Limits & & & 0.002 & 0.1 & 0.002 \\
\hline Reporting Limit (MI & L*3.18) & & & & 0.01 & 0.3 & 0.01 \\
\hline Hood Canal & HC-GC-1 & Graceful Crab & $2838-43$ & 021908-6100A & 1.11 & 17.6 & 2.42 \\
\hline Hood Canal & HC-GC-1 & Graceful Crab & 2838-43 DUP & 041608-6100 & 1.03 & 16.9 & 2.30 \\
\hline & MEAN & & & & 1.07 & 17.3 & 2.36 \\
\hline & RPD & & & & $7 \%$ & $4 \%$ & $5 \%$ \\
\hline Sinclair Inlet & SI-RF-1 & Spotted Ratfish & $2838-83$ & 022008-6100A & 2.25 & 29.2 & 0.115 \\
\hline Sinclair Inlet & SI-RF-1 & Spotted Ratfish & 2838-83 DUP & 041608-6100 & 2.06 & 27.1 & 0.112 \\
\hline & MEAN & & & & 2.16 & 28.2 & 0.114 \\
\hline & RPD & & & & $9 \%$ & $7 \%$ & $3 \%$ \\
\hline Elliott Bay & EB-RF-3 & Spotted Ratfish & $2838-50$ & 021908-6100A & 3.08 & 24.7 & 0.0780 \\
\hline Elliott Bay & EB-RF-3 & Spotted Ratfish & 2838-50 DUP & 041708-6100 & 2.02 & 25.5 & 0.0706 \\
\hline & MEAN & & & & 2.55 & 25.1 & 0.0743 \\
\hline & RPD & & & & $42 \% *$ & $3 \%$ & $10 \%$ \\
\hline Sinclair Inlet & SI-ES-2 & English Sole & $2838-59$ & 022008-6100A & 0.0272 & 17.6 & 0.0843 \\
\hline Sinclair Inlet & SI-ES-2 & English Sole & 2838-59 DUP & 041708-6100 & 0.0269 & 18.4 & 0.0781 \\
\hline & MEAN & & & & 0.0271 & 18.0 & 0.0812 \\
\hline & RPD & & & & $1 \%$ & $4 \%$ & $8 \%$ \\
\hline Sinclair Inlet & SI-RS-4 & Rock sole & $2838-70$ & 022008-6100A & $0.002 \mathrm{U}$ & 32.9 & 0.0343 \\
\hline Sinclair Inlet & SI-RS-4 & Rock sole & 2838-70 DUP & 041708-6100 & $0.002 \mathrm{U}$ & 37.8 & 0.0369 \\
\hline & MEAN & & & & NA & 35.4 & 0.0356 \\
\hline & RPD & & & & NA & $14 \%$ & $7 \%$ \\
\hline Sinclair Inlet & SI-SSc-1/2 & Staghorn sculpin (2) & $2838-77-78$ & 022008-6100A & 0.137 & 8.39 & 0.0682 \\
\hline Sinclair Inlet & SI-SSc-1/2 & Staghorn sculpin (2) & 2838-77-78 DUP & 041708-6100 & 0.116 & 9.09 & 0.0625 \\
\hline & MEAN & & & & 0.127 & 8.74 & 0.0654 \\
\hline & RPD & & & & $17 \%$ & $8 \%$ & $9 \%$ \\
\hline Nisqually & NIS-RF-1 & Spotted Ratfish & $2838-107$ & 022008-6100A & 4.90 & 34.8 & 0.243 \\
\hline Nisqually & NIS-RF-1 & Spotted Ratfish & 2838-107 DUP & 041708-6100 & 5.33 & 40.1 & 0.248 \\
\hline & MEAN & & & & 5.12 & 37.5 & 0.246 \\
\hline & RPD & & & & $8 \%$ & $14 \%$ & $2 \%$ \\
\hline Nisqually & NIS-RF-3 & Spotted Ratfish & 2838-109 & 022008-6100A & 2.14 & 27.2 & 0.531 \\
\hline Nisqually & NIS-RF-3 & Spotted Ratfish & 2838-109 DUP & 041708-6100 & 1.84 & 30.7 & 0.505 \\
\hline & MEAN & & & & 1.99 & 29.0 & 0.518 \\
\hline & RPD & & & & $15 \%$ & $12 \%$ & $5 \%$ \\
\hline Commencement Bay & CB-ES-3 & English Sole & 2838-112 & 022008-6100A & 0.0638 & 19.7 & 0.0939 \\
\hline Commencement Bay & CB-ES-3 & English Sole & 2838-112 DUP & 041708-6100 & 0.0645 & 21.7 & 0.0873 \\
\hline & MEAN & & & & 0.0642 & 20.7 & 0.0906 \\
\hline & RPD & & & & $1 \%$ & $10 \%$ & $7 \%$ \\
\hline
\end{tabular}




\begin{tabular}{|c|c|c|c|c|c|c|c|}
\hline Client Code & Client Code & & & & Uni & $\lg / \mathrm{g}$ dry & \\
\hline Station & PSAMP Code & Organism & MSL Code & $\begin{array}{l}\text { ICP-MS QC } \\
\text { Batch ID }\end{array}$ & Ag & As & Cd \\
\hline & & & & Instrument: & ICP-MS & ICP-MS & ICP-MS \\
\hline Laboratory & Method Detec & tion Limits & & & 0.002 & 0.1 & 0.002 \\
\hline Reporting I & L*3.18) & & & & 0.01 & 0.3 & 0.01 \\
\hline Standard R & Material & & & & & & \\
\hline SRM 1 & & Dogfish Muscle & DORM-2 0131 & 020608-6100A & NA & 18.1 & 0.0431 \\
\hline SRM 1 & & Dogfish Muscle & DORM-2 0205 & 021908-6100A & NA & 17.5 & 0.0433 \\
\hline SRM 1 & & Dogfish Muscle & DORM-2 0207 & 022008-6100A & NA & 17.4 & 0.0464 \\
\hline SRM 1 & & Dogfish Muscle & DORM-2 0214 & 022008-6100A & NA & 18.1 & 0.0433 \\
\hline SRM 1 & & Dogfish Muscle & DORM-2 0411 & 041608-6100 & NA & 17.0 & 0.0426 \\
\hline SRM 1 & & Dogfish Muscle & DORM-2 0415 & 041708-6100 & NA & 18.9 & 0.0457 \\
\hline & Certified Value & & & & NA & 18 & 0.043 \\
\hline & Range & & & & NA & \pm 1.1 & \pm 0.008 \\
\hline & Percent Differ & ence & & & NA & $1 \%$ & 0\% \\
\hline & Percent Differ & ence & & & NA & $3 \%$ & $1 \%$ \\
\hline & Percent Differ & ence & & & NA & $3 \%$ & $8 \%$ \\
\hline & Percent Differ & ence & & & NA & $1 \%$ & $1 \%$ \\
\hline & Percent Differ & ence & & & NA & $6 \%$ & $1 \%$ \\
\hline & Percent Differ & ence & & & NA & $5 \%$ & $6 \%$ \\
\hline SRM 2 & & Oyster Tissue & 1566b 013108 & 020608-6100A & 0.580 & 7.10 & 2.42 \\
\hline SRM 2 & & Oyster Tissue & $1566 \mathrm{~b} 020508$ & 021908-6100A & 0.604 & 6.94 & 2.52 \\
\hline SRM 2 & & Oyster Tissue & $1566 b 020708$ & 022008-6100A & 0.586 & 7.18 & 2.46 \\
\hline SRM 2 & & Oyster Tissue & $1566 \mathrm{~b} 021408$ & 022008-6100A & 0.600 & 7.32 & 2.43 \\
\hline SRM 2 & & Oyster Tissue & 1566b 041408 & 041608-6100 & 0.583 & 7.62 & 2.36 \\
\hline SRM 2 & & Oyster Tissue & 1566b 041508 & 041708-6100 & 0.612 & 7.14 & 2.48 \\
\hline & Certified Value & & & & 0.666 & 7.65 & 2.48 \\
\hline & Range & & & & \pm 0.01 & \pm 0.65 & \pm 0.08 \\
\hline & Percent Differ & ence & & & $13 \%$ & $7 \%$ & $2 \%$ \\
\hline & Percent Differ & ence & & & $9 \%$ & $9 \%$ & $2 \%$ \\
\hline & Percent Differ & ence & & & $12 \%$ & $6 \%$ & $1 \%$ \\
\hline & Percent Differ & ence & & & $10 \%$ & $4 \%$ & $2 \%$ \\
\hline & Percent Differ & ence & & & $12 \%$ & $0 \%$ & $5 \%$ \\
\hline & Percent Differ & ence & & & $8 \%$ & $7 \%$ & $0 \%$ \\
\hline
\end{tabular}




\begin{tabular}{|c|c|c|c|c|c|c|c|c|}
\hline Client Code & Client Code & & & & & nits $=\mu \mathrm{g} / \mathrm{g}$ & ry weight & \\
\hline Station & PSAMP Code & Organism & MSL Code & \begin{tabular}{|l} 
ICP-MS QC \\
Batch ID
\end{tabular} & $\mathrm{Cu}$ & $\mathbf{N i}$ & $\mathbf{P b}$ & Zn \\
\hline & & & & Instrument: & $I C P-M S$ & ICP-MS & $I C P-M S$ & ICP-MS \\
\hline Laboratory & I Method Detec & ion Limits & & & 0.09 & 0.04 & 0.003 & 0.1 \\
\hline Reporting I & L*3.18) & & & & 0.3 & 0.1 & 0.01 & 0.3 \\
\hline Procedural & & & & & & & & \\
\hline MB 1 & & & Blank 013108 & 020608-6100A & $0.09 \mathrm{U}$ & $0.04 \mathrm{U}$ & $0.0086 \mathrm{~J}$ & $0.1 \mathrm{U}$ \\
\hline MB 2 & & & Blank 020508 & 021908-6100A & $0.09 \mathrm{U}$ & $0.04 \mathrm{U}$ & $0.003 \mathrm{U}$ & 0.343 \\
\hline MB 3 & & & Blank 020708 & 022008-6100A & $0.09 \mathrm{U}$ & $0.04 \mathrm{U}$ & $0.003 \mathrm{U}$ & $0.1 \mathrm{U}$ \\
\hline MB 4 & & & Blank 021408 & 022008-6100A & $0.09 \mathrm{U}$ & $0.04 \mathrm{U}$ & $0.003 \mathrm{U}$ & $0.1 \mathrm{U}$ \\
\hline MB 5 & & & Blank 041108 & 041608-6100 & $0.09 \mathrm{U}$ & $0.04 \mathrm{U}$ & $0.003 \mathrm{U}$ & 0.621 \\
\hline MB 6 & & & Blank 041508 & 041708-6100 & $0.09 \mathrm{U}$ & $0.04 \mathrm{U}$ & $0.003 \mathrm{U}$ & $0.1 \mathrm{U}$ \\
\hline Laboratory & Sample Results & & & & & & & \\
\hline LCS 1 & & & LCS 013108 & 020608-6100A & 5.05 & 4.99 & 5.05 & 4.89 \\
\hline MB 1 & & & Blank 013108 & 020608-6100A & $0.09 \mathrm{U}$ & $0.04 \mathrm{U}$ & $0.0086 \mathrm{~J}$ & $0.05 \mathrm{U}$ \\
\hline & Spike concentrc & tion & & & 5 & 5 & 5 & 5 \\
\hline & PERCENT RE & COVERY & & & $101 \%$ & $100 \%$ & $101 \%$ & $98 \%$ \\
\hline LCS 2 & & & LCS 020508 & 021908-6100A & 5.08 & 4.94 & 5.03 & 5.80 \\
\hline MB 2 & & & Blank 020508 & 021908-6100A & $0.09 \mathrm{U}$ & $0.04 \mathrm{U}$ & $0.003 \mathrm{U}$ & 0.343 \\
\hline & Spike concentrc & ition & & & 5 & 5 & 5 & 5 \\
\hline & PERCENT RE & COVERY & & & $102 \%$ & $99 \%$ & $101 \%$ & $109 \%$ \\
\hline LCS 3 & & & LCS 020708 & 022008-6100A & 2.67 & 2.62 & 2.63 & 2.71 \\
\hline MB 3 & & & Blank 020708 & 022008-6100A & $0.09 \mathrm{U}$ & $0.04 \mathrm{U}$ & $0.003 \mathrm{U}$ & $0.1 \mathrm{U}$ \\
\hline & Spike concentro & ition & & & 2.5 & 2.5 & 2.5 & 2.5 \\
\hline & PERCENT RE & COVERY & & & $107 \%$ & $105 \%$ & $105 \%$ & $108 \%$ \\
\hline LCS 4 & & & LCS 021408 & 022008-6100A & 5.02 & 5.00 & 4.93 & 5.26 \\
\hline MB 4 & & & Blank 021408 & 022008-6100A & $0.09 \mathrm{U}$ & $0.04 \mathrm{U}$ & $0.003 \mathrm{U}$ & $0.1 \mathrm{U}$ \\
\hline & Spike concentrc & ition & & & 5 & 5 & 5 & 5 \\
\hline & PERCENT RE & COVERY & & & $100 \%$ & $100 \%$ & $99 \%$ & $105 \%$ \\
\hline LCS 5 & & & Blank 041108 & 041608-6100 & 5.22 & 5.15 & 5.10 & 5.79 \\
\hline MB 5 & & & Blank 041108 & $041608-6100$ & $0.09 \mathrm{U}$ & $0.04 \mathrm{U}$ & $0.003 \mathrm{U}$ & 0.621 \\
\hline & Spike concentrc & ition & & & 5 & 5 & 5 & 5 \\
\hline & PERCENT RE & COVERY & & & $104 \%$ & $103 \%$ & $102 \%$ & $103 \%$ \\
\hline LCS 6 & & & LCS 041508 & 041708-6100 & 5.50 & 5.36 & 5.26 & 5.60 \\
\hline MB 6 & & & Blank 041508 & $041708-6100$ & $0.09 \mathrm{U}$ & $0.04 \mathrm{U}$ & $0.003 \mathrm{U}$ & $0.1 \mathrm{U}$ \\
\hline & Spike concentrc & ition & & & 5 & 5 & 5 & 5 \\
\hline & PERCENT RE & COVERY & & & $110 \%$ & $107 \%$ & $105 \%$ & $112 \%$ \\
\hline Matrix Spik & & & & & & & & \\
\hline MS 1 & V-ES-5 & English Sole & 2838-5 MS & 020608-6100A & 25.6 & 24.8 & 2.92 & 151 \\
\hline MSD 1 & V-ES-5 & English Sole & 2838-5 MSD & 020608-6100A & 26.0 & 24.7 & 2.85 & 154 \\
\hline Vendovi & V-ES-5 & English Sole & $2838-5$ & 020608-6100A & 3.43 & 2.07 & 0.683 & 58.8 \\
\hline & Spike concentrc & tion, $M S$ & & & 24.8 & 24.8 & 2.10 & 104 \\
\hline & Spike concentrc & Ition, $M S D$ & & & 24.6 & 24.6 & 2.04 & 103 \\
\hline & PERCENT RE & COVERY, MS & & & $89 \%$ & $92 \%$ & $107 \%$ & $89 \%$ \\
\hline & PERCENT RE & COVERY, MSD & & & $92 \%$ & $92 \%$ & $106 \%$ & $92 \%$ \\
\hline & RPD & & & & $3 \%$ & 0\% & $1 \%$ & $3 \%$ \\
\hline
\end{tabular}




\begin{tabular}{|c|c|c|c|c|c|c|c|c|}
\hline Client Code & Client Code & & & & & Inits $=\mu \mathrm{g} / \mathrm{g}$ & dry weight & \\
\hline Station & PSAMP Code & Organism & MSL Code & \begin{tabular}{|l} 
ICP-MS QC \\
Batch ID
\end{tabular} & $\mathbf{C u}$ & $\mathbf{N i}$ & $\mathbf{P b}$ & Zn \\
\hline & & & & Instrument: & $I C P-M S$ & ICP-MS & $I C P-M S$ & $I C P-M S$ \\
\hline Laboratory Acl & I Method Detec & ion Limits & & & 0.09 & 0.04 & 0.003 & 0.1 \\
\hline Reporting Limi & L*3.18) & & & & 0.3 & 0.1 & 0.01 & 0.3 \\
\hline MS 2 & HC-RS-1 & Rock sole & 2838-36 MS & 021908-6100A & 26.0 & 25.4 & 2.30 & 167 \\
\hline MSD 2 & HC-RS-1 & Rock sole & 2838-36 MSD & 021908-6100A & 26.5 & 26.6 & 2.22 & 172 \\
\hline Hood Canal & HC-RS-1 & Rock sole & $2838-36$ & 021908-6100A & 2.09 & 1.77 & 0.158 & 67.0 \\
\hline & Spike concentrc & tion, $M S$ & & & 25.2 & 25.2 & 1.96 & 106 \\
\hline & Spike concentrc & Ition, MSD & & & 24.5 & 24.5 & 1.98 & 103 \\
\hline & PERCENT RE & COVERY, MS & & & $95 \%$ & $94 \%$ & $109 \%$ & $94 \%$ \\
\hline & PERCENT RE & COVERY, MSD & & & $100 \%$ & $101 \%$ & $104 \%$ & $102 \%$ \\
\hline & RPD & & & & $5 \%$ & $7 \%$ & $5 \%$ & $8 \%$ \\
\hline MS 3 & SI-SS-3 & Sand sole & 2838-73 MS & 022008-6100A & 28.6 & 26.9 & 2.40 & 180 \\
\hline MSD 3 & SI-SS-3 & Sand sole & 2838-73 MSD & 022008-6100A & 28.4 & 27.5 & 2.38 & 182 \\
\hline Sinclair Inlet & SI-SS-3 & Sand sole & $2838-73$ & 022008-6100A & 4.09 & 2.40 & 0.323 & 77.6 \\
\hline & Spike concentrc & Ition, MS & & & 24.9 & 24.9 & 1.99 & 105 \\
\hline & Spike concentrc & ition, $M S D$ & & & 25.2 & 25.2 & 1.98 & 106 \\
\hline & PERCENT RE & COVERY, MS & & & $98 \%$ & $98 \%$ & $104 \%$ & $98 \%$ \\
\hline & PERCENT RE & COVERY, MSD & & & $96 \%$ & $100 \%$ & $104 \%$ & $98 \%$ \\
\hline & RPD & & & & $2 \%$ & $2 \%$ & $0 \%$ & 0\% \\
\hline MS 4 & NIS-RF-2 & Spotted Ratfish & 2838-108 MS & 022008-6100A & 28.9 & 2.24 & 2.12 & 129 \\
\hline MSD 4 & NIS-RF-2 & Spotted Ratfish & 2838-108 MSD & 022008-6100A & 29.9 & 2.17 & 2.03 & 133 \\
\hline Nisqually & NIS-RF-2 & Spotted Ratfish & $2838-108$ & 022008-6100A & 3.60 & 0.215 & 0.123 & 24.3 \\
\hline & Spike concentrc & ition, $M S$ & & & 24.1 & 2.00 & 2.0 & 102 \\
\hline & Spike concentrc & ition, $M S D$ & & & 24.6 & 1.91 & 1.9 & 104 \\
\hline & PERCENT RE & COVERY, MS & & & $105 \%$ & $101 \%$ & $100 \%$ & $103 \%$ \\
\hline & PERCENT RE & COVERY, MSD & & & $107 \%$ & $102 \%$ & $100 \%$ & $105 \%$ \\
\hline & RPD & & & & $2 \%$ & $1 \%$ & $\mathbf{0 \%}$ & $2 \%$ \\
\hline MS 5 & DF-EM-1 & Dogfish 1 (EM) & 2838-126 MS & 041608-6100 & 28.5 & 2.29 & 2.09 & 147 \\
\hline MSD 5 & DF-EM-1 & Dogfish 1 (EM) & 2838-126 MSD & $041608-6100$ & 27.9 & 2.31 & 2.13 & 147 \\
\hline Admiralty Inlet & DF-EM-1 & Dogfish 1 (EM) & $2838-126$ & 041608-6100 & 1.96 & $0.04 \mathrm{U}$ & 0.0106 & 24.5 \\
\hline & Spike concentrc & ition, MS & & & 25.0 & 2.01 & 2.01 & 105 \\
\hline & Spike concentrc & ition, $M S D$ & & & 24.7 & 1.97 & 1.97 & 104 \\
\hline & PERCENT RE & COVERY, MS & & & $106 \%$ & $114 \%$ & $103 \%$ & $117 \%$ \\
\hline & PERCENT RE & COVERY, MSD & & & $105 \%$ & $117 \%$ & $108 \%$ & $118 \%$ \\
\hline & RPD & & & & $1 \%$ & $3 \%$ & $5 \%$ & $1 \%$ \\
\hline MS 6 & DF-LV-6 & Dogfish 6 & 2838-137 MS & 041708-6100 & 30.9 & 1.81 & 1.83 & 135 \\
\hline MSD 6 & DF-LV-6 & Dogfish 6 & 2838-137 MSD & 041708-6100 & 31.5 & 2.39 & 2.32 & 142 \\
\hline Admiralty Inlet & DF-LV-6 & Dogfish 6 & $2838-137$ & 041708-6100 & 5.82 & $0.04 \mathrm{U}$ & $0.00316 \mathrm{~J}$ & 25.3 \\
\hline & Spike concentrc & tion, $M S$ & & & 25.0 & 1.97 & 1.97 & 105 \\
\hline & Spike concentrc & ition, MSD & & & 24.7 & 2.02 & 2.02 & 104 \\
\hline & PERCENT RE & COVERY, MS & & & $100 \%$ & $92 \%$ & $93 \%$ & $104 \%$ \\
\hline & PERCENT RE & COVERY, MSD & & & $104 \%$ & $118 \%$ & $115 \%$ & $112 \%$ \\
\hline & RPD & & & & $4 \%$ & $25 \%$ & $21 \%$ & $7 \%$ \\
\hline
\end{tabular}




\begin{tabular}{|c|c|c|c|c|c|c|c|c|}
\hline Client Code & Client Code & & & & & nits $=\mu \mathrm{g} / \mathrm{g}$ & dry weight & \\
\hline Station & PSAMP Code & Organism & MSL Code & $\begin{array}{l}\text { ICP-MS QC } \\
\text { Batch ID } \\
\end{array}$ & $\mathbf{C u}$ & Ni & $\mathbf{P b}$ & Zn \\
\hline & & & & Instrument: & ICP-MS & ICP-MS & ICP-MS & ICP-MS \\
\hline Laboratory Ach & Method Detect & tion Limits & & & 0.09 & 0.04 & 0.003 & 0.1 \\
\hline Reporting Limi & OL*3.18) & & & & 0.3 & 0.1 & 0.01 & 0.3 \\
\hline Laboratory Du & e Results & & & & & & & \\
\hline Vendovi & V-SSc-3 & Staghorn sculpin & 2838-15 R1 & 020608-6100A & 3.75 & 1.28 & 0.0601 & 59.1 \\
\hline Vendovi & V-SSc-3 & Staghorn sculpin & 2838-15 R2 & 020608-6100A & 4.17 & 1.18 & 0.0594 & 60.7 \\
\hline & MEAN & & & & 3.96 & 1.23 & 0.0598 & 59.9 \\
\hline & RPD & & & & $11 \%$ & $8 \%$ & $1 \%$ & $3 \%$ \\
\hline Elliot Bay & EB-RF-1 & Spotted Ratfish & 2838-48 R1 & 021908-6100A & 4.30 & 0.435 & 0.233 & 23.7 \\
\hline Elliot Bay & EB-RF-1 & Spotted Ratfish & $2838-48 \mathrm{R} 2$ & 021908-6100A & 3.83 & & 0.252 & 23.5 \\
\hline Elliot Bay & EB-RF-1 & Spotted Ratfish & 2838-48 DUP & 041608-6100 & 3.88 & 0.464 & 0.222 & 24.1 \\
\hline & MEAN & & & & 4.00 & 0.450 & 0.236 & 23.8 \\
\hline & RSD or RPD (N & & & & $6 \%$ & $6 \%$ & $6 \%$ & $1 \%$ \\
\hline Sinclair Inlet & SI-ES-8 & English Sole & 2838-65 R1 & 022008-6100A & 4.22 & 1.76 & 1.85 & 77.7 \\
\hline Sinclair Inlet & SI-ES-8 & English Sole & $2838-65$ R2 & 022008-6100A & 4.10 & 1.97 & 1.84 & 77.7 \\
\hline & MEAN & & & & 4.16 & 1.87 & 1.85 & 77.7 \\
\hline & RPD & & & & $3 \%$ & $11 \%$ & $1 \%$ & 0\% \\
\hline Sinclair Inlet & SI-GC-2/6 & Graceful Crab (2) & 2838-93-97 R1 & 022008-6100A & 66.1 & 4.82 & 5.82 & 152 \\
\hline Sinclair Inlet & SI-GC-2/6 & Graceful Crab (2) & 2838-93-97 R2 & 022008-6100A & 58.8 & 5.04 & 5.46 & 138 \\
\hline & MEAN & & & & 62.5 & 4.93 & 5.64 & 145 \\
\hline & RPD & & & & $12 \%$ & $4 \%$ & $6 \%$ & $10 \%$ \\
\hline Admiralty Inlet & DF-WBWC-1 & Dogfish 1 & 2838-177 R1 & 041608-6100 & 1.54 & 0.156 & $0.00832 \mathrm{~J}$ & 21.4 \\
\hline Admiralty Inlet & DF-WBWC-1 & Dogfish 1 & 2838-177 R2 & 041608-6100 & 1.57 & 0.101 & $0.00811 \mathrm{~J}$ & 21.8 \\
\hline & MEAN & & & & 1.56 & 0.129 & $0.00822 \mathrm{~J}$ & 21.6 \\
\hline & RPD & & & & $2 \%$ & $43 \% *$ & $3 \%$ & $2 \%$ \\
\hline Admiralty Inlet & DF-WBWC-6 & Dogfish 6 & 2838-182 R1 & 041708-6100 & 2.72 & 0.555 & 0.0543 & 49.9 \\
\hline Admiralty Inlet & DF-WBWC-6 & Dogfish 6 & 2838-182 R2 & 041708-6100 & 2.14 & 0.558 & 0.0591 & 44.2 \\
\hline & MEAN & & & & 2.43 & 0.557 & 0.0567 & 47.1 \\
\hline & RPD & & & & $24 \%$ & $1 \%$ & $8 \%$ & $12 \%$ \\
\hline Vendovi & V-SSc-1 & Staghorn sculpin & $2838-13$ & 020608-6100A & 10.2 & 1.31 & 0.133 & 61.8 \\
\hline Vendovi & V-SSc-1 & Staghorn sculpin & 2838-13 DUP & 041608-6100 & 9.58 & 1.28 & 0.136 & 56.5 \\
\hline & MEAN & & & & 9.89 & 1.30 & 0.135 & 59.2 \\
\hline & RPD & & & & $6 \%$ & $2 \%$ & $2 \%$ & $9 \%$ \\
\hline Hood Canal & HC-RF-1 & Spotted Ratfish & $2838-37$ & 021908-6100A & 3.61 & 0.866 & NA & 27.7 \\
\hline Hood Canal & HC-RF-1 & Spotted Ratfish & 2838-37 DUP & 041608-6100 & 4.22 & 0.750 & 0.0927 & 24.1 \\
\hline & MEAN & & & & 3.92 & 0.808 & NA & 25.9 \\
\hline & RPD & & & & $16 \%$ & $14 \%$ & NA & $14 \%$ \\
\hline Hood Canal & HC-RF-3 & Spotted Ratfish & 2838-39 & 021908-6100A & 4.60 & 0.129 & 0.0417 & 26.2 \\
\hline Hood Canal & HC-RF-3 & Spotted Ratfish & 2838-39 DUP & 041608-6100 & 3.91 & 0.112 & 0.0410 & 22.5 \\
\hline & MEAN & & & & 4.26 & 0.121 & 0.0414 & 24.4 \\
\hline & RPD & & & & $16 \%$ & $14 \%$ & $2 \%$ & $15 \%$ \\
\hline
\end{tabular}




\begin{tabular}{|c|c|c|c|c|c|c|c|c|}
\hline Client Code & Client Code & & & & & Inits $=\mu \mathrm{g} /$ & ry weigh & \\
\hline Station & PSAMP Code & Organism & MSL Code & \begin{tabular}{|l} 
ICP-MS QC \\
Batch ID
\end{tabular} & $\mathrm{Cu}$ & $\mathbf{N i}$ & $\mathbf{P b}$ & Zn \\
\hline & & & & Instrument: & $I C P-M S$ & $I C P-M S$ & $I C P-M S$ & $I C P-M S$ \\
\hline Laboratory Achievec & d Method Detect & tion Limits & & & 0.09 & 0.04 & 0.003 & 0.1 \\
\hline Reporting Limit (MI & DL*3.18) & & & & 0.3 & 0.1 & 0.01 & 0.3 \\
\hline Hood Canal & HC-GC-1 & Graceful Crab & $2838-43$ & 021908-6100A & 46.9 & 4.58 & 0.298 & 80.6 \\
\hline Hood Canal & HC-GC-1 & Graceful Crab & 2838-43 DUP & 041608-6100 & 43.3 & 4.16 & 0.299 & 70.4 \\
\hline & MEAN & & & & 45.1 & 4.37 & 0.299 & 75.5 \\
\hline & RPD & & & & $8 \%$ & $10 \%$ & 0\% & $14 \%$ \\
\hline Sinclair Inlet & SI-RF-1 & Spotted Ratfish & $2838-83$ & 022008-6100A & 4.64 & 0.272 & 0.222 & 23.1 \\
\hline Sinclair Inlet & SI-RF-1 & Spotted Ratfish & 2838-83 DUP & $041608-6100$ & 4.46 & 0.244 & 0.201 & 21.6 \\
\hline & MEAN & & & & 4.55 & 0.258 & 0.212 & 22.4 \\
\hline & RPD & & & & $4 \%$ & $11 \%$ & $10 \%$ & $7 \%$ \\
\hline Elliott Bay & EB-RF-3 & Spotted Ratfish & $2838-50$ & 021908-6100A & 4.66 & 0.106 & 0.141 & 25.0 \\
\hline Elliott Bay & EB-RF-3 & Spotted Ratfish & 2838-50 DUP & 041708-6100 & 4.54 & 0.127 & 0.125 & 23.0 \\
\hline & MEAN & & & & 4.60 & 0.117 & 0.133 & 24.0 \\
\hline & RPD & & & & $3 \%$ & $18 \%$ & $12 \%$ & $8 \%$ \\
\hline Sinclair Inlet & SI-ES-2 & English Sole & $2838-59$ & 022008-6100A & 4.37 & 1.15 & 5.87 & 66.2 \\
\hline Sinclair Inlet & SI-ES-2 & English Sole & 2838-59 DUP & 041708-6100 & 5.45 & 1.30 & NA & 61.1 \\
\hline & MEAN & & & & 4.91 & 1.23 & NA & 63.7 \\
\hline & RPD & & & & $22 \%$ & $12 \%$ & NA & $8 \%$ \\
\hline Sinclair Inlet & SI-RS-4 & Rock sole & $2838-70$ & 022008-6100A & 1.78 & 3.38 & 2.43 & 111 \\
\hline Sinclair Inlet & SI-RS-4 & Rock sole & 2838-70 DUP & $041708-6100$ & 1.98 & 3.00 & 2.68 & 102 \\
\hline & MEAN & & & & 1.88 & 3.19 & 2.56 & 107 \\
\hline & RPD & & & & $11 \%$ & $12 \%$ & $10 \%$ & $8 \%$ \\
\hline Sinclair Inlet & SI-SSc-1/2 & Staghorn sculpin (2) & $2838-77-78$ & 022008-6100A & 9.87 & 2.12 & 1.17 & 73.4 \\
\hline Sinclair Inlet & SI-SSc-1/2 & Staghorn sculpin (2) & 2838-77-78 DUP & 041708-6100 & 9.40 & 1.93 & 1.14 & 65.9 \\
\hline & MEAN & & & & 9.64 & 2.03 & 1.16 & 69.7 \\
\hline & RPD & & & & $5 \%$ & $9 \%$ & $3 \%$ & $11 \%$ \\
\hline Nisqually & NIS-RF-1 & Spotted Ratfish & $2838-107$ & 022008-6100A & 4.76 & $0.0905 \mathrm{~J}$ & 0.108 & 22.5 \\
\hline Nisqually & NIS-RF-1 & Spotted Ratfish & 2838-107 DUP & 041708-6100 & 4.70 & $0.0955 \mathrm{~J}$ & 0.121 & 23.1 \\
\hline & MEAN & & & & 4.73 & $0.0930 \mathrm{~J}$ & 0.115 & 22.8 \\
\hline & RPD & & & & $1 \%$ & $5 \%$ & $11 \%$ & $3 \%$ \\
\hline Nisqually & NIS-RF-3 & Spotted Ratfish & 2838-109 & 022008-6100A & 6.17 & 0.296 & 0.0609 & 28.0 \\
\hline Nisqually & NIS-RF-3 & Spotted Ratfish & 2838-109 DUP & 041708-6100 & 6.41 & 0.345 & 0.0661 & 28.8 \\
\hline & MEAN & & & & 6.29 & 0.321 & 0.0635 & 28.4 \\
\hline & RPD & & & & $4 \%$ & $15 \%$ & $\mathbf{8 \%}$ & $3 \%$ \\
\hline Commencement Bay & CB-ES-3 & English Sole & $2838-112$ & 022008-6100A & 6.51 & 2.01 & 2.30 & 74.3 \\
\hline Commencement Bay & CB-ES-3 & English Sole & 2838-112 DUP & $041708-6100$ & 6.06 & 2.29 & 2.29 & 69.4 \\
\hline & MEAN & & & & 6.29 & 2.15 & 2.30 & 71.9 \\
\hline & RPD & & & & $7 \%$ & $13 \%$ & $0 \%$ & $7 \%$ \\
\hline
\end{tabular}




\begin{tabular}{|c|c|c|c|c|c|c|c|c|}
\hline Client Code & Client Code & & & & \multicolumn{4}{|c|}{ Units $=\mu \mathrm{g} / \mathrm{g}$ dry weight } \\
\hline \multirow[t]{2}{*}{ Station } & PSAMP Code & Organism & \multirow[t]{2}{*}{ MSL Code } & $\begin{array}{l}\text { ICP-MS QC } \\
\text { Batch ID }\end{array}$ & $\mathbf{C u}$ & $\mathrm{Ni}$ & $\mathbf{P b}$ & Zn \\
\hline & & & & Instrument: & $I C P-M S$ & $I C P-M S$ & $I C P-M S$ & ICP-MS \\
\hline \multicolumn{5}{|c|}{ Laboratory Achieved Method Detection Limits } & 0.09 & 0.04 & 0.003 & 0.1 \\
\hline \multicolumn{5}{|c|}{ Reporting Limit (MDL* 3.18) } & 0.3 & 0.1 & 0.01 & 0.3 \\
\hline \multicolumn{9}{|c|}{ Standard Reference Material } \\
\hline SRM 1 & & Dogfish Muscle & \multicolumn{2}{|c|}{ DORM-2 013108 020608-6100A } & 2.19 & 17.7 & 0.0493 & 25.9 \\
\hline SRM 1 & & Dogfish Muscle & \multicolumn{2}{|c|}{ DORM-2 020508 021908-6100A } & 2.07 & 18.1 & 0.0576 & 26.4 \\
\hline SRM 1 & & Dogfish Muscle & \multicolumn{2}{|c|}{ DORM-2 020708 022008-6100A } & 2.23 & 17.3 & 0.0544 & 25.9 \\
\hline SRM 1 & & Dogfish Muscle & \multicolumn{2}{|c|}{ DORM-2 021408 022008-6100A } & 2.44 & 19.0 & 0.0765 & 25.5 \\
\hline SRM 1 & & Dogfish Muscle & \multicolumn{2}{|c|}{ DORM-2 041108 041608-6100 } & 2.12 & 18.5 & 0.0512 & 25.7 \\
\hline \multirow[t]{9}{*}{ SRM 1} & & Dogfish Muscle & DORM-2 04150 & $041708-6100$ & 2.13 & 18.7 & 0.0611 & 26.0 \\
\hline & \multicolumn{2}{|l|}{ Certified Value } & & & 2.34 & 19.4 & 0.065 & 25.6 \\
\hline & \multicolumn{2}{|l|}{ Range } & & & \pm 0.16 & \pm 3.10 & \pm 0.007 & \pm 2.3 \\
\hline & \multicolumn{2}{|c|}{ Percent Difference } & & & $6 \%$ & $9 \%$ & $24 \% \&$ & $1 \%$ \\
\hline & \multicolumn{2}{|c|}{ Percent Difference } & & & $12 \%$ & $7 \%$ & $11 \%$ & $3 \%$ \\
\hline & \multicolumn{2}{|c|}{ Percent Difference } & & & $5 \%$ & $11 \%$ & $16 \%$ & $1 \%$ \\
\hline & \multicolumn{2}{|c|}{ Percent Difference } & & & $4 \%$ & $2 \%$ & $18 \%$ & $0 \%$ \\
\hline & \multicolumn{2}{|c|}{ Percent Difference } & & & $9 \%$ & $5 \%$ & $21 \% \&$ & $0 \%$ \\
\hline & \multicolumn{2}{|c|}{ Percent Difference } & & & $9 \%$ & $4 \%$ & $6 \%$ & $2 \%$ \\
\hline SRM 2 & & Oyster Tissue & 1566b 013108 & 020608-6100A & 66.4 & 1.04 & 0.295 & 1502 \\
\hline SRM 2 & & Oyster Tissue & 1566b 020508 & 021908-6100A & 67.2 & 0.935 & 0.296 & 1469 \\
\hline SRM 2 & & Oyster Tissue & 1566b 020708 & 022008-6100A & 68.2 & 0.934 & 0.294 & 1499 \\
\hline SRM 2 & & Oyster Tissue & 1566b 021408 & 022008-6100A & 69.8 & 0.945 & 0.280 & 1540 \\
\hline SRM 2 & & Oyster Tissue & 1566b 041408 & 041608-6100 & 70.6 & 1.05 & 0.284 & 1628 \\
\hline \multirow[t]{9}{*}{ SRM 2} & & Oyster Tissue & $1566 b 041508$ & $041708-6100$ & 67.7 & 0.964 & 0.281 & 1474 \\
\hline & Certified Value & & & & 71.6 & 1.04 & 0.308 & 1424 \\
\hline & Range & & & & \pm 1.60 & \pm 0.09 & \pm 0.01 & \pm 46.00 \\
\hline & Percent Differ & ence & & & $7 \%$ & 0\% & $4 \%$ & $5 \%$ \\
\hline & Percent Differ & ence & & & $6 \%$ & $10 \%$ & $4 \%$ & $3 \%$ \\
\hline & Percent Differ & ence & & & $5 \%$ & $10 \%$ & $5 \%$ & $5 \%$ \\
\hline & Percent Differ & ence & & & $3 \%$ & $9 \%$ & $9 \%$ & $8 \%$ \\
\hline & Percent Differ & ence & & & $1 \%$ & $1 \%$ & $8 \%$ & $14 \%$ \\
\hline & Percent Differ & ence & & & $5 \%$ & $7 \%$ & $9 \%$ & $4 \%$ \\
\hline
\end{tabular}


CVAA QC DATA

1529 West Sequim Bay Road

Sequim, Washington 98382-9099

Biota Studies

360/681-4564

2007 PSAMP Trawl Biota

Metals in Whole Organisms

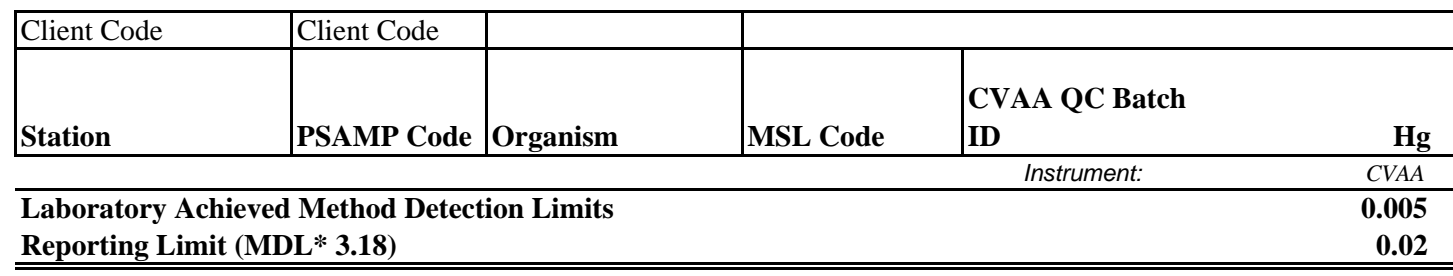

\section{Procedural Blanks}

MB 1

MB 2

MB 3

MB 4

MB 5

MB 6

Spike concentration

PERCENT RECOVERY

LCS 2

MB 2

Spike concentration

PERCENT RECOVERY

LCS 3

MB 3

Spike concentration

PERCENT RECOVERY

LCS 4

MB 4

Spike concentration

PERCENT RECOVERY

LCS 5

MB 5

Spike concentration

PERCENT RECOVERY

LCS 6

MB 6

Spike concentration

PERCENT RECOVERY

Matrix Spike Results

\begin{tabular}{lll}
\hline MS 1 & V-ES-5 & English Sole \\
MSD 1 & V-ES-5 & English Sole \\
Vendovi & V-ES-5 & English Sole \\
& Spike concentration, MS \\
& \multicolumn{2}{l}{ Spike concentration, MSD } \\
& PERCENT RECOVERY, MS \\
& PERCENT RECOVERY, MSD \\
& RPD
\end{tabular}

CVAA QC Data, Dry WT
Blank 013108

Blank 020508

Blank 020708

Blank 021408

Blank 041108

Blank 041508

LCS 013108

Blank 013108

031208HGBL

031208HGBL

032608HGBL

032608HGBL

042508HGBL

042508HGBL

$0.00550 \mathrm{~J}$

$0.005 \mathrm{U}$

$0.00595 \mathrm{~J}$

$0.005 \mathrm{U}$

$0.005 \mathrm{U}$

$0.0161 \mathrm{~J}$

$\begin{array}{ll}\text { LCS 020508 } & \text { 031208HGBL } \\ \text { Blank 020508 } & \text { 031208HGBL }\end{array}$

$0.005 \mathrm{U}$

$99 \%$

LCS 020708

032608HGBL

2.03

Blank 020708

032608HGBL

$0.00595 \mathrm{~J}$

$101 \%$

$\begin{array}{ll}\text { LCS 021408 } & \text { 032608HGBL } \\ \text { Blank 021408 } & \text { 032608HGBL }\end{array}$

2.06

$0.005 \mathrm{U}$

2

$103 \%$

Blank 041108

042508HGBL

2.03

Blank 041108

042508HGBL

$0.005 \mathrm{U}$

$102 \%$

LCS 041508 042508HGBL

1.95

Blank 041508

042508HGBL

$0.0161 \mathrm{~J}$

2

97\%

$\begin{array}{lrr}2838-5 \text { MS } & \text { 031208HGBL } & 2.17 \\ 2838-5 \text { MSD } & \text { 031208HGBL } & 2.17 \\ 2838-5 & 031208 \text { HGBL } & 0.228 \\ & & 2.10 \\ & 2.04 \\ & \mathbf{9 2 \%} \\ \mathbf{9 5 \%} \\ \mathbf{3 \%}\end{array}$


CVAA QC DATA

1529 West Sequim Bay Road

Sequim, Washington 98382-9099

Biota Studies

360/681-4564

2007 PSAMP Trawl Biota

Metals in Whole Organisms

\begin{tabular}{|c|c|c|c|c|c|}
\hline Client Code & Client Code & & & & \\
\hline Station & PSAMP Code & Organism & MSL Code & $\begin{array}{l}\text { CVAA QC Batch } \\
\text { ID }\end{array}$ & Hg \\
\hline & & & & Instrument: & CVAA \\
\hline Laboratory Ach & Method Detec & ion Limits & & & 0.005 \\
\hline Reporting Limi & $L * 3.18)$ & & & & 0.02 \\
\hline MS 2 & HC-RS-1 & Rock sole & 2838-36 MS & 031208HGBL & 2.11 \\
\hline MSD 2 & HC-RS-1 & Rock sole & 2838-36 MSD & 031208HGBL & 2.15 \\
\hline Hood Canal & HC-RS-1 & Rock sole & $2838-36$ & 031208HGBL & 0.216 \\
\hline & Spike concentrc & tion, $M S$ & & & 1.96 \\
\hline & Spike concentrc & tion, $M S D$ & & & 1.98 \\
\hline & PERCENT RE & COVERY, MS & & & $97 \%$ \\
\hline & PERCENT RE & COVERY, MSD & & & $98 \%$ \\
\hline & RPD & & & & $1 \%$ \\
\hline MS 3 & SI-SS-3 & Sand sole & 2838-73 MS & 032608HGBL & 2.55 \\
\hline MSD 3 & SI-SS-3 & Sand sole & 2838-73 MSD & 032608HGBL & 2.50 \\
\hline Sinclair Inlet & SI-SS-3 & Sand sole & $2838-73$ & 032608HGBL & 0.480 \\
\hline & Spike concentrc & tion, $M S$ & & & 1.99 \\
\hline & Spike concentrc & tion, $M S D$ & & & 1.98 \\
\hline & PERCENT RE & COVERY, MS & & & $104 \%$ \\
\hline & PERCENT RE & COVERY, MSD & & & $102 \%$ \\
\hline & RPD & & & & $2 \%$ \\
\hline MS 4 & NIS-RF-2 & Spotted Ratfish & 2838-108 MS & 032608HGBL & 2.64 \\
\hline MSD 4 & NIS-RF-2 & Spotted Ratfish & 2838-108 MSD & 032608HGBL & 2.49 \\
\hline Nisqually & NIS-RF-2 & Spotted Ratfish & 2838-108 & 032608HGBL & 0.485 \\
\hline & Spike concentrc & tion, $M S$ & & & 2.00 \\
\hline & Spike concentrc & tion, $M S D$ & & & 1.91 \\
\hline & PERCENT RE & COVERY, MS & & & $108 \%$ \\
\hline & PERCENT RE & COVERY, MSD & & & $105 \%$ \\
\hline & RPD & & & & $3 \%$ \\
\hline MS 5 & DF-EM-1 & Dogfish 1 (EM) & 2838-126 MS & 042508HGBL & 2.01 \\
\hline MSD 5 & DF-EM-1 & Dogfish 1 (EM) & 2838-126 MSD & 042508HGBL & 1.98 \\
\hline Admiralty Inlet & DF-EM-1 & Dogfish 1 (EM) & $2838-126$ & 042508HGBL & 0.0368 \\
\hline & Spike concentrc & tion, $M S$ & & & 2.01 \\
\hline & Spike concentrc & tion, $M S D$ & & & 1.97 \\
\hline & PERCENT RE & COVERY, MS & & & $98 \%$ \\
\hline & PERCENT RE & COVERY, MSD & & & $99 \%$ \\
\hline & RPD & & & & $1 \%$ \\
\hline MS 6 & DF-LV-6 & Dogfish 6 & 2838-137 MS & 042508HGBL & 2.15 \\
\hline MSD 6 & DF-LV-6 & Dogfish 6 & 2838-137 MSD & 042508HGBL & 2.20 \\
\hline Admiralty Inlet & DF-LV-6 & Dogfish 6 & $2838-137$ & 042508HGBL & 0.242 \\
\hline & Spike concentrc & tion, $M S$ & & & 1.97 \\
\hline & Spike concentrc & tion, MSD & & & 2.02 \\
\hline & PERCENT RE & COVERY, MS & & & $97 \%$ \\
\hline & PERCENT RE & COVERY, MSD & & & $97 \%$ \\
\hline & RPD & & & & 0\% \\
\hline
\end{tabular}




\begin{tabular}{|c|c|c|c|c|c|}
\hline Client Code & Client Code & & & & \\
\hline Station & PSAMP Code & Organism & MSL Code & $\begin{array}{l}\text { CVAA QC Batch } \\
\text { ID }\end{array}$ & Hg \\
\hline & & & & Instrument: & CVAA \\
\hline Laboratory Ach & Method Detect & tion Limits & & & 0.005 \\
\hline Reporting Limi & L*3.18) & & & & 0.02 \\
\hline Laboratory Du & e Results & & & & \\
\hline Vendovi & $\overline{V-S S c-3}$ & Staghorn sculpin & 2838-15 R1 & 031208HGBL & 0.353 \\
\hline Vendovi & V-SSc-3 & Staghorn sculpin & 2838-15 R2 & 031208HGBL & 0.368 \\
\hline & MEAN & & & & 0.361 \\
\hline & RPD & & & & $4 \%$ \\
\hline Elliot Bay & EB-RF-1 & Spotted Ratfish & 2838-48 R1 & 031208HGBL & 0.734 \\
\hline Elliot Bay & EB-RF-1 & Spotted Ratfish & $2838-48$ R2 & 031208HGBL & 0.712 \\
\hline Elliot Bay & EB-RF-1 & Spotted Ratfish & 2838-48 DUP & 042508HGBL & 0.763 \\
\hline & MEAN & & & & 0.736 \\
\hline & RSD & & & & $3 \%$ \\
\hline Sinclair Inlet & SI-ES-8 & English Sole & 2838-65 R1 & 032608HGBL & 0.180 \\
\hline Sinclair Inlet & SI-ES-8 & English Sole & $2838-65$ R2 & 032608HGBL & 0.174 \\
\hline & MEAN & & & & 0.177 \\
\hline & RPD & & & & $3 \%$ \\
\hline Sinclair Inlet & SI-GC-2/6 & Graceful Crab (2) & 2838-93-97 R1 & 032608HGBL & 0.217 \\
\hline Sinclair Inlet & SI-GC-2/6 & Graceful Crab (2) & 2838-93-97 R2 & 032608HGBL & 0.206 \\
\hline & MEAN & & & & 0.212 \\
\hline & RPD & & & & $5 \%$ \\
\hline Admiralty Inlet & DF-WBWC-1 & Dogfish 1 & 2838-177 R1 & 042508HGBL & 1.45 \\
\hline Admiralty Inlet & DF-WBWC-1 & Dogfish 1 & 2838-177 R2 & 042508HGBL & 1.46 \\
\hline & MEAN & & & & 1.46 \\
\hline & RPD & & & & $1 \%$ \\
\hline Admiralty Inlet & DF-WBWC-6 & Dogfish 6 & 2838-182 R1 & 042508HGBL & 1.35 \\
\hline Admiralty Inlet & DF-WBWC-6 & Dogfish 6 & 2838-182 R2 & 042508HGBL & 1.34 \\
\hline & MEAN & & & & 1.35 \\
\hline & RPD & & & & $1 \%$ \\
\hline Vendovi & V-SSc-1 & Staghorn sculpin & $2838-13$ & 031208HGBL & 0.300 \\
\hline Vendovi & V-SSc-1 & Staghorn sculpin & 2838-13 DUP & 042508HGBL & 0.307 \\
\hline & MEAN & & & & 0.304 \\
\hline & RPD & & & & $2 \%$ \\
\hline Hood Canal & HC-RF-1 & Spotted Ratfish & $2838-37$ & 031208HGBL & 0.298 \\
\hline Hood Canal & HC-RF-1 & Spotted Ratfish & 2838-37 DUP & 042508HGBL & 0.363 \\
\hline & MEAN & & & & 0.331 \\
\hline & RPD & & & & $20 \%$ \\
\hline Hood Canal & HC-RF-3 & Spotted Ratfish & $2838-39$ & 031208HGBL & 0.593 \\
\hline Hood Canal & HC-RF-3 & Spotted Ratfish & 2838-39 DUP & 042508HGBL & 0.598 \\
\hline & MEAN & & & & 0.596 \\
\hline & RPD & & & & $1 \%$ \\
\hline
\end{tabular}




\begin{tabular}{|c|c|c|c|c|c|}
\hline Client Code & Client Code & & & & \\
\hline Station & PSAMP Code & Organism & MSL Code & $\begin{array}{l}\text { CVAA QC Batch } \\
\text { ID }\end{array}$ & $\mathbf{H g}$ \\
\hline & & & & Instrument: & CVAA \\
\hline Laboratory Achieved & I Method Detect & tion Limits & & & 0.005 \\
\hline Reporting Limit (MI & L*3.18) & & & & 0.02 \\
\hline Hood Canal & HC-GC-1 & Graceful Crab & $2838-43$ & 031208HGBL & 0.0902 \\
\hline Hood Canal & HC-GC-1 & Graceful Crab & 2838-43 DUP & 042508HGBL & 0.0897 \\
\hline & MEAN & & & & 0.0900 \\
\hline & RPD & & & & $1 \%$ \\
\hline Sinclair Inlet & SI-RF-1 & Spotted Ratfish & $2838-83$ & 032608HGBL & 0.788 \\
\hline Sinclair Inlet & SI-RF-1 & Spotted Ratfish & 2838-83 DUP & 042508HGBL & 0.796 \\
\hline & MEAN & & & & 0.792 \\
\hline & RPD & & & & $1 \%$ \\
\hline Elliott Bay & EB-RF-3 & Spotted Ratfish & $2838-50$ & 031208HGBL & 0.776 \\
\hline Elliott Bay & EB-RF-3 & Spotted Ratfish & 2838-50 DUP & 042508HGBL & 0.753 \\
\hline & MEAN & & & & 0.765 \\
\hline & RPD & & & & $3 \%$ \\
\hline Sinclair Inlet & SI-ES-2 & English Sole & $2838-59$ & 032608HGBL & 0.126 \\
\hline Sinclair Inlet & SI-ES-2 & English Sole & 2838-59 DUP & 042508HGBL & 0.129 \\
\hline & MEAN & & & & 0.128 \\
\hline & RPD & & & & $2 \%$ \\
\hline Sinclair Inlet & SI-RS-4 & Rock sole & $2838-70$ & 032608HGBL & 0.410 \\
\hline Sinclair Inlet & SI-RS-4 & Rock sole & 2838-70 DUP & 042508HGBL & 0.402 \\
\hline & MEAN & & & & 0.406 \\
\hline & RPD & & & & $2 \%$ \\
\hline Sinclair Inlet & SI-SSc-1/2 & Staghorn sculpin (2) & $2838-77-78$ & 032608HGBL & 0.250 \\
\hline Sinclair Inlet & SI-SSc-1/2 & Staghorn sculpin (2) & 2838-77-78 DUP & 042508HGBL & 0.230 \\
\hline & MEAN & & & & 0.240 \\
\hline & RPD & & & & $8 \%$ \\
\hline Nisqually & NIS-RF-1 & Spotted Ratfish & $2838-107$ & 032608HGBL & 0.739 \\
\hline Nisqually & NIS-RF-1 & Spotted Ratfish & 2838-107 DUP & 042508HGBL & 0.758 \\
\hline & MEAN & & & & 0.749 \\
\hline & RPD & & & & $3 \%$ \\
\hline Nisqually & NIS-RF-3 & Spotted Ratfish & 2838-109 & 032608HGBL & 0.449 \\
\hline Nisqually & NIS-RF-3 & Spotted Ratfish & 2838-109 DUP & 042508HGBL & 0.424 \\
\hline & MEAN & & & & 0.437 \\
\hline & RPD & & & & $6 \%$ \\
\hline Commencement Bay & CB-ES-3 & English Sole & $2838-112$ & 032608HGBL & 0.229 \\
\hline Commencement Bay & CB-ES-3 & English Sole & 2838-112 DUP & 042508HGBL & 0.213 \\
\hline & MEAN & & & & 0.221 \\
\hline & RPD & & & & $7 \%$ \\
\hline
\end{tabular}


CVAA QC DATA

1529 West Sequim Bay Road

Sequim, Washington 98382-9099

Biota Studies

360/681-4564

2007 PSAMP Trawl Biota

Metals in Whole Organisms

\begin{tabular}{l|l|l|l|lr}
\hline Client Code & Client Code & & \multicolumn{2}{l}{} \\
\hline & & & CVAA QC Batch \\
Station & & MSL Code & ID & Instrument: & CVAA \\
\hline \multicolumn{2}{l}{ PSAMP Code } & Organism & & $\mathbf{0 . 0 0 5}$ \\
\hline Laboratory Achieved Method Detection Limits & & $\mathbf{0 . 0 2}$ \\
\hline
\end{tabular}

Standard Reference Material

SRM 1

SRM 1

SRM 1

SRM 1

SRM 1

SRM 1

\section{Dogfish Muscle \\ Dogfish Muscle \\ Dogfish Muscle \\ Dogfish Muscle \\ Dogfish Muscle \\ Dogfish Muscle}

Certified Value

Range

Percent Difference

Percent Difference

Percent Difference

Percent Difference

Percent Difference

Percent Difference
DORM-2 013108 031208HGBL

DORM-2 020508 031208HGBL

DORM-2 020708 032608HGBL

DORM-2 021408 032608HGBL

DORM-2 041108 042508HGBL

DORM-2 041508 042508HGBL
4.01
4.05
4.19
4.38
4.20
4.12
4.64
$\pm 0.26$
$14 \%$
$13 \%$
$10 \%$
$6 \%$
$\mathbf{9 \%}$
$11 \%$ 


\begin{tabular}{|c|c|c|c|c|c|}
\hline Client Code & Client Code & & & & \\
\hline \multirow[t]{2}{*}{ Station } & PSAMP Code & Organism & MSL Code & $\begin{array}{l}\text { ICP-OES QC } \\
\text { Batch ID }\end{array}$ & $\mathbf{C r}$ \\
\hline & & & & Instrument: & ICP-OES \\
\hline \multicolumn{5}{|c|}{ Laboratory Achieved Method Detection Limits } & 0.04 \\
\hline \multicolumn{5}{|c|}{ Reporting Limit (MDL* 3.18) } & 0.1 \\
\hline
\end{tabular}

\section{Procedural Blanks}

MB 1

MB 2

MB 3

MB 4

MB 5

MB 6

Laboratory Control Sample Results

LCS 1

MB 1

Spike concentration

PERCENT RECOVERY

LCS 2

MB 2

Spike concentration

PERCENT RECOVERY

LCS 3

MB 3

Spike concentration

PERCENT RECOVERY

LCS 4

MB 4

Spike concentration

PERCENT RECOVERY

LCS 5

MB 5

Spike concentration

PERCENT RECOVERY

LCS 6

MB 6

Spike concentration

PERCENT RECOVERY

Matrix Spike Results

\begin{tabular}{lll}
\hline MS 1 & V-ES-5 & English Sole \\
MSD 1 & V-ES-5 & English Sole \\
Vendovi & V-ES-5 & English Sole \\
& Spike concentration, MS \\
& Spike concentration, MSD \\
& PERCENT RECOVERY, MS \\
& PERCENT RECOVERY, MSD \\
& RPD
\end{tabular}

ICP-OES QC Results, Dry Wt
Blank 013108

Blank 020508

Blank 020708

Blank 021408

Blank 041108

Blank 041508

I040408A

I040408A

I040708A

I040708A

I051208C

I051208C

LCS 013108 I040408A

Blank $013108 \quad$ I040408A

4.62

$0.04 \mathrm{U}$

5

$92 \%$

LCS $020508 \quad$ I040408A

5.03

$0.04 \mathrm{U}$

5

$101 \%$

LCS $020708 \quad$ I040708A

2.62

Blank 020708

I040708A

$0.04 \mathrm{U}$

2.5

$105 \%$

$\begin{array}{ll}\text { LCS } 021408 & \text { I040708A } \\ & \end{array}$

5.11

$0.04 \mathrm{U}$

5

$102 \%$

Blank $041108 \quad$ I051208C

4.96

Blank $041108 \quad$ I051208C

$0.04 \mathrm{U}$

5

$99 \%$

LCS 041508 I051208C 5.08

Blank $041508 \quad$ I051208C

$0.04 \mathrm{U}$

$102 \%$

$\begin{array}{lrr}2838-5 \text { MS } & \text { I040408A } & 3.11 \\ 2838-5 \text { MSD } & \text { I040408A } & 3.01 \\ 2838-5 & \text { I040408A } & 1.18 \\ & & 2.10 \\ & & 2.04 \\ & & \mathbf{9 2 \%} \\ & & \mathbf{9 0 \%} \\ & & \mathbf{2 \%}\end{array}$

Page 1 of 4 


\begin{tabular}{|c|c|c|c|c|c|}
\hline Client Code & Client Code & & & & \\
\hline Station & PSAMP Code & Organism & MSL Code & $\begin{array}{l}\text { ICP-OES QC } \\
\text { Batch ID }\end{array}$ & $\mathrm{Cr}$ \\
\hline & & & & Instrument: & ICP-OES \\
\hline Laboratory Ach & Method Detect & tion Limits & & & 0.04 \\
\hline Reporting Limi & $L * 3.18)$ & & & & 0.1 \\
\hline MS 2 & HC-RS-1 & Rock sole & 2838-36 MS & I040408A & 2.82 \\
\hline MSD 2 & HC-RS-1 & Rock sole & 2838-36 MSD & I040408A & 2.89 \\
\hline Hood Canal & HC-RS-1 & Rock sole & $2838-36$ & I040408A & 0.684 \\
\hline & Spike concentrc & ation, MS & & & 1.96 \\
\hline & Spike concentrc & ation, MSD & & & 1.98 \\
\hline & PERCENT RE & COVERY, MS & & & $109 \%$ \\
\hline & PERCENT RE & COVERY, MSD & & & $111 \%$ \\
\hline & RPD & & & & $2 \%$ \\
\hline MS 3 & SI-SS-3 & Sand sole & 2838-73 MS & I040708A & 2.81 \\
\hline MSD 3 & SI-SS-3 & Sand sole & 2838-73 MSD & I040708A & 2.88 \\
\hline Sinclair Inlet & SI-SS-3 & Sand sole & $2838-73$ & I040708A & 0.810 \\
\hline & Spike concentrc & ation, MS & & & 1.99 \\
\hline & Spike concentrc & ation, MSD & & & 1.98 \\
\hline & PERCENT RE & COVERY, MS & & & $101 \%$ \\
\hline & PERCENT RE & COVERY, MSD & & & $105 \%$ \\
\hline & RPD & & & & $4 \%$ \\
\hline MS 4 & NIS-RF-2 & Spotted Ratfish & 2838-108 MS & I040708A & 2.17 \\
\hline MSD 4 & NIS-RF-2 & Spotted Ratfish & 2838-108 MSD & I040708A & 2.06 \\
\hline Nisqually & NIS-RF-2 & Spotted Ratfish & $2838-108$ & I040708A & 0.201 \\
\hline & Spike concentrc & ation, $M S$ & & & 2.00 \\
\hline & Spike concentrc & ation, $M S D$ & & & 1.91 \\
\hline & PERCENT RE & COVERY, MS & & & $98 \%$ \\
\hline & PERCENT RE & COVERY, MSD & & & $97 \%$ \\
\hline & RPD & & & & $1 \%$ \\
\hline MS 5 & DF-EM-1 & Dogfish 1 (EM) & 2838-126 MS & I051208C & 2.28 \\
\hline MSD 5 & DF-EM-1 & Dogfish 1 (EM) & 2838-126 MSD & I051208C & 2.23 \\
\hline Admiralty Inlet & DF-EM-1 & Dogfish 1 (EM) & $2838-126$ & I051208C & 0.198 \\
\hline & Spike concentrc & ation, $M S$ & & & 2.01 \\
\hline & Spike concentrc & ation, MSD & & & 1.97 \\
\hline & PERCENT RE & COVERY, MS & & & $104 \%$ \\
\hline & PERCENT RE & COVERY, MSD & & & $103 \%$ \\
\hline & RPD & & & & $1 \%$ \\
\hline MS 6 & DF-LV-6 & Dogfish 6 & 2838-137 MS & I051208C & 1.92 \\
\hline MSD 6 & DF-LV-6 & Dogfish 6 & 2838-137 MSD & I051208C & 2.47 \\
\hline Admiralty Inlet & DF-LV-6 & Dogfish 6 & $2838-137$ & I051208C & $0.0710 \mathrm{~J}$ \\
\hline & Spike concentrc & ation, MS & & & 1.97 \\
\hline & Spike concentrc & ation, MSD & & & 2.02 \\
\hline & PERCENT RE & COVERY, MS & & & $94 \%$ \\
\hline & PERCENT RE & COVERY, MSD & & & $119 \%$ \\
\hline & RPD & & & & $23 \%$ \\
\hline
\end{tabular}




\begin{tabular}{|c|c|c|c|c|c|}
\hline Client Code & Client Code & & & & \\
\hline Station & PSAMP Code & Organism & MSL Code & \begin{tabular}{|l} 
ICP-OES QC \\
Batch ID
\end{tabular} & $\mathrm{Cr}$ \\
\hline & & & & Instrument: & ICP-OES \\
\hline Laboratory Ach & I Method Detect & tion Limits & & & 0.04 \\
\hline Reporting Limi & L* 3.18) & & & & 0.1 \\
\hline Laboratory Du & e Results & & & & \\
\hline Vendovi & V-SSc-3 & Staghorn sculpin & 2838-15 R1 & I040408A & 0.680 \\
\hline Vendovi & V-SSc-3 & Staghorn sculpin & 2838-15 R2 & I040408A & 0.777 \\
\hline & MEAN & & & & 0.729 \\
\hline & RPD & & & & $13 \%$ \\
\hline Elliot Bay & EB-RF-1 & Spotted Ratfish & 2838-48 R1 & I040408A & 0.292 \\
\hline Elliot Bay & EB-RF-1 & Spotted Ratfish & $2838-48$ R2 & I040408A & 0.286 \\
\hline Elliot Bay & EB-RF-1 & Spotted Ratfish & 2838-48 DUP & I051208C & 0.364 \\
\hline & MEAN & & & & 0.314 \\
\hline & RSD & & & & $14 \%$ \\
\hline Sinclair Inlet & SI-ES-8 & English Sole & 2838-65 R1 & I040708A & 1.61 \\
\hline Sinclair Inlet & SI-ES-8 & English Sole & $2838-65$ R2 & I040708A & 1.60 \\
\hline & MEAN & & & & 1.61 \\
\hline & RPD & & & & $1 \%$ \\
\hline Sinclair Inlet & SI-GC-2/6 & Graceful Crab (2) & 2838-93-97 R1 & I040708A & 2.44 \\
\hline Sinclair Inlet & SI-GC-2/6 & Graceful Crab (2) & 2838-93-97 R2 & I040708A & 2.42 \\
\hline & MEAN & & & & 2.43 \\
\hline & RPD & & & & $1 \%$ \\
\hline Admiralty Inlet & DF-WBWC-1 & Dogfish 1 & 2838-177 R1 & I051208C & 0.170 \\
\hline Admiralty Inlet & DF-WBWC-1 & Dogfish 1 & 2838-177 R2 & I051208C & 0.157 \\
\hline & MEAN & & & & 0.164 \\
\hline & RPD & & & & $8 \%$ \\
\hline Vendovi & V-SSc-1 & Staghorn sculpin & $2838-13$ & I040408A & 1.33 \\
\hline Vendovi & V-SSc-1 & Staghorn sculpin & 2838-13 DUP & I051208C & 1.26 \\
\hline & MEAN & & & & 1.30 \\
\hline & RPD & & & & $5 \%$ \\
\hline Hood Canal & HC-RF-1 & Spotted Ratfish & $2838-37$ & I040408A & 0.317 \\
\hline Hood Canal & HC-RF-1 & Spotted Ratfish & 2838-37 DUP & I051208C & 0.328 \\
\hline & MEAN & & & & 0.323 \\
\hline & RPD & & & & $3 \%$ \\
\hline Hood Canal & HC-RF-3 & Spotted Ratfish & $2838-39$ & I040408A & 0.135 \\
\hline Hood Canal & HC-RF-3 & Spotted Ratfish & 2838-39 DUP & I051208C & 0.144 \\
\hline & MEAN & & & & 0.140 \\
\hline & RPD & & & & $6 \%$ \\
\hline Hood Canal & HC-GC-1 & Graceful Crab & $2838-43$ & I040408A & 1.42 \\
\hline Hood Canal & HC-GC-1 & Graceful Crab & 2838-43 DUP & I051208C & 1.43 \\
\hline & MEAN & & & & 1.43 \\
\hline & RPD & & & & $1 \%$ \\
\hline
\end{tabular}




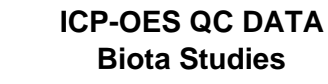

2007 PSAMP Trawl Biota

360/681-4564

\section{Metals in Whole Organisms}

\begin{tabular}{|c|c|c|c|c|c|}
\hline \multirow[t]{2}{*}{ Client Code } & \multicolumn{5}{|l|}{ Client Code } \\
\hline & PSAMP Code & Organism & MSL Code & $\begin{array}{l}\text { ICP-OES QC } \\
\text { Batch ID }\end{array}$ & $\mathrm{Cr}$ \\
\hline & & & & Instrument: & ICP-OES \\
\hline \multirow{2}{*}{\multicolumn{5}{|c|}{$\begin{array}{l}\text { Laboratory Achieved Method Detection Limits } \\
\text { Reporting Limit (MDL*3.18) }\end{array}$}} & 0.04 \\
\hline & & & & & 0.1 \\
\hline Sinclair Inlet & SI-RF-1 & Spotted Ratfish & $2838-83$ & I040708A & 0.317 \\
\hline \multirow[t]{3}{*}{ Sinclair Inlet } & SI-RF-1 & Spotted Ratfish & 2838-83 DUP & I051208C & 0.308 \\
\hline & MEAN & & & & 0.313 \\
\hline & RPD & & & & $3 \%$ \\
\hline \multirow{4}{*}{$\begin{array}{l}\text { Elliott Bay } \\
\text { Elliott Bay }\end{array}$} & EB-RF-3 & Spotted Ratfish & $2838-50$ & I040408A & 0.179 \\
\hline & EB-RF-3 & Spotted Ratfish & 2838-50 DUP & I051208C & 0.188 \\
\hline & MEAN & & & & 0.184 \\
\hline & RPD & & & & $5 \%$ \\
\hline \multirow{4}{*}{$\begin{array}{l}\text { Sinclair Inlet } \\
\text { Sinclair Inlet }\end{array}$} & SI-ES-2 & English Sole & $2838-59$ & I040708A & 1.21 \\
\hline & SI-ES-2 & English Sole & 2838-59 DUP & I051208C & 1.58 \\
\hline & MEAN & & & & 1.40 \\
\hline & RPD & & & & $26 \%$ \\
\hline \multirow{4}{*}{$\begin{array}{l}\text { Sinclair Inlet } \\
\text { Sinclair Inlet }\end{array}$} & SI-RS-4 & Rock sole & $2838-70$ & I040708A & 1.11 \\
\hline & SI-RS-4 & Rock sole & 2838-70 DUP & I051208C & 1.52 \\
\hline & MEAN & & & & 1.32 \\
\hline & RPD & & & & $31 \% *$ \\
\hline \multirow{4}{*}{$\begin{array}{l}\text { Sinclair Inlet } \\
\text { Sinclair Inlet }\end{array}$} & SI-SSc-1/2 & Staghorn sculpin (2) & $2838-77-78$ & I040708A & 1.45 \\
\hline & SI-SSc-1/2 & Staghorn sculpin (2) & 2838-77-78 DUP & I051208C & 1.62 \\
\hline & MEAN & & & & 1.54 \\
\hline & RPD & & & & $11 \%$ \\
\hline \multirow{4}{*}{$\begin{array}{l}\text { Commencement Bay } \\
\text { Commencement Bay }\end{array}$} & CB-ES-3 & English Sole & $2838-112$ & I040708A & 1.47 \\
\hline & CB-ES-3 & English Sole & 2838-112 DUP & I051208C & 1.78 \\
\hline & MEAN & & & & 1.63 \\
\hline & RPD & & & & $19 \%$ \\
\hline \multicolumn{6}{|c|}{ Standard Reference Material } \\
\hline \multirow{14}{*}{$\begin{array}{l}\text { SRM } 1 \\
\text { SRM } 1 \\
\text { SRM } 1 \\
\text { SRM } 1 \\
\text { SRM } 1 \\
\text { SRM } 1\end{array}$} & & Dogfish Muscle & DORM-2 013108 & I040408A & 30.1 \\
\hline & & Dogfish Muscle & DORM-2 020508 & I040408A & 33.7 \\
\hline & & Dogfish Muscle & DORM-2 020708 & I040708A & 32.3 \\
\hline & & Dogfish Muscle & DORM-2 021408 & I040708A & 34.6 \\
\hline & & Dogfish Muscle & DORM-2 041108 & I051208C & 33.6 \\
\hline & & Dogfish Muscle & DORM-2 041508 & I051208C & 34.4 \\
\hline & \multicolumn{4}{|l|}{ Certified Value } & 34.7 \\
\hline & \multicolumn{4}{|l|}{ Range } & \pm 5.5 \\
\hline & \multicolumn{4}{|c|}{ Percent Difference } & $13 \%$ \\
\hline & \multicolumn{4}{|c|}{ Percent Difference } & $3 \%$ \\
\hline & \multicolumn{4}{|c|}{ Percent Difference } & $7 \%$ \\
\hline & \multicolumn{4}{|c|}{ Percent Difference } & $\mathbf{0 \%}$ \\
\hline & \multicolumn{4}{|c|}{ Percent Difference } & $3 \%$ \\
\hline & \multicolumn{4}{|c|}{ Percent Difference } & $1 \%$ \\
\hline
\end{tabular}


BATTELLE MARINE SCIENCE LABORATORIES

1529 West Sequim Bay Road

Sequim, Washington 98382-9099

360/681-4564

\section{ENVVEST, SINCLAIR AND DYES INLET BIOTA STUDY \\ 2007 PSAMP Trawl Biota \\ Metals in Whole Organisms and Dogfish Parts}

\section{Data Qualifiers}

c Exceeds DQO but meets contingency criteria of either:

1 SRM certified $<10 x$ MDL

2 Insufficient spiking level relative to native sample concentrations

3 Sample concentration $<10 x$ MDL

$\mathrm{U}$ Analyte not detected at or above the MDL, MDL reported

J Analyte detected above the MDL, but less than the RL

-- Not analyzed

NA Not applicable/available

N Spiked sample recovery outside QC criterion of 70-130\%

$\&$ Accuracy result outside QC criterion of $\leq 20 \%$ PD

* Precision result outside QC criterion of $<30 \%$

NS Sample not spiked for this analyte

B Analyte detected in the method blank $>$ RL and sample concentration $<10$ times detected blank value

b Data are blank corrected using the batch specific procedural blank

\section{Legend}

WBC Whole Body Composite of one organism

COMP Composite of several organisms

EM Embryos

LV Liver

DIG Digestive tract + gut contents

SWC Section weighted composite

WBWC Whole body weighted composite

Weight and length of multiple organism complosites are given as the average of individuals incorporated into the com 


\section{QC Sample Results: PCBs in Biota Tissue}

\section{Wet Weight Basis}


BATTELLE MARINE SCIENCE LABORATORIES

1529 West Sequim Bay Road

Sequim, Washington 98382-9099

360/681-4564

\section{SINCLAIR AND DYES INLET \\ 2007 PSAMP Trawl Biota \\ PCBs in Whole Organisms}

\begin{tabular}{|c|c|c|c|c|c|c|}
\hline Sample Name: & V-ES-5 & V-ES-6 & V-ES-8 & V-RS-1 & V-RS-2 & V-RS-3 \\
\hline Station: & Vendovi & Vendovi & Vendovi & Vendovi & Vendovi & Vendovi \\
\hline Organism ID: & ES & ES & ES & $\mathrm{RS}$ & $\mathrm{RS}$ & RS \\
\hline Batch ID: & $08-0004$ & $08-0004$ & $08-0004$ & $08-0004$ & $08-0004$ & 08-0004 \\
\hline Sample Weight (g): & 30.01 & 30.34 & 31.04 & 30.18 & 30 & 30.36 \\
\hline \%Moisture & 81.41 & 82.21 & 77.36 & 78.21 & 78.77 & 79.70 \\
\hline \%Lipids & 0.65 & 0.49 & 1.47 & 0.63 & 0.48 & 0.75 \\
\hline Collection Date: & $05 / 01 / 2007$ & $05 / 01 / 2007$ & $05 / 01 / 2007$ & $05 / 01 / 2007$ & $05 / 01 / 2007$ & $05 / 01 / 2007$ \\
\hline Extraction Date: & 01/10/2008 & 01/10/2008 & 01/10/2008 & 01/10/2008 & 01/10/2008 & 01/10/2008 \\
\hline Analysis Date: & 01/28/2008 & 01/29/2008 & 01/30/2008 & 01/29/2008 & 01/29/2008 & 01/30/2008 \\
\hline Units (wet wt): & ng/g_WET & ng/g_WET & ng/g_WET & ng/g_WET & ng/g_WET & ng/g_WET \\
\hline $\mathrm{Cl} 2(8)$ & $0.02 \mathrm{~J}$ & $0.05 \mathrm{U}$ & $0.05 \mathrm{U}$ & $0.05 \mathrm{U}$ & $0.05 \mathrm{U}$ & $0.05 \mathrm{U}$ \\
\hline $\mathrm{Cl} 3(18)$ & $0.02 \mathrm{~J}$ & $0.06 \mathrm{U}$ & 0.04 & $0.06 \mathrm{U}$ & $0.06 \mathrm{U}$ & $0.06 \mathrm{U}$ \\
\hline $\mathrm{Cl} 3(28)$ & 0.07 & 0.03 & 0.05 & $0.06 \mathrm{U}$ & $0.06 \mathrm{U}$ & $0.06 \mathrm{U}$ \\
\hline $\mathrm{Cl} 4(44)$ & 0.03 & $0.02 \mathrm{~J}$ & 0.05 & $0.12 \mathrm{U}$ & $0.12 \mathrm{U}$ & $0.12 \mathrm{U}$ \\
\hline $\mathrm{Cl} 4(52)$ & 0.09 & 0.05 & 0.13 & $0.12 \mathrm{U}$ & 0.06 & $0.12 \mathrm{U}$ \\
\hline $\mathrm{Cl} 4(66)$ & 0.06 & $0.02 \mathrm{~J}$ & 0.06 & $0.07 \mathrm{U}$ & 0.05 & 0.09 \\
\hline $\mathrm{Cl} 4(77)$ & $0.05 \mathrm{U}$ & $0.05 \mathrm{U}$ & $0.05 \mathrm{U}$ & $0.05 \mathrm{U}$ & $0.05 \mathrm{U}$ & $0.05 \mathrm{U}$ \\
\hline $\mathrm{Cl} 5(101)$ & 0.22 & 0.10 & 0.23 & 0.11 & 0.11 & 0.1 \\
\hline $\mathrm{Cl} 5(105)$ & 0.07 & $0.05 \mathrm{U}$ & 0.07 & 0.08 & $0.05 \mathrm{U}$ & 0.06 \\
\hline $\mathrm{Cl} 5(118)$ & 0.22 & 0.08 & 0.18 & 0.20 & 0.18 & 0.24 \\
\hline $\mathrm{Cl} 5(126)$ & $0.07 \mathrm{U}$ & $0.07 \mathrm{U}$ & $0.07 \mathrm{U}$ & $0.07 \mathrm{U}$ & $0.07 \mathrm{U}$ & $0.07 \mathrm{U}$ \\
\hline $\mathrm{Cl6}(128)$ & 0.05 & $0.02 \mathrm{~J}$ & 0.07 & 0.07 & 0.06 & 0.07 \\
\hline $\mathrm{Cl6}(138)$ & 0.35 & 0.13 & 0.32 & 0.29 & 0.28 & 0.38 \\
\hline $\mathrm{Cl6}(153)$ & 0.66 & 0.24 & 0.58 & 0.47 & 0.49 & 0.71 \\
\hline $\mathrm{Cl} 7(170)$ & 0.09 & 0.03 & 0.07 & 0.07 & 0.06 & 0.10 \\
\hline $\mathrm{Cl}(187)$ & 0.30 & 0.12 & 0.23 & 0.11 & 0.15 & 0.13 \\
\hline $\mathrm{Cl}(188)$ & $0.02 \mathrm{U}$ & $0.02 \mathrm{U}$ & $0.02 \mathrm{U}$ & $0.02 \mathrm{U}$ & $0.02 \mathrm{U}$ & $0.02 \mathrm{U}$ \\
\hline $\mathrm{Cl}(195)$ & $0.05 \mathrm{U}$ & $0.05 \mathrm{U}$ & $0.05 \mathrm{U}$ & $0.05 \mathrm{U}$ & $0.05 \mathrm{U}$ & $0.05 \mathrm{U}$ \\
\hline $\mathrm{Cl}(200)$ & $0.04 \mathrm{U}$ & $0.04 \mathrm{U}$ & $0.04 \mathrm{U}$ & $0.04 \mathrm{U}$ & $0.04 \mathrm{U}$ & $0.04 \mathrm{U}$ \\
\hline $\mathrm{Cl9}(206)$ & 0.06 & $0.04 \mathrm{U}$ & 0.03 & $0.04 \mathrm{U}$ & $0.04 \mathrm{U}$ & $0.04 \mathrm{U}$ \\
\hline Cl10(209) & 0.03 & $0.07 \mathrm{U}$ & $0.02 \mathrm{~J}$ & $0.07 \mathrm{U}$ & $0.07 \mathrm{U}$ & $0.07 \mathrm{U}$ \\
\hline Total PCBs & 5.14 & 2.68 & 4.82 & 4.44 & 4.24 & 5.26 \\
\hline LOC 1 & $0.08 \mathrm{U}$ & $0.02 \mathrm{~J}$ & $0.08 \mathrm{U}$ & $0.08 \mathrm{U}$ & $0.08 \mathrm{U}$ & $0.08 \mathrm{U}$ \\
\hline LOC 2 & $0.05 \mathrm{~J}$ & $0.52 \mathrm{U}$ & $0.52 \mathrm{U}$ & $0.52 \mathrm{U}$ & $0.52 \mathrm{U}$ & $0.52 \mathrm{U}$ \\
\hline LOC 3 & $0.24 \mathrm{~J}$ & $0.07 \mathrm{~J}$ & $0.22 \mathrm{~J}$ & $0.75 \mathrm{U}$ & $0.75 \mathrm{U}$ & $0.75 \mathrm{U}$ \\
\hline LOC 4 & $0.45 \mathrm{~J}$ & $0.2 \mathrm{~J}$ & $0.58 \mathrm{~J}$ & $1.63 \mathrm{U}$ & $0.15 \mathrm{~J}$ & $0.19 \mathrm{~J}$ \\
\hline LOC 5 & 1.15 & $0.42 \mathrm{~J}$ & 1.17 & $0.63 \mathrm{~J}$ & $0.58 \mathrm{~J}$ & 0.76 \\
\hline LOC 6 & 1.82 & $0.64 \mathrm{~J}$ & 1.67 & 1.03 & 1.15 & 1.37 \\
\hline LOC 7 & 1.07 & $0.43 \mathrm{~J}$ & 0.86 & $0.42 \mathrm{~J}$ & $0.54 \mathrm{~J}$ & 0.68 \\
\hline LOC 8 & 0.34 & $0.51 \mathrm{U}$ & $0.12 \mathrm{~J}$ & $0.51 \mathrm{U}$ & $0.51 \mathrm{U}$ & $0.51 \mathrm{U}$ \\
\hline LOC 9 & $0.08 \mathrm{~J}$ & $0.16 \mathrm{U}$ & $0.03 \mathrm{~J}$ & $0.16 \mathrm{U}$ & $0.16 \mathrm{U}$ & $0.16 \mathrm{U}$ \\
\hline Total PCBs & 5.28 & 2.97 & 5.25 & 5.73 & 4.44 & 5.02 \\
\hline $\mathrm{Cl} 3(34)$ & 80 & 41 & 64 & 93 & 76 & 94 \\
\hline $\mathrm{Cl}(152)$ & 82 & 42 & 67 & 83 & 74 & 82 \\
\hline
\end{tabular}


1529 West Sequim Bay Road

Sequim, Washington 98382-9099

360/681-4564

\section{SINCLAIR AND DYES INLET 2007 PSAMP Trawl Biota PCBs in Whole Organisms}

\begin{tabular}{|c|c|c|c|c|}
\hline Sample Name: & V-SSc-1 & V-SSc-2 & V-SSc-3 & SG-ES-3 \\
\hline Station: & Vendovi & Vendovi & Vendovi & Strait of Georgia \\
\hline Organism ID: & STS & STS & STS & ES \\
\hline Batch ID: & $08-0004$ & $08-0004$ & $08-0004$ & $08-0004$ \\
\hline Sample Weight (g): & 30.34 & 30.14 & 31.02 & 30.92 \\
\hline \%Moisture & 80.46 & 79.46 & 80.10 & 79.46 \\
\hline \%Lipids & 0.64 & 0.84 & 0.95 & 1.81 \\
\hline Collection Date: & $05 / 01 / 2007$ & $05 / 01 / 2007$ & $05 / 01 / 2007$ & $05 / 02 / 2007$ \\
\hline Extraction Date: & 01/10/2008 & 01/10/2008 & 01/10/2008 & 01/10/2008 \\
\hline Analysis Date: & 01/29/2008 & 01/29/2008 & 01/29/2008 & 01/29/2008 \\
\hline Units (wet wt): & ng/g_WET & ng/g_WET & ng/g_WET & ng/g_WET \\
\hline $\mathrm{Cl} 2(8)$ & 0.04 & $0.05 \mathrm{U}$ & $0.05 \mathrm{U}$ & $0.02 \mathrm{~J}$ \\
\hline $\mathrm{Cl} 3(18)$ & 0.04 & $0.02 \mathrm{~J}$ & $0.06 \mathrm{U}$ & 0.05 \\
\hline $\mathrm{Cl} 3(28)$ & 0.05 & 0.08 & 0.04 & 0.11 \\
\hline Cl4(44) & 0.04 & 0.04 & 0.03 & 0.10 \\
\hline $\mathrm{Cl} 4(52)$ & 0.07 & 0.14 & 0.06 & 0.24 \\
\hline $\mathrm{Cl} 4(66)$ & 0.05 & 0.14 & 0.06 & 0.15 \\
\hline Cl4(77) & $0.05 \mathrm{U}$ & $0.05 \mathrm{U}$ & $0.05 \mathrm{U}$ & $0.05 \mathrm{U}$ \\
\hline Cl5(101) & 0.17 & 0.34 & 0.19 & 0.49 \\
\hline $\mathrm{Cl5}(105)$ & 0.09 & 0.28 & 0.11 & 0.15 \\
\hline $\mathrm{Cl5}(118)$ & 0.20 & 0.54 & 0.26 & 0.44 \\
\hline $\mathrm{Cl5}(126)$ & $0.07 \mathrm{U}$ & $0.07 \mathrm{U}$ & $0.07 \mathrm{U}$ & $0.07 \mathrm{U}$ \\
\hline $\mathrm{Cl6}(128)$ & 0.07 & 0.19 & 0.10 & 0.13 \\
\hline $\mathrm{Cl6}(138)$ & 0.34 & 0.81 & 0.42 & 0.60 \\
\hline $\mathrm{Cl} 6(153)$ & 0.63 & 1.24 & 0.79 & 1.10 \\
\hline $\mathrm{Cl} 1(170)$ & 0.07 & 0.17 & 0.07 & 0.13 \\
\hline Cl7(187) & 0.19 & 0.37 & 0.22 & 0.40 \\
\hline Cl7(188) & $0.02 \mathrm{U}$ & $0.02 \mathrm{U}$ & $0.02 \mathrm{U}$ & $0.02 \mathrm{U}$ \\
\hline Cl8(195) & $0.05 \mathrm{U}$ & $0.05 \mathrm{U}$ & $0.05 \mathrm{U}$ & $0.05 \mathrm{U}$ \\
\hline $\mathrm{Cl} 18(200)$ & $0.04 \mathrm{U}$ & $0.04 \mathrm{U}$ & $0.04 \mathrm{U}$ & $0.04 \mathrm{U}$ \\
\hline $\mathrm{Cl9}(206)$ & $0.04 \mathrm{U}$ & $0.04 \mathrm{U}$ & $0.04 \mathrm{U}$ & 0.07 \\
\hline Cl10(209) & $0.07 \mathrm{U}$ & $0.07 \mathrm{U}$ & $0.07 \mathrm{U}$ & $0.07 \mathrm{U}$ \\
\hline Total PCBs & 4.78 & 9.50 & 5.60 & 8.96 \\
\hline LOC 1 & $0.08 \mathrm{U}$ & $0.08 \mathrm{U}$ & $0.08 \mathrm{U}$ & $0.08 \mathrm{U}$ \\
\hline LOC 2 & $0.12 \mathrm{~J}$ & $0.52 \mathrm{U}$ & $0.05 \mathrm{~J}$ & $0.02 \mathrm{~J}$ \\
\hline LOC 3 & $0.22 \mathrm{~J}$ & $0.19 \mathrm{~J}$ & $0.07 \mathrm{~J}$ & $0.38 \mathrm{~J}$ \\
\hline LOC 4 & $0.35 \mathrm{~J}$ & $0.75 \mathrm{~J}$ & $0.31 \mathrm{~J}$ & 1.34 \\
\hline LOC 5 & 1.15 & 2.35 & 1.17 & 2.63 \\
\hline LOC 6 & 1.57 & 3.51 & 1.87 & 3.35 \\
\hline LOC 7 & 0.69 & 1.41 & 0.83 & 1.52 \\
\hline LOC 8 & $0.51 \mathrm{U}$ & $0.15 \mathrm{~J}$ & $0.08 \mathrm{~J}$ & 0.32 \\
\hline LOC 9 & $0.16 \mathrm{U}$ & $0.16 \mathrm{U}$ & $0.16 \mathrm{U}$ & $0.07 \mathrm{~J}$ \\
\hline Total PCBs & 4.85 & 9.12 & 4.62 & 9.71 \\
\hline $\mathrm{Cl} 3(34)$ & 60 & 69 & 60 & 56 \\
\hline Cl6(152) & 64 & 69 & 59 & 57 \\
\hline
\end{tabular}


1529 West Sequim Bay Road

Sequim, Washington 98382-9099

360/681-4564

\section{SINCLAIR AND DYES INLET \\ 2007 PSAMP Trawl Biota \\ PCBs in Whole Organisms}

\begin{tabular}{|c|c|c|c|c|c|}
\hline Sample Name: & SG-ES-4 & SG-ES-5 & HC-GC-1 & SI-ES-1 & SI-ES-2 \\
\hline Station: & 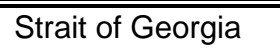 & 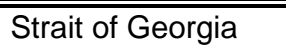 & Hood Canal & Sinclair Inlet & Sinclair Inlet \\
\hline Organism ID: & ES & ES & GC & ES & ES \\
\hline Batch ID: & $08-0004$ & $08-0004$ & 08-0004 & $08-0004$ & 08-0004 \\
\hline Sample Weight (g): & 30.34 & 30.2 & 30.46 & 30.78 & 30.74 \\
\hline \%Moisture & 79.05 & 78.61 & 80.86 & 77.81 & 80.65 \\
\hline \%Lipids & 1.18 & 1.98 & 0.41 & 2.23 & 2.20 \\
\hline Collection Date: & $05 / 02 / 2007$ & $05 / 02 / 2007$ & $05 / 03 / 2007$ & $05 / 17 / 2007$ & $05 / 17 / 2007$ \\
\hline Extraction Date: & $01 / 10 / 2008$ & $01 / 10 / 2008$ & $01 / 10 / 2008$ & $01 / 10 / 2008$ & $01 / 10 / 2008$ \\
\hline Analysis Date: & $01 / 30 / 2008$ & $01 / 29 / 2008$ & $01 / 29 / 2008$ & $01 / 30 / 2008$ & $01 / 30 / 2008$ \\
\hline Units (wet wt): & ng/g_WET & ng/g_WET & ng/g_WET & ng/g_WET & ng/g_WET \\
\hline $\mathrm{Cl} 2(8)$ & $0.05 \mathrm{U}$ & $0.02 \mathrm{~J}$ & $0.05 \mathrm{U}$ & 0.05 & 0.04 \\
\hline $\mathrm{Cl} 3(18)$ & $0.06 \mathrm{U}$ & $0.06 \mathrm{U}$ & $0.06 \mathrm{U}$ & 0.18 & 0.17 \\
\hline $\mathrm{Cl} 3(28)$ & $0.06 \mathrm{U}$ & $0.06 \mathrm{U}$ & $0.06 \mathrm{U}$ & 0.41 & 0.49 \\
\hline $\mathrm{Cl} 4(44)$ & $0.12 \mathrm{U}$ & $0.12 \mathrm{U}$ & $0.12 \mathrm{U}$ & 0.59 & 0.63 \\
\hline $\mathrm{Cl} 4(52)$ & 0.14 & 0.19 & $0.12 \mathrm{U}$ & 2.00 & 2.68 \\
\hline $\mathrm{Cl} 4(66)$ & 0.11 & 0.13 & $0.07 \mathrm{U}$ & 1.17 & 1.53 \\
\hline $\mathrm{Cl}(77)$ & $0.05 \mathrm{U}$ & $0.05 \mathrm{U}$ & $0.05 \mathrm{U}$ & 0.06 & $0.02 \mathrm{~J}$ \\
\hline $\mathrm{Cl5}(101)$ & 0.33 & 0.38 & 0.21 & $4.51 \mathrm{D}$ & $5.88 \mathrm{D}$ \\
\hline $\mathrm{Cl5}(105)$ & 0.11 & 0.13 & 0.08 & 2.19 & 2.80 \\
\hline $\mathrm{Cl5}(118)$ & 0.31 & 0.37 & 0.19 & $6.40 \mathrm{D}$ & $7.89 \mathrm{D}$ \\
\hline $\mathrm{Cl} 5(126)$ & $0.07 \mathrm{U}$ & $0.07 \mathrm{U}$ & $0.07 \mathrm{U}$ & $0.07 \mathrm{U}$ & $0.07 \mathrm{U}$ \\
\hline $\mathrm{Cl}(128)$ & 0.09 & 0.11 & 0.08 & 2.12 & 2.52 \\
\hline Cl6(138) & 0.46 & 0.49 & 0.34 & $5.82 \mathrm{D}$ & $7.72 \mathrm{D}$ \\
\hline Cl6(153) & 0.84 & 0.86 & 0.51 & $10.03 \mathrm{D}$ & $13.98 \mathrm{D}$ \\
\hline $\mathrm{Cl}(170)$ & 0.11 & 0.13 & 0.06 & 2.33 & 3.41 \\
\hline Cl7(187) & 0.32 & 0.33 & 0.16 & 6.65 & $4.82 \mathrm{D}$ \\
\hline Cl7(188) & $0.02 \mathrm{U}$ & $0.02 \mathrm{U}$ & $0.02 \mathrm{U}$ & 0.03 & 0.04 \\
\hline $\mathrm{Cl}(195)$ & $0.05 \mathrm{U}$ & $0.05 \mathrm{U}$ & $0.05 \mathrm{U}$ & 0.46 & 0.61 \\
\hline $\mathrm{Cl}(200)$ & $0.04 \mathrm{U}$ & $0.04 \mathrm{U}$ & $0.04 \mathrm{U}$ & 0.18 & 0.20 \\
\hline $\mathrm{Cl}(206)$ & 0.07 & $0.04 \mathrm{U}$ & $0.04 \mathrm{U}$ & 1.82 & 3.66 \\
\hline Cl10(209) & 0.03 & $0.07 \mathrm{U}$ & $0.07 \mathrm{U}$ & 0.53 & 1.00 \\
\hline Total PCBs & 6.88 & 7.44 & 4.90 & 95.2 & 120.32 \\
\hline LOC 1 & $0.08 \mathrm{U}$ & $0.08 \mathrm{U}$ & $0.08 \mathrm{U}$ & $0.08 \mathrm{U}$ & $0.08 \mathrm{U}$ \\
\hline LOC 2 & $0.52 \mathrm{U}$ & $0.02 \mathrm{~J}$ & $0.52 \mathrm{U}$ & $0.05 \mathrm{~J}$ & $0.06 \mathrm{~J}$ \\
\hline LOC 3 & $0.75 \mathrm{U}$ & $0.75 \mathrm{U}$ & $0.75 \mathrm{U}$ & 0.98 & 1.21 \\
\hline LOC 4 & $0.57 \mathrm{~J}$ & $0.74 \mathrm{~J}$ & $1.63 \mathrm{U}$ & 9.74 & 12.37 \\
\hline LOC 5 & 1.75 & 2.20 & 0.76 & 37.17 & 41.07 \\
\hline LOC 6 & 2.48 & 2.73 & 1.55 & 43.68 & 54.44 \\
\hline LOC 7 & 1.18 & 1.27 & $0.58 \mathrm{~J}$ & 28.47 & 32.37 \\
\hline LOC 8 & $0.15 \mathrm{~J}$ & $0.12 \mathrm{~J}$ & $0.51 \mathrm{U}$ & 9.26 & 14.62 \\
\hline LOC 9 & $0.07 \mathrm{~J}$ & $0.16 \mathrm{U}$ & $0.16 \mathrm{U}$ & 2.41 & 4.58 \\
\hline Total PCBs & 7.55 & 8.07 & 6.54 & 131.84 & 160.8 \\
\hline $\mathrm{Cl} 3(34)$ & 75 & 59 & 92 & 76 & 70 \\
\hline $\mathrm{Cl6}(152)$ & 62 & 55 & 83 & 76 & 74 \\
\hline
\end{tabular}


BATTELLE MARINE SCIENCE LABORATORIES

1529 West Sequim Bay Road

Sequim, Washington 98382-9099

360/681-4564

\section{SINCLAIR AND DYES INLET 2007 PSAMP Trawl Biota PCBs in Whole Organisms}

\begin{tabular}{|c|c|c|c|c|c|}
\hline Sample Name: & SI-ES-4 & SI-ES-6 & SI-ES-7 & SI-ES-8 & SI-RS-3 \\
\hline Station: & Sinclair Inlet & Sinclair Inlet & Sinclair Inlet & Sinclair Inlet & Sinclair Inlet \\
\hline Organism ID: & ES & ES & ES & ES & RS \\
\hline Batch ID: & 08-0004 & 08-0004 & $08-0004$ & $08-0004$ & $08-0004$ \\
\hline Sample Weight (g): & 30.2 & 30.29 & 31.18 & 30.34 & 30.16 \\
\hline \%Moisture & 59.34 & 79.69 & 79.49 & 79.29 & 78.88 \\
\hline \%Lipids & 1.01 & 1.18 & 1.05 & 1.54 & 0.71 \\
\hline Collection Date: & $05 / 17 / 2007$ & $05 / 17 / 2007$ & $05 / 17 / 2007$ & $05 / 17 / 2007$ & $05 / 17 / 2007$ \\
\hline Extraction Date: & 01/10/2008 & 01/10/2008 & 01/10/2008 & 01/10/2008 & 01/10/2008 \\
\hline Analysis Date: & 01/29/2008 & 01/30/2008 & 01/30/2008 & 01/30/2008 & 01/29/2008 \\
\hline Units (wet wt): & ng/g_WET & ng/g_WET & ng/g_WET & ng/g_WET & ng/g_WET \\
\hline $\mathrm{Cl} 2(8)$ & 0.04 & $0.02 \mathrm{~J}$ & $0.02 \mathrm{~J}$ & 0.04 & $0.05 \mathrm{U}$ \\
\hline $\mathrm{Cl} 3(18)$ & 0.09 & 0.07 & $0.06 \mathrm{U}$ & 0.19 & $0.06 \mathrm{U}$ \\
\hline $\mathrm{Cl} 3(28)$ & 0.23 & 0.17 & $0.06 \mathrm{U}$ & 0.50 & $0.06 \mathrm{U}$ \\
\hline $\mathrm{Cl} 4(44)$ & 0.30 & 0.22 & 0.21 & 0.80 & $0.12 \mathrm{U}$ \\
\hline $\mathrm{Cl} 4(52)$ & 1.24 & 0.83 & 0.82 & 2.68 & 0.32 \\
\hline $\mathrm{Cl} 4(66)$ & 0.73 & 0.52 & 0.50 & 1.48 & 0.23 \\
\hline $\mathrm{Cl} 4(77)$ & $0.05 \mathrm{U}$ & $0.02 \mathrm{~J}$ & $0.05 \mathrm{U}$ & $0.05 \mathrm{U}$ & $0.05 \mathrm{U}$ \\
\hline $\mathrm{Cl} 5(101)$ & $2.58 \mathrm{D}$ & 4.92 & 5.41 & $4.85 \mathrm{D}$ & 0.97 \\
\hline $\mathrm{Cl}$ (105) & 1.35 & 1.22 & 1.36 & 2.69 & 0.32 \\
\hline $\mathrm{Cl} 5(118)$ & 4.56 & 4.31 & 4.67 & $6.21 \mathrm{D}$ & 1.26 \\
\hline Cl5(126) & $0.07 \mathrm{U}$ & $0.07 \mathrm{U}$ & $0.07 \mathrm{U}$ & $0.07 \mathrm{U}$ & $0.07 \mathrm{U}$ \\
\hline $\mathrm{Cl6}(128)$ & 1.29 & 1.32 & 2.19 & 2.54 & 0.30 \\
\hline $\mathrm{Cl} 6(138)$ & $3.20 \mathrm{D}$ & $3.48 \mathrm{D}$ & $5.36 \mathrm{D}$ & $6.06 \mathrm{D}$ & 1.63 \\
\hline Cl6(153) & $6.48 \mathrm{D}$ & $6.94 \mathrm{D}$ & $9.6 \mathrm{D}$ & $11.71 \mathrm{D}$ & 2.98 \\
\hline $\mathrm{Cl}(170)$ & 1.26 & 1.40 & 2.71 & 2.79 & 0.26 \\
\hline $\mathrm{Cl} 7(187)$ & 4.08 & 4.30 & $4.76 \mathrm{D}$ & $4.28 \mathrm{D}$ & 1.03 \\
\hline $\mathrm{Cl} 7(188)$ & $0.02 \mathrm{~J}$ & $0.02 \mathrm{~J}$ & 0.08 & 0.05 & $0.02 \mathrm{U}$ \\
\hline $\mathrm{Cl}(195)$ & 0.24 & 0.28 & 0.75 & 0.56 & $0.05 \mathrm{U}$ \\
\hline $\mathrm{Cl} 8(200)$ & 0.11 & 0.12 & 0.29 & 0.25 & $0.04 \mathrm{U}$ \\
\hline $\mathrm{Cl9}(206)$ & 0.8 & 1.06 & 2.28 & 2.01 & 0.16 \\
\hline Cl10(209) & 0.34 & 0.37 & 0.96 & 0.73 & 0.09 \\
\hline Total PCBs & 58.12 & 63.32 & 84.42 & 101.08 & 20.14 \\
\hline LOC 1 & $0.08 \mathrm{U}$ & $0.08 \mathrm{U}$ & $0.08 \mathrm{U}$ & $0.08 \mathrm{U}$ & $0.08 \mathrm{U}$ \\
\hline LOC 2 & $0.05 \mathrm{~J}$ & $0.02 \mathrm{~J}$ & $0.08 \mathrm{~J}$ & $0.04 \mathrm{~J}$ & $0.52 \mathrm{U}$ \\
\hline LOC 3 & 0.75 & $0.37 \mathrm{~J}$ & $0.75 \mathrm{U}$ & 1.22 & $0.75 \mathrm{U}$ \\
\hline LOC 4 & 5.99 & 4.09 & 4.29 & 12.4 & 1.21 \\
\hline LOC 5 & 23.01 & 24.19 & 27.6 & 35.62 & 4.96 \\
\hline LOC 6 & 27.25 & 28.31 & 40.29 & 50.48 & 7.99 \\
\hline LOC 7 & 16.69 & 17.77 & 29.37 & 27.41 & 3.70 \\
\hline LOC 8 & 4.76 & 5.62 & 14.49 & 11.19 & 0.98 \\
\hline LOC 9 & 1.17 & 1.46 & 3.45 & 2.86 & 0.22 \\
\hline Total PCBs & 79.75 & 81.91 & 120.4 & 141.3 & 20.41 \\
\hline $\mathrm{Cl} 3(34)$ & 63 & 74 & 94 & 73 & 92 \\
\hline $\mathrm{Cl}(152)$ & 67 & 73 & 89 & 72 & 78 \\
\hline
\end{tabular}




\begin{tabular}{|c|c|c|c|c|c|}
\hline Sample Name: & V-SP-1 & V-SP-2 & V-SP-3 & HC-SP1 & HC-SP2 \\
\hline Station: & Vendovi & Vendovi & Vendovi & Hood Canal & Hood Canal \\
\hline Organism ID: & SP & SP & SP & SP & SP \\
\hline Batch ID: & $08-0013$ & 08-0013 & $08-0013$ & $08-0013$ & $08-0013$ \\
\hline Sample Weight (g): & 7.47 & 7.45 & 7.56 & 8.07 & 10.17 \\
\hline \%Moisture & 76.72 & 77.81 & 75.87 & 72.16 & 65.95 \\
\hline \%Lipids & 4.15 & 2.79 & 3.98 & 5.35 & 6.71 \\
\hline Collection Date: & $05 / 01 / 2007$ & $05 / 01 / 2007$ & $05 / 01 / 2007$ & $05 / 03 / 2007$ & $05 / 03 / 2007$ \\
\hline Extraction Date: & $01 / 22 / 2008$ & $01 / 22 / 2008$ & $01 / 22 / 2008$ & $01 / 22 / 2008$ & $01 / 22 / 2008$ \\
\hline Analysis Date: & $02 / 05 / 2008$ & $02 / 05 / 2008$ & 02/05/2008 & 02/05/2008 & $02 / 05 / 2008$ \\
\hline Units (wet wt): & ng/g_WET & ng/g_WET & ng/g_WET & ng/g_WET & ng/g_WET \\
\hline $\mathrm{Cl} 2(8)$ & $0.12 \mathrm{~J}$ & $0.05 \mathrm{U}$ & $0.16 \mathrm{~J}$ & $0.21 \mathrm{~J}$ & $0.06 \mathrm{~J}$ \\
\hline $\mathrm{Cl} 3(18)$ & $0.12 \mathrm{~J}$ & $0.06 \mathrm{U}$ & $0.15 \mathrm{~J}$ & 0.26 & $0.06 \mathrm{U}$ \\
\hline $\mathrm{Cl} 3(28)$ & $0.21 \mathrm{~J}$ & $0.13 \mathrm{~J}$ & 0.34 & 0.29 & $0.06 \mathrm{U}$ \\
\hline $\mathrm{Cl} 4(44)$ & $0.17 \mathrm{~J}$ & $0.11 \mathrm{~J}$ & 0.27 & $0.18 \mathrm{~J}$ & $0.10 \mathrm{~J}$ \\
\hline $\mathrm{Cl} 4(52)$ & 0.52 & 0.32 & 0.73 & 0.57 & 0.35 \\
\hline $\mathrm{Cl} 4(66)$ & $0.19 \mathrm{~J}$ & $0.16 \mathrm{~J}$ & 0.48 & $0.14 \mathrm{~J}$ & $0.10 \mathrm{~J}$ \\
\hline $\mathrm{Cl} 4(77)$ & $0.05 \mathrm{U}$ & $0.05 \mathrm{U}$ & $0.08 \mathrm{~J}$ & $0.05 \mathrm{~J}$ & $0.05 \mathrm{U}$ \\
\hline $\mathrm{Cl5}(101)$ & 1.06 & 0.84 & 1.23 & 2.61 & 1.62 \\
\hline $\mathrm{Cl5}(105)$ & 0.38 & 0.31 & 0.39 & 0.61 & 0.38 \\
\hline $\mathrm{Cl5}(118)$ & 1.58 & 1.42 & 1.84 & 3.74 & 2.62 \\
\hline $\mathrm{Cl5}(126)$ & $0.07 \mathrm{U}$ & $0.07 \mathrm{U}$ & $0.07 \mathrm{U}$ & $0.07 \mathrm{U}$ & $0.07 \mathrm{U}$ \\
\hline Cl6(128) & $0.18 \mathrm{~J}$ & $0.18 \mathrm{~J}$ & $0.23 \mathrm{~J}$ & 0.36 & 0.28 \\
\hline Cl6(138) & 0.92 & 0.93 & 1.02 & 2.61 & 2.27 \\
\hline $\mathrm{Cl6}(153)$ & 1.63 & 1.67 & 1.68 & 4.79 & 4.57 \\
\hline $\mathrm{Cl}(170)$ & $0.17 \mathrm{~J}$ & $0.15 \mathrm{~J}$ & $0.18 \mathrm{~J}$ & 0.58 & 0.47 \\
\hline $\mathrm{Cl}(187)$ & 0.48 & 0.53 & 0.52 & 1.62 & 1.51 \\
\hline $\mathrm{Cl} 7(188)$ & $0.02 \mathrm{U}$ & $0.02 \mathrm{~J}$ & $0.02 \mathrm{U}$ & $0.02 \mathrm{U}$ & $0.02 \mathrm{U}$ \\
\hline $\mathrm{Cl}(195)$ & $0.05 \mathrm{U}$ & $0.05 \mathrm{U}$ & $0.05 \mathrm{U}$ & $0.05 \mathrm{~J}$ & $0.08 \mathrm{~J}$ \\
\hline $\mathrm{Cl}(200)$ & $0.04 \mathrm{U}$ & $0.04 \mathrm{U}$ & $0.04 \mathrm{U}$ & $0.04 \mathrm{U}$ & $0.04 \mathrm{U}$ \\
\hline $\mathrm{Cl9}(206)$ & $0.04 \mathrm{U}$ & $0.04 \mathrm{U}$ & $0.04 \mathrm{U}$ & $0.19 \mathrm{~J}$ & $0.17 \mathrm{~J}$ \\
\hline Cl10(209) & $0.07 \mathrm{U}$ & $0.07 \mathrm{U}$ & $0.07 \mathrm{U}$ & $0.09 \mathrm{~J}$ & $0.06 \mathrm{~J}$ \\
\hline Total PCBs & 16.14 & 14.40 & 19.18 & 38.16 & 29.88 \\
\hline LOC 1 & $0.08 \mathrm{U}$ & $0.08 \mathrm{U}$ & $0.08 \mathrm{U}$ & $0.04 \mathrm{~J}$ & $0.08 \mathrm{U}$ \\
\hline LOC 2 & $0.12 \mathrm{~J}$ & $0.17 \mathrm{~J}$ & $0.16 \mathrm{~J}$ & $0.37 \mathrm{~J}$ & $0.10 \mathrm{~J}$ \\
\hline LOC 3 & $0.48 \mathrm{~J}$ & $0.20 \mathrm{~J}$ & $0.72 \mathrm{~J}$ & $0.79 \mathrm{~J}$ & $0.75 \mathrm{U}$ \\
\hline LOC 4 & $1.88 \mathrm{~J}$ & $1.37 \mathrm{~J}$ & $3.38 \mathrm{~J}$ & $2.20 \mathrm{~J}$ & $1.31 \mathrm{~J}$ \\
\hline LOC 5 & 6.52 & $4.95 \mathrm{~J}$ & 7.34 & 13.07 & 8.03 \\
\hline LOC 6 & $4.68 \mathrm{~J}$ & $4.71 \mathrm{~J}$ & $5.09 \mathrm{~J}$ & 14.00 & 11.94 \\
\hline LOC 7 & $1.72 \mathrm{~J}$ & $1.68 \mathrm{~J}$ & $1.87 \mathrm{~J}$ & 6.05 & 5.40 \\
\hline LOC 8 & $0.29 \mathrm{~J}$ & $0.1 \mathrm{~J}$ & $0.37 \mathrm{~J}$ & $1.08 \mathrm{~J}$ & $1.33 \mathrm{~J}$ \\
\hline LOC 9 & $0.16 \mathrm{U}$ & $0.16 \mathrm{U}$ & $0.16 \mathrm{U}$ & $0.27 \mathrm{~J}$ & $0.23 \mathrm{~J}$ \\
\hline Total PCBs & 15.93 & 13.42 & 19.17 & 37.87 & 29.17 \\
\hline $\mathrm{Cl} 3(34)$ & 77 & 61 & 66 & 47 & 58 \\
\hline $\mathrm{Cl6}(152)$ & 80 & 72 & 69 & 45 & 49 \\
\hline
\end{tabular}


1529 West Sequim Bay Road

Sequim, Washington 98382-9099

360/681-4564

\section{SINCLAIR AND DYES INLET \\ 2007 PSAMP Trawl Biota \\ PCBs in Whole Organisms}

\begin{tabular}{|c|c|c|c|c|c|}
\hline Sample Name: & SI-RS-1_2 & SI-RS-4 & SI-SS-1 & SI-SS-2 & SI-SS-3 \\
\hline Station: & Sinclair Inlet & Sinclair Inlet & Sinclair Inlet & Sinclair Inlet & Sinclair Inlet \\
\hline Organism ID: & RS & RS & SS & SS & SS \\
\hline Batch ID: & 08-0013 & $08-0013$ & $08-0013$ & $08-0013$ & $08-0013$ \\
\hline Sample Weight (g): & 17.6 & 30 & 30.63 & 30.55 & 30.01 \\
\hline \%Moisture & 75.72 & 78.83 & 78.34 & 77.87 & 78.95 \\
\hline \%Lipids & 3.10 & 0.47 & 0.85 & 0.72 & 0.54 \\
\hline Collection Date: & $05 / 17 / 2007$ & $05 / 17 / 2007$ & $05 / 17 / 2007$ & $05 / 17 / 2007$ & $05 / 17 / 2007$ \\
\hline Extraction Date: & $01 / 22 / 2008$ & $01 / 22 / 2008$ & $01 / 22 / 2008$ & $01 / 22 / 2008$ & $01 / 22 / 2008$ \\
\hline Analysis Date: & $02 / 12 / 2008$ & $02 / 05 / 2008$ & $02 / 13 / 2008$ & $02 / 14 / 2008$ & $02 / 14 / 2008$ \\
\hline Units (wet wt): & ng/g_WET & ng/g_WET & ng/g_WET & ng/g_WET & ng/g_WET \\
\hline $\mathrm{Cl} 2(8)$ & $0.05 \mathrm{U}$ & $0.05 \mathrm{U}$ & $0.05 \mathrm{U}$ & $0.05 \mathrm{U}$ & $0.05 \mathrm{U}$ \\
\hline $\mathrm{Cl} 3(18)$ & $0.06 \mathrm{U}$ & $0.06 \mathrm{U}$ & $0.06 \mathrm{U}$ & $0.06 \mathrm{U}$ & $0.06 \mathrm{U}$ \\
\hline $\mathrm{Cl} 3(28)$ & $0.06 \mathrm{U}$ & $0.06 \mathrm{~J}$ & 0.29 & 0.23 & $0.06 \mathrm{U}$ \\
\hline $\mathrm{Cl} 4(44)$ & 0.23 & $0.12 \mathrm{U}$ & 0.12 & 0.10 & $0.12 \mathrm{U}$ \\
\hline $\mathrm{Cl} 4(52)$ & 1.09 & 0.29 & 2.21 & 2.11 & 1.00 \\
\hline $\mathrm{Cl} 4(66)$ & 0.62 & 0.49 & 0.94 & 0.54 & $0.07 \mathrm{U}$ \\
\hline $\mathrm{Cl} 4(77)$ & $0.05 \mathrm{U}$ & $0.03 \mathrm{~J}$ & 0.04 & $0.05 \mathrm{U}$ & $0.05 \mathrm{U}$ \\
\hline $\mathrm{Cl5}(101)$ & 3.91 & 1.53 & 8.99 & 7.10 & 3.36 \\
\hline $\mathrm{Cl5}(105)$ & 0.50 & 1.38 & 1.44 & 1.12 & 0.40 \\
\hline $\mathrm{Cl} 5(118)$ & 3.18 & $3.78 \mathrm{D}$ & 8.98 & 7.00 & 3.14 \\
\hline $\mathrm{Cl} 5(126)$ & $0.07 \mathrm{U}$ & $0.07 \mathrm{U}$ & $0.07 \mathrm{U}$ & $0.07 \mathrm{U}$ & $0.07 \mathrm{U}$ \\
\hline $\mathrm{Cl6}(128)$ & 0.59 & 2.21 & 1.20 & 0.93 & $0.05 \mathrm{U}$ \\
\hline Cl6(138) & 3.41 & $7.58 \mathrm{D}$ & 9.59 & 6.17 & 2.43 \\
\hline Cl6(153) & 8.31 & $14.25 \mathrm{D}$ & $8.89 \mathrm{D}$ & 14.31 & 5.96 \\
\hline $\mathrm{Cl}(170)$ & 0.71 & 2.71 & 1.90 & 1.14 & 0.66 \\
\hline $\mathrm{Cl} 7(187)$ & 3.21 & 1.79 & 6.65 & 4.65 & 2.08 \\
\hline $\mathrm{Cl} 7(188)$ & $0.02 \mathrm{U}$ & 0.10 & $0.02 \mathrm{U}$ & 0.05 & $0.02 \mathrm{U}$ \\
\hline $\mathrm{Cl} 8(195)$ & $0.05 \mathrm{U}$ & 0.68 & 0.25 & 0.18 & $0.05 \mathrm{U}$ \\
\hline $\mathrm{Cl}(200)$ & $0.07 \mathrm{~J}$ & $0.02 \mathrm{~J}$ & $0.04 \mathrm{U}$ & $0.04 \mathrm{U}$ & $0.04 \mathrm{U}$ \\
\hline $\mathrm{Cl}(206)$ & 0.36 & 2.44 & 1.07 & 0.51 & $0.04 \mathrm{U}$ \\
\hline Cl10(209) & 0.19 & 1.12 & 0.42 & 0.22 & 0.16 \\
\hline Total PCBs & 53.48 & 81.52 & 106.44 & 93.26 & 39.74 \\
\hline LOC 1 & $0.08 \mathrm{U}$ & $0.08 \mathrm{U}$ & $0.08 \mathrm{U}$ & $0.08 \mathrm{U}$ & $0.08 \mathrm{U}$ \\
\hline LOC 2 & $0.52 \mathrm{U}$ & $0.52 \mathrm{U}$ & $0.52 \mathrm{U}$ & $0.52 \mathrm{U}$ & $0.52 \mathrm{U}$ \\
\hline LOC 3 & $0.75 \mathrm{U}$ & $0.06 \mathrm{~J}$ & 0.50 & 0.55 & $0.75 \mathrm{U}$ \\
\hline LOC 4 & 4.37 & $1.91 \mathrm{~J}$ & 7.96 & 7.01 & 2.47 \\
\hline LOC 5 & 19.7 & 15.78 & 43.49 & 32.36 & 13.83 \\
\hline LOC 6 & 25.66 & 33.36 & 45.28 & 39.06 & 16.25 \\
\hline LOC 7 & 11.17 & 17.8 & 24.66 & 15.94 & 8.19 \\
\hline LOC 8 & 3.28 & 9.36 & 7.76 & 3.96 & 0.79 \\
\hline LOC 9 & 0.62 & 3.15 & 1.65 & 0.78 & $0.16 \mathrm{U}$ \\
\hline Total PCBs & 66.15 & 82.02 & 131.9 & 100.26 & 43.04 \\
\hline $\mathrm{Cl} 3(34)$ & 100 & 65 & 106 & 91 & 94 \\
\hline Cl6(152) & 65 & 63 & 60 & 64 & 60 \\
\hline
\end{tabular}




\section{SINCLAIR AND DYES INLET 2007 PSAMP Trawl Biota PCBs in Whole Organisms}

\begin{tabular}{|c|c|c|c|c|c|}
\hline Sample Name: & SI-SSc-1_2 & SI-SSc-3_5 & SI-SSc-4_6 & SI-SP-4_5 & SI-SP-6_2 \\
\hline Station: & Sinclair Inlet & Sinclair Inlet & Sinclair Inlet & Sinclair Inlet & Sinclair Inlet \\
\hline Organism ID: & STS & STS & STS & $\mathrm{SP}$ & $\mathrm{SP}$ \\
\hline Batch ID: & $08-0013$ & $08-0013$ & $08-0013$ & $08-0013$ & $08-0013$ \\
\hline Sample Weight (g): & 30.46 & 30.6 & 33.78 & 10.65 & 10.01 \\
\hline \%Moisture & 79.34 & 79.97 & 80.49 & 75.53 & 75.32 \\
\hline \%Lipids & 1.14 & 1.01 & 1.17 & 4.01 & 2.83 \\
\hline Collection Date: & $05 / 17 / 2007$ & $05 / 17 / 2007$ & $05 / 17 / 2007$ & $05 / 17 / 2007$ & $05 / 17 / 2007$ \\
\hline Extraction Date: & $01 / 22 / 2008$ & $01 / 22 / 2008$ & 01/22/2008 & $01 / 22 / 2008$ & $01 / 22 / 2008$ \\
\hline Analysis Date: & $02 / 14 / 2008$ & $02 / 15 / 2008$ & 02/14/2008 & 03/08/2008 & $02 / 12 / 2008$ \\
\hline Units (wet wt): & ng/g_WET & ng/g_WET & ng/g_WET & ng/g_WET & ng/g_WET \\
\hline $\mathrm{Cl} 2(8)$ & $0.05 \mathrm{U}$ & $0.05 \mathrm{U}$ & $0.05 \mathrm{U}$ & $0.05 \mathrm{UT}$ & $0.05 \mathrm{U}$ \\
\hline $\mathrm{Cl} 3(18)$ & $0.06 \mathrm{U}$ & $0.06 \mathrm{U}$ & 0.06 & $0.05 \mathrm{~T}$ & $0.06 \mathrm{U}$ \\
\hline $\mathrm{Cl} 3(28)$ & $0.06 \mathrm{U}$ & 0.07 & 0.12 & $0.17 \mathrm{~T}$ & 0.29 \\
\hline Cl4(44) & $0.12 \mathrm{U}$ & 0.15 & 0.16 & $0.19 \mathrm{~T}$ & $0.12 \mathrm{U}$ \\
\hline Cl4(52) & 0.42 & 0.65 & 0.59 & $1.19 \mathrm{~T}$ & 1.29 \\
\hline Cl4(66) & 0.20 & 0.21 & 0.22 & $0.18 \mathrm{~T}$ & $0.18 \mathrm{~J}$ \\
\hline Cl4(77) & $0.05 \mathrm{U}$ & $0.05 \mathrm{U}$ & $0.01 \mathrm{~J}$ & $0.07 \mathrm{~T}$ & $0.03 \mathrm{~J}$ \\
\hline $\mathrm{Cl} 5(101)$ & 1.66 & 2.38 & 1.69 & $5.80 \mathrm{~T}$ & 4.96 \\
\hline Cl5(105) & 0.38 & 0.54 & 0.41 & $1.16 \mathrm{~T}$ & 0.75 \\
\hline Cl5(118) & 2.78 & 3.49 & 2.43 & $5.32 \mathrm{~T}$ & 4.89 \\
\hline $\mathrm{Cl} 15(126)$ & $0.07 \mathrm{U}$ & $0.07 \mathrm{U}$ & $0.07 \mathrm{U}$ & $0.07 \mathrm{UT}$ & $0.07 \mathrm{U}$ \\
\hline $\mathrm{Cl6}(128)$ & 0.39 & $0.05 \mathrm{U}$ & 0.31 & $0.59 \mathrm{~T}$ & 0.51 \\
\hline $\mathrm{Cl6}(138)$ & 2.42 & 3.11 & 2.02 & $6.04 \mathrm{~T}$ & 4.05 \\
\hline Cl6(153) & 6.09 & 6.68 & 4.12 & $15.52 \mathrm{~T}$ & 11.19 \\
\hline $\mathrm{Cl} 7(170)$ & 0.67 & 0.58 & 0.49 & $0.96 \mathrm{~T}$ & 0.73 \\
\hline Cl7(187) & 2.76 & 2.63 & 1.70 & $5.79 \mathrm{~T}$ & 3.55 \\
\hline Cl7(188) & $0.02 \mathrm{U}$ & 0.04 & $0.02 \mathrm{U}$ & $0.02 \mathrm{UT}$ & $0.02 \mathrm{U}$ \\
\hline Cl8(195) & 0.09 & 0.14 & 0.08 & $0.05 \mathrm{UT}$ & $0.05 \mathrm{U}$ \\
\hline $\mathrm{Cl} 8(200)$ & $0.04 \mathrm{U}$ & $0.04 \mathrm{U}$ & $0.04 \mathrm{U}$ & $0.04 \mathrm{UT}$ & $0.04 \mathrm{U}$ \\
\hline $\mathrm{Cl} 9(206)$ & 0.50 & 0.34 & 0.28 & $0.72 \mathrm{~T}$ & 0.37 \\
\hline Cl10(209) & 0.17 & 0.13 & 0.09 & $0.34 \mathrm{~T}$ & 0.23 \\
\hline Total PCBs & 38.00 & 42.92 & 29.92 & 88.64 & 66.86 \\
\hline LOC 1 & $0.08 \mathrm{U}$ & $0.08 \mathrm{U}$ & $0.08 \mathrm{U}$ & $0.08 \mathrm{UT}$ & $0.08 \mathrm{U}$ \\
\hline LOC 2 & $0.52 \mathrm{U}$ & $0.52 \mathrm{U}$ & $0.52 \mathrm{U}$ & $0.15 \mathrm{~T}$ & $0.52 \mathrm{U}$ \\
\hline LOC 3 & $0.75 \mathrm{U}$ & 0.07 & $0.24 \mathrm{~J}$ & $0.56 \mathrm{~T}$ & $0.29 \mathrm{~J}$ \\
\hline LOC 4 & 1.36 & 2.12 & 1.94 & $3.35 \mathrm{~T}$ & $3.87 \mathrm{~J}$ \\
\hline LOC 5 & 10.47 & 14.07 & 9.60 & $24.89 \mathrm{~T}$ & 21.67 \\
\hline LOC 6 & 17.44 & 18.91 & 12.83 & $36.28 \mathrm{~T}$ & 27.61 \\
\hline LOC 7 & 9.62 & 9.05 & 6.49 & $16.75 \mathrm{~T}$ & 11.43 \\
\hline LOC 8 & 3.08 & 2.54 & 2.05 & $5.43 \mathrm{~T}$ & 2.69 \\
\hline LOC 9 & 0.72 & 0.54 & 0.41 & $1.17 \mathrm{~T}$ & 0.62 \\
\hline Total PCBs & 44.04 & 47.9 & 34.16 & 88.66 & 68.78 \\
\hline $\mathrm{Cl} 3(34)$ & 82 & 65 & 66 & 70 & 106 \\
\hline Cl6(152) & 59 & 49 & 52 & 74 & 68 \\
\hline
\end{tabular}


1529 West Sequim Bay Road

Sequim, Washington 98382-9099

360/681-4564

\section{SINCLAIR AND DYES INLET 2007 PSAMP Trawl Biota PCBs in Whole Organisms}

\begin{tabular}{|c|c|c|c|c|c|}
\hline Sample Name: & SI-SP-7_3 & SI-GC-2_6 & SI-GC-3_4 & SI-GC-7_1 & SI-GC-5_8 \\
\hline Station: & Sinclair Inlet & Sinclair Inlet & Sinclair Inlet & Sinclair Inlet & Sinclair Inlet \\
\hline Organism ID: & SP & GC & GC & $\mathrm{GC}$ & $\mathrm{GC}$ \\
\hline Batch ID: & $08-0013$ & 08-0013 & $08-0013$ & $08-0013$ & $08-0013$ \\
\hline Sample Weight (g): & 9.07 & 31.73 & 30.43 & 30.81 & 30.07 \\
\hline$\%$ Moisture & 74.88 & 71.25 & 66.40 & 45.15 & 69.11 \\
\hline \%Lipids & 3.16 & 0.92 & 0.94 & 0.92 & 1.00 \\
\hline Collection Date: & $05 / 17 / 2007$ & $05 / 17 / 2007$ & $05 / 17 / 2007$ & $05 / 17 / 2007$ & $05 / 17 / 2007$ \\
\hline Extraction Date: & 01/22/2008 & 01/22/2008 & 01/22/2008 & 01/22/2008 & 01/22/2008 \\
\hline Analysis Date: & 02/12/2008 & 03/08/2008 & 02/12/2008 & 03/08/2008 & 02/12/2008 \\
\hline Units (wet wt): & ng/g_WET & ng/g_WET & ng/g_WET & ng/g_WET & ng/g_WET \\
\hline $\mathrm{Cl} 2(8)$ & $0.05 \mathrm{U}$ & $0.05 \mathrm{UT}$ & $0.05 \mathrm{U}$ & $0.05 \mathrm{UT}$ & $0.05 \mathrm{U}$ \\
\hline $\mathrm{Cl} 3(18)$ & $0.19 \mathrm{~J}$ & $0.06 \mathrm{UT}$ & $0.06 \mathrm{U}$ & $0.06 \mathrm{UT}$ & $0.06 \mathrm{U}$ \\
\hline $\mathrm{Cl} 3(28)$ & 0.34 & $0.22 \mathrm{~T}$ & 0.13 & $0.13 \mathrm{~T}$ & 0.12 \\
\hline Cl4(44) & 0.60 & $0.30 \mathrm{~T}$ & $0.12 \mathrm{U}$ & $0.12 \mathrm{UT}$ & 0.13 \\
\hline $\mathrm{Cl} 4(52)$ & 2.09 & $1.07 \mathrm{~T}$ & 0.67 & $0.64 \mathrm{~T}$ & 0.43 \\
\hline $\mathrm{Cl} 4(66)$ & 0.37 & $0.49 \mathrm{~T}$ & 0.57 & $0.40 \mathrm{~T}$ & 0.26 \\
\hline $\mathrm{Cl} 4(77)$ & $0.05 \mathrm{U}$ & $0.06 \mathrm{~T}$ & $0.05 \mathrm{U}$ & $0.05 \mathrm{UT}$ & $0.05 \mathrm{U}$ \\
\hline $\mathrm{Cl} 5(101)$ & 7.09 & $3.90 \mathrm{~T}$ & 2.93 & $2.74 \mathrm{~T}$ & 1.44 \\
\hline $\mathrm{Cl} 5(105)$ & 1.00 & $0.80 \mathrm{~T}$ & 0.58 & $0.56 \mathrm{~T}$ & 0.26 \\
\hline $\mathrm{Cl5}(118)$ & 5.76 & $3.69 \mathrm{~T}$ & 2.91 & $2.67 \mathrm{~T}$ & 1.40 \\
\hline $\mathrm{Cl} 5(126)$ & $0.07 \mathrm{U}$ & $0.07 \mathrm{UT}$ & $0.07 \mathrm{U}$ & $0.07 \mathrm{UT}$ & $0.07 \mathrm{U}$ \\
\hline $\mathrm{Cl6}(128)$ & 0.60 & $0.49 \mathrm{~T}$ & 0.43 & $0.46 \mathrm{~T}$ & 0.24 \\
\hline Cl6(138) & 4.91 & $3.38 \mathrm{~T}$ & 2.22 & $3.00 \mathrm{~T}$ & 1.25 \\
\hline Cl6(153) & 12.38 & $6.21 \mathrm{~T}$ & 5.10 & $6.12 \mathrm{~T}$ & 2.46 \\
\hline $\mathrm{Cl} 7(170)$ & 0.96 & $0.57 \mathrm{~T}$ & 0.51 & $0.58 \mathrm{~T}$ & 0.25 \\
\hline $\mathrm{Cl7}(187)$ & 3.82 & $2.31 \mathrm{~T}$ & 2.24 & $2.35 \mathrm{~T}$ & 1.22 \\
\hline $\mathrm{Cl7}(188)$ & $0.02 \mathrm{U}$ & $0.02 \mathrm{UT}$ & $0.02 \mathrm{U}$ & $0.02 \mathrm{UT}$ & $0.03 \mathrm{~J}$ \\
\hline $\mathrm{Cl}(195)$ & $0.05 \mathrm{U}$ & $0.05 \mathrm{UT}$ & 0.08 & $0.05 \mathrm{UT}$ & $0.05 \mathrm{U}$ \\
\hline $\mathrm{Cl}(200)$ & $0.04 \mathrm{U}$ & $0.11 \mathrm{~T}$ & $0.06 \mathrm{~J}$ & $0.04 \mathrm{UT}$ & $0.03 \mathrm{~J}$ \\
\hline $\mathrm{Cl} 9(206)$ & 0.62 & $0.45 \mathrm{~T}$ & 0.36 & $0.42 \mathrm{~T}$ & 0.22 \\
\hline Cl10(209) & 0.25 & $0.23 \mathrm{~T}$ & 0.16 & $0.19 \mathrm{~T}$ & 0.15 \\
\hline Total PCBs & 82.52 & 49.06 & 38.64 & 41.44 & 20.34 \\
\hline LOC 1 & $0.08 \mathrm{U}$ & $0.08 \mathrm{UT}$ & $0.08 \mathrm{U}$ & $0.08 \mathrm{UT}$ & $0.08 \mathrm{U}$ \\
\hline LOC 2 & $0.52 \mathrm{U}$ & $0.52 \mathrm{UT}$ & $0.52 \mathrm{U}$ & $0.52 \mathrm{UT}$ & $0.52 \mathrm{U}$ \\
\hline LOC 3 & $0.82 \mathrm{~J}$ & $0.38 \mathrm{~T}$ & $0.22 \mathrm{~J}$ & $0.22 \mathrm{~T}$ & $0.18 \mathrm{~J}$ \\
\hline LOC 4 & 7.02 & $3.87 \mathrm{~T}$ & 2.43 & $1.88 \mathrm{~T}$ & $1.63 \mathrm{~J}$ \\
\hline LOC 5 & 31.08 & $17.13 \mathrm{~T}$ & 12.16 & $12.08 \mathrm{~T}$ & 7.32 \\
\hline LOC 6 & 34.03 & $19.68 \mathrm{~T}$ & 17.04 & $17.71 \mathrm{~T}$ & 9.25 \\
\hline LOC 7 & 13.53 & $9.25 \mathrm{~T}$ & 7.76 & $8.96 \mathrm{~T}$ & 4.28 \\
\hline LOC 8 & 3.12 & $2.62 \mathrm{~T}$ & 2.11 & $2.68 \mathrm{~T}$ & 1.39 \\
\hline LOC 9 & 0.80 & $0.65 \mathrm{~T}$ & 0.50 & $0.56 \mathrm{~T}$ & 0.36 \\
\hline Total PCBs & 91 & 54.18 & 42.82 & 44.69 & 25.01 \\
\hline $\mathrm{Cl} 3(34)$ & 70 & 89 & 81 & 58 & 78 \\
\hline $\mathrm{Cl6}(152)$ & 44 & 84 & 52 & 45 & 48 \\
\hline
\end{tabular}




\section{SINCLAIR AND DYES INLET 2007 PSAMP Trawl Biota PCBs in Whole Organisms}

\begin{tabular}{|c|c|c|c|c|c|}
\hline Sample Name: & V-SC-COMP & SG-RF-1 & SG-RF-2 & SG-RF-3 & SG-SC-COMP \\
\hline Station: & Vendovi & Strait of Georgia & Strait of Georgia & 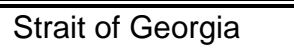 & $\overline{\text { Strait of Georgia }}$ \\
\hline Organism ID: & SC & RF & RF & $\mathrm{RF}$ & $\mathrm{SC}$ \\
\hline Batch ID: & 08-0015 & 08-0015 & 08-0015 & 08-0015 & 08-0015 \\
\hline Sample Weight (g): & 27.63 & 29.92 & 30.42 & 29.92 & 31.26 \\
\hline \%Moisture & 86.80 & 80.14 & 72.01 & 66.36 & 92.30 \\
\hline \%Lipids & 0.10 & 4.06 & 11.50 & 15.36 & 0.34 \\
\hline Collection Date: & $05 / 01 / 2007$ & $05 / 02 / 2007$ & $05 / 02 / 2007$ & $05 / 02 / 2007$ & $05 / 02 / 2007$ \\
\hline Extraction Date: & $01 / 28 / 2008$ & 01/28/2008 & $01 / 28 / 2008$ & $01 / 28 / 2008$ & $01 / 28 / 2008$ \\
\hline Analysis Date: & $02 / 16 / 2008$ & $02 / 16 / 2008$ & $02 / 16 / 2008$ & $02 / 16 / 2008$ & $02 / 16 / 2008$ \\
\hline Units (wet wt): & ng/g_WET & ng/g_WET & ng/g_WET & ng/g_WET & ng/g_WET \\
\hline $\mathrm{Cl} 2(8)$ & $0.05 \mathrm{U}$ & $0.05 \mathrm{U}$ & $0.01 \mathrm{~J}$ & $0.04 \mathrm{~J}$ & $0.05 \mathrm{U}$ \\
\hline $\mathrm{Cl} 3(18)$ & $0.01 \mathrm{~J}$ & $0.01 \mathrm{~J}$ & $0.01 \mathrm{~J}$ & $0.06 \mathrm{U}$ & $0.06 \mathrm{U}$ \\
\hline $\mathrm{Cl} 3(28)$ & $0.02 \mathrm{~J}$ & $0.04 \mathrm{~J}$ & 0.12 & 0.28 & $0.06 \mathrm{U}$ \\
\hline $\mathrm{Cl} 4(44)$ & $0.04 \mathrm{~J}$ & $0.12 \mathrm{U}$ & $0.12 \mathrm{U}$ & $0.12 \mathrm{U}$ & $0.12 \mathrm{U}$ \\
\hline $\mathrm{Cl} 4(52)$ & $0.03 \mathrm{~J}$ & $0.01 \mathrm{~J}$ & $0.01 \mathrm{~J}$ & 0.11 & $0.12 \mathrm{U}$ \\
\hline $\mathrm{Cl} 4(66)$ & $0.07 \mathrm{U}$ & 0.11 & 0.30 & 0.29 & $0.07 \mathrm{U}$ \\
\hline Cl4(77) & $0.05 \mathrm{U}$ & $0.05 \mathrm{U}$ & $0.05 \mathrm{U}$ & $0.05 \mathrm{U}$ & $0.05 \mathrm{U}$ \\
\hline Cl5(101) & $0.02 \mathrm{~J}$ & $0.02 \mathrm{~J}$ & $0.02 \mathrm{~J}$ & 0.09 & 0.06 \\
\hline $\mathrm{Cl} 5(105)$ & $0.05 \mathrm{U}$ & 0.15 & 0.29 & 0.46 & $0.05 \mathrm{U}$ \\
\hline $\mathrm{Cl5}(118)$ & $0.04 \mathrm{~J}$ & 0.66 & 1.45 & 1.17 & 0.09 \\
\hline $\mathrm{Cl5}(126)$ & $0.07 \mathrm{U}$ & $0.07 \mathrm{U}$ & $0.07 \mathrm{U}$ & $0.07 \mathrm{U}$ & $0.07 \mathrm{U}$ \\
\hline $\mathrm{Cl6}(128)$ & $0.05 \mathrm{U}$ & 0.18 & 0.29 & 0.46 & $0.05 \mathrm{U}$ \\
\hline Cl6(138) & $0.03 \mathrm{~J}$ & 0.78 & 1.29 & 1.97 & 0.15 \\
\hline $\mathrm{Cl6}(153)$ & $0.06 \mathrm{~J}$ & 1.31 & 1.97 & 3.43 & 0.27 \\
\hline $\mathrm{Cl}(170)$ & $0.04 \mathrm{U}$ & 0.20 & 0.33 & 0.43 & $0.04 \mathrm{U}$ \\
\hline Cl7(187) & $0.03 \mathrm{~J}$ & $0.02 \mathrm{~J}$ & $0.05 \mathrm{~J}$ & 0.14 & 0.13 \\
\hline $\mathrm{Cl}(188)$ & $0.02 \mathrm{U}$ & $0.02 \mathrm{U}$ & $0.02 \mathrm{U}$ & $0.02 \mathrm{U}$ & $0.02 \mathrm{U}$ \\
\hline $\mathrm{Cl}(195)$ & $0.05 \mathrm{U}$ & $0.04 \mathrm{~J}$ & 0.08 & $0.05 \mathrm{U}$ & $0.05 \mathrm{U}$ \\
\hline $\mathrm{Cl} 8(200)$ & $0.04 \mathrm{U}$ & $0.04 \mathrm{U}$ & $0.04 \mathrm{U}$ & $0.04 \mathrm{U}$ & $0.04 \mathrm{U}$ \\
\hline $\mathrm{Cl9}(206)$ & $0.04 \mathrm{U}$ & 0.08 & 0.17 & 0.18 & $0.04 \mathrm{U}$ \\
\hline Cl10(209) & $0.07 \mathrm{U}$ & $0.03 \mathrm{~J}$ & 0.07 & $0.06 \mathrm{~J}$ & $0.07 \mathrm{U}$ \\
\hline Total PCBs & 1.76 & 7.98 & 13.52 & 19.04 & 3.32 \\
\hline LOC 1 & $0.08 \mathrm{U}$ & $0.08 \mathrm{U}$ & $0.08 \mathrm{U}$ & $0.08 \mathrm{U}$ & $0.08 \mathrm{U}$ \\
\hline LOC 2 & $0.52 \mathrm{U}$ & $0.52 \mathrm{U}$ & $0.01 \mathrm{~J}$ & $0.04 \mathrm{~J}$ & $0.52 \mathrm{U}$ \\
\hline LOC 3 & $0.07 \mathrm{~J}$ & $0.06 \mathrm{~J}$ & $0.17 \mathrm{~J}$ & $0.50 \mathrm{~J}$ & $0.75 \mathrm{U}$ \\
\hline LOC 4 & $0.12 \mathrm{~J}$ & $0.31 \mathrm{~J}$ & $0.81 \mathrm{~J}$ & $1.61 \mathrm{~J}$ & $1.63 \mathrm{U}$ \\
\hline LOC 5 & $0.11 \mathrm{~J}$ & $1.34 \mathrm{~J}$ & 2.83 & 3.06 & $0.30 \mathrm{~J}$ \\
\hline LOC 6 & $0.15 \mathrm{~J}$ & 2.70 & 4.23 & 6.71 & $0.62 \mathrm{~J}$ \\
\hline LOC 7 & $0.07 \mathrm{~J}$ & $1.14 \mathrm{~J}$ & 1.78 & 2.29 & $0.34 \mathrm{~J}$ \\
\hline LOC 8 & $0.51 \mathrm{U}$ & $0.31 \mathrm{~J}$ & $0.55 \mathrm{~J}$ & $0.51 \mathrm{U}$ & $0.51 \mathrm{U}$ \\
\hline LOC 9 & $0.16 \mathrm{U}$ & $0.10 \mathrm{~J}$ & $0.20 \mathrm{~J}$ & $0.18 \mathrm{~J}$ & $0.16 \mathrm{U}$ \\
\hline Total PCBs & 1.79 & 6.56 & 10.66 & 14.98 & 4.91 \\
\hline $\mathrm{Cl} 3(34)$ & 64 & 60 & 54 & 80 & 78 \\
\hline Cl6(152) & 70 & 55 & 51 & 88 & 96 \\
\hline
\end{tabular}


1529 West Sequim Bay Road

Sequim, Washington 98382-9099

360/681-4564

\section{SINCLAIR AND DYES INLET 2007 PSAMP Trawl Biota PCBs in Whole Organisms}

\begin{tabular}{|c|c|c|c|c|}
\hline Sample Name: & HC-ES-1 & HC-ES-2 & HC-ES-3 & HC-RS-1 \\
\hline Station: & Hood Canal & Hood Canal & Hood Canal & Hood Canal \\
\hline Organism ID: & ES & ES & ES & RS \\
\hline Batch ID: & 08-0015 & 08-0015 & 08-0015 & 08-0015 \\
\hline Sample Weight (g): & 30.79 & 30.26 & 30.27 & 30.05 \\
\hline \%Moisture & 82.09 & 79.72 & 78.81 & 77.93 \\
\hline \%Lipids & 0.52 & 1.69 & 1.63 & 0.79 \\
\hline Collection Date: & $05 / 03 / 2007$ & $05 / 03 / 2007$ & $05 / 03 / 2007$ & $05 / 03 / 2007$ \\
\hline Extraction Date: & $01 / 28 / 2008$ & $01 / 28 / 2008$ & $01 / 28 / 2008$ & $01 / 28 / 2008$ \\
\hline Analysis Date: & $02 / 16 / 2008$ & $02 / 16 / 2008$ & $02 / 17 / 2008$ & $02 / 17 / 2008$ \\
\hline Units (wet wt): & ng/g_WET & ng/g_WET & ng/g_WET & ng/g_WET \\
\hline $\mathrm{Cl} 2(8)$ & $0.05 \mathrm{U}$ & $0.05 \mathrm{U}$ & $0.01 \mathrm{~J}$ & $0.05 \mathrm{U}$ \\
\hline $\mathrm{Cl} 3(18)$ & $0.06 \mathrm{U}$ & $0.06 \mathrm{U}$ & $0.03 \mathrm{~J}$ & $0.06 \mathrm{U}$ \\
\hline $\mathrm{Cl} 3(28)$ & $0.02 \mathrm{~J}$ & $0.06 \mathrm{~J}$ & 0.07 & $0.03 \mathrm{~J}$ \\
\hline $\mathrm{Cl} 4(44)$ & $0.12 \mathrm{U}$ & $0.06 \mathrm{~J}$ & 0.09 & $0.12 \mathrm{U}$ \\
\hline $\mathrm{Cl} 4(52)$ & $0.03 \mathrm{~J}$ & 0.16 & 0.25 & 0.09 \\
\hline $\mathrm{Cl} 4(66)$ & $0.02 \mathrm{~J}$ & 0.09 & 0.12 & $0.04 \mathrm{~J}$ \\
\hline $\mathrm{Cl} 4(77)$ & $0.05 \mathrm{U}$ & $0.05 \mathrm{U}$ & $0.05 \mathrm{U}$ & $0.05 \mathrm{U}$ \\
\hline $\mathrm{Cl5}(101)$ & 0.12 & 0.44 & 0.72 & 0.18 \\
\hline $\mathrm{Cl} 15(105)$ & $0.05 \mathrm{U}$ & 0.16 & 0.21 & 0.08 \\
\hline $\mathrm{Cl5}(118)$ & 0.13 & 0.53 & 0.77 & 0.32 \\
\hline $\mathrm{Cl} 5(126)$ & $0.07 \mathrm{U}$ & $0.07 \mathrm{U}$ & $0.07 \mathrm{U}$ & $0.07 \mathrm{U}$ \\
\hline $\mathrm{Cl6}(128)$ & $0.04 \mathrm{~J}$ & 0.15 & 0.16 & 0.12 \\
\hline Cl6(138) & 0.27 & 0.88 & 1.13 & 0.67 \\
\hline Cl6(153) & 0.56 & 1.72 & 2.33 & 1.16 \\
\hline $\mathrm{Cl} 7(170)$ & 0.09 & 0.20 & 0.26 & 0.12 \\
\hline $\mathrm{Cl} 7(187)$ & 0.27 & 0.72 & 0.89 & 0.38 \\
\hline Cl7(188) & $0.02 \mathrm{U}$ & $0.02 \mathrm{U}$ & $0.02 \mathrm{U}$ & $0.02 \mathrm{U}$ \\
\hline $\mathrm{Cl}(195)$ & $0.05 \mathrm{U}$ & $0.05 \mathrm{U}$ & $0.05 \mathrm{U}$ & $0.05 \mathrm{U}$ \\
\hline $\mathrm{Cl} 8(200)$ & $0.04 \mathrm{U}$ & $0.04 \mathrm{U}$ & $0.04 \mathrm{U}$ & $0.04 \mathrm{U}$ \\
\hline $\mathrm{Cl9}(206)$ & $0.04 \mathrm{~J}$ & 0.09 & 0.13 & 0.09 \\
\hline Cl10(209) & $0.04 \mathrm{~J}$ & $0.03 \mathrm{~J}$ & $0.05 \mathrm{~J}$ & $0.03 \mathrm{~J}$ \\
\hline Total PCBs & 4.28 & 11.26 & 14.90 & 7.54 \\
\hline LOC 1 & $0.08 \mathrm{U}$ & $0.08 \mathrm{U}$ & $0.08 \mathrm{U}$ & $0.08 \mathrm{U}$ \\
\hline LOC 2 & $0.52 \mathrm{U}$ & $0.52 \mathrm{U}$ & $0.01 \mathrm{~J}$ & $0.52 \mathrm{U}$ \\
\hline LOC 3 & $0.02 \mathrm{~J}$ & $0.11 \mathrm{~J}$ & $0.19 \mathrm{~J}$ & $0.06 \mathrm{~J}$ \\
\hline LOC 4 & $0.09 \mathrm{~J}$ & $0.71 \mathrm{~J}$ & $1.07 \mathrm{~J}$ & $0.30 \mathrm{~J}$ \\
\hline LOC 5 & $0.48 \mathrm{~J}$ & 2.56 & 3.98 & $1.07 \mathrm{~J}$ \\
\hline LOC 6 & $1.26 \mathrm{~J}$ & 4.61 & 6.57 & 2.63 \\
\hline LOC 7 & $0.91 \mathrm{~J}$ & 2.52 & 3.22 & 1.51 \\
\hline LOC 8 & $0.51 \mathrm{U}$ & $0.67 \mathrm{~J}$ & 0.96 & $0.47 \mathrm{~J}$ \\
\hline LOC 9 & $0.04 \mathrm{~J}$ & $0.13 \mathrm{~J}$ & 0.22 & $0.13 \mathrm{~J}$ \\
\hline Total PCBs & 3.91 & 11.91 & 16.3 & 6.77 \\
\hline $\mathrm{Cl} 3(34)$ & 78 & 67 & 71 & 78 \\
\hline Cl6(152) & 89 & 73 & 73 & 84 \\
\hline
\end{tabular}


1529 West Sequim Bay Road

Sequim, Washington 98382-9099

360/681-4564
SINCLAIR AND DYES INLET

2007 PSAMP Trawl Biota

PCBs in Whole Organisms

\begin{tabular}{|c|c|c|c|c|c|c|}
\hline Sample Name: & HC-RF-1 & HC-RF-2 & HC-RF-3 & EB-ES-1_2 & EB-ES-3 & EB-RF-1 \\
\hline Station: & Hood Canal & Hood Canal & Hood Canal & Elliot Bay & Elliot Bay & Elliot Bay \\
\hline Organism ID: & $\mathrm{RF}$ & $\mathrm{RF}$ & $\mathrm{RF}$ & ES & ES & RF \\
\hline Batch ID: & 08-0015 & $08-0015$ & 08-0015 & $08-0015$ & $08-0015$ & $08-0015$ \\
\hline Sample Weight (g): & 30.98 & 30.58 & 30.38 & 21 & 30.33 & 30.99 \\
\hline \%Moisture & 74.37 & 72.96 & 72.39 & 78.56 & 80.28 & 73.57 \\
\hline \%Lipids & 7.66 & 12.00 & 12.70 & 0.69 & 0.81 & 10.60 \\
\hline Collection Date: & $05 / 03 / 2007$ & $05 / 03 / 2007$ & $05 / 03 / 2007$ & $05 / 16 / 2007$ & $05 / 16 / 2007$ & $05 / 16 / 2007$ \\
\hline Extraction Date: & $01 / 28 / 2008$ & 01/28/2008 & 01/28/2008 & 01/28/2008 & 01/28/2008 & 01/28/2008 \\
\hline Analysis Date: & 02/17/2008 & 02/17/2008 & 02/17/2008 & 02/17/2008 & $02 / 17 / 2008$ & $02 / 17 / 2008$ \\
\hline Units (wet wt): & ng/g_WET & ng/g_WET & ng/g_WET & ng/g_WET & ng/g_WET & ng/g_WET \\
\hline $\mathrm{Cl} 2(8)$ & $0.01 \mathrm{~J}$ & $0.02 \mathrm{~J}$ & $0.03 \mathrm{~J}$ & $0.05 \mathrm{U}$ & $0.05 \mathrm{U}$ & $0.04 \mathrm{~J}$ \\
\hline $\mathrm{Cl} 3(18)$ & $0.06 \mathrm{U}$ & $0.06 \mathrm{U}$ & $0.06 \mathrm{U}$ & $0.06 \mathrm{U}$ & 0.09 & $0.06 \mathrm{U}$ \\
\hline $\mathrm{Cl} 3(28)$ & 0.19 & 0.18 & 0.27 & $0.07 \mathrm{~J}$ & 0.20 & 3.74 \\
\hline $\mathrm{Cl} 4(44)$ & $0.12 \mathrm{U}$ & $0.12 \mathrm{U}$ & $0.12 \mathrm{U}$ & $0.08 \mathrm{~J}$ & 0.37 & $0.12 \mathrm{U}$ \\
\hline $\mathrm{Cl} 4(52)$ & $0.04 \mathrm{~J}$ & $0.12 \mathrm{U}$ & $0.12 \mathrm{U}$ & 0.20 & 1.26 & 0.27 \\
\hline $\mathrm{Cl} 4(66)$ & 0.48 & 0.38 & 1.23 & 0.15 & 0.79 & $21.14 \mathrm{D}$ \\
\hline $\mathrm{Cl}(77)$ & $0.05 \mathrm{U}$ & $0.05 \mathrm{U}$ & $0.05 \mathrm{U}$ & $0.05 \mathrm{U}$ & $0.03 \mathrm{~J}$ & $0.05 \mathrm{U}$ \\
\hline $\mathrm{Cl5}(101)$ & $0.03 \mathrm{~J}$ & $0.05 \mathrm{U}$ & $0.05 \mathrm{~J}$ & 0.69 & 7.56 & 1.11 \\
\hline $\mathrm{Cl} 5(105)$ & 1.06 & 0.76 & 2.82 & 0.26 & 2.03 & $62.2 \mathrm{D}$ \\
\hline $\mathrm{Cl5}(118)$ & 2.82 & 1.87 & 7.36 & 0.92 & 6.70 & 186.45 D \\
\hline $\mathrm{Cl} 5(126)$ & $0.07 \mathrm{U}$ & $0.07 \mathrm{U}$ & $0.07 \mathrm{U}$ & $0.07 \mathrm{U}$ & $0.07 \mathrm{U}$ & $0.07 \mathrm{U}$ \\
\hline $\mathrm{Cl}(128)$ & 1.45 & 1.04 & 3.89 & 0.28 & 2.76 & $85.04 \mathrm{D}$ \\
\hline Cl6(138) & 6.68 & 3.65 & 13.34 & 1.55 & 12.04 & $412.93 \mathrm{D}$ \\
\hline $\mathrm{Cl6}(153)$ & 10.3 & 5.65 & $27.65 \mathrm{D}$ & 2.97 & $29.96 \mathrm{D}$ & $604.75 \mathrm{D}$ \\
\hline $\mathrm{Cl}(170)$ & 1.59 & 0.81 & 4.68 & 0.38 & 4.91 & $128.18 \mathrm{D}$ \\
\hline $\mathrm{Cl}(187)$ & 0.06 & 0.07 & 0.14 & 1.02 & 10.53 & 3.24 \\
\hline $\mathrm{Cl}(188)$ & $0.02 \mathrm{U}$ & $0.02 \mathrm{U}$ & $0.02 \mathrm{U}$ & $0.02 \mathrm{U}$ & $0.04 \mathrm{~J}$ & 0.10 \\
\hline $\mathrm{Cl} 8(195)$ & 0.23 & 0.15 & 1.01 & $0.05 \mathrm{U}$ & 0.92 & $23.1 \mathrm{D}$ \\
\hline $\mathrm{Cl}(200)$ & $0.04 \mathrm{U}$ & $0.04 \mathrm{U}$ & $0.04 \mathrm{U}$ & $0.04 \mathrm{U}$ & 0.36 & $0.04 \mathrm{U}$ \\
\hline $\mathrm{Cl} 9(206)$ & 0.63 & 0.39 & 1.96 & 0.18 & 2.34 & $29.33 \mathrm{D}$ \\
\hline Cl10(209) & 0.29 & 0.19 & 0.80 & $0.05 \mathrm{~J}$ & 1.02 & 8.59 \\
\hline Total PCBs & 52.44 & 31.38 & 131.42 & 18.28 & 168.06 & 3141.10 \\
\hline LOC 1 & $0.08 \mathrm{U}$ & $0.08 \mathrm{U}$ & $0.08 \mathrm{U}$ & $0.08 \mathrm{U}$ & $0.08 \mathrm{U}$ & $0.08 \mathrm{U}$ \\
\hline LOC 2 & $0.01 \mathrm{~J}$ & $0.02 \mathrm{~J}$ & $0.03 \mathrm{~J}$ & $0.52 \mathrm{U}$ & $0.52 \mathrm{U}$ & $0.04 \mathrm{~J}$ \\
\hline LOC 3 & $0.22 \mathrm{~J}$ & $0.21 \mathrm{~J}$ & $0.31 \mathrm{~J}$ & $0.11 \mathrm{~J}$ & $0.45 \mathrm{~J}$ & 3.96 \\
\hline LOC 4 & $1.49 \mathrm{~J}$ & $1.10 \mathrm{~J}$ & 3.77 & $1.09 \mathrm{~J}$ & 6.96 & 62.8 \\
\hline LOC 5 & 6.43 & 5.06 & 17.33 & 4.95 & 41.16 & 475.97 \\
\hline LOC 6 & 20.59 & 11.86 & 50.62 & 8.87 & 90.81 & 1261.94 \\
\hline LOC 7 & 8.43 & 4.35 & 23.73 & 4.48 & 52.38 & 707.03 \\
\hline LOC 8 & 2.31 & 1.23 & 8.03 & $0.97 \mathrm{~J}$ & 14.61 & 197.02 \\
\hline LOC 9 & 0.72 & 0.44 & 2.20 & $0.18 \mathrm{~J}$ & 3.35 & 32.82 \\
\hline Total PCBs & 40.28 & 24.35 & 106.1 & 21.25 & 210.32 & 2741.66 \\
\hline $\mathrm{Cl} 3(34)$ & 70 & 78 & 78 & 76 & 80 & 75 \\
\hline Cl6(152) & 75 & 77 & 79 & 78 & 83 & 76 \\
\hline
\end{tabular}




\begin{tabular}{|c|c|c|c|c|c|}
\hline Sample Name: & EB-RF-2 & EB-RF-3 & EH-ES-2 & EH-ES-3 & EH-ES-4 \\
\hline Station: & Elliot Bay & Elliot Bay & Eagle Harbor & Eagle Harbor & Eagle Harbor \\
\hline Organism ID: & $\mathrm{RF}$ & RF & ES & ES & ES \\
\hline Batch ID: & 08-0015 & 08-0015 & 08-0015 & 08-0015 & 08-0015 \\
\hline Sample Weight (g): & 30.03 & 30.65 & 31.54 & 20.71 & 30.45 \\
\hline \%Moisture & 72.77 & 72.89 & 82.65 & 76.62 & 78.31 \\
\hline \%Lipids & 12.10 & 9.17 & 0.31 & 0.79 & 1.31 \\
\hline Collection Date: & $05 / 16 / 2007$ & $05 / 16 / 2007$ & $05 / 19 / 2007$ & $05 / 19 / 2007$ & $05 / 19 / 2007$ \\
\hline Extraction Date: & $01 / 28 / 2008$ & 01/28/2008 & $01 / 28 / 2008$ & 01/28/2008 & 01/28/2008 \\
\hline Analysis Date: & $02 / 17 / 2008$ & $02 / 28 / 2008$ & $02 / 17 / 2008$ & $02 / 17 / 2008$ & $02 / 17 / 2008$ \\
\hline Units (wet wt): & ng/g_WET & ng/g_WET & ng/g_WET & ng/g_WET & ng/g_WET \\
\hline $\mathrm{Cl} 2(8)$ & $0.04 \mathrm{~J}$ & 0.11 & $0.05 \mathrm{U}$ & $0.05 \mathrm{U}$ & $0.05 \mathrm{U}$ \\
\hline $\mathrm{Cl} 3(18)$ & $0.06 \mathrm{U}$ & $0.06 \mathrm{U}$ & $0.06 \mathrm{U}$ & $0.06 \mathrm{U}$ & $0.06 \mathrm{U}$ \\
\hline $\mathrm{Cl} 3(28)$ & 1.65 & 4.29 & 0.09 & 0.21 & 0.12 \\
\hline Cl4(44) & $0.12 \mathrm{U}$ & $0.12 \mathrm{U}$ & 0.09 & 0.51 & 0.18 \\
\hline $\mathrm{Cl} 4(52)$ & 0.23 & 0.70 & 0.52 & 2.27 & 0.97 \\
\hline $\mathrm{Cl} 4(66)$ & 4.94 & $6.48 \mathrm{D}$ & 0.31 & 1.32 & 0.51 \\
\hline $\mathrm{Cl} 4(77)$ & $0.05 \mathrm{U}$ & $0.05 \mathrm{U}$ & $0.05 \mathrm{U}$ & $0.02 \mathrm{~J}$ & $0.01 \mathrm{~J}$ \\
\hline $\mathrm{Cl} 5(101)$ & 0.76 & 2.64 & 2.43 & 7.85 & 4.14 \\
\hline $\mathrm{Cl5}(105)$ & 8.58 & $11.88 \mathrm{D}$ & 0.79 & 2.54 & 1.58 \\
\hline $\mathrm{Cl} 5(118)$ & $21.6 \mathrm{D}$ & $36.87 \mathrm{D}$ & 2.94 & 9.46 & 5.12 \\
\hline $\mathrm{Cl} 5(126)$ & $0.07 \mathrm{U}$ & $0.07 \mathrm{U}$ & $0.07 \mathrm{U}$ & $0.07 \mathrm{U}$ & $0.07 \mathrm{U}$ \\
\hline $\mathrm{Cl} 6(128)$ & 7.74 & $13.34 \mathrm{D}$ & 1.01 & 2.62 & 1.46 \\
\hline $\mathrm{Cl6}(138)$ & $43.35 \mathrm{D}$ & $78.74 \mathrm{D}$ & 4.85 & 9.71 & 5.48 \\
\hline $\mathrm{Cl6}(153)$ & $67.83 \mathrm{D}$ & $146.65 \mathrm{ED}$ & 9.52 & 19.48 & 9.71 \\
\hline $\mathrm{Cl}(170)$ & 8.94 & $27.46 \mathrm{D}$ & 1.57 & 2.92 & 1.13 \\
\hline $\mathrm{Cl}(187)$ & 1.89 & 3.86 & 4.92 & 8.30 & 3.01 \\
\hline $\mathrm{Cl} 7(188)$ & $0.02 \mathrm{~J}$ & 0.12 & $0.04 \mathrm{~J}$ & $0.05 \mathrm{~J}$ & $0.03 \mathrm{~J}$ \\
\hline $\mathrm{Cl}(195)$ & 1.14 & 8.06 & 0.41 & 0.72 & 0.18 \\
\hline $\mathrm{Cl}(200)$ & $0.04 \mathrm{U}$ & $0.04 \mathrm{U}$ & 0.14 & 0.29 & 0.08 \\
\hline $\mathrm{Cl}(206)$ & 2.09 & 9.29 & 1.77 & 2.91 & 1.34 \\
\hline Cl10(209) & 0.95 & 3.50 & 0.78 & 1.29 & 0.96 \\
\hline Total PCBs & 344.18 & 708.66 & 64.82 & 145.30 & 72.38 \\
\hline LOC 1 & $0.08 \mathrm{U}$ & $0.08 \mathrm{U}$ & $0.08 \mathrm{U}$ & $0.08 \mathrm{U}$ & $0.08 \mathrm{U}$ \\
\hline LOC 2 & $0.04 \mathrm{~J}$ & 0.11 & $0.52 \mathrm{U}$ & $0.52 \mathrm{U}$ & $0.52 \mathrm{U}$ \\
\hline LOC 3 & 2.15 & 6.57 & $0.14 \mathrm{~J}$ & $0.45 \mathrm{~J}$ & $0.17 \mathrm{~J}$ \\
\hline LOC 4 & 14.74 & 39.13 & 2.66 & 10.33 & 3.94 \\
\hline LOC 5 & 65.12 & 116.03 & 14.75 & 49.41 & 24.09 \\
\hline LOC 6 & 144.2 & 307.12 & 28.02 & 61.42 & 30.17 \\
\hline LOC 7 & 63.91 & 173.16 & 19.34 & 36.17 & 12.88 \\
\hline LOC 8 & 12.0 & 50.77 & 7.62 & 12.7 & 4.24 \\
\hline LOC 9 & 2.41 & 11.03 & 2.53 & 4.12 & 2.01 \\
\hline Total PCBs & 304.65 & 704 & 75.66 & 175.2 & 78.1 \\
\hline $\mathrm{Cl} 3(34)$ & 79 & 79 & 78 & 54 & 75 \\
\hline $\mathrm{Cl6}(152)$ & 78 & 66 & 76 & 75 & 75 \\
\hline
\end{tabular}




\begin{tabular}{|c|c|c|c|c|c|}
\hline Sample Name: & EH-RF-1 & EH-RF-2 & EH-RF-3 & SI-RF-1 & SI-RF-2 \\
\hline Station: & Eagle Harbor & Eagle Harbor & Eagle Harbor & Sinclair Inlet & Sinclair Inlet \\
\hline Organism ID: & $\mathrm{RF}$ & $\mathrm{RF}$ & $\mathrm{RF}$ & $\mathrm{RF}$ & $\mathrm{RF}$ \\
\hline Batch ID: & $08-0016$ & 08-0016 & 08-0016 & 08-0016 & 08-0016 \\
\hline Sample Weight (g): & 30.03 & 30.44 & 30.67 & 30.79 & 31.19 \\
\hline \%Moisture & 73.50 & 76.61 & 68.32 & 67.33 & 73.39 \\
\hline \%Lipids & 12.30 & 10.00 & 13.50 & 13.40 & 10.20 \\
\hline Collection Date: & $05 / 19 / 2007$ & $05 / 19 / 2007$ & $05 / 19 / 2007$ & $05 / 17 / 2007$ & $05 / 17 / 2007$ \\
\hline Extraction Date: & $01 / 28 / 2008$ & 01/28/2008 & $01 / 28 / 2008$ & $01 / 28 / 2008$ & 01/28/2008 \\
\hline Analysis Date: & 03/14/2008 & $02 / 27 / 2008$ & 03/15/2008 & 03/14/2008 & 03/14/2008 \\
\hline Units (wet wt): & ng/g_WET & ng/g_WET & ng/g_WET & ng/g_WET & ng/g_WET \\
\hline $\mathrm{Cl} 2(8)$ & $0.05 \mathrm{UT}$ & $0.02 \mathrm{~J}$ & 0.05 UTME & $0.06 \mathrm{JT}$ & $0.05 \mathrm{UT}$ \\
\hline $\mathrm{Cl} 3(18)$ & $0.06 \mathrm{UT}$ & $0.06 \mathrm{U}$ & 0.06 UTME & $0.06 \mathrm{UT}$ & $0.06 \mathrm{UT}$ \\
\hline $\mathrm{Cl} 3(28)$ & $0.44 \mathrm{~T}$ & 0.62 & $1.72 \mathrm{TME}$ & $1.60 \mathrm{~T}$ & $0.61 \mathrm{~T}$ \\
\hline $\mathrm{Cl} 4(44)$ & $0.12 \mathrm{UT}$ & $0.12 \mathrm{U}$ & 0.12 UTME & $0.12 \mathrm{UT}$ & $0.12 \mathrm{UT}$ \\
\hline $\mathrm{Cl} 4(52)$ & $0.12 \mathrm{UT}$ & 0.20 & 0.12 UTME & $0.29 \mathrm{~T}$ & $0.12 \mathrm{UT}$ \\
\hline $\mathrm{Cl} 4(66)$ & $1.01 \mathrm{~T}$ & 2.63 & 4.13 TME & $5.71 \mathrm{~T}$ & $2.31 \mathrm{~T}$ \\
\hline $\mathrm{Cl} 4(77)$ & $0.05 \mathrm{UT}$ & $0.05 \mathrm{U}$ & 0.05 UTME & $0.05 \mathrm{UT}$ & $0.05 \mathrm{UT}$ \\
\hline $\mathrm{Cl} 5(101)$ & $0.29 \mathrm{~T}$ & 0.57 & 0.6 TME & $1.30 \mathrm{~T}$ & $0.28 \mathrm{~T}$ \\
\hline $\mathrm{Cl5}(105)$ & $1.06 \mathrm{~T}$ & 3.11 & 11.72 TME & $11.58 \mathrm{~T}$ & $3.39 \mathrm{~T}$ \\
\hline $\mathrm{Cl} 5(118)$ & $4.94 \mathrm{~T}$ & 13.46 & $11.32 \mathrm{TME}$ & $21.39 \mathrm{DT}$ & $12.87 \mathrm{~T}$ \\
\hline $\mathrm{Cl} 5(126)$ & $0.07 \mathrm{UT}$ & $0.07 \mathrm{U}$ & 0.07 UTME & $0.07 \mathrm{UT}$ & $0.07 \mathrm{UT}$ \\
\hline $\mathrm{Cl6}(128)$ & $0.10 \mathrm{~T}$ & 3.03 & 7.1 TME & $13.07 \mathrm{~T}$ & $3.72 \mathrm{~T}$ \\
\hline Cl6(138) & $11.32 \mathrm{~T}$ & $7.96 \mathrm{D}$ & 19.44 TME & $38.6 \mathrm{DT}$ & $10.37 \mathrm{DT}$ \\
\hline Cl6(153) & $22.61 \mathrm{~T}$ & $17.57 \mathrm{D}$ & 56.99 TME & $82.8 \mathrm{DT}$ & 19.71 DT \\
\hline $\mathrm{Cl}(170)$ & $1.94 \mathrm{~T}$ & 3.22 & $2.96 \mathrm{TME}$ & $14.32 \mathrm{~T}$ & $4.26 \mathrm{~T}$ \\
\hline $\mathrm{Cl} 7(187)$ & $0.30 \mathrm{~T}$ & 0.65 & $0.98 \mathrm{TME}$ & $1.77 \mathrm{~T}$ & $0.32 \mathrm{~T}$ \\
\hline $\mathrm{Cl} 7(188)$ & $0.04 \mathrm{JT}$ & 0.07 & 0.02 UTME & $0.12 \mathrm{~T}$ & $0.09 \mathrm{~T}$ \\
\hline $\mathrm{Cl}(195)$ & $0.41 \mathrm{~T}$ & 0.63 & 0.05 UTME & $3.15 \mathrm{~T}$ & $1.02 \mathrm{~T}$ \\
\hline $\mathrm{Cl}(200)$ & $0.04 \mathrm{UT}$ & $0.04 \mathrm{U}$ & 0.04 UTME & 0.04 UT & $0.04 \mathrm{UT}$ \\
\hline $\mathrm{Cl} 9(206)$ & $0.92 \mathrm{~T}$ & 1.69 & $1.58 \mathrm{TME}$ & $7.34 \mathrm{~T}$ & $3.98 \mathrm{~T}$ \\
\hline Cl10(209) & $0.48 \mathrm{~T}$ & 1.05 & $0.98 \mathrm{TME}$ & $3.51 \mathrm{~T}$ & $2.97 \mathrm{~T}$ \\
\hline Total PCBs & 92.74 & 113.64 & 240.20 & 413.90 & 132.82 \\
\hline LOC 1 & $0.08 \mathrm{UT}$ & $0.08 \mathrm{U}$ & 0.08 UTME & $0.08 \mathrm{UT}$ & $0.08 \mathrm{UT}$ \\
\hline LOC 2 & $0.52 \mathrm{UT}$ & $0.02 \mathrm{~J}$ & 0.52 UTME & $0.06 \mathrm{JT}$ & $0.52 \mathrm{UT}$ \\
\hline LOC 3 & $0.44 \mathrm{JT}$ & $0.69 \mathrm{~J}$ & 1.72 TME & $2.23 \mathrm{~T}$ & $0.61 \mathrm{JT}$ \\
\hline LOC 4 & $4.56 \mathrm{~T}$ & 8.05 & 12.29 TME & $18.11 \mathrm{~T}$ & $7.97 \mathrm{~T}$ \\
\hline LOC 5 & $16.22 \mathrm{~T}$ & 39.13 & 47.19 TME & $74.92 \mathrm{~T}$ & $38.35 \mathrm{~T}$ \\
\hline LOC 6 & $37.89 \mathrm{~T}$ & 38.55 & 101.42 TME & $165.32 \mathrm{~T}$ & $44.16 \mathrm{~T}$ \\
\hline LOC 7 & $14.79 \mathrm{~T}$ & 22.24 & $22.56 \mathrm{TME}$ & $70.13 \mathrm{~T}$ & $29.71 \mathrm{~T}$ \\
\hline LOC 8 & $4.01 \mathrm{~T}$ & 7.15 & 0.51 UTME & $30.43 \mathrm{~T}$ & $10.6 \mathrm{~T}$ \\
\hline LOC 9 & $1.05 \mathrm{~T}$ & 2.13 & $1.58 \mathrm{TME}$ & $8.75 \mathrm{~T}$ & $4.66 \mathrm{~T}$ \\
\hline Total PCBs & 79.56 & 118.04 & 187.87 & 370.03 & 136.66 \\
\hline $\mathrm{Cl} 3(34)$ & 88 & 86 & $117 \mathrm{ME}$ & 66 & 82 \\
\hline Cl6(152) & 66 & 64 & 147 \#ME & 67 & 65 \\
\hline
\end{tabular}


1529 West Sequim Bay Road

Sequim, Washington 98382-9099

360/681-4564

\section{SINCLAIR AND DYES INLET 2007 PSAMP Trawl Biota PCBs in Whole Organisms}

\begin{tabular}{|c|c|c|c|c|}
\hline Sample Name: & SI-SC-COMP & PG-ES-1 & PG-ES-2 & PG-ES-3 \\
\hline Station: & "Sinclair Inlet & Port Gardner & Port Gardner & Port Gardner \\
\hline Organism ID: & $\mathrm{SC}$ & ES & ES & ES \\
\hline Batch ID: & $08-0016$ & 08-0016 & 08-0016 & 08-0016 \\
\hline Sample Weight (g): & 11.12 & 30.75 & 30.34 & 31.51 \\
\hline \%Moisture & 90.48 & 80.77 & 79.18 & 80.05 \\
\hline \%Lipids & 0.21 & 0.81 & 0.48 & 0.73 \\
\hline Collection Date: & $05 / 17 / 2007$ & $05 / 29 / 2007$ & $05 / 29 / 2007$ & $05 / 29 / 2007$ \\
\hline Extraction Date: & 01/28/2008 & 01/28/2008 & 01/28/2008 & 01/28/2008 \\
\hline Analysis Date: & 02/28/2008 & $02 / 28 / 2008$ & $02 / 28 / 2008$ & 02/28/2008 \\
\hline Units (wet wt): & ng/g_WET & ng/g_WET & ng/g_WET & ng/g_WET \\
\hline $\mathrm{Cl} 2(8)$ & $0.05 \mathrm{U}$ & $0.05 \mathrm{U}$ & $0.05 \mathrm{U}$ & $0.05 \mathrm{U}$ \\
\hline $\mathrm{Cl} 3(18)$ & $0.06 \mathrm{U}$ & $0.06 \mathrm{U}$ & $0.06 \mathrm{U}$ & $0.06 \mathrm{U}$ \\
\hline $\mathrm{Cl} 3(28)$ & $0.06 \mathrm{U}$ & $0.04 \mathrm{~J}$ & $0.06 \mathrm{U}$ & $0.05 \mathrm{~J}$ \\
\hline $\mathrm{Cl} 4(44)$ & $0.12 \mathrm{U}$ & 0.13 & $0.12 \mathrm{U}$ & 0.08 \\
\hline $\mathrm{Cl} 4(52)$ & $0.12 \mathrm{U}$ & 0.51 & 0.14 & 0.22 \\
\hline $\mathrm{Cl} 4(66)$ & $0.07 \mathrm{U}$ & 0.22 & 0.10 & 0.25 \\
\hline $\mathrm{Cl} 4(77)$ & $0.05 \mathrm{U}$ & $0.02 \mathrm{~J}$ & $0.05 \mathrm{U}$ & $0.04 \mathrm{~J}$ \\
\hline $\mathrm{Cl} 5(101)$ & 0.33 & 1.93 & 0.92 & 1.04 \\
\hline $\mathrm{Cl5}(105)$ & $0.13 \mathrm{~J}$ & 0.44 & 0.20 & 0.28 \\
\hline $\mathrm{Cl5}(118)$ & 0.33 & 1.89 & 0.76 & 1.01 \\
\hline $\mathrm{Cl} 5(126)$ & $0.07 \mathrm{U}$ & $0.07 \mathrm{U}$ & $0.07 \mathrm{U}$ & $0.07 \mathrm{U}$ \\
\hline Cl6(128) & 0.20 & 0.30 & 0.22 & 0.26 \\
\hline Cl6(138) & 0.60 & 2.01 & 1.65 & 1.49 \\
\hline Cl6(153) & 1.11 & 4.84 & 3.83 & 3.47 \\
\hline $\mathrm{Cl} 7(170)$ & $0.11 \mathrm{~J}$ & 0.55 & 0.55 & 0.55 \\
\hline Cl7(187) & 0.53 & 1.61 & 1.52 & 1.26 \\
\hline $\mathrm{Cl}(188)$ & $0.02 \mathrm{U}$ & $0.02 \mathrm{U}$ & $0.01 \mathrm{~J}$ & $0.02 \mathrm{U}$ \\
\hline Cl8(195) & $0.05 \mathrm{U}$ & 0.11 & 0.10 & 0.09 \\
\hline $\mathrm{Cl}(200)$ & $0.04 \mathrm{U}$ & $0.04 \mathrm{U}$ & $0.03 \mathrm{~J}$ & $0.04 \mathrm{U}$ \\
\hline $\mathrm{Cl9}(206)$ & $0.10 \mathrm{~J}$ & 0.13 & 0.08 & 0.12 \\
\hline Cl10(209) & $0.05 \mathrm{~J}$ & $0.04 \mathrm{~J}$ & $0.03 \mathrm{~J}$ & $0.03 \mathrm{~J}$ \\
\hline Total PCBs & 8.40 & 30.02 & 21.10 & 20.96 \\
\hline LOC 1 & $0.08 \mathrm{U}$ & $0.08 \mathrm{U}$ & $0.08 \mathrm{U}$ & $0.08 \mathrm{U}$ \\
\hline LOC 2 & $0.52 \mathrm{U}$ & $0.52 \mathrm{U}$ & $0.52 \mathrm{U}$ & $0.52 \mathrm{U}$ \\
\hline LOC 3 & $0.75 \mathrm{U}$ & $0.07 \mathrm{~J}$ & $0.02 \mathrm{~J}$ & $0.11 \mathrm{~J}$ \\
\hline LOC 4 & $0.05 \mathrm{~J}$ & 2.16 & $0.63 \mathrm{~J}$ & $1.41 \mathrm{~J}$ \\
\hline LOC 5 & $1.93 \mathrm{~J}$ & 10.48 & 4.29 & 5.67 \\
\hline LOC 6 & $3.23 \mathrm{~J}$ & 13.5 & 10.59 & 9.73 \\
\hline LOC 7 & $1.72 \mathrm{~J}$ & 7.42 & 7.21 & 6.41 \\
\hline LOC 8 & $0.43 \mathrm{~J}$ & 1.55 & 1.49 & 1.48 \\
\hline LOC 9 & $0.10 \mathrm{~J}$ & $0.19 \mathrm{~J}$ & $0.13 \mathrm{~J}$ & $0.15 \mathrm{~J}$ \\
\hline Total PCBs & 8.81 & 35.97 & 24.96 & 25.56 \\
\hline $\mathrm{Cl} 3(34)$ & 69 & 72 & 67 & 70 \\
\hline $\mathrm{Cl6}(152)$ & 73 & 77 & 72 & 73 \\
\hline
\end{tabular}


BATTELLE MARINE SCIENCE LABORATORIES

1529 West Sequim Bay Road

Sequim, Washington 98382-9099

360/681-4564

SINCLAIR AND DYES INLET

2007 PSAMP Trawl Biota

PCBs in Whole Organisms

\begin{tabular}{|c|c|c|c|c|c|c|}
\hline Sample Name: & NIS-ES-1 & NIS-ES-2 & NIS-ES-3 & NIS-RF-1 & NIS-RF-2 & NIS-RF-3 \\
\hline Station: & Nisqually & Nisqually & Nisqually & Nisqually & Nisqually & Nisqually \\
\hline Organism ID: & ES & ES & ES & RF & RF & RF \\
\hline Batch ID: & 08-0016 & 08-0016 & $08-0016$ & 08-0016 & $08-0016$ & 08-0016 \\
\hline Sample Weight (g): & 30.59 & 30.71 & 30.24 & 31.44 & 30.88 & 30.69 \\
\hline \%Moisture & 79.33 & 78.48 & 78.39 & 68.79 & 67.47 & 70.65 \\
\hline \%Lipids & 1.09 & 1.71 & 1.57 & 13.40 & 13.45 & 16.62 \\
\hline Collection Date: & $05 / 30 / 2007$ & $05 / 30 / 2007$ & $05 / 30 / 2007$ & $05 / 30 / 2007$ & $05 / 30 / 2007$ & $05 / 30 / 2007$ \\
\hline Extraction Date: & $01 / 28 / 2008$ & $01 / 28 / 2008$ & $01 / 28 / 2008$ & $01 / 28 / 2008$ & $01 / 28 / 2008$ & $01 / 28 / 2008$ \\
\hline Analysis Date: & $02 / 28 / 2008$ & $02 / 28 / 2008$ & $02 / 28 / 2008$ & $03 / 14 / 2008$ & $03 / 14 / 2008$ & $03 / 14 / 2008$ \\
\hline Units (wet wt): & ng/g_WET & ng/g_WET & ng/g_WET & ng/g_WET & ng/g_WET & ng/g_WET \\
\hline $\mathrm{Cl} 2(8)$ & $0.05 \mathrm{U}$ & $0.05 \mathrm{U}$ & $0.05 \mathrm{U}$ & $0.05 \mathrm{UT}$ & $0.05 \mathrm{UT}$ & $0.05 \mathrm{UT}$ \\
\hline $\mathrm{Cl} 3(18)$ & $0.06 \mathrm{U}$ & $0.06 \mathrm{U}$ & $0.06 \mathrm{U}$ & $0.06 \mathrm{UT}$ & $0.06 \mathrm{UT}$ & $0.06 \mathrm{UT}$ \\
\hline $\mathrm{Cl} 3(28)$ & $0.06 \mathrm{U}$ & 0.08 & $0.05 \mathrm{~J}$ & $0.80 \mathrm{~T}$ & $0.50 \mathrm{~T}$ & $0.88 \mathrm{~T}$ \\
\hline $\mathrm{Cl} 4(44)$ & $0.12 \mathrm{U}$ & 0.18 & 0.07 & $0.12 \mathrm{UT}$ & $0.12 \mathrm{UT}$ & $0.12 \mathrm{UT}$ \\
\hline $\mathrm{Cl} 4(52)$ & 0.10 & 0.26 & 0.21 & $0.12 \mathrm{UT}$ & $0.12 \mathrm{UT}$ & $0.12 \mathrm{UT}$ \\
\hline $\mathrm{Cl} 4(66)$ & 0.08 & 0.18 & 0.14 & $5.67 \mathrm{~T}$ & $1.26 \mathrm{~T}$ & $1.92 \mathrm{~T}$ \\
\hline $\mathrm{Cl}(77)$ & $0.05 \mathrm{U}$ & $0.05 \mathrm{U}$ & $0.05 \mathrm{U}$ & $0.05 \mathrm{UT}$ & $0.05 \mathrm{UT}$ & $0.05 \mathrm{UT}$ \\
\hline $\mathrm{Cl5}(101)$ & 0.51 & 1.12 & 1.00 & $0.05 \mathrm{UT}$ & $0.05 \mathrm{UT}$ & $0.28 \mathrm{~T}$ \\
\hline $\mathrm{Cl} 5(105)$ & 0.12 & 0.24 & 0.25 & $9.20 \mathrm{~T}$ & $1.70 \mathrm{~T}$ & $2.40 \mathrm{~T}$ \\
\hline $\mathrm{Cl} 5(118)$ & 0.50 & 0.98 & 0.94 & 16.77 DT & $7.20 \mathrm{~T}$ & $9.37 \mathrm{~T}$ \\
\hline $\mathrm{Cl} 5(126)$ & $0.07 \mathrm{U}$ & $0.07 \mathrm{U}$ & $0.07 \mathrm{U}$ & $0.07 \mathrm{UT}$ & $0.07 \mathrm{UT}$ & $0.07 \mathrm{UT}$ \\
\hline $\mathrm{Cl6}(128)$ & 0.15 & 0.27 & 0.31 & $10.85 \mathrm{~T}$ & $1.75 \mathrm{~T}$ & $2.85 \mathrm{~T}$ \\
\hline Cl6(138) & 1.03 & 1.97 & 1.88 & $29.91 \mathrm{DT}$ & $15.7 \mathrm{~T}$ & $27.56 \mathrm{~T}$ \\
\hline Cl6(153) & 2.43 & 4.54 & 4.51 & $70.43 \mathrm{DT}$ & $24.97 \mathrm{~T}$ & 18.87 DT \\
\hline $\mathrm{Cl} 7(170)$ & 0.26 & 0.45 & 0.41 & $14.05 \mathrm{~T}$ & $2.62 \mathrm{~T}$ & $3.49 \mathrm{~T}$ \\
\hline $\mathrm{Cl}(187)$ & 1.39 & 2.16 & 2.10 & $0.23 \mathrm{~T}$ & $0.37 \mathrm{~T}$ & $0.46 \mathrm{~T}$ \\
\hline $\mathrm{Cl}(188)$ & $0.01 \mathrm{~J}$ & $0.01 \mathrm{~J}$ & $0.02 \mathrm{U}$ & $0.09 \mathrm{~T}$ & $0.06 \mathrm{~T}$ & $0.07 \mathrm{~T}$ \\
\hline $\mathrm{Cl} 8(195)$ & 0.09 & 0.13 & 0.12 & $3.65 \mathrm{~T}$ & $0.75 \mathrm{~T}$ & $0.93 \mathrm{~T}$ \\
\hline $\mathrm{Cl}(200)$ & $0.04 \mathrm{U}$ & $0.04 \mathrm{~J}$ & $0.04 \mathrm{~J}$ & $0.04 \mathrm{UT}$ & 0.04 UT & $0.04 \mathrm{UT}$ \\
\hline $\mathrm{Cl}(206)$ & 0.27 & 0.36 & 0.37 & $10.6 \mathrm{~T}$ & $2.69 \mathrm{~T}$ & $3.11 \mathrm{~T}$ \\
\hline Cl10(209) & 0.14 & 0.15 & 0.16 & $5.96 \mathrm{~T}$ & $1.51 \mathrm{~T}$ & $1.71 \mathrm{~T}$ \\
\hline Total PCBs & 15.06 & 26.70 & 25.62 & 357.54 & 123.28 & 148.82 \\
\hline LOC 1 & $0.08 \mathrm{U}$ & $0.08 \mathrm{U}$ & $0.08 \mathrm{U}$ & $0.08 \mathrm{UT}$ & $0.08 \mathrm{UT}$ & $0.08 \mathrm{UT}$ \\
\hline LOC 2 & $0.52 \mathrm{U}$ & $0.52 \mathrm{U}$ & $0.52 \mathrm{U}$ & $0.52 \mathrm{UT}$ & $0.52 \mathrm{UT}$ & $0.52 \mathrm{UT}$ \\
\hline LOC 3 & $0.75 \mathrm{U}$ & $0.08 \mathrm{~J}$ & $0.08 \mathrm{~J}$ & $0.80 \mathrm{JT}$ & $0.50 \mathrm{JT}$ & $0.88 \mathrm{JT}$ \\
\hline LOC 4 & $0.36 \mathrm{~J}$ & $1.16 \mathrm{~J}$ & $1.00 \mathrm{~J}$ & $17.69 \mathrm{~T}$ & $4.18 \mathrm{~T}$ & $6.39 \mathrm{~T}$ \\
\hline LOC 5 & 2.44 & 5.89 & 5.17 & $52.55 \mathrm{~T}$ & $20.12 \mathrm{~T}$ & $29.63 \mathrm{~T}$ \\
\hline LOC 6 & 6.14 & 12.2 & 11.82 & $134.88 \mathrm{~T}$ & $47.85 \mathrm{~T}$ & $57.90 \mathrm{~T}$ \\
\hline LOC 7 & 4.41 & 7.13 & 7.12 & $71.6 \mathrm{~T}$ & $18.2 \mathrm{~T}$ & $25.83 \mathrm{~T}$ \\
\hline LOC 8 & 1.80 & 2.63 & 2.50 & $33.55 \mathrm{~T}$ & $7.82 \mathrm{~T}$ & $10.32 \mathrm{~T}$ \\
\hline LOC 9 & 0.42 & 0.53 & 0.54 & $12.01 \mathrm{~T}$ & $3.18 \mathrm{~T}$ & $3.65 \mathrm{~T}$ \\
\hline Total PCBs & 16.92 & 30.22 & 28.83 & 323.68 & 102.45 & 135.2 \\
\hline $\mathrm{Cl} 3(34)$ & 66 & 70 & 67 & 77 & 65 & 84 \\
\hline Cl6(152) & 70 & 73 & 73 & 60 & 55 & 70 \\
\hline
\end{tabular}


BATTELLE MARINE SCIENCE LABORATORIES

1529 West Sequim Bay Road

Sequim, Washington 98382-9099

360/681-4564

\section{SINCLAIR AND DYES INLET \\ 2007 PSAMP Trawl Biota \\ PCBs in Whole Organisms}

\begin{tabular}{|c|c|c|c|}
\hline Sample Name: & CB-ES-1 & CB-ES-2 & CB-ES-3 \\
\hline Station: & Commencement Bay & Commencement Bay & Commencement Bay \\
\hline Organism ID: & ES & ES & ES \\
\hline Batch ID: & $08-0016$ & 08-0016 & 08-0016 \\
\hline Sample Weight (g): & 30.87 & 30.54 & 30.7 \\
\hline \%Moisture & 78.50 & 76.32 & 76.84 \\
\hline \%Lipids & 0.74 & 2.11 & 2.41 \\
\hline Collection Date: & $05 / 31 / 2007$ & $05 / 31 / 2007$ & $05 / 31 / 2007$ \\
\hline Extraction Date: & 01/28/2008 & $01 / 28 / 2008$ & 01/28/2008 \\
\hline Analysis Date: & 02/28/2008 & $02 / 28 / 2008$ & 02/28/2008 \\
\hline Units (wet wt): & ng/g_WET & ng/g_WET & ng/g_WET \\
\hline $\mathrm{Cl} 2(8)$ & $0.05 \mathrm{U}$ & $0.05 \mathrm{U}$ & $0.05 \mathrm{U}$ \\
\hline $\mathrm{Cl} 3(18)$ & $0.06 \mathrm{U}$ & $0.06 \mathrm{U}$ & $0.06 \mathrm{U}$ \\
\hline $\mathrm{Cl} 3(28)$ & 0.13 & 0.16 & 0.49 \\
\hline $\mathrm{Cl} 4(44)$ & $0.12 \mathrm{U}$ & 0.48 & 1.11 \\
\hline $\mathrm{Cl} 4(52)$ & 0.60 & 0.66 & 1.59 \\
\hline $\mathrm{Cl} 4(66)$ & 0.50 & 0.59 & 1.13 \\
\hline $\mathrm{Cl}(77)$ & $0.03 \mathrm{~J}$ & $0.05 \mathrm{U}$ & $0.05 \mathrm{U}$ \\
\hline $\mathrm{Cl5}(101)$ & 4.46 & 2.64 & 8.20 \\
\hline $\mathrm{Cl} 5(105)$ & 1.34 & 0.89 & 2.42 \\
\hline $\mathrm{Cl5}(118)$ & 3.86 & 2.40 & 6.88 \\
\hline $\mathrm{Cl} 5(126)$ & $0.07 \mathrm{U}$ & $0.07 \mathrm{U}$ & $0.07 \mathrm{U}$ \\
\hline $\mathrm{Cl6}(128)$ & 1.19 & 0.68 & 2.43 \\
\hline $\mathrm{Cl6}(138)$ & $6.24 \mathrm{D}$ & 3.56 & $11.71 \mathrm{D}$ \\
\hline $\mathrm{Cl} 6(153)$ & $13.06 \mathrm{D}$ & 7.20 & $27.78 \mathrm{D}$ \\
\hline $\mathrm{Cl}(170)$ & 2.93 & 0.98 & 3.03 \\
\hline $\mathrm{Cl}(187)$ & 6.83 & 3.10 & $12.94 \mathrm{D}$ \\
\hline $\mathrm{Cl}(188)$ & $0.02 \mathrm{~J}$ & $0.01 \mathrm{~J}$ & 0.13 \\
\hline $\mathrm{Cl}(195)$ & 0.56 & 0.20 & 0.78 \\
\hline $\mathrm{Cl}(200)$ & 0.18 & 0.08 & 0.49 \\
\hline $\mathrm{Cl9}(206)$ & 2.38 & 0.53 & 3.20 \\
\hline Cl10(209) & 0.47 & 0.18 & 1.19 \\
\hline Total PCBs & 90.16 & 49.14 & 171.46 \\
\hline LOC 1 & $0.08 \mathrm{U}$ & $0.08 \mathrm{U}$ & $0.08 \mathrm{U}$ \\
\hline LOC 2 & $0.52 \mathrm{U}$ & $0.52 \mathrm{U}$ & $0.52 \mathrm{U}$ \\
\hline LOC 3 & $0.13 \mathrm{~J}$ & $0.16 \mathrm{~J}$ & $0.49 \mathrm{~J}$ \\
\hline LOC 4 & 2.90 & 3.97 & 8.86 \\
\hline LOC 5 & 23.64 & 16.87 & 42.91 \\
\hline LOC 6 & 40.99 & 22.67 & 80.59 \\
\hline LOC 7 & 34.98 & 13.52 & 50.76 \\
\hline LOC 8 & 12.85 & 4.17 & 19.28 \\
\hline LOC 9 & 2.94 & 0.75 & 4.29 \\
\hline Total PCBs & 119.03 & 62.71 & 207.78 \\
\hline $\mathrm{Cl} 3(34)$ & 69 & 68 & 69 \\
\hline Cl6(152) & 76 & 69 & 75 \\
\hline
\end{tabular}


1529 West Sequim Bay Road

Sequim, Washington 98382-9099

360/681-4564

\section{SINCLAIR AND DYES INLET 2007 PSAMP Trawl Biota \\ PCBs in Whole Organisms}

\begin{tabular}{|c|c|c|c|}
\hline Sample Name: & CB-RF-1 & CB-RF-2 & CB-RF-3 \\
\hline Station: & Commencement Bay & Commencement Bay & Commencement Bay \\
\hline Organism ID: & RF & RF & RF \\
\hline Batch ID: & 08-0016 & 08-0016 & 08-0017 \\
\hline Sample Weight (g): & 30.09 & 31.65 & 29.73 \\
\hline \%Moisture & 69.83 & 71.46 & 69.42 \\
\hline \%Lipids & 16.10 & 12.40 & 15.52 \\
\hline Collection Date: & $05 / 31 / 2007$ & $05 / 31 / 2007$ & $05 / 31 / 2007$ \\
\hline Extraction Date: & $01 / 28 / 2008$ & $01 / 28 / 2008$ & 02/07/2008 \\
\hline Analysis Date: & $02 / 28 / 2008$ & 03/14/2008 & $02 / 29 / 2008$ \\
\hline Units (wet wt): & ng/g_WET & ng/g_WET & ng/g_WET \\
\hline $\mathrm{Cl} 2(8)$ & $0.05 \mathrm{U}$ & $0.05 \mathrm{UT}$ & $0.03 \mathrm{~J}$ \\
\hline $\mathrm{Cl} 3(18)$ & $0.06 \mathrm{U}$ & $0.06 \mathrm{UT}$ & $0.06 \mathrm{U}$ \\
\hline $\mathrm{Cl} 3(28)$ & 0.56 & $0.76 \mathrm{~T}$ & 0.77 \\
\hline $\mathrm{Cl} 4(44)$ & $0.12 \mathrm{U}$ & $0.12 \mathrm{UT}$ & $0.12 \mathrm{U}$ \\
\hline $\mathrm{Cl} 4(52)$ & $0.12 \mathrm{U}$ & $0.17 \mathrm{~T}$ & 0.10 \\
\hline $\mathrm{Cl} 4(66)$ & 1.50 & $3.18 \mathrm{~T}$ & 3.02 \\
\hline $\mathrm{Cl}(77)$ & $0.05 \mathrm{U}$ & $0.05 \mathrm{UT}$ & $0.05 \mathrm{U}$ \\
\hline $\mathrm{Cl5}(101)$ & 0.47 & $0.54 \mathrm{~T}$ & 0.20 \\
\hline $\mathrm{Cl5}(105)$ & 2.56 & $7.16 \mathrm{~T}$ & $4.19 \mathrm{D}$ \\
\hline $\mathrm{Cl5}(118)$ & 5.77 & $18.5 \mathrm{DT}$ & $10.04 \mathrm{D}$ \\
\hline $\mathrm{Cl} 5(126)$ & $0.07 \mathrm{U}$ & $0.07 \mathrm{UT}$ & $0.07 \mathrm{U}$ \\
\hline Cl6(128) & 2.27 & $8.81 \mathrm{~T}$ & $4.46 \mathrm{D}$ \\
\hline Cl6(138) & $10.2 \mathrm{D}$ & $43.00 \mathrm{DT}$ & $20.19 \mathrm{D}$ \\
\hline $\mathrm{Cl6}(153)$ & $19.09 \mathrm{D}$ & $83.12 \mathrm{DT}$ & $32.57 \mathrm{D}$ \\
\hline $\mathrm{Cl}(170)$ & 1.88 & $8.35 \mathrm{~T}$ & $4.81 \mathrm{D}$ \\
\hline $\mathrm{Cl}(187)$ & 0.37 & $0.48 \mathrm{~T}$ & 0.22 \\
\hline $\mathrm{Cl}(188)$ & $0.03 \mathrm{~J}$ & $0.05 \mathrm{JT}$ & $0.02 \mathrm{~J}$ \\
\hline Cl8(195) & 0.44 & $2.01 \mathrm{~T}$ & 1.38 \\
\hline $\mathrm{Cl}(200)$ & $0.04 \mathrm{U}$ & 0.04 UT & $0.04 \mathrm{U}$ \\
\hline $\mathrm{Cl9}(206)$ & 1.30 & $6.29 \mathrm{~T}$ & $3.77 \mathrm{D}$ \\
\hline Cl10(209) & 0.82 & $4.19 \mathrm{~T}$ & 2.43 \\
\hline Total PCBs & 95.54 & 374 & 177.08 \\
\hline LOC 1 & $0.08 \mathrm{U}$ & $0.08 \mathrm{UT}$ & $0.08 \mathrm{U}$ \\
\hline LOC 2 & $0.52 \mathrm{U}$ & $0.52 \mathrm{UT}$ & $0.03 \mathrm{~J}$ \\
\hline LOC 3 & $0.56 \mathrm{~J}$ & $0.76 \mathrm{JT}$ & $0.77 \mathrm{~J}$ \\
\hline LOC 4 & 4.69 & $9.96 \mathrm{~T}$ & 8.23 \\
\hline LOC 5 & 20.54 & $53.14 \mathrm{~T}$ & 30.2 \\
\hline LOC 6 & 38.43 & $151.45 \mathrm{~T}$ & 67.15 \\
\hline LOC 7 & 12.62 & $56.35 \mathrm{~T}$ & 27.21 \\
\hline LOC 8 & 4.35 & $17.31 \mathrm{~T}$ & 14.2 \\
\hline LOC 9 & 1.57 & $7.19 \mathrm{~T}$ & 4.22 \\
\hline Total PCBs & 83.36 & 296.76 & 152.09 \\
\hline $\mathrm{Cl} 3(34)$ & 52 & 73 & 79 \\
\hline Cl6(152) & 50 & 84 & 68 \\
\hline
\end{tabular}


BATTELLE MARINE SCIENCE LABORATORIES

1529 West Sequim Bay Road

Sequim, Washington 98382-9099

360/681-4564

\section{SINCLAIR AND DYES INLET 2007 PSAMP Trawl Biota PCBs in Whole Organisms}

\begin{tabular}{|c|c|c|c|}
\hline Sample Name: & DU-ES-1 & DU-ES-2 & DU-ES-3 \\
\hline Station: & Duwamish & Duwamish & Duwamish \\
\hline Organism ID: & ES & ES & ES \\
\hline Batch ID: & 08-0017 & $08-0017$ & 08-0017 \\
\hline Sample Weight (g): & 20.82 & 30.12 & 30.56 \\
\hline \%Moisture & 77.05 & 79.68 & 77.00 \\
\hline \%Lipids & 2.27 & 0.77 & 3.75 \\
\hline Collection Date: & $05 / 18 / 2007$ & $05 / 18 / 2007$ & $05 / 18 / 2007$ \\
\hline Extraction Date: & $02 / 07 / 2008$ & 02/07/2008 & 02/07/2008 \\
\hline Analysis Date: & $02 / 29 / 2008$ & 02/29/2008 & $02 / 29 / 2008$ \\
\hline Units (wet wt): & ng/g_WET & ng/g_WET & ng/g_WET \\
\hline $\mathrm{Cl} 2(8)$ & $0.09 \mathrm{~J}$ & $0.03 \mathrm{~J}$ & 0.31 \\
\hline $\mathrm{Cl} 3(18)$ & 0.76 & 0.12 & 2.80 \\
\hline $\mathrm{Cl} 3(28)$ & 2.42 & 0.34 & $7.90 \mathrm{D}$ \\
\hline $\mathrm{Cl} 4(44)$ & 3.01 & 0.42 & $9.52 \mathrm{D}$ \\
\hline $\mathrm{Cl} 4(52)$ & 11.84 & 1.17 & $29.25 \mathrm{D}$ \\
\hline $\mathrm{Cl} 4(66)$ & 9.97 & 0.79 & $31.64 \mathrm{D}$ \\
\hline $\mathrm{Cl} 4(77)$ & 0.20 & $0.04 \mathrm{~J}$ & 0.48 \\
\hline $\mathrm{Cl5}(101)$ & $27.83 \mathrm{D}$ & 3.30 & $62.38 \mathrm{D}$ \\
\hline $\mathrm{Cl} 5(105)$ & $9.33 \mathrm{D}$ & 0.92 & $30.85 \mathrm{D}$ \\
\hline $\mathrm{Cl5}(118)$ & $19.73 \mathrm{D}$ & 2.92 & $53.93 \mathrm{D}$ \\
\hline $\mathrm{Cl} 5(126)$ & $0.07 \mathrm{U}$ & $0.07 \mathrm{U}$ & $0.07 \mathrm{U}$ \\
\hline Cl6(128) & 7.69 & 0.83 & $16.43 \mathrm{D}$ \\
\hline $\mathrm{Cl6}(138)$ & $32.45 \mathrm{D}$ & 4.13 & $68.59 \mathrm{D}$ \\
\hline Cl6(153) & $80.63 \mathrm{D}$ & $7.08 \mathrm{D}$ & $146.87 \mathrm{D}$ \\
\hline $\mathrm{Cl} 7(170)$ & $9.64 \mathrm{D}$ & 1.16 & 18.64 D \\
\hline $\mathrm{Cl}(187)$ & $21.51 \mathrm{D}$ & 3.09 & $36.27 \mathrm{D}$ \\
\hline $\mathrm{Cl} 7(188)$ & $0.05 \mathrm{~J}$ & $0.01 \mathrm{~J}$ & 0.08 \\
\hline Cl8(195) & 2.08 & 0.20 & 3.46 \\
\hline $\mathrm{Cl}(200)$ & 0.61 & 0.08 & 1.14 \\
\hline $\mathrm{Cl9}(206)$ & 1.63 & 0.47 & 2.81 \\
\hline Cl10(209) & 0.28 & 0.17 & 0.49 \\
\hline Total PCBs & 483.64 & 54.68 & 1047.82 \\
\hline LOC 1 & $0.08 \mathrm{U}$ & $0.08 \mathrm{U}$ & $0.08 \mathrm{U}$ \\
\hline LOC 2 & $0.09 \mathrm{~J}$ & $0.03 \mathrm{~J}$ & $0.45 \mathrm{~J}$ \\
\hline LOC 3 & 7.81 & 1.14 & 25.93 \\
\hline LOC 4 & 67.03 & 6.37 & 190.66 \\
\hline LOC 5 & 159.96 & 20.01 & 432.25 \\
\hline LOC 6 & 246.18 & 24.41 & 483.21 \\
\hline LOC 7 & 117.24 & 14.09 & 202.88 \\
\hline LOC 8 & 28.66 & 3.87 & 34.28 \\
\hline LOC 9 & 2.26 & 0.67 & 3.84 \\
\hline Total PCBs & 629.31 & 70.67 & 1373.58 \\
\hline $\mathrm{Cl} 3(34)$ & 88 & 71 & 73 \\
\hline $\mathrm{Cl}(152)$ & 81 & 67 & 67 \\
\hline
\end{tabular}


BATTELLE MARINE SCIENCE LABORATORIES

1529 West Sequim Bay Road

Sequim, Washington 98382-9099

360/681-4564

\section{SINCLAIR AND DYES INLET \\ 2007 PSAMP Trawl Biota \\ PCBs in Whole Organisms}

\begin{tabular}{|c|c|c|c|c|}
\hline Sample Name: & DF-EM-1 & DF-EM-2 & DF-EM-4 & DF-EM-6 \\
\hline Station: & Embryo & Embryo & Embryo & $\overline{\text { Embryo }}$ \\
\hline Organism ID: & DF1 & DF & DF & DF \\
\hline Batch ID: & $08-0017$ & 08-0017 & 08-0017 & 08-0017 \\
\hline Sample Weight (g): & 10.52 & 10.59 & 10.53 & 10.44 \\
\hline \%Moisture & 47.03 & 47.13 & 47.41 & 43.67 \\
\hline \%Lipids & 22.40 & 23.05 & 23.00 & 26.30 \\
\hline Collection Date: & 09/18/2007 & $09 / 18 / 2007$ & $09 / 18 / 2007$ & $09 / 18 / 2007$ \\
\hline Extraction Date: & 02/07/2008 & 02/07/2008 & $02 / 07 / 2008$ & 02/07/2008 \\
\hline Analysis Date: & 03/01/2008 & 03/01/2008 & 03/01/2008 & 03/01/2008 \\
\hline Units (wet wt): & ng/g_WET & ng/g_WET & ng/g_WET & ng/g_WET \\
\hline $\mathrm{Cl} 2(8)$ & $0.05 \mathrm{U}$ & $0.05 \mathrm{U}$ & $0.05 \mathrm{U}$ & $0.05 \mathrm{U}$ \\
\hline Cl3(18) & $0.06 \mathrm{U}$ & $0.06 \mathrm{U}$ & $0.06 \mathrm{U}$ & $0.06 \mathrm{U}$ \\
\hline Cl3(28) & 0.51 & 0.41 & 0.28 & 0.52 \\
\hline Cl4(44) & 0.23 & $0.12 \mathrm{U}$ & $0.12 \mathrm{U}$ & 0.37 \\
\hline $\mathrm{Cl} 4(52)$ & 1.93 & 1.42 & 1.08 & 2.38 \\
\hline $\mathrm{Cl} 14(66)$ & 0.97 & 0.72 & 0.25 & 1.39 \\
\hline $\mathrm{Cl} 4(77)$ & $0.05 \mathrm{U}$ & $0.05 \mathrm{U}$ & $0.05 \mathrm{U}$ & $0.05 \mathrm{U}$ \\
\hline Cl5(101) & 3.75 & 2.73 & 1.19 & 7.37 \\
\hline $\mathrm{Cl5}(105)$ & 1.08 & 0.66 & $0.18 \mathrm{~J}$ & 1.68 \\
\hline $\mathrm{Cl5}(118)$ & 5.43 & 4.19 & 0.91 & 7.96 \\
\hline $\mathrm{Cl5}(126)$ & $0.07 \mathrm{U}$ & $0.07 \mathrm{U}$ & $0.07 \mathrm{U}$ & $0.07 \mathrm{U}$ \\
\hline Cl6(128) & 0.78 & 0.43 & $0.06 \mathrm{~J}$ & 1.05 \\
\hline Cl6(138) & 6.33 & 4.18 & 0.79 & 8.07 \\
\hline Cl6(153) & 13.69 & 9.18 & 1.86 & 16.99 \\
\hline $\mathrm{Cl} 7(170)$ & 1.34 & 0.72 & $0.14 \mathrm{~J}$ & 1.41 \\
\hline Cl7(187) & 2.27 & 2.62 & 0.36 & 4.97 \\
\hline Cl7(188) & $0.02 \mathrm{U}$ & $0.02 \mathrm{U}$ & $0.02 \mathrm{U}$ & $0.02 \mathrm{U}$ \\
\hline $\mathrm{Cl}(195)$ & $0.05 \mathrm{U}$ & $0.05 \mathrm{U}$ & $0.05 \mathrm{U}$ & $0.05 \mathrm{U}$ \\
\hline $\mathrm{Cl} 18(200)$ & $0.04 \mathrm{U}$ & $0.04 \mathrm{U}$ & $0.04 \mathrm{U}$ & $0.04 \mathrm{U}$ \\
\hline $\mathrm{Cl9}(206)$ & 0.28 & $0.17 \mathrm{~J}$ & $0.04 \mathrm{U}$ & 0.29 \\
\hline Cl10(209) & $0.16 \mathrm{~J}$ & $0.08 \mathrm{~J}$ & $0.07 \mathrm{U}$ & $0.10 \mathrm{~J}$ \\
\hline Total PCBs & 78.18 & 55.94 & 15.34 & 109.78 \\
\hline LOC 1 & $0.08 \mathrm{U}$ & $0.08 \mathrm{U}$ & $0.08 \mathrm{U}$ & $0.08 \mathrm{U}$ \\
\hline LOC 2 & $0.52 \mathrm{U}$ & $0.52 \mathrm{U}$ & $0.52 \mathrm{U}$ & $0.52 \mathrm{U}$ \\
\hline LOC 3 & $0.51 \mathrm{~J}$ & $0.41 \mathrm{~J}$ & $0.28 \mathrm{~J}$ & $0.52 \mathrm{~J}$ \\
\hline LOC 4 & 5.82 & $3.85 \mathrm{~J}$ & $2.21 \mathrm{~J}$ & 7.96 \\
\hline LOC 5 & 22.01 & 14.57 & 4.88 & 35.94 \\
\hline LOC 6 & 29.94 & 20.68 & $3.88 \mathrm{~J}$ & 44.31 \\
\hline LOC 7 & 12.9 & 9.81 & $1.27 \mathrm{~J}$ & 18.71 \\
\hline LOC 8 & 3.05 & 2.39 & $0.51 \mathrm{U}$ & 3.92 \\
\hline LOC 9 & $0.36 \mathrm{~J}$ & $0.31 \mathrm{~J}$ & $0.16 \mathrm{U}$ & $0.45 \mathrm{~J}$ \\
\hline Total PCBs & 75.19 & 52.62 & 13.79 & 112.41 \\
\hline $\mathrm{Cl} 3(34)$ & 93 & 74 & 85 & 90 \\
\hline Cl6(152) & 70 & 52 & 68 & 65 \\
\hline
\end{tabular}


BATTELLE MARINE SCIENCE LABORATORIES

1529 West Sequim Bay Road

Sequim, Washington 98382-9099

360/681-4564

\section{SINCLAIR AND DYES INLET \\ 2007 PSAMP Trawl Biota \\ PCBs in Whole Organisms}

\begin{tabular}{|c|c|c|c|c|}
\hline Sample Name: & DF-LV-1 & DF-LV-2 & $\begin{array}{l}\text { DF-LV-4 } \\
\end{array}$ & $\begin{array}{l}\text { DF-LV-6 } \\
\end{array}$ \\
\hline Station: & Liver & Liver & Liver & Liver \\
\hline Organism ID: & DF & DF & DF & DF \\
\hline Batch ID: & 08-0017 & $08-0017$ & 08-0017 & $08-0017$ \\
\hline Sample Weight (g): & 5.03 & 5.49 & 5.7 & 5.42 \\
\hline \%Moisture & 44.79 & 47.05 & 22.82 & 68.07 \\
\hline \%Lipids & 44.76 & 62.26 & 63.43 & 70.50 \\
\hline Collection Date: & $09 / 18 / 2007$ & $09 / 18 / 2007$ & $09 / 18 / 2007$ & $09 / 18 / 2007$ \\
\hline Extraction Date: & $02 / 07 / 2008$ & 02/07/2008 & $02 / 07 / 2008$ & $02 / 07 / 2008$ \\
\hline Analysis Date: & $02 / 29 / 2008$ & 02/29/2008 & $02 / 29 / 2008$ & 03/01/2008 \\
\hline Units (wet wt): & ng/g_WET & ng/g_WET & ng/g_WET & ng/g_WET \\
\hline $\mathrm{Cl} 2(8)$ & $0.05 \mathrm{U}$ & $0.05 \mathrm{U}$ & $0.05 \mathrm{U}$ & $0.05 \mathrm{U}$ \\
\hline $\mathrm{Cl} 3(18)$ & $0.06 \mathrm{U}$ & $0.06 \mathrm{U}$ & $0.06 \mathrm{U}$ & $0.06 \mathrm{U}$ \\
\hline $\mathrm{Cl} 3(28)$ & 1.25 & 1.77 & 1.10 & 2.19 \\
\hline $\mathrm{Cl} 4(44)$ & 0.51 & 0.66 & 0.41 & 1.41 \\
\hline $\mathrm{Cl} 4(52)$ & 2.69 & 4.53 & 3.16 & 7.13 \\
\hline $\mathrm{Cl} 4(66)$ & 3.45 & 4.58 & 1.36 & 7.45 \\
\hline $\mathrm{Cl}(777)$ & $0.21 \mathrm{~J}$ & 0.37 & $0.05 \mathrm{~J}$ & 0.45 \\
\hline $\mathrm{Cl5}(101)$ & 7.07 & 12.39 & 4.32 & 26.8 \\
\hline $\mathrm{Cl} 5(105)$ & 6.88 & 10.16 & 1.64 & 15.82 \\
\hline $\mathrm{Cl5}(118)$ & 18.42 & $19.59 \mathrm{D}$ & 4.59 & $21.52 \mathrm{D}$ \\
\hline $\mathrm{Cl} 5(126)$ & $0.26 \mathrm{~J}$ & $0.32 \mathrm{~J}$ & $0.07 \mathrm{U}$ & 0.37 \\
\hline $\mathrm{Cl}(128)$ & 5.67 & 6.30 & $0.23 \mathrm{~J}$ & 10.09 \\
\hline Cl6(138) & $23.01 \mathrm{D}$ & $30.44 \mathrm{D}$ & 4.67 & $38.9 \mathrm{D}$ \\
\hline $\mathrm{Cl6}(153)$ & $43.05 \mathrm{D}$ & $65.42 \mathrm{D}$ & 10.61 & $87.52 \mathrm{D}$ \\
\hline $\mathrm{Cl}(170)$ & 6.64 & 10.57 & 0.86 & 11.31 \\
\hline $\mathrm{Cl7}(187)$ & 6.86 & 21.22 & 2.22 & $26.3 \mathrm{D}$ \\
\hline $\mathrm{Cl}(188)$ & $0.05 \mathrm{~J}$ & $0.07 \mathrm{~J}$ & $0.02 \mathrm{U}$ & $0.08 \mathrm{~J}$ \\
\hline Cl8(195) & 1.02 & 1.29 & $0.05 \mathrm{U}$ & 1.14 \\
\hline $\mathrm{Cl}(200)$ & $0.04 \mathrm{U}$ & $0.04 \mathrm{U}$ & $0.04 \mathrm{U}$ & $0.25 \mathrm{~J}$ \\
\hline $\mathrm{Cl9}(206)$ & 1.11 & 2.17 & $0.11 \mathrm{~J}$ & 1.56 \\
\hline Cl10(209) & $0.30 \mathrm{~J}$ & 0.63 & $0.05 \mathrm{~J}$ & $0.34 \mathrm{~J}$ \\
\hline Total PCBs & 257.20 & 385.26 & 71.34 & 521.48 \\
\hline LOC 1 & $0.08 \mathrm{U}$ & $0.08 \mathrm{U}$ & $0.08 \mathrm{U}$ & $0.08 \mathrm{U}$ \\
\hline LOC 2 & $0.52 \mathrm{U}$ & $0.06 \mathrm{~J}$ & $0.52 \mathrm{U}$ & $0.52 \mathrm{U}$ \\
\hline LOC 3 & $1.25 \mathrm{~J}$ & $1.77 \mathrm{~J}$ & $1.27 \mathrm{~J}$ & $2.19 \mathrm{~J}$ \\
\hline LOC 4 & 13.88 & 19.62 & $9.18 \mathrm{~J}$ & 32.7 \\
\hline LOC 5 & 65.18 & 83.98 & 22.49 & 144.64 \\
\hline LOC 6 & 100.77 & 156.14 & 25.33 & 235.61 \\
\hline LOC 7 & 49.6 & 93.17 & 8.48 & 102.91 \\
\hline LOC 8 & 11.42 & 22.79 & $0.47 \mathrm{~J}$ & 20.97 \\
\hline LOC 9 & 1.41 & 3.08 & $0.11 \mathrm{~J}$ & 2.20 \\
\hline Total PCBs & 244.11 & 380.69 & 67.93 & 541.82 \\
\hline $\mathrm{Cl} 3(34)$ & 63 & 73 & 75 & 77 \\
\hline Cl6(152) & 62 & 69 & 72 & 72 \\
\hline
\end{tabular}




\section{SINCLAIR AND DYES INLET 2007 PSAMP Trawl Biota PCBs in Whole Organisms}

\begin{tabular}{|c|c|c|c|c|}
\hline Sample Name: & DF-DIG-1 & DF-DIG-2 & DF-DIG-4 & DF-DIG-6 \\
\hline Station: & Digestive & Digestive & Digestive & Digestive \\
\hline Organism ID: & DF & DF & DF & DF \\
\hline Batch ID: & 08-0017 & 08-0017 & 08-0017 & 08-0017 \\
\hline Sample Weight (g): & 18.5 & 19.38 & 10.68 & 19.14 \\
\hline \%Moisture & 77.33 & 78.84 & 84.41 & 82.94 \\
\hline \%Lipids & 12.40 & 3.53 & 1.75 & 2.63 \\
\hline Collection Date: & $09 / 18 / 2007$ & $09 / 18 / 2007$ & $09 / 18 / 2007$ & $09 / 18 / 2007$ \\
\hline Extraction Date: & $02 / 07 / 2008$ & 02/07/2008 & 02/07/2008 & $02 / 07 / 2008$ \\
\hline Analysis Date: & 03/01/2008 & 03/01/2008 & 03/01/2008 & 03/01/2008 \\
\hline Units (wet wt): & ng/g_WET & ng/g_WET & ng/g_WET & ng/g_WET \\
\hline $\mathrm{Cl} 2(8)$ & $0.02 \mathrm{~J}$ & $0.01 \mathrm{~J}$ & $0.03 \mathrm{~J}$ & $0.05 \mathrm{U}$ \\
\hline $\mathrm{Cl} 3(18)$ & $0.02 \mathrm{~J}$ & $0.02 \mathrm{~J}$ & $0.06 \mathrm{U}$ & $0.06 \mathrm{U}$ \\
\hline $\mathrm{Cl} 3(28)$ & $0.09 \mathrm{~J}$ & $0.04 \mathrm{~J}$ & $0.07 \mathrm{~J}$ & $0.04 \mathrm{~J}$ \\
\hline $\mathrm{Cl} 4(44)$ & $0.12 \mathrm{U}$ & $0.12 \mathrm{U}$ & $0.12 \mathrm{U}$ & $0.12 \mathrm{U}$ \\
\hline $\mathrm{Cl} 4(52)$ & $0.05 \mathrm{~J}$ & $0.05 \mathrm{~J}$ & $0.09 \mathrm{~J}$ & 0.12 \\
\hline $\mathrm{Cl} 4(66)$ & 0.18 & $0.06 \mathrm{~J}$ & $0.06 \mathrm{~J}$ & 0.15 \\
\hline $\mathrm{Cl} 4(77)$ & $0.02 \mathrm{~J}$ & $0.05 \mathrm{U}$ & $0.04 \mathrm{~J}$ & $0.05 \mathrm{~J}$ \\
\hline $\mathrm{Cl} 5(101)$ & $0.05 \mathrm{~J}$ & $0.06 \mathrm{~J}$ & $0.06 \mathrm{~J}$ & 0.32 \\
\hline $\mathrm{Cl} 5(105)$ & 0.35 & $0.07 \mathrm{~J}$ & $0.06 \mathrm{~J}$ & 0.33 \\
\hline $\mathrm{Cl} 5(118)$ & 0.72 & 0.10 & $0.07 \mathrm{~J}$ & 0.53 \\
\hline $\mathrm{Cl} 5(126)$ & $0.07 \mathrm{U}$ & $0.07 \mathrm{U}$ & $0.07 \mathrm{U}$ & $0.07 \mathrm{U}$ \\
\hline $\mathrm{Cl}(128)$ & 0.30 & $0.05 \mathrm{U}$ & $0.05 \mathrm{U}$ & $0.07 \mathrm{~J}$ \\
\hline $\mathrm{Cl6}(138)$ & 1.49 & 0.10 & $0.07 \mathrm{~J}$ & 0.80 \\
\hline Cl6(153) & 2.74 & 0.20 & $0.13 \mathrm{~J}$ & 1.70 \\
\hline $\mathrm{Cl7}(170)$ & 0.33 & $0.04 \mathrm{U}$ & $0.04 \mathrm{U}$ & 0.18 \\
\hline Cl7(187) & $0.06 \mathrm{~J}$ & $0.05 \mathrm{~J}$ & $0.05 \mathrm{~J}$ & 0.51 \\
\hline $\mathrm{Cl}(188)$ & $0.02 \mathrm{U}$ & $0.02 \mathrm{U}$ & $0.02 \mathrm{U}$ & $0.02 \mathrm{U}$ \\
\hline $\mathrm{Cl}(195)$ & $0.05 \mathrm{U}$ & $0.05 \mathrm{U}$ & $0.05 \mathrm{U}$ & $0.05 \mathrm{U}$ \\
\hline $\mathrm{Cl} 18(200)$ & $0.04 \mathrm{U}$ & $0.04 \mathrm{U}$ & $0.04 \mathrm{U}$ & $0.04 \mathrm{U}$ \\
\hline $\mathrm{Cl9}(206)$ & 0.13 & $0.04 \mathrm{U}$ & $0.04 \mathrm{U}$ & $0.07 \mathrm{~J}$ \\
\hline Cl10(209) & $0.05 \mathrm{~J}$ & $0.07 \mathrm{U}$ & $0.07 \mathrm{U}$ & $0.06 \mathrm{~J}$ \\
\hline Total PCBs & 13.80 & 2.62 & 2.58 & 10.68 \\
\hline LOC 1 & $0.08 \mathrm{U}$ & $0.08 \mathrm{U}$ & $0.08 \mathrm{U}$ & $0.08 \mathrm{U}$ \\
\hline LOC 2 & $0.02 \mathrm{~J}$ & $0.01 \mathrm{~J}$ & $0.03 \mathrm{~J}$ & $0.52 \mathrm{U}$ \\
\hline LOC 3 & $0.19 \mathrm{~J}$ & $0.12 \mathrm{~J}$ & $0.12 \mathrm{~J}$ & $0.05 \mathrm{~J}$ \\
\hline LOC 4 & $0.58 \mathrm{~J}$ & $0.19 \mathrm{~J}$ & $0.29 \mathrm{~J}$ & $0.6 \mathrm{~J}$ \\
\hline LOC 5 & $2.21 \mathrm{~J}$ & $0.35 \mathrm{~J}$ & $0.26 \mathrm{~J}$ & 2.36 \\
\hline LOC 6 & 5.24 & $0.45 \mathrm{~J}$ & $0.28 \mathrm{~J}$ & 4.08 \\
\hline LOC 7 & $1.82 \mathrm{~J}$ & $0.18 \mathrm{~J}$ & $0.10 \mathrm{~J}$ & $2.00 \mathrm{~J}$ \\
\hline LOC 8 & $0.57 \mathrm{~J}$ & $0.51 \mathrm{U}$ & $0.51 \mathrm{U}$ & $0.47 \mathrm{~J}$ \\
\hline LOC 9 & $0.13 \mathrm{~J}$ & $0.16 \mathrm{U}$ & $0.16 \mathrm{U}$ & $0.09 \mathrm{~J}$ \\
\hline Total PCBs & 10.84 & 2.05 & 1.83 & 10.25 \\
\hline $\mathrm{Cl} 3(34)$ & 50 & 41 & 71 & 66 \\
\hline Cl6(152) & 57 & 45 & 77 & 74 \\
\hline
\end{tabular}


1529 West Sequim Bay Road

Sequim, Washington 98382-9099

360/681-4564

\section{SINCLAIR AND DYES INLET \\ 2007 PSAMP Trawl Biota \\ PCBs in Whole Organisms}

\begin{tabular}{|c|c|c|c|c|c|}
\hline Sample Name: & DF-SWC-1 & DF-SWC-2 & DF-SWC-4 & DF-SWC-6 & DF-WBWC-1 \\
\hline Station: & Sec. Comp. & Sec. Comp. & Sec. Comp. & Sec. Comp. & W.Body Comp. \\
\hline Organism ID: & DF & DF & DF & DF & DF \\
\hline Batch ID: & 08-0017 & 08-0017 & 08-0018 & $08-0017$ & 08-0017 \\
\hline Sample Weight (g): & 15.32 & 15.35 & 15.5 & 15.78 & 15.57 \\
\hline \%Moisture & 62.81 & 71.19 & 70.16 & 64.77 & 60.24 \\
\hline \%Lipids & 15.96 & 13.00 & 14.45 & 16.83 & 19.90 \\
\hline Collection Date: & 09/18/2007 & $09 / 18 / 2007$ & $09 / 18 / 2007$ & $09 / 18 / 2007$ & $09 / 18 / 2007$ \\
\hline Extraction Date: & $02 / 07 / 2008$ & 02/07/2008 & 02/07/2008 & 02/07/2008 & 02/07/2008 \\
\hline Analysis Date: & 03/01/2008 & 03/01/2008 & $02 / 29 / 2008$ & 03/01/2008 & 03/01/2008 \\
\hline Units (wet wt): & ng/g_WET & ng/g_WET & ng/g_WET & ng/g_WET & ng/g_WET \\
\hline $\mathrm{Cl} 2(8)$ & $0.02 \mathrm{~J}$ & $0.01 \mathrm{~J}$ & $0.05 \mathrm{U}$ & $0.05 \mathrm{U}$ & $0.05 \mathrm{U}$ \\
\hline $\mathrm{Cl} 3(18)$ & $0.06 \mathrm{U}$ & $0.06 \mathrm{U}$ & $0.06 \mathrm{U}$ & $0.04 \mathrm{~J}$ & $0.06 \mathrm{U}$ \\
\hline $\mathrm{Cl} 3(28)$ & 0.65 & 0.41 & 0.23 & 0.52 & 0.31 \\
\hline $\mathrm{Cl} 4(44)$ & 0.24 & 0.17 & $0.08 \mathrm{~J}$ & 0.41 & 0.13 \\
\hline $\mathrm{Cl} 4(52)$ & 1.47 & 1.22 & 0.58 & 1.94 & 0.72 \\
\hline $\mathrm{Cl} 4(66)$ & 1.60 & 0.86 & 0.19 & 1.66 & 0.84 \\
\hline $\mathrm{Cl} 4(77)$ & $0.05 \mathrm{U}$ & $0.02 \mathrm{~J}$ & $0.05 \mathrm{U}$ & $0.01 \mathrm{~J}$ & $0.04 \mathrm{~J}$ \\
\hline $\mathrm{Cl5}(101)$ & 3.55 & 2.66 & 0.83 & 6.81 & 1.76 \\
\hline $\mathrm{Cl5}(105)$ & 2.94 & 1.94 & 0.26 & 3.55 & 1.75 \\
\hline $\mathrm{Cl5}(118)$ & 8.13 & 5.39 & 0.72 & $4.22 \mathrm{D}$ & 4.19 \\
\hline $\mathrm{Cl} 5(126)$ & $0.07 \mathrm{U}$ & $0.07 \mathrm{U}$ & $0.07 \mathrm{U}$ & $0.07 \mathrm{U}$ & $0.07 \mathrm{U}$ \\
\hline $\mathrm{Cl6}(128)$ & 2.25 & 1.04 & 0.14 & 2.23 & 1.35 \\
\hline Cl6(138) & $8.18 \mathrm{D}$ & 7.79 & 0.86 & $8.02 \mathrm{D}$ & 7.37 \\
\hline $\mathrm{Cl} 6(153)$ & $15.92 \mathrm{D}$ & $9.71 \mathrm{D}$ & 1.74 & $15.59 \mathrm{D}$ & $9.52 \mathrm{D}$ \\
\hline $\mathrm{Cl} 7(170)$ & 1.77 & 1.25 & $0.09 \mathrm{~J}$ & 2.18 & 1.21 \\
\hline $\mathrm{Cl} 7(187)$ & 2.40 & 3.30 & 0.29 & 5.88 & 1.51 \\
\hline $\mathrm{Cl}(188)$ & $0.01 \mathrm{~J}$ & $0.02 \mathrm{U}$ & $0.02 \mathrm{U}$ & $0.02 \mathrm{~J}$ & $0.02 \mathrm{U}$ \\
\hline $\mathrm{Cl}(195)$ & 0.23 & $0.05 \mathrm{U}$ & $0.05 \mathrm{U}$ & 0.19 & 0.18 \\
\hline $\mathrm{Cl} 8(200)$ & $0.04 \mathrm{U}$ & $0.04 \mathrm{U}$ & $0.04 \mathrm{U}$ & $0.04 \mathrm{U}$ & $0.04 \mathrm{U}$ \\
\hline $\mathrm{Cl9}(206)$ & 0.15 & 0.19 & $0.04 \mathrm{U}$ & 0.27 & 0.15 \\
\hline Cl10(209) & $0.04 \mathrm{~J}$ & $0.05 \mathrm{~J}$ & $0.07 \mathrm{U}$ & $0.10 \mathrm{~J}$ & $0.05 \mathrm{~J}$ \\
\hline Total PCBs & 99.54 & 72.50 & 12.92 & 107.60 & 62.64 \\
\hline LOC 1 & $0.08 \mathrm{U}$ & $0.08 \mathrm{U}$ & $0.08 \mathrm{U}$ & $0.08 \mathrm{U}$ & $0.08 \mathrm{U}$ \\
\hline LOC 2 & $0.02 \mathrm{~J}$ & $0.01 \mathrm{~J}$ & $0.52 \mathrm{U}$ & $0.52 \mathrm{U}$ & $0.52 \mathrm{U}$ \\
\hline LOC 3 & $0.82 \mathrm{~J}$ & $0.45 \mathrm{~J}$ & $0.23 \mathrm{~J}$ & $0.56 \mathrm{~J}$ & $0.31 \mathrm{~J}$ \\
\hline LOC 4 & 7.07 & 4.43 & $1.48 \mathrm{~J}$ & 8.07 & $3.60 \mathrm{~J}$ \\
\hline LOC 5 & 30.57 & 18.71 & 3.80 & 34.56 & 15.57 \\
\hline LOC 6 & 38.82 & 29.2 & 4.06 & 48.68 & 25.68 \\
\hline LOC 7 & 14.36 & 13.18 & $1.10 \mathrm{~J}$ & 23.78 & 9.70 \\
\hline LOC 8 & 2.38 & 2.31 & $0.51 \mathrm{U}$ & 4.09 & 1.86 \\
\hline LOC 9 & $0.20 \mathrm{~J}$ & $0.26 \mathrm{~J}$ & $0.16 \mathrm{U}$ & 0.41 & $0.19 \mathrm{~J}$ \\
\hline Total PCBs & 94.32 & 68.63 & 11.94 & 120.75 & 57.51 \\
\hline $\mathrm{Cl} 3(34)$ & 72 & 63 & 75 & 72 & 37 \# \\
\hline $\mathrm{Cl}(152)$ & 75 & 72 & 80 & 73 & 45 \\
\hline
\end{tabular}




\begin{tabular}{|c|c|c|c|c|c|}
\hline Sample Name: & DF-WBWC-2 & DF-WBWC-3 & DF-WBWC-4 & DF-WBWC-5 & DF-WBWC-6 \\
\hline Station: & W.Body Comp. & W.Body Comp. & W.Body Comp. & W.Body Comp. & W.Body Comp. \\
\hline Organism ID: & DF & DF & DF & DF & DF \\
\hline Batch ID: & 08-0018 & 08-0018 & 08-0018 & $08-0018$ & $08-0018$ \\
\hline Sample Weight (g): & 15.65 & 15.1 & 14.94 & 15.65 & 15.71 \\
\hline \%Moisture & 64.17 & 63.30 & 59.64 & 58.97 & 62.97 \\
\hline \%Lipids & 15.32 & 18.06 & 18.09 & 22.21 & 22.21 \\
\hline Collection Date: & 09/18/2007 & $09 / 18 / 2007$ & 09/18/2007 & 09/18/2007 & $09 / 18 / 2007$ \\
\hline Extraction Date: & $02 / 07 / 2008$ & $02 / 07 / 2008$ & $02 / 07 / 2008$ & 02/07/2008 & $02 / 07 / 2008$ \\
\hline Analysis Date: & $02 / 29 / 2008$ & $02 / 29 / 2008$ & $02 / 29 / 2008$ & 02/29/2008 & $02 / 29 / 2008$ \\
\hline Units (wet wt): & ng/g_WET & ng/g_WET & ng/g_WET & ng/g_WET & ng/g_WET \\
\hline $\mathrm{Cl} 2(8)$ & $0.05 \mathrm{U}$ & $0.05 \mathrm{U}$ & $0.05 \mathrm{U}$ & $0.05 \mathrm{U}$ & $0.05 \mathrm{U}$ \\
\hline $\mathrm{Cl} 3(18)$ & $0.06 \mathrm{U}$ & $0.06 \mathrm{U}$ & $0.06 \mathrm{U}$ & $0.06 \mathrm{U}$ & $0.06 \mathrm{U}$ \\
\hline $\mathrm{Cl} 3(28)$ & 0.49 & 0.41 & 0.36 & 0.60 & 0.48 \\
\hline $\mathrm{Cl} 4(44)$ & 0.18 & 0.18 & 0.14 & 0.18 & 0.33 \\
\hline $\mathrm{Cl} 4(52)$ & 1.20 & 1.46 & 0.96 & 0.78 & 1.71 \\
\hline $\mathrm{Cl} 4(66)$ & 0.90 & 1.02 & 0.43 & 2.06 & 1.51 \\
\hline $\mathrm{Cl} 4(77)$ & $0.05 \mathrm{U}$ & $0.05 \mathrm{U}$ & $0.05 \mathrm{U}$ & $0.05 \mathrm{U}$ & 0.13 \\
\hline $\mathrm{Cl5}(101)$ & 3.17 & 4.79 & 1.53 & 2.90 & 6.94 \\
\hline $\mathrm{Cl} 5(105)$ & 1.68 & 2.21 & 0.51 & 4.31 & 3.17 \\
\hline $\mathrm{Cl} 5(118)$ & 5.66 & 6.68 & 1.38 & 12.98 & 8.84 \\
\hline $\mathrm{Cl} 5(126)$ & $0.07 \mathrm{U}$ & $0.07 \mathrm{U}$ & $0.07 \mathrm{U}$ & $0.07 \mathrm{U}$ & $0.07 \mathrm{U}$ \\
\hline $\mathrm{Cl6}(128)$ & 1.08 & 1.45 & 0.21 & 3.99 & 2.10 \\
\hline $\mathrm{Cl6}(138)$ & 8.60 & 9.35 & 1.61 & $15.08 \mathrm{D}$ & 11.95 \\
\hline $\mathrm{Cl6}(153)$ & $11.58 \mathrm{D}$ & $11.89 \mathrm{D}$ & 3.18 & $29.47 \mathrm{D}$ & $13.90 \mathrm{D}$ \\
\hline $\mathrm{Cl}(170)$ & 1.37 & 1.63 & 0.23 & 4.09 & 1.90 \\
\hline $\mathrm{Cl}(187)$ & 3.54 & 4.50 & 0.63 & 3.64 & 5.79 \\
\hline $\mathrm{Cl}(188)$ & $0.01 \mathrm{~J}$ & $0.02 \mathrm{~J}$ & $0.02 \mathrm{U}$ & $0.02 \mathrm{~J}$ & $0.03 \mathrm{~J}$ \\
\hline $\mathrm{Cl}(195)$ & 0.16 & 0.23 & $0.05 \mathrm{U}$ & 0.50 & 0.27 \\
\hline $\mathrm{Cl}(200)$ & $0.04 \mathrm{U}$ & $0.04 \mathrm{U}$ & $0.04 \mathrm{U}$ & $0.04 \mathrm{U}$ & $0.04 \mathrm{U}$ \\
\hline $\mathrm{Cl9}(206)$ & 0.22 & 0.29 & $0.04 \mathrm{U}$ & 0.44 & 0.33 \\
\hline Cl10(209) & $0.08 \mathrm{~J}$ & 0.13 & $0.07 \mathrm{U}$ & $0.12 \mathrm{~J}$ & $0.11 \mathrm{~J}$ \\
\hline Total PCBs & 80.38 & 93.02 & 23.24 & 162.86 & 119.42 \\
\hline LOC 1 & $0.08 \mathrm{U}$ & $0.08 \mathrm{U}$ & $0.08 \mathrm{U}$ & $0.08 \mathrm{U}$ & $0.08 \mathrm{U}$ \\
\hline LOC 2 & $0.52 \mathrm{U}$ & $0.52 \mathrm{U}$ & $0.52 \mathrm{U}$ & $0.52 \mathrm{U}$ & $0.52 \mathrm{U}$ \\
\hline LOC 3 & $0.56 \mathrm{~J}$ & $0.41 \mathrm{~J}$ & $0.41 \mathrm{~J}$ & $0.60 \mathrm{~J}$ & $0.48 \mathrm{~J}$ \\
\hline LOC 4 & 4.73 & 5.65 & $3.09 \mathrm{~J}$ & 6.87 & 6.35 \\
\hline LOC 5 & 18.98 & 25.72 & 7.06 & 38.55 & 36.00 \\
\hline LOC 6 & 31.86 & 37.12 & 7.72 & 64.09 & 49.53 \\
\hline LOC 7 & 13.84 & 17.48 & $2.19 \mathrm{~J}$ & 29.11 & 22.86 \\
\hline LOC 8 & 2.65 & 3.70 & $0.51 \mathrm{U}$ & 6.22 & 4.08 \\
\hline LOC 9 & $0.31 \mathrm{~J}$ & 0.41 & $0.16 \mathrm{U}$ & 0.59 & 0.49 \\
\hline Total PCBs & 73.53 & 91.09 & 21.74 & 146.63 & 120.39 \\
\hline $\mathrm{Cl} 3(34)$ & 78 & 72 & 75 & 62 & 68 \\
\hline $\mathrm{Cl}(152)$ & 81 & 71 & 73 & 61 & 68 \\
\hline
\end{tabular}


BATTELLE MARINE SCIENCE LABORATORIES

1529 West Sequim Bay Road

Sequim, Washington 98382-9099

360/681-4564

\section{SINCLAIR AND DYES INLET \\ 2007 PSAMP Trawl Biota \\ PCBs in Whole Organisms}

\section{Data Qualifiers}

U Not detected; MDL reported

$\mathrm{J}$ Analyte concentration is less than RL

D Results determined from dilution

\& Outside Project DQO guidelines for spike recovery (40-120\%), replicate analysis (<30\%) or SRM percent different ( $<30 \%$ for concentrations $>10 \times \mathrm{MDL})$

C Quality Control (QC) value is outside the accuracy or precision Data Quality Objective (DQO), but meets the contingency criteria.

\# Outside Project DQO guidelines for SIS recovery (40-120\%)

$\mathrm{T} \quad$ Exceeds suggested hold time; see narrative

ME Results are estimates do to matrix effects; see narrative 
QA Narrative
Metals in Biota Tissue 


\section{QA/QC NARRATIVE}

PROJECT:

PARAMETER:

LABORATORY:

MATRIX:

SAMPLE CUSTODY AND PROCESSING:
2007 Puget Sound Area Monitoring Program (PSAMP) Trawl Biota, ENVVEST Biota Studies

Metals (Ag, As, Cd, Cr, Cu, Hg, Ni, Pb, Zn)

Battelle Marine Sciences Laboratory (MSL), Sequim, Washington

Marine Invertebrates and Fish

Specimen samples were received by MSL and stored frozen until sample selection and processing. Specimens were collected during the 2007 PSAMP trawls and the dogfish samples were collected by University of Washington. All samples were received in good condition (i.e., all sample containers were intact). A subset of the specimens were selected for analysis and assigned a Battelle Central File (CF) identification number (2838). Each specimen's weight, length, and photo were entered into Battelle's laboratory information and sample tracking system.

The following lists information on sample receipt and processing activities:

\begin{tabular}{ll} 
MSL Lab ID & $\begin{array}{l}106 \text { samples processed, see table for } \\
\text { specific sample IDs }\end{array}$ \\
Collection dates & May 2007 and $09 / 19 / 07$ \\
Digestion (aqua regia) & $01 / 31 / 08,02 / 05 / 08,02 / 07 / 08,02 / 14 / 08$, \\
CVAA analysis $(\mathrm{Hg})$ & $04 / 11 / 08$, and $04 / 15 / 08$ \\
ICP-MS analysis & $03 / 12 / 08,03 / 26 / 08$, and $04 / 25 / 08$ \\
(Ag, As, Cd, Cu, $\mathrm{Ni}, \mathrm{Pb}, \mathrm{Zn})$ & $02 / 06 / 08,02 / 19 / 08,02 / 20 / 08,04 / 16 / 08$, \\
ICP-OES analysis $(\mathrm{Cr})$ & and $04 / 17 / 08$ \\
\hline
\end{tabular}

\section{QA/QC METHOD QUALITY CRITERIA:}

\begin{tabular}{lcccccc} 
Analyte & $\begin{array}{c}\text { Analytical } \\
\text { Method }\end{array}$ & $\begin{array}{c}\text { Range of } \\
\text { Recovery }\end{array}$ & $\begin{array}{c}\text { SRM } \\
\text { Accuracy }\end{array}$ & $\begin{array}{c}\text { Relative } \\
\text { Precision }\end{array}$ & $\begin{array}{c}\text { Detection Limits ( } \mathbf{\mu g} / \mathbf{g} \text { ) } \\
\text { Achieved } \\
\text { MDL } \\
\text { (dry wt.) }\end{array}$ & $\begin{array}{c}\text { Reporting Limit } \\
\text { (dry wt.) }^{(3)}\end{array}$ \\
\hline Silver (Ag) & ICP-MS & $70 \%-130 \%$ & $\leq 20 \%^{(1)}$ & $<30 \%$ & 0.002 & 0.01 \\
Arsenic (As) & ICP-MS & $70 \%-130 \%$ & $\leq 20 \%^{(1)}$ & $<30 \%$ & 0.1 & 0.3 \\
Cadmium (Cd) & ICP-MS & $70 \%-130 \%$ & $\leq 20 \%^{(1)}$ & $<30 \%$ & 0.002 & 0.01 \\
Chromium (Cr) & ICP-OES & $70 \%-130 \%$ & $\leq 20 \%^{(1)}$ & $<30 \%$ & 0.04 & 0.1 \\
Copper (Cu) & ICP-MS & $70 \%-130 \%$ & $\leq 20 \%^{(1)}$ & $<30 \%$ & 0.09 & 0.3 \\
Mercury (Hg) & CVAA & $70 \%-130 \%$ & $\leq 20 \%^{(1)}$ & $<30 \%$ & 0.005 & 0.02 \\
Nickel (Ni) & ICP-MS & $70 \%-130 \%$ & $\leq 20 \%^{(1)}$ & $<30 \%$ & 0.04 & 0.1 \\
Lead (Pb) & ICP-MS & $70 \%-130 \%$ & $\leq 20 \%^{(1)}$ & $<30 \%$ & 0.003 & 0.01 \\
Zinc (Zn) & ICP-MS & $70 \%-130 \%$ & $\leq 20 \%^{(1)}$ & $<30 \%$ & 0.1 & 0.3 \\
\hline
\end{tabular}

(1) Evaluated for SRMs certified greater than 10 times the achieved MDL.

(2) Achieved MDL reported from the Annual Tissue MDL Study.

(3) Reporting Limit $=3.18$ times MDL 


\section{QA/QC NARRATIVE}

SAMPLE PREPARATION:

METHODS:

\section{Sample Preparation and Homogenization Procedures:}

- Excess liquid collected during thawing was discarded prior to homogenizing.

- Ceramic cutting knives and a Teflon block were used to cut fish into pieces small enough to fit into the specialized tissue homogenizer equipped with titanium blades to prevent metals contamination.

- All homogenization equipment was decontaminated between each sample using a laboratory detergent, hot water rinse, methanol rinse (if necessary due to oily samples), and $3 \mathrm{x}$ deionized water rinse. Gloves and work surface papers were changed between samples.

- Homogenized samples were placed into three separate containers

1) approximately $30 \mathrm{~g}$ into a precleaned $4 \mathrm{oz}$. glass jar for PCBs

2) approximately $20 \mathrm{~g}$ in a tared, precleaned $4 \mathrm{oz}$. polyprolyene jar for metals

3) approximately $5 \mathrm{~g}$ in a tared $2 \mathrm{oz}$. polyprolyene jar for isotopes

\section{Fish Preparation:}

- At least three specimens for each species catch and sampling location were selected for chemical analyses. Specimens were selected with similar weight or length to approximate relative age. In general, individual fish specimen were homogenized to create a whole body composite (WBC) for each specimen. Due to sample size limitations, a few specimens were combined into a single sample. These samples are noted on the table as COMP sample type or composite sample type.

- Fish were rinsed with DI water to remove external debris and patted dry with paper towel.

- Fish were sectioned using ceramic tools into portions that fit into the tissue homogenizer.

- The samples were homogenized to a uniform color.

\section{Sea Cucumber Preparation:}

- During PSAMP specimen collection, the sea cucumbers were stored in a single zip-top bag and frozen. Therefore, individual specimens were not achievable and the entire bag was used to generate a composite sample for each location. The number of individual specimens in each composite sample is noted on the table.

\section{Crab Preparation:}

- Whole individual crabs were thawed, rinsed with DI water, and homogenized to a uniform color.

- The crabs collected from Sinclair Inlet were too small for individual analyses. Two crabs were composited for this location (see table).

Composite, homogenized tissue samples were freeze-dried and milled to provide an additional homogenization in accordance with MSL-C-003, Percent Dry Weight and Homogenizing Dry Sediment, Soil, and Tissue. Dried tissue samples were analyzed for nine metals: $\mathrm{Ag}, \mathrm{As}, \mathrm{Cd}, \mathrm{Cr}, \mathrm{Cu}, \mathrm{Pb}, \mathrm{Hg}, \mathrm{Ni}$, and $\mathrm{Zn}$. Tissue samples were digested according to Battelle SOP MSL-I-024, Mixed Acid Tissue Digestion. An approximately 500-mg aliquot of each dried, homogeneous sample was combined with nitric and hydrochloric acids (aqua regia) in a Teflon vessel and heated in an oven at $130^{\circ} \mathrm{C}$ $\left( \pm 10^{\circ} \mathrm{C}\right)$ for a minimum of 8 hours. After heating and cooling, deionized water was added to the acid-digested tissue to achieve analysis volume and the digestates were submitted for analysis by three methods.

Digested samples were analyzed for $\mathrm{Hg}$ by cold-vapor atomic absorption spectroscopy 


\section{QA/QC NARRATIVE}

METHODS, cont.:

HOLDING TIMES:

DETECTION LIMITS:

METHOD BLANKS:

\section{LABORATORY \\ CONTROL SAMPLE/BLANK SPIKE ACCURACY:}

(CVAA) according to Battelle SOP MSL-I-016, Total Mercury in Tissues and Sediments by Cold Vapor Atomic Absorption, which is based on EPA Method 245.6, Determination of Mercury in Tissue by Cold Vapor Atomic Absorption Spectrometry.

Digested samples were analyzed for $\mathrm{Ag}, \mathrm{As}, \mathrm{Cd}, \mathrm{Cu}, \mathrm{Ni}, \mathrm{Pb}$, and $\mathrm{Zn}$ using inductively coupled plasma-mass spectrometry (ICP-MS) according to Battelle SOP MSL-I-022, Determination of Elements in Aqueous and Digestate Samples by ICP/MS. This procedure is based on two methods modified and adapted for analysis of solid sample digestates: EPA Method 1638, Determination of Trace Elements in Ambient Waters by Inductively Coupled Plasma-Mass Spectrometry and EPA Method 200.8, Determination of Trace Elements in Water and Wastes by Inductively Coupled PlasmaMass Spectrometry.

Digested samples were analyzed for $\mathrm{Cr}$ using inductively coupled plasma-optical emissions spectrometry (ICP-OES) according to Battelle SOP MSL-I-027, Determination of Elements in Aqueous and Digestate Samples by ICP/OES.

All results were determined and reported in units of $\mu \mathrm{g} / \mathrm{g}$ on a dry-weight basis. Field sample concentrations were converted to $\mu \mathrm{g} / \mathrm{g}$ wet weight using the percent moisture determined for each sample.

The recommended holding time for metals analyses in fish is 1 year frozen. The recommended holding time was achieved for all samples.

Achieved method detection limits are reported from the Annual Tissue MDL study. Reporting limits were determined as 3.18 times the achieved MDL. Sample concentrations were evaluated and flagged to the following criteria:

U Analyte not detected at or above the MDL, MDL reported for all sample values

J Analyte detected above the MDL, but less than the RL

N Spiked sample recovery outside QC criterion of 70-130\%

\& Accuracy result outside QC criterion of $\leq 20 \%$ PD

* Precision result outside QC criterion of $<30 \%$

B Analyte detected in the method blank $>$ RL and sample concentration $<10$ times detected blank value

c Exceeds DQO but meets contingency criteria of either:

1 SRM certified $<10 x$ MDL

2 Insufficient spiking level relative to native sample concentrations

3 Sample concentration $<10 \mathrm{x}$ MDL

Six method blanks were analyzed with this set of samples. Analytes in the blank were generally not detected above the MDL or less than the RL. Two blanks contained detectable $\mathrm{Zn}$ concentrations, but samples concentrations were at least 1-2 orders of magnitude higher than the detected blank. The data are not considered impacted.

Six replicates of a blank spike/laboratory control samples (LCS) were analyzed with this set of samples. All LCS recoveries were within the QC acceptance criterion of $70 \%$ to $130 \%$. 


\section{QA/QC NARRATIVE}

MATRIX SPIKE ACCURACY:

REPLICATE PRECISION:

STANDARD REFERENCE MATERIAL ACCURACY:
Six tissue samples of different species were selected for matrix spike/matrix spike duplicate samples. All MS/MSD recoveries were within the QC acceptance criterion of $70 \%$ to $130 \%$.

Two types of duplicates were used to evaluate laboratory precision: 1) laboratory duplicates and 2) matrix spike duplicates. Replicate precision was expressed as the relative percent difference (RPD) of replicate results. If triplicates were analyzed the precision was expressed as the relative standard deviation (RSD). Eighteen sets of laboratory duplicates were analyzed with this set of samples. The RPDs for laboratory duplicates were within the QC acceptance criterion of $<30 \%$ RPD for all metals except one duplicate (ratfish) for Ag (RPD 42\%) and one dogfish replicate for Ni (42\%). Acceptable precision was demonstrated for these metals and species on all other measures of precision.

The RPD values for all MS/MSD pairs were within the QC acceptance criterion for all metals.

SRM accuracy was expressed as the percent difference (PD) between the measured and certified SRM concentrations. Recovery of a particular analyte exceeded the QC criterion if the PD exceeded 20\%. SRMs are reported for analytes certified greater than 10 times the achieved MDL.

Six replicates of SRMs DORM-2 Dogfish Muscle Tissue and 1566b Oyster Tissue were analyzed with the set of samples. Multiple SRMs were selected because no single SRM is certified for all metals of interest at appropriate concentration ranges.

The percent differences for analytes reported from SRM 1566b were all within the QC criterion. The percent differences for analytes reported from SRM DORM-2 were all within the QC criterion of $\leq 20 \%$ percent difference except two replicates for $\mathrm{Pb}(24 \%$ and $21 \%$ ). Acceptable recovery for Pb was demonstrated by the SRM 1566b, matrix spikes, and blank spikes. Data were not significantly impacted. 


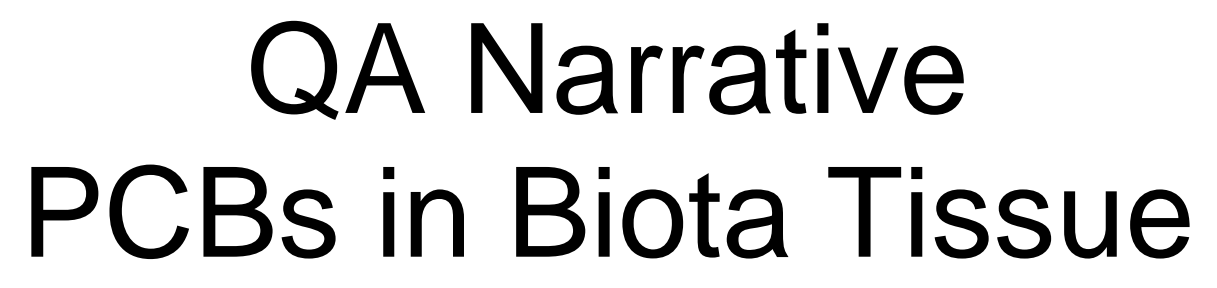




\title{
PCB - TISSUE QA/QC SUMMARY QC Batches 08-0004
}

\author{
PROJECT: \\ PARAMETER: \\ Sinclair \& Dyes Inlet 2008 \\ LABORATORY: \\ PCB \\ MATRIX: \\ Battelle, Duxbury, MA \\ SAMPLE CUSTODY: \\ Tissues - Fish \\ Tissue samples were collected 5/1/2007 - 5/3/2007, and 5/17/2007. They were \\ transferred from MSL on 1/7/2008. The shipment was received at Battelle Duxbury \\ on $1 / 8 / 2008$. Upon arrival, the cooler temperature was recorded at $0.2^{\circ} \mathrm{C}$. This is \\ above the upper limit of $-10^{\circ} \mathrm{C}$, for frozen tissue samples. Additionally two samples \\ did not have complete sample ID labels on the jars. The laboratory documented the \\ occurrences and proceeded as usual. No other custody issues were noted. The samples \\ were logged into the Battelle LIMS system and assigned unique IDs. Samples were \\ stored at $-10^{\circ} \mathrm{C}$ in access-controlled walk-in freezer until sample preparation could \\ begin.
}

\section{QA/QC DATA QUALITY OBJECTIVES:}

\begin{tabular}{|c|c|c|c|c|c|c|c|}
\hline & $\begin{array}{l}\text { Reference } \\
\text { Method } \\
\end{array}$ & $\begin{array}{c}\text { Method } \\
\text { Blank } \\
\end{array}$ & $\begin{array}{l}\text { Surrogate } \\
\text { Recovery }\end{array}$ & $\begin{array}{l}\text { LCS/MS } \\
\text { Recovery }\end{array}$ & $\begin{array}{c}\text { SRM } \\
\text { \% Diff. }\end{array}$ & $\begin{array}{c}\text { Sample } \\
\text { Replicate } \\
\text { Relative } \\
\text { Precision } \\
\end{array}$ & $\begin{array}{c}\text { Detection } \\
\text { Limits } \\
\text { (ng/g wet) }\end{array}$ \\
\hline \multirow[t]{2}{*}{ PCB } & $\begin{array}{l}\text { General } \\
\text { NS\&T }\end{array}$ & $<5 \times$ MDL & $40-120 \%$ & $\begin{array}{l}40-120 \% \\
\text { Recovery }\end{array}$ & $\begin{array}{c}\leq 30 \% \text { PD on } \\
\text { average }\end{array}$ & $\leq 30 \%$ RPD & $\begin{array}{c}\sim 0.04- \\
1.63\end{array}$ \\
\hline & & & & $\begin{array}{l}\text { (target spike must } \\
\text { be }>5 \mathrm{x} \text { native } \\
\text { conc.) }\end{array}$ & $\begin{array}{l}\text { (for analytes > }>5 x \\
\text { MDL) }\end{array}$ & $\begin{array}{l}\text { (for analytes }>5 \\
x \text { MDL) }\end{array}$ & \\
\hline
\end{tabular}

METHOD: Tissue samples were extracted for PCB following general NS\&T methods. For this analytical batch of tissue samples, $\sim 30 \mathrm{~g}$ of tissue was spiked with surrogates and extracted three times with methylene chloride using tissuemizer and shaker table techniques. The combined extract was dried over anhydrous sodium sulfate, concentrated, processed through florisil cleanup column, concentrated, and acid-cleaned. Because of the high lipid content associated with these samples, additional clean-up procedures were performed. Extracts were again processed through a florisil clean-up column, acid cleaned, and further purified by GPC/HPLC. The postHPLC extract was concentrated, fortified with RIS and submitted for analysis. Extracts intended for PCB analysis were analyzed using gas chromatography/mass spectrometry (GC/MS), following Battelle SOP 5-315 which is based on key components of the PCB congener analysis approach described in EPA Method 1668A. Sample data were quantified by the method of internal standards, using the Internal Standard (RIS) compounds.

HOLDING TIMES:

Tissue samples were stored frozen until sample preparation could begin. Samples were prepared for analysis in one analytical batch and were extracted within the 1-year holding time for sample collection and analyzed within 40 days of extraction.

\begin{tabular}{lcr} 
Batch & Extraction Date & Analysis Date \\
\hline $08-0004$ & $1 / 10 / 2008$ & $1 / 28 / 2008-2 / 7 / 2008$
\end{tabular}




\section{PCB - TISSUE QA/QC SUMMARY QC Batches 08-0004}

BLANKS:

\section{LABORATORY CONTROL SAMPLE:}

\section{MATRIX SPIKES:}

STANDARD REFERENCE MATERIAL:
A procedural blank $(\mathrm{PB})$ was prepared with the analytical batch. Blanks were analyzed to ensure the sample extraction and analysis methods were free of contamination.

08-0004 - No exceedences noted.

Comments - None.

A laboratory control sample (LCS) was prepared the analytical batch. The percent recoveries of PCB were calculated to measure data quality in terms of accuracy.

08-0004 - No exceedence noted.

Comments - All percent recoveries for spiked analytes were within the laboratory control limits (40-120\%).

A matrix spike (MS)/matrix spike duplicate (MSD) pair was prepared with the analytical batch. The percent recoveries of PCB were calculated to measure data quality in terms of accuracy; RPDs were calculated to measure quality in terms of precision.

08-0004 - No exceedences noted.

Comments - All percent recoveries for spiked analytes were within the laboratory control limits (40-120\%). All RPDs were within the laboratory control limits $(\leq 30 \%)$.

A standard reference material (SRM, NIST 2977) was prepared with each analytical batch. The percent difference (PD) between the measured value and the certified range was calculated to measure data quality in terms of accuracy.

08-0004 - 1exceedence noted.

Comments - All percent differences were within the laboratory control limits ( $<30 \%$ PD plus variance), except Cl6(138). This compound was under-recovered and had a PD of $57 \%$. Chromatography and calculations were reviewed, no discrepancies were found. Since accuracy for this compound was demonstrated in both the LCS and MS/MSD quality control samples, it appears this exceedence is isolated to the SRM sample and does not have a significant impact on the data. The exceedence was qualified with an " $\&$ ". No further corrective action was taken.

SURROGATES: Two surrogate compounds were added prior to extraction, including PCB 34 and PCB 152 . The recovery of each surrogate compound was calculated to measure data quality in terms of accuracy (extraction efficiency).

08-0004 - No exceedences noted.

Comments - None. 


\section{PCB - TISSUE QA/QC SUMMARY QC Batches 08-0004}

CALIBRATION: The GC/MS is calibrated with a minimum of a 6-point initial calibration curve (ICAL). The $\mathrm{r}^{2}$ value for the quadratic calibration must be $>0.995$ for all analytes. An Instrument Calibration Check (ICC), prepared from a different source from that used for the ICAL, was analyzed after the ICAL. The calculated concentration of target analytes in the ICC should be $\leq 25$ percent different (PD) from the true concentration in the ICC standard. Continuing calibration verification (CCV) standards were analyzed every 24-hr (minimally) to verify that the instrument response remains in calibration. The PD between response factors from the CCV and ICAL should be $\leq 25 \%$ for individual analytes.

08-0004 - ICAL: No exceedences noted. ICC: One exceedence noted. CCV: No exceedences noted.

Comments - In the ICC sample from run SD0645 PCB 81 was under-recovered with a PD of $33 \%$. Chromatography and calculations were reviewed. No discrepancies were found. This compound passed all other quality controls, indicating the exceedence does not have a significant impact on the data. The PCB 81 under-recovery is believed to be due to a contribution from PCB 166 in the ICAL. The exceedence was qualified with an "N" on the ICC report. No further corrective action was taken. Additionally PCB 81 was under-recovered fro the ICC from run SF0451. However the run was only for dilutions, and the PCB 81 exceedence did not pertain to any data collected from this sequence. 


\title{
PCB - TISSUE QAIQC SUMMARY QC Batches 08-0013
}

\author{
PROJECT: \\ PARAMETER: \\ Sinclair \& Dyes Inlet 2008 \\ LABORATORY: \\ PCB \\ MATRIX: \\ Battelle, Duxbury, MA \\ SAMPLE CUSTODY: \\ Tissues \\ Tissue samples were collected 5/1/2007 - 5/3/2007, and 5/17/2007. They were \\ transferred from MSL on 1/7/2008. The shipment was received at Battelle Duxbury \\ on $1 / 8 / 2008$. Upon arrival, the cooler temperature was recorded at $0.2^{\circ} \mathrm{C}$. This is \\ above the upper limit of $-10^{\circ} \mathrm{C}$, for frozen tissue samples. Additionally two samples \\ did not have complete sample ID labels on the jars. The laboratory documented the \\ occurrences and proceeded as usual. No other custody issues were noted. The samples \\ were logged into the Battelle LIMS system and assigned unique IDs. Samples were \\ stored at $-10^{\circ} \mathrm{C}$ in access-controlled walk-in freezer until sample preparation could \\ begin.
}

\section{QA/QC DATA QUALITY OBJECTIVES:}

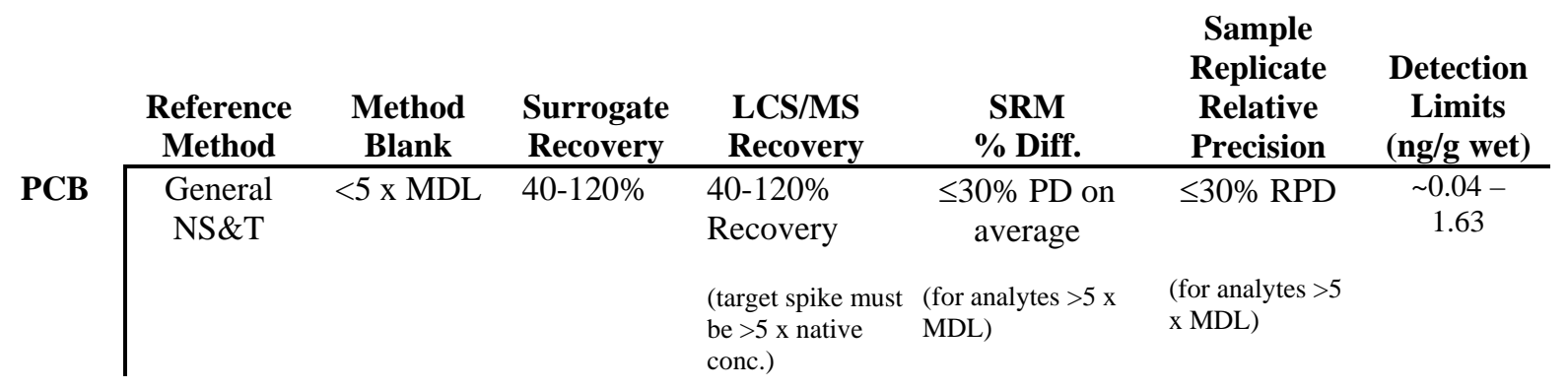

METHOD:

Tissue samples were extracted for PCB following general NS\&T methods. For this analytical batch of tissue samples, $\sim 30 \mathrm{~g}$ of tissue was spiked with surrogates and extracted three times with methylene chloride using tissuemizer and shaker table techniques. The combined extract was dried over anhydrous sodium sulfate, concentrated, processed through florisil cleanup column, concentrated, and acid-cleaned. Because of the high lipid content associated with these samples, additional clean-up procedures were performed. Extracts were again processed through a florisil clean-up column, acid cleaned, and further purified by GPC/HPLC. The postHPLC extract was concentrated, fortified with RIS and submitted for analysis. Extracts intended for PCB analysis were analyzed using gas chromatography/mass spectrometry (GC/MS), following Battelle SOP 5-315 which is based on key components of the PCB congener analysis approach described in EPA Method 1668A. Sample data were quantified by the method of internal standards, using the Internal Standard (RIS) compounds.

HOLDING TIMES:

Tissue samples were stored frozen until sample preparation could begin. Samples were prepared for analysis in one analytical batch and were extracted within the 1-year holding time for sample collection. Because of the high lipid content of these samples, samples were run numerous times, with numerous calibrations. Because of this not all samples were analyzed within the 40-day holding time for sample extracts. Data that was not acquired for within this holding time has been qualified with a "T". No further corrective action was taken.

\begin{tabular}{lcr} 
Batch & Extraction Date & Analysis Date \\
\hline $08-0013$ & $1 / 22 / 2008$ & $2 / 5 / 2008-3 / 11 / 2008$
\end{tabular}




\section{PCB - TISSUE QA/QC SUMMARY QC Batches 08-0013}

BLANKS: $\quad$ A procedural blank (PB) was prepared with the analytical batch. Blanks were analyzed to ensure the sample extraction and analysis methods were free of contamination.

08-0013 - No exceedences noted.

Comments - None.

LABORATORY CONTROL SAMPLE:

MATRIX SPIKES:

STANDARD REFERENCE MATERIAL:
A laboratory control sample (LCS) was prepared the analytical batch. The percent recoveries of PCB were calculated to measure data quality in terms of accuracy.

08-0013 - No exceedence noted.

Comments - All percent recoveries for spiked analytes were within the laboratory control limits $(40-120 \%)$.

A matrix spike (MS)/matrix spike duplicate (MSD) pair was prepared with the analytical batch. The percent recoveries of PCB were calculated to measure data quality in terms of accuracy; RPDs were calculated to measure quality in terms of precision.

08-0013 - No exceedences noted.

Comments - All percent recoveries for spiked analytes were within the laboratory control limits (40-120\%) for analytes spiked at a concentration great enough to be used for data quality assessment ( $>5 \mathrm{x}$ background). All RPDs were within the laboratory control limits $(\leq 30 \%)$.

A standard reference material (SRM, NIST 2977) was prepared with each analytical batch. The percent difference (PD) between the measured value and the certified range was calculated to measure data quality in terms of accuracy.

08-0013 - 2 exceedences noted.

Comments - All percent differences were within the laboratory control limits ( $<30 \%$ PD plus variance), except $\mathrm{Cl5}$ (118) and $\mathrm{Cl6}(138)$. These compounds were over-recovered and under-recovered, respectively. Chromatography and calculations were reviewed, no discrepancies were found. Accuracy for these compounds was demonstrated in the LCS sample. The exceedences were qualified with an " $\&$ ". No further corrective action was taken.

SURROGATES: Two surrogate compounds were added prior to extraction, including PCB 34 and PCB 152 . The recovery of each surrogate compound was calculated to measure data quality in terms of accuracy (extraction efficiency).

08-0013 - No exceedences noted.

Comments - None. 


\section{PCB - TISSUE QA/QC SUMMARY QC Batches 08-0013}

CALIBRATION: The GC/MS is calibrated with a minimum of a 6-point initial calibration curve (ICAL). The $\mathrm{r}^{2}$ value for the quadratic calibration must be $>0.995$ for all analytes. An Instrument Calibration Check (ICC), prepared from a different source from that used for the ICAL, was analyzed after the ICAL. The calculated concentration of target analytes in the ICC should be $\leq 25$ percent different (PD) from the true concentration in the ICC standard. Continuing calibration verification (CCV) standards were analyzed every 24-hr (minimally) to verify that the instrument response remains in calibration. The PD between response factors from the CCV and ICAL should be $\leq 25 \%$ for individual analytes.

08-0013 - ICAL: No exceedences noted. ICC: 6 exceedences noted. CCV: 6 exceedences noted.

Comments - Because of the high lipid content of these samples, extracts were analyzed numerous times with multiple methods. Each method is associated with its own ICAL, CCV, and ICC. In total, 4 calibrations were used. In all four ICC samples PCB 81 was under-recovered. Chromatography and calculations were reviewed. No discrepancies were found. The PCB 81 under-recovery is believed to be due to a contribution from PCB 166 in the ICAL. The exceedence was qualified with an "N" on the ICC report. No further corrective action was taken. Additionally PCB 209 failed low in ICC F9962 at $27.8 \%$ and PCB 205 failed low in ICC F0002 at 28\%. Samples potentially impacted by these exceedences include - Q1545, Q1555, Q1556, Q1558, Q1560 (for PCB 209) and Q1553 (for PCB 205), however the overall impact on the data is believed to be minimal. PCB 206 and PCB 209 failed low in 3 CCV samples. All impacted samples were re-run and bracketed by passing CCVs. 


\title{
PCB - TISSUE QAIQC SUMMARY QC Batches 08-0015
}

\author{
PROJECT: \\ PARAMETER: \\ Sinclair \& Dyes Inlet 2008 \\ LABORATORY: \\ PCB \\ MATRIX: \\ Battelle, Duxbury, MA \\ SAMPLE CUSTODY: \\ Tissues \\ Tissue samples were collected 5/1/2007 - 5/3/2007, and 5/16/2007 - 5/19/2007. They \\ were transferred from MSL on 1/10/2008. The shipment was received at Battelle \\ Duxbury on $1 / 12 / 2008$. Upon arrival, the cooler temperature was recorded at $-0.8^{\circ} \mathrm{C}$. \\ This is above the upper limit of $-10^{\circ} \mathrm{C}$, for frozen tissue samples. No other custody \\ issues were noted. The samples were logged into the Battelle LIMS system and \\ assigned unique IDs. Samples were stored at $-10^{\circ} \mathrm{C}$ in access-controlled walk-in \\ freezer until sample preparation could begin.
}

\section{QA/QC DATA QUALITY OBJECTIVES:}

\begin{tabular}{|c|c|c|c|c|c|c|c|}
\hline \multirow{3}{*}{ PCB } & $\begin{array}{l}\text { Reference } \\
\text { Method }\end{array}$ & $\begin{array}{c}\text { Method } \\
\text { Blank } \\
\end{array}$ & $\begin{array}{l}\text { Surrogate } \\
\text { Recovery }\end{array}$ & $\begin{array}{l}\text { LCS/MS } \\
\text { Recovery }\end{array}$ & $\begin{array}{l}\text { SRM } \\
\text { \% Diff. }\end{array}$ & $\begin{array}{c}\text { Sample } \\
\text { Replicate } \\
\text { Relative } \\
\text { Precision }\end{array}$ & $\begin{array}{c}\text { Detection } \\
\text { Limits } \\
\text { (ng/g wet) }\end{array}$ \\
\hline & $\begin{array}{c}\text { General } \\
\text { NS\&T }\end{array}$ & $<5 \times \mathrm{MDL}$ & $40-120 \%$ & $\begin{array}{l}40-120 \% \\
\text { Recovery }\end{array}$ & $\begin{array}{c}\leq 30 \% \text { PD on } \\
\text { average }\end{array}$ & $\leq 30 \% \mathrm{RPD}$ & $\sim 0.02-0.1$ \\
\hline & & & & $\begin{array}{l}\text { (target spike must } \\
\text { be }>5 \mathrm{x} \text { native } \\
\text { conc.) }\end{array}$ & $\begin{array}{l}\text { (for analytes }>5 \mathrm{x} \\
\text { MDL) }\end{array}$ & $\begin{array}{l}\text { (for analytes }>5 \\
x \text { MDL) }\end{array}$ & \\
\hline
\end{tabular}

METHOD: Tissue samples were extracted for PCB following general NS\&T methods. For this analytical batch of tissue samples, $\sim 30 \mathrm{~g}$ of tissue was spiked with surrogates and extracted three times with methylene chloride using tissuemizer and shaker table techniques. The combined extract was dried over anhydrous sodium sulfate, concentrated, processed through florisil cleanup column, concentrated, and acid-cleaned. Because of the high lipid content associated with these samples, additional clean-up procedures were performed. Extracts were again processed through a florisil clean-up column, acid cleaned, and further purified by GPC/HPLC. The postHPLC extract was concentrated, fortified with RIS and submitted for analysis. Extracts intended for PCB analysis were analyzed using gas chromatography/mass spectrometry (GC/MS), following Battelle SOP 5-315 which is based on key components of the PCB congener analysis approach described in EPA Method 1668A. Sample data were quantified by the method of internal standards, using the Internal Standard (RIS) compounds.

HOLDING TIMES:
Tissue samples were stored frozen until sample preparation could begin. Samples were prepared for analysis in one analytical batch and were extracted within the 1-year holding time for sample collection and analyzed within 40 days of extraction.

\begin{tabular}{lcr} 
Batch & Extraction Date & Analysis Date \\
\hline $08-0015$ & $1 / 28 / 2008$ & $2 / 16 / 2008-3 / 2 / 2008$
\end{tabular}




\section{PCB - TISSUE QA/QC SUMMARY QC Batches 08-0015}

BLANKS:

A procedural blank $(\mathrm{PB})$ was prepared with the analytical batch. Blanks were analyzed to ensure the sample extraction and analysis methods were free of contamination.

08-0015 - No exceedences noted.

Comments - None.

LABORATORY CONTROL SAMPLE:

MATRIX

SPIKES:

STANDARD REFERENCE MATERIAL:

\section{SURROGATES:}

Two surrogate compounds were added prior to extraction, including PCB 34 and PCB 152. The recovery of each surrogate compound was calculated to measure data quality in terms of accuracy (extraction efficiency).

08-0015 - No exceedences noted.

Comments - None.

A laboratory control sample (LCS) was prepared the analytical batch. The percent recoveries of PCB were calculated to measure data quality in terms of accuracy.

08-0015 - No exceedence noted.

Comments - All percent recoveries for spiked analytes were within the laboratory control limits $(40-120 \%)$.

A matrix spike (MS)/matrix spike duplicate (MSD) pair was prepared with the analytical batch. The percent recoveries of PCB were calculated to measure data quality in terms of accuracy; RPDs were calculated to measure quality in terms of precision.

08-0015 - No exceedences noted.

Comments - All percent recoveries for spiked analytes were within the laboratory control limits (40-120\%). All RPDs were within the laboratory control limits $(\leq 30 \%)$.

A standard reference material (SRM, NIST 2977) was prepared with each analytical batch. The percent difference (PD) between the measured value and the certified range was calculated to measure data quality in terms of accuracy.

08-0015 - No exceedence noted.

Comments - All percent differences were within the laboratory control limits $(<30 \%$ PD plus variance) for reported congeners. 


\section{PCB - TISSUE QA/QC SUMMARY QC Batches 08-0015}

CALIBRATION: The GC/MS is calibrated with a minimum of a 6-point initial calibration curve (ICAL). The $\mathrm{r}^{2}$ value for the quadratic calibration must be $>0.995$ for all analytes. An Instrument Calibration Check (ICC), prepared from a different source from that used for the ICAL, was analyzed after the ICAL. The calculated concentration of target analytes in the ICC should be $\leq 25$ percent different (PD) from the true concentration in the ICC standard. Continuing calibration verification (CCV) standards were analyzed every 24-hr (minimally) to verify that the instrument response remains in calibration. The PD between response factors from the CCV and ICAL should be $\leq 25 \%$ for individual analytes.

08-0015 - ICAL: No exceedences noted. ICC: 2 exceedences noted. CCV: 15 exceedences noted.

Comments - Data from this batch was acquired over 2 sequences. In both ICC samples PCB 81 was under-recovered. Chromatography and calculations were reviewed. No discrepancies were found. This compound passed all other quality controls, indicating the exceedence does not have a significant impact on the data. The PCB 81 underrecovery is believed to be due to a contribution from PCB 166 in the ICAL. The exceedence was qualified with an "N" on the ICC report. No further corrective action was taken.

In CCV F0030 PCB 1 was under-recovered. The QC samples bracketed by this failing mid had acceptable PCB 8 recoveries, indicating the failure had minimal impact on the data. Additionally PCB 205 was over-recovered, however this compound was not detected in any field sample bracketed by the mid, so this exceedence has no impact on the data. Although PCB 1and PCB 205 are not reported as individual PCB congeners on the data tables, it is used in the calculation for LOCs.

In CCV F0044 PCB 3, 4, 5, 6, 7, 8, 9, 11, 12, 13, 15, 16, and 30 were all over-recovered. Samples Q1616 - Q1628 (excluding Q1625) are associated with this mid. All of the above over-recoveries are not detected in the field samples, except for PCB 8, which is detected below the reporting limit, indicating the exceedences have minimal impact on the data. None of the above mentioned congeners, except for PCB 8, are reported as individual congeners; however they are used in the calculations for LOCs. 


\title{
PCB - TISSUE QA/QC SUMMARY QC Batches 08-0016
}

\author{
PROJECT: \\ PARAMETER: \\ Sinclair \& Dyes Inlet 2008 \\ LABORATORY: \\ PCB \\ MATRIX: \\ Battelle, Duxbury, MA \\ SAMPLE CUSTODY: \\ Tissues \\ Tissue samples were collected in May 2007. They were transferred from MSL on \\ $1 / 7 / 2008$. The shipment was received at Battelle Duxbury on $1 / 12 / 2008$. Upon \\ arrival, the cooler temperature was recorded at $-0.8^{\circ} \mathrm{C}$. This is above the upper limit of \\ $-10^{\circ} \mathrm{C}$, for frozen tissue samples. The laboratory documented the occurrences and \\ proceeded as usual. No other custody issues were noted. The samples were logged \\ into the Battelle LIMS system and assigned unique IDs. Samples were stored at $-10^{\circ} \mathrm{C}$ \\ in access-controlled walk-in freezer until sample preparation could begin.
}

\section{QA/QC DATA QUALITY OBJECTIVES:}

\begin{tabular}{|c|c|c|c|c|c|c|c|}
\hline & $\begin{array}{l}\text { Reference } \\
\text { Method } \\
\end{array}$ & $\begin{array}{c}\text { Method } \\
\text { Blank }\end{array}$ & $\begin{array}{l}\text { Surrogate } \\
\text { Recovery }\end{array}$ & $\begin{array}{l}\text { LCS/MS } \\
\text { Recovery }\end{array}$ & $\begin{array}{l}\text { SRM } \\
\text { \% Diff. }\end{array}$ & $\begin{array}{c}\text { Sample } \\
\text { Replicate } \\
\text { Relative } \\
\text { Precision } \\
\end{array}$ & $\begin{array}{c}\text { Detection } \\
\text { Limits } \\
\text { (ng/g wet) }\end{array}$ \\
\hline \multirow[t]{2}{*}{ PCB } & $\begin{array}{l}\text { General } \\
\text { NS\&T }\end{array}$ & $<5 \times \mathrm{MDL}$ & $40-120 \%$ & $\begin{array}{l}40-120 \% \\
\text { Recovery }\end{array}$ & $\begin{array}{c}\leq 30 \% \text { PD on } \\
\text { average }\end{array}$ & $\leq 30 \%$ RPD & $\begin{array}{c}\sim 0.04- \\
1.63\end{array}$ \\
\hline & & & & $\begin{array}{l}\text { (target spike must } \\
\text { be }>5 \times \text { native } \\
\text { conc.) }\end{array}$ & $\begin{array}{l}\text { (for analytes >5 } \mathrm{x} \\
\text { MDL) }\end{array}$ & $\begin{array}{l}\text { (for analytes }>5 \\
x \text { MDL) }\end{array}$ & \\
\hline
\end{tabular}

METHOD: $\quad$ Tissue samples were extracted for PCB following general NS\&T methods. For this analytical batch of tissue samples, $\sim 30 \mathrm{~g}$ of tissue was spiked with surrogates and extracted three times with methylene chloride using tissuemizer and shaker table techniques. The combined extract was dried over anhydrous sodium sulfate, concentrated, processed through florisil cleanup column, concentrated, and acid-cleaned. Because of the high lipid content associated with these samples, additional clean-up procedures were performed. Extracts were again processed through a florisil clean-up column, acid cleaned, and further purified by GPC/HPLC. The postHPLC extract was concentrated, fortified with RIS and submitted for analysis. Extracts intended for PCB analysis were analyzed using gas chromatography/mass spectrometry (GC/MS), following Battelle SOP 5-315 which is based on key components of the PCB congener analysis approach described in EPA Method 1668A. Sample data were quantified by the method of internal standards, using the Internal Standard (RIS) compounds. Because of the complexity of the sample matrix, which resulted in $\mathrm{CCV}$ failures, samples extracts ran multiple times.

HOLDING TIMES:
Tissue samples were stored frozen until sample preparation could begin. Samples were prepared for analysis in one analytical batch and were extracted within the 1-year holding time for sample collection. Because of the high lipid content of these samples, samples were run numerous times. Because of this not all samples were analyzed within the 40-day holding time for sample extracts. Data that was not acquired for within this holding time has been qualified with a "T". No further corrective action was taken.

\begin{tabular}{lcr} 
Batch & Extraction Date & Analysis Date \\
\hline $08-0016$ & $1 / 28 / 2008$ & $2 / 27 / 2008-3 / 15 / 2008$
\end{tabular}




\section{PCB - TISSUE QA/QC SUMMARY QC Batches 08-0016}

BLANKS:

\section{LABORATORY CONTROL SAMPLE:}

\section{MATRIX SPIKES:}

A procedural blank (PB) was prepared with the analytical batch. Blanks were analyzed to ensure the sample extraction and analysis methods were free of contamination.

08-0016 - No exceedences noted.

Comments - None.

A laboratory control sample (LCS) was prepared the analytical batch. The percent recoveries of PCB were calculated to measure data quality in terms of accuracy.

08-0016 - No exceedence noted.

Comments - All percent recoveries for spiked analytes were within the laboratory control limits (40-120\%).

A matrix spike (MS)/matrix spike duplicate (MSD) pair was prepared with the analytical batch. The percent recoveries of PCB were calculated to measure data quality in terms of accuracy; RPDs were calculated to measure quality in terms of precision.

08-0016 - 1 percent recovery exceedence noted. No RPD exceedences noted.

Comments - All percent recoveries, for analytes spiked at a concentration great enough to be used for data quality assessment, were within the laboratory control limits (40-120\%), except for PCB 209. This compound was under-recovered in sample Q1639MSD (background EH-RF-MSD). Chromatography and calculations were reviewed. No discrepancies were found. The analyst notes that the complex nature of these sample extracts lead to the under-recovery of PCB 209. The data was qualified with an " \&". No further corrective action was taken.

All RPDs, for analytes spiked at a concentration great enough to be used for data quality assessment, were within the laboratory control limits $(\leq 30 \%)$, except for The RPD between PCB 209 and LOC 9 percent recoveries. Chromatography and calculations were reviewed. No discrepancies were found. Again the analyst notes that the complex nature of these sample extracts lead to these exceedences. The data was qualified with an " $\&$ ". No further corrective action was taken.

A standard reference material (SRM, NIST 2977) was prepared with each analytical batch. The percent difference (PD) between the measured value and the certified range was calculated to measure data quality in terms of accuracy.

08-0016 - 2exceedence noted.

Comments - All percent differences were within the laboratory control limits $(<30 \%$ PD plus variance), except Cl4(44) and Cl6(138). These compounds were over-recovered and under-recovered, respectively. Chromatography and calculations were reviewed, no discrepancies were found. The exceedence was qualified with an "\&". No further corrective action was taken. 


\section{PCB - TISSUE QAIQC SUMMARY QC Batches 08-0016}

SURROGATES: $\quad$ Two surrogate compounds were added prior to extraction, including PCB 34 and PCB 152 . The recovery of each surrogate compound was calculated to measure data quality in terms of accuracy (extraction efficiency).

08-0016 - 1 exceedence noted.

Comments - All percent recoveries for surrogate compounds were within the laboratory control limits (40-120\%), except for PCB 152 in sample Q1631. This surrogate was overrecovered at $147 \%$. The chromatography and calculations were reviewed. This sample was particularly difficult to analyze and all the data have been qualified with an "ME" to indicate the results are estimates do to matrix effects. Additionally the exceedence was qualified with an "\#”. No further corrective action was taken.

CALIBRATION: The GC/MS is calibrated with a minimum of a 6-point initial calibration curve (ICAL). The $r^{2}$ value for the quadratic calibration must be $>0.995$ for all analytes. An Instrument Calibration Check (ICC), prepared from a different source from that used for the ICAL, was analyzed after the ICAL. The calculated concentration of target analytes in the ICC should be $\leq 25$ percent different (PD) from the true concentration in the ICC standard. Continuing calibration verification (CCV) standards were analyzed every 24-hr (minimally) to verify that the instrument response remains in calibration. The PD between response factors from the CCV and ICAL should be $\leq 25 \%$ for individual analytes.

08-0016 - ICAL: No exceedences noted. ICC: One exceedence noted. CCV: No exceedences noted.

Comments - In the ICC sample, PCB 81 was under-recovered with a PD of $32.6 \%$. Chromatography and calculations were reviewed. No discrepancies were found. This compound passed all other quality controls, indicating the exceedence does not have a significant impact on the data. The PCB 81 under-recovery is believed to be due to a contribution from PCB 166 in the ICAL. The exceedence was qualified with an "N" on the ICC report. No further corrective action was taken.

In CCV D7802 PCB 209 failed low with a PD of 30.4\%. Samples bracketed by this mid were run numerous times with similar results (PCB $209 \mathrm{CCV}$ failures). Therefore the data was reported as is. This failure impacted samples Q1644, Q1645, Q1646, and Q1647. In CCV D7852, 60 PCB compounds failed (see CCV report for specifics). Chromatography and calculations were reviewed. The only sample bracketed by this CCV was sample Q1631. This sample ran numerous times, yet was always bracketed by failing CCVs, due to the complex nature of the extract. Since this sample ran numerous times with similar results, and the extract was sent back to the prep lab for additional cleanup numerous times, yet CCV results were not improved, the data bracketed by the failing CCV was reported. All concentrations were qualified with an "ME" to indicate they are estimates due to matrix effect. 


\title{
PCB - TISSUE QA/QC SUMMARY QC Batches 08-0017
}

\author{
PROJECT: \\ PARAMETER: \\ Sinclair \& Dyes Inlet 2008 \\ LABORATORY: \\ PCB \\ MATRIX: \\ Battelle, Duxbury, MA \\ SAMPLE CUSTODY: \\ Tissues \\ Tissue samples were collected in May and September 2007. They were transferred \\ from MSL in two shipments on 1/10/2008 and 1/21/2008. Each shipment was \\ received at Battelle Duxbury on the following day. Upon arrival, the cooler \\ temperatures were recorded at $-0.8^{\circ} \mathrm{C}$ and $1.2^{\circ} \mathrm{C}$, respectively. They are both above the \\ upper limit of $-10^{\circ} \mathrm{C}$, for frozen tissue samples. No other custody issues were noted. \\ The samples were logged into the Battelle LIMS system and assigned unique IDs. \\ Samples were stored at $-10^{\circ} \mathrm{C}$ in access-controlled walk-in freezer until sample \\ preparation could begin.
}

QA/QC DATA QUALITY OBJECTIVES:

\begin{tabular}{|c|c|c|c|c|c|c|c|}
\hline & $\begin{array}{c}\text { Reference } \\
\text { Method }\end{array}$ & $\begin{array}{l}\text { Method } \\
\text { Blank }\end{array}$ & $\begin{array}{l}\text { Surrogate } \\
\text { Recovery }\end{array}$ & $\begin{array}{l}\text { LCS/MS } \\
\text { Recovery }\end{array}$ & $\begin{array}{l}\text { SRM } \\
\text { \% Diff. }\end{array}$ & $\begin{array}{c}\text { Sample } \\
\text { Replicate } \\
\text { Relative } \\
\text { Precision }\end{array}$ & $\begin{array}{l}\text { Detection } \\
\text { Limits } \\
\text { (ng/g wet) }\end{array}$ \\
\hline \multirow[t]{2}{*}{ PCB } & $\begin{array}{c}\text { General } \\
\text { NS\&T }\end{array}$ & $<5 \times \mathrm{MDL}$ & $40-120 \%$ & $\begin{array}{l}40-120 \% \\
\text { Recovery }\end{array}$ & $\begin{array}{c}\leq 30 \% \text { PD on } \\
\text { average }\end{array}$ & $\leq 30 \%$ RPD & $\begin{array}{l}\sim 0.04- \\
1.63\end{array}$ \\
\hline & & & & $\begin{array}{l}\text { (target spike must } \\
\text { be }>5 \times \text { native } \\
\text { conc.) }\end{array}$ & $\begin{array}{l}\text { (for analytes }>5 \mathrm{x} \\
\text { MDL) }\end{array}$ & $\begin{array}{l}\text { (for analytes }>5 \\
x \text { MDL) }\end{array}$ & \\
\hline
\end{tabular}

METHOD: Tissue samples were extracted for PCB following general NS\&T methods. For this analytical batch of tissue samples, $\sim 30 \mathrm{~g}$ of tissue was spiked with surrogates and extracted three times with methylene chloride using tissuemizer and shaker table techniques. The combined extract was dried over anhydrous sodium sulfate, concentrated, processed through florisil cleanup column, concentrated, and acid-cleaned. Because of the high lipid content associated with these samples, additional clean-up procedures were performed. Extracts were again processed through a florisil clean-up column, acid cleaned, and further purified by GPC/HPLC. The postHPLC extract was concentrated, fortified with RIS and submitted for analysis. Extracts intended for PCB analysis were analyzed using gas chromatography/mass spectrometry (GC/MS), following Battelle SOP 5-315 which is based on key components of the PCB congener analysis approach described in EPA Method 1668A. Sample data were quantified by the method of internal standards, using the Internal Standard (RIS) compounds. Because of the high lipid content of these samples, extracts were analyzed numerous times.

HOLDING Tissue samples were stored frozen until sample preparation could begin. Samples were TIMES: prepared for analysis in one analytical batch and were extracted within the 1-year holding time for sample collection and analyzed within 40 days of extraction.

$\begin{array}{lcr}\text { Batch } & \text { Extraction Date } & \text { Analysis Date } \\ 08-0017 & 2 / 7 / 2008 & 2 / 29 / 2008-3 / 7 / 2008\end{array}$




\section{PCB - TISSUE QA/QC SUMMARY QC Batches 08-0017}

BLANKS:

LABORATORY CONTROL SAMPLE:

\section{MATRIX \\ SPIKES:}

STANDARD REFERENCE MATERIAL:
A procedural blank $(\mathrm{PB})$ was prepared with the analytical batch. Blanks were analyzed to ensure the sample extraction and analysis methods were free of contamination.

08-0017 - No exceedences noted.

Comments - None.

A laboratory control sample (LCS) was prepared the analytical batch. The percent recoveries of PCB were calculated to measure data quality in terms of accuracy.

08-0017 - No exceedence noted.

Comments - All percent recoveries for spiked analytes were within the laboratory control limits (40-120\%).

A matrix spike (MS)/matrix spike duplicate (MSD) pair was prepared with the analytical batch. The percent recoveries of PCB were calculated to measure data quality in terms of accuracy; RPDs were calculated to measure quality in terms of precision.

08-0017 - No exceedences noted.

Comments - All percent recoveries for compounds spiked at a concentration great enough to be used for data quality assessment ( $5 \mathrm{x}$ background) were within the laboratory control limits (40-120\%). All RPDs were within the laboratory control limits $(\leq 30 \%)$.

A standard reference material (SRM, NIST 2977) was prepared with each analytical batch. The percent difference (PD) between the measured value and the certified range was calculated to measure data quality in terms of accuracy.

08-0017 - 1exceedence noted.

Comments - All percent differences were within the laboratory control limits ( $<30 \%$ PD plus variance), except Cl6(138). This compound was under-recovered. Chromatography and calculations were reviewed, no discrepancies were found. Since accuracy for this compound was demonstrated in LCS, it appears this exceedence is isolated to the SRM sample and does not have a significant impact on the data. The exceedence was qualified with an "\&". No further corrective action was taken. 


\section{PCB - TISSUE QA/QC SUMMARY QC Batches 08-0017}

SURROGATES: $\quad$ Two surrogate compounds were added prior to extraction, including PCB 34 and PCB 152 . The recovery of each surrogate compound was calculated to measure data quality in terms of accuracy (extraction efficiency).

08-0017 - 1 exceedence noted.

Comments - All percent recoveries for surrogate compounds were within the laboratory control limits (40-120\%), except for PCB 34 in sample Q1692 (DF-WBWC-1). This surrogate was under-recovered at $37 \%$. Chromatography and calculations were reviewed. No discrepancies were found. Multiple analysis of the same extract provided similar results. The exceedence was qualified with an "\#" and no further corrective action was taken.

CALIBRATION: The GC/MS is calibrated with a minimum of a 6-point initial calibration curve (ICAL). The $\mathrm{r}^{2}$ value for the quadratic calibration must be $>0.995$ for all analytes. An Instrument Calibration Check (ICC), prepared from a different source from that used for the ICAL, was analyzed after the ICAL. The calculated concentration of target analytes in the ICC should be $\leq 25$ percent different (PD) from the true concentration in the ICC standard. Continuing calibration verification (CCV) standards were analyzed every 24-hr (minimally) to verify that the instrument response remains in calibration. The PD between response factors from the CCV and ICAL should be $\leq 25 \%$ for individual analytes.

08-0017 - ICAL: No exceedences noted. ICC: 1 exceedence noted.

CCV: 3 exceedences noted.

Comments -In the ICC sample PCB 81 was under-recovered. Chromatography and calculations were reviewed. No discrepancies were found. The PCB 81 under-recovery is believed to be due to a contribution from PCB 166 in the ICAL. The exceedence was qualified with an "N" on the ICC report. No further corrective action was taken. Additionally PCB 209 failed low in 3 CCV samples. Samples were re-run, however PCB 209 continuously failed in some mids. This resulted on samples Q1690, Q1691, Q1692, Q1677, Q1678, Q1679, and Q1680 having PCB 209 concentrations reported from data bracketed by a failing CCV. 


\title{
PCB - TISSUE QAIQC SUMMARY QC Batches 08-0018
}

\author{
PROJECT: \\ PARAMETER: \\ Sinclair \& Dyes Inlet 2008 \\ LABORATORY: \\ MATRIX: \\ PCB \\ SAMPLE CUSTODY: \\ Battelle, Duxbury, MA \\ Tissues \\ Tissue samples were collected in September 2007. They were transferred from MSL \\ on 1/21/2008. The shipment was received at Battelle Duxbury on the following day. \\ Upon arrival, the cooler temperature was recorded at $1.2^{\circ} \mathrm{C}$. This is above the upper \\ limit of $-10^{\circ} \mathrm{C}$, for frozen tissue samples. No other custody issues were noted. The \\ samples were logged into the Battelle LIMS system and assigned unique IDs. Samples \\ were stored at $-10^{\circ} \mathrm{C}$ in access-controlled walk-in freezer until sample preparation \\ could begin.
}

\section{QA/QC DATA QUALITY OBJECTIVES:}

\begin{tabular}{|c|c|c|c|c|c|c|c|}
\hline \multirow{3}{*}{ PCB } & $\begin{array}{l}\text { Reference } \\
\text { Method }\end{array}$ & $\begin{array}{c}\text { Method } \\
\text { Blank } \\
\end{array}$ & $\begin{array}{l}\text { Surrogate } \\
\text { Recovery }\end{array}$ & $\begin{array}{l}\text { LCS/MS } \\
\text { Recovery }\end{array}$ & $\begin{array}{l}\text { SRM } \\
\text { \% Diff. }\end{array}$ & $\begin{array}{c}\text { Sample } \\
\text { Replicate } \\
\text { Relative } \\
\text { Precision }\end{array}$ & $\begin{array}{c}\text { Detection } \\
\text { Limits } \\
\text { (ng/g wet) }\end{array}$ \\
\hline & $\begin{array}{c}\text { General } \\
\text { NS\&T }\end{array}$ & $<5 \times \mathrm{MDL}$ & $40-120 \%$ & $\begin{array}{l}40-120 \% \\
\text { Recovery }\end{array}$ & $\begin{array}{c}\leq 30 \% \text { PD on } \\
\text { average }\end{array}$ & $\leq 30 \%$ RPD & $\begin{array}{c}\sim 0.04- \\
1.63\end{array}$ \\
\hline & & & & $\begin{array}{l}\text { (target spike must } \\
\text { be }>5 \mathrm{x} \text { native } \\
\text { conc.) }\end{array}$ & $\begin{array}{l}\text { (for analytes }>5 \mathrm{x} \\
\text { MDL) }\end{array}$ & $\begin{array}{l}\text { (for analytes }>5 \\
x \text { MDL) }\end{array}$ & \\
\hline
\end{tabular}

METHOD: Tissue samples were extracted for PCB following general NS\&T methods. For this analytical batch of tissue samples, $\sim 30 \mathrm{~g}$ of tissue was spiked with surrogates and extracted three times with methylene chloride using tissuemizer and shaker table techniques. The combined extract was dried over anhydrous sodium sulfate, concentrated, processed through florisil cleanup column, concentrated, and acid-cleaned. Because of the high lipid content associated with these samples, additional clean-up procedures were performed. Extracts were again processed through a florisil clean-up column, acid cleaned, and further purified by GPC/HPLC. The postHPLC extract was concentrated, fortified with RIS and submitted for analysis. Extracts intended for PCB analysis were analyzed using gas chromatography/mass spectrometry (GC/MS), following Battelle SOP 5-315 which is based on key components of the PCB congener analysis approach described in EPA Method 1668A. Sample data were quantified by the method of internal standards, using the Internal Standard (RIS) compounds. Because problems related to the sample matrix, extracts were analyzed numerous times.

HOLDING Tissue samples were stored frozen until sample preparation could begin. Samples were TIMES: prepared for analysis in one analytical batch and were extracted within the 1-year holding time for sample collection and analyzed within 40 days of extraction.

\begin{tabular}{lcr} 
Batch & Extraction Date & Analysis Date \\
\hline $08-0018$ & $2 / 7 / 2008$ & $2 / 29 / 2008-3 / 11 / 2008$
\end{tabular}




\section{PCB - TISSUE QA/QC SUMMARY QC Batches 08-0018}

BLANKS:

\section{LABORATORY CONTROL SAMPLE:}

\section{MATRIX SPIKES:}

STANDARD REFERENCE MATERIAL:
A procedural blank $(\mathrm{PB})$ was prepared with the analytical batch. Blanks were analyzed to ensure the sample extraction and analysis methods were free of contamination.

08-0018 - No exceedences noted.

Comments - None.

A laboratory control sample (LCS) was prepared the analytical batch. The percent recoveries of PCB were calculated to measure data quality in terms of accuracy.

08-0018 - No exceedence noted.

Comments - All percent recoveries for spiked analytes were within the laboratory control limits (40-120\%).

A matrix spike (MS)/matrix spike duplicate (MSD) pair was prepared with the analytical batch. The percent recoveries of PCB were calculated to measure data quality in terms of accuracy; RPDs were calculated to measure quality in terms of precision.

08-0018 - No exceedences noted.

Comments - All percent recoveries for spiked analytes were within the laboratory control limits (40-120\%), except for PCB 153 in sample Q1649MS (background DF-WBWC-3); however this analyte was not spiked at a concentration great enough to be used for data quality assessment. All RPDs were within the laboratory control limits $(\leq 30 \%)$.

A standard reference material (SRM, NIST 2977) was prepared with each analytical batch. The percent difference (PD) between the measured value and the certified range was calculated to measure data quality in terms of accuracy.

08-0018 - 1exceedence noted.

Comments - All percent differences were within the laboratory control limits ( $<30 \%$ PD plus variance), except Cl6(138). This compound was under-recovered. Chromatography and calculations were reviewed, no discrepancies were found. Since accuracy for this compound was demonstrated in both the LCS and MS/MSD quality control samples, it appears this exceedence is isolated to the SRM sample and does not have a significant impact on the data. The exceedence was qualified with an "\&". No further corrective action was taken.

SURROGATES: $\quad$ Two surrogate compounds were added prior to extraction, including PCB 34 and PCB 152 . The recovery of each surrogate compound was calculated to measure data quality in terms of accuracy (extraction efficiency).

08-0018 - No exceedences noted.

Comments - All percent recoveries for surrogate compounds were within the laboratory control limits $(40-120 \%)$. 


\section{PCB - TISSUE QA/QC SUMMARY QC Batches 08-0018}

CALIBRATION: The GC/MS is calibrated with a minimum of a 6-point initial calibration curve (ICAL). The $\mathrm{r}^{2}$ value for the quadratic calibration must be $>0.995$ for all analytes. An Instrument Calibration Check (ICC), prepared from a different source from that used for the ICAL, was analyzed after the ICAL. The calculated concentration of target analytes in the ICC should be $\leq 25$ percent different (PD) from the true concentration in the ICC standard. Continuing calibration verification (CCV) standards were analyzed every 24-hr (minimally) to verify that the instrument response remains in calibration. The PD between response factors from the CCV and ICAL should be $\leq 25 \%$ for individual analytes.

08-0018 - ICAL: No exceedences noted.

ICC: 2 exceedences noted.

CCV: No exceedences noted.

Comments - Two calibrations are associated with this batch. In both ICC samples PCB 81 was under-recovered with a PD of $28 \%$ and $38.2 \%$ Chromatography and calculations were reviewed. No discrepancies were found. This compound passed all other quality controls, indicating the exceedence does not have a significant impact on the data. The PCB 81 under-recovery is believed to be due to a contribution from PCB 166 in the ICAL. The exceedence was qualified with an "N" on the ICC report. 


\section{QA Narrative Stable Isotopes in Biota Tissue}


PROJECT:

PARAMETER:

LABORATORY:

MATRIX:

SAMPLE CUSTODY AND PROCESSING:
2007 Puget Sound Area Monitoring Program (PSAMP) Trawl Biota, ENVVEST Biota Studies

Stable Isotopes of Carbon $\left(\delta^{13} \mathrm{C}\right)$ and nitrogen $\left(\delta^{15} \mathrm{~N}\right)$

Dr. Jay Brandes, Skidaway Institute for Oceanography

Muscle Tissue from Marine Invertebrates and Fish

Specimen samples were received by MSL and stored frozen until sample selection and processing. Specimens were collected during the 2007 PSAMP trawls and the dogfish samples were collected by University of Washington. All samples were received in good condition (i.e., all sample containers were intact). Each specimen was assigned a Battelle Central File (CF) identification number (2838). Each specimen's weight, length, and photo were entered into Battelle's laboratory information and sample tracking system. Homogenized muscle tissue and whole body weighted composites for the dogfish were sent to Skidaway Institute of Oceanography.

The following lists information on sample receipt and processing activities:

MSL Lab ID See Table

Collection dates May 2007 and 09/19/07

\section{Sample Preparation and Homogenization Procedures:}

- Excess liquid collected during thawing was discarded prior to homogenizing.

- Ceramic cutting knives and a Teflon block were used to cut muscle tissue for each specimen.

- All homogenization equipment was decontaminated between each sample using a laboratory detergent, hot water rinse, methanol rinse (if necessary due to oily samples), and $3 x$ deionized water rinse. Gloves and work surface papers were changed between samples.

- Homogenized samples were placed into in a tarred $2 \mathrm{oz}$. polyprolyene jar for isotopes

- Samples were frozen to $-70^{\circ} \mathrm{C}$, freeze dried, and ground using a sediment miller.

\section{PSAMP Fish Preparation:}

- Muscle tissue from each specimens provided by PSAMP was collected for isotope analysis.

- Fish were rinsed with DI water to remove external debris and patted dry with paper towel.

- A ceramic knife was used to collect muscle tissue.

\section{Dogfish Preparation:}

- Samples from the dogfish were collected as section-weighted composited, which are primarily muscle tissue.

- A second set of samples was collected as whole body weighted composited, which included the internal organs.

\section{Sea Cucumber Preparation:}

- During PSAMP specimen collection, the sea cucumbers were stored in a single zip-top bag and frozen. Therefore, individual specimens were not achievable and the entire bag was used to generate a composite sample for each location. The number of individual specimens in each composite sample is noted on the table. 


\section{Crab Preparation:}

- Whole individual crabs were thawed, rinsed with DI water, and soft tissue was extracted using a ceramic knife.

METHODS/Discussion: Carbon $\left(\delta^{13} \mathrm{C}\right)$ and nitrogen $\left(\delta^{15} \mathrm{~N}\right)$ analyses were performed of freeze dried, ground samples using a ThermoFinnigan Delta V plus stable isotope mass spectrometer coupled to a Thermo Flash elemental analyzer. Internal laboratory standards composed of marine chitin (Fisher Scientific) and calibrated to NIST standards were employed to correct sample data to international reference scales. Typical sample sizes analyzed were 0.5 to $1.0 \mathrm{mg}$. Typical precision of repeated chitin internal standards was $0.1 \%$ o for $\mathrm{C}$ and $0.2 \%$ for $\mathrm{N}(1 \mathrm{SD})$. Values are given vs. $\mathrm{vPDB}(\mathrm{C})$ and air $(\mathrm{N})$ standard scales.

Sample isotopic values are plotted in Figure 1. Most samples fall along a group with a trend of co-varying $\mathrm{C}$ and $\mathrm{N}$ values. Literature values for isotopic changes between trophic levels predict a shift of $+0-1 \%$ for $\mathrm{C}$ and 2-4\%o for N (Fry 1991; Hansson et al. 1997; Fantle et al. 1999; Benstead et al. 2006). The lines presented on the graph are relationship between $\mathrm{N}$ and $\mathrm{C}$ for marine samples (3:1 ) and a best fit line (3:2). The regression line on all the data except the eelgrass and 174-183 samples (dogfish) is 1.46:1 N to C, or 3:2. Several conclusions can be drawn from this dataset. First, most organisms appear to be using a marine planktonic food source of around -20 to -22 per mil in $\mathrm{C}$ and 6-8 in $\mathrm{N}$. Both these numbers are reasonable, the $\mathrm{C}$ is the average isotopic value for phytoplankton (Fry 1996) and the N value matches well with values of 5-6 measured for marine nitrate isotopes in the region (Brandes 1997). If the assumption is made that this is the food source, then most of the samples are in the 2nd or third trophic level, basically anything from about $11-13$ in $\delta^{15} \mathrm{~N}$ is in the second level and 14$16 \delta^{15} \mathrm{~N}$ is in the third. There is a lot of overlap suggesting that several organisms are mixotrophs. Figure 2 plots the isotopic values with species identification.

There are two noticeable outlier groups. The benthic primary producer seagrass isotopic values are enriched in ${ }^{13} \mathrm{C}$ and depleted in ${ }^{15} \mathrm{~N}$. This value is distinct enough in carbon isotopic value to strongly influence consumers utilizing this food source. Most of the samples do not appear to reflect such utilization with the exception of the sample points 16-18 (Shiner Perch from Vendovi).

The other outlier is the group 174-183 (dogfish). Judging from the data there are two possible interpretations. First, this group could be assimilating $\mathrm{C}$ with little isotopic discrimination. In this case, which has been observed often in the literature (Fantle et al. 1999), they are eating a marine food source, most likely phytoplankton, of isotopic value $-21 \%$ o $\left(\delta^{13} \mathrm{C}\right)$. The high $\mathrm{N}$ enrichment value argues against a direct assimilation of phytoplankton; however, as the organism looks to be on the third trophic level by $\mathrm{N}$ value. It could be eating some food source not sampled, which, in turn does not fractionate.

The second possibility is that the samples are biased by their high lipid content. Future work should include a lipid extraction on these to see how this changes the isotopic value of the samples. In any case the ${ }^{15} \mathrm{~N}$ value should be unaffected. 


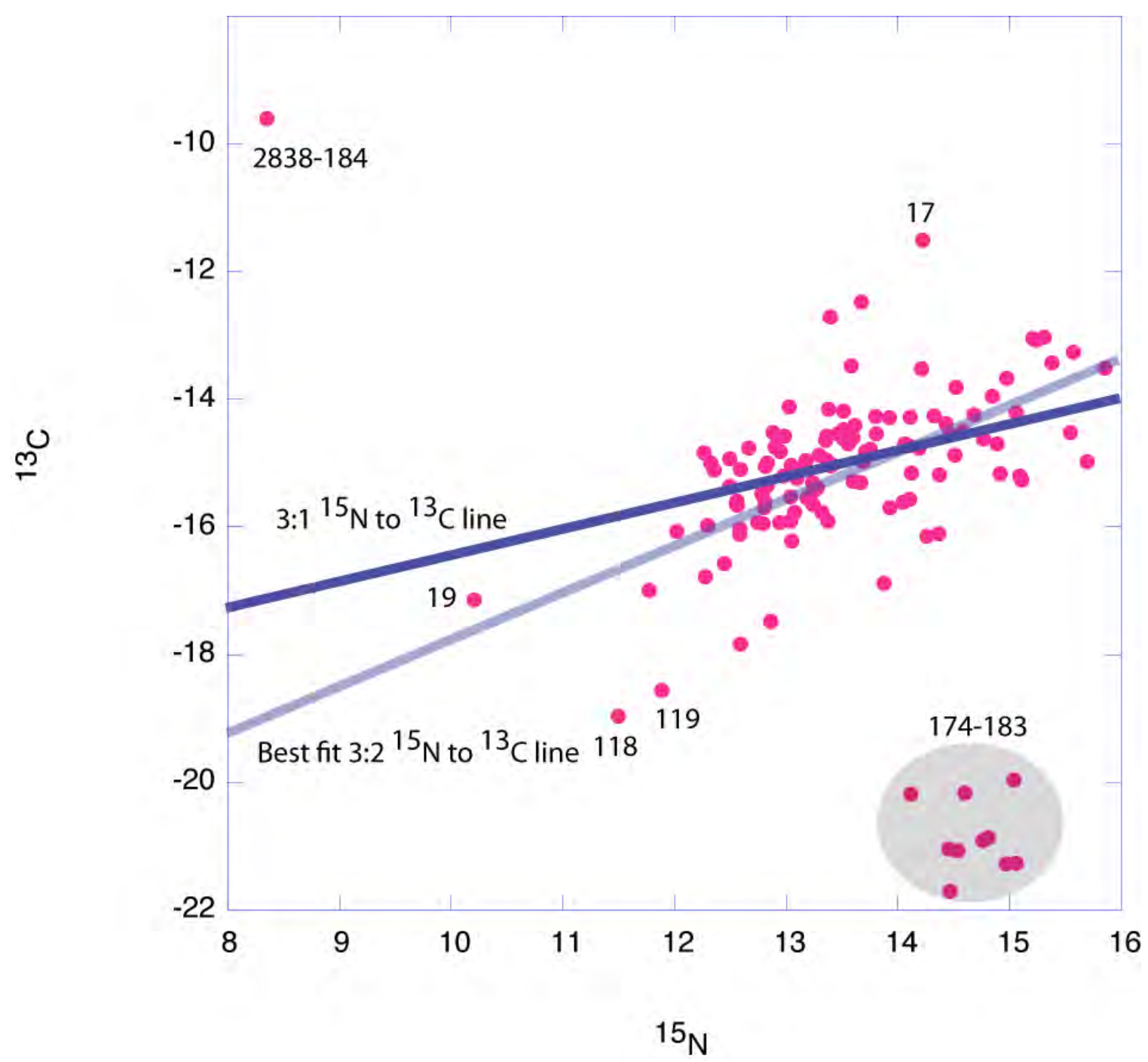

Figure 1. 2007 fish and invertebrate sample isotopic values. 


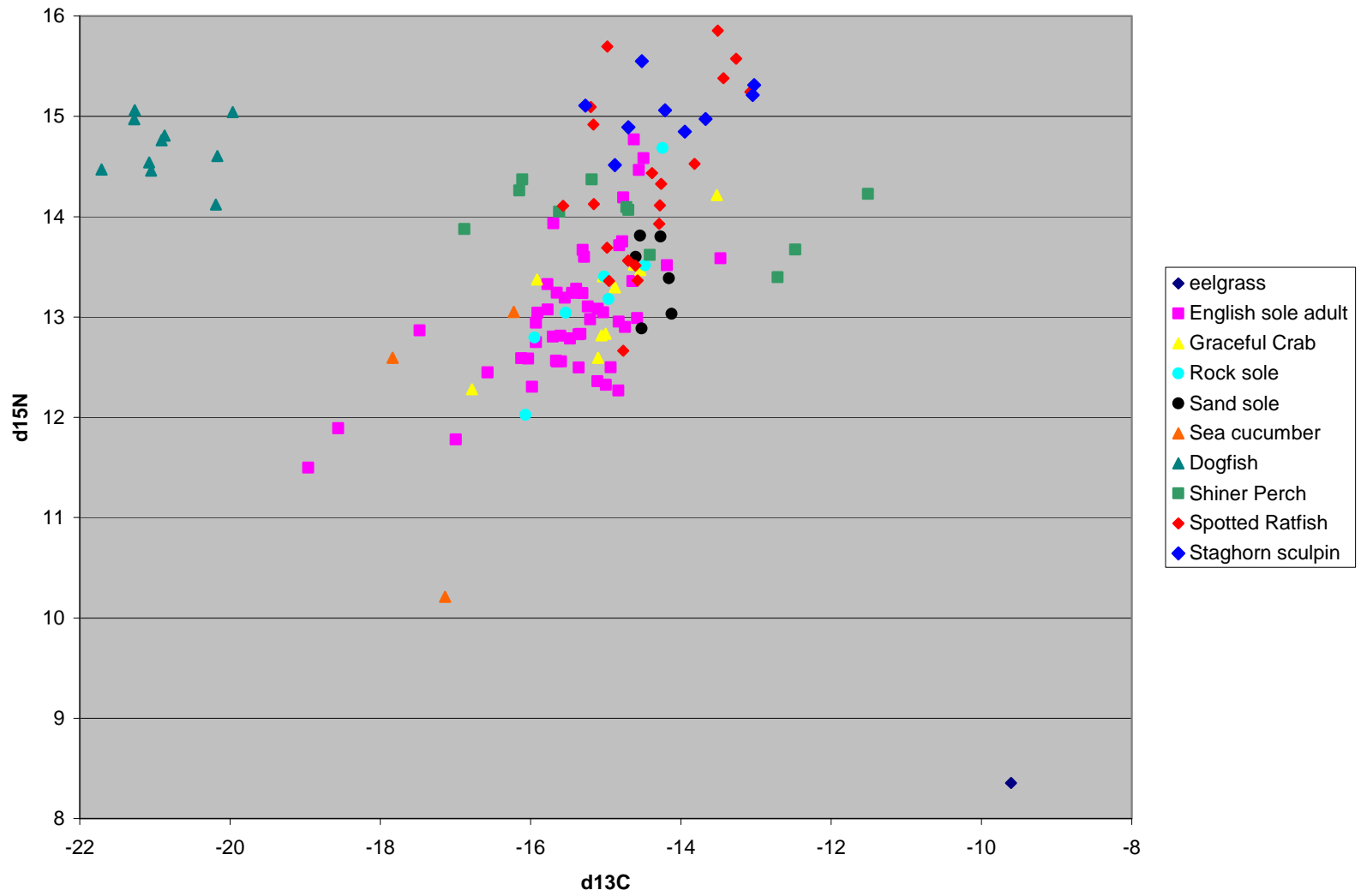

Figure 2. 2007 sample isotopic values with species identification. 


\section{Sample Custody Records}

- Field Collection Worksheets

- Sample Selection

- Sample Login 
PSAMP Tow Information:

\begin{tabular}{|c|l|l|l|r|l|}
\hline Year & SurveyID & StationID & EffortID & EffortDate & GearType \\
\hline 2007 & TRWL0705 & VENDOVI & 07VD-H01 & $5 / 1 / 2007$ & OtterTrawl-Eastern400 \\
\hline 2007 & TRWL0705 & STRTGEOR & 07SG-H02 & $5 / 2 / 2007$ & OtterTrawl-Eastern400 \\
\hline 2007 & TRWL0705 & HDCANAL & 07HC-H03 & $5 / 3 / 2007$ & OtterTrawl-Eastern400 \\
\hline 2007 & TRWL0705 & ELLTBAY & 07EB-H04 & $5 / 16 / 2007$ & OtterTrawl-Eastern400 \\
\hline 2007 & TRWL0705 & SCLINLET & 07SI-H06 & $5 / 17 / 2007$ & OtterTrawl-Eastern400 \\
\hline 2007 & TRWL0705 & EGLHARBR & 07EH-H05 & $5 / 17 / 2007$ & OtterTrawl-Eastern400 \\
\hline 2007 & TRWL0705 & DUWAMISH & 07DU-H07[A] & $5 / 18 / 2007$ & OtterTrawl-Eastern400 \\
\hline 2007 & TRWL0705 & DUWAMISH & 07DU-H07[B] & $5 / 18 / 2007$ & OtterTrawl-Eastern400 \\
\hline 2007 & TRWL0705 & PTGARDNR & 07PG-H08[A] & $5 / 29 / 2007$ & OtterTrawl-Eastern400 \\
\hline 2007 & TRWL0705 & PTGARDNR & 07PG-H08[B] & $5 / 29 / 2007$ & OtterTrawl-Eastern400 \\
\hline 2007 & TRWL0705 & NISQUALY & 07NQ-H09[A] & $5 / 30 / 2007$ & OtterTrawl-Eastern400 \\
\hline 2007 & TRWL0705 & NISQUALY & 07NQ-H09[B] & $5 / 30 / 2007$ & OtterTrawl-Eastern400 \\
\hline 2007 & TRWL0705 & COMMBAY & 07CB-H10[A] & $5 / 31 / 2007$ & OtterTrawl-Eastern400 \\
\hline 2007 & TRWL0705 & COMMBAY & 07CB-H10[B] & $5 / 31 / 2007$ & OtterTrawl-Eastern400 \\
\hline 2007 & TRWL0705 & COMMBAY & 07CB-H10[C] & $5 / 31 / 2007$ & OtterTrawl-Eastern400 \\
\hline
\end{tabular}


PSAMP Tow Information (cont.):

\begin{tabular}{|l|c|c|c|c|}
\hline \multirow{2}{*}{ StationDescription } & \multicolumn{4}{|c|}{ Degrees - Decimal minutes } \\
\cline { 2 - 5 } & \multicolumn{2}{|c|}{ Start } & \multicolumn{2}{c|}{ End } \\
\cline { 2 - 5 } & Latitude & Longitude & Latitude & Longitude \\
\hline Vendovi Island & 4838.901 & 12238.993 & 4838.837 & 12238.156 \\
\hline Strait of Georgia & 4851.064 & 12256.935 & 4852.005 & 12257.889 \\
\hline Hood Canal North & 4750.204 & 12238.341 & 4750.133 & 12238.929 \\
\hline Elliott Bay - Seattle Waterfront & 4736.228 & 12220.542 & 4736.482 & 12221.278 \\
\hline Sinclair Inlet - Bremerton Waterfront & 4732.699 & 12239.502 & 4733.004 & 12238.053 \\
\hline Eagle Harbor & 4737.168 & 12230.596 & 4737.271 & 12230.412 \\
\hline Duwamish River & 4733.727 & 12220.808 & 4733.298 & 12220.565 \\
\hline Duwamish River & 4733.717 & 12220.772 & 4733.238 & 12220.527 \\
\hline Port Gardner & 4758.960 & 12214.159 & 4759.245 & 12215.136 \\
\hline Port Gardner & 4759.000 & 12214.201 & 4759.169 & 12215.233 \\
\hline Nisqually Reach & 4709.013 & 12239.958 & 4709.608 & 12240.173 \\
\hline Nisqually Reach & 4709.205 & 12240.001 & 4709.713 & 12239.911 \\
\hline Commencement Bay - Thea Foss & 4715.290 & 12226.016 & 4715.790 & 12226.304 \\
\hline Commencement Bay - Thea Foss & 4715.287 & 12226.015 & 4715.803 & 12226.372 \\
\hline Commencement Bay - Thea Foss & 4715.296 & 12226.016 & 4715.841 & 12226.356 \\
\hline
\end{tabular}


PSAMP Tow Information (cont.):

\begin{tabular}{|c|c|c|c|c|c|c|c|c|c|c|c|c|}
\hline \multicolumn{4}{|c|}{ Decimal Degrees } & \multirow{2}{*}{\multicolumn{2}{|c|}{$\begin{array}{c}\text { Decimal Degrees } \\
\text { Mid-point }\end{array}$}} & \multicolumn{4}{|c|}{ Depth } & \multirow{3}{*}{$\begin{array}{c}\text { Time } \\
\text { Min }\end{array}$} & \multirow{3}{*}{$\begin{array}{c}\text { Wire } \\
\text { out }\end{array}$} & \multirow{3}{*}{$\begin{array}{c}\text { Net } \\
\text { width }\end{array}$} \\
\hline \multicolumn{2}{|c|}{ Start } & \multicolumn{2}{|c|}{ End } & & & \multicolumn{2}{|c|}{ Fathoms } & \multicolumn{2}{|c|}{ Meters } & & & \\
\hline Latitude & Longitude & Latitude & Longitude & Latitude & Longitude & Min & Max & Min & $\operatorname{Max}$ & & & \\
\hline 48.64835 & 122.64988 & 48.64728 & 122.63593 & 48.64782 & 122.64291 & 26.0 & 30.0 & 47.5 & 54.9 & 22 & 75 & 12.24 \\
\hline 47.83673 & 122.63902 & 47.83555 & 122.64882 & 47.83614 & 122.64392 & 25.0 & 29.0 & 45.7 & 53.0 & 14 & 55 & 10.71 \\
\hline 47.60380 & 122.34237 & 47.60803 & 122.35463 & 47.60592 & 122.34850 & 10.0 & 30.0 & 18.3 & 54.9 & 15 & 45 & 11.55 \\
\hline 47.56212 & 122.34680 & 47.55497 & 122.34275 & 47.55854 & 122.34478 & 3.8 & 4.6 & 6.9 & 8.4 & 13 & 7 & 8.74 \\
\hline 47.56195 & 122.34620 & 47.55397 & 122.34212 & 47.55796 & 122.34416 & 4.1 & 4.5 & 7.5 & 8.2 & 14 & 7 & 8.74 \\
\hline 47.98267 & 122.23598 & 47.98742 & 122.25227 & 47.98504 & 122.24413 & 6.0 & 10.0 & 11.0 & 18.3 & 16 & 35 & 10.79 \\
\hline 47.98333 & 122.23668 & 47.98615 & 122.25388 & 47.98474 & 122.24528 & 20.0 & 30.0 & 36.6 & 54.9 & 13 & 50 & 10.49 \\
\hline 47.15022 & 122.66597 & 47.16013 & 122.66955 & 47.15518 & 122.66776 & 70.0 & 75.0 & 128.0 & 137.2 & 19 & 200 & 13.42 \\
\hline 47.25478 & 122.43358 & 47.26338 & 122.43953 & 47.25908 & 122.43656 & 3.5 & 15.0 & 6.4 & 27.4 & 15 & 15 & 8.74 \\
\hline 47.25493 & 122.43360 & 47.26402 & 122.43927 & 47.25948 & 122.43643 & 3.5 & 18.0 & 6.4 & 32.9 & 16 & 15 & 8.74 \\
\hline
\end{tabular}




\begin{tabular}{|c|c|c|c|c|c|c|c|c|c|c|c|c|c|}
\hline \multicolumn{14}{|c|}{ 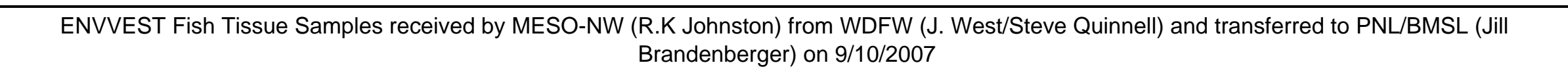 } \\
\hline \multirow[t]{2}{*}{ tag\# } & \multirow[t]{2}{*}{ haul } & \multirow[t]{2}{*}{ date } & \multirow[t]{2}{*}{ Location } & \multicolumn{9}{|c|}{ Total Specimens (all sizes) } & \multirow[t]{2}{*}{ ( } \\
\hline & & & & 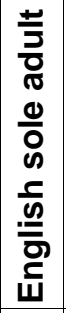 & $\begin{array}{l}0 \\
0 \\
0 \\
i \\
0 \\
0\end{array}$ & 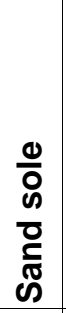 & 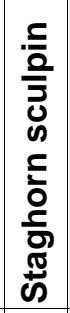 & 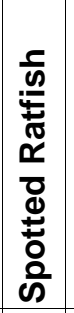 & 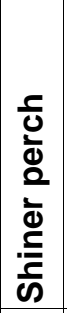 & 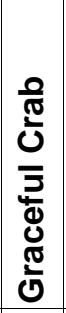 & 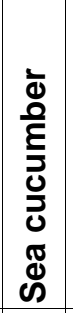 & 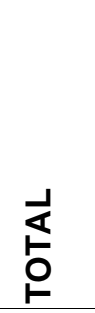 & \\
\hline 1762 & 1 & $5 / 1 / 2007$ & Vendovi & 9 & 3 & & 3 & & 3 & & 3 & 21 & $\begin{array}{l}\text { at least } 6 \text { good size ES; could not separate individual sea } \\
\text { cucumbers }\end{array}$ \\
\hline \multirow[t]{2}{*}{1462} & & $5 / 2 / 2007$ & Strait of Georgia & 9 & & & & 3 & & & 6 & 18 & $\begin{array}{l}\text { no haul number; at least } 7 \text { good size ES; could not separate } \\
\text { individual sea cucumbers }\end{array}$ \\
\hline & 3 & $5 / 3 / 2007$ & Hood Canal & 3 & 1 & & & 3 & 3 & 2 & & 12 & no tag\#; 2 large surf perch one small; 1 large male crab and 1 small \\
\hline \multirow[t]{2}{*}{3382} & 4 & $5 / 16 / 2007$ & Elliott Bay & 3 & & & & 3 & & & & 6 & 1 large and 2 small ES; \\
\hline & 5 & $5 / 19 / 2007$ & Eagle Harbor & 4 & & & & 3 & & & & 7 & No tag\#; 3 large and 1 small ES \\
\hline 3388 & 6 & $5 / 17 / 2007$ & Sinclair Inlet & 9 & 4 & 6 & 6 & 2 & 7 & 8 & 6 & 48 & $\begin{array}{l}9 \text { good size ES; } 3 \text { large Rock Sole } 1 \text { too small; } 2 \text { large and } 4 \text { small } \\
\text { surf pearch; } 8 \text { crabs male and female w/eggs } 2 \text { crabs large enough } \\
\text { may need to composite smaller ones; may not be able to separate } \\
\text { individual sea cucumbers; }\end{array}$ \\
\hline 1481 & & $5 / 29 / 2007$ & Port Gardner & 3 & & & & & & & & 3 & no haul number \\
\hline 3322 & $9 A / 9 B$ & $5 / 30 / 2007$ & Nisqually & 3 & & & & 3 & & & & 6 & \\
\hline 3310 & $10 A$ & $5 / 31 / 2007$ & Commencement Bay & 3 & & & & 3 & & & & 6 & \\
\hline \multirow[t]{3}{*}{3277} & 7B & $5 / 18 / 2007$ & Duwamish & 4 & & & & & & & & 4 & 3 large and 1 small ES \\
\hline & & & & & & & & & & & & & \\
\hline & & 2005 & Sinclair Inlet & 6 & & & & & & & & & $\begin{array}{l}\text { viscera only, these are the ES viscera of the missing SI samples } \\
\text { from } 2005\end{array}$ \\
\hline
\end{tabular}


Samples obtained from Jon Reum, UW on 19 SEP 2007. Specimens were caught in Admiralty Inlet of the Puget Sound on 18 SEP 2007.

parameter

embryo mass (g)

liver ( $\mathrm{g}$ )

digestive tract all (g)

reproductive organ?

1 head $(\mathrm{g})$

2 pectral area $(\mathrm{g})$

3 midsecton ( $\mathrm{g}$ )

4 tail (g)

kidney (g)

parameter

embryo mass (g)

liver ( $\mathrm{g}$ )

digestive tract all (g)

reproductive organ?

1 head ( $g$ )

2 pectral area $(\mathrm{g})$

3 midsecton ( $\mathrm{g}$ )

4 tail (g)

kidney (g)

$\begin{array}{rr}\text { ADUW-DOG01 } & \% \\ 410 & 7.59 \% \\ 498 & 9.22 \% \\ 319 & 5.91 \% \\ 47.2 & 0.87 \% \\ 613.3 & 11.36 \% \\ 1027 & 19.02 \% \\ 1590 & 29.44 \% \\ 670 & 12.41 \% \\ 14.4 & 0.27 \%\end{array}$

\begin{tabular}{rr} 
ADUW-DOG05 & \multicolumn{1}{c}{$\%$} \\
151.6 & $5.02 \%$ \\
382.9 & $12.68 \%$ \\
190.6 & $6.31 \%$ \\
49.1 & $1.63 \%$ \\
510 & $16.89 \%$ \\
420 & $13.91 \%$ \\
900 & $29.80 \%$ \\
320 & $10.60 \%$ \\
12.7 & $0.42 \%$
\end{tabular}

$\begin{array}{rr}\text { ADUW-DOG02 } & \% \\ 440 & 10.26 \% \\ 260 & 6.06 \% \\ 194 & 4.52 \% \\ 48.8 & 1.14 \% \\ 730 & 17.02 \% \\ 720 & 16.78 \% \\ 1240 & 28.90 \% \\ 450 & 10.49 \% \\ 10.5 & 0.24 \% \\ & \\ & \\ & \\ \text { ADUW-DOG06 } & \% \\ 321.7 & 7.87 \% \\ 433.5 & 10.60 \% \\ 326.7 & 7.99 \% \\ 46.7 & 1.14 \% \\ 510 & 12.47 \% \\ 620 & 15.16 \% \\ 1370 & 33.50 \% \\ 450 & 11.00 \% \\ & 0.00 \%\end{array}$

specimen ID

\begin{tabular}{rrrr} 
ADUW-DOG03 & \multicolumn{1}{c}{$\%$} & ADUW-DOG04 & $\%$ \\
97 & $3.09 \%$ & 188 & $3.47 \%$ \\
318 & $10.13 \%$ & 790 & $14.58 \%$ \\
288.8 & $9.20 \%$ & 320 & $5.90 \%$ \\
37.7 & $1.20 \%$ & 65.2 & $1.20 \%$ \\
490 & $15.61 \%$ & 790 & $14.58 \%$ \\
510 & $16.24 \%$ & 610 & $11.25 \%$ \\
940 & $29.94 \%$ & 1800 & $33.21 \%$ \\
320 & $10.19 \%$ & 600 & $11.07 \%$ \\
8.2 & $0.26 \%$ & 18.5 & $0.34 \%$
\end{tabular}


Supplemental Information for Dogfish Sample Labeling:

\begin{tabular}{|c|c|c|c|c|c|c|c|}
\hline $\begin{array}{l}\text { SAMPLE } \\
\text { LABEL }\end{array}$ & Location Site & SITE_LABEL & Site Total & Sub-Sample & $\begin{array}{l}\text { TISSUE_ } \\
\text { TYPE }\end{array}$ & Rep & $\begin{array}{l}\text { MSL } \\
\text { Code }\end{array}$ \\
\hline DF-EM-1 & ADUW-DOG01 & Admiralty Inlet & Dogfish 1 & embryo mass & EM & 1 & $2838-126$ \\
\hline DF-LV-1 & ADUW-DOG01 & Admiralty Inlet & Dogfish 1 & liver & LV & 1 & $2838-132$ \\
\hline DF-DIG-1 & ADUW-DOG01 & Admiralty Inlet & Dogfish 1 & $\begin{array}{l}\text { digestive tract } \\
\text { - All }\end{array}$ & DIG & 1 & 2838-138 \\
\hline DF-SWC-1 & ADUW-DOG01 & Admiralty Inlet & Dogfish 1 & \begin{tabular}{|l|} 
Section \\
Weighted- \\
Composite \\
\end{tabular} & SWC & 1 & 2838-174 \\
\hline DF-WBWC-1 & ADUW-DOG01 & Admiralty Inlet & Dogfish 1 & $\begin{array}{l}\text { Whole Body } \\
\text { Weighted- } \\
\text { Composite }\end{array}$ & WBWC & 1 & 2838-177 \\
\hline DF-EM-2 & ADUW-DOG02 & Admiralty Inlet & Dogfish 2 & embryo mass & EM & 2 & 2838-127 \\
\hline DF-LV-2 & ADUW-DOG02 & Admiralty Inlet & Dogfish 2 & liver & $\mathrm{LV}$ & 2 & $2838-133$ \\
\hline DF-DIG-2 & ADUW-DOG02 & Admiralty Inlet & Dogfish 2 & $\begin{array}{l}\text { digestive tract } \\
\text { - All }\end{array}$ & DIG & 2 & 2838-139 \\
\hline DF-SWC-2 & ADUW-DOG02 & Admiralty Inlet & Dogfish 2 & $\begin{array}{l}\text { Section } \\
\text { Weighted- } \\
\text { Composite }\end{array}$ & SWC & 2 & 2838-175 \\
\hline DF-WBWC-2 & ADUW-DOG02 & Admiralty Inlet & Dogfish 2 & $\begin{array}{l}\text { Whole Body } \\
\text { Weighted- } \\
\text { Composite }\end{array}$ & WBWC & 2 & 2838-178 \\
\hline DF-WBWC-3 & ADUW-DOG03 & Admiralty Inlet & Dogfish 3 & $\begin{array}{l}\text { Whole Body } \\
\text { Weighted- } \\
\text { Composite }\end{array}$ & WBWC & 3 & 2838-179 \\
\hline DF-EM-4 & ADUW-DOG04 & Admiralty Inlet & Dogfish 4 & embryo mass & EM & 4 & $2838-129$ \\
\hline DF-LV-4 & ADUW-DOG04 & Admiralty Inlet & Dogfish 4 & liver & LV & 4 & 2838-135 \\
\hline DF-DIG-4 & ADUW-DOG04 & Admiralty Inlet & Dogfish 4 & $\begin{array}{l}\text { digestive tract } \\
\text { - All }\end{array}$ & DIG & 4 & 2838-141 \\
\hline DF-SWC-4 & ADUW-DOG04 & Admiralty Inlet & Dogfish 4 & $\begin{array}{l}\text { Section } \\
\text { Weighted- } \\
\text { Composite }\end{array}$ & SWC & 4 & 2838-183 \\
\hline DF-WBWC-4 & ADUW-DOG04 & Admiralty Inlet & Dogfish 4 & $\begin{array}{l}\text { Whole Body } \\
\text { Weighted- } \\
\text { Composite }\end{array}$ & WBWC & 4 & 2838-180 \\
\hline DF-WBWC-5 & ADUW-DOG05 & Admiralty Inlet & Dogfish 5 & $\begin{array}{l}\text { Whole Body } \\
\text { Weighted- } \\
\text { Composite }\end{array}$ & WBWC & 5 & 2838-181 \\
\hline DF-EM-6 & ADUW-DOG06 & Admiralty Inlet & Dogfish 6 & embryo mass & EM & 6 & 2838-131 \\
\hline DF-LV-6 & ADUW-DOG06 & Admiralty Inlet & Dogfish 6 & liver & LV & 6 & 2838-137 \\
\hline DF-DIG-6 & ADUW-DOG06 & Admiralty Inlet & Dogfish 6 & $\begin{array}{l}\text { digestive tract } \\
\text { - All }\end{array}$ & DIG & 6 & 2838-143 \\
\hline DF-SWC-6 & ADUW-DOG06 & Admiralty Inlet & Dogfish 6 & \begin{tabular}{|l|} 
Section \\
Weighted- \\
Composite
\end{tabular} & SWC & 3 & 2838-176 \\
\hline DF-WBWC-6 & ADUW-DOG06 & Admiralty Inlet & Dogfish 6 & $\begin{array}{l}\text { Whole Body } \\
\text { Weighted- } \\
\text { Composite }\end{array}$ & WBWC & 6 & 2838-182 \\
\hline
\end{tabular}


Final Sample Selection List for Metals Analysis

\begin{tabular}{|c|c|c|c|c|c|c|c|c|}
\hline $\begin{array}{c}\text { Location } \\
\text { Code }\end{array}$ & Location Site & $\begin{array}{c}\text { Collection } \\
\text { Date }\end{array}$ & Species & Rep & Site Total & Client Code & MSL Code & Metals \\
\hline 1762 & Vendovi & $5 / 1 / 2007$ & English sole adult & 5 & & V-ES-5 & $2838-5$ & 1 \\
\hline 1762 & Vendovi & $5 / 1 / 2007$ & English sole adult & 6 & & V-ES-6 & $2838-6$ & 1 \\
\hline 1762 & Vendovi & $5 / 1 / 2007$ & English sole adult & 8 & & V-ES-8 & 2838-8 & 1 \\
\hline 1762 & Vendovi & $5 / 1 / 2007$ & Rock sole & 1 & & V-RS-1 & $2838-10$ & 1 \\
\hline 1762 & Vendovi & $5 / 1 / 2007$ & Rock sole & 2 & & V-RS-2 & 2838-11 & 1 \\
\hline 1762 & Vendovi & $5 / 1 / 2007$ & Rock sole & 3 & & V-RS-3 & 2838-12 & 1 \\
\hline 1762 & Vendovi & $5 / 1 / 2007$ & Staghorn sculpin & 1 & & V-SSc-1 & $2838-13$ & 1 \\
\hline 1762 & Vendovi & $5 / 1 / 2007$ & Staghorn sculpin & 2 & & V-SSc-2 & $2838-14$ & 1 \\
\hline 1762 & Vendovi & $5 / 1 / 2007$ & Staghorn sculpin & 3 & & V-SSc-3 & $2838-15$ & 1 \\
\hline 1762 & Vendovi & $5 / 1 / 2007$ & Shiner perch & 1 & & V-SP-1 & $2838-16$ & 1 \\
\hline 1762 & Vendovi & $5 / 1 / 2007$ & Shiner perch & 2 & & V-SP-2 & $2838-17$ & 1 \\
\hline 1762 & Vendovi & $5 / 1 / 2007$ & Shiner perch & 3 & & V-SP-3 & $2838-18$ & 1 \\
\hline 1762 & Vendovi & $5 / 1 / 2007$ & Sea cucumber (3) & COMP & 19 & V-SC-COMP & 2838-19 & 1 \\
\hline 1462 & Strait of Georgia & $5 / 2 / 2007$ & English sole adult & 3 & & SG-ES-3 & $2838-22$ & 1 \\
\hline 1462 & Strait of Georgia & $5 / 2 / 2007$ & English sole adult & 4 & & SG-ES-4 & $2838-23$ & 1 \\
\hline 1462 & Strait of Georgia & $5 / 2 / 2007$ & English sole adult & 5 & & SG-ES-5 & $2838-24$ & 1 \\
\hline 1462 & Strait of Georgia & $5 / 2 / 2007$ & Spotted Ratfish & 1 & & SG-RF-1 & 2838-29 & 1 \\
\hline 1462 & Strait of Georgia & $5 / 2 / 2007$ & Spotted Ratfish & 2 & & SG-RF-2 & $2838-30$ & 1 \\
\hline 1462 & Strait of Georgia & $5 / 2 / 2007$ & Spotted Ratfish & 3 & & SG-RF-3 & 2838-31 & 1 \\
\hline \multirow[t]{11}{*}{1462} & Strait of Georgia & $5 / 2 / 2007$ & Sea cucumber (6) & COMP & 13 & SG-SC-COMP & $2838-32$ & 1 \\
\hline & Hood Canal & $5 / 3 / 2007$ & English sole adult & 1 & & HC-ES-1 & $2838-33$ & 1 \\
\hline & Hood Canal & $5 / 3 / 2007$ & English sole adult & 2 & & HC-ES-2 & 2838-34 & 1 \\
\hline & Hood Canal & $5 / 3 / 2007$ & English sole adult & 3 & & $\mathrm{HC}-\mathrm{ES}-3$ & $2838-35$ & 1 \\
\hline & Hood Canal & $5 / 3 / 2007$ & Rock sole & 1 & & HC-RS-1 & $2838-36$ & 1 \\
\hline & Hood Canal & $5 / 3 / 2007$ & Spotted Ratfish & 1 & & HC-RF-1 & $2838-37$ & 1 \\
\hline & Hood Canal & $5 / 3 / 2007$ & Spotted Ratfish & 2 & & HC-RF-2 & $2838-38$ & 1 \\
\hline & Hood Canal & $5 / 3 / 2007$ & Spotted Ratfish & 3 & & HC-RF-3 & 2838-39 & 1 \\
\hline & Hood Canal & $5 / 3 / 2007$ & Shiner perch & 1 & & HC-SP1 & $2838-40$ & 1 \\
\hline & Hood Canal & $5 / 3 / 2007$ & Shiner perch & 2 & & $\mathrm{HC}-\mathrm{SP} 2$ & 2838-41 & 1 \\
\hline & Hood Canal & $5 / 3 / 2007$ & Graceful Crab & 1 & & HC-GC-1 & $2838-43$ & 1 \\
\hline
\end{tabular}


Final Sample Selection List for Metals Analysis

\begin{tabular}{|c|c|c|c|c|c|c|c|c|}
\hline $\begin{array}{c}\text { Location } \\
\text { Code }\end{array}$ & Location Site & $\begin{array}{c}\text { Collection } \\
\text { Date }\end{array}$ & Species & Rep & Site Total & Client Code & MSL Code & Metals \\
\hline 3382 & Elliott Bay & $5 / 16 / 2007$ & English sole adult & & & EB-ES-1/2 & 2838-45-46 & 1 \\
\hline 3382 & Elliott Bay & $5 / 16 / 2007$ & English sole adult & 3 & & EB-ES-3 & $2838-47$ & 1 \\
\hline 3382 & Elliott Bay & $5 / 16 / 2007$ & Spotted Ratfish & 1 & & EB-RF-1 & 2838-48 & 1 \\
\hline 3382 & Elliott Bay & $5 / 16 / 2007$ & Spotted Ratfish & 2 & & EB-RF-2 & $2838-49$ & 1 \\
\hline \multirow[t]{7}{*}{3382} & Elliott Bay & $5 / 16 / 2007$ & Spotted Ratfish & 3 & 6 & EB-RF-3 & $2838-50$ & 1 \\
\hline & Eagle Harbor & $5 / 19 / 2007$ & English sole adult & 2 & & EH-ES-2 & $2838-52$ & 1 \\
\hline & Eagle Harbor & $5 / 19 / 2007$ & English sole adult & 3 & & EH-ES-3 & $2838-53$ & 1 \\
\hline & Eagle Harbor & $5 / 19 / 2007$ & English sole adult & 4 & & EH-ES-4 & $2838-54$ & 1 \\
\hline & Eagle Harbor & $5 / 19 / 2007$ & Spotted Ratfish & 1 & & EH-RF-1 & $2838-55$ & 1 \\
\hline & Eagle Harbor & $5 / 19 / 2007$ & Spotted Ratfish & 2 & & EH-RF-2 & $2838-56$ & 1 \\
\hline & Eagle Harbor & $5 / 19 / 2007$ & Spotted Ratfish & 3 & 7 & EH-RF-3 & $2838-57$ & 1 \\
\hline 3388 & Sinclair Inlet & $5 / 17 / 2007$ & English sole adult & 1 & & SI-ES-1 & $2838-58$ & 1 \\
\hline 3388 & Sinclair Inlet & $5 / 17 / 2007$ & English sole adult & 2 & & SI-ES-2 & $2838-59$ & 1 \\
\hline 3388 & Sinclair Inlet & $5 / 17 / 2007$ & English sole adult & 4 & & SI-ES-4 & 2838-61 & 1 \\
\hline 3388 & Sinclair Inlet & $5 / 17 / 2007$ & English sole adult & 6 & & SI-ES-6 & $2838-63$ & 1 \\
\hline 3388 & Sinclair Inlet & $5 / 17 / 2007$ & English sole adult & 7 & & SI-ES-7 & $2838-64$ & 1 \\
\hline 3388 & Sinclair Inlet & $5 / 17 / 2007$ & English sole adult & 8 & & SI-ES-8 & $2838-65$ & 1 \\
\hline 3388 & Sinclair Inlet & $5 / 17 / 2007$ & Rock sole & & & SI-RS-1/2 & 2838-67-68 & 1 \\
\hline 3388 & Sinclair Inlet & $5 / 17 / 2007$ & Rock sole & 3 & & SI-RS-3 & $2838-69$ & 1 \\
\hline 3388 & Sinclair Inlet & $5 / 17 / 2007$ & Rock sole & 4 & & SI-RS-4 & $2838-70$ & 1 \\
\hline 3388 & Sinclair Inlet & $5 / 17 / 2007$ & Sand sole & 1 & & SI-SS-1 & 2838-71 & 1 \\
\hline 3388 & Sinclair Inlet & $5 / 17 / 2007$ & Sand sole & 2 & & SI-SS-2 & $2838-72$ & 1 \\
\hline 3388 & Sinclair Inlet & $5 / 17 / 2007$ & Sand sole & 3 & & SI-SS-3 & $2838-73$ & 1 \\
\hline 3388 & Sinclair Inlet & $5 / 17 / 2007$ & Staghorn sculpin & & & SI-SSc-1/2 & $2838-77-78$ & 1 \\
\hline 3388 & Sinclair Inlet & $5 / 17 / 2007$ & Staghorn sculpin & & & SI-SSc-3/5 & 2838-79-81 & 1 \\
\hline 3388 & Sinclair Inlet & $5 / 17 / 2007$ & Staghorn sculpin & & & SI-SSc-4/6 & $2838-80-82$ & 1 \\
\hline 3388 & Sinclair Inlet & $5 / 17 / 2007$ & Spotted Ratfish & 1 & & SI-RF-1 & $2838-83$ & 1 \\
\hline 3388 & Sinclair Inlet & $5 / 17 / 2007$ & Spotted Ratfish & 2 & & SI-RF-2 & $2838-84$ & 1 \\
\hline 3388 & Sinclair Inlet & $5 / 17 / 2007$ & Shiner perch & & & SI-SP-4/5 & 2838-88-89 & 1 \\
\hline 3388 & Sinclair Inlet & $5 / 17 / 2007$ & Shiner perch & & & SI-SP-6/2 & $2838-90-86$ & 1 \\
\hline
\end{tabular}


Final Sample Selection List for Metals Analysis

\begin{tabular}{|c|c|c|c|c|c|c|c|c|}
\hline $\begin{array}{c}\text { Location } \\
\text { Code }\end{array}$ & Location Site & $\begin{array}{c}\text { Collection } \\
\text { Date }\end{array}$ & Species & Rep & Site Total & Client Code & MSL Code & Metals \\
\hline 3388 & Sinclair Inlet & $5 / 17 / 2007$ & Shiner perch & & & SI-SP-7/3 & 2838-91-87 & 1 \\
\hline 3388 & Sinclair Inlet & $5 / 17 / 2007$ & Graceful Crab & & & SI-GC-2/6 & $2838-93-97$ & 1 \\
\hline 3388 & Sinclair Inlet & $5 / 17 / 2007$ & Graceful Crab & & & SI-GC-3/4 & 2838-94-95 & 1 \\
\hline 3388 & Sinclair Inlet & $5 / 17 / 2007$ & Graceful Crab & 7 & & SI-GC-7/1 & $2838-98-92$ & 1 \\
\hline 3388 & Sinclair Inlet & $5 / 17 / 2007$ & Graceful Crab & & & SI-GC-5/8 & 2838-96-99 & 1 \\
\hline 3388 & Sinclair Inlet & $5 / 17 / 2007$ & Sea cucumber (6) & COMP & 43 & SI-SC-COMP & $2838-100$ & 1 \\
\hline 1481 & Port Gardner & $5 / 29 / 2007$ & English sole adult & 1 & & PG-ES-1 & 2838-101 & 1 \\
\hline 1481 & Port Gardner & $5 / 29 / 2007$ & English sole adult & 2 & & PG-ES-2 & $2838-102$ & 1 \\
\hline 1481 & Port Gardner & $5 / 29 / 2007$ & English sole adult & 3 & 3 & PG-ES-3 & $2838-103$ & 1 \\
\hline 3322 & Nisqually & $5 / 30 / 2007$ & English sole adult & 1 & & NIS-ES-1 & 2838-104 & 1 \\
\hline 3322 & Nisqually & $5 / 30 / 2007$ & English sole adult & 2 & & NIS-ES-2 & 2838-105 & 1 \\
\hline 3322 & Nisqually & $5 / 30 / 2007$ & English sole adult & 3 & & NIS-ES-3 & $2838-106$ & 1 \\
\hline 3322 & Nisqually & $5 / 30 / 2007$ & Spotted Ratfish & 1 & & NIS-RF-1 & $2838-107$ & 1 \\
\hline 3322 & Nisqually & $5 / 30 / 2007$ & Spotted Ratfish & 2 & & NIS-RF-2 & $2838-108$ & 1 \\
\hline 3322 & Nisqually & $5 / 30 / 2007$ & Spotted Ratfish & 3 & 6 & NIS-RF-3 & 2838-109 & 1 \\
\hline 3310 & $\begin{array}{l}\text { Commencement } \\
\text { Bay }\end{array}$ & $5 / 31 / 2007$ & English sole adult & 1 & & CB-ES-1 & $2838-110$ & 1 \\
\hline 3310 & $\begin{array}{l}\text { Commencement } \\
\text { Bay }\end{array}$ & $5 / 31 / 2007$ & English sole adult & 2 & & CB-ES-2 & 2838-111 & 1 \\
\hline 3310 & $\begin{array}{l}\text { Commencement } \\
\text { Bay } \\
\end{array}$ & $5 / 31 / 2007$ & English sole adult & 3 & & CB-ES-3 & 2838-112 & 1 \\
\hline 3310 & $\begin{array}{l}\text { Commencement } \\
\text { Bay }\end{array}$ & $5 / 31 / 2007$ & Spotted Ratfish & 1 & & CB-RF-1 & $2838-113$ & 1 \\
\hline 3310 & $\begin{array}{l}\text { Commencement } \\
\text { Bay }\end{array}$ & $5 / 31 / 2007$ & Spotted Ratfish & 2 & & CB-RF-2 & $2838-114$ & 1 \\
\hline 3310 & $\begin{array}{l}\text { Commencement } \\
\text { Bay } \\
\end{array}$ & $5 / 31 / 2007$ & Spotted Ratfish & 3 & 6 & CB-RF-3 & $2838-115$ & 1 \\
\hline 3277 & Duwamish & $5 / 18 / 2007$ & English sole adult & 1 & & DU-ES-1 & 2838-116 & 1 \\
\hline 3277 & Duwamish & $5 / 18 / 2007$ & English sole adult & 2 & & DU-ES-2 & $2838-117$ & 1 \\
\hline \multirow[t]{4}{*}{3277} & Duwamish & $5 / 18 / 2007$ & English sole adult & 3 & & DU-ES-3 & $2838-118$ & 1 \\
\hline & ADUW-DOG01 & & embryo mass (g) & 1 & Dogfish 1 & DF-EM-1 & $2838-126$ & 1 \\
\hline & ADUW-DOG01 & & liver $(\mathrm{g})$ & 1 & Dogfish 1 & DF-LV-1 & 2838-132 & 1 \\
\hline & ADUW-DOG01 & & digestive tract - All & 1 & Dogfish 1 & DF-DIG-1 & 2838-138 & 1 \\
\hline
\end{tabular}


Final Sample Selection List for Metals Analysis

\begin{tabular}{|c|c|c|c|c|c|c|c|c|}
\hline $\begin{array}{c}\text { Location } \\
\text { Code }\end{array}$ & Location Site & $\begin{array}{l}\text { Collection } \\
\text { Date }\end{array}$ & Species & Rep & Site Total & Client Code & MSL Code & Metals \\
\hline & ADUW-DOG01 & & Section Weighted-Composite & 1 & Dogfish 1 & DF-SWC-1 & 2838-174 & 1 \\
\hline & ADUW-DOG01 & & $\begin{array}{l}\text { Whole Body Weighted- } \\
\text { Composite }\end{array}$ & 1 & Dogfish 1 & DF-WBWC-1 & $2838-177$ & 1 \\
\hline & ADUW-DOG02 & & embryo mass $(\mathrm{g})$ & 2 & Dogfish 2 & DF-EM-2 & $2838-127$ & 1 \\
\hline & ADUW-DOG02 & & liver (g) & 2 & Dogfish 2 & DF-LV-2 & 2838-133 & 1 \\
\hline & ADUW-DOG02 & & digestive tract - All & 2 & Dogfish 2 & DF-DIG-2 & 2838-139 & 1 \\
\hline & ADUW-DOG02 & & Section Weighted-Composite & 2 & Dogfish 2 & DF-SWC-2 & $2838-175$ & 1 \\
\hline & ADUW-DOG02 & & $\begin{array}{l}\text { Whole Body Weighted- } \\
\text { Composite }\end{array}$ & 2 & Dogfish 2 & DF-WBWC-2 & $2838-178$ & 1 \\
\hline & ADUW-DOG03 & & $\begin{array}{l}\text { Whole Body Weighted- } \\
\text { Composite }\end{array}$ & 3 & Dogfish 3 & DF-WBWC-3 & 2838-179 & 1 \\
\hline & ADUW-DOG04 & & embryo mass (g) & 4 & Dogfish 4 & DF-EM-4 & $2838-129$ & 1 \\
\hline & ADUW-DOG04 & & liver (g) & 4 & Dogfish 4 & DF-LV-4 & $2838-135$ & 1 \\
\hline & ADUW-DOG04 & & digestive tract - All & 4 & \begin{tabular}{|l|} 
Dogfish 4 \\
\end{tabular} & DF-DIG-4 & $2838-141$ & 1 \\
\hline & ADUW-DOG04 & & Section Weighted-Composite & 4 & Dogfish 4 & DF-SWC-4 & 2838-183 & 1 \\
\hline & ADUW-DOG04 & & $\begin{array}{l}\text { Whole Body Weighted- } \\
\text { Composite }\end{array}$ & 4 & Dogfish 4 & DF-WBWC-4 & $2838-180$ & 1 \\
\hline & ADUW-DOG05 & & $\begin{array}{l}\text { Whole Body Weighted- } \\
\text { Composite } \\
\end{array}$ & 5 & Dogfish 5 & DF-WBWC-5 & 2838-181 & 1 \\
\hline & ADUW-DOG06 & & embryo mass (g) & 6 & Dogfish 6 & DF-EM-6 & 2838-131 & 1 \\
\hline & ADUW-DOG06 & & liver $(\mathrm{g})$ & 6 & Dogfish 6 & DF-LV-6 & $2838-137$ & 1 \\
\hline & ADUW-DOG06 & & digestive tract - All & 6 & Dogfish 6 & DF-DIG-6 & 2838-143 & 1 \\
\hline & ADUW-DOG06 & & Section Weighted-Composite & 3 & Dogfish 6 & DF-SWC-6 & $2838-176$ & 1 \\
\hline & ADUW-DOG06 & & $\begin{array}{l}\text { Whole Body Weighted- } \\
\text { Composite }\end{array}$ & 6 & Dogfish 6 & DF-WBWC-6 & 2838-182 & 1 \\
\hline
\end{tabular}


Final Sample Selection List for Organics Analysis

\begin{tabular}{|c|c|c|c|c|c|c|c|c|c|}
\hline $\begin{array}{c}\text { Location } \\
\text { Code }\end{array}$ & Location Site & $\begin{array}{c}\text { Collection } \\
\text { Date }\end{array}$ & Species & Rep & Site Total & Client Code & MSL Code & $\begin{array}{l}\text { PCBs/ } \\
\text { Lipids }\end{array}$ & $\begin{array}{c}\text { PCB Shipped } \\
\text { to Duxbury }\end{array}$ \\
\hline 1762 & Vendovi & $5 / 1 / 2007$ & English sole adult & 5 & & V-ES-5 & $2838-5$ & 1 & $1 / 7 / 2008$ \\
\hline 1762 & Vendovi & $5 / 1 / 2007$ & English sole adult & 6 & & V-ES-6 & $2838-6$ & 1 & $1 / 7 / 2008$ \\
\hline 1762 & Vendovi & $5 / 1 / 2007$ & English sole adult & 8 & & V-ES-8 & $2838-8$ & 1 & $1 / 7 / 2008$ \\
\hline 1762 & Vendovi & $5 / 1 / 2007$ & Rock sole & 1 & & V-RS-1 & 2838-10 & 1 & $1 / 7 / 2008$ \\
\hline 1762 & Vendovi & $5 / 1 / 2007$ & Rock sole & 2 & & V-RS-2 & $2838-11$ & 1 & $1 / 7 / 2008$ \\
\hline 1762 & Vendovi & $5 / 1 / 2007$ & Rock sole & 3 & & V-RS-3 & $2838-12$ & 1 & $1 / 7 / 2008$ \\
\hline 1762 & Vendovi & $5 / 1 / 2007$ & Staghorn sculpin & 1 & & V-SSc-1 & $2838-13$ & 1 & $1 / 7 / 2008$ \\
\hline 1762 & Vendovi & $5 / 1 / 2007$ & Staghorn sculpin & 2 & & V-SSc-2 & 2838-14 & 1 & $1 / 7 / 2008$ \\
\hline 1762 & Vendovi & $5 / 1 / 2007$ & Staghorn sculpin & 3 & & V-SSc-3 & 2838-15 & 1 & $1 / 7 / 2008$ \\
\hline 1762 & Vendovi & $5 / 1 / 2007$ & Shiner perch & 1 & & V-SP-1 & $2838-16$ & 1 & $1 / 7 / 2008$ \\
\hline 1762 & Vendovi & $5 / 1 / 2007$ & Shiner perch & 2 & & V-SP-2 & $2838-17$ & 1 & $1 / 7 / 2008$ \\
\hline 1762 & Vendovi & $5 / 1 / 2007$ & Shiner perch & 3 & & V-SP-3 & $2838-18$ & 1 & $1 / 7 / 2008$ \\
\hline 1762 & Vendovi & $5 / 1 / 2007$ & Sea cucumber (3) & COMP & 19 & V-SC-COMP & 2838-19 & 1 & $1 / 10 / 2008$ \\
\hline 1462 & Strait of Georgia & $5 / 2 / 2007$ & English sole adult & 3 & & SG-ES-3 & $2838-22$ & 1 & $1 / 7 / 2008$ \\
\hline 1462 & Strait of Georgia & $5 / 2 / 2007$ & English sole adult & 4 & & SG-ES-4 & $2838-23$ & 1 & $1 / 7 / 2008$ \\
\hline 1462 & Strait of Georgia & $5 / 2 / 2007$ & English sole adult & 5 & & SG-ES-5 & 2838-24 & 1 & $1 / 7 / 2008$ \\
\hline 1462 & Strait of Georgia & $5 / 2 / 2007$ & Spotted Ratfish & 1 & & SG-RF-1 & $2838-29$ & 1 & $1 / 10 / 2008$ \\
\hline 1462 & Strait of Georgia & $5 / 2 / 2007$ & Spotted Ratfish & 2 & & SG-RF-2 & $2838-30$ & 1 & $1 / 10 / 2008$ \\
\hline 1462 & Strait of Georgia & $5 / 2 / 2007$ & Spotted Ratfish & 3 & & SG-RF-3 & $2838-31$ & 1 & $1 / 10 / 2008$ \\
\hline \multirow[t]{8}{*}{1462} & Strait of Georgia & $5 / 2 / 2007$ & Sea cucumber (6) & COMP & 13 & SG-SC-COMP & 2838-32 & 1 & $1 / 10 / 2008$ \\
\hline & Hood Canal & $5 / 3 / 2007$ & English sole adult & 1 & & HC-ES-1 & 2838-33 & 1 & $1 / 10 / 2008$ \\
\hline & Hood Canal & $5 / 3 / 2007$ & English sole adult & 2 & & HC-ES-2 & $2838-34$ & 1 & $1 / 10 / 2008$ \\
\hline & Hood Canal & $5 / 3 / 2007$ & English sole adult & 3 & & HC-ES-3 & $2838-35$ & 1 & $1 / 10 / 2008$ \\
\hline & Hood Canal & $5 / 3 / 2007$ & Rock sole & 1 & & HC-RS-1 & $2838-36$ & 1 & $1 / 10 / 2008$ \\
\hline & Hood Canal & $5 / 3 / 2007$ & Spotted Ratfish & 1 & & HC-RF-1 & $2838-37$ & 1 & $1 / 10 / 2008$ \\
\hline & Hood Canal & $5 / 3 / 2007$ & Spotted Ratfish & 2 & & HC-RF-2 & 2838-38 & 1 & $1 / 10 / 2008$ \\
\hline & Hood Canal & $5 / 3 / 2007$ & Spotted Ratfish & 3 & & HC-RF-3 & 2838-39 & 1 & $1 / 10 / 2008$ \\
\hline
\end{tabular}


Final Sample Selection List for Organics Analysis

\begin{tabular}{|c|c|c|c|c|c|c|c|c|c|}
\hline $\begin{array}{c}\text { Location } \\
\text { Code }\end{array}$ & Location Site & $\begin{array}{c}\text { Collection } \\
\text { Date }\end{array}$ & Species & Rep & Site Total & Client Code & MSL Code & $\begin{array}{l}\text { PCBs/ } \\
\text { Lipids }\end{array}$ & $\begin{array}{c}\text { PCB Shipped } \\
\text { to Duxbury }\end{array}$ \\
\hline & Hood Canal & $5 / 3 / 2007$ & Shiner perch & 1 & & HC-SP1 & $2838-40$ & 1 & $1 / 7 / 2008$ \\
\hline & Hood Canal & $5 / 3 / 2007$ & Shiner perch & 2 & & HC-SP2 & 2838-41 & 1 & $1 / 7 / 2008$ \\
\hline & Hood Canal & $5 / 3 / 2007$ & Graceful Crab & 1 & & HC-GC-1 & 2838-43 & 1 & $1 / 7 / 2008$ \\
\hline 3382 & Elliott Bay & $5 / 16 / 2007$ & English sole adult & & & EB-ES-1/2 & $2838-45-46$ & 1 & $1 / 10 / 2008$ \\
\hline 3382 & Elliott Bay & $5 / 16 / 2007$ & English sole adult & 3 & & EB-ES-3 & $2838-47$ & 1 & $1 / 10 / 2008$ \\
\hline 3382 & Elliott Bay & $5 / 16 / 2007$ & Spotted Ratfish & 1 & & EB-RF-1 & $2838-48$ & 1 & $1 / 10 / 2008$ \\
\hline 3382 & Elliott Bay & $5 / 16 / 2007$ & Spotted Ratfish & 2 & & EB-RF-2 & 2838-49 & 1 & $1 / 10 / 2008$ \\
\hline 3382 & Elliott Bay & $5 / 16 / 2007$ & Spotted Ratfish & 3 & 6 & EB-RF-3 & $2838-50$ & 1 & $1 / 10 / 2008$ \\
\hline & Eagle Harbor & $5 / 19 / 2007$ & English sole adult & 2 & & EH-ES-2 & $2838-52$ & 1 & $1 / 10 / 2008$ \\
\hline & Eagle Harbor & $5 / 19 / 2007$ & English sole adult & 3 & & EH-ES-3 & $2838-53$ & 1 & $1 / 10 / 2008$ \\
\hline & Eagle Harbor & $5 / 19 / 2007$ & English sole adult & 4 & & EH-ES-4 & $2838-54$ & 1 & $1 / 10 / 2008$ \\
\hline & Eagle Harbor & $5 / 19 / 2007$ & Spotted Ratfish & 1 & & EH-RF-1 & $2838-55$ & 1 & $1 / 10 / 2008$ \\
\hline & Eagle Harbor & $5 / 19 / 2007$ & Spotted Ratfish & 2 & & EH-RF-2 & $2838-56$ & 1 & $1 / 10 / 2008$ \\
\hline & Eagle Harbor & $5 / 19 / 2007$ & Spotted Ratfish & 3 & 7 & EH-RF-3 & $2838-57$ & 1 & $1 / 10 / 2008$ \\
\hline 3388 & Sinclair Inlet & $5 / 17 / 2007$ & English sole adult & 1 & & SI-ES-1 & $2838-58$ & 1 & $1 / 7 / 2008$ \\
\hline 3388 & Sinclair Inlet & $5 / 17 / 2007$ & English sole adult & 2 & & SI-ES-2 & $2838-59$ & 1 & $1 / 7 / 2008$ \\
\hline 3388 & Sinclair Inlet & $5 / 17 / 2007$ & English sole adult & 4 & & SI-ES-4 & $2838-61$ & 1 & $1 / 7 / 2008$ \\
\hline 3388 & Sinclair Inlet & $5 / 17 / 2007$ & English sole adult & 6 & & SI-ES-6 & $2838-63$ & 1 & $1 / 7 / 2008$ \\
\hline 3388 & Sinclair Inlet & $5 / 17 / 2007$ & English sole adult & 7 & & SI-ES-7 & 2838-64 & 1 & $1 / 7 / 2008$ \\
\hline 3388 & Sinclair Inlet & $5 / 17 / 2007$ & English sole adult & 8 & & SI-ES-8 & $2838-65$ & 1 & $1 / 7 / 2008$ \\
\hline 3388 & Sinclair Inlet & $5 / 17 / 2007$ & Rock sole & & & SI-RS-1/2 & $2838-67-68$ & 1 & $1 / 7 / 2008$ \\
\hline 3388 & Sinclair Inlet & $5 / 17 / 2007$ & Rock sole & 3 & & SI-RS-3 & $2838-69$ & 1 & $1 / 7 / 2008$ \\
\hline 3388 & Sinclair Inlet & $5 / 17 / 2007$ & Rock sole & 4 & & SI-RS-4 & $2838-70$ & 1 & $1 / 7 / 2008$ \\
\hline 3388 & Sinclair Inlet & $5 / 17 / 2007$ & Sand sole & 1 & & SI-SS-1 & $2838-71$ & 1 & $1 / 7 / 2008$ \\
\hline 3388 & Sinclair Inlet & $5 / 17 / 2007$ & Sand sole & 2 & & SI-SS-2 & $2838-72$ & 1 & $1 / 7 / 2008$ \\
\hline 3388 & Sinclair Inlet & $5 / 17 / 2007$ & Sand sole & 3 & & SI-SS-3 & $2838-73$ & 1 & $1 / 7 / 2008$ \\
\hline 3388 & Sinclair Inlet & $5 / 17 / 2007$ & Staghorn sculpin & & & SI-SSc-1/2 & $2838-77-78$ & 1 & $1 / 7 / 2008$ \\
\hline
\end{tabular}


Final Sample Selection List for Organics Analysis

\begin{tabular}{|c|c|c|c|c|c|c|c|c|c|}
\hline $\begin{array}{c}\text { Location } \\
\text { Code }\end{array}$ & Location Site & $\begin{array}{c}\text { Collection } \\
\text { Date }\end{array}$ & Species & Rep & Site Total & Client Code & MSL Code & $\begin{array}{l}\text { PCBs/ } \\
\text { Lipids } \\
\end{array}$ & $\begin{array}{c}\text { PCB Shipped } \\
\text { to Duxbury }\end{array}$ \\
\hline 3388 & Sinclair Inlet & $5 / 17 / 2007$ & Staghorn sculpin & & & SI-SSc-3/5 & 2838-79-81 & 1 & $1 / 7 / 2008$ \\
\hline 3388 & Sinclair Inlet & $5 / 17 / 2007$ & Staghorn sculpin & & & SI-SSc-4/6 & 2838-80-82 & 1 & $1 / 7 / 2008$ \\
\hline 3388 & Sinclair Inlet & $5 / 17 / 2007$ & Spotted Ratfish & 1 & & SI-RF-1 & $2838-83$ & 1 & $1 / 10 / 2008$ \\
\hline 3388 & Sinclair Inlet & $5 / 17 / 2007$ & Spotted Ratfish & 2 & & SI-RF-2 & 2838-84 & 1 & $1 / 10 / 2008$ \\
\hline 3388 & Sinclair Inlet & $5 / 17 / 2007$ & Shiner perch & & & SI-SP-4/5 & 2838-88-89 & 1 & $1 / 7 / 2008$ \\
\hline 3388 & Sinclair Inlet & $5 / 17 / 2007$ & Shiner perch & & & SI-SP-6/2 & 2838-90-86 & 1 & $1 / 7 / 2008$ \\
\hline 3388 & Sinclair Inlet & $5 / 17 / 2007$ & Shiner perch & & & SI-SP-7/3 & 2838-91-87 & 1 & $1 / 7 / 2008$ \\
\hline 3388 & Sinclair Inlet & $5 / 17 / 2007$ & Graceful Crab & & & SI-GC-2/6 & 2838-93-97 & 1 & $1 / 7 / 2008$ \\
\hline 3388 & Sinclair Inlet & $5 / 17 / 2007$ & Graceful Crab & & & SI-GC-3/4 & 2838-94-95 & 1 & $1 / 7 / 2008$ \\
\hline 3388 & Sinclair Inlet & $5 / 17 / 2007$ & Graceful Crab & & & SI-GC-7/1 & 2838-98-92 & 1 & $1 / 7 / 2008$ \\
\hline 3388 & Sinclair Inlet & $5 / 17 / 2007$ & Graceful Crab & & & SI-GC-5/8 & 2838-96-99 & 1 & $1 / 7 / 2008$ \\
\hline 3388 & Sinclair Inlet & $5 / 17 / 2007$ & Sea cucumber (6) & COMP & 43 & SI-SC-COMP & 2838-100 & 1 & $1 / 10 / 2008$ \\
\hline 1481 & Port Gardner & $5 / 29 / 2007$ & English sole adult & 1 & & PG-ES-1 & 2838-101 & 1 & $1 / 10 / 2008$ \\
\hline 1481 & Port Gardner & $5 / 29 / 2007$ & English sole adult & 2 & & PG-ES-2 & 2838-102 & 1 & $1 / 10 / 2008$ \\
\hline 1481 & Port Gardner & $5 / 29 / 2007$ & English sole adult & 3 & 3 & PG-ES-3 & 2838-103 & 1 & $1 / 10 / 2008$ \\
\hline 3322 & Nisqually & $5 / 30 / 2007$ & English sole adult & 1 & & NIS-ES-1 & 2838-104 & 1 & $1 / 10 / 2008$ \\
\hline 3322 & Nisqually & $5 / 30 / 2007$ & English sole adult & 2 & & NIS-ES-2 & 2838-105 & 1 & $1 / 10 / 2008$ \\
\hline 3322 & Nisqually & $5 / 30 / 2007$ & English sole adult & 3 & & NIS-ES-3 & 2838-106 & 1 & $1 / 10 / 2008$ \\
\hline 3322 & Nisqually & $5 / 30 / 2007$ & Spotted Ratfish & 1 & & NIS-RF-1 & 2838-107 & 1 & $1 / 10 / 2008$ \\
\hline 3322 & Nisqually & $5 / 30 / 2007$ & Spotted Ratfish & 2 & & NIS-RF-2 & 2838-108 & 1 & $1 / 10 / 2008$ \\
\hline 3322 & Nisqually & $5 / 30 / 2007$ & Spotted Ratfish & 3 & 6 & NIS-RF-3 & 2838-109 & 1 & $1 / 10 / 2008$ \\
\hline 3310 & Commencement Ba & $5 / 31 / 2007$ & English sole adult & 1 & & CB-ES-1 & 2838-110 & 1 & $1 / 10 / 2008$ \\
\hline 3310 & Commencement Ba & $5 / 31 / 2007$ & English sole adult & 2 & & CB-ES-2 & 2838-111 & 1 & $1 / 10 / 2008$ \\
\hline 3310 & Commencement Ba & $5 / 31 / 2007$ & English sole adult & 3 & & CB-ES-3 & 2838-112 & 1 & $1 / 10 / 2008$ \\
\hline 3310 & Commencement Ba & $5 / 31 / 2007$ & Spotted Ratfish & 1 & & CB-RF-1 & $2838-113$ & 1 & $1 / 10 / 2008$ \\
\hline 3310 & Commencement Ba & $5 / 31 / 2007$ & Spotted Ratfish & 2 & & CB-RF-2 & 2838-114 & 1 & $1 / 10 / 2008$ \\
\hline 3310 & Commencement Ba & $5 / 31 / 2007$ & Spotted Ratfish & 3 & 6 & CB-RF-3 & 2838-115 & 1 & $1 / 10 / 2008$ \\
\hline
\end{tabular}


Final Sample Selection List for Organics Analysis

\begin{tabular}{|c|c|c|c|c|c|c|c|c|c|}
\hline $\begin{array}{c}\text { Location } \\
\text { Code }\end{array}$ & Location Site & $\begin{array}{c}\text { Collection } \\
\text { Date }\end{array}$ & Species & Rep & Site Total & Client Code & MSL Code & $\begin{array}{l}\text { PCBs/ } \\
\text { Lipids }\end{array}$ & $\begin{array}{c}\text { PCB Shipped } \\
\text { to Duxbury }\end{array}$ \\
\hline 3277 & Duwamish & $5 / 18 / 2007$ & English sole adult & 1 & & DU-ES-1 & 2838-116 & 1 & $1 / 10 / 2008$ \\
\hline 3277 & Duwamish & $5 / 18 / 2007$ & English sole adult & 2 & & DU-ES-2 & $2838-117$ & 1 & $1 / 10 / 2008$ \\
\hline \multirow[t]{23}{*}{3277} & Duwamish & $5 / 18 / 2007$ & English sole adult & 3 & & DU-ES-3 & $2838-118$ & 1 & $1 / 10 / 2008$ \\
\hline & ADUW-DOG01 & & embryo mass (g) & 1 & Dogfish 1 & DF-EM-1 & 2838-126 & 1 & $1 / 21 / 2008$ \\
\hline & ADUW-DOG01 & & liver $(\mathrm{g})$ & 1 & Dogfish 1 & DF-LV-1 & 2838-132 & 1 & $1 / 21 / 2008$ \\
\hline & ADUW-DOG01 & & digestive tract - All & 1 & Dogfish 1 & DF-DIG-1 & 2838-138 & 1 & $1 / 21 / 2008$ \\
\hline & ADUW-DOG01 & & Section Weighted-Composite & 1 & Dogfish 1 & DF-SWC-1 & 2838-174 & 1 & $1 / 21 / 2008$ \\
\hline & ADUW-DOG01 & & Whole Body Weighted-Composite & 1 & Dogfish 1 & DF-WBWC-1 & $2838-177$ & 1 & $1 / 21 / 2008$ \\
\hline & ADUW-DOG02 & & embryo mass (g) & 2 & Dogfish 2 & DF-EM-2 & $2838-127$ & 1 & $1 / 21 / 2008$ \\
\hline & ADUW-DOG02 & & liver $(g)$ & 2 & Dogfish 2 & DF-LV-2 & 2838-133 & 1 & $1 / 21 / 2008$ \\
\hline & ADUW-DOG02 & & digestive tract - All & 2 & Dogfish 2 & DF-DIG-2 & 2838-139 & 1 & $1 / 21 / 2008$ \\
\hline & ADUW-DOG02 & & Section Weighted-Composite & 2 & Dogfish 2 & DF-SWC-2 & 2838-175 & 1 & $1 / 21 / 2008$ \\
\hline & ADUW-DOG02 & & Whole Body Weighted-Composite & 2 & Dogfish 2 & DF-WBWC-2 & 2838-178 & 1 & $1 / 21 / 2008$ \\
\hline & ADUW-DOG03 & & Whole Body Weighted-Composite & 3 & Dogfish 3 & DF-WBWC-3 & 2838-179 & 1 & $1 / 21 / 2008$ \\
\hline & ADUW-DOG04 & & embryo mass $(\mathrm{g})$ & 4 & Dogfish 4 & DF-EM-4 & $2838-129$ & 1 & $1 / 21 / 2008$ \\
\hline & ADUW-DOG04 & & liver $(\mathrm{g})$ & 4 & Dogfish 4 & DF-LV-4 & 2838-135 & 1 & $1 / 21 / 2008$ \\
\hline & ADUW-DOG04 & & digestive tract - All & 4 & Dogfish 4 & DF-DIG-4 & 2838-141 & 1 & $1 / 21 / 2008$ \\
\hline & ADUW-DOG04 & & Section Weighted-Composite & 4 & Dogfish 4 & DF-SWC-4 & 2838-183 & 1 & $1 / 21 / 2008$ \\
\hline & ADUW-DOG04 & & Whole Body Weighted-Composite & 4 & Dogfish 4 & DF-WBWC-4 & $2838-180$ & 1 & $1 / 21 / 2008$ \\
\hline & ADUW-DOG05 & & Whole Body Weighted-Composite & 5 & Dogfish 5 & DF-WBWC-5 & 2838-181 & 1 & $1 / 21 / 2008$ \\
\hline & ADUW-DOG06 & & embryo mass (g) & 6 & Dogfish 6 & DF-EM-6 & 2838-131 & 1 & $1 / 21 / 2008$ \\
\hline & ADUW-DOG06 & & liver $(\mathrm{g})$ & 6 & Dogfish 6 & DF-LV-6 & $2838-137$ & 1 & $1 / 21 / 2008$ \\
\hline & ADUW-DOG06 & & digestive tract - All & 6 & Dogfish 6 & DF-DIG-6 & 2838-143 & 1 & $1 / 21 / 2008$ \\
\hline & ADUW-DOG06 & & Section Weighted-Composite & 3 & Dogfish 6 & DF-SWC-6 & 2838-176 & 1 & $1 / 21 / 2008$ \\
\hline & ADUW-DOG06 & & Whole Body Weighted-Composite & 6 & Dogfish 6 & DF-WBWC-6 & 2838-182 & 1 & $1 / 21 / 2008$ \\
\hline
\end{tabular}


Final Sample Selection List for Stable Isotope Analysis

\begin{tabular}{|c|c|c|c|c|c|c|c|c|}
\hline $\begin{array}{l}\text { Location } \\
\text { Code }\end{array}$ & Location Site & $\begin{array}{c}\text { Collection } \\
\text { Date }\end{array}$ & Species & Rep & Total Org & Client Code & MSL Code & Isotopes \\
\hline 1762 & Vendovi & $5 / 1 / 2007$ & English sole adult & 1 & & V-ES-1 & 2838-1 & 1 \\
\hline 1762 & Vendovi & $5 / 1 / 2007$ & English sole adult & 2 & & V-ES-2 & $2838-2$ & 1 \\
\hline 1762 & Vendovi & $5 / 1 / 2007$ & English sole adult & 3 & & V-ES-3 & $2838-3$ & 1 \\
\hline 1762 & Vendovi & $5 / 1 / 2007$ & English sole adult & 4 & & V-ES-4 & $2838-4$ & 1 \\
\hline 1762 & Vendovi & $5 / 1 / 2007$ & English sole adult & 5 & & V-ES-5 & $2838-5$ & 1 \\
\hline 1762 & Vendovi & $5 / 1 / 2007$ & English sole adult & 6 & & V-ES-6 & $2838-6$ & 1 \\
\hline 1762 & Vendovi & $5 / 1 / 2007$ & English sole adult & 7 & & V-ES-7 & $2838-7$ & 1 \\
\hline 1762 & Vendovi & $5 / 1 / 2007$ & English sole adult & 8 & & V-ES-8 & 2838-8 & 1 \\
\hline 1762 & Vendovi & $5 / 1 / 2007$ & English sole adult & 9 & & V-ES-9 & $2838-9$ & 1 \\
\hline 1762 & Vendovi & $5 / 1 / 2007$ & Rock sole & 1 & & V-RS-1 & $2838-10$ & 1 \\
\hline 1762 & Vendovi & $5 / 1 / 2007$ & Rock sole & 2 & & V-RS-2 & 2838-11 & 1 \\
\hline 1762 & Vendovi & $5 / 1 / 2007$ & Rock sole & 3 & & V-RS-3 & 2838-12 & 1 \\
\hline 1762 & Vendovi & $5 / 1 / 2007$ & Staghorn sculpin & 1 & & V-SSc-1 & $2838-13$ & 1 \\
\hline 1762 & Vendovi & $5 / 1 / 2007$ & Staghorn sculpin & 2 & & V-SSc-2 & $2838-14$ & 1 \\
\hline 1762 & Vendovi & $5 / 1 / 2007$ & Staghorn sculpin & 3 & & V-SSc-3 & $2838-15$ & 1 \\
\hline 1762 & Vendovi & $5 / 1 / 2007$ & Shiner perch & 1 & & V-SP-1 & $2838-16$ & 1 \\
\hline 1762 & Vendovi & $5 / 1 / 2007$ & Shiner perch & 2 & & V-SP-2 & $2838-17$ & 1 \\
\hline 1762 & Vendovi & $5 / 1 / 2007$ & Shiner perch & 3 & & V-SP-3 & 2838-18 & 1 \\
\hline 1762 & Vendovi & $5 / 1 / 2007$ & Sea cucumber (3) & COMP & 19 & V-SC-COMP & 2838-19 & 1 \\
\hline 1462 & Strait of Georgia & $5 / 2 / 2007$ & English sole adult & 1 & & SG-ES-1 & $2838-20$ & 1 \\
\hline 1462 & Strait of Georgia & $5 / 2 / 2007$ & English sole adult & 2 & & SG-ES-2 & $2838-21$ & 1 \\
\hline 1462 & Strait of Georgia & $5 / 2 / 2007$ & English sole adult & 3 & & SG-ES-3 & 2838-22 & 1 \\
\hline 1462 & Strait of Georgia & $5 / 2 / 2007$ & English sole adult & 4 & & SG-ES-4 & 2838-23 & 1 \\
\hline 1462 & Strait of Georgia & $5 / 2 / 2007$ & English sole adult & 5 & & SG-ES-5 & $2838-24$ & 1 \\
\hline 1462 & Strait of Georgia & $5 / 2 / 2007$ & English sole adult & 6 & & SG-ES-6 & $2838-25$ & 1 \\
\hline 1462 & Strait of Georgia & $5 / 2 / 2007$ & English sole adult & 7 & & SG-ES-7 & $2838-26$ & 1 \\
\hline 1462 & Strait of Georgia & $5 / 2 / 2007$ & English sole adult & 8 & & SG-ES-8 & $2838-27$ & 1 \\
\hline 1462 & Strait of Georgia & $5 / 2 / 2007$ & English sole adult & 9 & & SG-ES-9 & $2838-28$ & 1 \\
\hline 1462 & Strait of Georgia & $5 / 2 / 2007$ & Spotted Ratfish & 1 & & SG-RF-1 & $2838-29$ & 1 \\
\hline 1462 & Strait of Georgia & $5 / 2 / 2007$ & Spotted Ratfish & 2 & & SG-RF-2 & $2838-30$ & 1 \\
\hline 1462 & Strait of Georgia & $5 / 2 / 2007$ & Spotted Ratfish & 3 & & SG-RF-3 & 2838-31 & 1 \\
\hline \multirow[t]{3}{*}{1462} & Strait of Georgia & $5 / 2 / 2007$ & Sea cucumber (6) & COMP & 13 & SG-SC-COMP & $2838-32$ & 1 \\
\hline & Hood Canal & $5 / 3 / 2007$ & English sole adult & 1 & & HC-ES-1 & 2838-33 & 1 \\
\hline & Hood Canal & $5 / 3 / 2007$ & English sole adult & 2 & & HC-ES-2 & $2838-34$ & 1 \\
\hline
\end{tabular}


Final Sample Selection List for Stable Isotope Analysis

\begin{tabular}{|c|c|c|c|c|c|c|c|c|}
\hline $\begin{array}{c}\text { Location } \\
\text { Code }\end{array}$ & Location Site & $\begin{array}{c}\text { Collection } \\
\text { Date }\end{array}$ & Species & Rep & Total Org & Client Code & MSL Code & Isotopes \\
\hline & Hood Canal & $5 / 3 / 2007$ & English sole adult & 3 & & HC-ES-3 & 2838-35 & 1 \\
\hline & Hood Canal & $5 / 3 / 2007$ & Rock sole & 1 & & HC-RS-1 & $2838-36$ & 1 \\
\hline & Hood Canal & $5 / 3 / 2007$ & Spotted Ratfish & 1 & & HC-RF-1 & 2838-37 & 1 \\
\hline & Hood Canal & $5 / 3 / 2007$ & Spotted Ratfish & 2 & & HC-RF-2 & $2838-38$ & 1 \\
\hline & Hood Canal & $5 / 3 / 2007$ & Spotted Ratfish & 3 & & HC-RF-3 & 2838-39 & 1 \\
\hline & Hood Canal & $5 / 3 / 2007$ & Shiner perch & 1 & & HC-SP1 & $2838-40$ & 1 \\
\hline & Hood Canal & $5 / 3 / 2007$ & Shiner perch & 2 & & $\mathrm{HC}-\mathrm{SP} 2$ & $2838-41$ & 1 \\
\hline & Hood Canal & $5 / 3 / 2007$ & Shiner perch & 3 & & $\mathrm{HC}-\mathrm{SP} 3$ & $2838-42$ & 1 \\
\hline & Hood Canal & $5 / 3 / 2007$ & Graceful Crab & 1 & & $\mathrm{HC}-\mathrm{GC}-1$ & $2838-43$ & 1 \\
\hline & Hood Canal & $5 / 3 / 2007$ & Graceful Crab & 2 & 12 & HC-GC-2 & $2838-44$ & 1 \\
\hline 3382 & Elliott Bay & $5 / 16 / 2007$ & English sole adult & 1 & & EB-ES-1 & $2838-45$ & 1 \\
\hline 3382 & Elliott Bay & $5 / 16 / 2007$ & English sole adult & 2 & & EB-ES-2 & $2838-46$ & 1 \\
\hline 3382 & Elliott Bay & $5 / 16 / 2007$ & English sole adult & 3 & & EB-ES-3 & $2838-47$ & 1 \\
\hline 3382 & Elliott Bay & $5 / 16 / 2007$ & Spotted Ratfish & 1 & & EB-RF-1 & $2838-48$ & 1 \\
\hline 3382 & Elliott Bay & $5 / 16 / 2007$ & Spotted Ratfish & 2 & & EB-RF-2 & $2838-49$ & 1 \\
\hline \multirow[t]{8}{*}{3382} & Elliott Bay & $5 / 16 / 2007$ & Spotted Ratfish & 3 & 6 & EB-RF-3 & $2838-50$ & 1 \\
\hline & Eagle Harbor & $5 / 19 / 2007$ & English sole adult & 1 & & EH-ES-1 & $2838-51$ & 1 \\
\hline & Eagle Harbor & $5 / 19 / 2007$ & English sole adult & 2 & & EH-ES-2 & $2838-52$ & 1 \\
\hline & Eagle Harbor & $5 / 19 / 2007$ & English sole adult & 3 & & EH-ES-3 & $2838-53$ & 1 \\
\hline & Eagle Harbor & $5 / 19 / 2007$ & English sole adult & 4 & & EH-ES-4 & $2838-54$ & 1 \\
\hline & Eagle Harbor & $5 / 19 / 2007$ & Spotted Ratfish & 1 & & EH-RF-1 & $2838-55$ & 1 \\
\hline & Eagle Harbor & $5 / 19 / 2007$ & Spotted Ratfish & 2 & & EH-RF-2 & $2838-56$ & 1 \\
\hline & Eagle Harbor & $5 / 19 / 2007$ & Spotted Ratfish & 3 & 7 & EH-RF-3 & $2838-57$ & 1 \\
\hline 3388 & Sinclair Inlet & $5 / 17 / 2007$ & English sole adult & 1 & & SI-ES-1 & $2838-58$ & 1 \\
\hline 3388 & Sinclair Inlet & $5 / 17 / 2007$ & English sole adult & 2 & & SI-ES-2 & $2838-59$ & 1 \\
\hline 3388 & Sinclair Inlet & $5 / 17 / 2007$ & English sole adult & 3 & & SI-ES-3 & $2838-60$ & 1 \\
\hline 3388 & Sinclair Inlet & $5 / 17 / 2007$ & English sole adult & 4 & & SI-ES-4 & $2838-61$ & 1 \\
\hline 3388 & Sinclair Inlet & $5 / 17 / 2007$ & English sole adult & 5 & & SI-ES-5 & $2838-62$ & 1 \\
\hline 3388 & Sinclair Inlet & $5 / 17 / 2007$ & English sole adult & 6 & & SI-ES-6 & 2838-63 & 1 \\
\hline 3388 & Sinclair Inlet & $5 / 17 / 2007$ & English sole adult & 7 & & SI-ES-7 & 2838-64 & 1 \\
\hline 3388 & Sinclair Inlet & $5 / 17 / 2007$ & English sole adult & 8 & & SI-ES-8 & 2838-65 & 1 \\
\hline 3388 & Sinclair Inlet & $5 / 17 / 2007$ & English sole adult & 9 & & SI-ES-9 & $2838-66$ & 1 \\
\hline 3388 & Sinclair Inlet & $5 / 17 / 2007$ & Rock sole & 1 & 2 & SI-RS-1 & $2838-67-68$ & 1 \\
\hline 3388 & Sinclair Inlet & $5 / 17 / 2007$ & Rock sole & 3 & & SI-RS-3 & 2838-69 & 1 \\
\hline
\end{tabular}


Final Sample Selection List for Stable Isotope Analysis

\begin{tabular}{|c|c|c|c|c|c|c|c|c|}
\hline $\begin{array}{l}\text { Location } \\
\text { Code }\end{array}$ & Location Site & $\begin{array}{c}\text { Collection } \\
\text { Date }\end{array}$ & Species & Rep & Total Org & Client Code & MSL Code & Isotopes \\
\hline 3388 & Sinclair Inlet & $5 / 17 / 2007$ & Rock sole & 4 & & SI-RS-4 & $2838-70$ & 1 \\
\hline 3388 & Sinclair Inlet & $5 / 17 / 2007$ & Sand sole & 1 & & SI-SS-1 & 2838-71 & 1 \\
\hline 3388 & Sinclair Inlet & $5 / 17 / 2007$ & Sand sole & 2 & & SI-SS-2 & $2838-72$ & 1 \\
\hline 3388 & Sinclair Inlet & $5 / 17 / 2007$ & Sand sole & 3 & & SI-SS-3 & $2838-73$ & 1 \\
\hline 3388 & Sinclair Inlet & $5 / 17 / 2007$ & Sand sole & 4 & & SI-SS-4 & $2838-74$ & 1 \\
\hline 3388 & Sinclair Inlet & $5 / 17 / 2007$ & Sand sole & 5 & & SI-SS-5 & $2838-75$ & 1 \\
\hline 3388 & Sinclair Inlet & $5 / 17 / 2007$ & Sand sole & 6 & & SI-SS-6 & $2838-76$ & 1 \\
\hline 3388 & Sinclair Inlet & $5 / 17 / 2007$ & Staghorn sculpin & 1 & & SI-SSc-1 & $2838-77$ & 1 \\
\hline 3388 & Sinclair Inlet & $5 / 17 / 2007$ & Staghorn sculpin & 2 & & SI-SSc-2 & $2838-78$ & 1 \\
\hline 3388 & Sinclair Inlet & $5 / 17 / 2007$ & Staghorn sculpin & 3 & & SI-SSc-3 & $2838-79$ & 1 \\
\hline 3388 & Sinclair Inlet & $5 / 17 / 2007$ & Staghorn sculpin & 5 & & SI-SSc-5 & $2838-81$ & 1 \\
\hline 3388 & Sinclair Inlet & $5 / 17 / 2007$ & Staghorn sculpin & 4 & & SI-SSc-4 & $2838-80$ & 1 \\
\hline 3388 & Sinclair Inlet & $5 / 17 / 2007$ & Staghorn sculpin & 6 & & SI-SSc-6 & $2838-82$ & 1 \\
\hline 3388 & Sinclair Inlet & $5 / 17 / 2007$ & Spotted Ratfish & 1 & & SI-RF-1 & $2838-83$ & 1 \\
\hline 3388 & Sinclair Inlet & $5 / 17 / 2007$ & Spotted Ratfish & 2 & & SI-RF-2 & $2838-84$ & 1 \\
\hline 3388 & Sinclair Inlet & $5 / 17 / 2007$ & Shiner perch & 4 & & SI-SP-4 & $2838-88$ & 1 \\
\hline 3388 & Sinclair Inlet & $5 / 17 / 2007$ & Shiner perch & 5 & & SI-SP-5 & $2838-89$ & 1 \\
\hline 3388 & Sinclair Inlet & $5 / 17 / 2007$ & Shiner perch & 6 & & SI-SP-6 & $2838-90$ & 1 \\
\hline 3388 & Sinclair Inlet & $5 / 17 / 2007$ & Shiner perch & 7 & & SI-SP-7 & 2838-91 & 1 \\
\hline 3388 & Sinclair Inlet & $5 / 17 / 2007$ & Shiner perch & 3 & & SI-SP-3 & $2838-87$ & 1 \\
\hline 3388 & Sinclair Inlet & $5 / 17 / 2007$ & Graceful Crab & 2 & & SI-GC-2 & $2838-93$ & 1 \\
\hline 3388 & Sinclair Inlet & $5 / 17 / 2007$ & Graceful Crab & 6 & & SI-GC-6 & $2838-97$ & 1 \\
\hline 3388 & Sinclair Inlet & $5 / 17 / 2007$ & Graceful Crab & 3 & & SI-GC-3 & $2838-94$ & 1 \\
\hline 3388 & Sinclair Inlet & $5 / 17 / 2007$ & Graceful Crab & 4 & & SI-GC-4 & $2838-95$ & 1 \\
\hline 3388 & Sinclair Inlet & $5 / 17 / 2007$ & Graceful Crab & 7 & & SI-GC-7 & $2838-98$ & 1 \\
\hline 3388 & Sinclair Inlet & $5 / 17 / 2007$ & Graceful Crab & 1 & & SI-GC-1 & $2838-92$ & 1 \\
\hline 3388 & Sinclair Inlet & $5 / 17 / 2007$ & Graceful Crab & 5 & & SI-GC-5 & $2838-96$ & 1 \\
\hline 3388 & Sinclair Inlet & $5 / 17 / 2007$ & Graceful Crab & 8 & & SI-GC-8 & $2838-99$ & 1 \\
\hline 3388 & Sinclair Inlet & $5 / 17 / 2007$ & Sea cucumber (6) & COMP & 43 & SI-SC-COMP & $2838-100$ & 1 \\
\hline 1481 & Port Gardner & $5 / 29 / 2007$ & English sole adult & 1 & & PG-ES-1 & 2838-101 & 1 \\
\hline 1481 & Port Gardner & $5 / 29 / 2007$ & English sole adult & 2 & & PG-ES-2 & 2838-102 & 1 \\
\hline 1481 & Port Gardner & $5 / 29 / 2007$ & English sole adult & 3 & 3 & PG-ES-3 & $2838-103$ & 1 \\
\hline 3322 & Nisqually & $5 / 30 / 2007$ & English sole adult & 1 & & NIS-ES-1 & 2838-104 & 1 \\
\hline 3322 & Nisqually & $5 / 30 / 2007$ & English sole adult & 2 & & NIS-ES-2 & 2838-105 & 1 \\
\hline
\end{tabular}


Final Sample Selection List for Stable Isotope Analysis

\begin{tabular}{|c|c|c|c|c|c|c|c|c|}
\hline $\begin{array}{c}\text { Location } \\
\text { Code }\end{array}$ & Location Site & $\begin{array}{c}\text { Collection } \\
\text { Date }\end{array}$ & Species & Rep & Total Org & Client Code & MSL Code & Isotopes \\
\hline 3322 & Nisqually & $5 / 30 / 2007$ & English sole adult & 3 & & NIS-ES-3 & 2838-106 & 1 \\
\hline 3322 & Nisqually & $5 / 30 / 2007$ & Spotted Ratfish & 1 & & NIS-RF-1 & 2838-107 & 1 \\
\hline 3322 & Nisqually & $5 / 30 / 2007$ & Spotted Ratfish & 2 & & NIS-RF-2 & 2838-108 & 1 \\
\hline 3322 & Nisqually & $5 / 30 / 2007$ & Spotted Ratfish & 3 & 6 & NIS-RF-3 & 2838-109 & 1 \\
\hline 3310 & Commencement Bay & $5 / 31 / 2007$ & English sole adult & 1 & & CB-ES-1 & $2838-110$ & 1 \\
\hline 3310 & Commencement Bay & $5 / 31 / 2007$ & English sole adult & 2 & & CB-ES-2 & $2838-111$ & 1 \\
\hline 3310 & Commencement Bay & $5 / 31 / 2007$ & English sole adult & 3 & & CB-ES-3 & $2838-112$ & 1 \\
\hline 3310 & Commencement Bay & $5 / 31 / 2007$ & Spotted Ratfish & 1 & & CB-RF-1 & $2838-113$ & 1 \\
\hline 3310 & Commencement Bay & $5 / 31 / 2007$ & Spotted Ratfish & 2 & & CB-RF-2 & $2838-114$ & 1 \\
\hline 3310 & Commencement Bay & $5 / 31 / 2007$ & Spotted Ratfish & 3 & 6 & CB-RF-3 & $2838-115$ & 1 \\
\hline 3277 & Duwamish & $5 / 18 / 2007$ & English sole adult & 1 & & DU-ES-1 & $2838-116$ & 1 \\
\hline 3277 & Duwamish & $5 / 18 / 2007$ & English sole adult & 2 & & DU-ES-2 & 2838-117 & 1 \\
\hline 3277 & Duwamish & $5 / 18 / 2007$ & English sole adult & 3 & & DU-ES-3 & 2838-118 & 1 \\
\hline \multirow[t]{12}{*}{3277} & Duwamish & $5 / 18 / 2007$ & English sole adult & 4 & 4 & DU-ES-4 & $2838-119$ & 1 \\
\hline & ADUW-DOG01 & & Section Weighted-Composite & 1 & Dogfish 1 & DF-SWC-1 & $2838-174$ & 1 \\
\hline & ADUW-DOG02 & & Section Weighted-Composite & 2 & Dogfish 2 & DF-SWC-2 & 2838-175 & 1 \\
\hline & ADUW-DOG06 & & Section Weighted-Composite & 3 & Dogfish 6 & DF-SWC-6 & $2838-176$ & 1 \\
\hline & ADUW-DOG01 & & Whole Body Weighted-Composite & 1 & Dogfish 1 & DF-WBWC-1 & $2838-177$ & 1 \\
\hline & ADUW-DOG02 & & Whole Body Weighted-Composite & 2 & Dogfish 2 & DF-WBWC-2 & $2838-178$ & 1 \\
\hline & ADUW-DOG03 & & Whole Body Weighted-Composite & 3 & Dogfish 3 & DF-WBWC-3 & 2838-179 & 1 \\
\hline & ADUW-DOG04 & & Whole Body Weighted-Composite & 4 & Dogfish 4 & DF-WBWC-4 & $2838-180$ & 1 \\
\hline & ADUW-DOG05 & & Whole Body Weighted-Composite & 5 & Dogfish 5 & DF-WBWC-5 & 2838-181 & 1 \\
\hline & ADUW-DOG06 & & Whole Body Weighted-Composite & 6 & Dogfish 6 & DF-WBWC-6 & 2838-182 & 1 \\
\hline & ADUW-DOG04 & & Section Weighted-Composite & 4 & Dogfish 4 & DF-SWC-4 & 2838-183 & 1 \\
\hline & Sequim Bay & & eelgrass shoots & 5 & & & $2838-184$ & 1 \\
\hline
\end{tabular}


cc: Project Manager/Central File Login File
SAMPLE LOGIN

(SOP\# MSL-A-001)
Project Manager: Brandenberger

Date Received: May, June, Sept. 2007

Batch: Multiple

PROJEC 2008 ENVVEST Biota

\begin{tabular}{|c|c|c|c|c|c|c|c|c|}
\hline $\begin{array}{c}\text { SPONSOR } \\
\text { CODE }\end{array}$ & Site Description & $\begin{array}{l}\text { BATTELLE } \\
\text { CODE }\end{array}$ & MATRIX & $\begin{array}{c}\text { STORAGE } \\
\text { LOCATION } \\
\end{array}$ & PARAMETERS REQUESTED* & \begin{tabular}{|c|}
$\begin{array}{c}\text { COLLECTION } \\
\text { DATE }\end{array}$ \\
\end{tabular} & INITIALS & Species/section \\
\hline V-ES-1 & Vendovi & $2838-1$ & Tissue & Walk-in Freezer & Isotopes, PCBs, Lipids, Metals & $5 / 1 / 2007$ & CS & English sole adult \\
\hline V-ES-2 & Vendovi & $2838-2$ & Tissue & Walk-in Freezer & Isotopes, PCBs, Lipids, Metals & $5 / 1 / 2007$ & CS & English sole adult \\
\hline V-ES-3 & Vendovi & $2838-3$ & Tissue & Walk-in Freezer & Isotopes, PCBs, Lipids, Metals & $5 / 1 / 2007$ & CS & English sole adult \\
\hline V-ES-4 & Vendovi & $2838-4$ & Tissue & Walk-in Freezer & Isotopes, PCBs, Lipids, Metals & $5 / 1 / 2007$ & CS & English sole adult \\
\hline V-ES-5 & Vendovi & $2838-5$ & Tissue & Walk-in Freezer & Isotopes, PCBs, Lipids, Metals & $5 / 1 / 2007$ & CS & English sole adult \\
\hline V-ES-6 & Vendovi & $2838-6$ & Tissue & Walk-in Freezer & Isotopes, PCBs, Lipids, Metals & $5 / 1 / 2007$ & CS & English sole adult \\
\hline V-ES-7 & Vendovi & 2838-7 & Tissue & Walk-in Freezer & Isotopes, PCBs, Lipids, Metals & $5 / 1 / 2007$ & CS & English sole adult \\
\hline V-ES-8 & Vendovi & $2838-8$ & Tissue & Walk-in Freezer & Isotopes, PCBs, Lipids, Metals & $5 / 1 / 2007$ & CS & English sole adult \\
\hline V-ES-9 & Vendovi & $2838-9$ & Tissue & Walk-in Freezer & Isotopes, PCBs, Lipids, Metals & $5 / 1 / 2007$ & CS & English sole adult \\
\hline V-RS-1 & Vendovi & $2838-10$ & Tissue & Walk-in Freezer & Isotopes, PCBs, Lipids, Metals & $5 / 1 / 2007$ & CS & Rock sole \\
\hline V-RS-2 & Vendovi & 2838-11 & Tissue & Walk-in Freezer & Isotopes, PCBs, Lipids, Metals & $5 / 1 / 2007$ & CS & Rock sole \\
\hline V-RS-3 & Vendovi & $2838-12$ & Tissue & Walk-in Freezer & Isotopes, PCBs, Lipids, Metals & $5 / 1 / 2007$ & CS & Rock sole \\
\hline V-SSc-1 & Vendovi & $2838-13$ & Tissue & Walk-in Freezer & Isotopes, PCBs, Lipids, Metals & $5 / 1 / 2007$ & CS & Staghorn sculpin \\
\hline V-SSc-2 & Vendovi & 2838-14 & Tissue & Walk-in Freezer & Isotopes, PCBs, Lipids, Metals & $5 / 1 / 2007$ & CS & Staghorn sculpin \\
\hline V-SSc-3 & Vendovi & 2838-15 & Tissue & Walk-in Freezer & Isotopes, PCBs, Lipids, Metals & $5 / 1 / 2007$ & CS & Staghorn sculpin \\
\hline V-SP-1 & Vendovi & $2838-16$ & Tissue & Walk-in Freezer & Isotopes, PCBs, Lipids, Metals & $5 / 1 / 2007$ & CS & Shiner perch \\
\hline V-SP-2 & Vendovi & $2838-17$ & Tissue & Walk-in Freezer & Isotopes, PCBs, Lipids, Metals & $5 / 1 / 2007$ & CS & Shiner perch \\
\hline V-SP-3 & Vendovi & $2838-18$ & Tissue & Walk-in Freezer & Isotopes, PCBs, Lipids, Metals & $5 / 1 / 2007$ & $\mathrm{CS}$ & Shiner perch \\
\hline V-SC-COMP & Vendovi & $2838-19$ & Tissue & Walk-in Freezer & Isotopes, PCBs, Lipids, Metals & $5 / 1 / 2007$ & $\mathrm{CS}$ & Sea cucumber (3) \\
\hline SG-ES-1 & Strait of Georgia & $2838-20$ & Tissue & Walk-in Freezer & Isotopes, PCBs, Lipids, Metals & $5 / 2 / 2007$ & $\mathrm{CS}$ & English sole adult \\
\hline SG-ES-2 & Strait of Georgia & $2838-21$ & Tissue & Walk-in Freezer & Isotopes, PCBs, Lipids, Metals & $5 / 2 / 2007$ & CS & English sole adult \\
\hline SG-ES-3 & Strait of Georgia & $2838-22$ & Tissue & Walk-in Freezer & Isotopes, PCBs, Lipids, Metals & $5 / 2 / 2007$ & CS & English sole adult \\
\hline SG-ES-4 & Strait of Georgia & $2838-23$ & Tissue & Walk-in Freezer & Isotopes, PCBs, Lipids, Metals & $5 / 2 / 2007$ & CS & English sole adult \\
\hline SG-ES-5 & Strait of Georgia & $2838-24$ & Tissue & Walk-in Freezer & Isotopes, PCBs, Lipids, Metals & $5 / 2 / 2007$ & CS & English sole adult \\
\hline SG-ES-6 & Strait of Georgia & $2838-25$ & Tissue & Walk-in Freezer & Isotopes, PCBs, Lipids, Metals & $5 / 2 / 2007$ & CS & English sole adult \\
\hline SG-ES-7 & Strait of Georgia & $2838-26$ & Tissue & Walk-in Freezer & Isotopes, PCBs, Lipids, Metals & $5 / 2 / 2007$ & CS & English sole adult \\
\hline SG-ES-8 & Strait of Georgia & $2838-27$ & Tissue & Walk-in Freezer & Isotopes, PCBs, Lipids, Metals & $5 / 2 / 2007$ & CS & English sole adult \\
\hline SG-ES-9 & Strait of Georgia & $2838-28$ & Tissue & Walk-in Freezer & Isotopes, PCBs, Lipids, Metals & $5 / 2 / 2007$ & CS & English sole adult \\
\hline
\end{tabular}


cc: Project Manager/Central File Login File
SAMPLE LOGIN

(SOP\# MSL-A-001)
Project Manager: Brandenberger

Date Received: May, June, Sept. 2007

Batch: Multiple

PROJEC 2008 ENVVEST Biota

\begin{tabular}{|c|c|c|c|c|c|c|c|c|}
\hline $\begin{array}{l}\text { SPONSOR } \\
\text { CODE }\end{array}$ & Site Description & $\begin{array}{l}\text { BATTELLE } \\
\text { CODE }\end{array}$ & MATRIX & $\begin{array}{l}\text { STORAGE } \\
\text { LOCATION }\end{array}$ & PARAMETERS REQUESTED* & \begin{tabular}{|c|} 
COLLECTION \\
DATE
\end{tabular} & INITIALS & Species/section \\
\hline SG-RF-1 & Strait of Georgia & $2838-29$ & Tissue & Walk-in Freezer & Isotopes, PCBs, Lipids, Metals & $5 / 2 / 2007$ & CS & Spotted Ratfish \\
\hline SG-RF-2 & Strait of Georgia & 2838-30 & Tissue & Walk-in Freezer & Isotopes, PCBs, Lipids, Metals & $5 / 2 / 2007$ & CS & Spotted Ratfish \\
\hline SG-RF-3 & Strait of Georgia & 2838-31 & Tissue & Walk-in Freezer & Isotopes, PCBs, Lipids, Metals & $5 / 2 / 2007$ & CS & Spotted Ratfish \\
\hline SG-SC-COMP & Strait of Georgia & 2838-32 & Tissue & Walk-in Freezer & Isotopes, PCBs, Lipids, Metals & $5 / 2 / 2007$ & CS & Sea cucumber (6) \\
\hline HC-ES-1 & Hood Canal & 2838-33 & Tissue & Walk-in Freezer & Isotopes, PCBs, Lipids, Metals & $5 / 3 / 2007$ & CS & English sole adult \\
\hline HC-ES-2 & Hood Canal & 2838-34 & Tissue & Walk-in Freezer & Isotopes, PCBs, Lipids, Metals & $5 / 3 / 2007$ & CS & English sole adult \\
\hline HC-ES-3 & Hood Canal & 2838-35 & Tissue & Walk-in Freezer & Isotopes, PCBs, Lipids, Metals & $5 / 3 / 2007$ & CS & English sole adult \\
\hline HC-RS-1 & Hood Canal & 2838-36 & Tissue & Walk-in Freezer & Isotopes, PCBs, Lipids, Metals & $5 / 3 / 2007$ & CS & Rock sole \\
\hline HC-RF-1 & Hood Canal & 2838-37 & Tissue & Walk-in Freezer & Isotopes, PCBs, Lipids, Metals & $5 / 3 / 2007$ & CS & Spotted Ratfish \\
\hline HC-RF-2 & Hood Canal & 2838-38 & Tissue & Walk-in Freezer & Isotopes, PCBs, Lipids, Metals & $5 / 3 / 2007$ & CS & Spotted Ratfish \\
\hline HC-RF-3 & Hood Canal & 2838-39 & Tissue & Walk-in Freezer & Isotopes, PCBs, Lipids, Metals & $5 / 3 / 2007$ & CS & Spotted Ratfish \\
\hline HC-SP1 & Hood Canal & $2838-40$ & Tissue & Walk-in Freezer & Isotopes, PCBs, Lipids, Metals & $5 / 3 / 2007$ & CS & Shiner perch \\
\hline HC-SP2 & Hood Canal & $2838-41$ & Tissue & Walk-in Freezer & Isotopes, PCBs, Lipids, Metals & $5 / 3 / 2007$ & CS & Shiner perch \\
\hline HC-SP3 & Hood Canal & $2838-42$ & Tissue & Walk-in Freezer & Isotopes, PCBs, Lipids, Metals & $5 / 3 / 2007$ & CS & Shiner perch \\
\hline HC-GC-1 & Hood Canal & $2838-43$ & Tissue & Walk-in Freezer & Isotopes, PCBs, Lipids, Metals & $5 / 3 / 2007$ & CS & Graceful Crab \\
\hline HC-GC-2 & Hood Canal & $2838-44$ & Tissue & Walk-in Freezer & Isotopes, PCBs, Lipids, Metals & $5 / 3 / 2007$ & CS & Graceful Crab \\
\hline EB-ES-1 & Elliott Bay & $2838-45$ & Tissue & Walk-in Freezer & Isotopes, PCBs, Lipids, Metals & $5 / 16 / 2007$ & CS & English sole adult \\
\hline EB-ES-2 & Elliott Bay & $2838-46$ & Tissue & Walk-in Freezer & Isotopes, PCBs, Lipids, Metals & $5 / 16 / 2007$ & CS & English sole adult \\
\hline EB-ES-3 & Elliott Bay & $2838-47$ & Tissue & Walk-in Freezer & Isotopes, PCBs, Lipids, Metals & $5 / 16 / 2007$ & CS & English sole adult \\
\hline EB-RF-1 & Elliott Bay & $2838-48$ & Tissue & Walk-in Freezer & Isotopes, PCBs, Lipids, Metals & $5 / 16 / 2007$ & CS & Spotted Ratfish \\
\hline EB-RF-2 & Elliott Bay & $2838-49$ & Tissue & Walk-in Freezer & Isotopes, PCBs, Lipids, Metals & $5 / 16 / 2007$ & CS & Spotted Ratfish \\
\hline EB-RF-3 & Elliott Bay & $2838-50$ & Tissue & Walk-in Freezer & Isotopes, PCBs, Lipids, Metals & $5 / 16 / 2007$ & CS & Spotted Ratfish \\
\hline EH-ES-1 & Eagle Harbor & $2838-51$ & Tissue & Walk-in Freezer & Isotopes, PCBs, Lipids, Metals & $5 / 19 / 2007$ & CS & English sole adult \\
\hline EH-ES-2 & Eagle Harbor & $2838-52$ & Tissue & Walk-in Freezer & Isotopes, PCBs, Lipids, Metals & $5 / 19 / 2007$ & CS & English sole adult \\
\hline EH-ES-3 & Eagle Harbor & $2838-53$ & Tissue & Walk-in Freezer & Isotopes, PCBs, Lipids, Metals & $5 / 19 / 2007$ & CS & English sole adult \\
\hline EH-ES-4 & Eagle Harbor & $2838-54$ & Tissue & Walk-in Freezer & Isotopes, PCBs, Lipids, Metals & $5 / 19 / 2007$ & CS & English sole adult \\
\hline EH-RF-1 & Eagle Harbor & $2838-55$ & Tissue & Walk-in Freezer & Isotopes, PCBs, Lipids, Metals & $5 / 19 / 2007$ & CS & Spotted Ratfish \\
\hline EH-RF-2 & Eagle Harbor & $2838-56$ & Tissue & Walk-in Freezer & Isotopes, PCBs, Lipids, Metals & $5 / 19 / 2007$ & CS & Spotted Ratfish \\
\hline
\end{tabular}


cc: Project Manager/Central File Login File
SAMPLE LOGIN

(SOP\# MSL-A-001)
Project Manager: Brandenberger

Date Received: May, June, Sept. 2007

Batch: Multiple

PROJEC 2008 ENVVEST Biota

\begin{tabular}{|c|c|c|c|c|c|c|c|c|}
\hline $\begin{array}{c}\text { SPONSOR } \\
\text { CODE }\end{array}$ & Site Description & $\begin{array}{c}\text { BATTELLE } \\
\text { CODE }\end{array}$ & MATRIX & $\begin{array}{l}\text { STORAGE } \\
\text { LOCATION }\end{array}$ & PARAMETERS REQUESTED* & \begin{tabular}{|c|} 
COLLECTION \\
DATE
\end{tabular} & INITIALS & Species/section \\
\hline EH-RF-3 & Eagle Harbor & $2838-57$ & Tissue & Walk-in Freezer & Isotopes, PCBs, Lipids, Metals & $5 / 19 / 2007$ & CS & Spotted Ratfish \\
\hline SI-ES-1 & Sinclair Inlet & $2838-58$ & Tissue & Walk-in Freezer & Isotopes, PCBs, Lipids, Metals & $5 / 17 / 2007$ & CS & English sole adult \\
\hline SI-ES-2 & Sinclair Inlet & $2838-59$ & Tissue & Walk-in Freezer & Isotopes, PCBs, Lipids, Metals & $5 / 17 / 2007$ & CS & English sole adult \\
\hline SI-ES-3 & Sinclair Inlet & $2838-60$ & Tissue & Walk-in Freezer & Isotopes, PCBs, Lipids, Metals & $5 / 17 / 2007$ & CS & English sole adult \\
\hline SI-ES-4 & Sinclair Inlet & 2838-61 & Tissue & Walk-in Freezer & Isotopes, PCBs, Lipids, Metals & $5 / 17 / 2007$ & CS & English sole adult \\
\hline SI-ES-5 & Sinclair Inlet & $2838-62$ & Tissue & Walk-in Freezer & Isotopes, PCBs, Lipids, Metals & $5 / 17 / 2007$ & CS & English sole adult \\
\hline SI-ES-6 & Sinclair Inlet & $2838-63$ & Tissue & Walk-in Freezer & Isotopes, PCBs, Lipids, Metals & $5 / 17 / 2007$ & CS & English sole adult \\
\hline SI-ES-7 & Sinclair Inlet & $2838-64$ & Tissue & Walk-in Freezer & Isotopes, PCBs, Lipids, Metals & $5 / 17 / 2007$ & CS & English sole adult \\
\hline SI-ES-8 & Sinclair Inlet & $2838-65$ & Tissue & Walk-in Freezer & Isotopes, PCBs, Lipids, Metals & $5 / 17 / 2007$ & CS & English sole adult \\
\hline SI-ES-9 & Sinclair Inlet & $2838-66$ & Tissue & Walk-in Freezer & Isotopes, PCBs, Lipids, Metals & $5 / 17 / 2007$ & CS & English sole adult \\
\hline SI-RS-1 & Sinclair Inlet & $2838-67$ & Tissue & Walk-in Freezer & Isotopes, PCBs, Lipids, Metals & $5 / 17 / 2007$ & CS & Rock sole \\
\hline SI-RS-2 & Sinclair Inlet & $2838-68$ & Tissue & Walk-in Freezer & Isotopes, PCBs, Lipids, Metals & $5 / 17 / 2007$ & CS & Rock sole \\
\hline SI-RS-3 & Sinclair Inlet & 2838-69 & Tissue & Walk-in Freezer & Isotopes, PCBs, Lipids, Metals & $5 / 17 / 2007$ & CS & Rock sole \\
\hline SI-RS-4 & Sinclair Inlet & $2838-70$ & Tissue & Walk-in Freezer & Isotopes, PCBs, Lipids, Metals & $5 / 17 / 2007$ & CS & Rock sole \\
\hline SI-SS-1 & Sinclair Inlet & 2838-71 & Tissue & Walk-in Freezer & Isotopes, PCBs, Lipids, Metals & $5 / 17 / 2007$ & CS & Sand sole \\
\hline SI-SS-2 & Sinclair Inlet & $2838-72$ & Tissue & Walk-in Freezer & Isotopes, PCBs, Lipids, Metals & $5 / 17 / 2007$ & CS & Sand sole \\
\hline SI-SS-3 & Sinclair Inlet & $2838-73$ & Tissue & Walk-in Freezer & Isotopes, PCBs, Lipids, Metals & $5 / 17 / 2007$ & CS & Sand sole \\
\hline SI-SS-4 & Sinclair Inlet & $2838-74$ & Tissue & Walk-in Freezer & Isotopes, PCBs, Lipids, Metals & $5 / 17 / 2007$ & CS & Sand sole \\
\hline SI-SS-5 & Sinclair Inlet & $2838-75$ & Tissue & Walk-in Freezer & Isotopes, PCBs, Lipids, Metals & $5 / 17 / 2007$ & CS & Sand sole \\
\hline SI-SS-6 & Sinclair Inlet & $2838-76$ & Tissue & Walk-in Freezer & Isotopes, PCBs, Lipids, Metals & $5 / 17 / 2007$ & CS & Sand sole \\
\hline SI-SSc-1 & Sinclair Inlet & $2838-77$ & Tissue & Walk-in Freezer & Isotopes, PCBs, Lipids, Metals & $5 / 17 / 2007$ & CS & Staghorn sculpin \\
\hline SI-SSc-2 & Sinclair Inlet & 2838-78 & Tissue & Walk-in Freezer & Isotopes, PCBs, Lipids, Metals & $5 / 17 / 2007$ & CS & Staghorn sculpin \\
\hline SI-SSc-3 & Sinclair Inlet & 2838-79 & Tissue & Walk-in Freezer & Isotopes, PCBs, Lipids, Metals & $5 / 17 / 2007$ & CS & Staghorn sculpin \\
\hline SI-SSc-5 & Sinclair Inlet & 2838-81 & Tissue & Walk-in Freezer & Isotopes, PCBs, Lipids, Metals & $5 / 17 / 2007$ & CS & Staghorn sculpin \\
\hline SI-SSc-4 & Sinclair Inlet & $2838-80$ & Tissue & Walk-in Freezer & Isotopes, PCBs, Lipids, Metals & $5 / 17 / 2007$ & $\mathrm{CS}$ & Staghorn sculpin \\
\hline SI-SSc-6 & Sinclair Inlet & 2838-82 & Tissue & Walk-in Freezer & Isotopes, PCBs, Lipids, Metals & $5 / 17 / 2007$ & $\mathrm{CS}$ & Staghorn sculpin \\
\hline SI-RF-1 & Sinclair Inlet & $2838-83$ & Tissue & Walk-in Freezer & Isotopes, PCBs, Lipids, Metals & $5 / 17 / 2007$ & CS & Spotted Ratfish \\
\hline SI-RF-2 & Sinclair Inlet & 2838-84 & Tissue & Walk-in Freezer & Isotopes, PCBs, Lipids, Metals & $5 / 17 / 2007$ & CS & Spotted Ratfish \\
\hline
\end{tabular}


cc: Project Manager/Central File Login File
SAMPLE LOGIN

(SOP\# MSL-A-001)
Project Manager: Brandenberger

Date Received: May, June, Sept. 2007

Batch: Multiple

PROJEC 2008 ENVVEST Biota

\begin{tabular}{|c|c|c|c|c|c|c|c|c|}
\hline $\begin{array}{c}\text { SPONSOR } \\
\text { CODE }\end{array}$ & Site Description & $\begin{array}{l}\text { BATTELLE } \\
\text { CODE }\end{array}$ & MATRIX & $\begin{array}{l}\text { STORAGE } \\
\text { LOCATION }\end{array}$ & PARAMETERS REQUESTED* & \begin{tabular}{|c|} 
COLLECTION \\
DATE
\end{tabular} & INITIALS & Species/section \\
\hline SI-SP-1 & Sinclair Inlet & $2838-85$ & Tissue & Walk-in Freezer & Isotopes, PCBs, Lipids, Metals & $5 / 17 / 2007$ & CS & Shiner perch \\
\hline SI-SP-4 & Sinclair Inlet & $2838-88$ & Tissue & Walk-in Freezer & Isotopes, PCBs, Lipids, Metals & $5 / 17 / 2007$ & CS & Shiner perch \\
\hline SI-SP-5 & Sinclair Inlet & 2838-89 & Tissue & Walk-in Freezer & Isotopes, PCBs, Lipids, Metals & $5 / 17 / 2007$ & CS & Shiner perch \\
\hline SI-SP-6 & Sinclair Inlet & $2838-90$ & Tissue & Walk-in Freezer & Isotopes, PCBs, Lipids, Metals & $5 / 17 / 2007$ & CS & Shiner perch \\
\hline SI-SP-2 & Sinclair Inlet & $2838-86$ & Tissue & Walk-in Freezer & Isotopes, PCBs, Lipids, Metals & $5 / 17 / 2007$ & CS & Shiner perch \\
\hline SI-SP-7 & Sinclair Inlet & 2838-91 & Tissue & Walk-in Freezer & Isotopes, PCBs, Lipids, Metals & $5 / 17 / 2007$ & CS & Shiner perch \\
\hline SI-SP-3 & Sinclair Inlet & $2838-87$ & Tissue & Walk-in Freezer & Isotopes, PCBs, Lipids, Metals & $5 / 17 / 2007$ & CS & Shiner perch \\
\hline SI-GC-2 & Sinclair Inlet & $2838-93$ & Tissue & Walk-in Freezer & Isotopes, PCBs, Lipids, Metals & $5 / 17 / 2007$ & CS & Graceful Crab \\
\hline SI-GC-6 & Sinclair Inlet & $2838-97$ & Tissue & Walk-in Freezer & Isotopes, PCBs, Lipids, Metals & $5 / 17 / 2007$ & CS & Graceful Crab \\
\hline SI-GC-3 & Sinclair Inlet & $2838-94$ & Tissue & Walk-in Freezer & Isotopes, PCBs, Lipids, Metals & $5 / 17 / 2007$ & CS & Graceful Crab \\
\hline SI-GC-4 & Sinclair Inlet & 2838-95 & Tissue & Walk-in Freezer & Isotopes, PCBs, Lipids, Metals & $5 / 17 / 2007$ & CS & Graceful Crab \\
\hline SI-GC-7 & Sinclair Inlet & 2838-98 & Tissue & Walk-in Freezer & Isotopes, PCBs, Lipids, Metals & $5 / 17 / 2007$ & CS & Graceful Crab \\
\hline SI-GC-1 & Sinclair Inlet & $2838-92$ & Tissue & Walk-in Freezer & Isotopes, PCBs, Lipids, Metals & $5 / 17 / 2007$ & CS & Graceful Crab \\
\hline SI-GC-5 & Sinclair Inlet & $2838-96$ & Tissue & Walk-in Freezer & Isotopes, PCBs, Lipids, Metals & $5 / 17 / 2007$ & CS & Graceful Crab \\
\hline SI-GC-8 & Sinclair Inlet & 2838-99 & Tissue & Walk-in Freezer & Isotopes, PCBs, Lipids, Metals & $5 / 17 / 2007$ & CS & Graceful Crab \\
\hline SI-SC-COMP & Sinclair Inlet & $2838-100$ & Tissue & Walk-in Freezer & Isotopes, PCBs, Lipids, Metals & $5 / 17 / 2007$ & CS & Sea cucumber (6) \\
\hline PG-ES-1 & Port Gardner & 2838-101 & Tissue & Walk-in Freezer & Isotopes, PCBs, Lipids, Metals & $5 / 29 / 2007$ & CS & English sole adult \\
\hline PG-ES-2 & Port Gardner & 2838-102 & Tissue & Walk-in Freezer & Isotopes, PCBs, Lipids, Metals & $5 / 29 / 2007$ & CS & English sole adult \\
\hline PG-ES-3 & Port Gardner & 2838-103 & Tissue & Walk-in Freezer & Isotopes, PCBs, Lipids, Metals & $5 / 29 / 2007$ & CS & English sole adult \\
\hline NIS-ES-1 & Nisqually & 2838-104 & Tissue & Walk-in Freezer & Isotopes, PCBs, Lipids, Metals & $5 / 30 / 2007$ & CS & English sole adult \\
\hline NIS-ES-2 & Nisqually & 2838-105 & Tissue & Walk-in Freezer & Isotopes, PCBs, Lipids, Metals & $5 / 30 / 2007$ & CS & English sole adult \\
\hline NIS-ES-3 & Nisqually & 2838-106 & Tissue & Walk-in Freezer & Isotopes, PCBs, Lipids, Metals & $5 / 30 / 2007$ & CS & English sole adult \\
\hline NIS-RF-1 & Nisqually & 2838-107 & Tissue & Walk-in Freezer & Isotopes, PCBs, Lipids, Metals & $5 / 30 / 2007$ & CS & Spotted Ratfish \\
\hline NIS-RF-2 & Nisqually & 2838-108 & Tissue & Walk-in Freezer & Isotopes, PCBs, Lipids, Metals & $5 / 30 / 2007$ & CS & Spotted Ratfish \\
\hline NIS-RF-3 & Nisqually & 2838-109 & Tissue & Walk-in Freezer & Isotopes, PCBs, Lipids, Metals & $5 / 30 / 2007$ & CS & Spotted Ratfish \\
\hline CB-ES-1 & Commencement Ba & $2838-110$ & Tissue & Walk-in Freezer & Isotopes, PCBs, Lipids, Metals & $5 / 31 / 2007$ & CS & English sole adult \\
\hline CB-ES-2 & Commencement Ba & $2838-111$ & Tissue & Walk-in Freezer & Isotopes, PCBs, Lipids, Metals & $5 / 31 / 2007$ & CS & English sole adult \\
\hline CB-ES-3 & Commencement $\mathrm{Ba}$ & $2838-112$ & Tissue & Walk-in Freezer & Isotopes, PCBs, Lipids, Metals & $5 / 31 / 2007$ & CS & English sole adult \\
\hline
\end{tabular}


cc: Project Manager/Central File Login File
SAMPLE LOGIN

(SOP\# MSL-A-001)
Project Manager: Brandenberger

Date Received: May, June, Sept. 2007

Batch: Multiple

PROJEC 2008 ENVVEST Biota

\begin{tabular}{|c|c|c|c|c|c|c|c|c|}
\hline $\begin{array}{c}\text { SPONSOR } \\
\text { CODE }\end{array}$ & Site Description & $\begin{array}{c}\text { BATTELLE } \\
\text { CODE }\end{array}$ & MATRIX & $\begin{array}{l}\text { STORAGE } \\
\text { LOCATION }\end{array}$ & PARAMETERS REQUESTED* & $\begin{array}{c}\text { COLLECTION } \\
\text { DATE }\end{array}$ & INITIALS & Species/section \\
\hline CB-RF-1 & \multicolumn{2}{|c|}{ Commencement Ba' 2838-113 } & Tissue & Walk-in Freezer & Isotopes, PCBs, Lipids, Metals & $5 / 31 / 2007$ & CS & Spotted Ratfish \\
\hline CB-RF-2 & \multicolumn{2}{|c|}{ Commencement Ba' 2838-114 } & Tissue & Walk-in Freezer & Isotopes, PCBs, Lipids, Metals & $5 / 31 / 2007$ & CS & Spotted Ratfish \\
\hline CB-RF-3 & \multicolumn{2}{|c|}{ Commencement Ba' 2838-115 } & Tissue & Walk-in Freezer & Isotopes, PCBs, Lipids, Metals & $5 / 31 / 2007$ & CS & Spotted Ratfish \\
\hline DU-ES-1 & Duwamish & $2838-116$ & Tissue & Walk-in Freezer & Isotopes, PCBs, Lipids, Metals & $5 / 18 / 2007$ & CS & English sole adult \\
\hline DU-ES-2 & Duwamish & $2838-117$ & Tissue & Walk-in Freezer & Isotopes, PCBs, Lipids, Metals & $5 / 18 / 2007$ & CS & English sole adult \\
\hline DU-ES-3 & Duwamish & 2838-118 & Tissue & Walk-in Freezer & Isotopes, PCBs, Lipids, Metals & $5 / 18 / 2007$ & CS & English sole adult \\
\hline DU-ES-4 & Duwamish & 2838-119 & Tissue & Walk-in Freezer & Isotopes, PCBs, Lipids, Metals & $5 / 18 / 2007$ & CS & English sole adult \\
\hline DF-EM-1 & ADUW-DOG01 & $2838-126$ & Tissue & Walk-in Freezer & Isotopes, PCBs, Lipids, Metals & $9 / 18 / 2007$ & CS & embryo mass (g) \\
\hline DF-EM-2 & ADUW-DOG02 & $2838-127$ & Tissue & Walk-in Freezer & Isotopes, PCBs, Lipids, Metals & $9 / 18 / 2007$ & CS & embryo mass (g) \\
\hline DF-EM-3 & ADUW-DOG03 & $2838-128$ & Tissue & Walk-in Freezer & Isotopes, PCBs, Lipids, Metals & $9 / 18 / 2007$ & CS & embryo mass (g) \\
\hline DF-EM-4 & ADUW-DOG04 & $2838-129$ & Tissue & Walk-in Freezer & Isotopes, PCBs, Lipids, Metals & $9 / 18 / 2007$ & CS & embryo mass (g) \\
\hline DF-EM-5 & ADUW-DOG05 & $2838-130$ & Tissue & Walk-in Freezer & Isotopes, PCBs, Lipids, Metals & 9/18/2007 & CS & embryo mass (g) \\
\hline DF-EM-6 & ADUW-DOG06 & 2838-131 & Tissue & Walk-in Freezer & Isotopes, PCBs, Lipids, Metals & 9/18/2007 & CS & embryo mass (g) \\
\hline DF-LV-1 & ADUW-DOG01 & $2838-132$ & Tissue & Walk-in Freezer & Isotopes, PCBs, Lipids, Metals & 9/18/2007 & CS & liver (g) \\
\hline DF-LV-2 & ADUW-DOG02 & $2838-133$ & Tissue & Walk-in Freezer & Isotopes, PCBs, Lipids, Metals & 9/18/2007 & CS & liver (g) \\
\hline DF-LV-3 & ADUW-DOG03 & $2838-134$ & Tissue & Walk-in Freezer & Isotopes, PCBs, Lipids, Metals & 9/18/2007 & CS & liver (g) \\
\hline DF-LV-4 & ADUW-DOG04 & 2838-135 & Tissue & Walk-in Freezer & Isotopes, PCBs, Lipids, Metals & 9/18/2007 & CS & liver (g) \\
\hline DF-LV-5 & ADUW-DOG05 & $2838-136$ & Tissue & Walk-in Freezer & Isotopes, PCBs, Lipids, Metals & $9 / 18 / 2007$ & CS & liver (g) \\
\hline DF-LV-6 & ADUW-DOG06 & $2838-137$ & Tissue & Walk-in Freezer & Isotopes, PCBs, Lipids, Metals & 9/18/2007 & CS & liver (g) \\
\hline DF-DIG-1 & ADUW-DOG01 & 2838-138 & Tissue & Walk-in Freezer & Isotopes, PCBs, Lipids, Metals & $9 / 18 / 2007$ & CS & digestive tract - All \\
\hline DF-DIG-2 & ADUW-DOG02 & 2838-139 & Tissue & Walk-in Freezer & Isotopes, PCBs, Lipids, Metals & $9 / 18 / 2007$ & CS & digestive tract - All \\
\hline DF-DIG-3 & ADUW-DOG03 & $2838-140$ & Tissue & Walk-in Freezer & Isotopes, PCBs, Lipids, Metals & $9 / 18 / 2007$ & CS & digestive tract - All \\
\hline DF-DIG-4 & ADUW-DOG04 & 2838-141 & Tissue & Walk-in Freezer & Isotopes, PCBs, Lipids, Metals & $9 / 18 / 2007$ & CS & digestive tract - All \\
\hline DF-DIG-5 & ADUW-DOG05 & 2838-142 & Tissue & Walk-in Freezer & Isotopes, PCBs, Lipids, Metals & 9/18/2007 & CS & digestive tract - All \\
\hline DF-DIG-6 & ADUW-DOG06 & $2838-143$ & Tissue & Walk-in Freezer & Isotopes, PCBs, Lipids, Metals & $9 / 18 / 2007$ & CS & digestive tract - All \\
\hline DF-REP-1 & ADUW-DOG01 & 2838-144 & Tissue & Walk-in Freezer & Isotopes, PCBs, Lipids, Metals & $9 / 18 / 2007$ & CS & reproductive organ? \\
\hline DF-REP-2 & ADUW-DOG02 & $2838-145$ & Tissue & Walk-in Freezer & Isotopes, PCBs, Lipids, Metals & $9 / 18 / 2007$ & CS & reproductive organ? \\
\hline DF-REP-3 & ADUW-DOG03 & $2838-146$ & Tissue & Walk-in Freezer & Isotopes, PCBs, Lipids, Metals & $9 / 18 / 2007$ & CS & reproductive organ? \\
\hline
\end{tabular}


cc: Project Manager/Central File Login File
SAMPLE LOGIN

(SOP\# MSL-A-001)
Project Manager: Brandenberger

Date Received: May, June, Sept. 2007

Batch: Multiple

PROJEC 2008 ENVVEST Biota

\begin{tabular}{|c|c|c|c|c|c|c|c|c|}
\hline $\begin{array}{c}\text { SPONSOR } \\
\text { CODE }\end{array}$ & Site Description & $\begin{array}{l}\text { BATTELLE } \\
\text { CODE }\end{array}$ & MATRIX & $\begin{array}{l}\text { STORAGE } \\
\text { LOCATION }\end{array}$ & PARAMETERS REQUESTED* & \begin{tabular}{|} 
COLLECTION \\
DATE
\end{tabular} & INITIALS & Species/section \\
\hline DF-REP-4 & ADUW-DOG04 & $2838-147$ & Tissue & Walk-in Freezer & Isotopes, PCBs, Lipids, Metals & $9 / 18 / 2007$ & $\mathrm{CS}$ & reproductive organ? \\
\hline DF-REP-5 & ADUW-DOG05 & 2838-148 & Tissue & Walk-in Freezer & Isotopes, PCBs, Lipids, Metals & $9 / 18 / 2007$ & CS & reproductive organ? \\
\hline DF-REP-6 & ADUW-DOG06 & 2838-149 & Tissue & Walk-in Freezer & Isotopes, PCBs, Lipids, Metals & 9/18/2007 & CS & reproductive organ? \\
\hline DF-HEAD-1 & ADUW-DOG01 & $2838-150$ & Tissue & Walk-in Freezer & Isotopes, PCBs, Lipids, Metals & $9 / 18 / 2007$ & CS & Head \\
\hline DF-HEAD-2 & ADUW-DOG02 & 2838-151 & Tissue & Walk-in Freezer & Isotopes, PCBs, Lipids, Metals & 9/18/2007 & CS & Head \\
\hline DF-HEAD-3 & ADUW-DOG03 & 2838-152 & Tissue & Walk-in Freezer & Isotopes, PCBs, Lipids, Metals & 9/18/2007 & CS & Head \\
\hline DF-HEAD-4 & ADUW-DOG04 & $2838-153$ & Tissue & Walk-in Freezer & Isotopes, PCBs, Lipids, Metals & 9/18/2007 & CS & Head \\
\hline DF-HEAD-5 & ADUW-DOG05 & 2838-154 & Tissue & Walk-in Freezer & Isotopes, PCBs, Lipids, Metals & 9/18/2007 & CS & Head \\
\hline DF-HEAD-6 & ADUW-DOG06 & 2838-155 & Tissue & Walk-in Freezer & Isotopes, PCBs, Lipids, Metals & 9/18/2007 & CS & Head \\
\hline DF-PEC-1 & ADUW-DOG01 & 2838-156 & Tissue & Walk-in Freezer & Isotopes, PCBs, Lipids, Metals & 9/18/2007 & CS & pectral area $(\mathrm{g})$ \\
\hline DF-PEC-2 & ADUW-DOG02 & 2838-157 & Tissue & Walk-in Freezer & Isotopes, PCBs, Lipids, Metals & 9/18/2007 & CS & pectral area $(\mathrm{g})$ \\
\hline DF-PEC-3 & ADUW-DOG03 & 2838-158 & Tissue & Walk-in Freezer & Isotopes, PCBs, Lipids, Metals & 9/18/2007 & CS & pectral area $(\mathrm{g})$ \\
\hline DF-PEC-4 & ADUW-DOG04 & 2838-159 & Tissue & Walk-in Freezer & Isotopes, PCBs, Lipids, Metals & 9/18/2007 & CS & pectral area $(\mathrm{g})$ \\
\hline DF-PEC-5 & ADUW-DOG05 & $2838-160$ & Tissue & Walk-in Freezer & Isotopes, PCBs, Lipids, Metals & 9/18/2007 & CS & pectral area $(\mathrm{g})$ \\
\hline DF-PEC-6 & ADUW-DOG06 & 2838-161 & Tissue & Walk-in Freezer & Isotopes, PCBs, Lipids, Metals & 9/18/2007 & CS & pectral area $(\mathrm{g})$ \\
\hline DF-MID-1 & ADUW-DOG01 & 2838-162 & Tissue & Walk-in Freezer & Isotopes, PCBs, Lipids, Metals & 9/18/2007 & CS & midsecton $(\mathrm{g})$ \\
\hline DF-MID-2 & ADUW-DOG02 & $2838-163$ & Tissue & Walk-in Freezer & Isotopes, PCBs, Lipids, Metals & $9 / 18 / 2007$ & CS & midsecton $(\mathrm{g})$ \\
\hline DF-MID-3 & ADUW-DOG03 & 2838-164 & Tissue & Walk-in Freezer & Isotopes, PCBs, Lipids, Metals & $9 / 18 / 2007$ & CS & midsecton $(\mathrm{g})$ \\
\hline DF-MID-4 & ADUW-DOG04 & 2838-165 & Tissue & Walk-in Freezer & Isotopes, PCBs, Lipids, Metals & $9 / 18 / 2007$ & CS & midsecton $(\mathrm{g})$ \\
\hline DF-MID-5 & ADUW-DOG05 & 2838-166 & Tissue & Walk-in Freezer & Isotopes, PCBs, Lipids, Metals & 9/18/2007 & CS & midsecton (g) \\
\hline DF-MID-6 & ADUW-DOG06 & $2838-167$ & Tissue & Walk-in Freezer & Isotopes, PCBs, Lipids, Metals & $9 / 18 / 2007$ & CS & midsecton (g) \\
\hline DF-TAIL-1 & ADUW-DOG01 & $2838-168$ & Tissue & Walk-in Freezer & Isotopes, PCBs, Lipids, Metals & $9 / 18 / 2007$ & CS & tail $(g)$ \\
\hline DF-TAIL-2 & ADUW-DOG02 & 2838-169 & Tissue & Walk-in Freezer & Isotopes, PCBs, Lipids, Metals & $9 / 18 / 2007$ & CS & tail (g) \\
\hline DF-TAIL-3 & ADUW-DOG03 & $2838-170$ & Tissue & Walk-in Freezer & Isotopes, PCBs, Lipids, Metals & $9 / 18 / 2007$ & CS & tail (g) \\
\hline DF-TAIL-4 & ADUW-DOG04 & $2838-171$ & Tissue & Walk-in Freezer & Isotopes, PCBs, Lipids, Metals & 9/18/2007 & CS & tail (g) \\
\hline DF-TAIL-5 & ADUW-DOG05 & 2838-172 & Tissue & Walk-in Freezer & Isotopes, PCBs, Lipids, Metals & $9 / 18 / 2007$ & CS & tail (g) \\
\hline DF-TAIL-6 & ADUW-DOG06 & $2838-173$ & Tissue & Walk-in Freezer & Isotopes, PCBs, Lipids, Metals & $9 / 18 / 2007$ & CS & tail $(g)$ \\
\hline DF-SWC-1 & ADUW-DOG01 & 2838-174 & Tissue & Walk-in Freezer & Isotopes, PCBs, Lipids, Metals & 9/18/2007 & CS & Section Weighted-Composite \\
\hline
\end{tabular}


cc: Project Manager/Central File Login File
SAMPLE LOGIN

(SOP\# MSL-A-001)
Project Manager: Brandenberger

Date Received: May, June, Sept. 2007

Batch: Multiple

\section{PROJEC 2008 ENVVEST Biota}

\begin{tabular}{|c|c|c|c|c|c|c|c|c|}
\hline $\begin{array}{l}\text { SPONSOR } \\
\text { CODE }\end{array}$ & Site Description & $\begin{array}{l}\text { BATTELLE } \\
\text { CODE }\end{array}$ & MATRIX & $\begin{array}{l}\text { STORAGE } \\
\text { LOCATION }\end{array}$ & PARAMETERS REQUESTED* & \begin{tabular}{|} 
COLLECTION \\
DATE
\end{tabular} & INITIALS & Species/section \\
\hline DF-SWC-2 & ADUW-DOG02 & $2838-175$ & Tissue & Walk-in Freezer & Isotopes, PCBs, Lipids, Metals & $9 / 18 / 2007$ & CS & Section Weighted-Composite \\
\hline DF-SWC-6 & ADUW-DOG06 & 2838-176 & Tissue & Walk-in Freezer & Isotopes, PCBs, Lipids, Metals & $9 / 18 / 2007$ & CS & Section Weighted-Composite \\
\hline DF-SWC-4 & ADUW-DOG04 & 2838-183 & Tissue & Walk-in Freezer & Isotopes, PCBs, Lipids, Metals & $9 / 18 / 2007$ & CS & Section Weighted-Composite \\
\hline DF-WBWC-1 & ADUW-DOG01 & 2838-177 & Tissue & Walk-in Freezer & Isotopes, PCBs, Lipids, Metals & 9/18/2007 & CS & Whole Body Weighted-Composite \\
\hline DF-WBWC-2 & ADUW-DOG02 & 2838-178 & Tissue & Walk-in Freezer & Isotopes, PCBs, Lipids, Metals & $9 / 18 / 2007$ & CS & Whole Body Weighted-Composite \\
\hline DF-WBWC-3 & ADUW-DOG03 & 2838-179 & Tissue & Walk-in Freezer & Isotopes, PCBs, Lipids, Metals & $9 / 18 / 2007$ & CS & Whole Body Weighted-Composite \\
\hline DF-WBWC-4 & ADUW-DOG04 & $2838-180$ & Tissue & Walk-in Freezer & Isotopes, PCBs, Lipids, Metals & $9 / 18 / 2007$ & CS & Whole Body Weighted-Composite \\
\hline DF-WBWC-5 & ADUW-DOG05 & 2838-181 & Tissue & Walk-in Freezer & Isotopes, PCBs, Lipids, Metals & $9 / 18 / 2007$ & CS & Whole Body Weighted-Composite \\
\hline DF-WBWC-6 & ADUW-DOG06 & 2838-182 & Tissue & Walk-in Freezer & Isotopes, PCBs, Lipids, Metals & $9 / 18 / 2007$ & CS & Whole Body Weighted-Composite \\
\hline DF-SWC-4 & ADUW-DOG04 & 2838-183 & Tissue & Walk-in Freezer & Isotopes, PCBs, Lipids, Metals & $9 / 18 / 2007$ & CS & Section Weighted-Composite \\
\hline
\end{tabular}

* actual analysis dependent on client approvals and amount of material available 


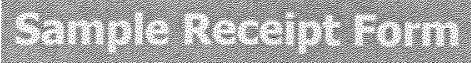

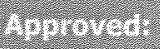

Project Number:

Client:

Received by:

Seyfert, Jeannine

Date/Time Received: Tuesday, January 08, 2008 12:00 AM

No. of Shipping Containers:

1

\section{SHIPMENT}

Method of Delivery:

coc Forms:

Commercial Carrier

Tracking Number: $\quad 7914-7145-6387$

\section{Cooler(s)/Box(es)}

\begin{tabular}{|c|c|c|c|c|c|c|c|}
\hline Whis & 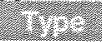 & 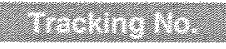 & 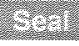 & 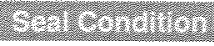 & 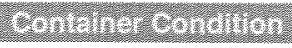 & 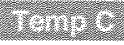 & $4 x^{2}+x^{2}$ \\
\hline 1 of 1 & Cooler & $7914-7145-6387$ & Tape & Intact & Intact & 0.2 & 40 \\
\hline
\end{tabular}

\section{Samples}

Sample Labels:

Container Seals:

Condition of Samples:
Sample labels agree with $\mathrm{COC}$ forms

Discrepancies (see Sample Custody Corrective Action Form)

$$
\begin{aligned}
& \square \text { Tape } \square \text { Custody Seals } \square \text { Other Seals (See sample Log) } \\
& \checkmark \text { Seals intact for each shipping container } \\
& \square \text { Seals broken (See sample log for impacted samples) }
\end{aligned}
$$

\section{No Forms}


coc Client:

COC Project: ENVVEST - 2008 Biota

COC Date: $1 / 8 / 20081: 23: 00$ PM

\section{Description of Problem:}

Explanation:

Client Id Either label or C-O-C cannot be verified

The jar for sample Q1537 (HC-SP2) does not have a label. Marker was used to write " 41 " on the lid of the jar. This most likely corresponds to the MSL Lab ID which is listed as "2838-41" on the COC.

The jar for sample Q1538 (HC-GC-1) does not have a label. Marker was used to write "43" on the lid of the jar. This most likely corresponds to the MSL Lab ID which is listed as "2838-43" on the COC.

Temperature Receipt temperature outside of acceptability and The cooler was received at a temperature Preservation of 0.2 degrees $C$. This is warmer than the 10 degree $C$ temperature cut off for frozen sample receipt.

Documentation of project manager notification

$\begin{array}{llll}\text { Sample Custodian } & \text { Seyfert, Jeannine } & \text { Date: } 1 / 8 / 2008 \text { 2:02:00 PM } \\ \text { Laboratory Manager: } & \text { Thorn, Jonathan } & \text { Date: } 1 / 29 / 2008 \text { 1:02:00 PM } \\ \text { Project Manager: } & \text { Peven-McCarthy, Carole } & \text { Date: } 1 / 29 / 2008 \text { 12:36:00 PM }\end{array}$

Documentation of client notification (should be completed by project manager within 24 hrs):

On

I contacted Brandenberger, Jill

at Aqua Survey

Results of communication with client (Describe any corrective action directed by the client):

PM contacted. Use hand-written ID. Temp discrepancy noted.

Date this form was received back to the custodian:

Reference Number: 


\section{Baftelle}

ShpNo

Project Number:

Client:

Received by:

Seyfert, Jeannine

Date/Time Received: Tuesday, January 08, 2008 12:00 AM

No. of Shipping Containers: 1

\begin{tabular}{|c|c|c|c|c|c|c|c|c|c|c|c|c|}
\hline BDO Id: & Client Sample ID: & Collection Date: & Login Date: & Ctrs: & Matrix: & Temp: & $\mathrm{pH}:$ & TRC: & VOC: & Stored In: & Loc: & No: Comments: \\
\hline Q1521 & V-ES-5 & $05 / 01 / 070: 00$ & $01 / 08 / 0813: 43$ & 1 & TISSUE & 0.2 & NA & NA & NA & F0002 (Walk-in) & $B I N$ & 22 \\
\hline Q1522 & V-ES-6 & $05 / 01 / 070: 00$ & $01 / 08 / 0813: 43$ & 1 & TISSUE & 0.2 & NA & NA & NA & Fo002 (Walk-in) & BIN & 22 \\
\hline Q1523 & V-ES-8 & $05 / 01 / 070: 00$ & 01/08/08 13:43 & 1 & TISSUE & 0.2 & NA & NA & NA & F0002 (Walk-in) & $B I N$ & 22 \\
\hline Q1524 & $V-R S-1$ & $05 / 01 / 070.00$ & $01 / 08 / 0813: 44$ & 1 & TISSUE & 0.2 & NA & NA & NA & Foo02 (Walk-in) & BIN & 22 \\
\hline Q1525 & V-RS-2 & $05 / 01 / 070: 00$ & $01 / 08 / 0813: 44$ & 1 & TISSUE & 0.2 & NA & NA & NA & F0002 (Walk-in) & $B I N$ & 22 \\
\hline Q1526 & V-RS-3 & $05 / 01 / 070: 00$ & $01 / 08 / 08.13: 44$ & 1 & TISSUE & 0.2 & NA & NA & NA & Foo02 (Walk-in) & $B I N$ & 22 \\
\hline Q1527 & $V-S S C-1$ & 05/01/07 0:00 & $01 / 08 / 0813: 44$ & 1 & TISSUE & 0.2 & NA & NA & NA & F0002 (Walk-in) & $B I N$ & 22 \\
\hline Q1529 & V-SSc-3 & 05/01/07 0:00 & $01 / 08 / 0813: 45$ & 1 & TISSUE & 0.2 & NA & NA & NA & F0002 (Walk-in) & $B I N$ & 22 \\
\hline Q1530 & $V-S P-1$ & 05/01/07 0:00 & $01 / 08 / 0813: 46$ & 1 & TISSUE & 0.2 & NA & NA & NA & F0002 (Walk-in) & BIN & 22 \\
\hline Q1531 & V-SP-2 & $05 / 01 / 070: 00$ & $01 / 08 / 0813: 46$ & 1 & TISSUE & 0.2 & NA & NA & NA & Foo02 (Walk-in) & BIN & 22 \\
\hline Q1532 & $V-S P-3$ & 05/01/07 0:00 & $01 / 08 / 08 \quad 13: 46$ & 1 & TISSUE & 0.2 & NA & NA & NA & F0002 (Walk-in) & BIN & 22 \\
\hline Q1533 & $S G-E S-3$ & $05 / 02 / 070: 00$ & $01 / 08 / 0813: 46$ & 1 & TISSUE & 0.2 & NA & NA & NA & F0002 (Walk-in) & $\mathrm{BIN}$ & 22 \\
\hline Q1534 & SG-ES-4 & $05 / 02 / 070: 00$ & $01 / 08 / 0813: 47$ & 1 & TISSUE & 0.2 & NA & NA & NA & F0002 (Walk-in) & BIN & 22 \\
\hline Q1535 & SG-ES-5 & $05 / 02 / 070: 00$ & $01 / 08 / 08 \quad 13: 47$ & 1 & TISSUE & 0.2 & NA & NA & NA & F0002 (Walk-in) & BIN & 22 \\
\hline Q1536 & HC-SP1 & $05 / 03 / 070: 00$ & $01 / 08 / 0813: 47$ & 1 & TISSUE & 0.2 & NA & NA & NA & F0002 (Walk-in) & BIN & 22 \\
\hline Q1538 & $\mathrm{HC}-\mathrm{GC}-1$ & 05/03/07 0:00 & $01 / 08 / 0813: 48$ & 1 & TISSUE & 0.2 & NA & NA & NA & F0002 (Walk-in) & BIN & 22 \\
\hline Q1539 & SI-ES-1 & $05 / 17 / 070: 00$ & 01/08/08 13:49 & 1 & TISSUE & 0.2 & NA & NA & NA & F0002 (Walk-in) & BIN & 22 \\
\hline Q1540 & SI-ES-2 & $05 / 17 / 070: 00$ & $01 / 08 / 0813: 49$ & 1 & TISSUE & 0.2 & NA & NA & NA & F0002 (Walk-in) & $B I N$ & 22 \\
\hline Q1541 & SI-ES-4 & $05 / 17 / 070: 00$ & $01 / 08 / 0813: 50$ & 1 & TISSUE & 0.2 & NA & NA & NA & F0002 (Walk-in) & $\mathrm{BIN}$ & 22 \\
\hline Q1542 & Sl-ES-6 & $05 / 17 / 070: 00$ & $01 / 08 / 0813: 50$ & 1 & TISSUE & 0.2 & NA & NA & NA & F0002 (Walk-in) & BIN & 22 \\
\hline Q1543 & SI-ES-7 & $05 / 17 / 070: 00$ & $01 / 08 / 0813: 50$ & 1 & TISSUE & 0.2 & NA & NA & NA & F0002 (Walk-in) & BIN & 22 \\
\hline Q1544 & SI-ES-8 & $05 / 17 / 070: 00$ & $01 / 08 / 0813: 50$ & 1 & TISSUE & 0.2 & NA & NA & NA & F0002 (Walk-in) & $B I N$ & 22 \\
\hline Q1545 & SI-RS-1_2 & $05 / 17 / 070: 00$ & $01 / 08 / 0813: 51$ & 1 & TISSUE & 0.2 & NA & NA & NA & F0002 (Walk-in) & BIN & 22 \\
\hline Q1546 & SI-RS-3 & $05 / 17 / 070: 00$ & $01 / 08 / 08 \quad 13: 51$ & 1 & TISSUE & 0.2 & NA & NA & NA & F0002 (Walk-in) & BIN & 22 \\
\hline Q1547 & SI-RS-4 & $05 / 17 / 070: 00$ & $01 / 08 / 0813: 51$ & 1 & TISSUE & 0.2 & NA & NA & NA & Foo02 (Walk-in) & $\mathrm{BIN}$ & 22 \\
\hline Q1548 & SI-SS-1 & $05 / 17 / 070: 00$ & $01 / 08 / 0813: 52$ & 1 & TISSUE & 0.2 & NA & NA & NA & F0002 (Walk-in) & BIN & 22 \\
\hline
\end{tabular}




\section{Battelle}

The Business of Innovation

ShpNo SHP-080108-01

Battelle Project No:

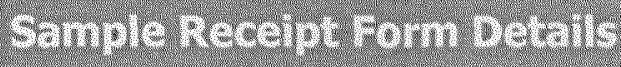

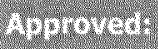

\section{Project Number:}

\section{Client:}

Received by:

Seyfert, Jeannine

Date/Time Received: Tuesday, January 08, 2008 12:00 AM

No. of Shipping Containers: 1

\begin{tabular}{|c|c|c|c|c|c|c|c|c|c|c|c|c|}
\hline BDO Id: & Client Sample ID: & Collection Date: & Login Date: & Ctrs: & Matrix: & Temp: & $\mathrm{pH}:$ & TRC: & VOC: & Stored In: & Loc: & No: Comments: \\
\hline Q1549 & SI-SS-2 & $05 / 17 / 070: 00$ & $01 / 08 / 08 \quad 13: 52$ & 1 & TISSUE & 0.2 & NA & NA & NA & F0002 (Walk-in) & BIN & 22 \\
\hline Q1550 & SI-SS-3 & $05 / 17 / 070: 00$ & $01 / 08 / 0813: 52$ & 1 & TISSUE & 0.2 & NA & NA & NA & F0002 (Walk-in) & BIN & 22 \\
\hline Q1551 & $S I-S S C-1 / 2$ & $05 / 17 / 070: 00$ & $01 / 08 / 08: 13: 52$ & 1 & TISSUE & 0.2 & NA & NA & NA & F0002 (Walk-in) & BIN & 22 \\
\hline Q1552 & SI-SSc-3_5 & $05 / 17 / 070: 00$ & $01 / 08 / 0813: 53$ & 1 & TISSUE & 0.2 & NA & NA & NA & F0002 (Walk-in) & BIN & 22 \\
\hline Q1553 & SI-SSc-4_6 & 05/17/07 0:00 & $01 / 08 / 0813: 53$ & 1 & TISSUE & 0.2 & NA & NA & NA & Fo002 (Walk-in) & $B I N$ & 22 \\
\hline Q1554 & SI-SP-4 5 & $05 / 17 / 070: 00$ & $01 / 08 / 08 \quad 13: 54$ & 1 & TISSUE & 0.2 & NA & NA & NA & F0002 (Walk-in) & $B I N$ & 22 \\
\hline Q1555 & SI-SP-6_2 & $05 / 17 / 070: 00$ & $01 / 08 / 08+13: 54$ & 1 & TISSUE & 0.2 & NA & NA & NA & F0002 (Walk-in) & BIN & 22 \\
\hline Q1557 & SI-GC-2 6 & $05 / 17 / 070: 00$ & $01 / 08 / 0813: 55$ & 1 & TISSUE & 0.2 & NA & NA & NA & F0002 (Walk-in) & $B I N$ & 22 \\
\hline Q1558 & SI-GC-3 4 & $05 / 17 / 070: 00$ & $01 / 08 / 0813: 55$ & 1 & TISSUE & 0.2 & NA & NA & NA & F0002 (Walk-in) & $\mathrm{BIN}$ & 22 \\
\hline Q1559 & SI-GC-7 1 & $05 / 17 / 070: 00$ & $01 / 08 / 0813: 55$ & 1 & TISSUE & 0.2 & NA & NA & NA & F0002 (Walk-in) & BIN & 22 \\
\hline Q1560 & SI-GC-5.8 & $05 / 17 / 070: 00$ & 01/08/08 13:56 & 1 & TISSUE & 0.2 & NA & NA & NA & F0002 (Walk-in) & $B I N$ & 22 \\
\hline
\end{tabular}

Total Samples:

40 


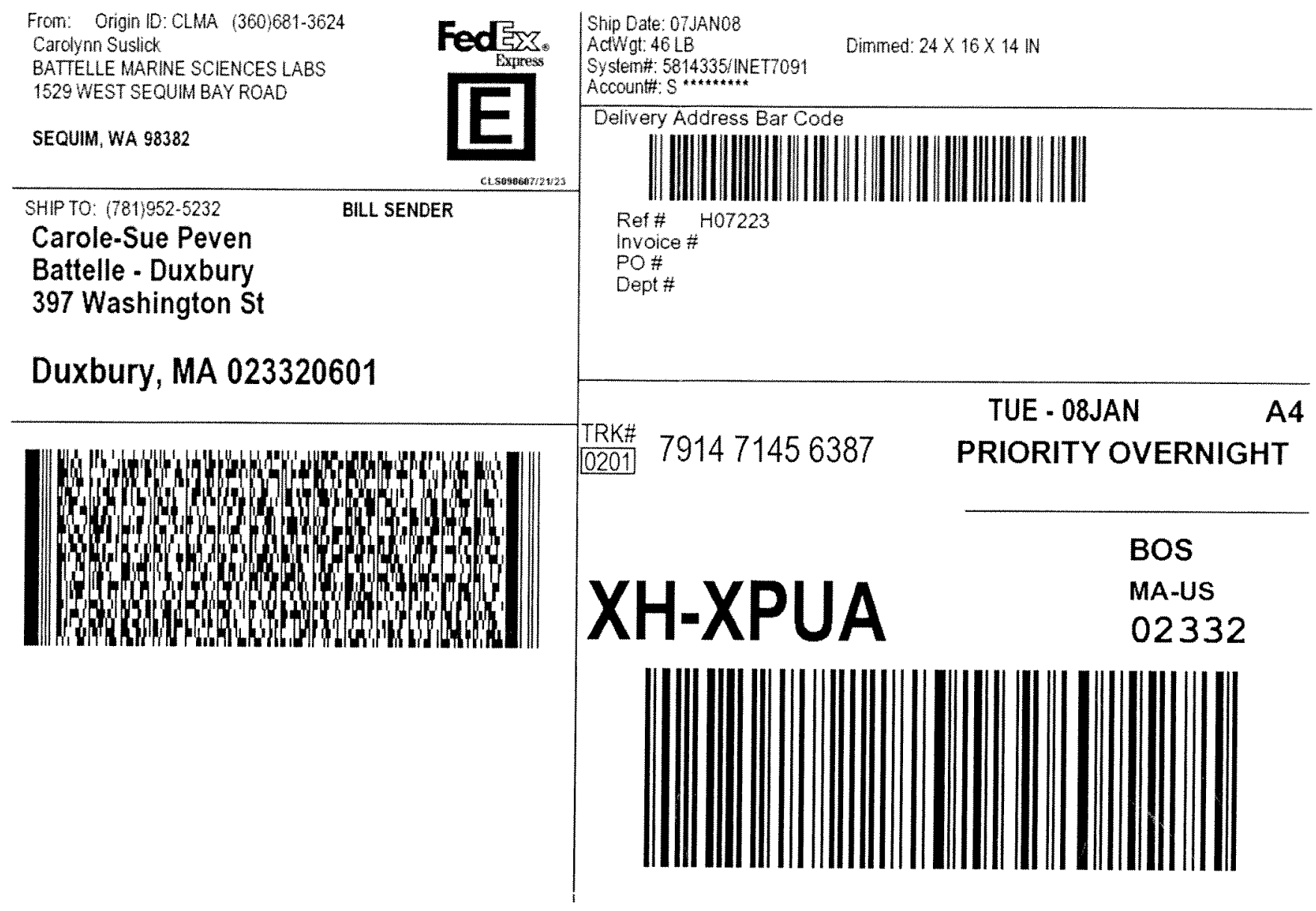

Shiphing Label: You whments is complete

1. Use the 'Print' feature from your browser to send this page to your laser or inkjet printer.

2. Fold the printed page along the horizontal line.

3. Place label in shipping pouch and affix it to your shipment so that the barcode portion of the label can be read and scanned.

Warning: Use only the printed original label for shipping. Using ahotocopy of this label for shipping purposes is fraudulent and could result in additional billing charges, along with the cancellation of your FedEx account number.

Use of this system constitutes your agreement to the service conditions in the current FedEx Service Guide, available on fedex.com.

FedEx will not be responsible for any claim in excess of $\$ 100$ per package, whether the result of loss, damage, delay, non-delivery, misdelivery, or misinformation, unless you declare a higher value, pay an additional charge, document your actual loss and file a timely claim. Limitations found in the current FedEx Service Guide apply. Your right to recover from FedEx for any loss, including intrinsic value of the package, loss of sales, income interest, profit, attorney's fees, costs, and other forms of damage whether direct, incidental, consequential, or special is limited to the greater of $\$ 100$ or the authorized declared value. Recovery cannot exceed actual documented loss. Maximum for items of extraordinary value is $\$ 500$, e.g. jewelry, precious metals, negotiable instruments and other items listed in our Service Guide. Written claims must be filed within strict time limits, see current FedEx Service Guide. 


\section{SAMPLE CUSTODY RECORD}

(SOP\#: MSL-A-001 \& MSL-A-002)

Date:

$1 / 7 / 2008$

Project Name: ENVVEST - 2008 Biota

Project Manager: J.Brandenberger

Phone Number: 360-681-4654

Shipment Method: Fedex Priority Overnight

Preservation: Frozen, on blue ice

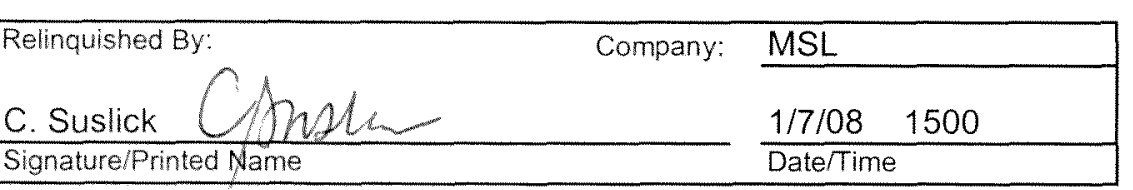

Signature/Printed Name

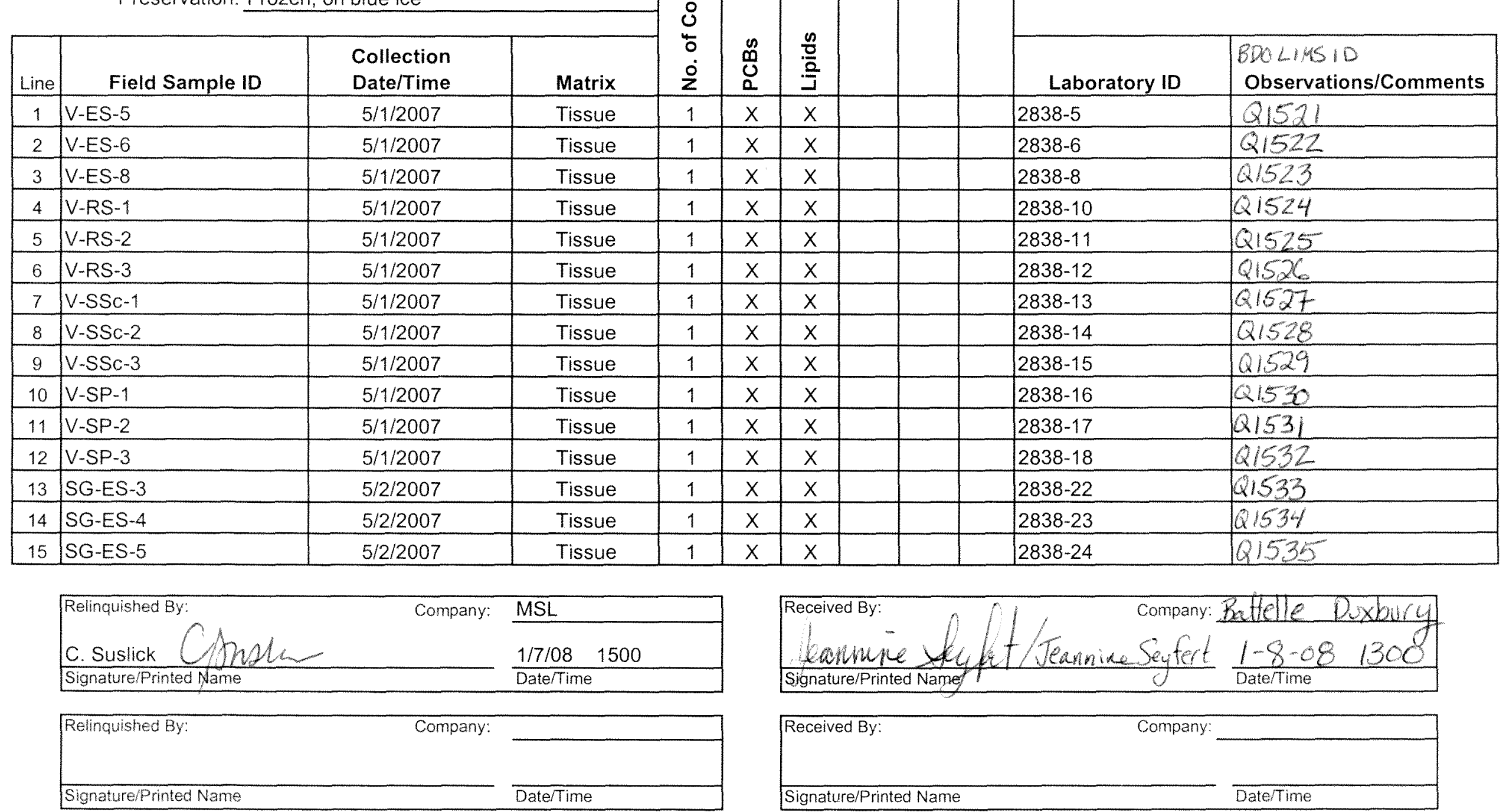

\section{Battelle}

.. Pulling Technology To Work

Pacific Northwest Division

Marine Sciences Laboratory

1529 West Sequim Bay Road

Sequim, Washington 98382

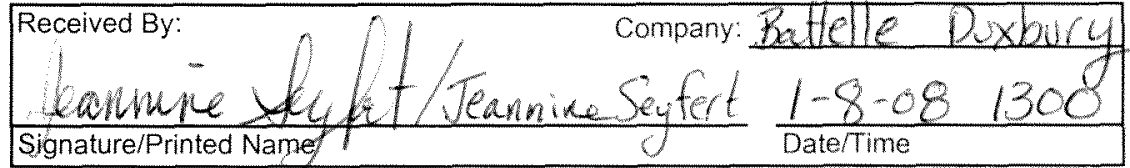

\begin{tabular}{|ll|}
\hline Received By: & Company: \\
\hline Signature/Printed Name & Date/Time \\
\hline
\end{tabular}




\section{SAMPLE CUSTODY RECORD}

(SOP\#: MSL-A-001 \& MSL-A-002)

Project Name: ENVVEST - 2008 Biota Project Manager: J. Brandenberger

Phone Number: $360-681-4654$

Shipment Method: Fedex Priority Overnight

Preservation: Frozen, on blue ice

\begin{tabular}{|c|c|c|c|c|}
\hline Line & Field Sample ID & $\begin{array}{l}\text { Collection } \\
\text { Date/Time }\end{array}$ & Matrix & i் \\
\hline 1 & HC-SP1 & $5 / 3 / 2007$ & Tissue & 1 \\
\hline 2 & HC-SP2 & $5 / 3 / 2007$ & Tissue & 1 \\
\hline 3 & HC-GC-1 & $5 / 3 / 2007$ & Tissue & 1 \\
\hline 4 & SI-ES-1 & $5 / 17 / 2007$ & Tissue & 1 \\
\hline 5 & SI-ES-2 & $5 / 17 / 2007$ & Tissue & 1 \\
\hline 6 & SI-ES-4 & $5 / 17 / 2007$ & Tissue & 1 \\
\hline 7 & SI-ES-6 & $5 / 17 / 2007$ & Tissue & 1 \\
\hline 8 & SI-ES-7 & $5 / 17 / 2007$ & Tissue & 1 \\
\hline 9 & SI-ES-8 & $5 / 17 / 2007$ & Tissue & 1 \\
\hline 10 & SI-RS-1 2 & $5 / 17 / 2007$ & Tissue & 1 \\
\hline 11 & SI-RS-3 & $5 / 17 / 2007$ & Tissue & 1 \\
\hline 12 & SI-RS-4 & $5 / 17 / 2007$ & Tissue & 1 \\
\hline 13 & SI-SS-1 & $5 / 17 / 2007$ & Tissue & 1 \\
\hline 14 & SI-SS-2 & $5 / 17 / 2007$ & Tissue & 1 \\
\hline 15 & SI-SS-3 & $5 / 17 / 2007$ & Tissue & 1 \\
\hline & Relinquished By: & Company: & MSL & \\
\hline & C. Suslick & & $1 / 7 / 08 \quad 1500$ & \\
\hline
\end{tabular}

\begin{tabular}{|ll|}
\hline Relinquished By: & Company: \\
\hline Signature/Printed Name & \\
\hline
\end{tabular}

BDO LIMS 10 Observations/Comments 2838-40 2838-41 2838-43 2838-58 2838-59 2838-61 2838-63 2838-64 2838-65 2838-67 68 2838-69 2838-70 2838-71 2838-72 2838-73

Pacific Northwest Division Marine Sciences Laboratory 1529 West Sequim Bay Road Sequim, Washington 98382

Q1536

Q1537

Q1538

Q1539

Q1540

Q1541

Q1542

Q1543

Q1544

Q1545

Q1546

Q1547

Q1548

Q1549

$Q 1550$

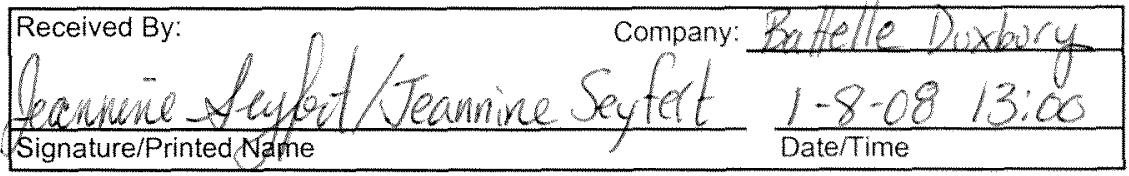

\begin{tabular}{|ll|}
\hline Received By: & Company: \\
\hline Signature/Printed Name & \\
\hline
\end{tabular}

Page $2 \quad 1873$ 


\section{SAMPLE CUSTODY RECORD}

(SOP\#: MSL-A-001 \& MSL-A-002)

Project Name: ENVVEST - 2008 Biota

Project Manager: J.Brandenberger

Phone Number: 360-681-4654

Shipment Method: Fedex Priority Overnight

Preservation: Frozen, on blue ice

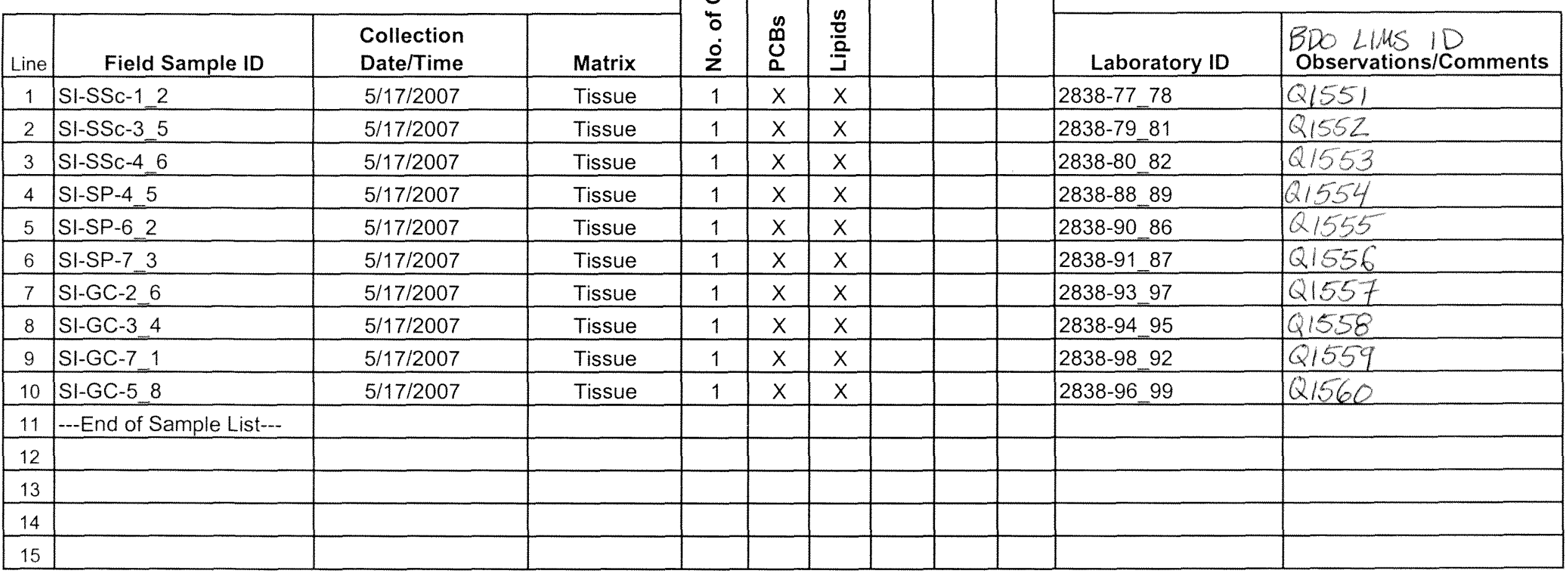

\begin{tabular}{|ll|}
\hline Relinquished By: & Company: \\
C. Suslick & MSL \\
\hline Signature/Printed Name & $\frac{1 / 7 / 08 \quad 1500}{\text { DaterTime }}$ \\
\hline
\end{tabular}

\begin{tabular}{|l|l|}
\hline Relinquished By: & Company: \\
\hline Signature/Printed Name & \\
\hline
\end{tabular}

\section{Battelle}

... Pulting Technology To Work

Pacific Northwest Division

Marine Sciences Laboratory

1529 West Sequim Bay Road

Sequim, Washington 98382
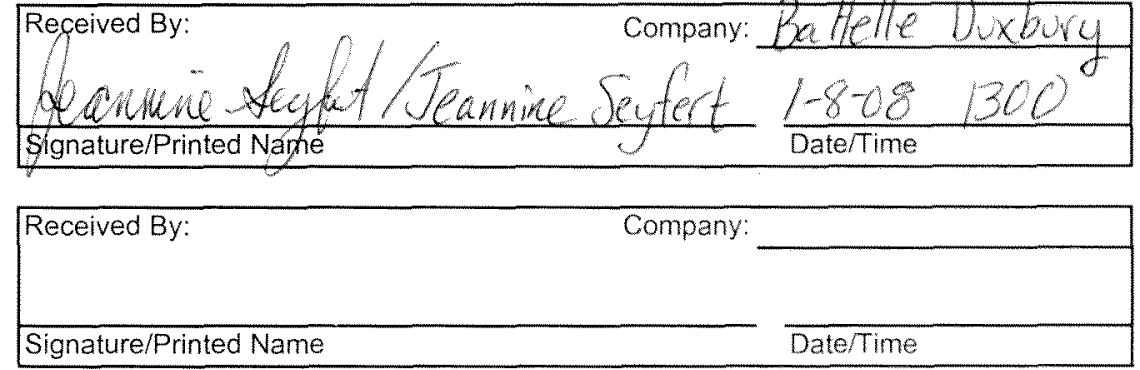
Project Number:

Received by: Seyfert, Jeannine

No. of Shipping Containers: 1

\section{SHIPMENT}

Method of Delivery:

COC Forms:

Commercial Carrier

Shipped with samples
Tracking Number:

$7914-7443-3536$

\section{No Forms}

\section{Cooler(s)/Box(es)}

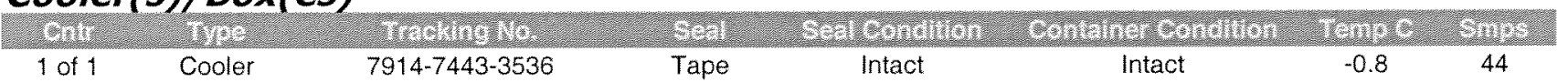

\section{Samples}

Sample Labels:

Container Seals:

Condition of Samples:
Sample labels agree with COC forms

Discrepancies (see Sample Custody Corrective Action Form)

$\square$ Tape $\square$ Custody Seals $\square$ Other Seals (See sample Log)

Seals intact for each shipping container

Seals broken (See sample log for impacted samples)

$\checkmark$ Sample containers intact

Sample containers broken/leaking (See Custody Corrective Action Form)

Temperature upon receipt $\left({ }^{\circ} \mathrm{C}\right): \quad-0.8 \quad$ Temperature Blank used $\square$ Yes $\square$ No

(Note: If temperature upon receipt differs from required conditions, see sample log comment field)

$\begin{array}{ll}\text { Samples Acidified: } & \square \text { Yes } \square \text { No } \square \text { Unknown } \\ \text { Initial pH 5-9?: } & \square \text { Yes } \square \text { No } \square \text { NA }\end{array}$

If no, individual sample adjustments on the Auxiliary Sample Receipt Form

Total Residual Chlorine Present?: $\square$ Yes $\square$ No $\square$ NA

If yes, individual sample adjustments on the Auxiliary Sample Receipt Form

Head Space $<1 \%$ in samples for water VOC analysis: $\square$ Yes $\square$ No $\square$ NA

Individual sample deviations noted on sample log

Samples Containers:

Samples returned in PC-grade jars: $\quad \overrightarrow{ }$ Yes $\square$ No $\square$ Unknown Lot No.: Unknown

$\begin{array}{llll}\text { Storage Location: } & \text { Chem North: Freezer - F0002 (Walk-in) } & \text { BDo IDs Assigned: } & \text { Q1609-Q1652 } \\ \text { Samples logged in by: } & \text { Seyfert, Jeannine } & \text { Date/Time: } & 01 / 12 / 200812: 00 \text { AM } \\ \text { Approved By: } & \text { Brackett, Roxanne } & \text { Approved On: 1/30/2008 3:42: } \\ \text { Authorized By: } & & \text { Authorized On: }\end{array}$




\section{Baftelle}

The Business of Innovation

ShpNo: SHP-080114-01

Remort contreane atctons

Battelle Project No: air and Dyes

Corrective Action No: 1 of 1

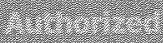

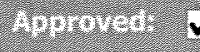

COC Client: Battelle MSL Sequim

COC Project: Sinclair and Dyes Tissue (ENVVEST-2008 Biota)

COC Date: $\quad$ 1/14/2008 9:18:00 AM

Description of Problem:

Explanation:

Temperature Receipt temperature outside of acceptability and The cooler was received at a temperature of -0.8 degrees Celcius which is warmer

Preservation than the -10 degree cut off temperature for frozen sample receipt.

Documentation of project manager notification

$\begin{array}{llll}\text { Sample Custodian } & \text { Seyfert, Jeannine } & \text { Date: } 1 / 14 / 2008 \text { 9:46:00 AM } \\ \text { Laboratory Manager: } & \text { Thorn, Jonathan } & \text { Date: } 1 / 29 / 2008 \text { 1:02:00 PM } \\ \text { Project Manager: } & \text { Peven-McCarthy, Carole } & \text { Date: } 1 / 29 / 2008 \text { 12:34:00 PM }\end{array}$

Documentation of client notification (should be completed by project manager within 24 hrs):

On

I contacted

at

Results of communication with client (Describe any corrective action directed by the client):

Sent PM a notification by email. Proceed with processing and analysis.

Date this form was received back to the custodian:

Reference Number: 


\section{Battelle}

Sample Recejpt Form Datils

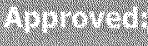

Project Number:

Client: Battelle MSL Sequim

Received by:

Seyfert, Jeannine

Date/Time Received: Saturday, January 12, 2008 12:00 AM

No. of Shipping Containers: 1

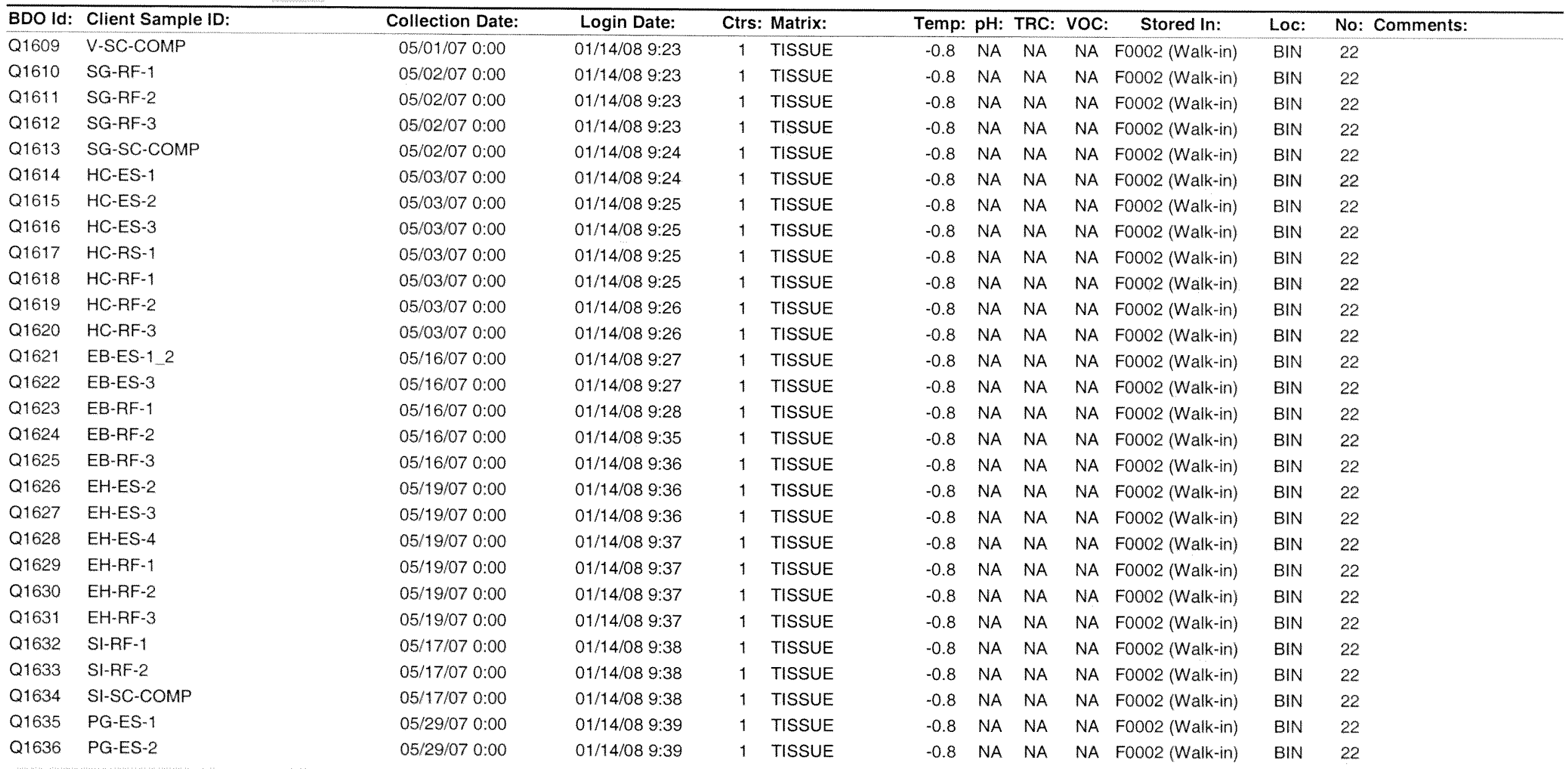




\section{Battelle}

ShpNo SHP 080114-01

The Business of Innovation

Battelle Project No:

Sample Receipt Form beths

Aprortate

\section{Project Number:}

\section{Received by:}

1

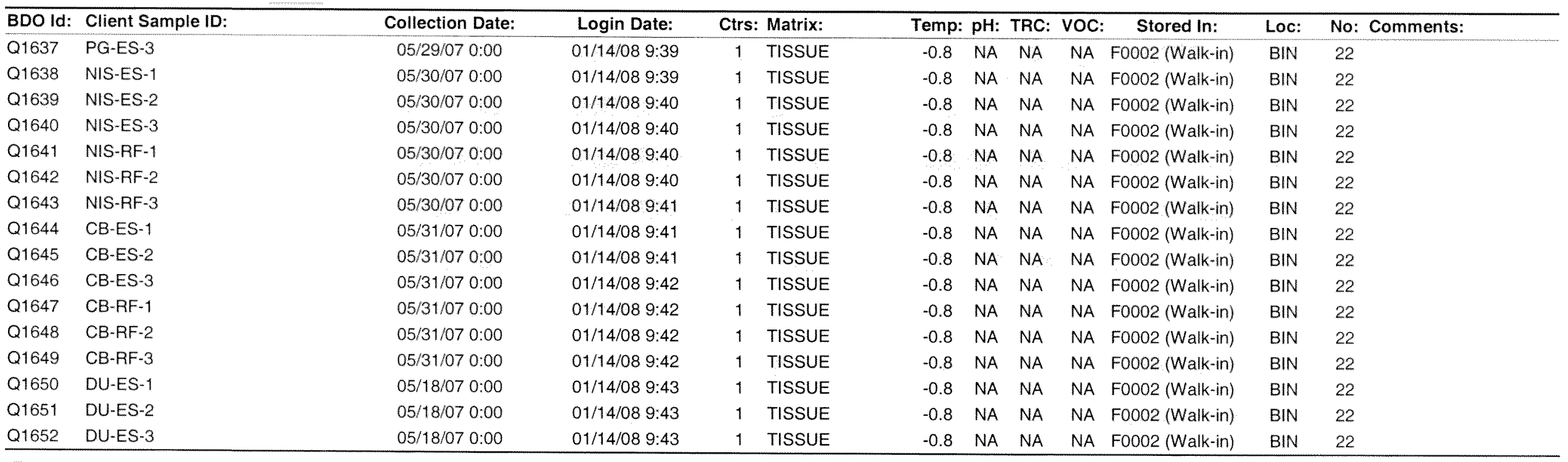

Total Samples:

Client: Battelle MSL Sequim

Date/Time Received: Saturday, January 12, 2008 12:00 AM

44 
SAMPLE CUSTODY RECORD

(SOP\#: MSL-A-001 \& MSL-A-002)

Date:

Project Name: ENVEST - 2008 Biota

Project Manager: J.Brandenberger

Phone Number: 360-681-4654

Shipment Method: Fedex Priority Overnight

Preservation: Frozen, on blue ice

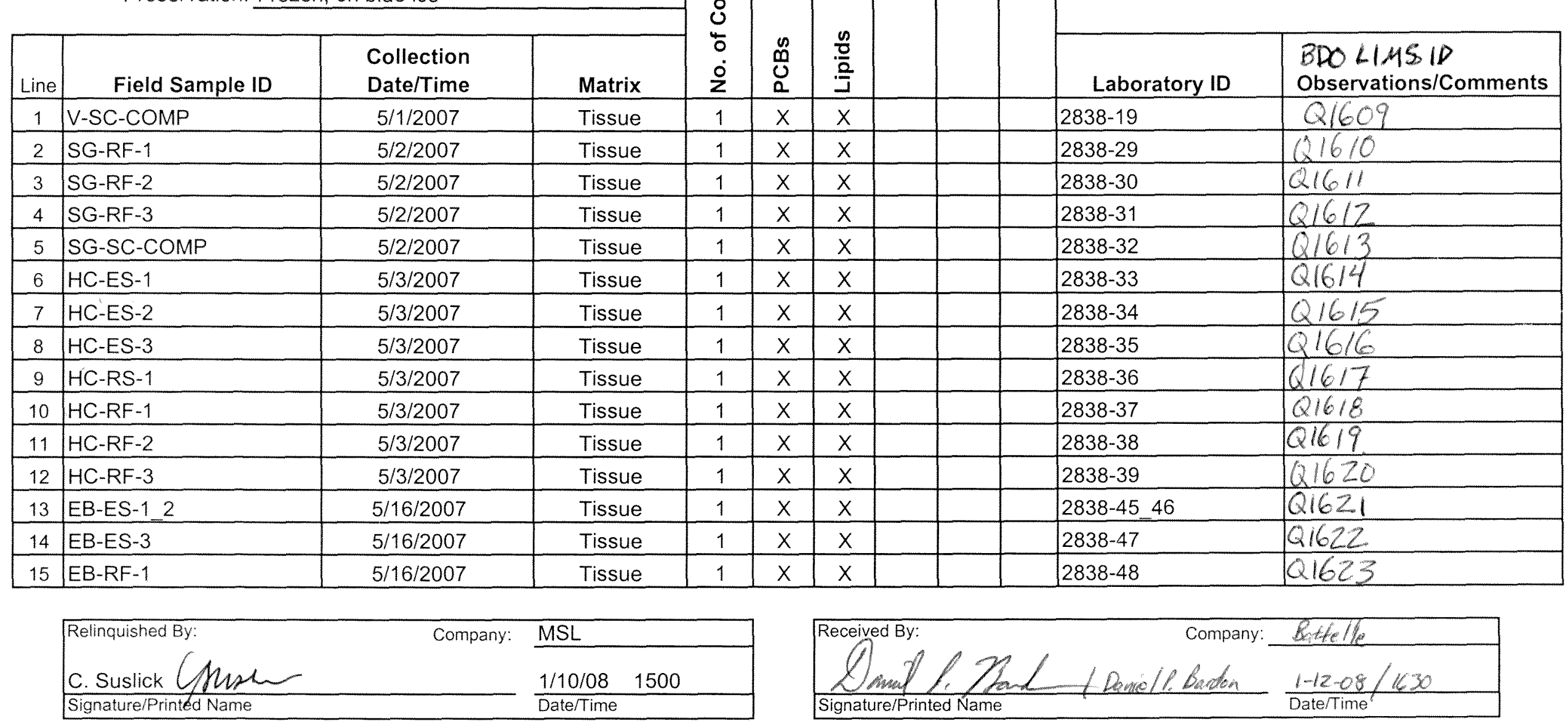

\section{Batte Balle}

.. Putting Technology To Work

Pacific Northwest Division

Marine Sciences Laboratory

1529 West Sequim Bay Road

Sequim, Washington 98382

\begin{tabular}{|ll|}
\hline Relinquished By: & Company: \\
\hline Signature/Printed Name & Date/Time \\
\hline
\end{tabular}

Rev. 020102000

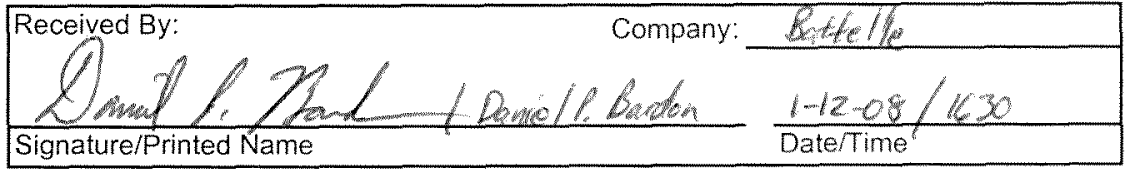

\begin{tabular}{|l|l|}
\hline Received By: & Company: \\
\hline Signafure/Printed Name & DaterTime \\
\hline
\end{tabular}




\section{SAMPLE CUSTODY RECORD}

(SOP\#: MSL-A-001 \& MSL-A-002)

Date:

$1 / 7 / 2008$

Project Name: ENVVEST - 2008 Biota Project Manager: J. Brandenberger

Phone Number: $360-681-4654$

Shipment Method: Fedex Priority Overnight

Preservation: Frozen, on blue ice

\begin{tabular}{|c|c|c|c|c|}
\hline Line & Field Sample ID & $\begin{array}{l}\text { Collection } \\
\text { Date/Time }\end{array}$ & Matrix & $\begin{array}{l}0 \\
\vdots \\
2\end{array}$ \\
\hline 1 & EB-RF-2 & $5 / 16 / 2007$ & Tissue & 1 \\
\hline 2 & EB-RF-3 & $5 / 16 / 2007$ & Tissue & 1 \\
\hline 3 & EH-ES-2 & $5 / 19 / 2007$ & Tissue & 1 \\
\hline 4 & EH-ES-3 & $5 / 19 / 2007$ & Tissue & 1 \\
\hline 5 & EH-ES-4 & $5 / 19 / 2007$ & Tissue & 1 \\
\hline 6 & EH-RF-1 & $5 / 19 / 2007$ & Tissue & 1 \\
\hline 7 & EH-RF-2 & $5 / 19 / 2007$ & Tissue & 1 \\
\hline 8 & EH-RF-3 & $5 / 19 / 2007$ & Tissue & 1 \\
\hline 9 & SI-RF-1 & $5 / 17 / 2007$ & Tissue & 1 \\
\hline 10 & SI-RF-2 & $5 / 17 / 2007$ & Tissue & 1 \\
\hline 11 & SI-SC-COMP & $5 / 17 / 2007$ & Tissue & 1 \\
\hline 12 & PG-ES-1 & $5 / 29 / 2007$ & Tissue & 1 \\
\hline 13 & PG-ES-2 & $5 / 29 / 2007$ & Tissue & 1 \\
\hline 14 & PG-ES-3 & $5 / 29 / 2007$ & Tissue & 1 \\
\hline 15 & NIS-ES-1 & $5 / 30 / 2007$ & Tissue & 1 \\
\hline & Relinquished By: & Company: & \multicolumn{2}{|l|}{ MSL } \\
\hline & C. Suslick & & \multicolumn{2}{|l|}{$1 / 10 / 08 \quad 1500$} \\
\hline & Signature/Printed Name & & \multicolumn{2}{|l|}{ Date/Time } \\
\hline
\end{tabular}

\begin{tabular}{|l|l|}
\hline Relinquished By: & Company: \\
\hline Signature/Printed Name & Date/Time \\
\hline
\end{tabular}

\section{Battelle}

... Putting Technology To Work

Pacific Northwest Division Marine Sciences Laboratory

1529 West Sequim Bay Road Sequim, Washington 98382

\begin{tabular}{|l|l|}
\hline \multicolumn{1}{|c|}{ Laboratory ID } & Observations/Comments \\
\hline $2838-49$ & Q 1624 \\
\hline $2838-50$ & $Q 1625$ \\
\hline $2838-52$ & $Q 1626$ \\
\hline $2838-53$ & $Q 1627$ \\
\hline $2838-54$ & $Q 1628$ \\
\hline $2838-55$ & $Q 1629$ \\
\hline $2838-56$ & $Q 1630$ \\
\hline $2838-57$ & $Q 1631$ \\
\hline $2838-83$ & $Q 1632$ \\
\hline $2838-84$ & $Q 1633$ \\
\hline $2838-100$ & $Q 1631$ \\
\hline $2838-101$ & $Q 1635$ \\
\hline $2838-102$ & $Q 1636$ \\
\hline $2838-103$ & $Q 1637$ \\
\hline $2838-104$ & $Q 1638$ \\
\hline
\end{tabular}

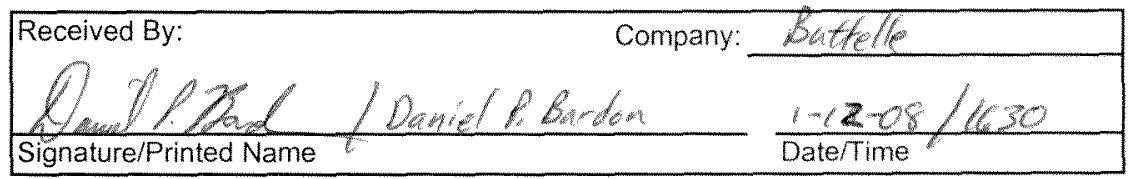

\begin{tabular}{|l|}
\hline Received By: Company: \\
\hline Signature/Printed Name \\
\hline
\end{tabular}




\section{SAMPLE CUSTODY RECORD}

(SOP\#: MSL-A-001 \& MSL-A-002)

Project Name: ENVVEST - 2008 Biota

Project Manager: J. Brandenberger

Phone Number: 360-681-4654

Shipment Method: Fedex Priority Overnight

Preservation: Frozen, on blue ice

\begin{tabular}{|ll|}
\hline Relinquished By: & Company: \\
\hline Signature/Printed Name & Date/Time \\
\hline
\end{tabular}

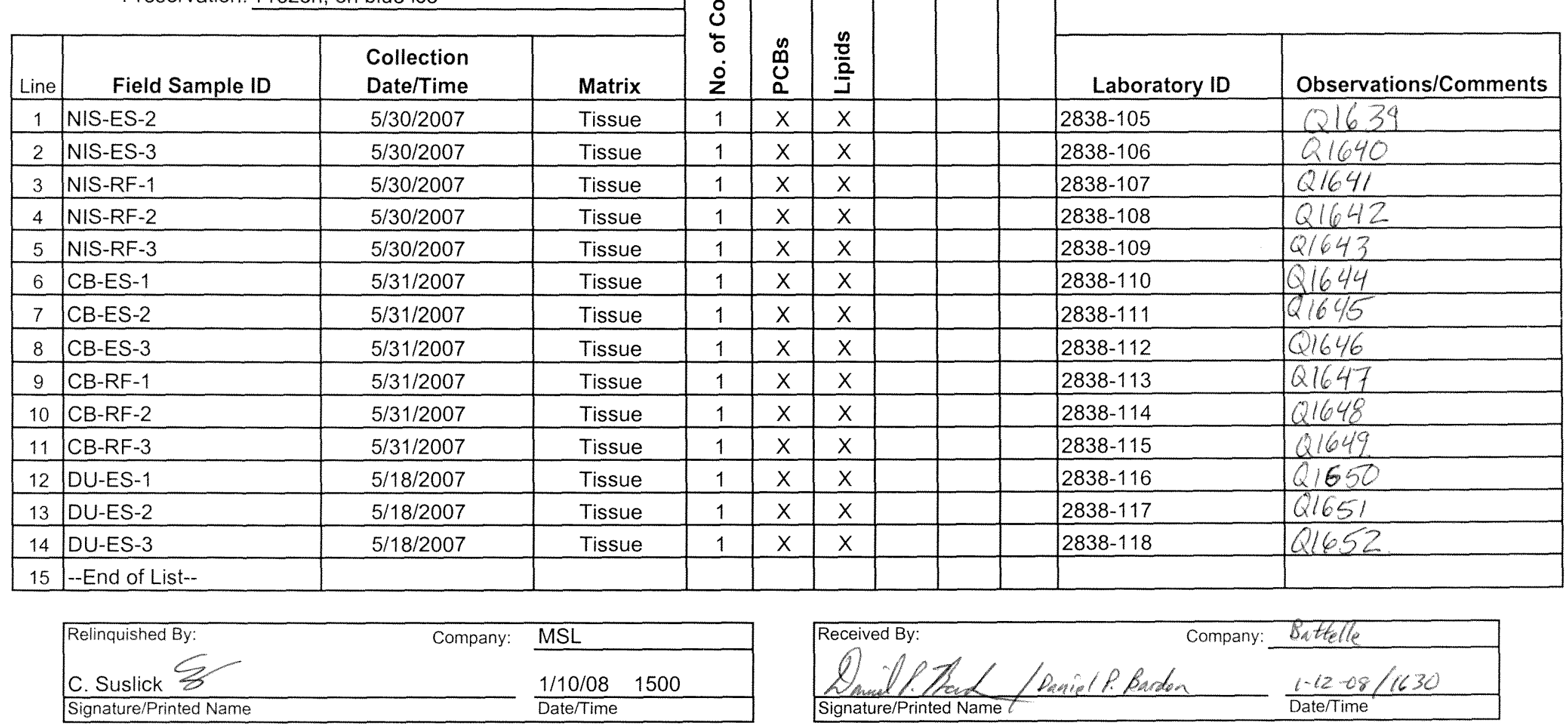

\section{Baftelle}

... Putting Technology To Work

Pacific Northwest Division

Marine Sciences Laboratory

1529 West Sequim Bay Road

Sequim, Washington 98382

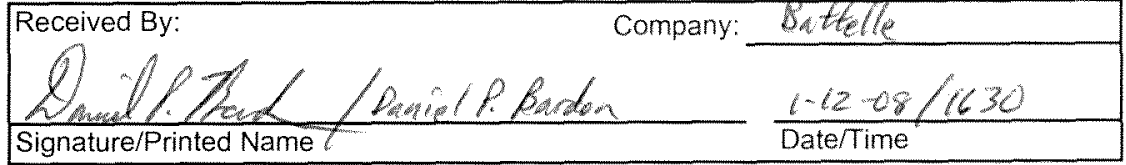

\begin{tabular}{|l|l|}
\hline Received By: & Company: \\
\hline Signature/Printed Name & Date/Time \\
\hline
\end{tabular}


The Business of Innovation

Battelle Project No:

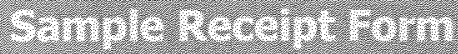

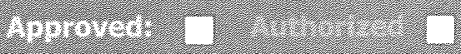

Project Number: $\quad$ G006010

Received by: Brackett, Roxanne

No. of Shipping Containers:

1

\section{SHIPMENT}

Method of Delivery: Commercial Carrier

coc Forms:

Shipped with samples
Tracking Number: 798355564751

\section{No Forms}

\section{Cooler(s)/Box(es)}

\begin{tabular}{|c|c|c|c|c|c|c|c|}
\hline (3) & the & mathiture & (x) & 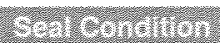 & 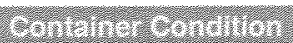 & 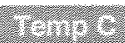 & $=x^{2}=$ \\
\hline 1 of 1 & Cooler & 798355564751 & Tape & Intact & Intact & 1.2 & 22 \\
\hline
\end{tabular}

\section{Samples}

Sample Labels:

Container Seals:

Condition of Samples:
Sample labels agree with COC forms

Discrepancies (see Sample Custody Corrective Action Form) $\checkmark$ Seals intact for each shipping container

Seals broken (See sample log for impacted samples)

\section{Condition of Samples:}

$\checkmark$ Sample containers intact

Sample containers broken/leaking (See Custody Corrective Action Form)

Temperature upon receipt $\left({ }^{\circ} \mathrm{C}\right): \quad 1.2 \quad$ Temperature Blank used $\square$ Yes $\square$ No

(Note: If temperature upon receipt differs from required conditions, see sample log comment field)

Samples Acidified:
$\begin{aligned} & \square \text { Yes } \square \text { No } \square \text { Unknown } \\ & \text { Initial pH 5-9?: } \\ & \text { If no, individual sample adjustments on the Auxiliary Sample Receipt Form }\end{aligned}$

Total Residual Chlorine Present?: $\square$ Yes $\square$ No $\square$ NA

If yes, individual sample adjustments on the Auxiliary Sample Receipt Form

Head Space $<1 \%$ in samples for water VOC analysis: $\square$ Yes $\square$ No $\checkmark$ NA Individual sample deviations noted on sample log

Samples Containers:

Samples returned in PC-grade jars: $\quad \checkmark$ Yes $\square$ No $\square$ Unknown /Lot No.: Unknown

Storage Location:

Samples logged in by:

Approved By:

Authorized By:

\section{Chem North: Freezer - F0002 (Walk-in)}

Brackett, Roxanne

BDO IDs Assigned: $\quad$ Q1677-Q1698

Date/Time: $\quad 01 / 22 / 2008$ 12:00 AM

Approved On:

Authorized On: 


\section{SAMPLE CUSTODY RECORD}

(SOP\#: MSL-A-001 \& MSL-A-002)

Date:

$1 / 21 / 2008$

Project Name: ENVVEST - 2008 Biota

Project Manager: J. Brandenberger

Phone Number: $360-681-4654$

Shipment Method: Fedex Priority Overnight

Preservation: Frozen, on blue ice

\begin{tabular}{|c|l|l|l|}
\hline Line & \multicolumn{1}{|c|}{ Field Sample ID } & $\begin{array}{c}\text { Collection } \\
\text { Date/Time }\end{array}$ & Matrix \\
\hline 1 & DF-WBWC-1 & & Tissue \\
\hline 2 & DF-WBWC-2 & & Tissue \\
\hline 3 & DF-WBWC-3 & & Tissue \\
\hline 4 & DF-WBWC-4 & & Tissue \\
\hline 5 & DF-WBWC-5 & & Tissue \\
\hline 6 & DF-WBWC-6 & & Tissue \\
\hline 7 & DF-SWC-4 & & Tissue \\
\hline 8 & -End of List-- & & \\
\hline 9 & & & \\
\hline 10 & & & \\
\hline 11 & & & \\
\hline 12 & & & \\
\hline 13 & & & \\
\hline 14 & & & \\
\hline 15 & & & \\
\hline
\end{tabular}

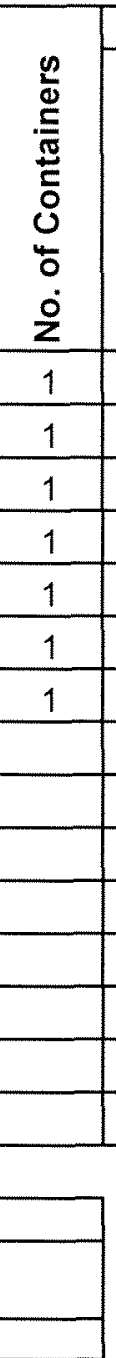

\begin{tabular}{|lll|}
\hline Relinquished By: & Company: & MSL \\
C. Suslick & $\frac{1 / 21 / 08}{1500}$ \\
\hline Signature/Printed Name & Date/Time \\
\hline
\end{tabular}

\begin{tabular}{|ll|}
\hline Relinquished By: & Company: \\
\hline Signature/Printed Name & Date/Time \\
\hline
\end{tabular}

\section{sattelle}

.. Putting Technology To Work

Pacific Northwest Division

Marine Sciences Laboratory

1529 West Sequim Bay Road

Sequim, Washington 98382

\begin{tabular}{|c|c|}
\hline Laboratory ID & $\begin{array}{l}\text { Batte } / L \text {, } 7 \\
\text { observations/Comments }\end{array}$ \\
\hline $2838-177$ & $Q 11892$ \\
\hline $2838-178$ & 91093 \\
\hline $2838-179$ & 01694 \\
\hline $2838-180$ & 01695 \\
\hline $2838-181$ & 01636 \\
\hline $2838-182$ & $6160^{2} 7$ \\
\hline $2838-183$ & 61698 \\
\hline & \\
\hline & \\
\hline & \\
\hline & \\
\hline & \\
\hline & \\
\hline & 22 Containers total \\
\hline
\end{tabular}

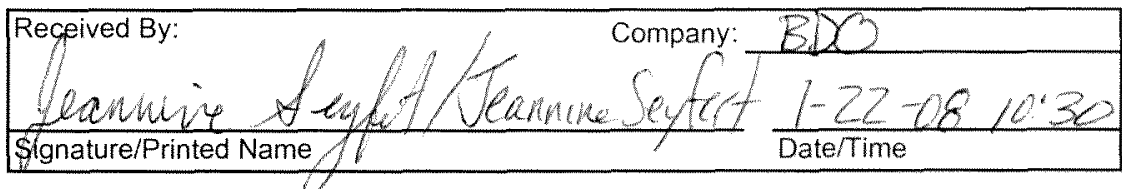

\begin{tabular}{|ll|}
\hline Received By: & Company: \\
\hline Signature/Printed Name & Date/Time \\
\hline
\end{tabular}


SAMPLE CUSTODY RECORD

(SOPH: MSL-A-001 \& MSL-A.002)

Date

$1 / 21 / 2008$

Project Name: ENVVEST - 2008 Biota

Project Manager: J.Brandenberger

Phone Number: 360-681-4654

Shipment Method: Fedex Priority Overnight

Preservation: Frozen, on blue ice

\begin{tabular}{|c|c|c|c|c|}
\hline Line & Field Sample ID & $\begin{array}{l}\text { Collection } \\
\text { Date/Time }\end{array}$ & Matrix & $\begin{array}{l}0 \\
0 \\
2\end{array}$ \\
\hline 1 & DF-EM-1 & $9 / 18 / 2007$ & Tissue & 1 \\
\hline 2 & DF-EM-2 & $9 / 18 / 2007$ & Tissue & 1 \\
\hline 3 & DF-EM-4 & $9 / 18 / 2007$ & Tissue & 1 \\
\hline 4 & DF-EM-6 & $9 / 18 / 2007$ & Tissue & 1 \\
\hline 5 & $D F-L V-1$ & $9 / 18 / 2007$ & Tissue & 1 \\
\hline 6 & DF-LV-2 & $9 / 18 / 2007$ & Tissue & 1 \\
\hline 7 & DF-LV-4 & $9 / 18 / 2007$ & Tissue & 1 \\
\hline 8 & $D F-L V-6$ & $9 / 18 / 2007$ & Tissue & 1 \\
\hline 9 & DF-DIG-1 & $9 / 18 / 2007$ & Tissue & 1 \\
\hline 10 & DF-DIG-2 & $9 / 18 / 2007$ & Tissue & 1 \\
\hline 11 & DF-DIG-4 & $9 / 18 / 2007$ & Tissue & 1 \\
\hline 12 & DF-DIG-6 & $9 / 18 / 2007$ & Tissue & 1 \\
\hline 13 & DF-SWC-1 & $9 / 18 / 2007$ & Tissue & 1 \\
\hline 14 & DF-SWC-2 & $9 / 18 / 2007$ & Tissue & 1 \\
\hline 15 & DF-SWC-6 & $9 / 18 / 2007$ & Tissue & 1 \\
\hline & Relinquished By: & Company: & \multicolumn{2}{|l|}{ MSL } \\
\hline & C. Suslick & & \multicolumn{2}{|l|}{$1 / 21 / 08 \quad 1500$} \\
\hline & Signature/Printed Name & & \multicolumn{2}{|l|}{ Date/Time } \\
\hline
\end{tabular}

\begin{tabular}{|ll|}
\hline Relinquished By: & Company: \\
\hline Signature/Printed Name & \\
\hline
\end{tabular}

\section{Batlelle}

Putting Technology To Work

Pacific Northwest Division

Marine Sciences Laboratory 1529 West Sequim Bay Road

Sequim, Washington 98382

\begin{tabular}{|l|l|}
\hline \multicolumn{1}{|c|}{ Laboratory ID } & Observations/Comments \\
\hline $2838-126$ & \\
\hline $2838-127$ & \\
\hline $2838-129$ & \\
\hline $2838-131$ & \\
\hline $2838-132$ & \\
\hline $2838-133$ & \\
\hline $2838-135$ & \\
\hline $2838-137$ & \\
\hline $2838-138$ & \\
\hline $2838-139$ & \\
\hline $2838-141$ & \\
\hline $2838-143$ & \\
\hline $2838-174$ & \\
\hline $2838-175$ & \\
\hline $2838-176$ & \\
\hline
\end{tabular}

Signature/Printed Name

\begin{tabular}{|l|l|}
\hline Received By: & Company: \\
\hline Signature/Printed Name & Date/Time \\
\hline
\end{tabular}




\section{SAMPLE CUSTODY RECORD}

(SOP\#: MSL-A-001 \& MSL-A-002)

Project Name: ENVVEST - 2008 Biota

Project Manager: J.Brandenberger

Phone Number: $360-681-4654$

Shipment Method: Fedex Priority Overnight

Preservation: Frozen, on blue ice

\begin{tabular}{|c|c|c|c|c|}
\hline Line & Field Sample ID & $\begin{array}{l}\text { Collection } \\
\text { Date/Time }\end{array}$ & Matrix & i \\
\hline 1 & DF-WBWC-1 & $9 / 18 / 2007$ & Tissue & 1 \\
\hline 2 & DF-WBWC-2 & $9 / 18 / 2007$ & Tissue & 1 \\
\hline 3 & DF-WBWC-3 & $9 / 18 / 2007$ & Tissue & 1 \\
\hline 4 & DF-WBWC-4 & $9 / 18 / 2007$ & Tissue & 1 \\
\hline 5 & DF-WBWC-5 & $9 / 18 / 2007$ & Tissue & 1 \\
\hline 6 & DF-WBWC-6 & $9 / 18 / 2007$ & Tissue & 1 \\
\hline 7 & DF-SWC-4 & $9 / 18 / 2007$ & Tissue & 1 \\
\hline 8 & -End of List-- & & & \\
\hline 9 & & & & \\
\hline 10 & & & & \\
\hline 11 & & & & \\
\hline 12 & & & & \\
\hline 13 & & & & \\
\hline 14 & & & & \\
\hline 15 & & & & \\
\hline & Relinquished By: & Company: & MSL & \\
\hline & C. Suslick & & $1 / 21 / 08$ & \\
\hline & Signature/Printed Name & & Date/Time & \\
\hline
\end{tabular}

\begin{tabular}{|ll|}
\hline Relinquished By: & Company: \\
\hline Signature/Printed Name & \\
\hline
\end{tabular}

Date:

\section{saltelle}

Pulling Technology To Work

Pacific Northwest Division

Marine Sciences Laboratory

1529 West Sequim Bay Road

Sequim, Washington 98382

\begin{tabular}{|l|l|}
\hline \multicolumn{1}{|c|}{ Laboratory ID } & Observations/Comments \\
\hline $2838-177$ & \\
\hline $2838-178$ & \\
\hline $2838-179$ & \\
\hline $2838-180$ & \\
\hline $2838-181$ & \\
\hline $2838-182$ & \\
\hline $2838-183$ & \\
\hline & \\
\hline & \\
\hline & \\
\hline & \\
\hline
\end{tabular}

\begin{tabular}{|lc|}
\hline Received By: & Company: \\
\hline Signature/Printed Name & Date/Time \\
\hline Received By: & Company: \\
\hline Signature/Printed Name & \\
\hline
\end{tabular}




\section{SAMPLE CUSTODY RECORD}

(SOP\#: MSL-A-001 \& MSL-A-002)

Date:

$1 / 21 / 2008$

Project Name: ENVVEST - 2008 Biota

Project Manager: J.Brandenberger

Phone Number: 360-681-4654

Shipment Method: Fedex Priority Overnight

Preservation: Frozen, on blue ice

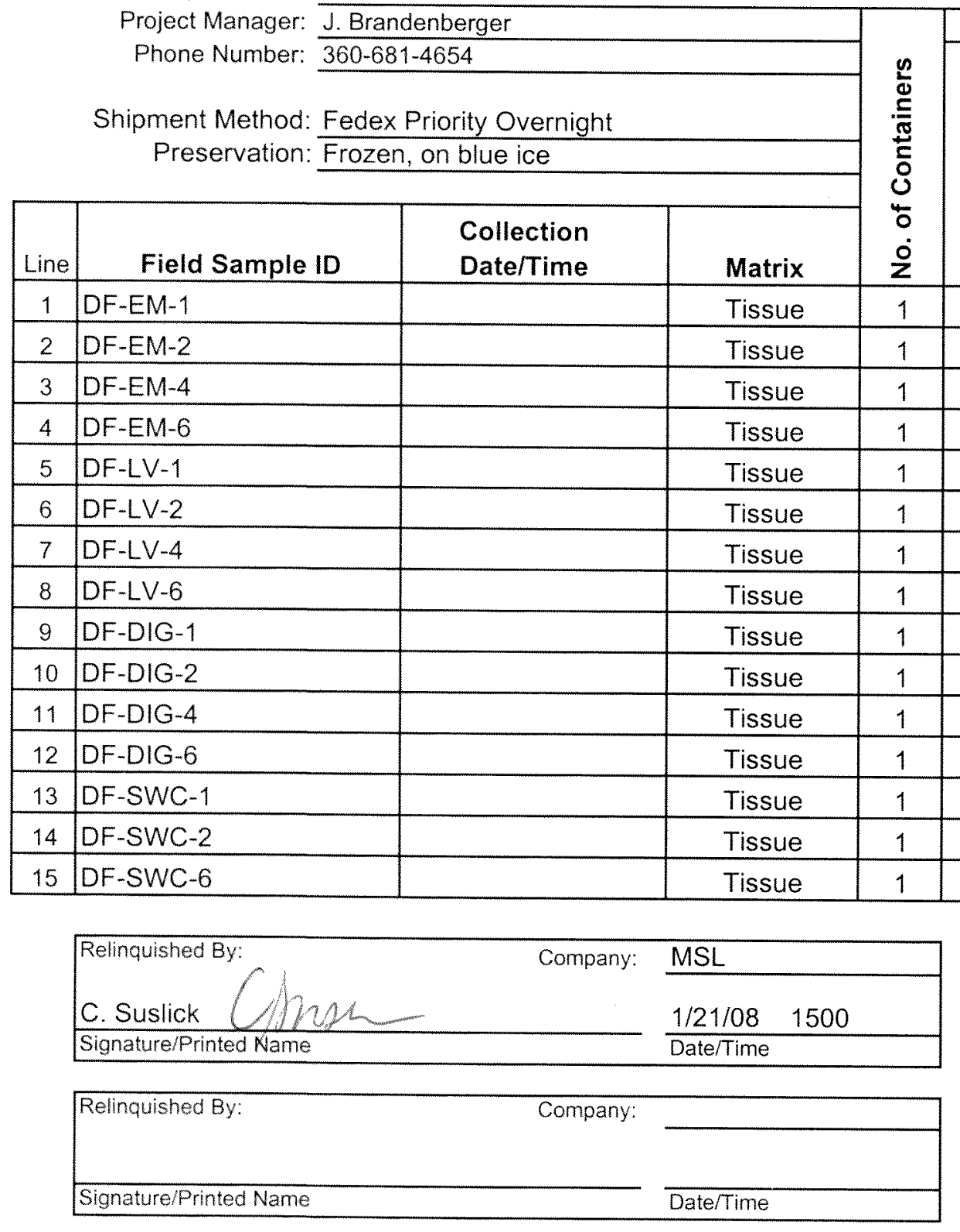

$x$

\section{柴:Batfelle}

... Putting Technology To Work

Pacific Northwest Division Marine Sciences Laboratory 1529 West Sequim Bay Road Sequim, Washington 98382

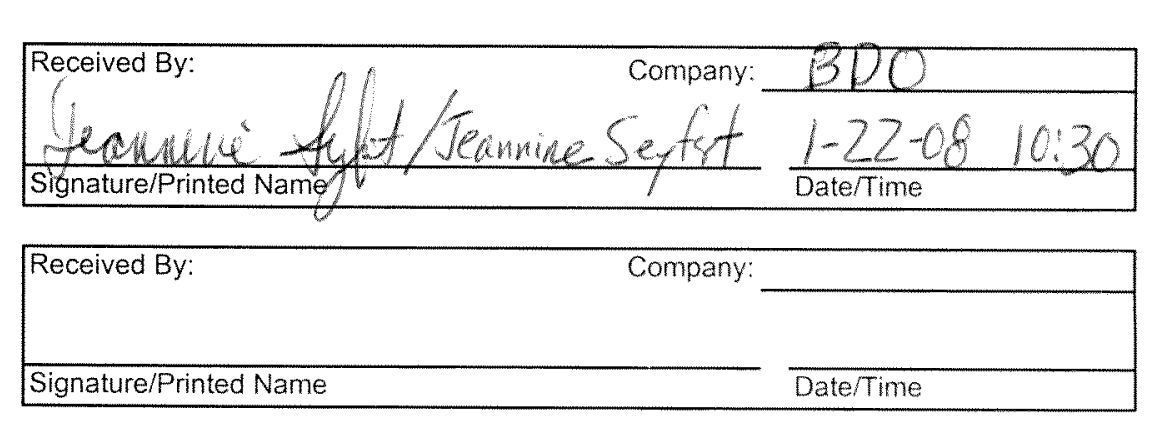

\begin{tabular}{|c|c|c|}
\hline Received By: & Company: & \\
\hline Signature/Printed Name & & Date/Time \\
\hline
\end{tabular}

\begin{tabular}{|l|c|}
\hline \multicolumn{1}{|c|}{ Laboratory ID } & $\begin{array}{c}\text { Battele } D D \\
\text { Observations/Comments }\end{array}$ \\
\hline $2838-126$ & Q1677 \\
\hline $2838-127$ & Q 1678 \\
\hline $2838-129$ & $Q 1679$ \\
\hline $2838-131$ & $Q 1680$ \\
\hline $2838-132$ & $Q 1681$ \\
\hline $2838-133$ & $Q 1682$ \\
\hline $2838-135$ & $Q 1683$ \\
\hline $2838-137$ & $Q 1684$ \\
\hline $2838-138$ & $Q 1685$ \\
\hline $2838-139$ & $Q 1686$ \\
\hline $2838-141$ & $Q 1687$ \\
\hline $2838-143$ & $Q 1688$ \\
\hline $2838-174$ & $Q 1689$ \\
\hline $2838-175$ & $Q 1690$ \\
\hline $2838-176$ & $Q 1691$ \\
\hline & \\
\hline
\end{tabular}




\section{Battelle}

Project Number: $\quad$ G006010

Received by: Brackett, Roxanne
Client: Sindair and Dyes (ENVEST - 2008 Biota)

Date/Time Received: Tuesday, January 22, 2008 12:00 AM

No. of Shipping Containers: 1

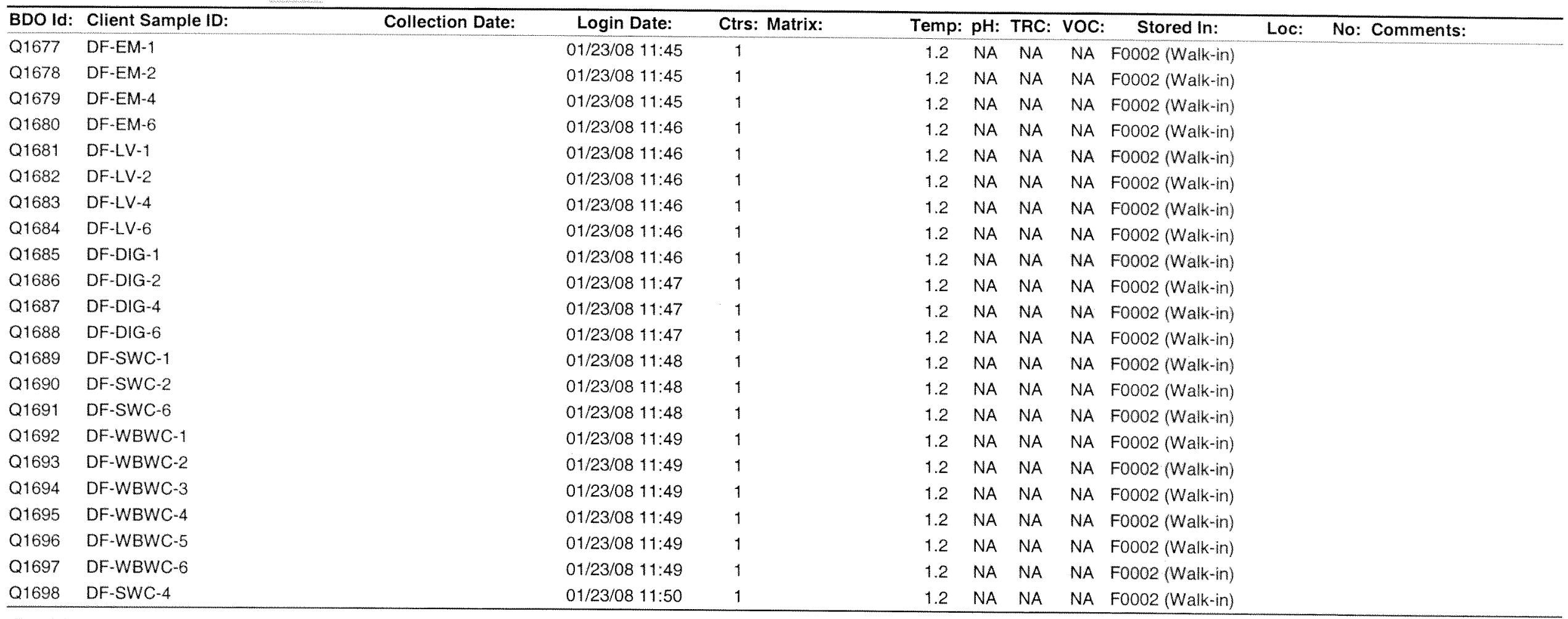

Total Samples: 


\section{References}




\section{References}

Benstead, J. P., J. G. March, B. Fry, K. C. Ewel and C. M. Pringle (2006). "Testing isosource: Stable isotope analysis of a tropical fishery with diverse organic matter sources." Ecology 87(2): 326-333.

Brandenberger, J.M. C. Suslick, C., and R.K. Johnston. 2003. Sinclair and Dyes Inlet TMDL Study: Biological Sampling and Analysis for Metals and PCBs. Pacific Northwest National Laboratory Data Report 43043.

Brandenberger, J.M. C.R. Suslick, C., and R.K. Johnston. 2006. Biological Sampling and Analysis in Sinclair and Dyes Inlets, Washington: Chemical Analyses for 2005 Puget Sound Biota Study. Pacific Northwest National Laboratory Report PNNL-15834.

Brandes, J. A. a. A. H. D. (1997). "Isotopic fractionation of oxygen and nitrogen in coastal marine sediments." Geochim. Cosmochim. Acta 61: 1793-1801.

Ecology (Washington State Department of Ecology) 1998. Final 1998 Section 303(d) List -WRIA 15: Kitsap Watershed. Available online: http://www.ecy.wa.gov/programs/wq/303d/1998/1998_by_wrias.html

Fantle, M. S., A. I. Dittel, S. M. Schwalm, C. E. Epifanio and M. L. Fogel (1999). "A food web analysis of the juvenile blue crab, Callinectes sapidus, using stable isotopes in whole animals and individual amino acids." Oecologia 120(3): 416-426.

Fry, B. (1991). "Stable isotope diagrams of fresh-water food webs." Ecology 72(6): 2293-2297.

Johnston, R.K., J.M. Brandenberger, E.A. Crecelius, S. Steinert, M.H. Salazar, and S.M. Salazar. 2005. Biological Sampling and Analysis in Sinclair and Dyes Inlets, Washington 2005 Quality Assurance Project Plan. Prepared by Puget Sound Naval Shipyard \& Intermediate Maintenance Facility Project ENVVEST.

Johnston RK, DE Leisle, JM Brandenberger, SA Steinert, M Salazar, and SM Salazar. 2007. Contaminate Residues in Demersal Fish, Invetebrates, and Deployed Mussels in Selected Areas of The Puget Sound, WA . 2007 Georgia Basin Puget Sound Conference, Vancouver, BC, Canada on March 29, 2007. Manuscript published in proceedings PNNL-SA-55152.

Steinert, S, R.K. Johnston, B.M. Davidson, M.H. Salazar, S.M. Salazar, and J.M. Brandenberger. 2006. Monitoring DNA Damage in Caged Mussels Deployed in Sinclair and Dyes Inlets, Puget Sound, Washington. PNNL-SA-50128. Presented at the 2006 Annual Pacific Northwest Society of Environmental Toxicology and Chemistry (SETAC) North America $27^{\text {th }}$ Annual Meeting, 5-7 November, Montreal, Quebec, Canada.

Salazar MH, SM Salazar, RK Johnston, B Davidson, SA Steinert, and JM Brandenberger. 2006. "Using Caged Mussels to Characterize Exposure and Effects Over Small Spatial Scales in Sinclair/Dyes Inlet, WA." Presented at PNW-SETAC 15th Annual Meeting, Port Townsend, WA. PNNL-SA-48869.

WDFW (Washington State Department of Fish and Wildlife). 2003. Washington State Department of Fish and Wildlife. Available online: http://www.wdfw.wa.gov/fish/psamp/index.htm 